WHOI-89-51

\title{
CTD Observations in the Great South Channel During the South Channel Ocean Productivity EXperiment, SCOPEX, May - June 1989
}

\author{
by \\ Richard Limeburner and Robert C. Beardsley \\ Woods Hole Oceanographic Institution \\ Woods Hole, Massachusetts 02543
}

November 1989

\section{Technical Report}

Funding was provided by the National Science Foundation through Grant Number 87-13988

Reproduction in whole or in part is permitted for any purpose of the United States Government. This report should be cited as:

Woods Hole Oceanog. Inst. Tech. Rept., WHOI-89-51.

Approved for publication; distribution unlimited.

Approved for Distribution:

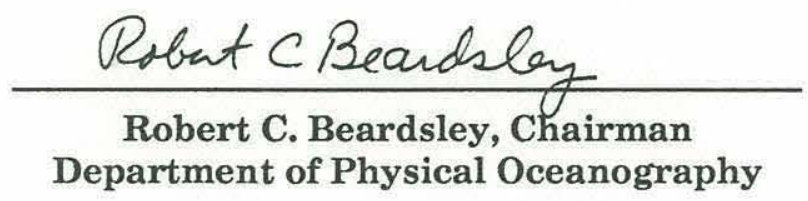




\title{
CTD OBSERVATIONS IN THE GREAT SOUTH CHANNEL DURING THE SOUTH CHANNEL OCEAN PRODUCTIVITY EXPERIMENT, SCOPEX, MAY - JUNE 1989
}

\begin{abstract}
CTD and acoustic Doppler current profiler (ADCP) observations were made in the Great South Channel (GSC) off the New England coast during R/V Endeavor cruise EN196 May 18 to June 12, 1989 as part of the South Channel Ocean Productivity EXperiment (SCOPEX). These observations were obtained using several sampling plans - a series of small-scale surveys in support of biological sampling and a large-scale survey of five cross-channel transects extending from Nantucket Shoals and the coast of Cape Cod to Georges Bank. The maximum sampling depth at each station was within a few meters of the bottom.

The primary objectives of the hydrographic measurement program were to a) observe and characterize the temperature, salinity, density, oxygen, fluorescence and light transmission fields and their spatial variability in the Great South Channel off the New England coast, (b) resolve the low salinity surface plume-like structure usually observed east of Cape Cod in late spring, (c) define the front or boundary between the vertically well-mixed water over Nantucket Shoals, the GSC, Georges Bank, and the stratified water in the deeper southwestern Gulf of Maine, and (d) characterize water properties in regions of enhanced biological productivity. This report presents a summary in graphic and tabular form of the hydrographic observations made during cruise EN196 on the R/V Endeavor.
\end{abstract}




\section{Table of Contents}

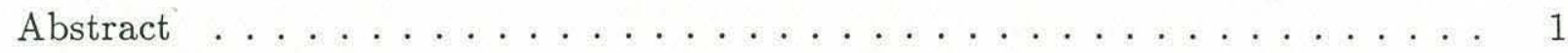

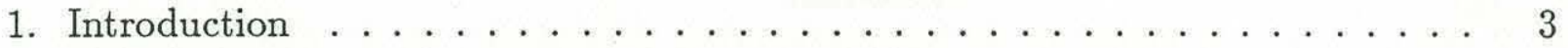

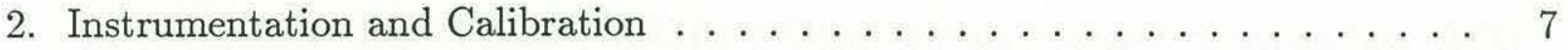

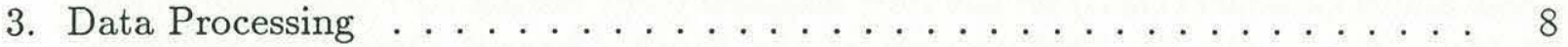

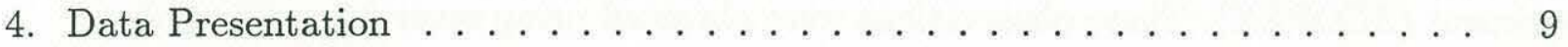

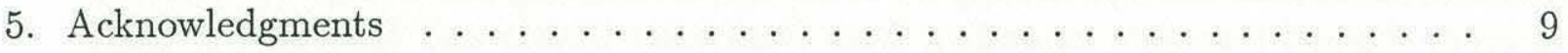

6. References............................... 10

7. Graphic Description of CTD Data . . . . . . . . . . . . . 11

A. Large-Scale Survey - Jun 7-12, 1989 - Stations 149 to 207 . . . . . 11

1. Station Locations . . . . . . . . . . . . . . . . 12

2. Section Locations . . . . . . . . . . . . . . 13

3. $\mathrm{T} / \mathrm{S}$ and Vertical Sections .................... 14

4. Surface Horizontal Sections . . . . . . . . . . . . . 34

8. Station Listings and Profiles ... . . . . . . . . . . . . 53 


\section{Introduction}

The South Channel Ocean Productivity EXperiment (SCOPEX) is a multidisciplinary and multi-institutional program to investigate springtime aggregations of right whales near the Great South Channel (GSC) off the New England coast, and the interactions between these whales and their main prey, the copepod Calanus finmarchicus. The right whale is an endangered species, with only a few hundred individuals left in the northwestern Atlantic population. Each spring, for at least the last ten years, almost the entire population of right whales has been present for about a month in a small area at the southern end of the Gulf of Maine (GOM) just north of the GSC. The SCOPEX program seeks to understand this phenomenon, as well as the broader issues of ecosystem "hot spots" and the implications of patchiness for trophic transfer. The purpose of the SCOPEX 1989 field program was to study whale/plankton/environment interactions, especially whale behavior in relation to copepod distributions, copepod aggregations and their structure and causes, primary and secondary production, and the role of currents and hydrography in forming and maintaining the aggregations.

Our basic underlying hypothesis is that right whales congregate in this limited area each spring because of unusually dense concentrations of their copepod food. Three sub-hypotheses concerning the causes of the copepod aggregations have been developed. The advection hypothesis states that the interaction between the water flow (especially the regional circulation and vertical stratification) and the behavior of the copepods (especially their vertical migration and depth preferences) passively concentrates the copepods. The productivity hypothesis states that the high copepod abundance is caused by a regional elevation of primary production and a response up the food chain. The innate behavior hypothesis is that a species-specific social behavior, such as swarming, creates the copepod aggregations. These hypotheses are not mutually exclusive, and it seems likely that elements of each may contribute to the observed phenomenon in the GSC. One particular mechanism suggested by the 1989 field observations is that the subduction of surface water carrying copepods beneath another water mass also containing copepods combined with a tendency for the copepods to migrate vertically back toward the surface can lead to high copepod concentrations near the surface front.

In order to examine these hypotheses, a coordinated field program involving two research vessels and an aircraft was conducted in the Great South Channel region during May and June, 1989. The aircraft and the R/V Marlin were used primarily to locate right whales in the GSC area and study their behavior while the R/V Endeavor was used to obtain environmental measurements both near the whales and on larger scales 
within the GSC. These measurements included hydrographic and current profiling with a CTD and an acoustic Doppler current profiler (ADCP) respectively, acoustic profiling for zooplankton distribution and biomass with a towed 150 and $200 \mathrm{KHz}$ acoustic fin, and biological sampling with MOCNESS (a multiple opening and closing environmental sampling system) and other towed nets.

We present here in graphic and tabular form a preliminary analysis of the CTD observations made in the GSC on board the R/V Endeavor during cruise EN196 May 18 to June 12,1989 . This cruise was split into three legs and the hydrographic observations were obtained using a wide variety of sampling plans, ranging from single stations in support of biological sampling to both shallow and deep tow-yos near feeding whales to one quasi-synoptic regional CTD/ADCP survey. A total of 207 CTD stations was made on EN196, and the following station summary is intended to provide a brief description of the sequence of CTD sampling during the cruise. Vertical and horizontal sections of water properties obtained from the regional CTD survey on Leg 3 June 7 to 12 are shown in Section 7. Listings of the CTD observations at standard depths for all stations are given in Section 8.

\section{CTD STATION LISTING SUMMARY}

\section{Leg 1 - May $18-30,1989$}

Stations 1-23 - May 18-21. Initial mesoscale survey of the south-central and western flank of the GSC with an acoustic fin to determine if a surface freshwater plume was present and to map the initial distribution of zooplankton.

Stations 24-26 - May 21. Short CTD/MOCNESS section east of Cape Cod through the freshest part of the surface freshwater plume.

Stations 27-37 - May 22. Small-scale acoustic survey (rectangular grid with CTD stations at corners) in the southeastern GSC in the general vicinity of concurrent right whale sightings.

Stations 38-46 - May 22-23. 24 hour time series (diel station 1 ) to study variability in the center of the whale sighting area in the southeastern GSC. CTD stations every $3 \mathrm{hrs}$ at the central site, some additional CTD stations made in connection with MOCNESS tows. CTD station 45 (Tow-yo 1) includes 22 casts made between the surface and $50 \mathrm{~m}$. 
Stations 47-57 - May 24. Combined CTD/MOCNESS east/west transect extending through the right whale area toward Georges Bank to examine day/night variations.

Stations 58-76 - May 24-25. Small-scale acoustic survey (rectangular grid with CTD stations at corners) in southeastern GSC.

Stations 77-86 - May 26. Combined CTD/MOCNESS transect across southern GSC through a tidal front onto Nantucket Shoals.

Stations 87-97 - May 26-27. $24 \mathrm{hr}$ time series (diel station 2) to study variability in south-central GSC. CTD stations made every $3 \mathrm{hrs}$ at central site with associated net tows, and some additional CTD stations made with MOCNESS tows. CTD station 93 (Tow-yo 2) includes 12 casts made between the surface and $60 \mathrm{~m}$. CTD station 95 (Towyo 3 ) includes 6 casts made between 70 and near bottom and 3 casts between surface and $60 \mathrm{~m}$.

Stations 98-104 - May 28. Combined CTD/MOCNESS cross-isobath transect on the western flank of GSC through tidal front.

Stations 104-111 - May 28-29. Shortened time series (diel station 3) of three hourly CTD and net tows to study variability in western GSC. Made several additional CTD/MOCNESS stations, including three 3 stations (106, 110, and 111) to examine deep increase in near-bottom fluorescence.

Station 112 - May 29. Single cast made before deep MOCNESS in area of whale sightings in southeastern GSC.

Station 113 - May 29. CTD station 113 (Tow-yo 4) includes 14 casts from surface to about $60 \mathrm{~m}$. CTD fish \#1 lost.

Station 114 - May 30. First cast with fish \#2 showed noisy $6500 \mathrm{db}$ transducer.

\section{Leg 2 - May 30-June 6, 1989}

Station 115 - May 30. Trial cast made to test fish \#2 with a new $1600 \mathrm{db}$ transducer and backup transmissometer and fluorometer. Pressure and fluorometer data ok, but transmissometer not working correctly. Replaced this unit with $\mathrm{K}$. Wishner's transmissometer normally mounted on the URI MOCNESS.

Station 116 - June 1. Single CTD station made at the end of $6 \mathrm{hr}$ small-scale acoustic fin survey (multiple triangular grid) in thecenter of whale aggregation. 
Stations $117-118$ - June 1 . Stations made near $41^{\circ} 41^{\prime} \mathrm{N}, 68^{\circ} 41^{\prime} \mathrm{W}$ while monitoring nighttime vertical migration with MOCNESS and acoustic fin.

Stations 119-127 - June 2. $24 \mathrm{hr}$ time series (diel station 4) to study variability in the center of whale sighting area in southeastern GSC. CTD stations made about every $3 \mathrm{hr}$ at central site near $41^{\circ} 27^{\prime} \mathrm{N}, 68^{\circ} 50^{\prime} \mathrm{W}$.

Stations 128-136 - June 2-3. CTD stations made during $7 \mathrm{hr}$ small-scale acoustic fin survey (multiple triangular grid) near $41^{\circ} 25^{\prime} \mathrm{N}, 68^{\circ} 47^{\prime} \mathrm{W}$ in center of recent whale sighting area. Also made $11 \mathrm{XBT}$ drops to help quickly document thermal structure during survey. CTD station 136 made at end of acoustic fin transect onto shallow flank of Little Georges within the sea surface temperature front.

Stations 137-138 - June 3. CTD stations made at beginning and end of westward acoustic fin transect between $41^{\circ} 39^{\prime} \mathrm{N}, 68^{\circ} 24^{\prime} \mathrm{W}$ in $21 \mathrm{~m}$ within SST front on Georges Bank and $41^{\circ} 39^{\prime} \mathrm{N}, 68^{\circ} 35^{\prime} \mathrm{W}$.

Station 139 - June 3. CTD station 139 (Tow-yo 5) made 16 casts to about $60 \mathrm{~m}$ and last cast to about $120 \mathrm{~m}$ (water depth $132 \mathrm{~m}$ ) while following whale surface feeding. Acoustic fin and MOCNESS data also collected during Tow-yo 5.

Station 140 - June 3. CTD station 140 (Tow-yo 6) made 21 casts to about $60 \mathrm{~m}$ and last cast to $136 \mathrm{~m}$ (water depth $158 \mathrm{~m}$ ) while following whale feeding both at surface and just below surface. Acoustic fin and MOCNESS data also collected during tow-yo.

Stations 141-147 - June 4-5. CTD and net tows made every $3 \mathrm{hrs}$ (diel station 5) to monitor variation near $42^{\circ} 02^{\prime} \mathrm{N}, 69^{\circ} 41^{\prime} \mathrm{W}$ on northwestern flank of GSC.

Station 148 - June 6. CTD station 148 made 11 casts to about $50 \mathrm{~m}$ to study surface slicks and internal wave packet observed on radar in general vicinity of feeding whale near $41^{\circ} 30^{\prime} \mathrm{N}, 68^{\circ} 57^{\prime} \mathrm{W}$. Acoustic fin data showed zooplankton advected vertically by internal waves.

Leg 3 - June 7-12, 1989

Stations 149-207 - June 7-12. 5 transects made with recovered CTD fish \#1. 


\section{Instrumentation and Calibration}

A Neil Brown Instrument Systems (NBIS) model MKIII CTD fish was used as the primary profiling instrument during R/V Endeavor cruise EN196. The instrument provided continuous sampling of temperature, conductivity, pressure, oxygen, fluorescence and light transmission. Salinity and density were subsequently derived from the measured variables. The instrument package consisted of the MKIII CTD underwater unit mounted one meter below a General Oceanics rosette sampler with 12 five-liter Niskin bottles. A Sea Tech $25 \mathrm{~cm}$ pathlength transmissometer and a Sea Tech fluorometer were mounted near the CTD fish. The fast response thermistor on the CTD fish was disabled and the instrument sampled at a rate of $32 \mathrm{hz}$. Lowering speeds were approximately $36 \mathrm{~m} / \mathrm{min}$ to match the response times of the temperature and conductivity sensors (Giles and McDougall, 1986).

The CTD underwater unit is part of the R/V Endeavor's scientific equipment. Water samples were normally collected at each station on the up cast at a depth where the vertical stratification was minimal. The calibration samples were then processed onboard during the cruise using a Guildline AutoSal mometer to determine salinity. The individual salinities were converted to in situ conductivity and compared to the conductivity output of the instrument.

The primary CTD underwater unit (fish \#1) was lost at station 113 on Leg 1 and a second underwater unit (fish \#2) was used on the rest of Leg 1 and on Leg 2 at stations 114 to 147 . The original CTD underwater unit was recovered with a grapnel bar near the end of Leg 2, found to be undamaged and was used on Leg 3 at stations 149 to 207. For Leg 1, a least-squares fit of the difference between the bottle and instrument conductivity gives

$$
C(B O T T L E)-C(C T D)=-0.0000726031 * X-0.0121143
$$

where $X$ is the station number which varied from 1 to 112 , and the sum of the squares of the residuals is $0.0121 \mathrm{mmho}^{2}$. This means that the CTD conductivity sensor drift and offset were small during the cruise and that the rms difference between the corrected CTD and bottle conductivity values was $\pm 0.010 \mathrm{mmho}$. 
For Leg 2, a least-squares fit of the difference between the bottle and instrument conductivity gives

$$
C(B O T T L E)-C(C T D)=-0.00016083 * X-0.008097
$$

where $X$ is the station number which varied from 114 to 147 , and the sum of the squares of the residuals is $0.00091 \mathrm{mmho}^{2}$. The rms difference between the corrected CTD and bottle conductivity values was $\pm 0.006 \mathrm{mmho}$.

For Leg 3, a least-squares fit of the difference between the bottle and instrument conductivity gives

$$
C(B O T T L E)-C(C T D)=-0.000264601 * X-0.0406355
$$

where $X$ is the station number which varied from 149 to 207 , and the sum of the squares of the residuals is $0.00214 \mathrm{mmho}^{2}$. The rms difference between the corrected CTD and bottle conductivity values was $\pm 0.007 \mathrm{mmho}$. The CTD conductivity for the entire cruise has been edited using the linear regression corrections given above.

A new Sea Tech transmissometer was used on Legs 1 and 3 and the data is given her as a percent of full scale. On Leg 2, a backup light transmissometer did not function properly at station 114 and a third transmissometer (which normally was mounted on the URI MOCNESS) was used at stations 115-147. This instrument used on Leg 2 had a full scale output which was reduced by $7 \%$ from the instrument used on Legs 1 and 3 . The transmissometer data listed here for Leg 2 have not been adjusted for this difference, but simply left in terms of percent full scale. Both fluorometers had been recently calibrated and the fluorescence data obtained on EN196 are given as a percent of full scale.

\section{Data Processing}

The CTD data were recorded at sea with a PC-based data acquisition system written by Howard Saklad of the University of Alaska, Institute of Marine Science. The program acquires the raw CTD data at $32 \mathrm{hz}$, displays the data in real time, postcast data averages, and prints lists of data at each station. Normally, only the down profile is processed at sea. During post cruise data processing, the conductivity calibration was 
first applied to the raw data. Then an exponential recursive filter (Middleton and Foster, 1980) described by Cox was applied to pressure and conductivity to match the amplitude and phase of the platinum temperature sensor. The Cox filter has the form

$$
c^{\prime}(t)=w_{0} c^{\prime}(t-d t)+\left(1-w_{0}\right) c(t)
$$

where $c^{\prime}(t)$ is the filtered conductivity at time $t, c^{\prime}(t-d t)$ is the previous filtered conductivity, and $c(t)$ is the original unfiltered conductivity at time $t$. The filter weight is given by

$$
w_{0}=e^{-d t / t l a g}
$$

where $d t=.032 \mathrm{sec}$ is the sampling period, and tlag $=.235 \mathrm{sec}$ is the time lag of the platinum temperature sensor. A uniform pressure series of $1 \mathrm{db}$ interval was then created from the filtered data, after spurious data observations (spikes) had been replaced with interpolated data. Salinity was calculated using Lewis (1980) and a value of 42.914 was used to convert to conductivity ratio (Culkin and Smith, 1980).

\section{Data Presentation}

The hydrographic data collected on Leg 3 as part of the regional CTD/ADCP survey are shown in the form of vertical sections, $\mathrm{T} / \mathrm{S}$ correlations and horizontal sections in Section 7. The CTD data collected on all three legs of EN196 are shown in Section 8 as vertical profiles and tabular lists at graduated depths.

\section{Acknowledgments}

Cruise EN196 on the R/V Endeavor was supported by the NSF Grant OCE8713988. Dr. Karen Wishner was the Chief Scientist on the multidisciplinary cruise. The work was a cooperative effort by R. Beardsley, R. Limeburner, C. Hilliard, K. Wishner, E. Durbin, A. Durbin, J. Schoenherr, J. Szelag and B. Fanning. Their assistance is greatly appreciated. Finally, the helpfulness of the officers and crew of the R/V Endeavor contributed significantly to the success of the cruise. 


\section{References}

Culkin, F. and N. D. Smith (1980) Determination of the concentration of potassium chloride having the same electrical conductivity, at $15^{\circ} \mathrm{C}$ and infinite frequency, as standard seawater of salinity $35.000 \mathrm{ppm}$ (chlorinity $19.37394 \mathrm{ppm}$ ). IEEE Journal of Ocean Engineering, OE-5, 22-23.

Giles, A. B., and T. J. McDougall (1986) Two methods for the reduction of salinity spiking of CTDs. Deep-Sea Research, 33(9), 1253-1274.

Lewis, L. L. (1980) The Practical Salinity Scale 1978 and its antecedents. IEEE Journal of Ocean Engineering, OE-5, 3-8.

Middleton, J. H., and T. D. Foster (1980) Fine structure measurements in a temperature compensated halocline. Journal of Geophysical Research, 85(C2), 1107-1122. 
7. Graphic Description of CTD Data

A. Large-Scale Survey - Jun 7-12, 1989 Stations 149 to 207 


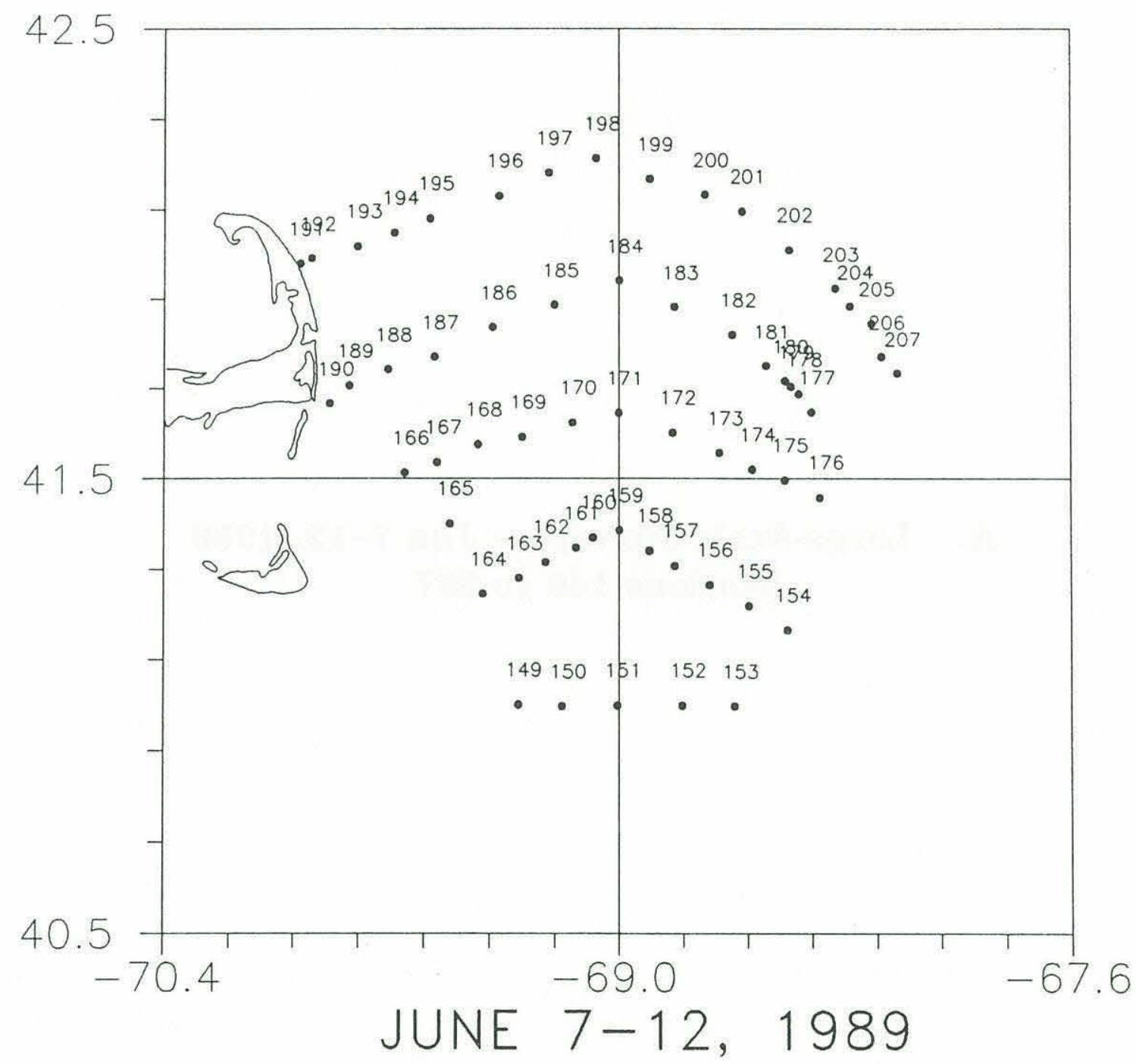




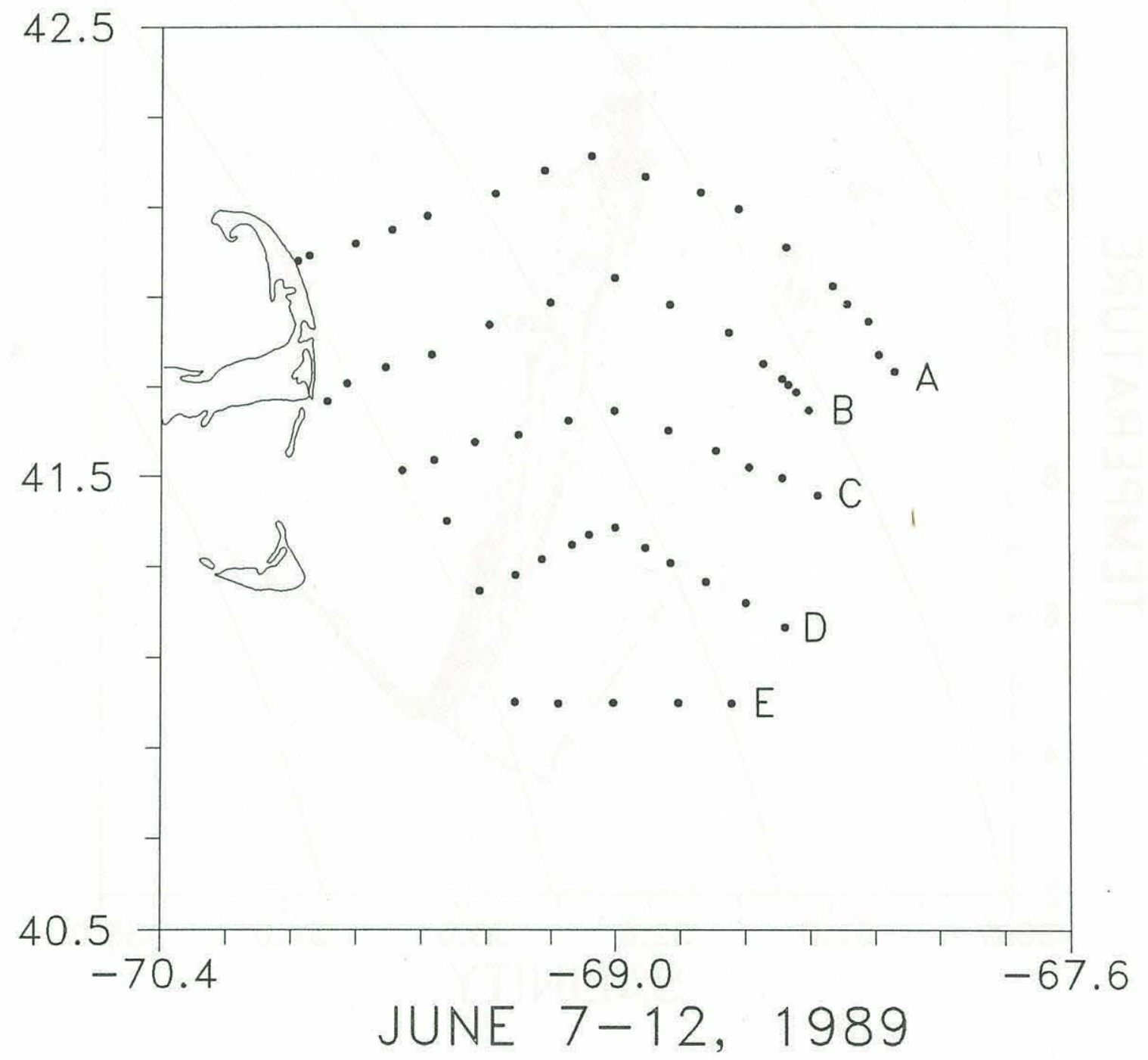




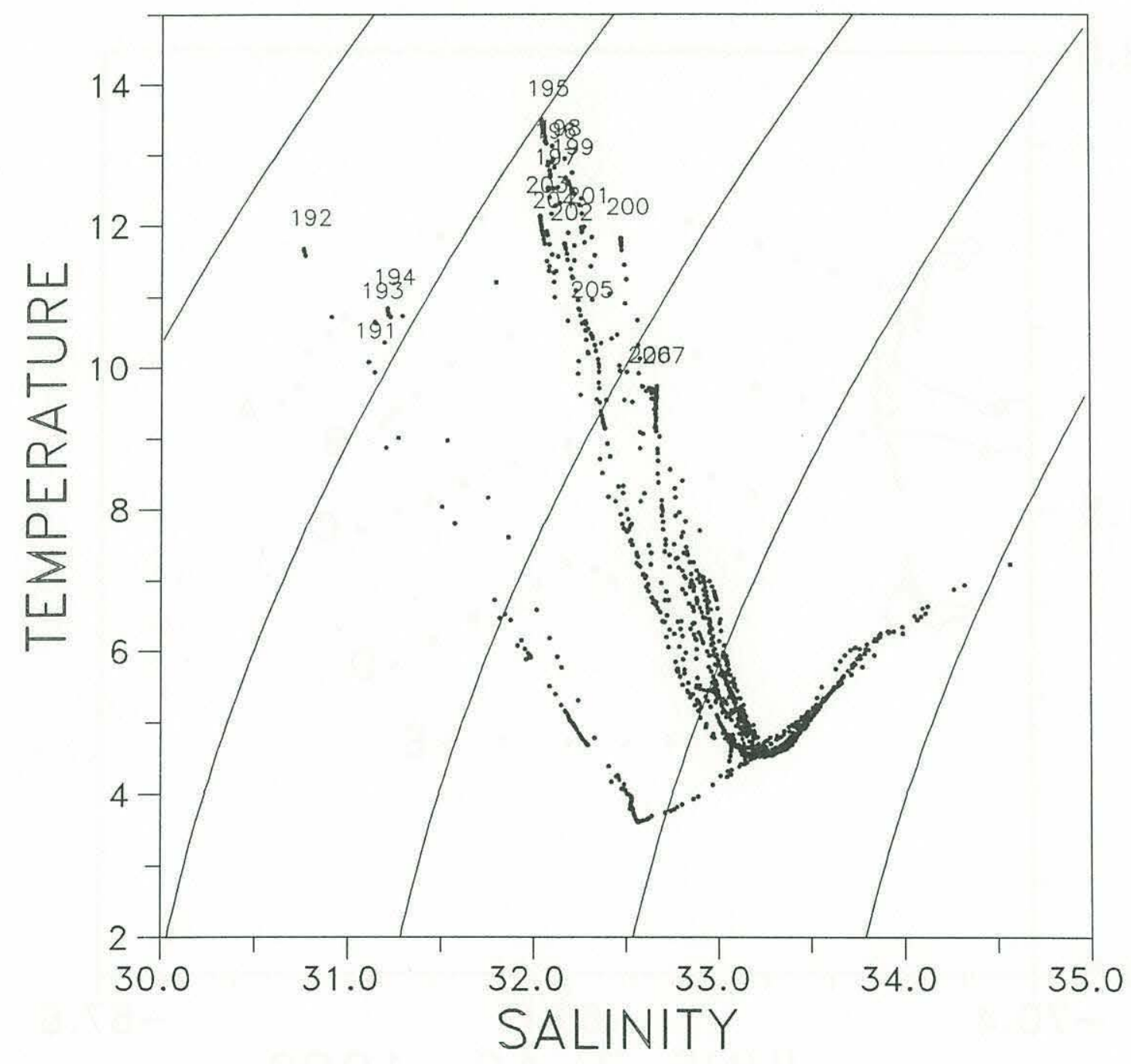




\section{A TEMPERATURE}

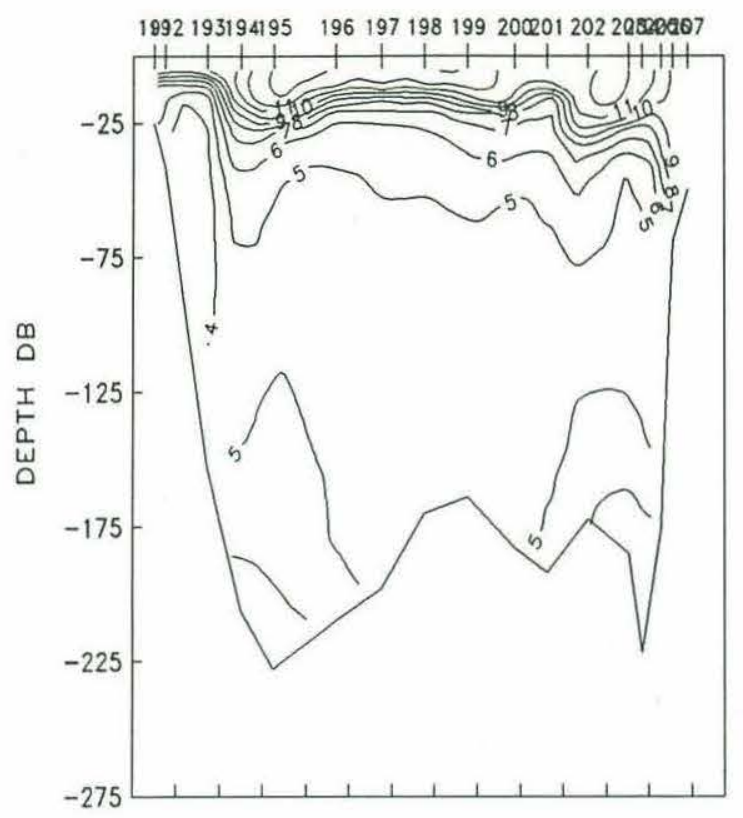

A SIGMA-T

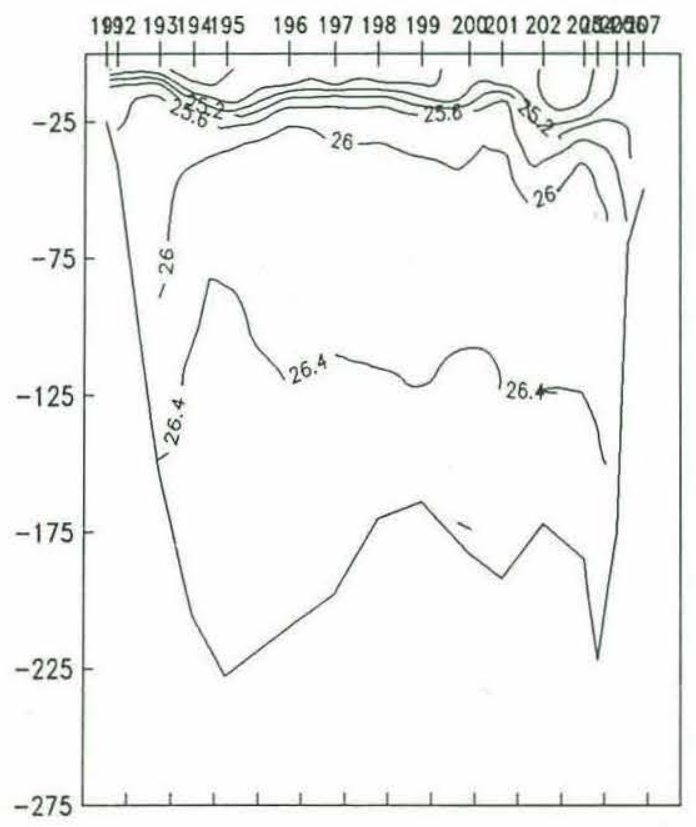

A SALINITY

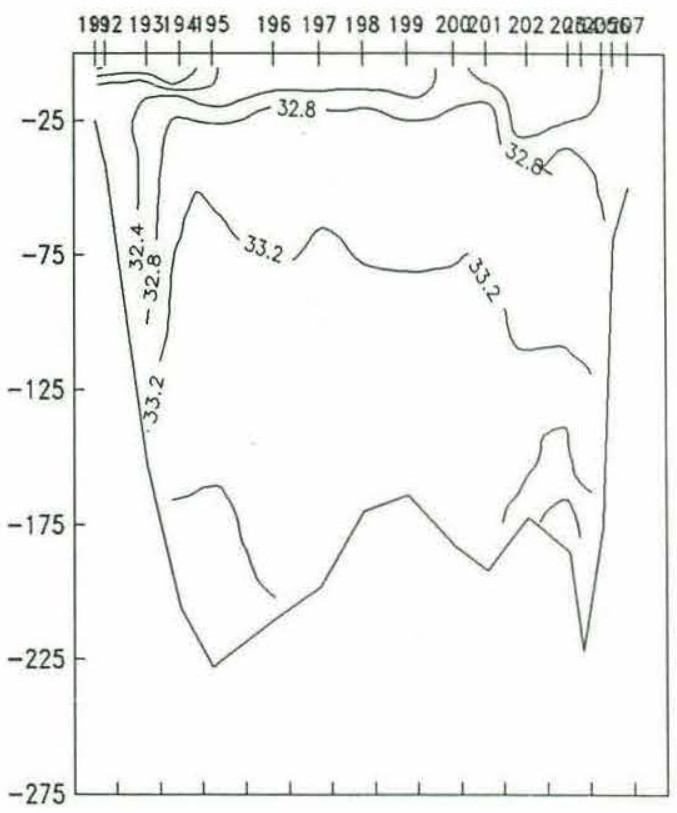

A LIGHT PCT

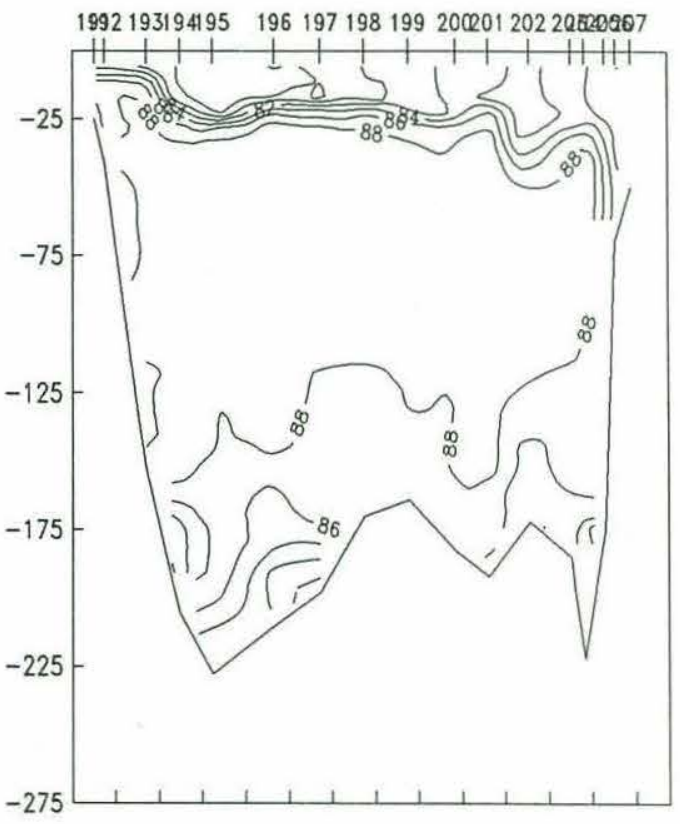



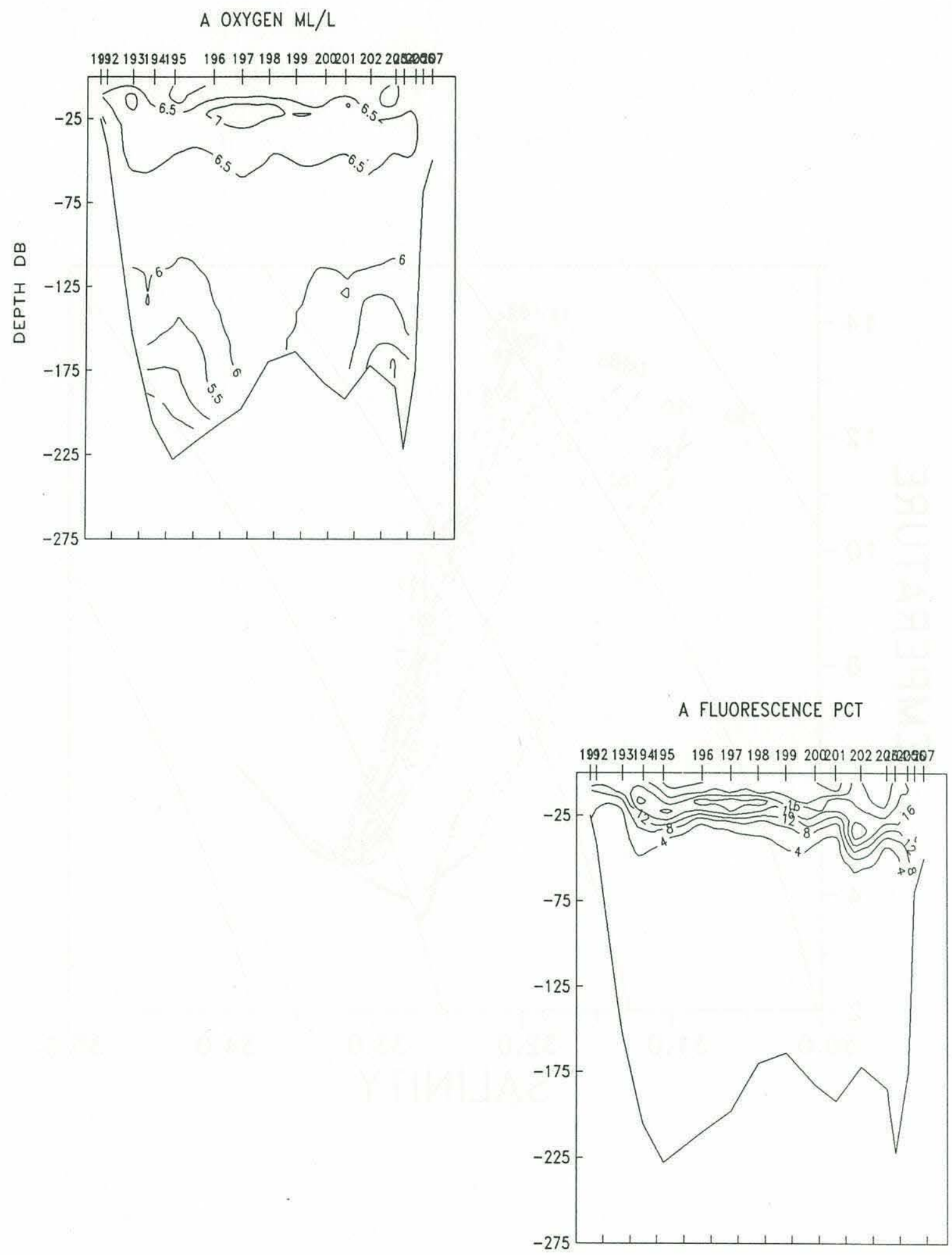


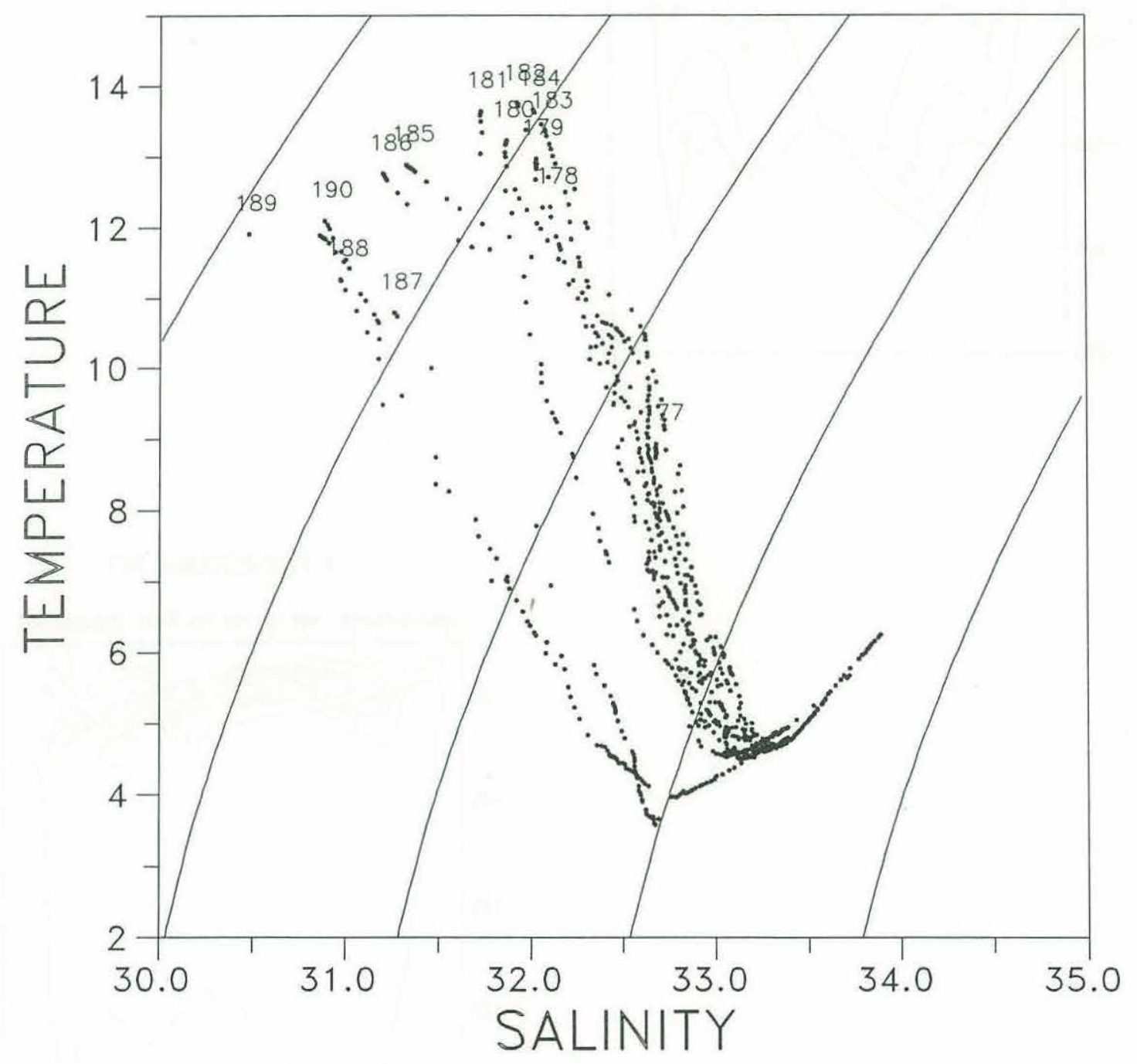


B TEMPERATURE

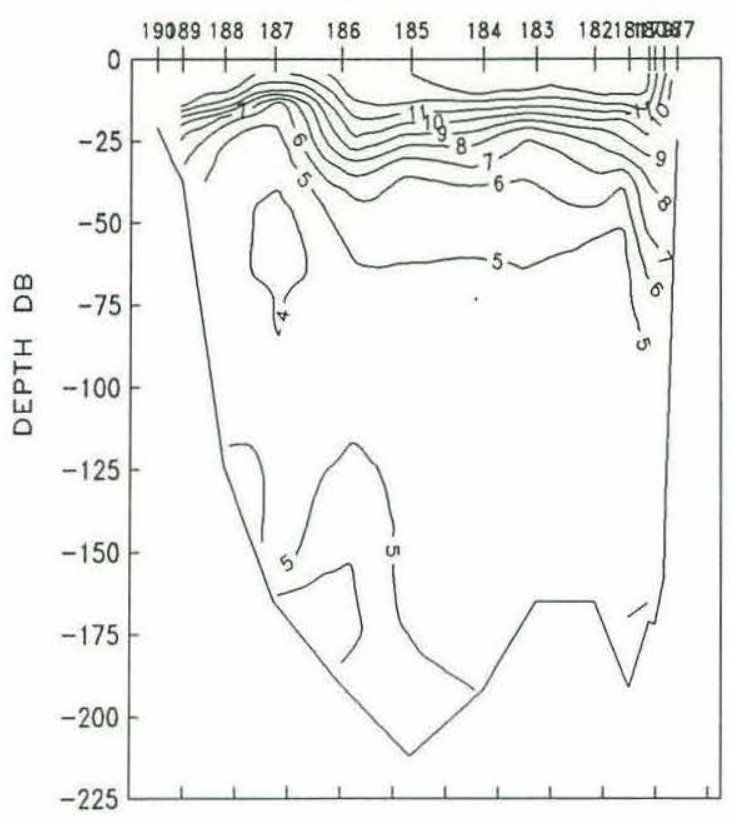

B SALINITY

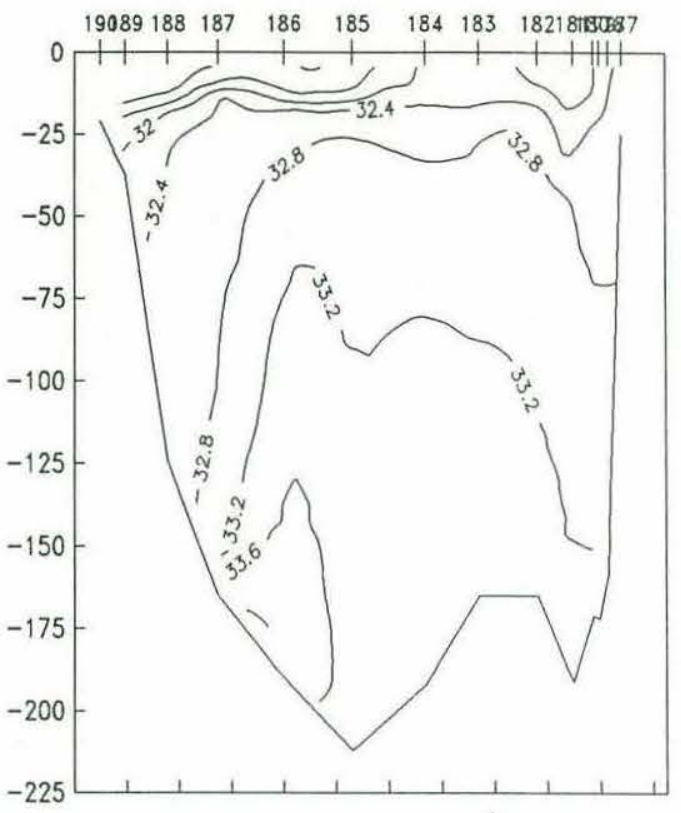

B SIGMA-T

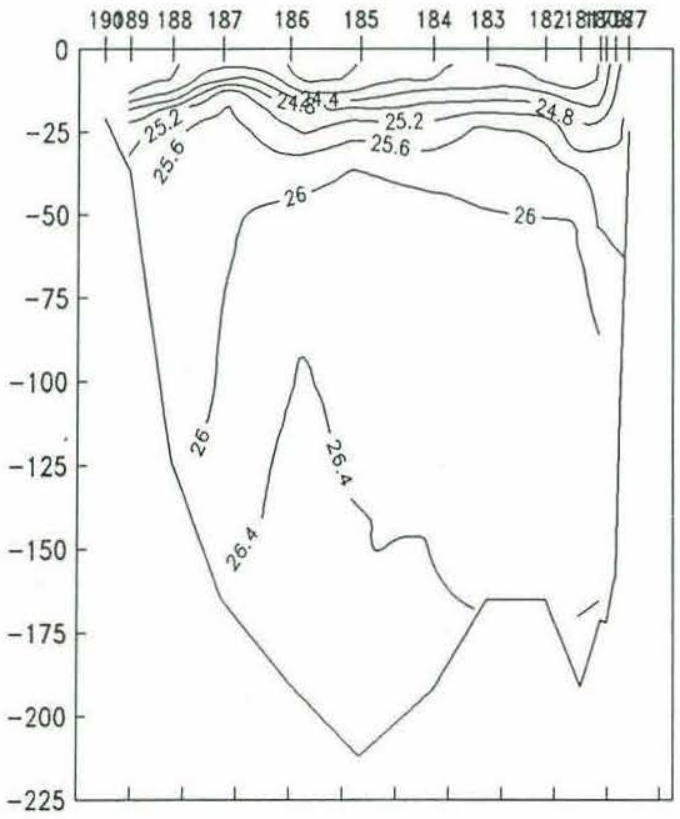

B LIGHT PCT

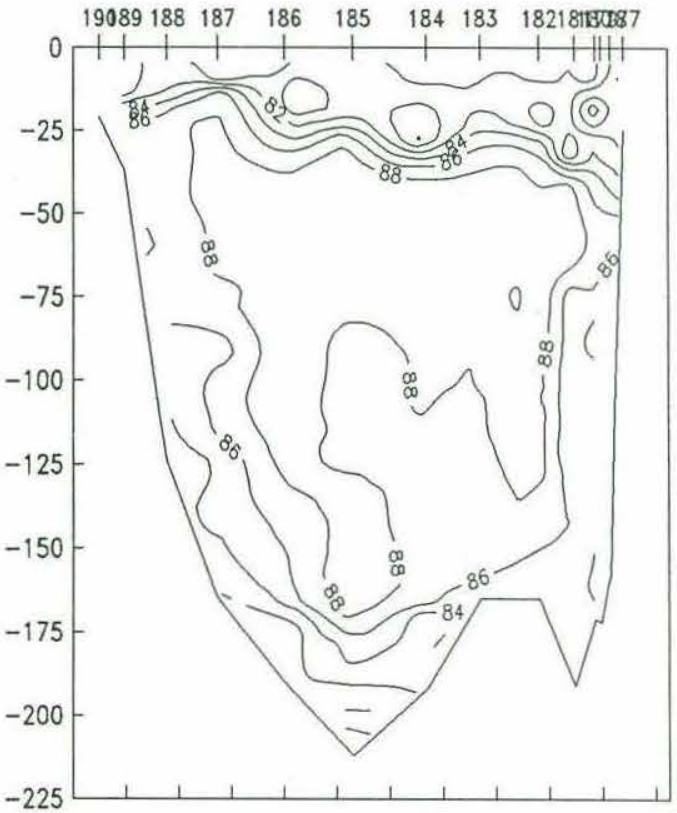


B OXYGEN ML/L

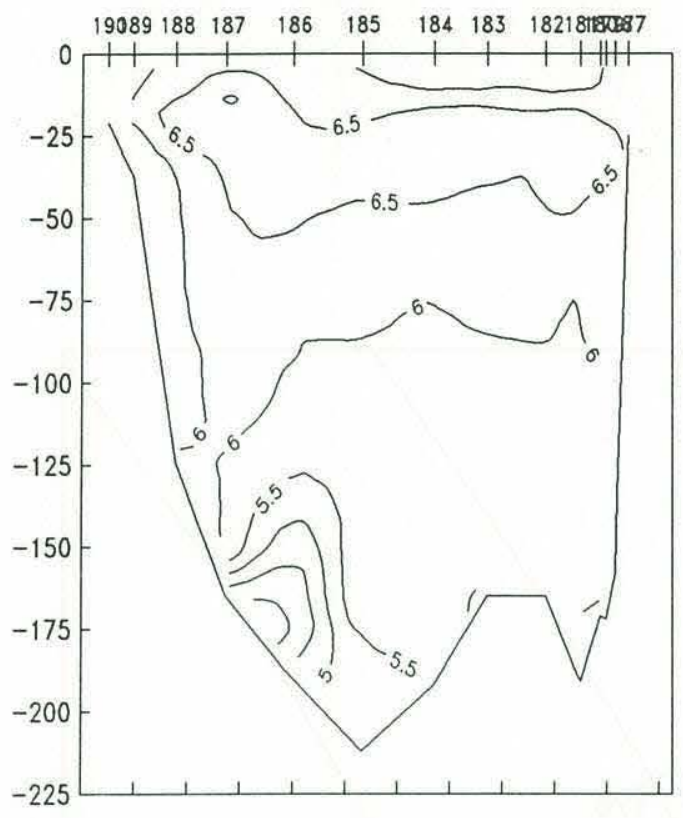

B FLUORESCENCE PCT

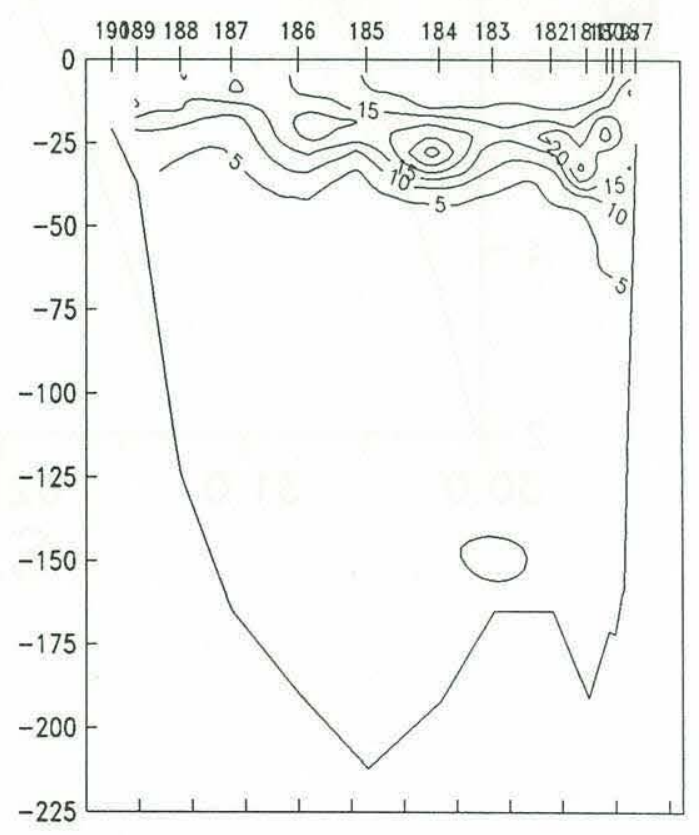




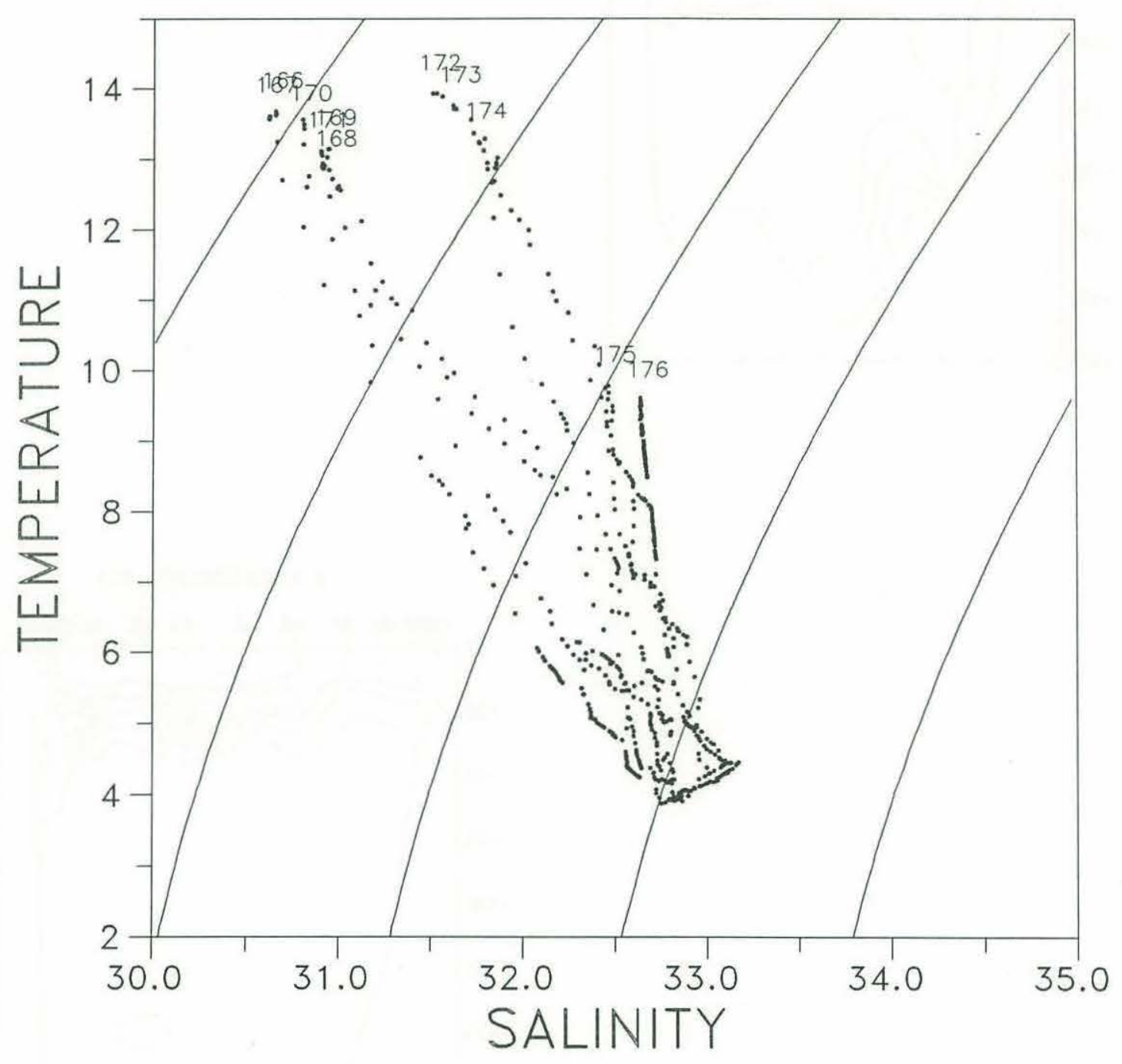




\section{TEMPERATURE}

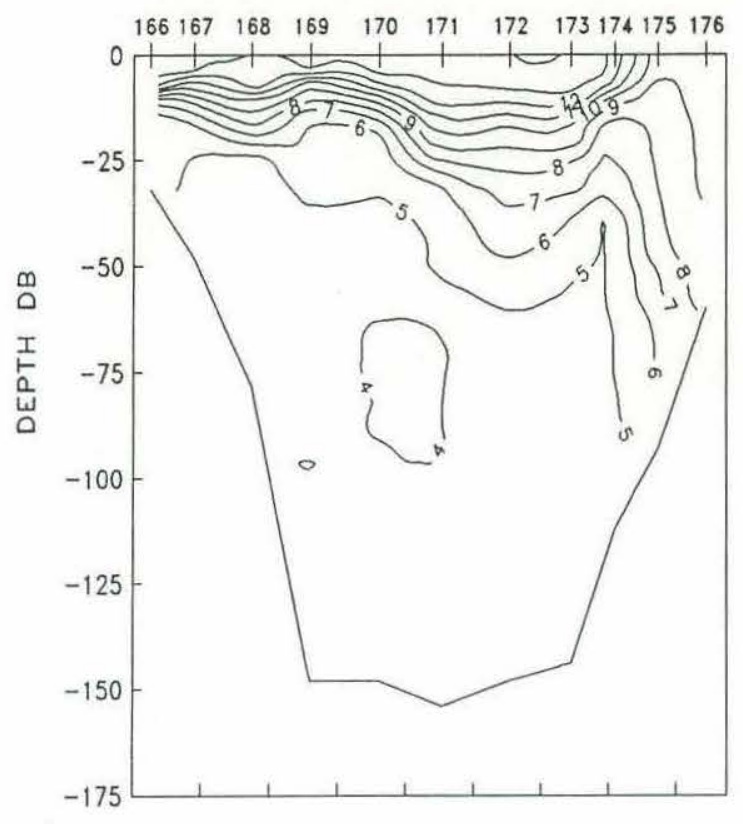

C SIGMA-T

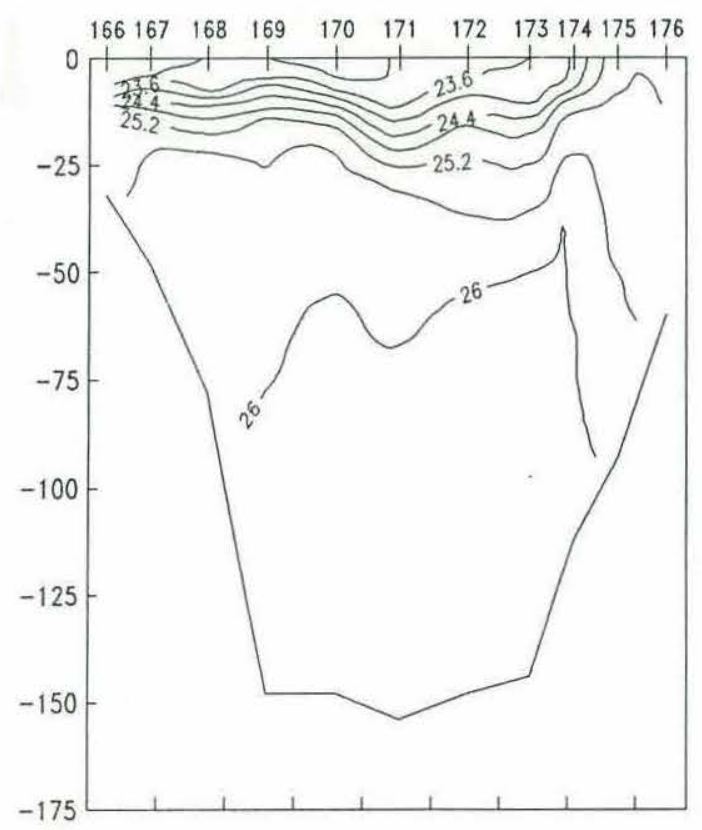

C SALINITY

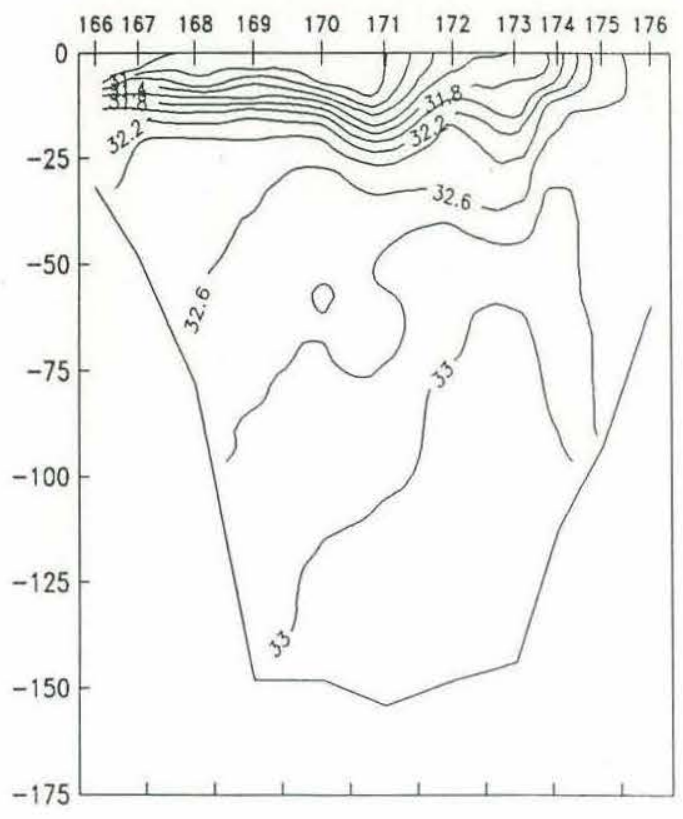

C LIGHT PCT

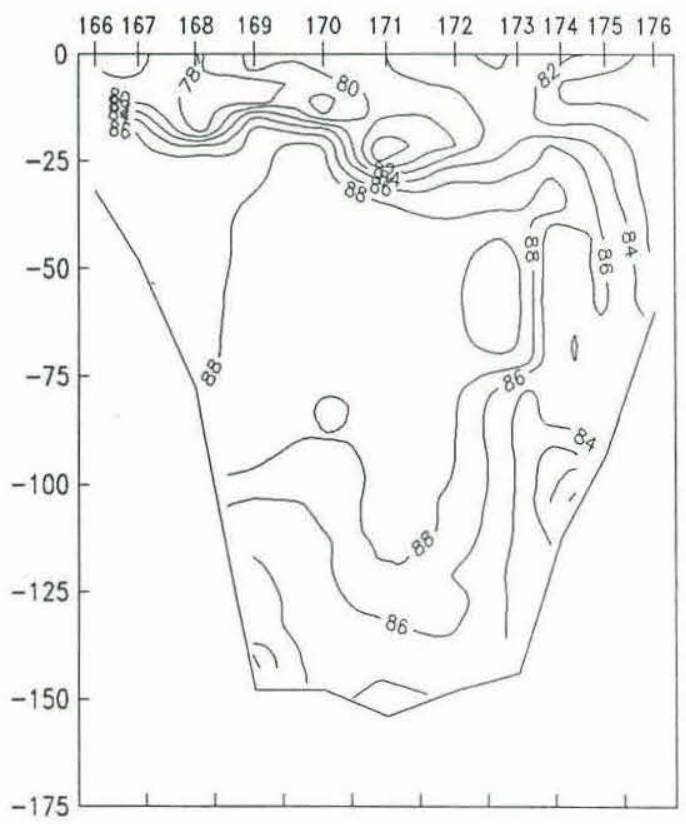




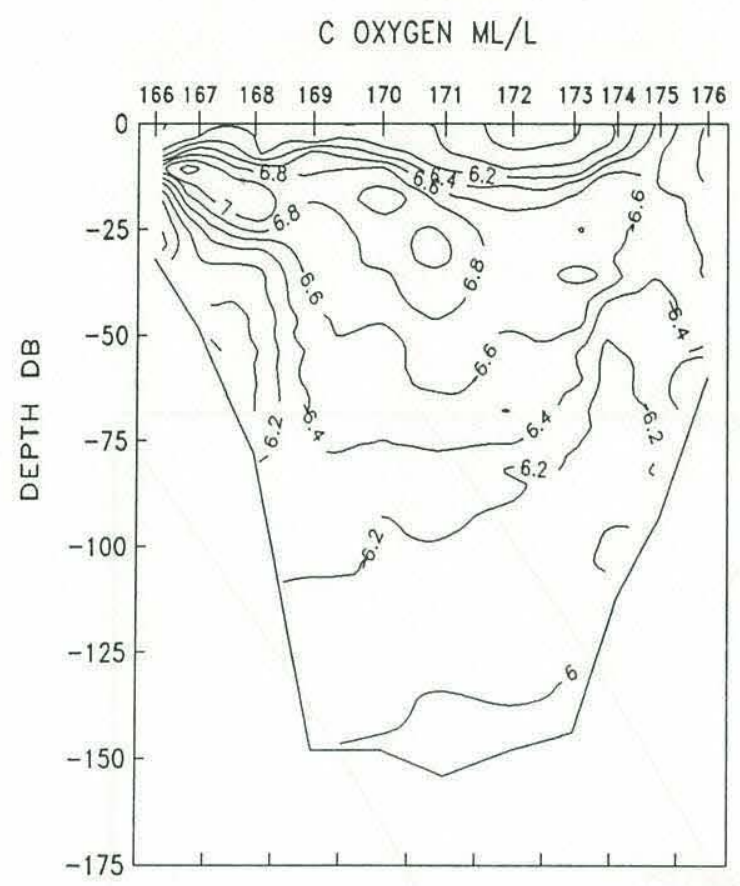

C FLUORESCENCE PCT

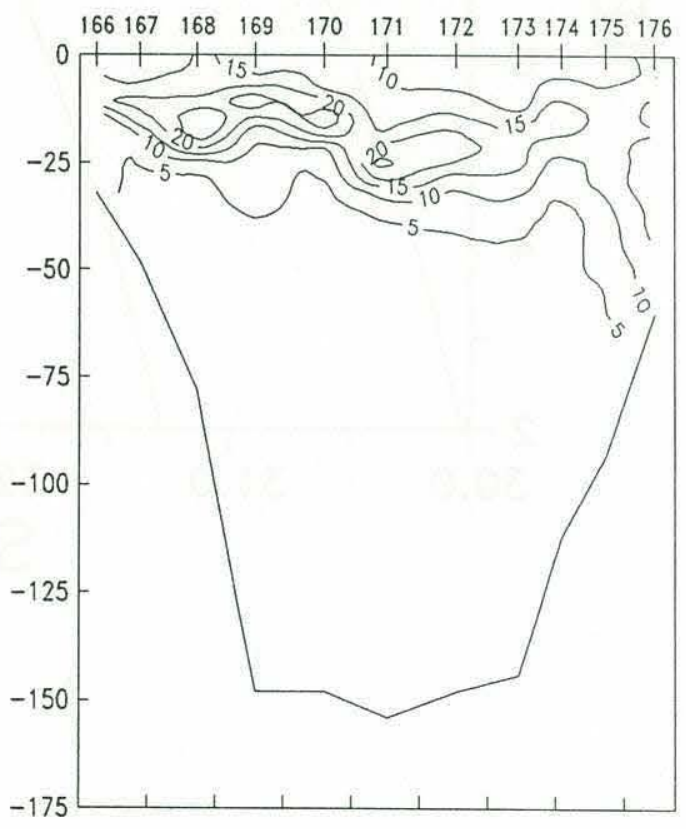




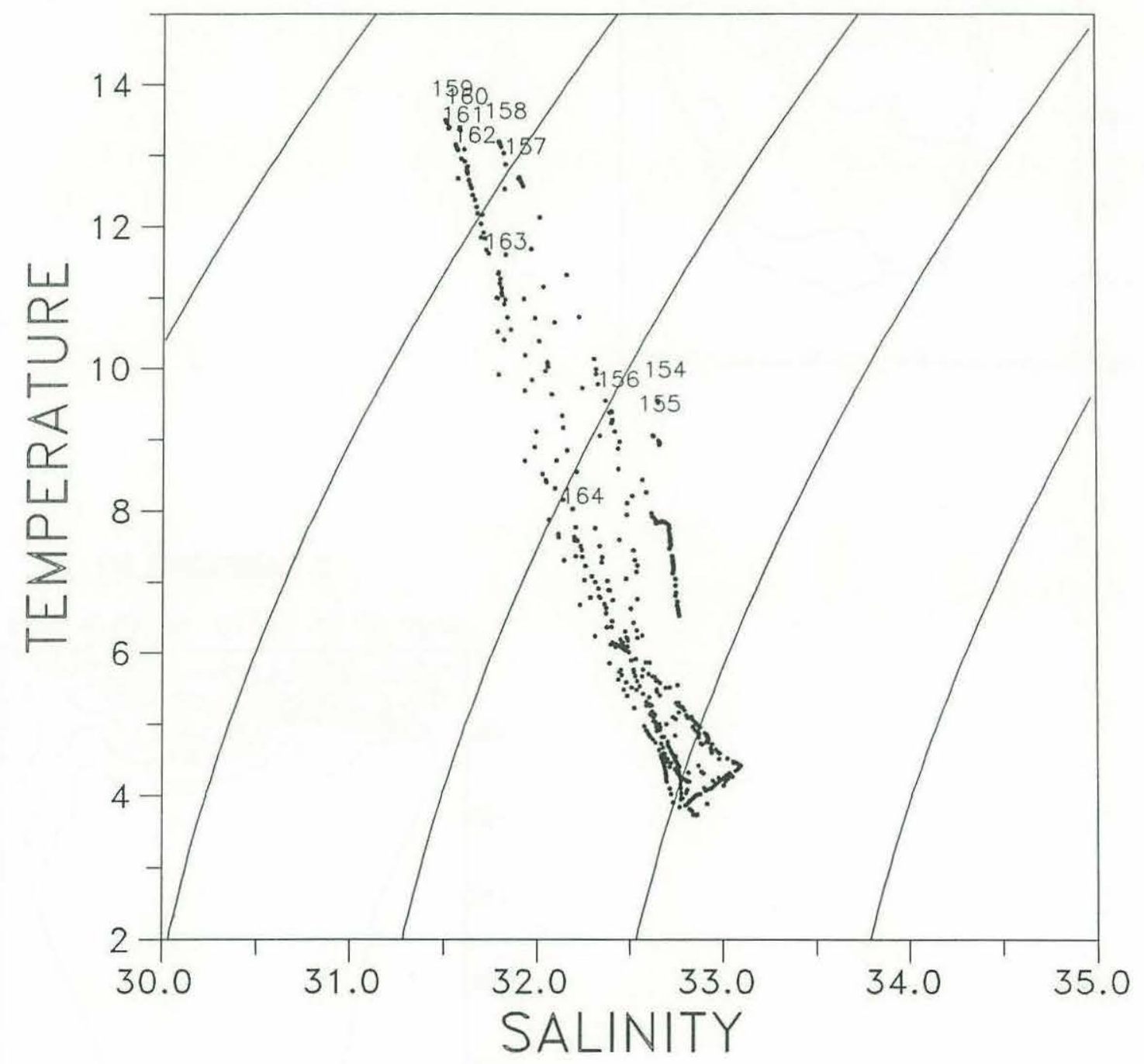


D TEMPERATURE

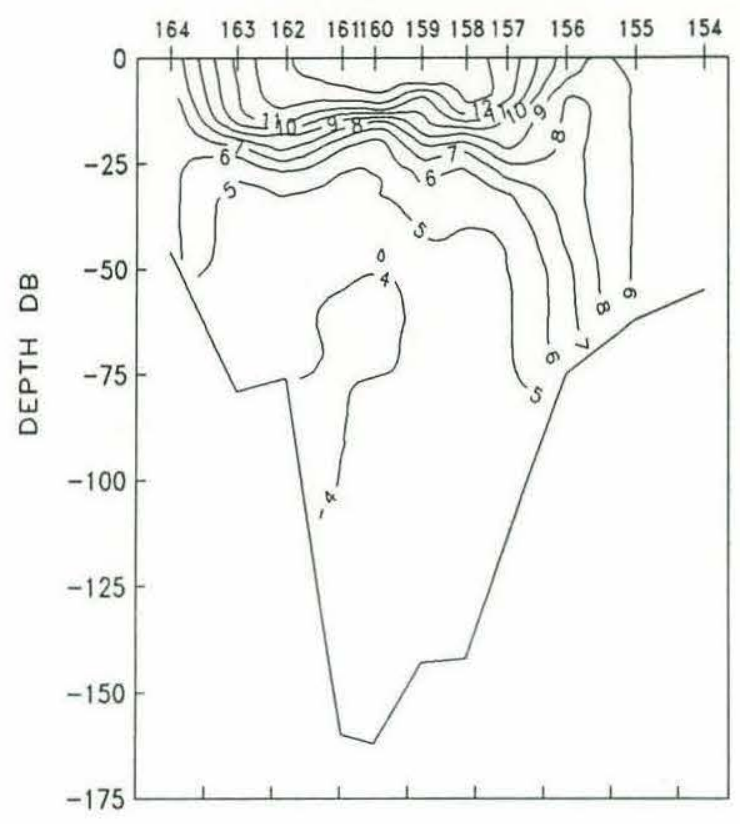

D SALINITY

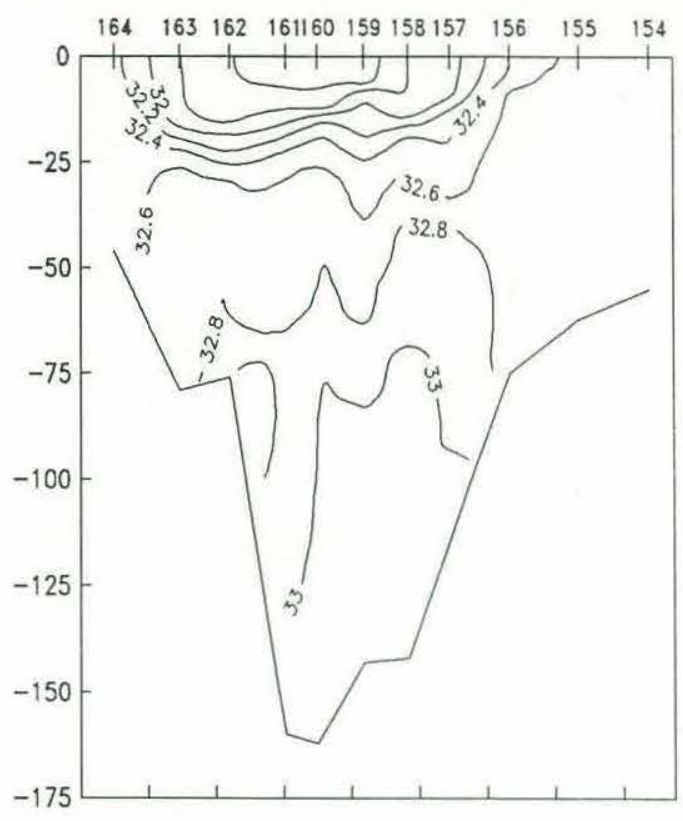

D SIGMA-T

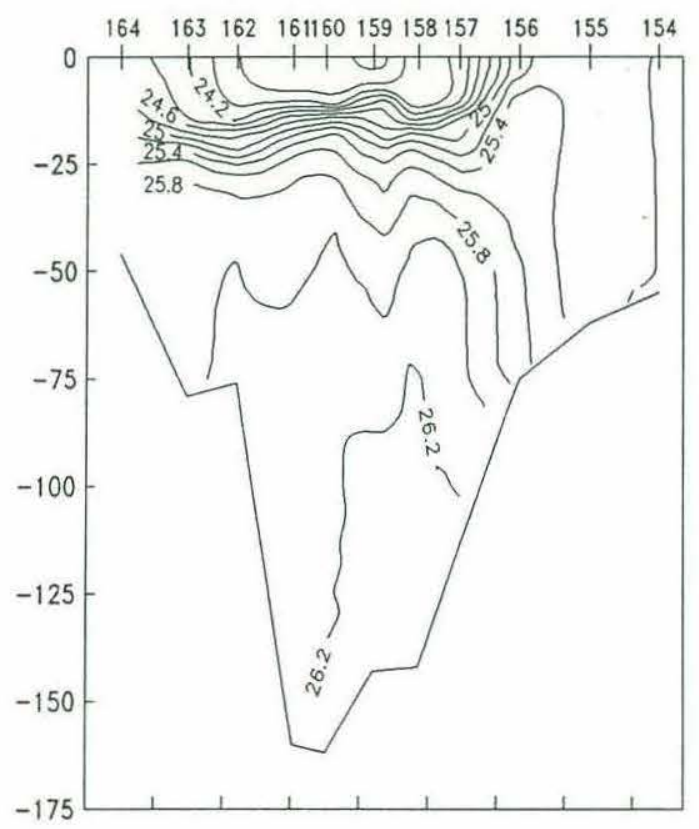

D LIGHT PCT

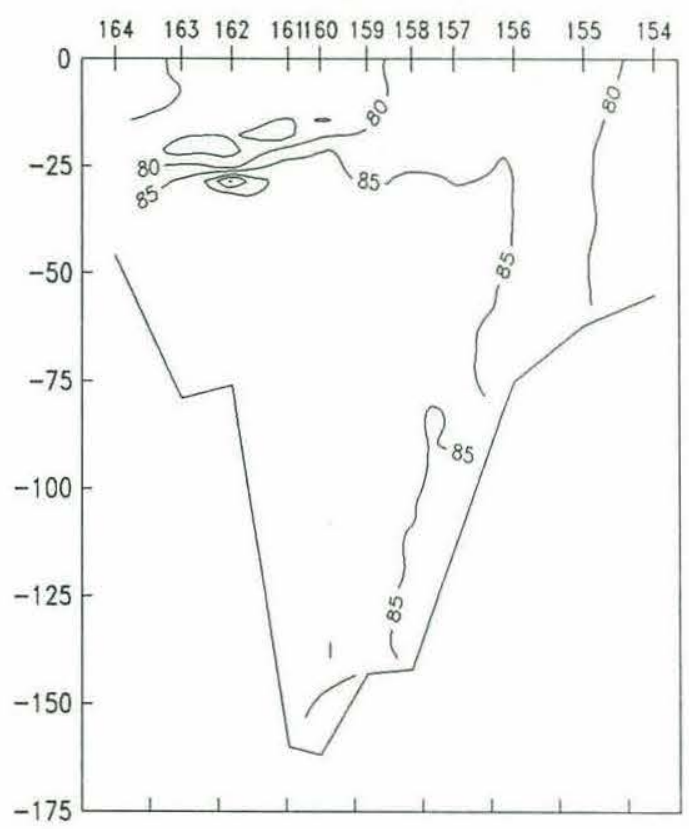



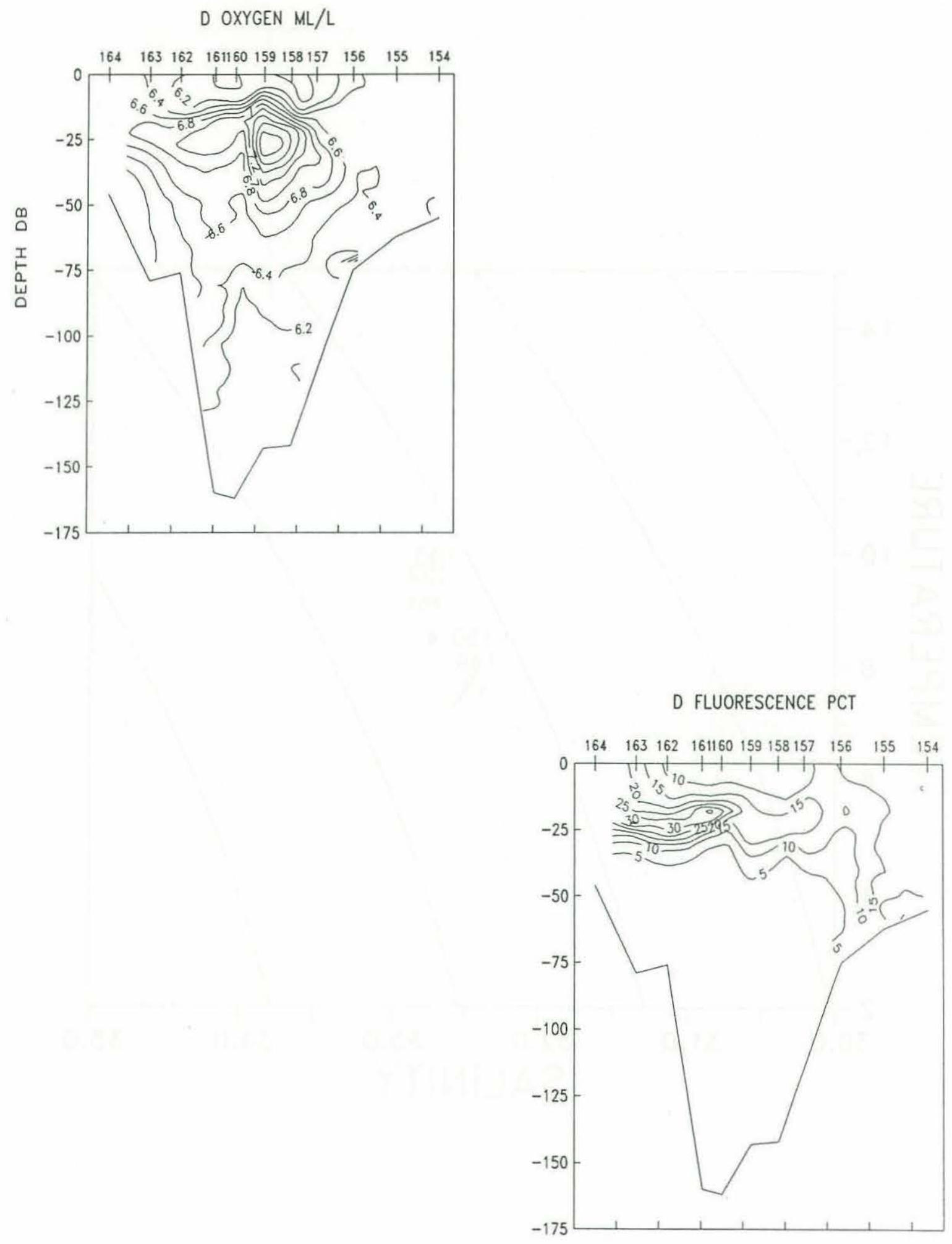


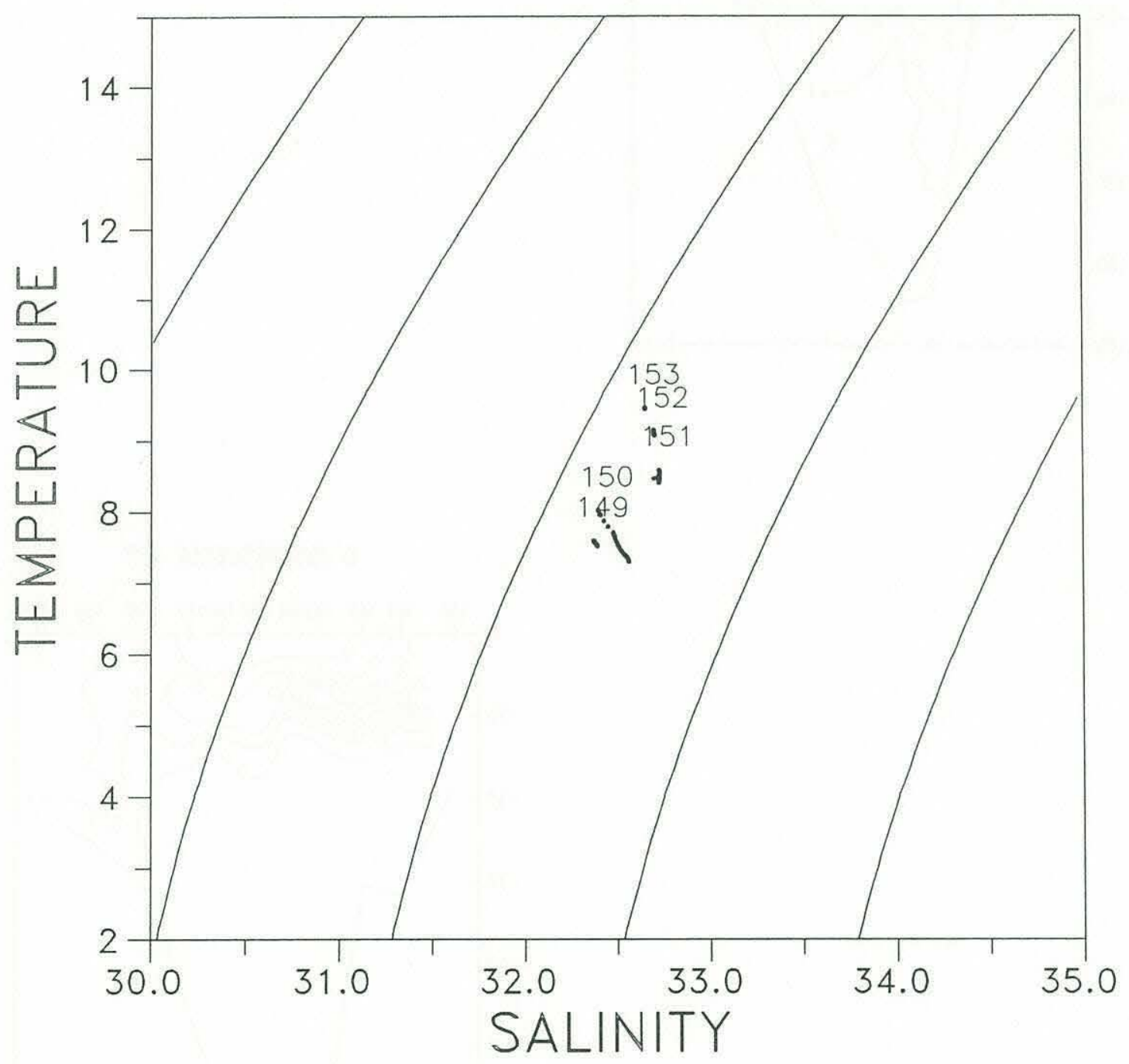


E TEMPERATURE

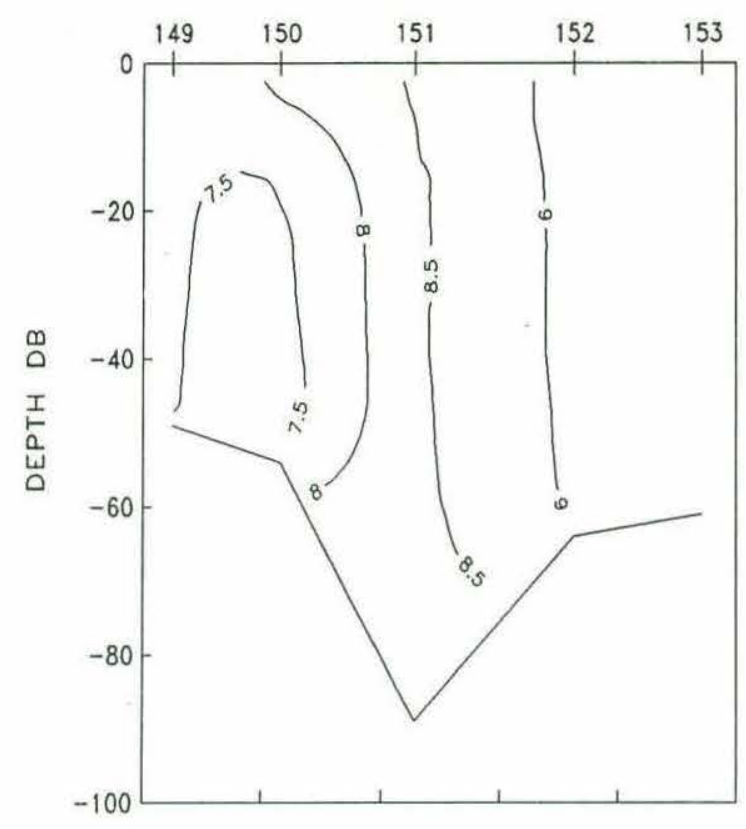

E SIGMA-T

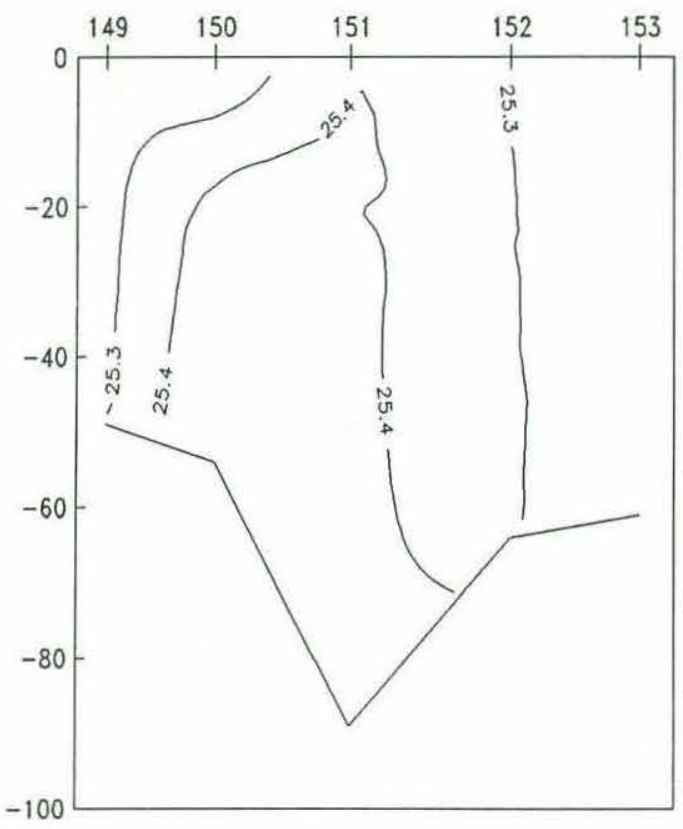

E SALINITY

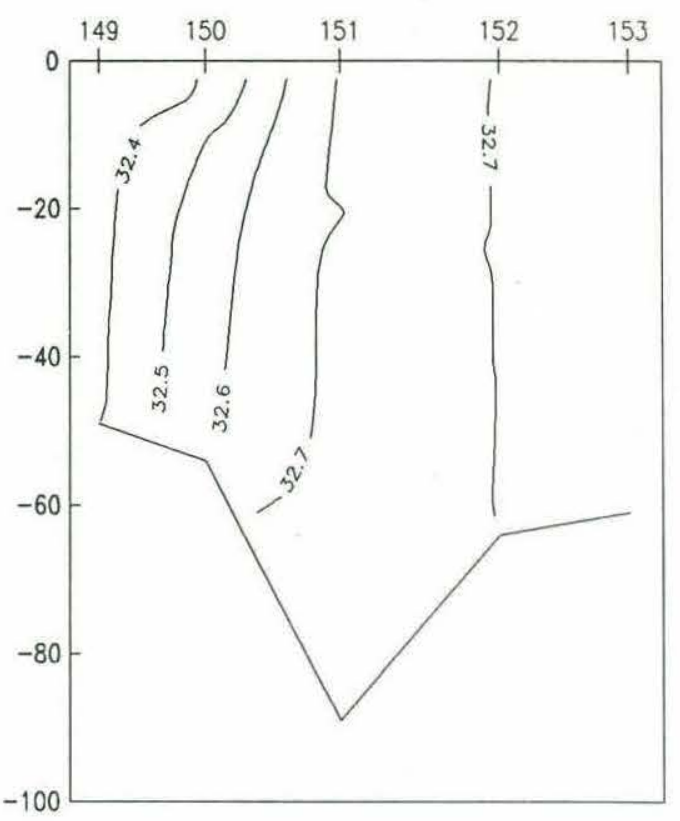

E LIGHT

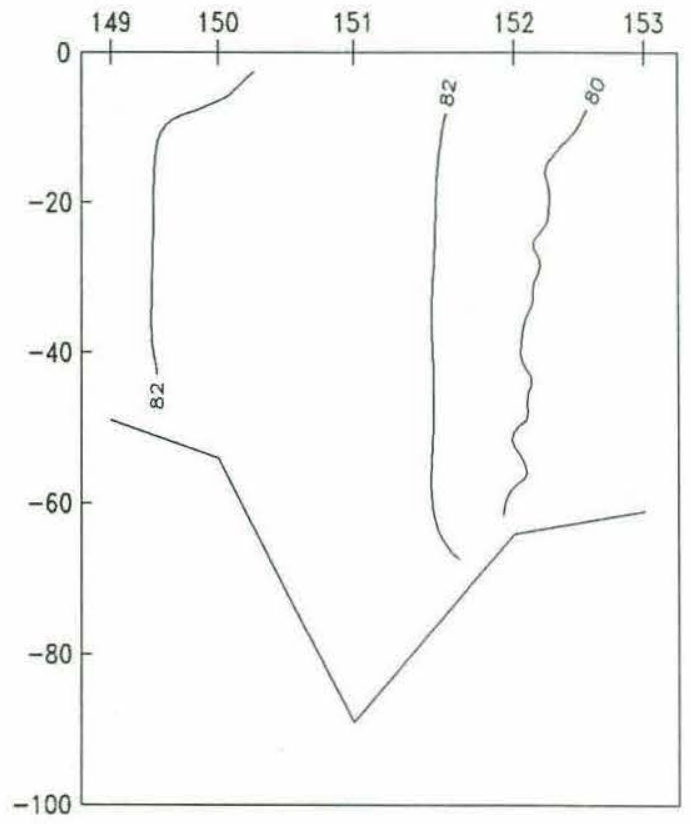



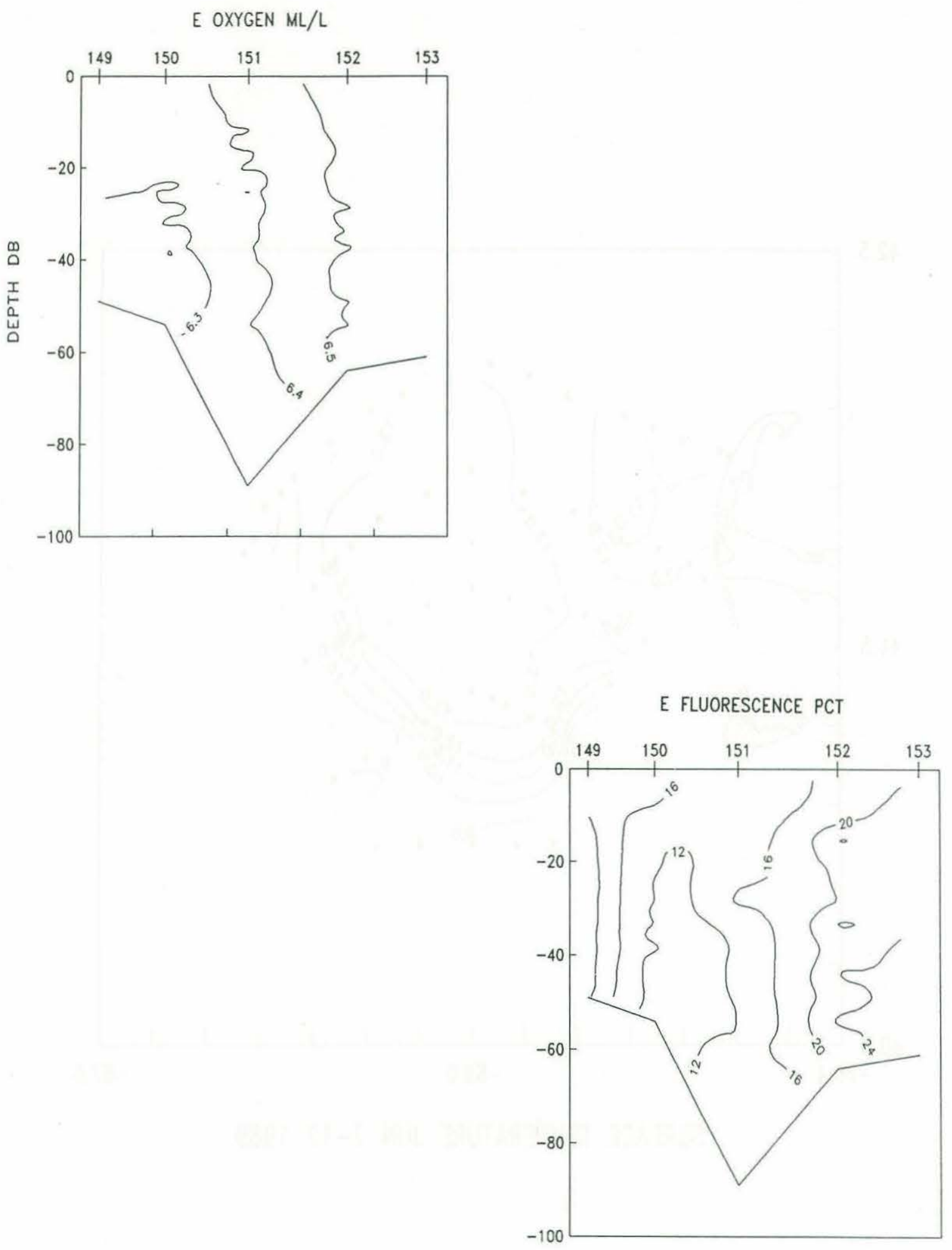


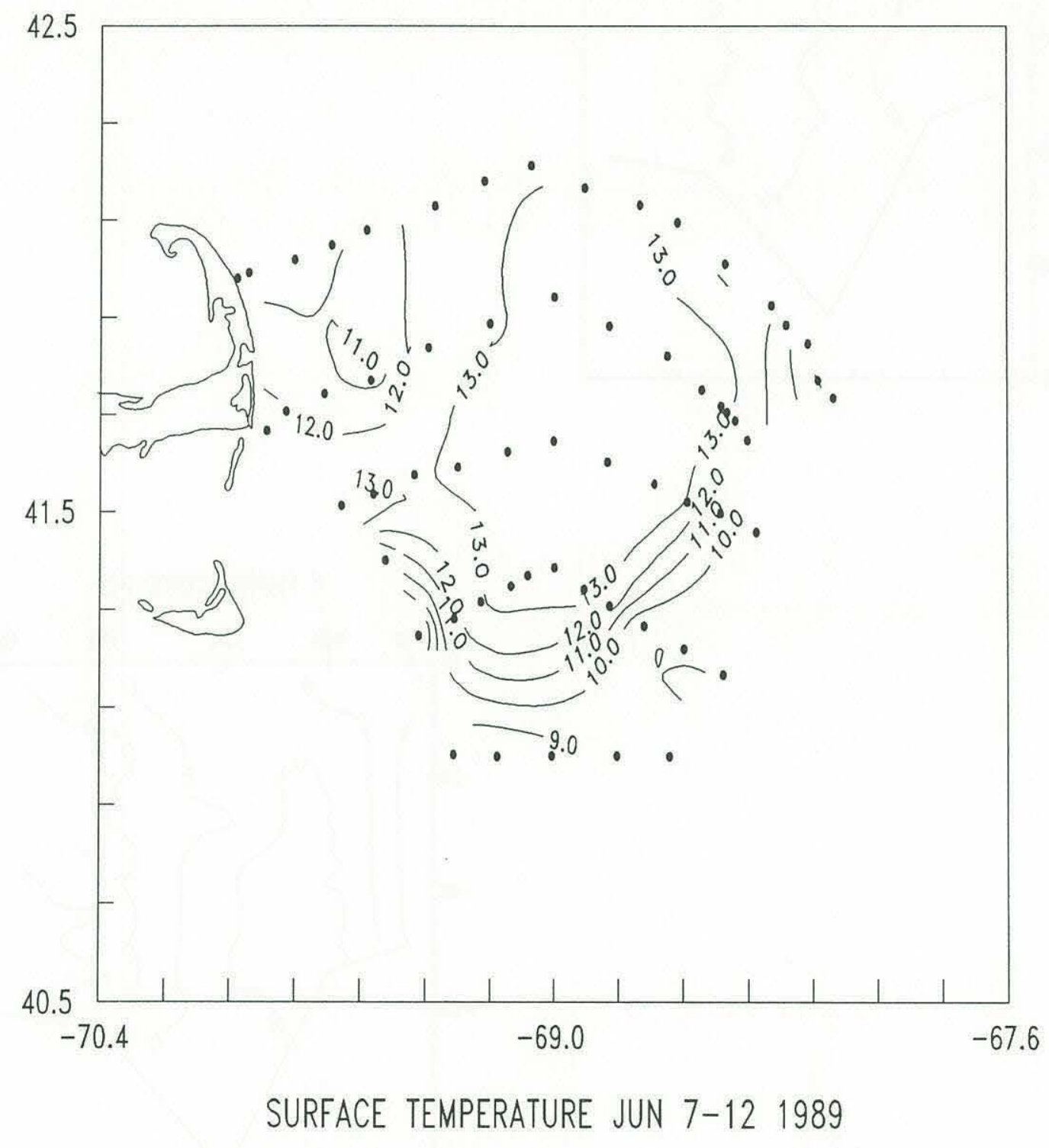




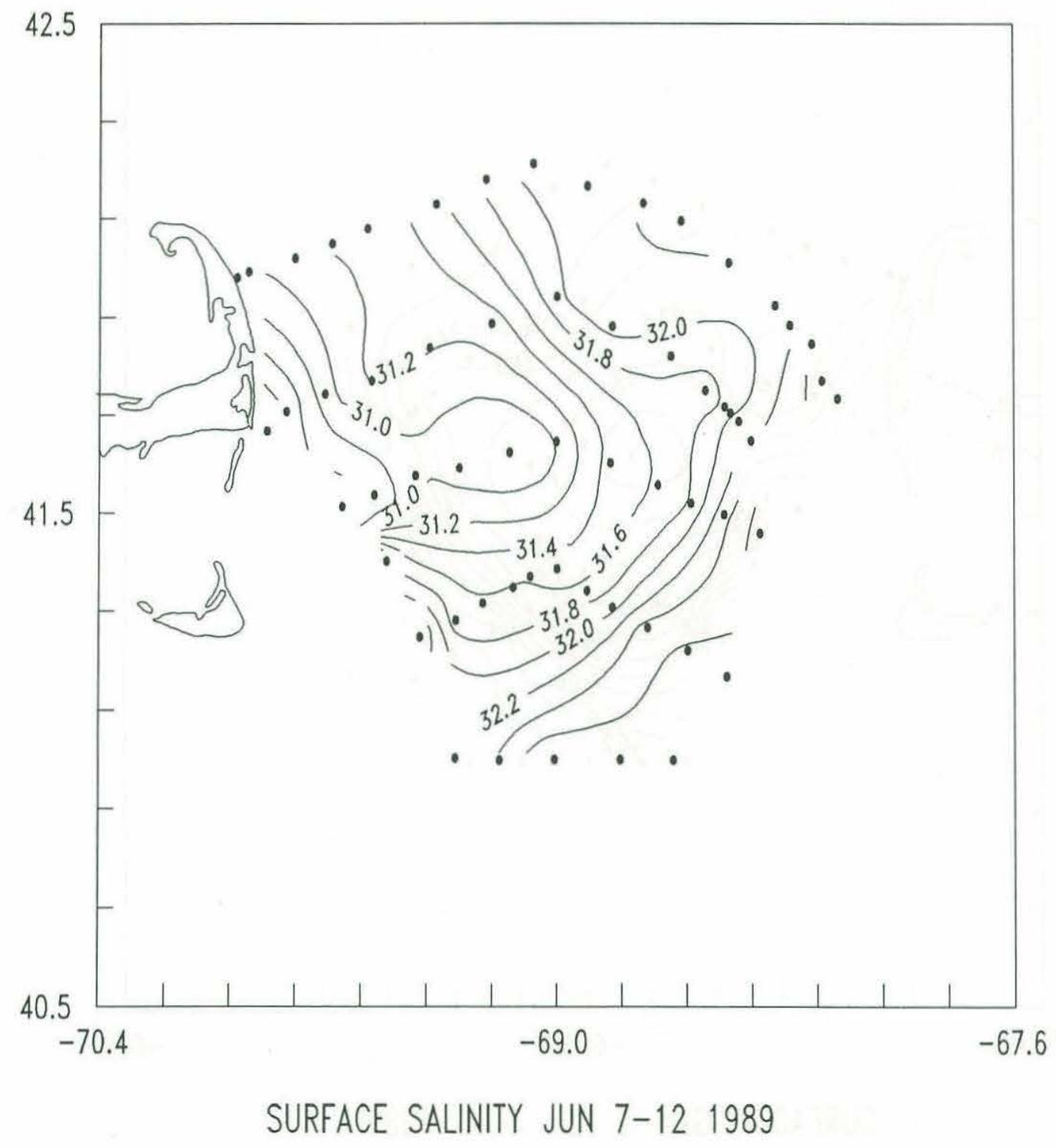




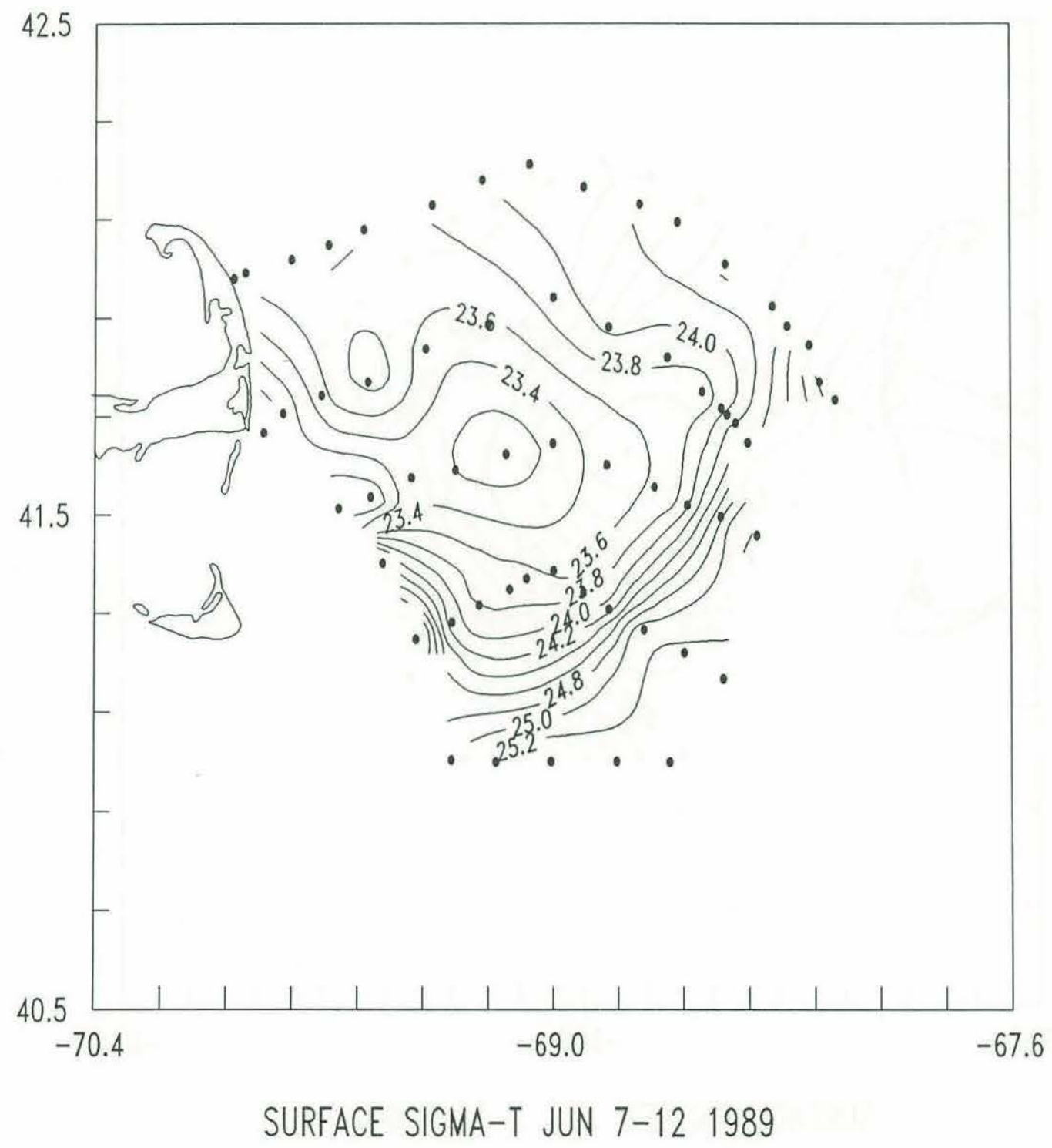




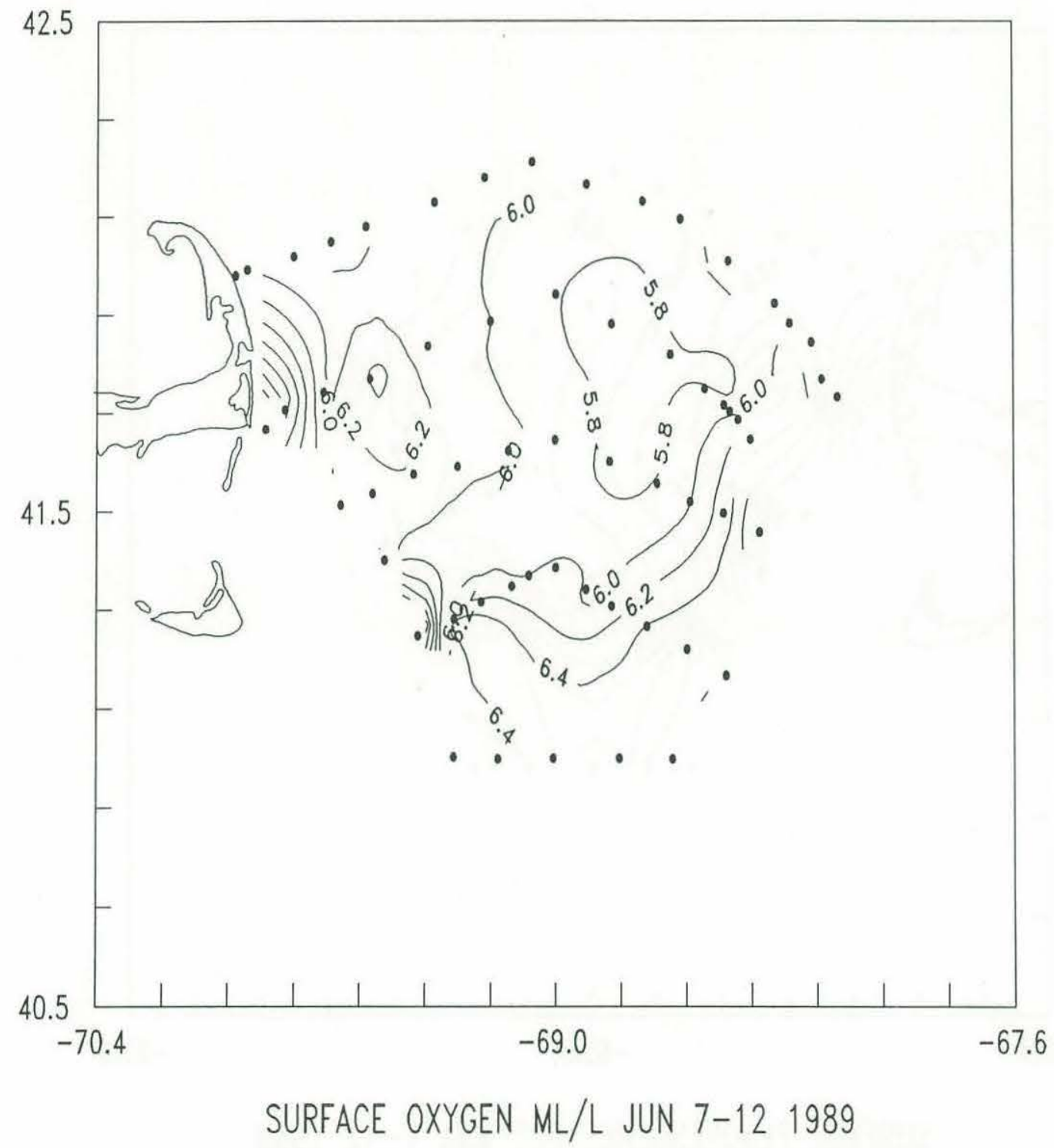




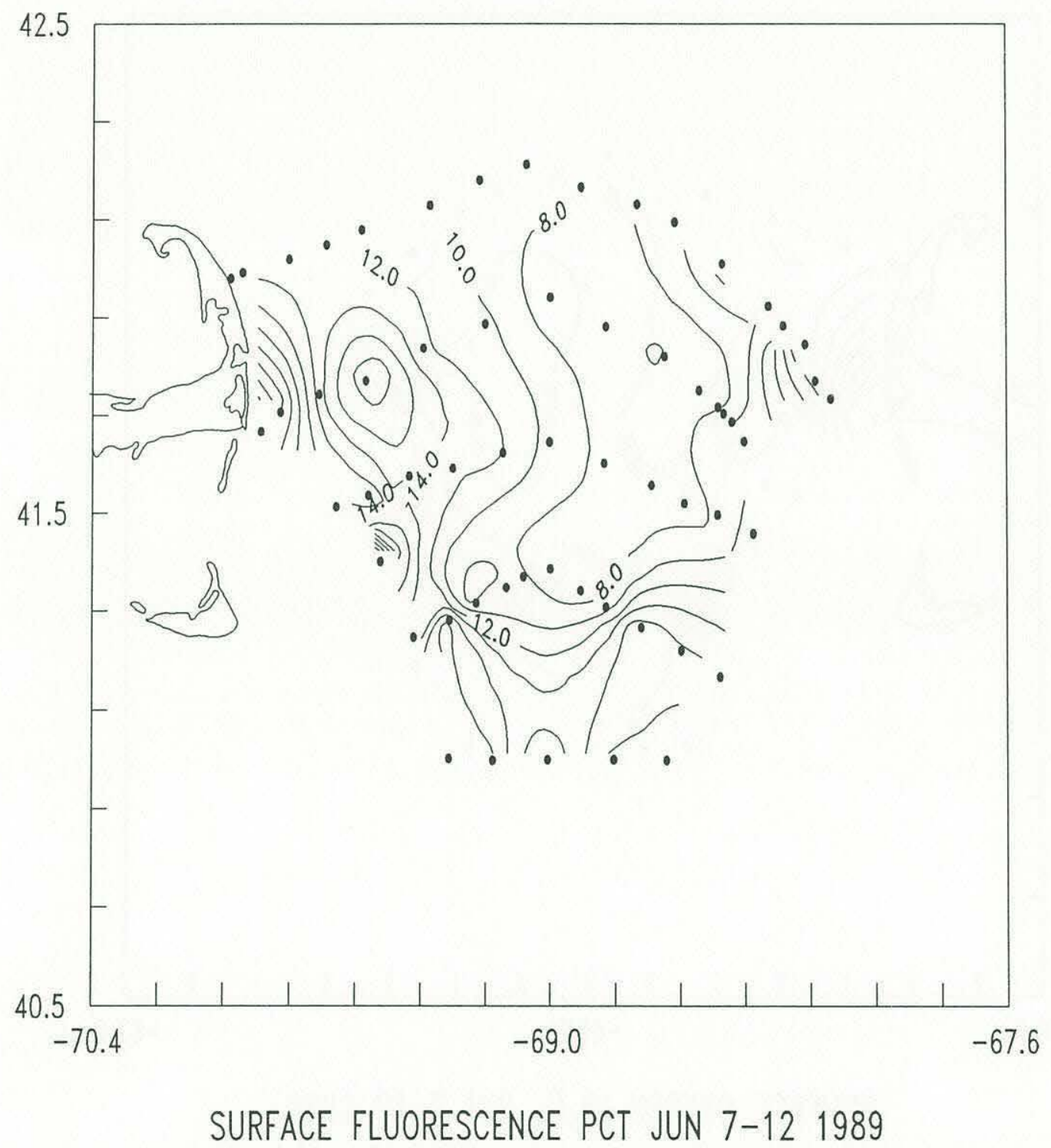




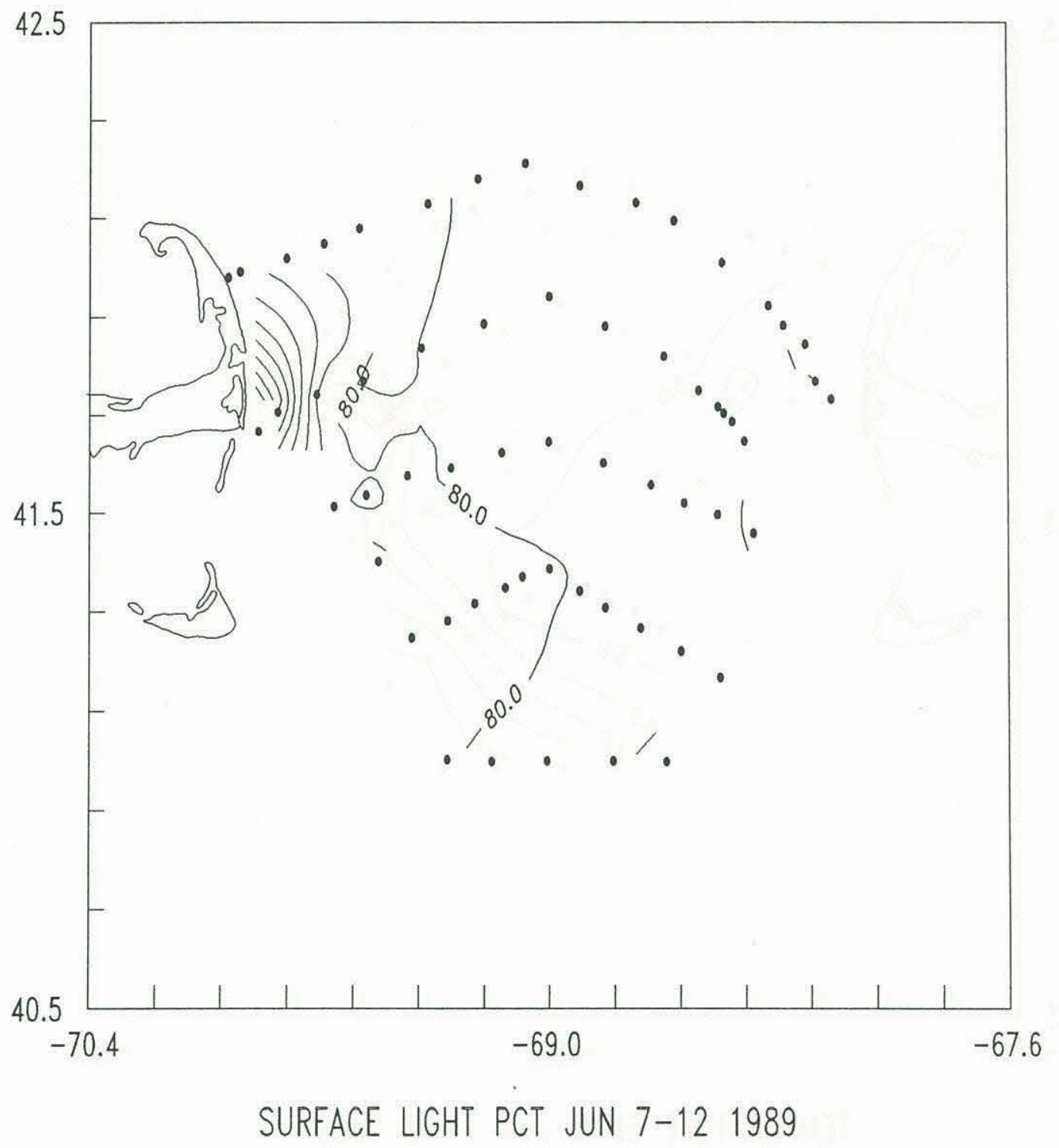




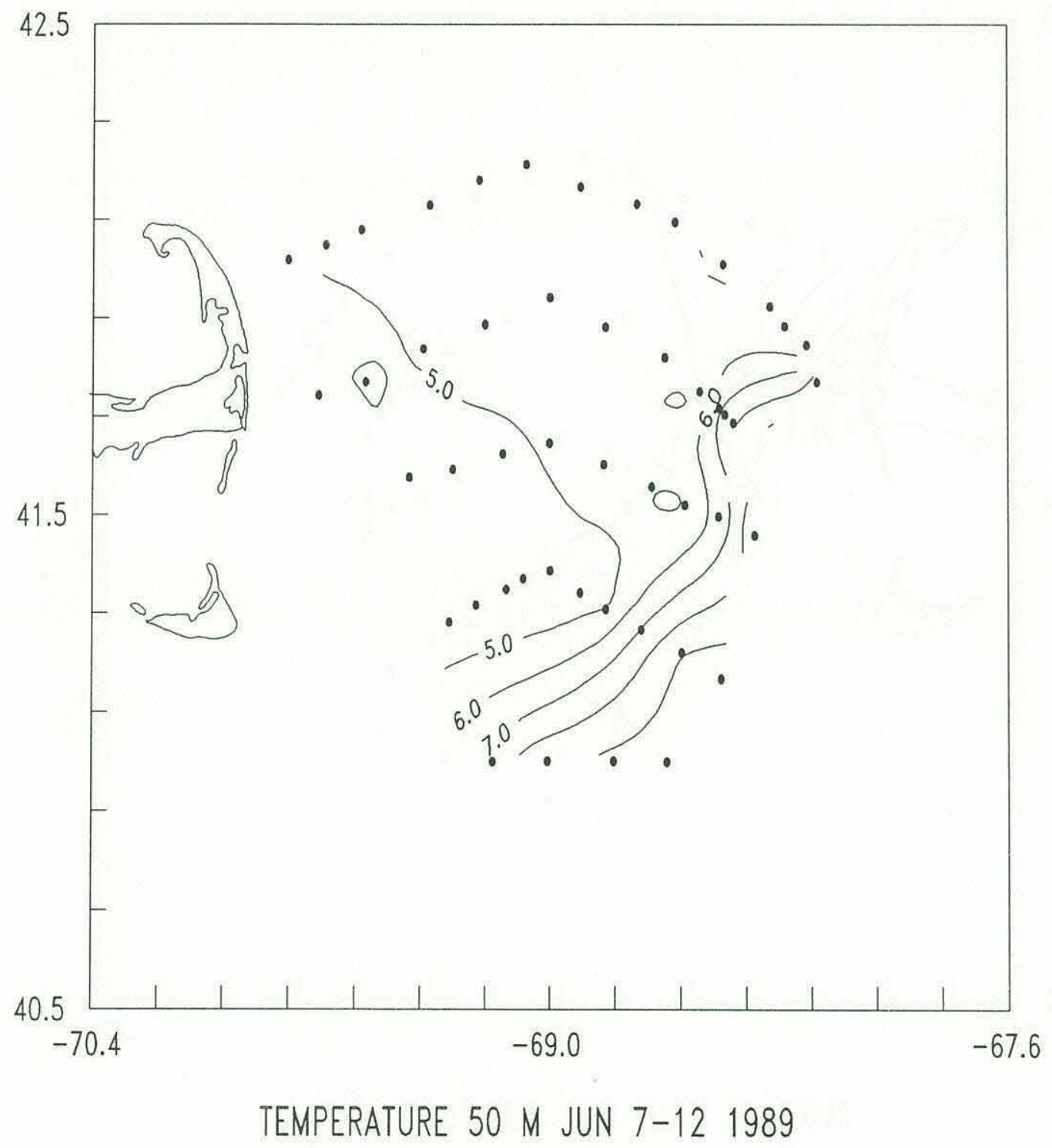




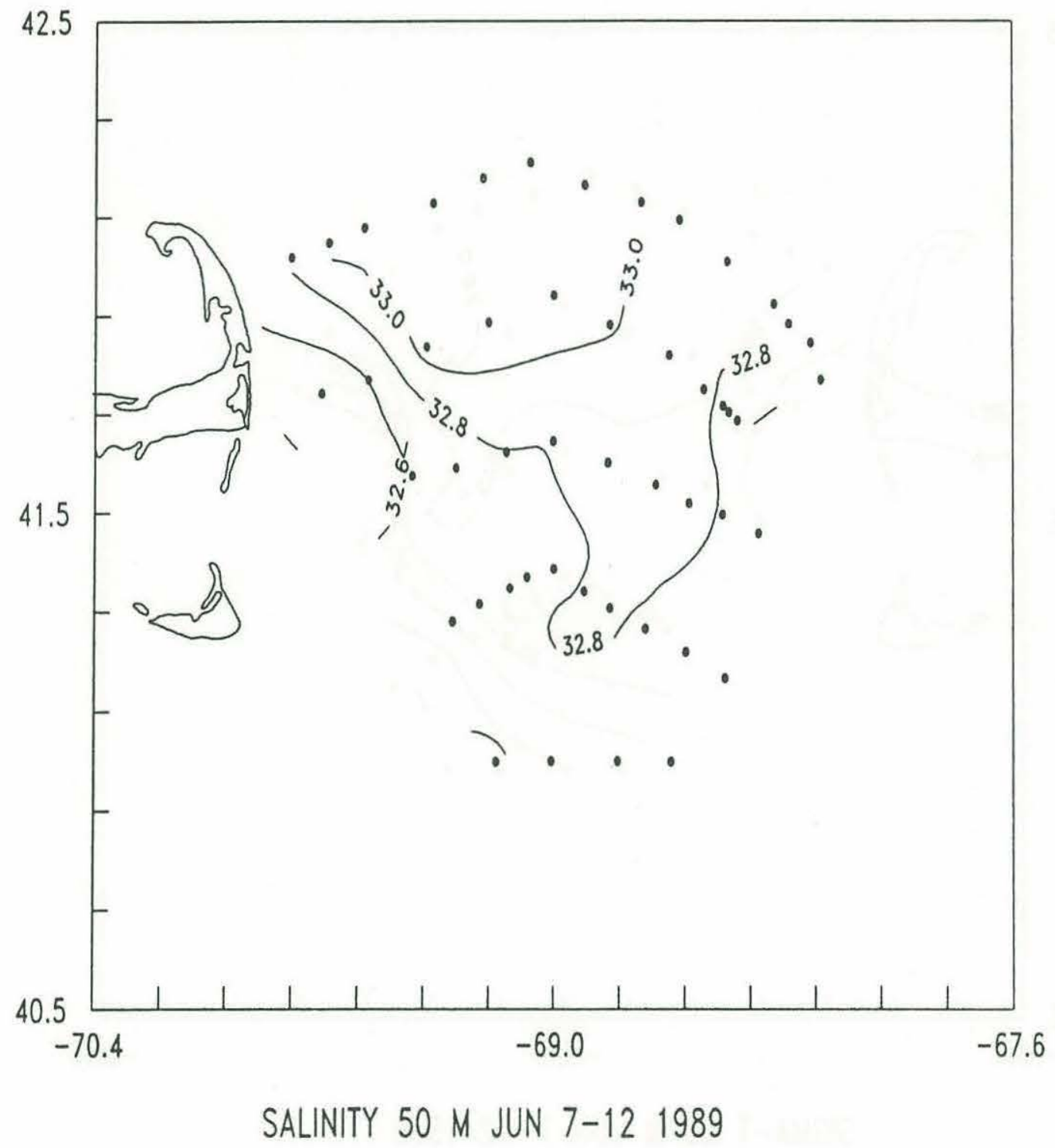




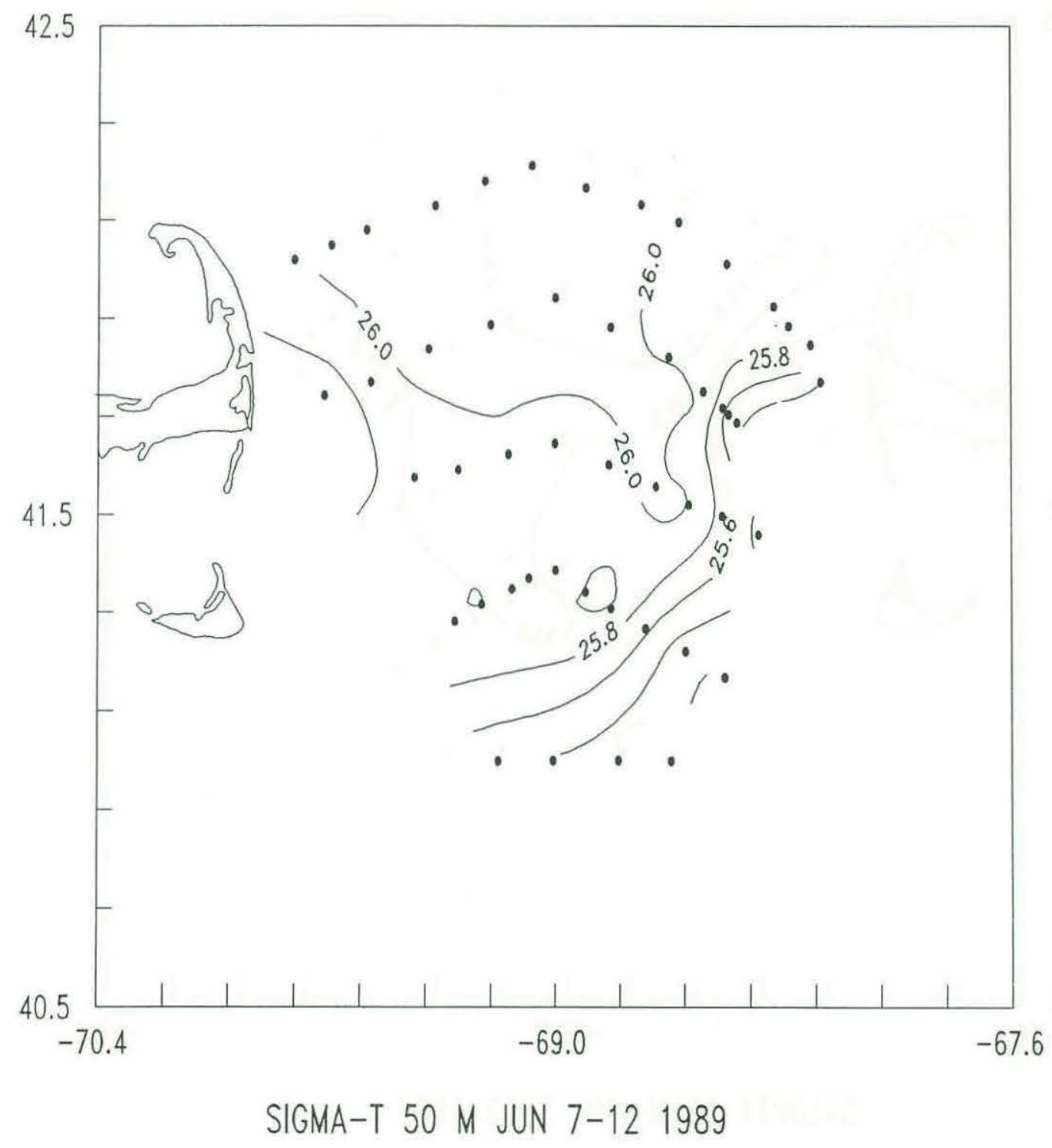




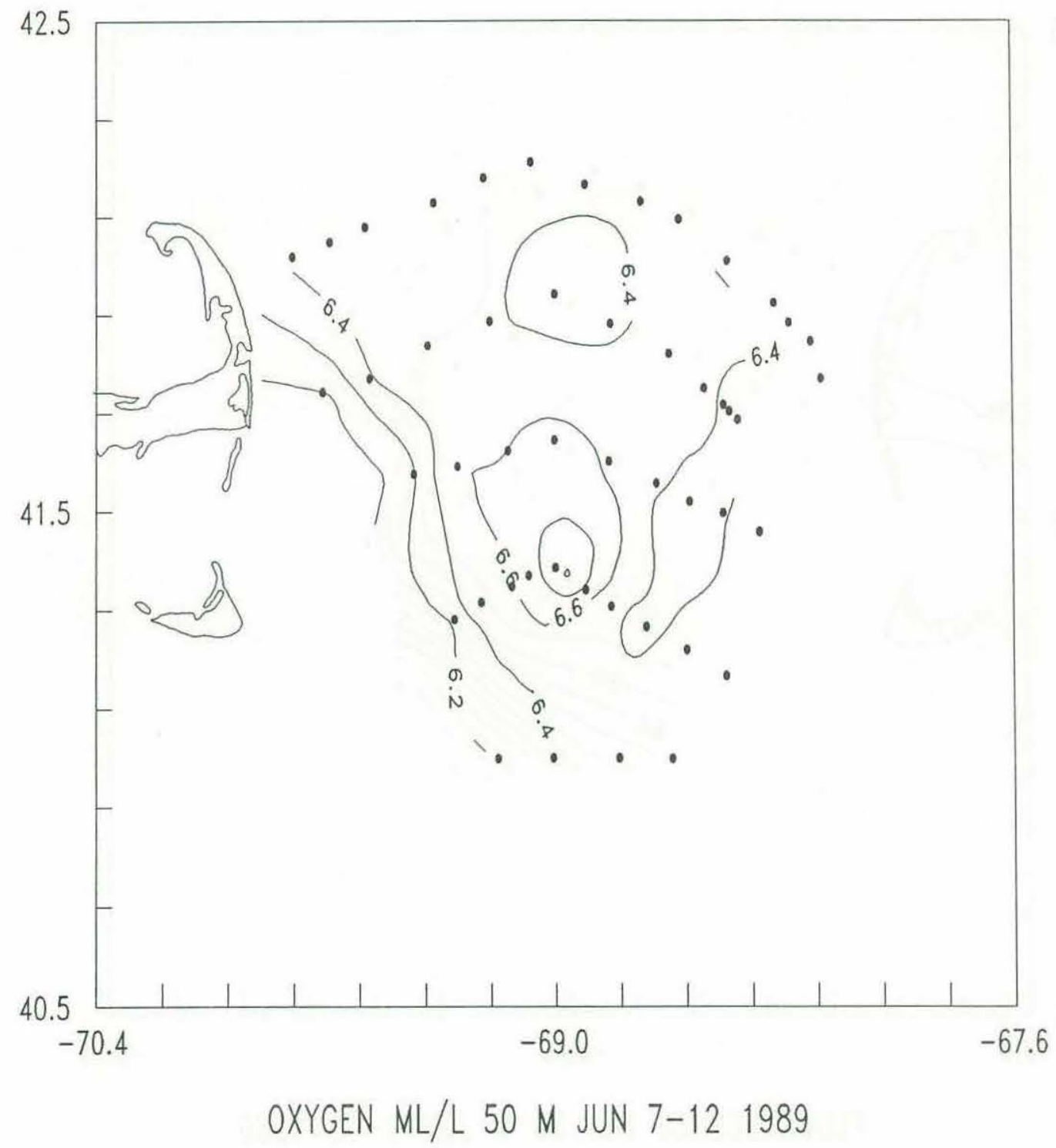




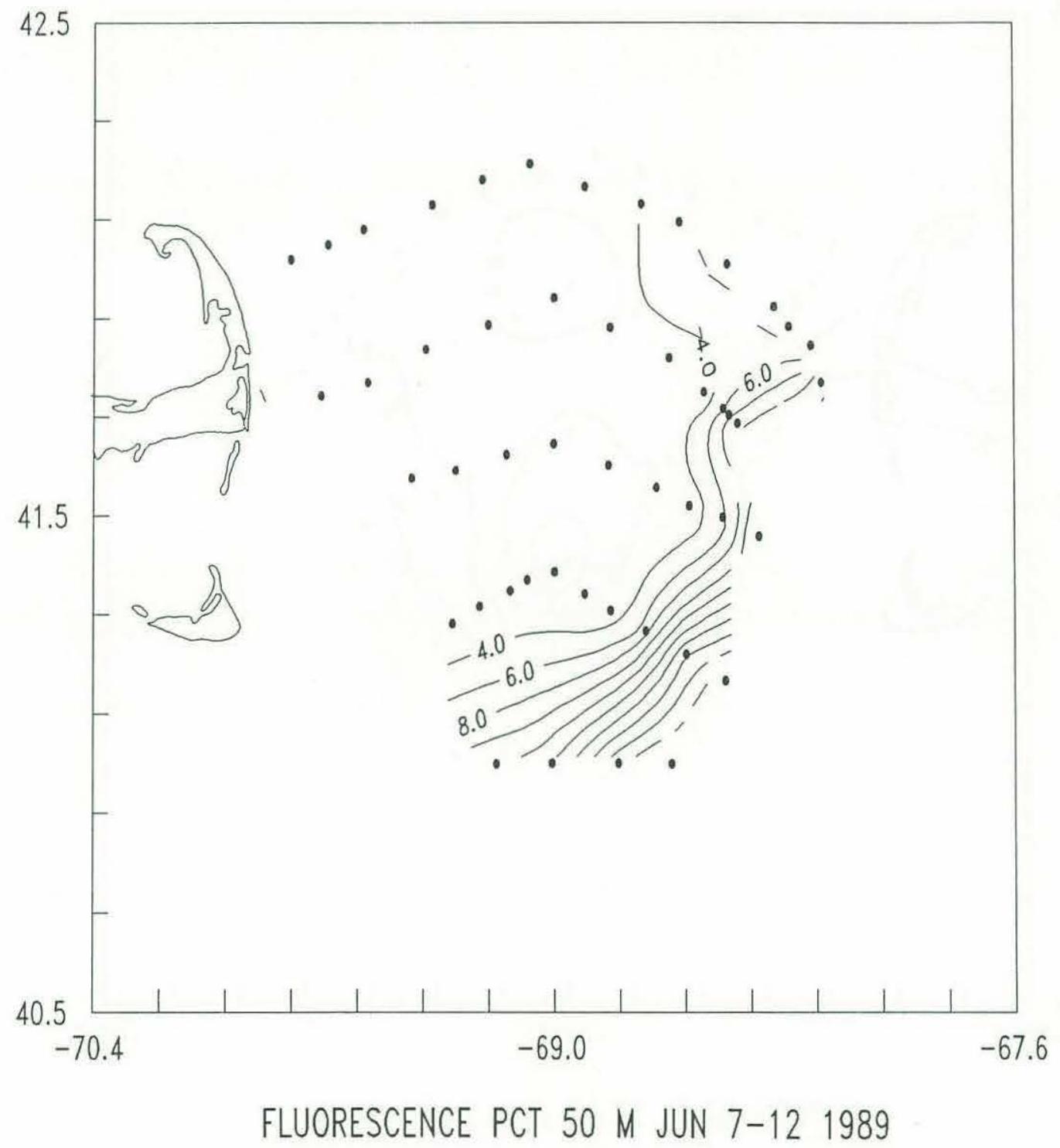




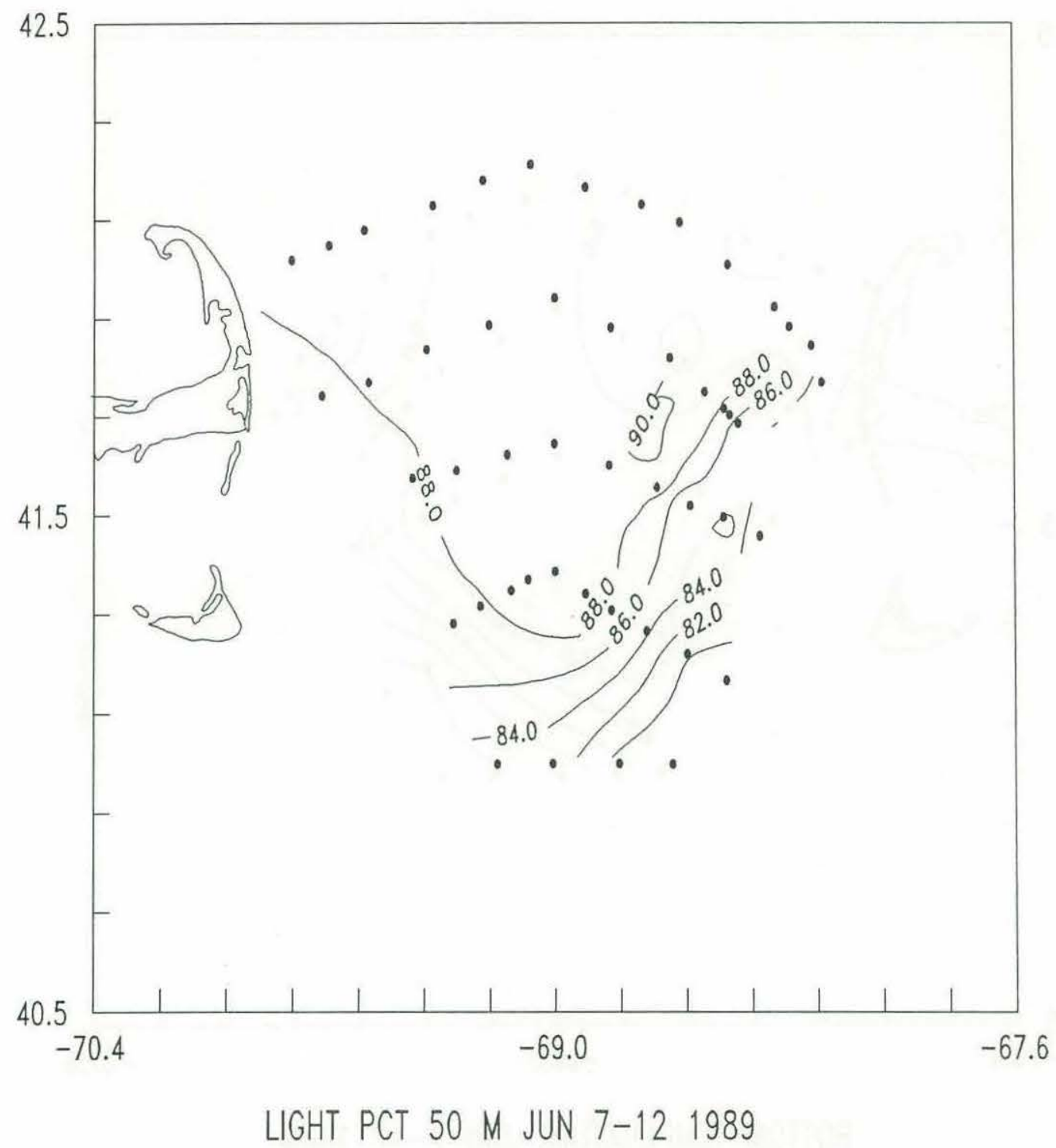




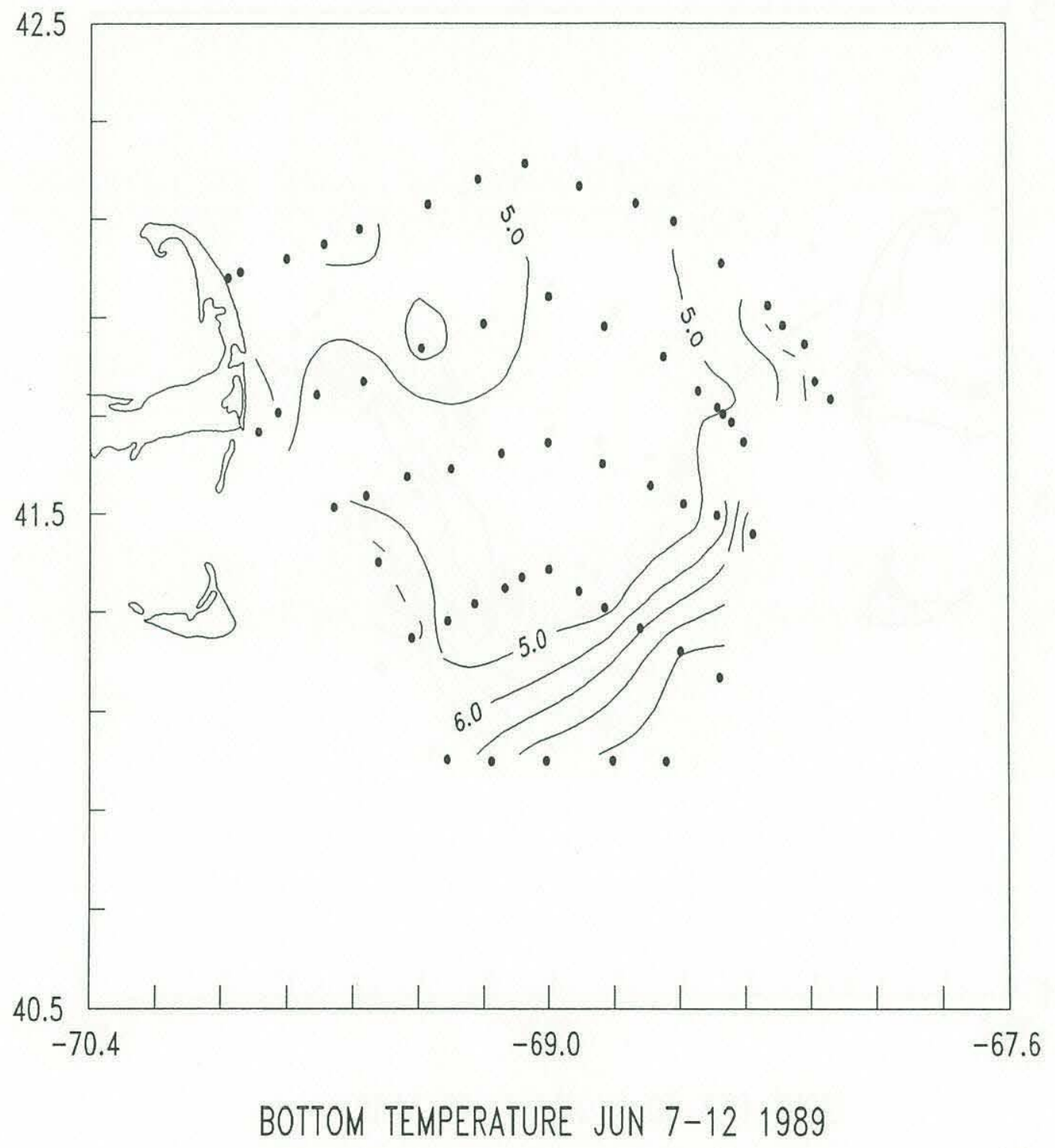




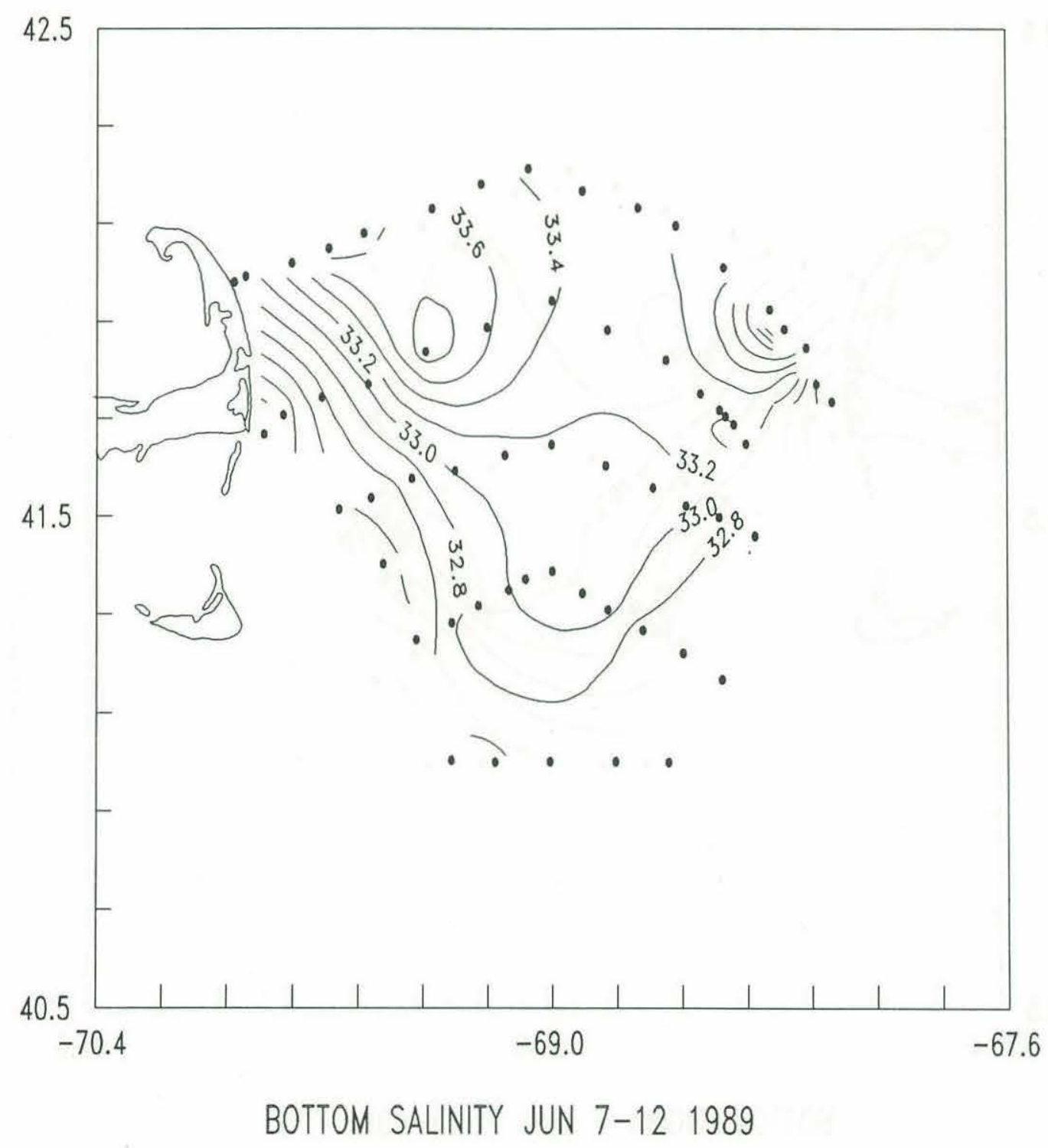




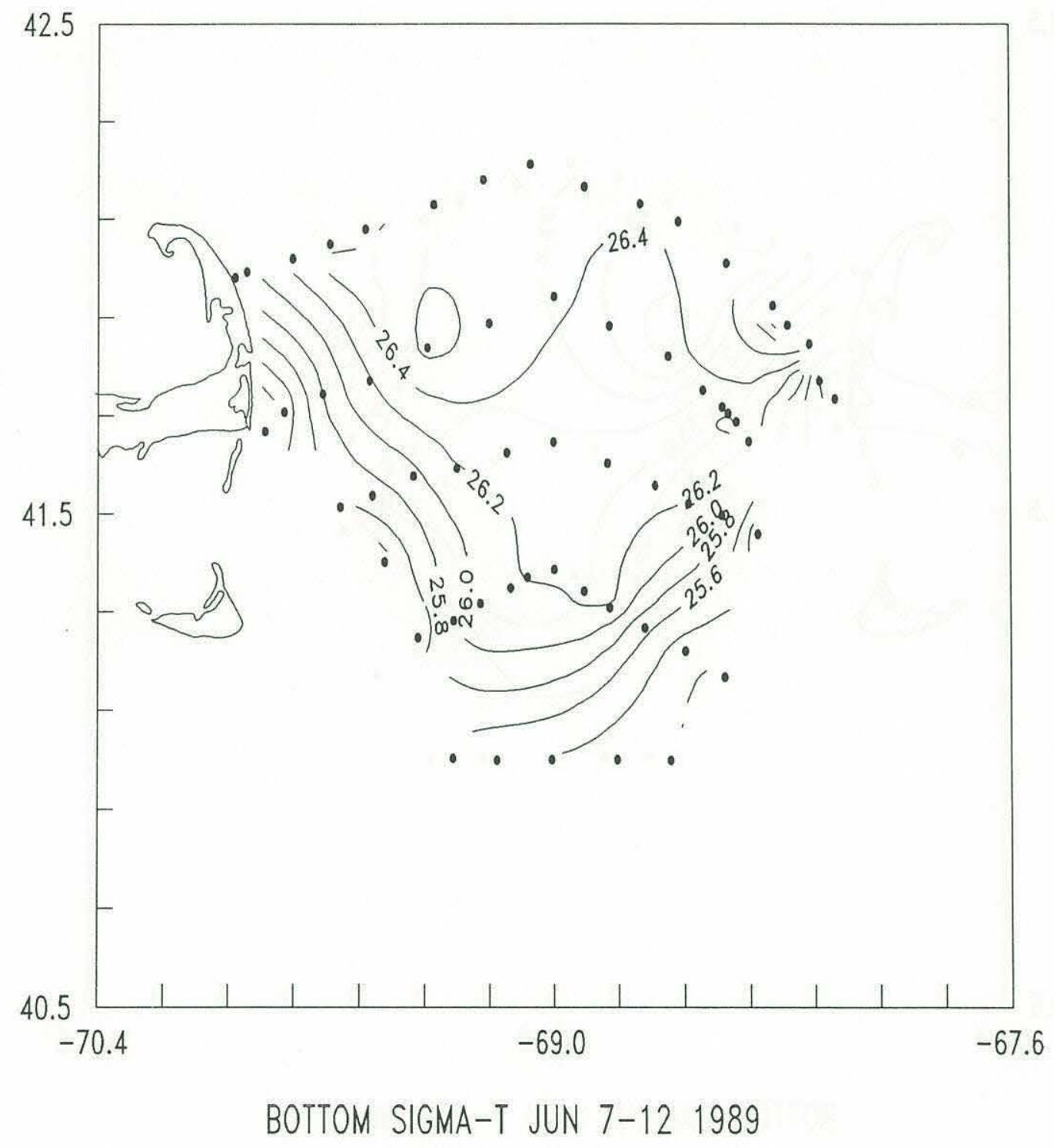




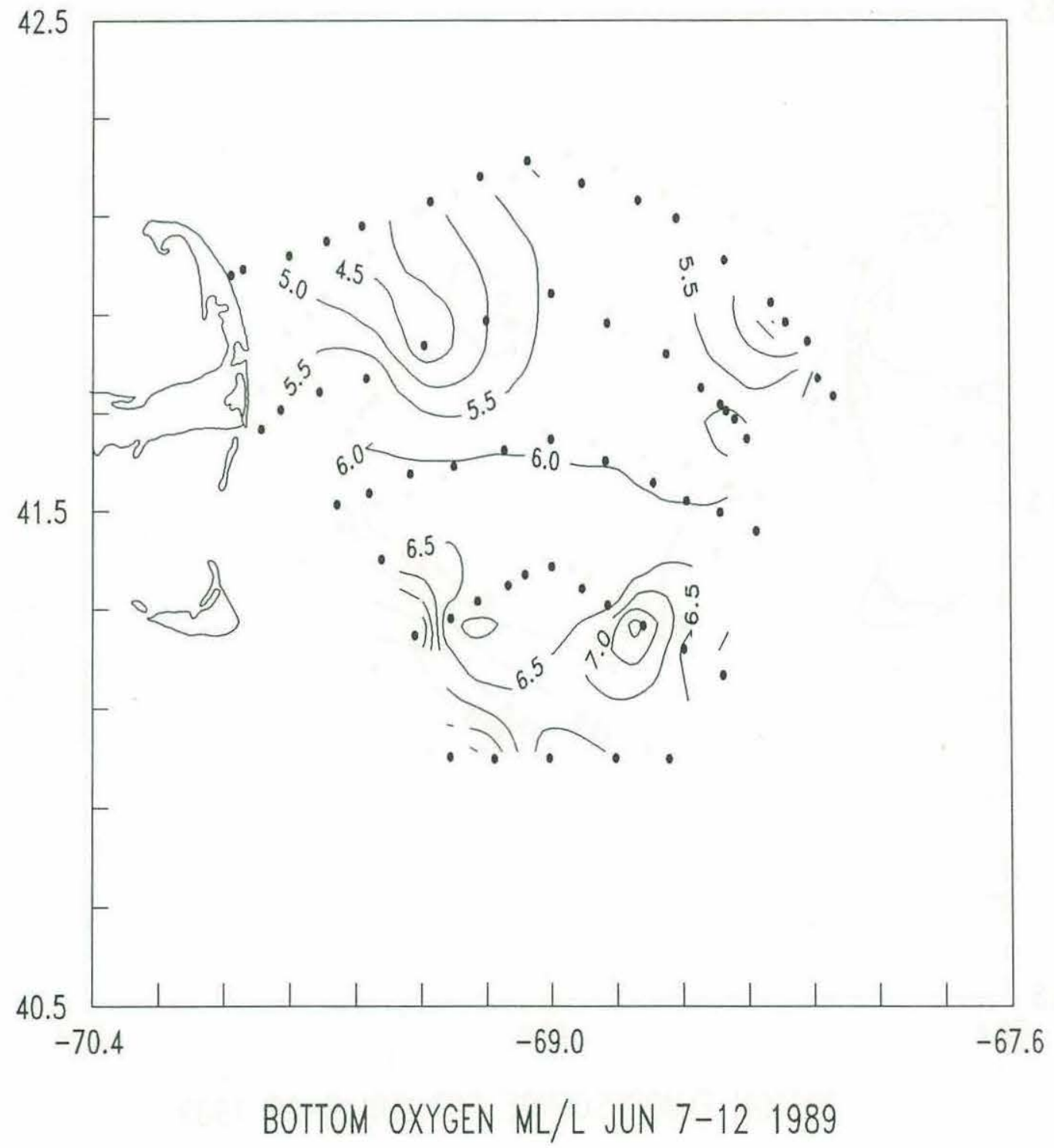




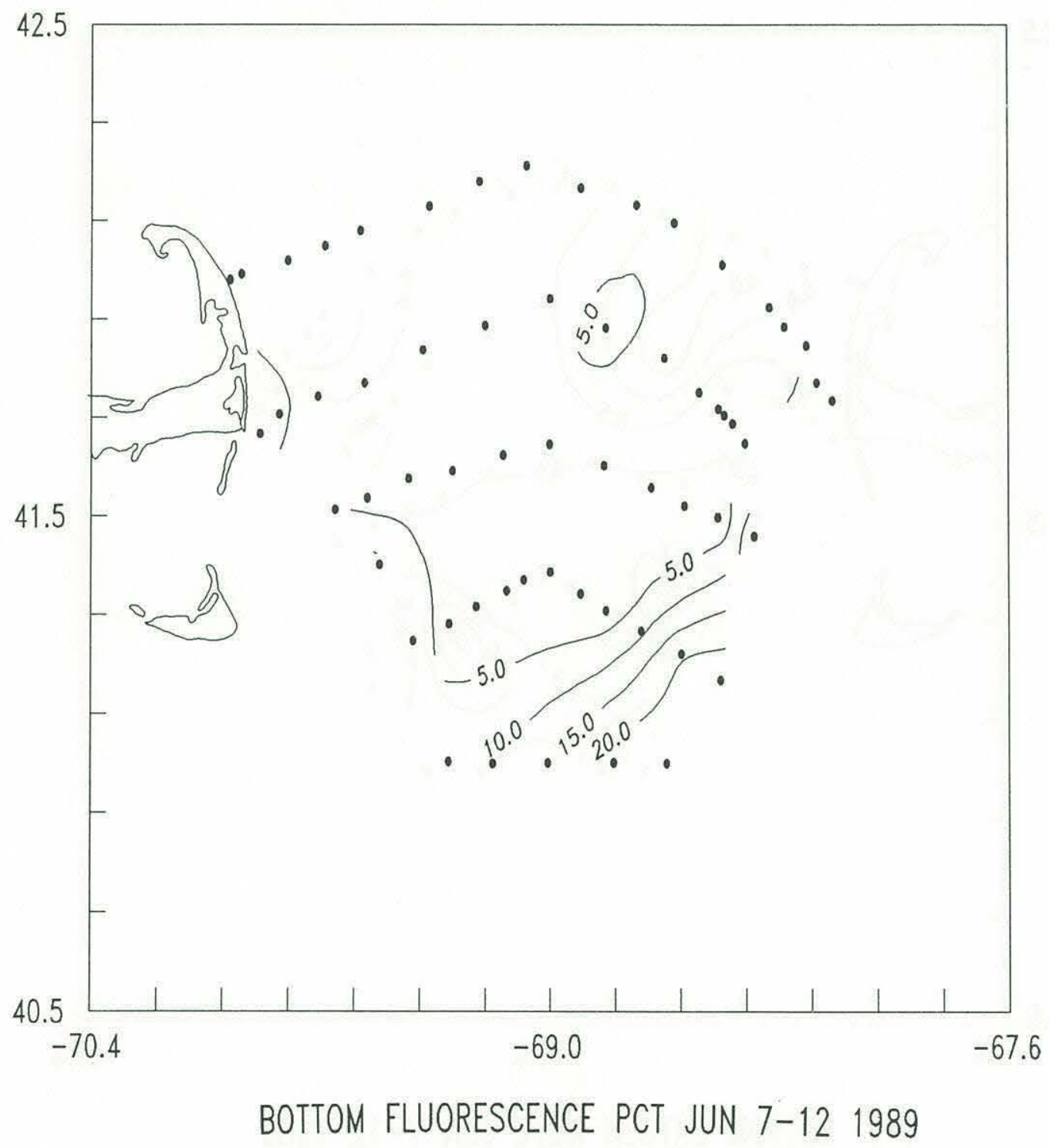




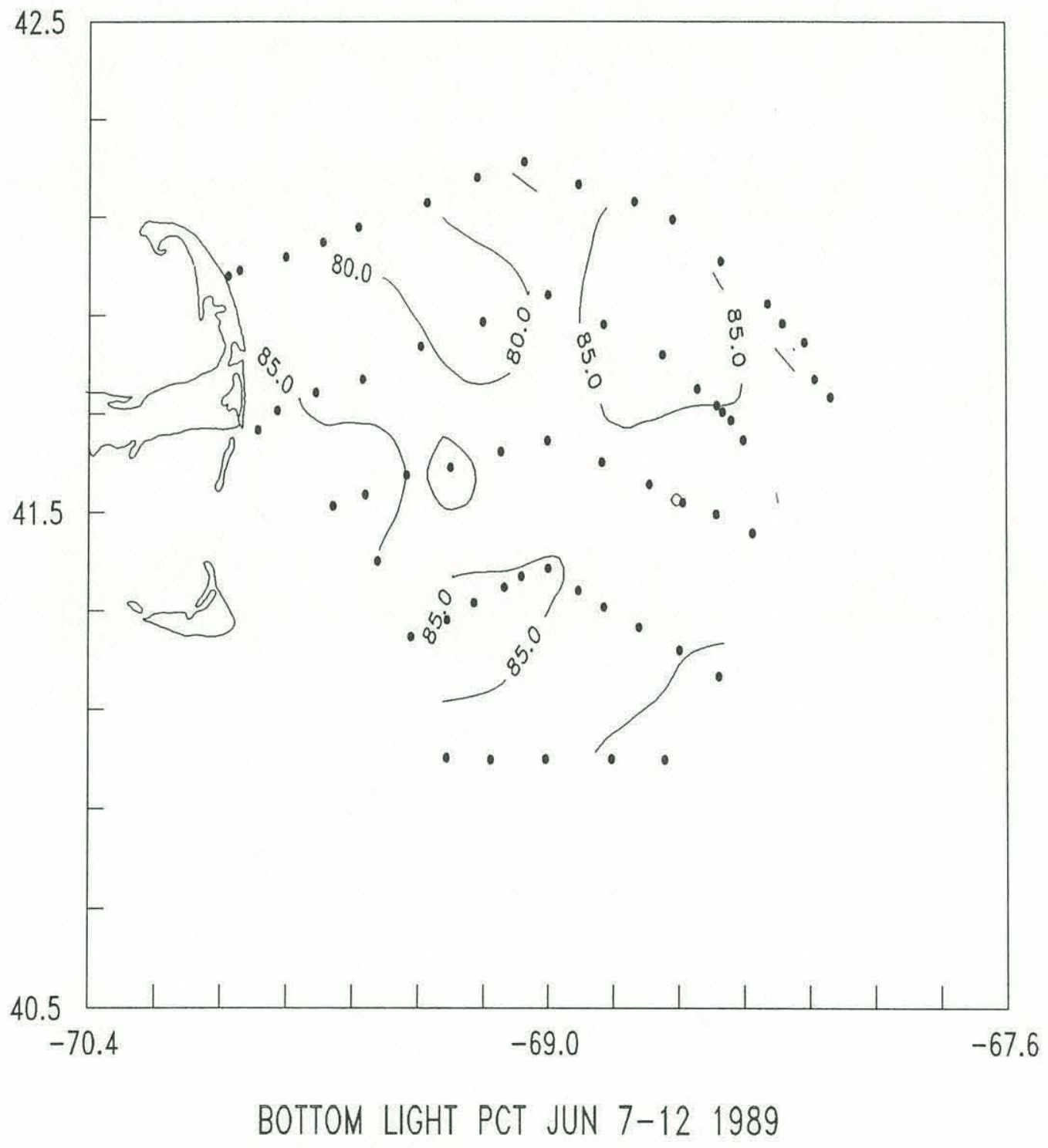


8. Station Listings and Profiles 


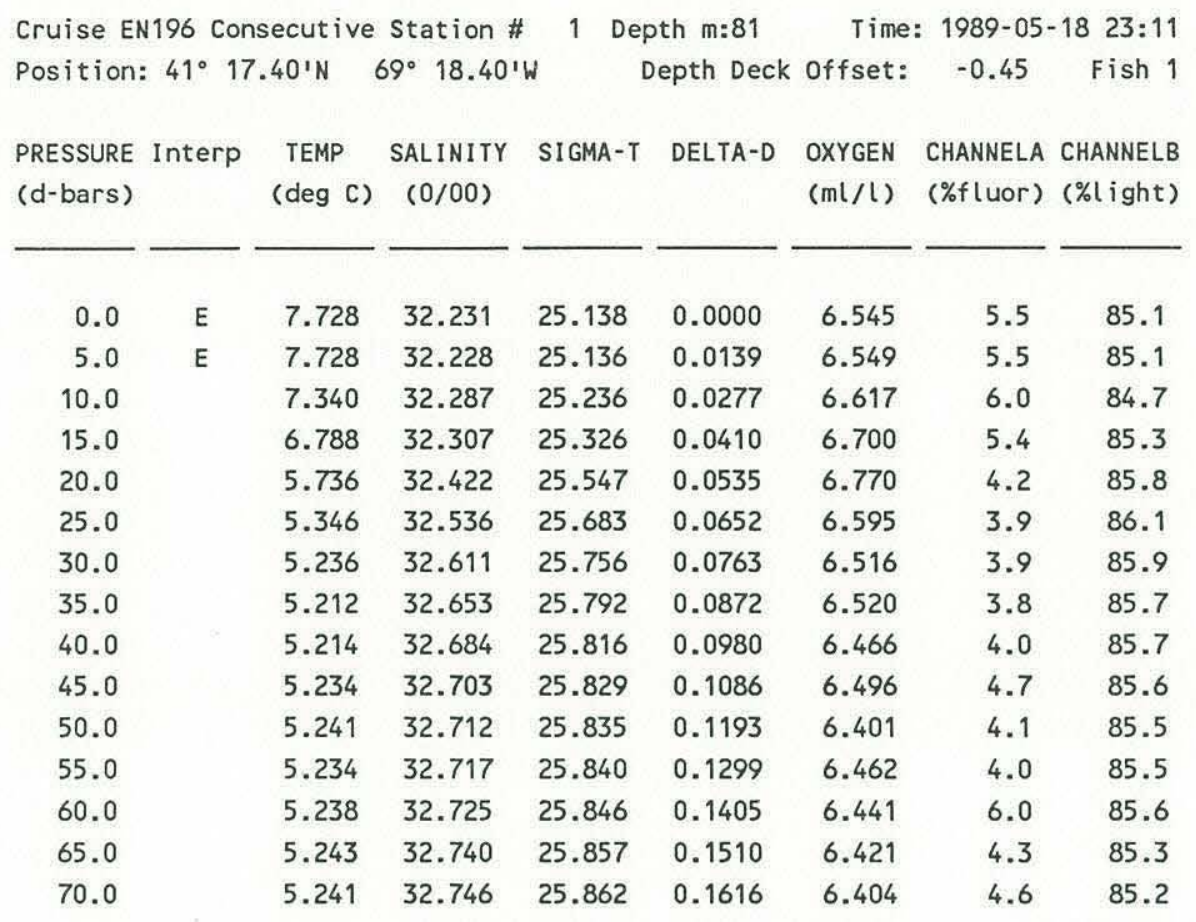
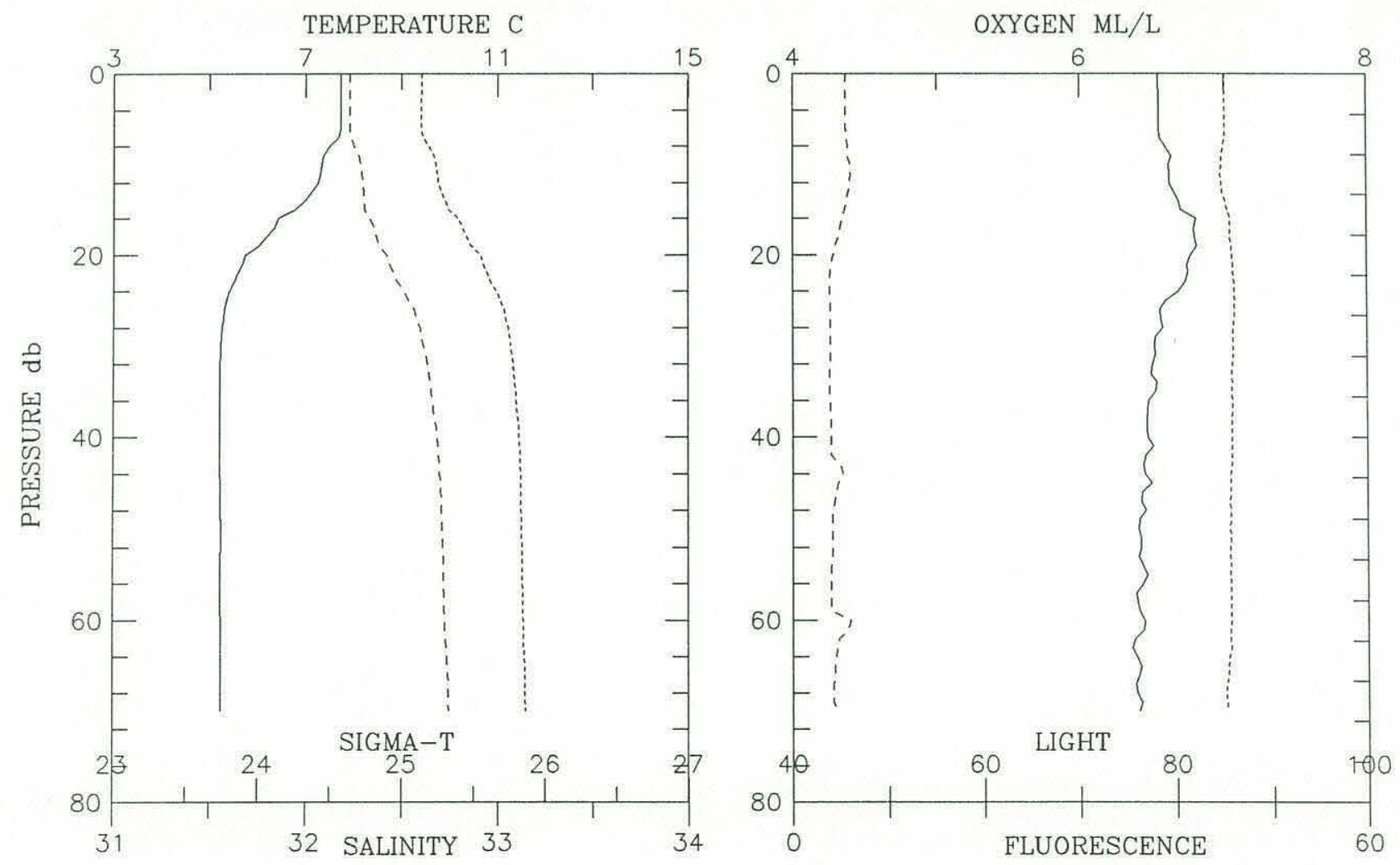


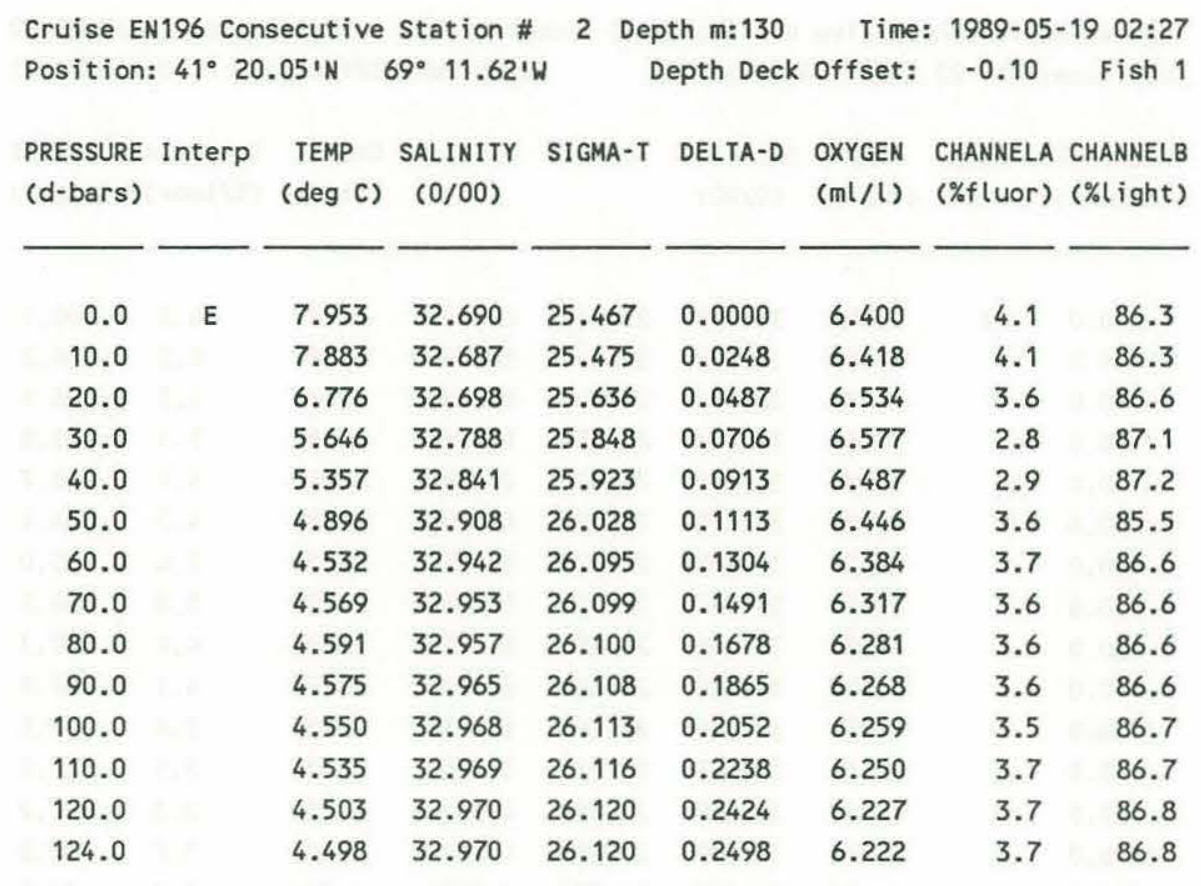

TEMPERATURE C

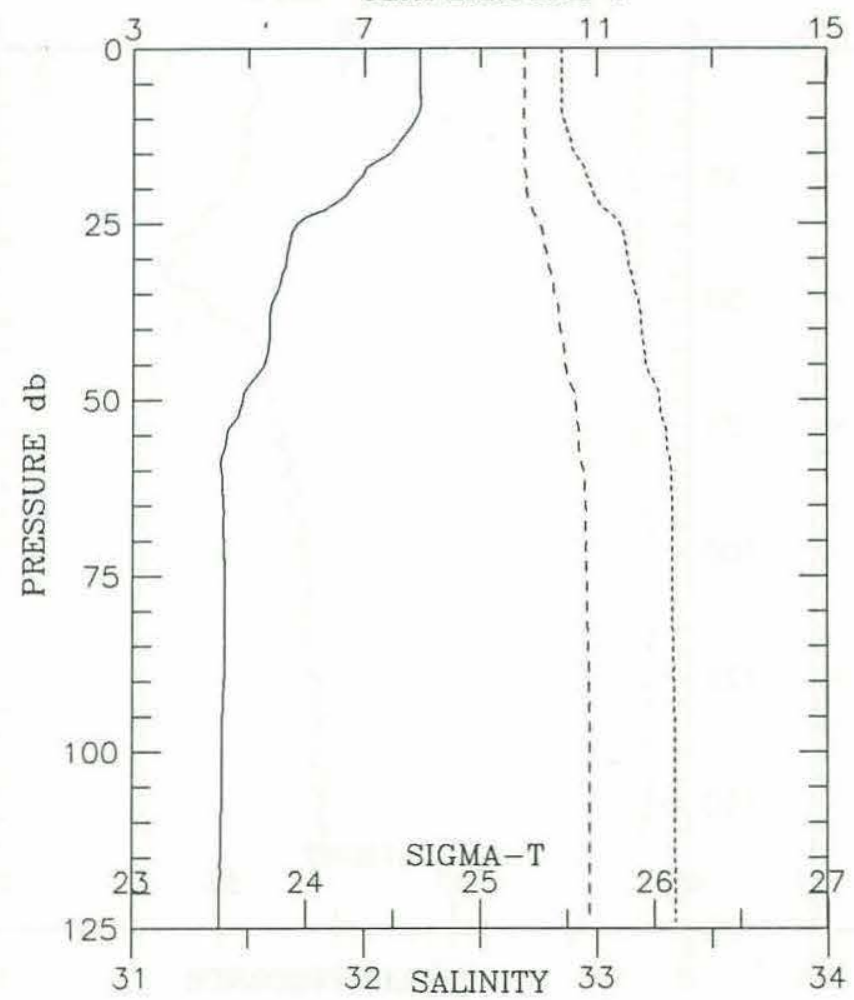

OXYGEN ML/L

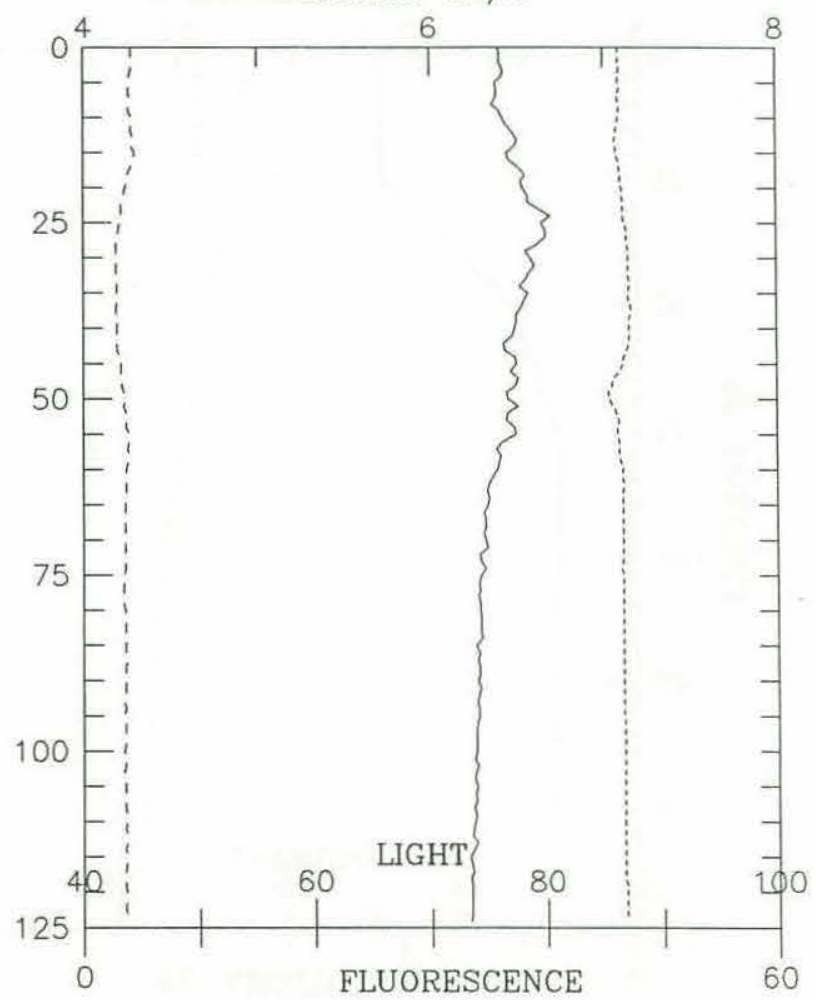




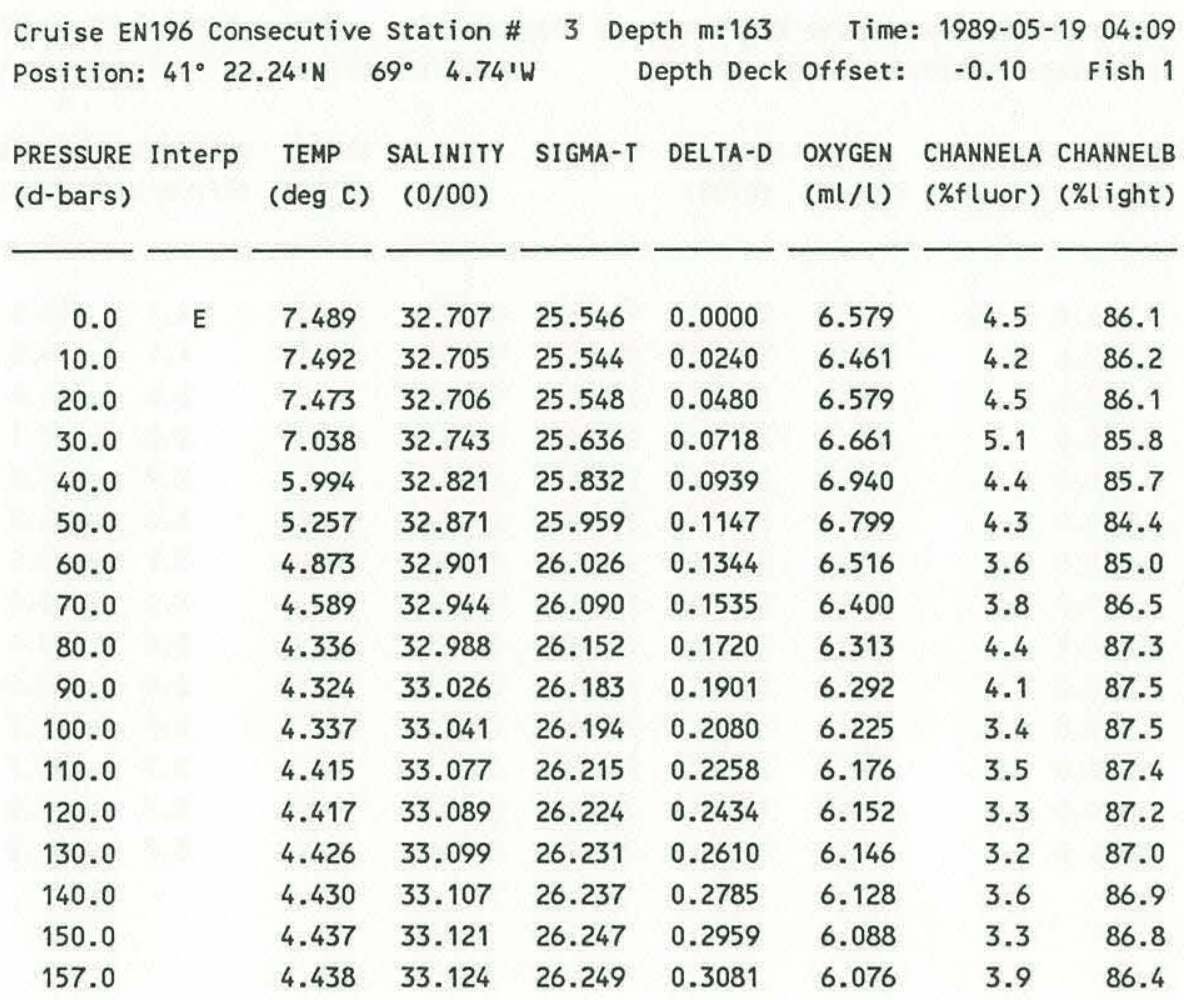
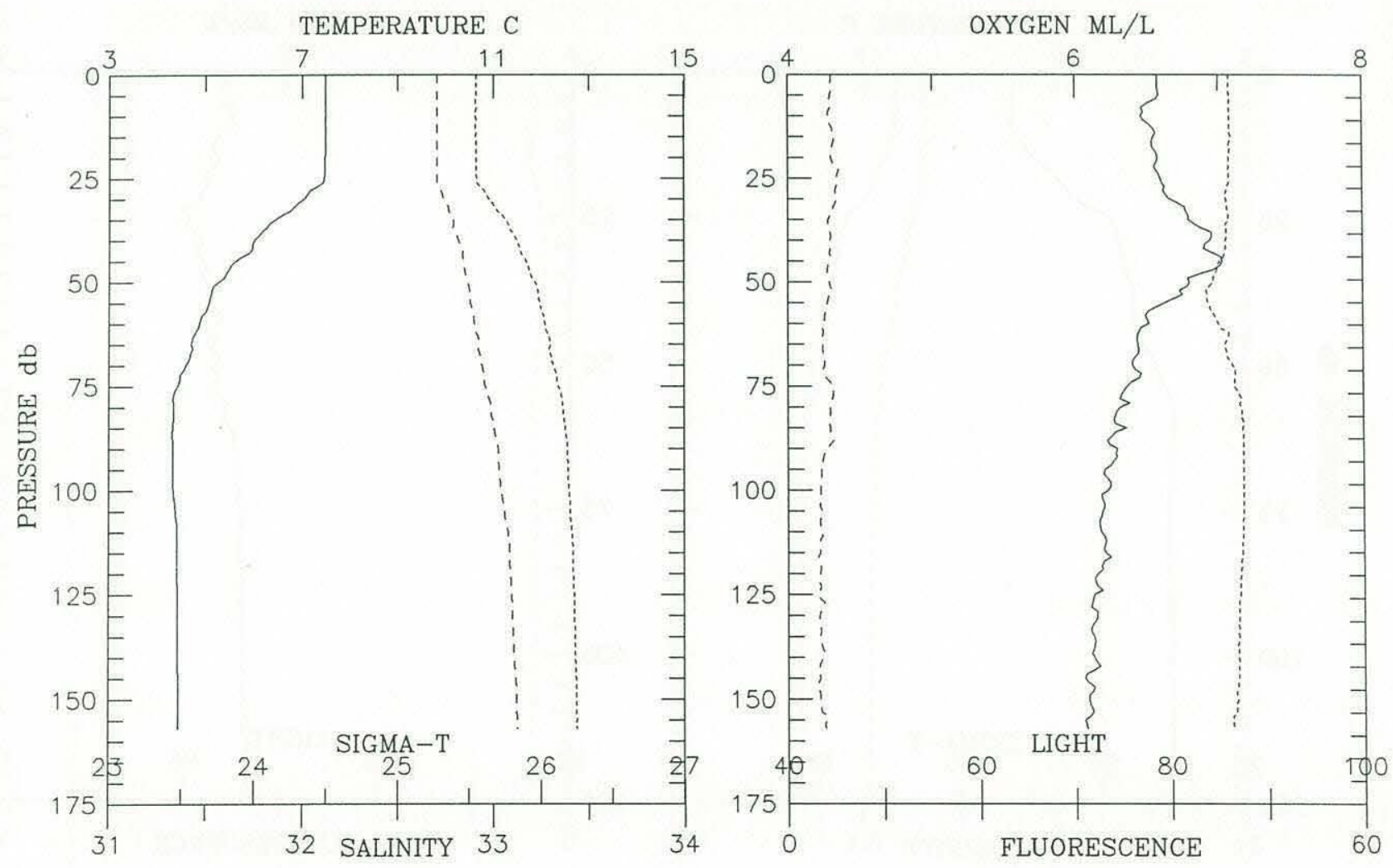


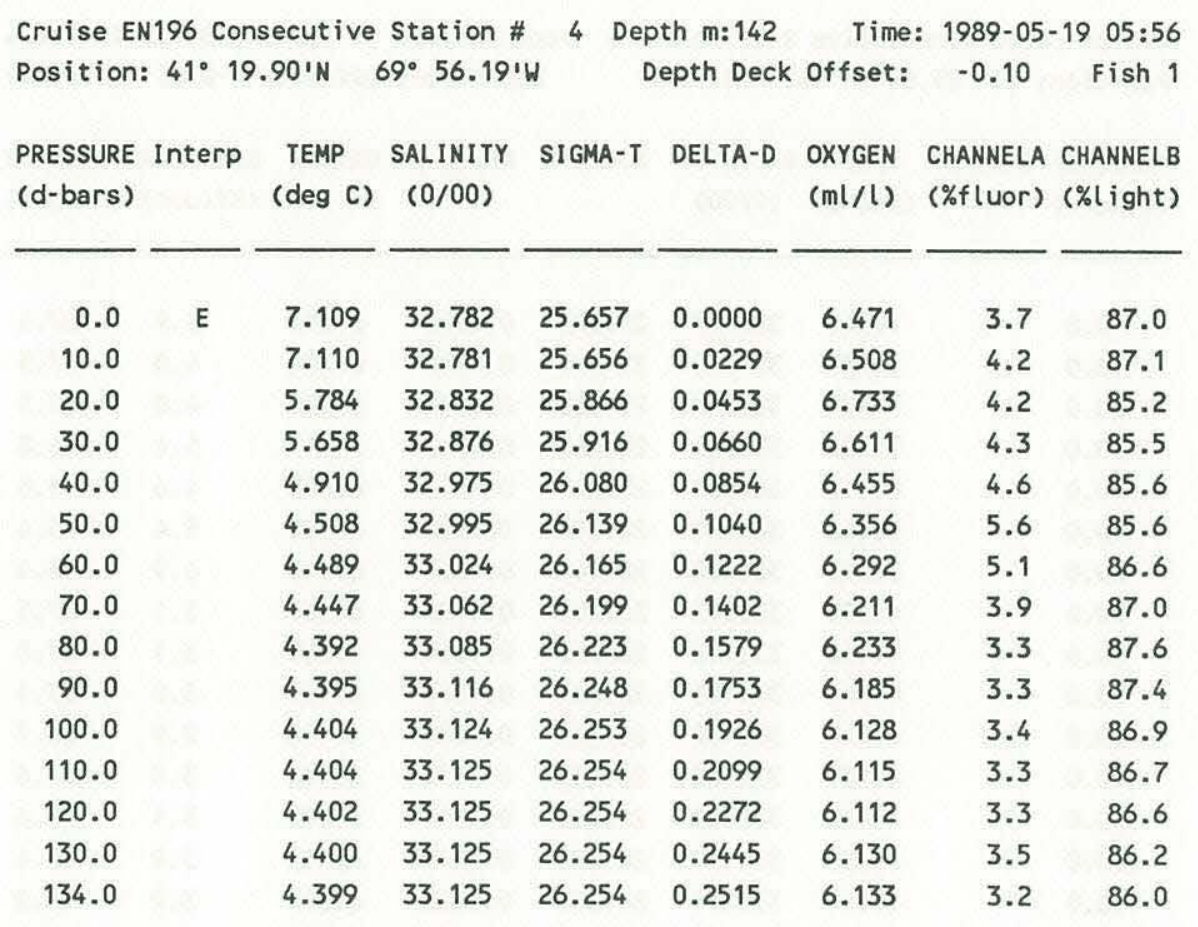
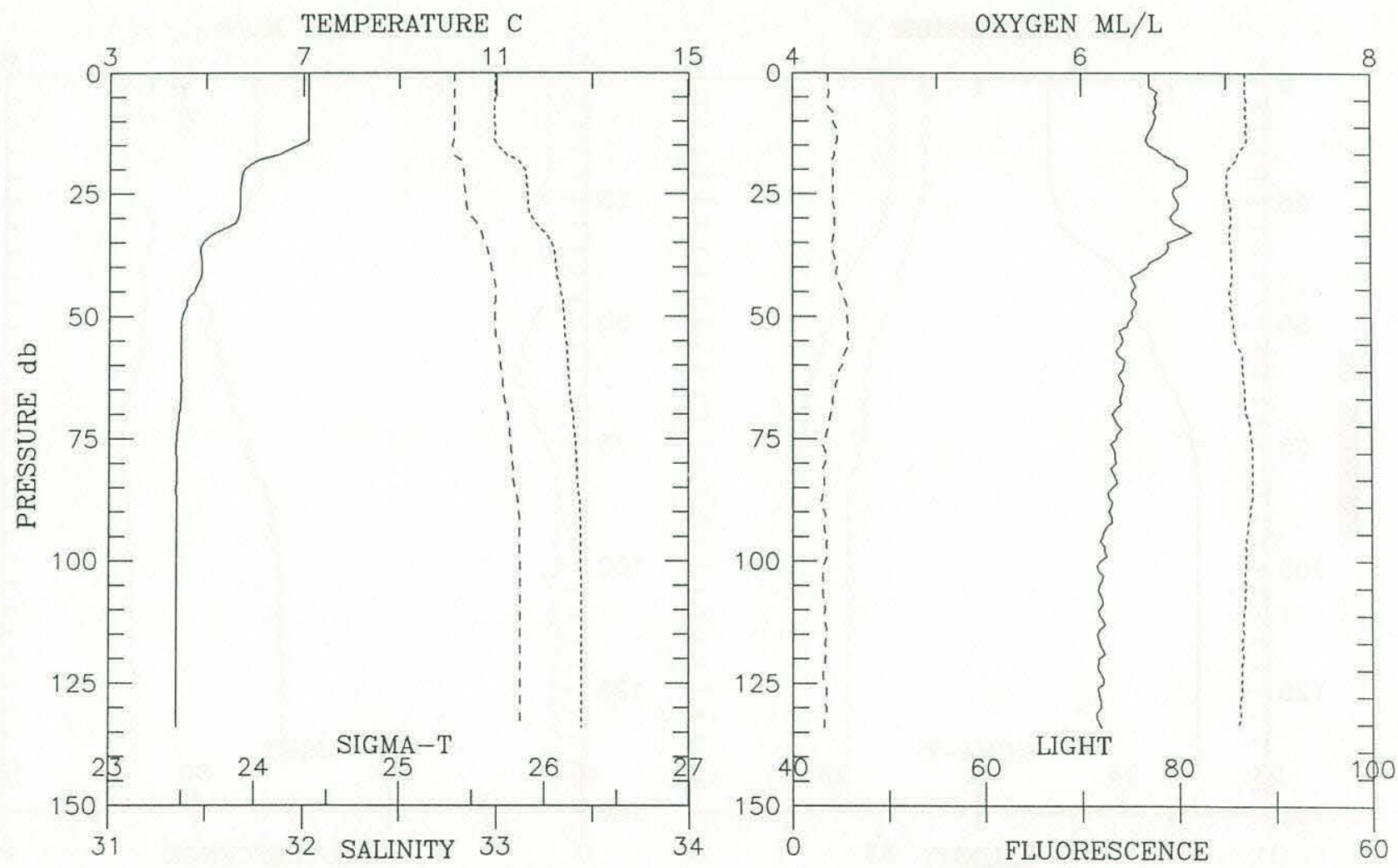
Cruise EN196 Consecutive Station \# 5 Depth m:145 Time: 1989-05-19 11:24 Position: $41^{\circ} 25.60^{\prime} \mathrm{N} \quad 69^{\circ} 51.75^{\prime} \mathrm{W} \quad$ Depth Deck offset: -0.25 Fish 1

\begin{tabular}{|c|c|c|c|c|c|c|c|c|}
\hline $\begin{array}{l}\text { PRESSURE } \\
\text { (d-bars) }\end{array}$ & Interp & $\begin{array}{c}\text { TEMP } \\
(\operatorname{deg} \mathrm{C})\end{array}$ & $\begin{array}{l}\text { SALINITY } \\
(0 / 00)\end{array}$ & SIGMA-T & DELTA-D & $\begin{array}{l}\text { OXYGEN } \\
(m l / l)\end{array}$ & $\begin{array}{l}\text { CHANNELA } \\
\text { (\%fluor) }\end{array}$ & $\begin{array}{l}\text { CHANNELB } \\
\text { (\%light) }\end{array}$ \\
\hline 0.0 & E & 7.549 & 32.777 & 25.593 & 0.0000 & 6.296 & 3.9 & 87.5 \\
\hline 10.0 & & 7.537 & 32.776 & 25.594 & 0.0235 & 6.278 & 4.0 & 87.5 \\
\hline 20.0 & & 7.495 & 32.780 & 25.602 & 0.0471 & 6.260 & 4.0 & 87.3 \\
\hline 30.0 & & 7.284 & 32.826 & 25.668 & 0.0703 & 6.417 & 5.4 & 84.8 \\
\hline 40.0 & & 6.012 & 32.901 & 25.893 & 0.0920 & 6.695 & 4.6 & 85.8 \\
\hline 50.0 & & 5.668 & 33.001 & 26.014 & 0.1120 & 6.581 & 5.4 & 85.4 \\
\hline 60.0 & & 5.093 & 33.082 & 26.144 & 0.1308 & 6.519 & 6.9 & 86.4 \\
\hline 70.0 & & 4.653 & 33.093 & 26.202 & 0.1489 & 6.366 & 5.1 & 87.5 \\
\hline 80.0 & & 4.468 & 33.148 & 26.265 & 0.1664 & 6.265 & 3.1 & 87.8 \\
\hline 90.0 & & 4.451 & 33.166 & 26.281 & 0.1834 & 6.153 & 3.0 & 87.1 \\
\hline 100.0 & & 4.442 & 33.169 & 26.285 & 0.2004 & 6.118 & 2.9 & 86.7 \\
\hline 110.0 & & 4.439 & 33.169 & 26.285 & 0.2174 & 6.112 & 3.0 & 86.6 \\
\hline 120.0 & & 4.437 & 33.170 & 26.286 & 0.2344 & 6.075 & 3.1 & 86.6 \\
\hline 130.0 & & 4.439 & 33.170 & 26.286 & 0.2514 & 6.063 & 3.0 & 86.4 \\
\hline 140.0 & & 4.441 & 33.171 & 26.286 & 0.2685 & 6.065 & 3.2 & 86.2 \\
\hline 141.0 & & 4.442 & 33.171 & 26.286 & 0.2702 & 6.053 & 3.3 & 86.2 \\
\hline
\end{tabular}
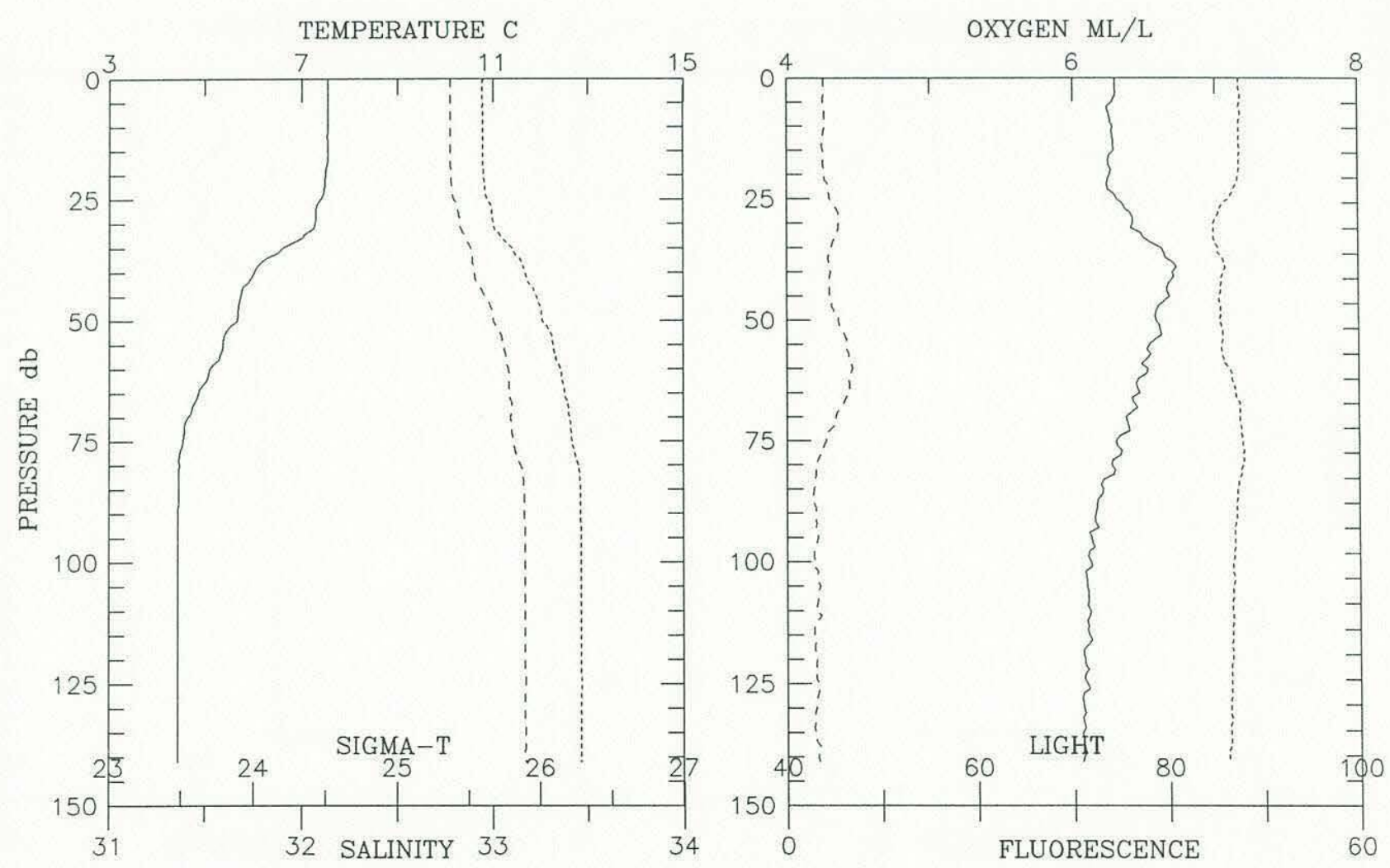


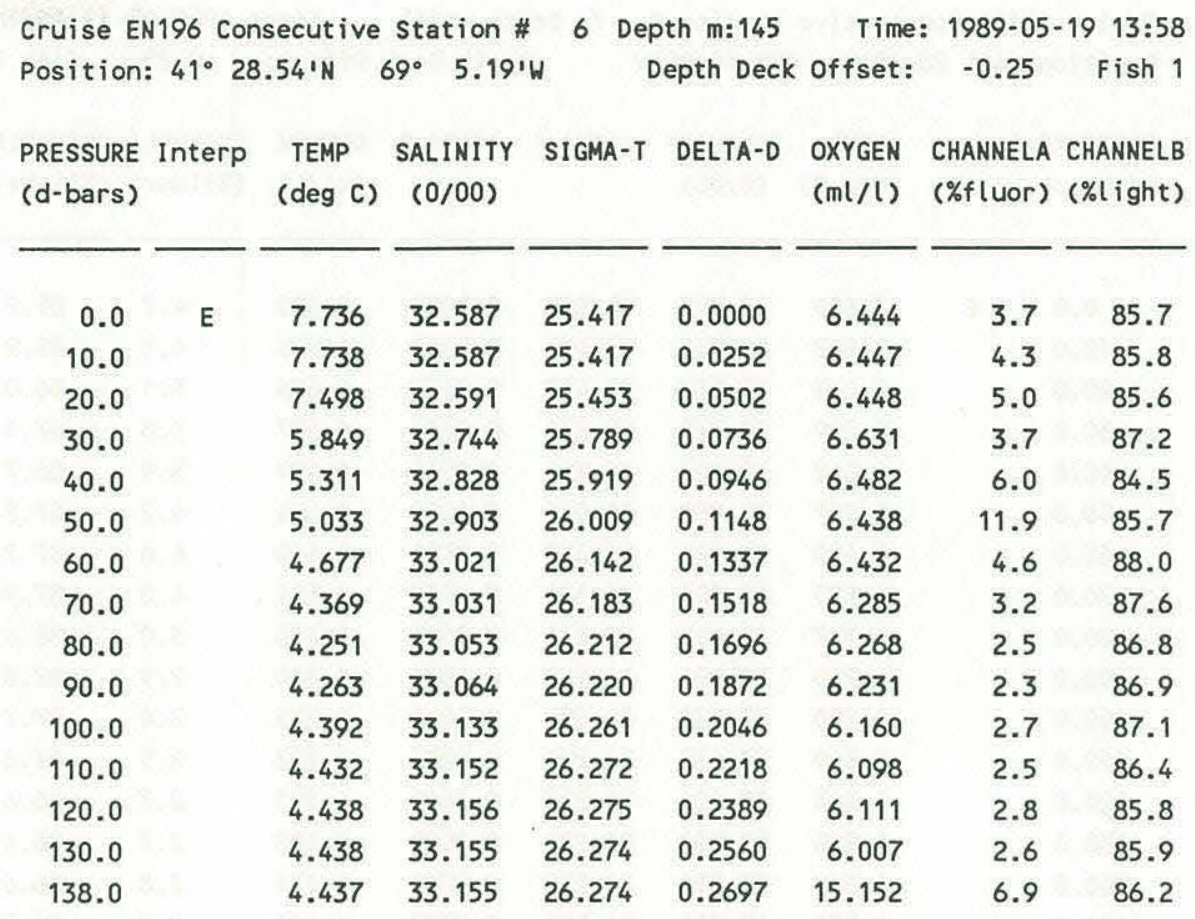
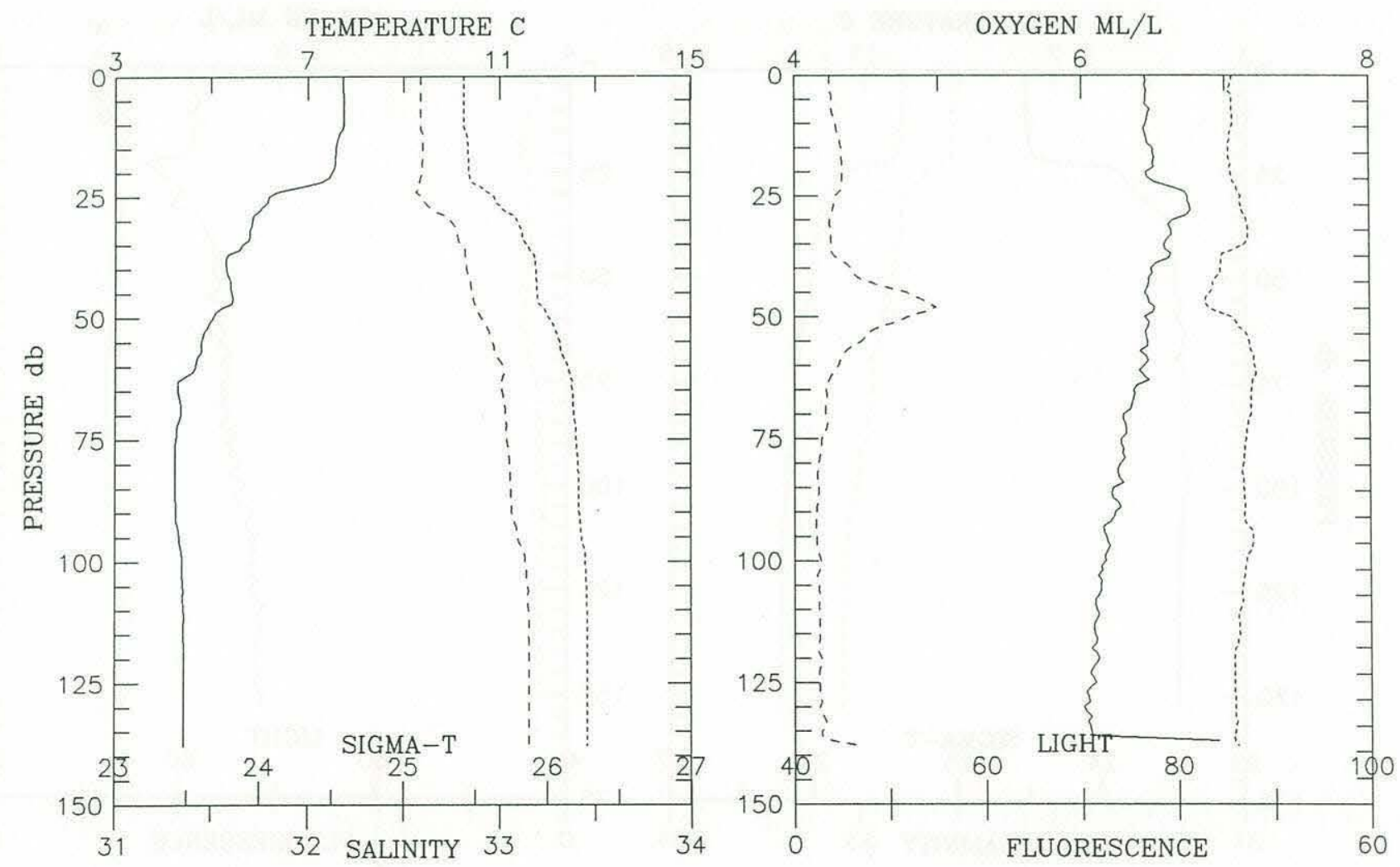


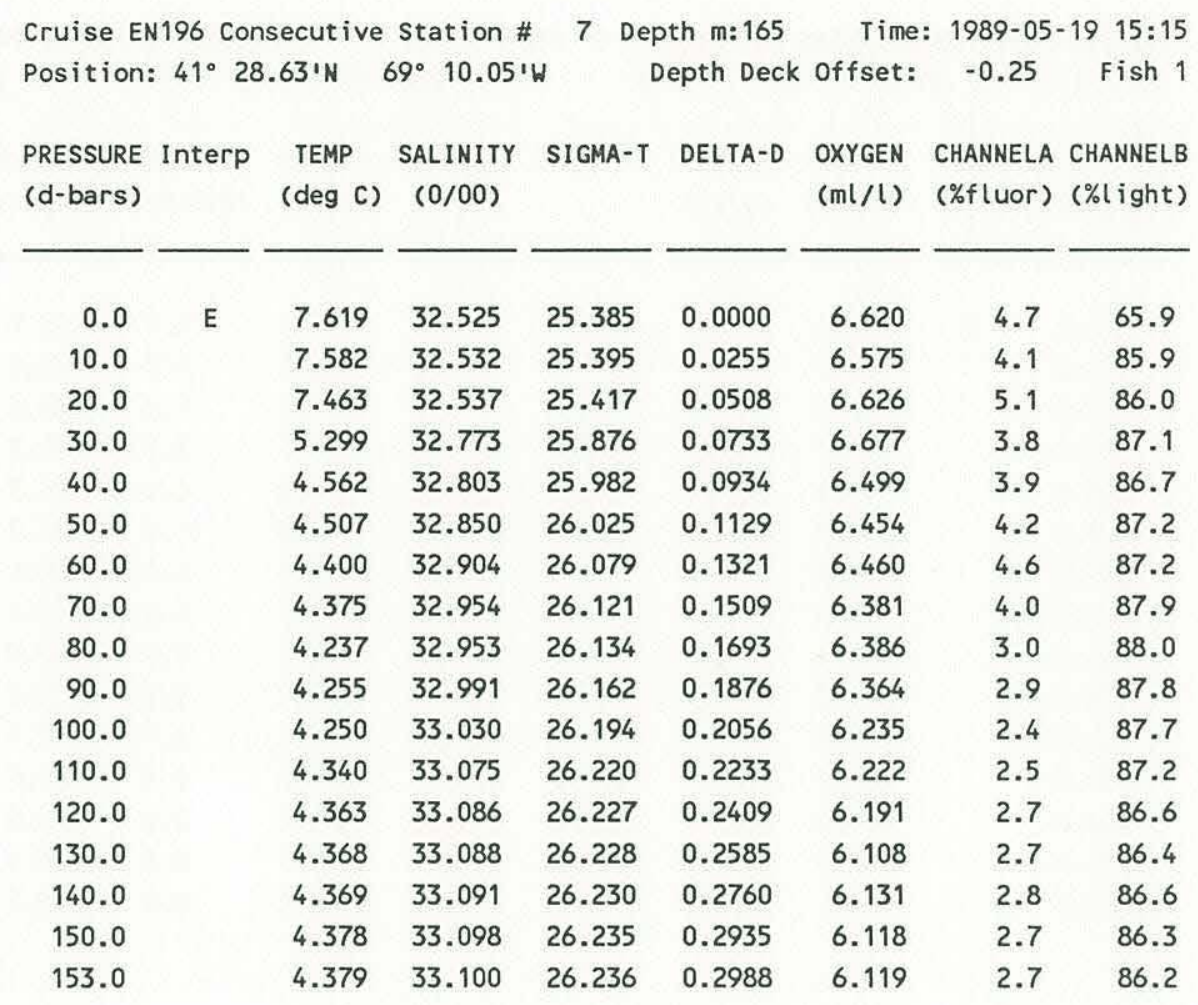
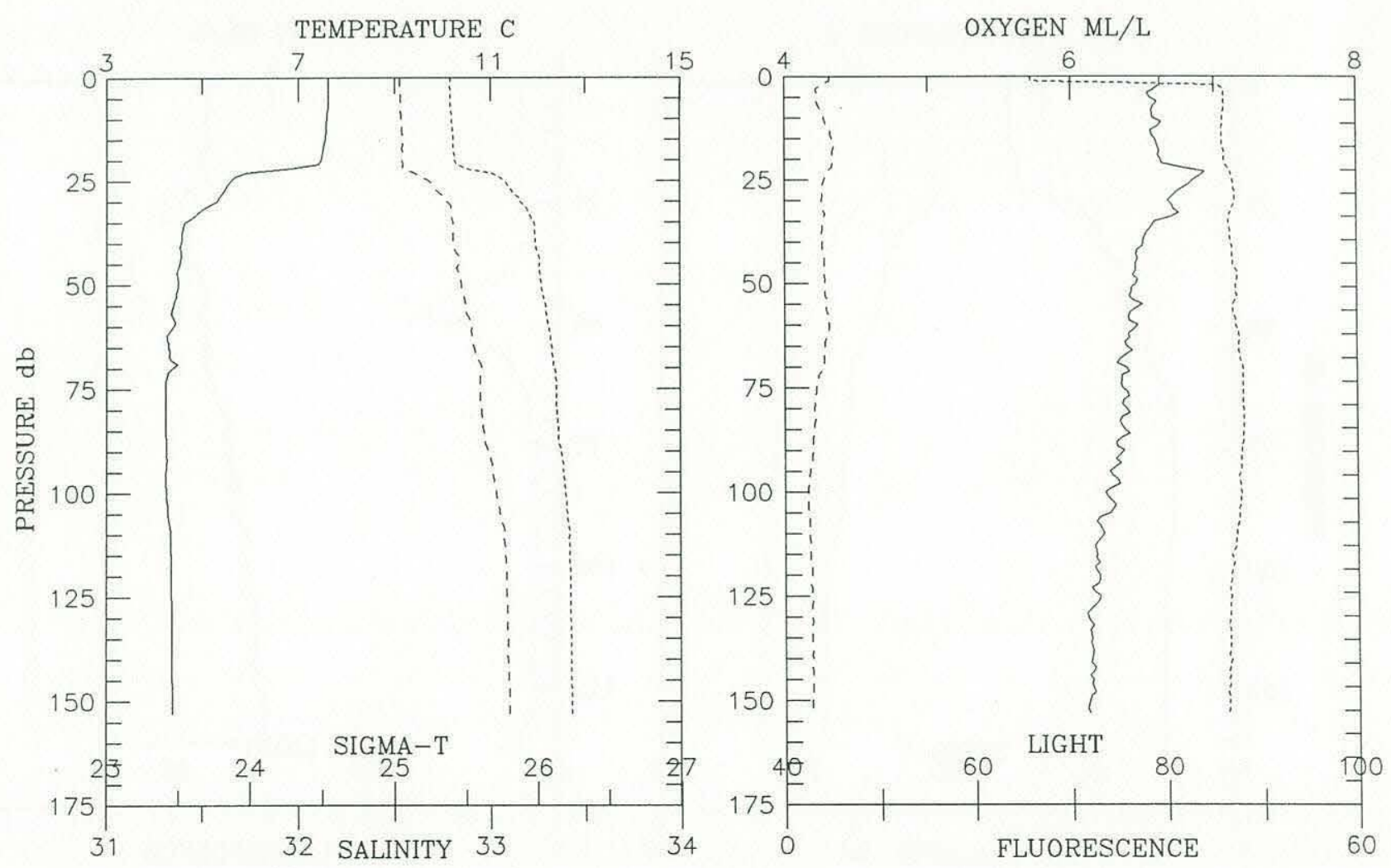


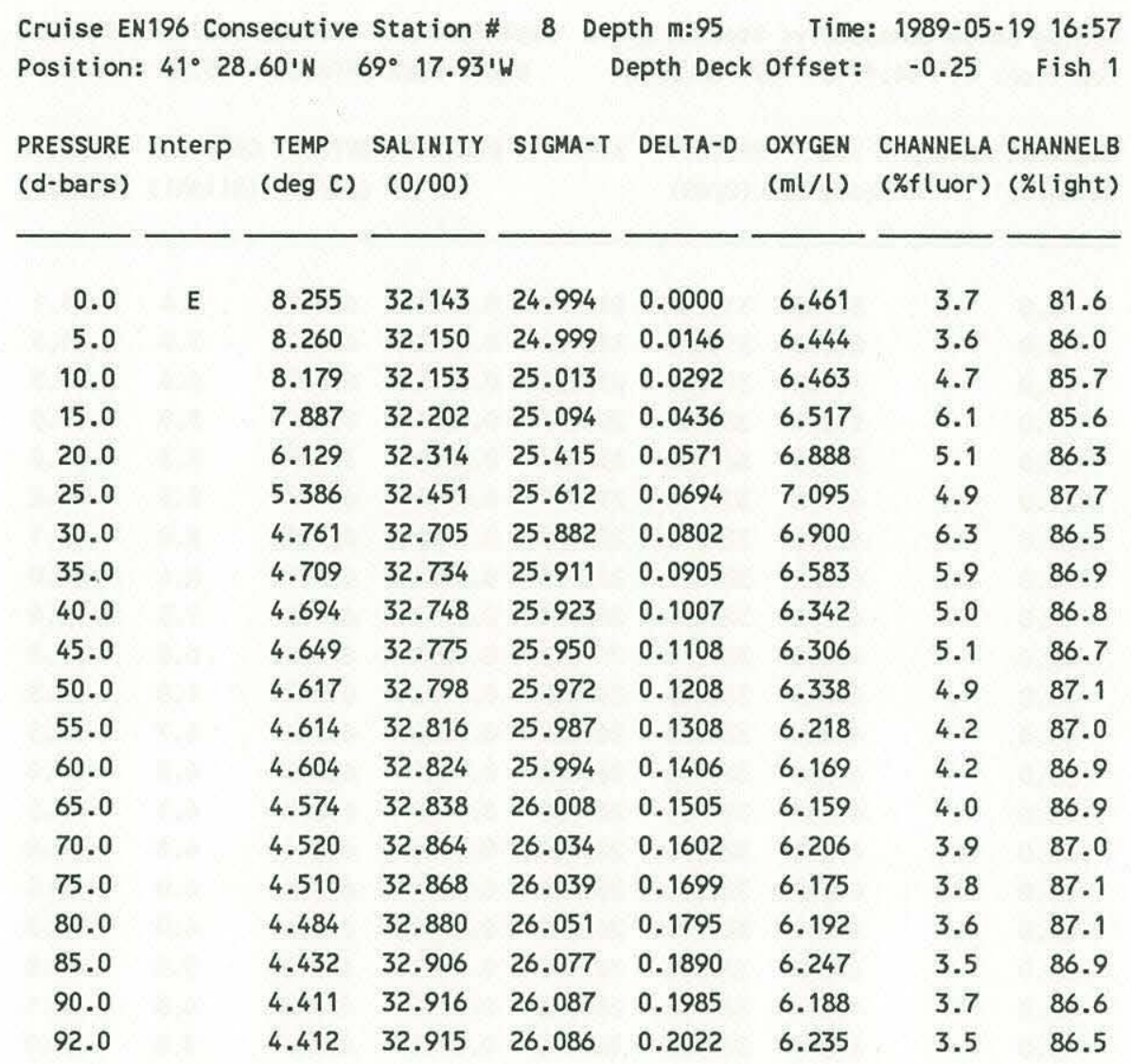
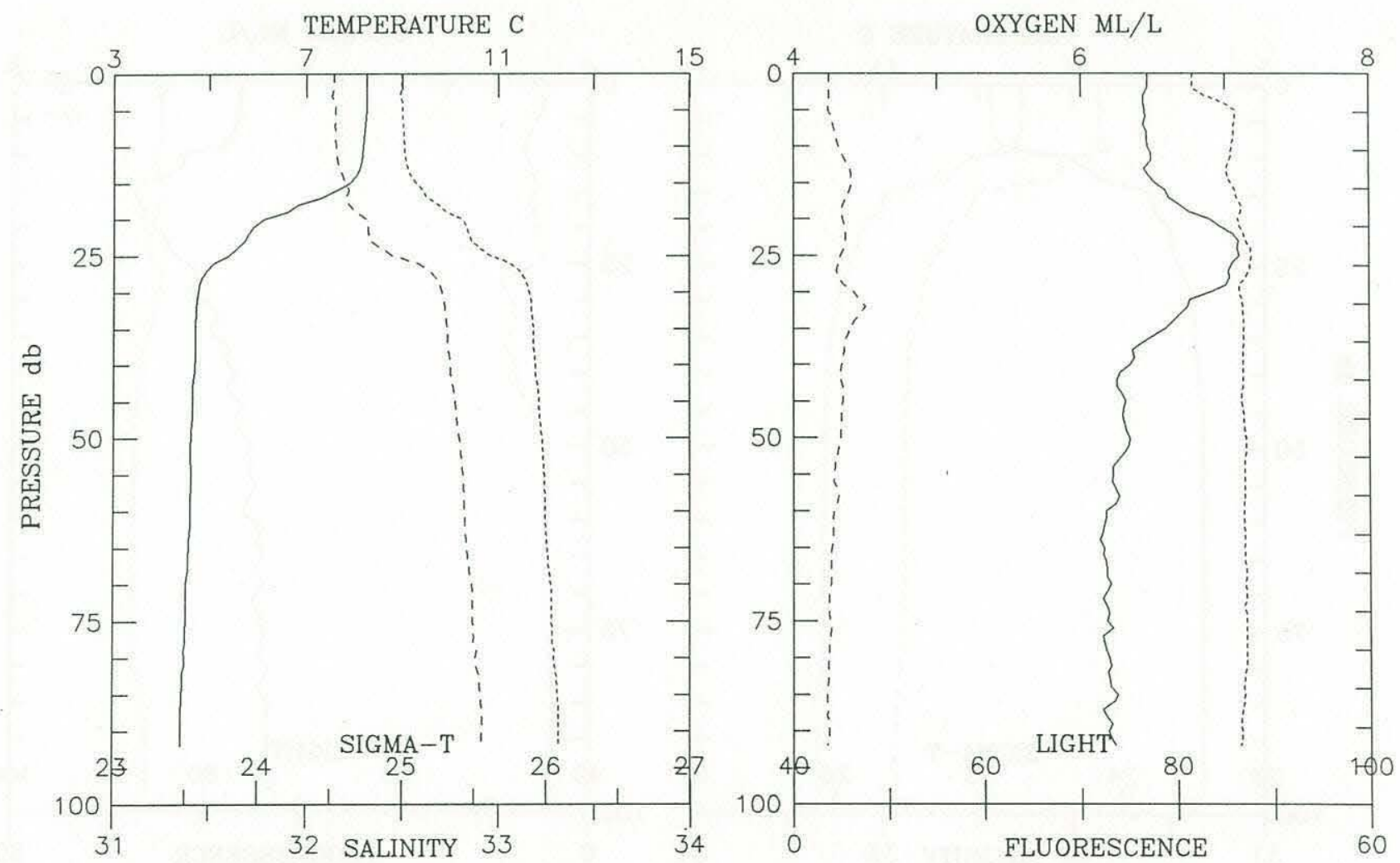


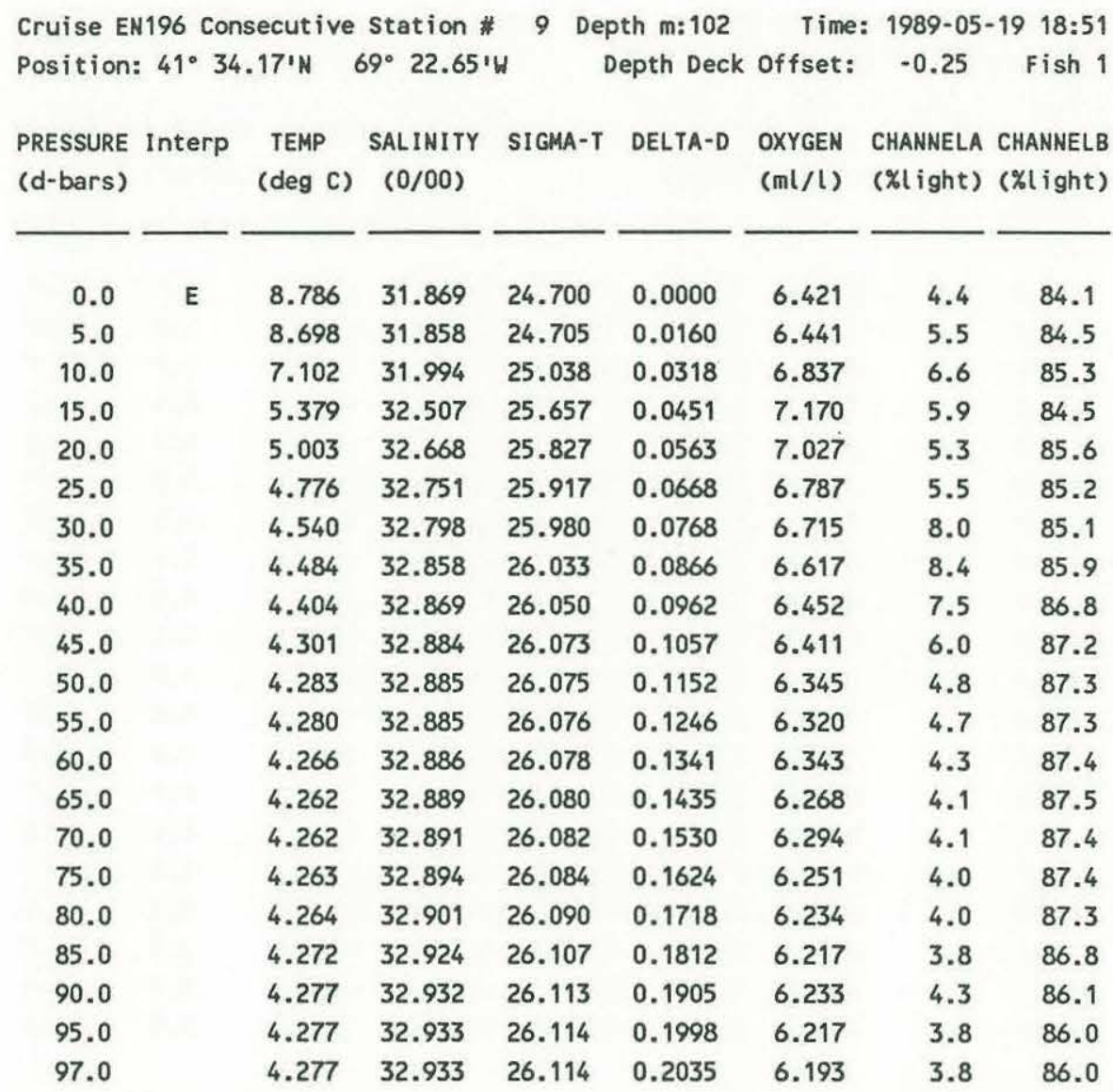
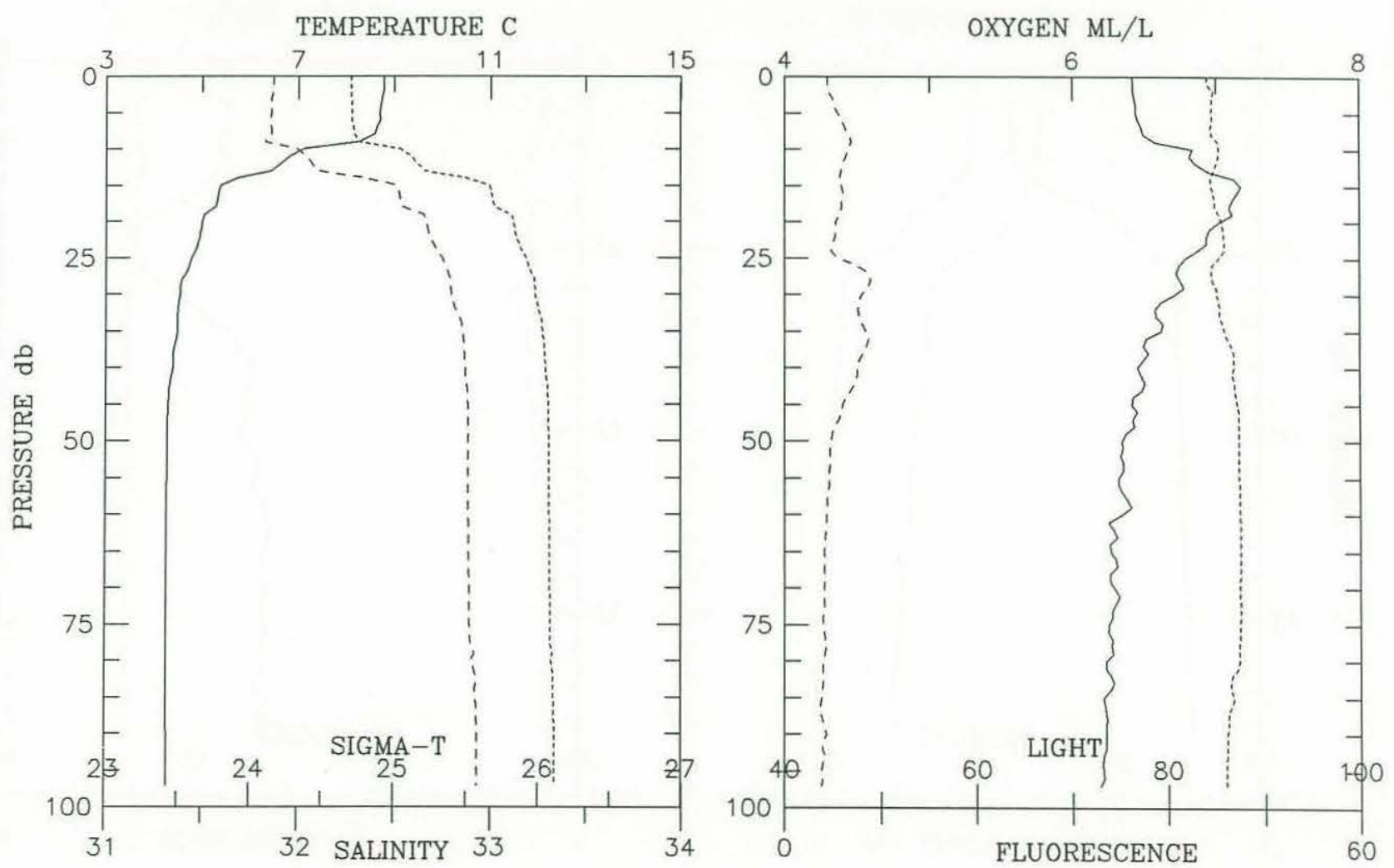


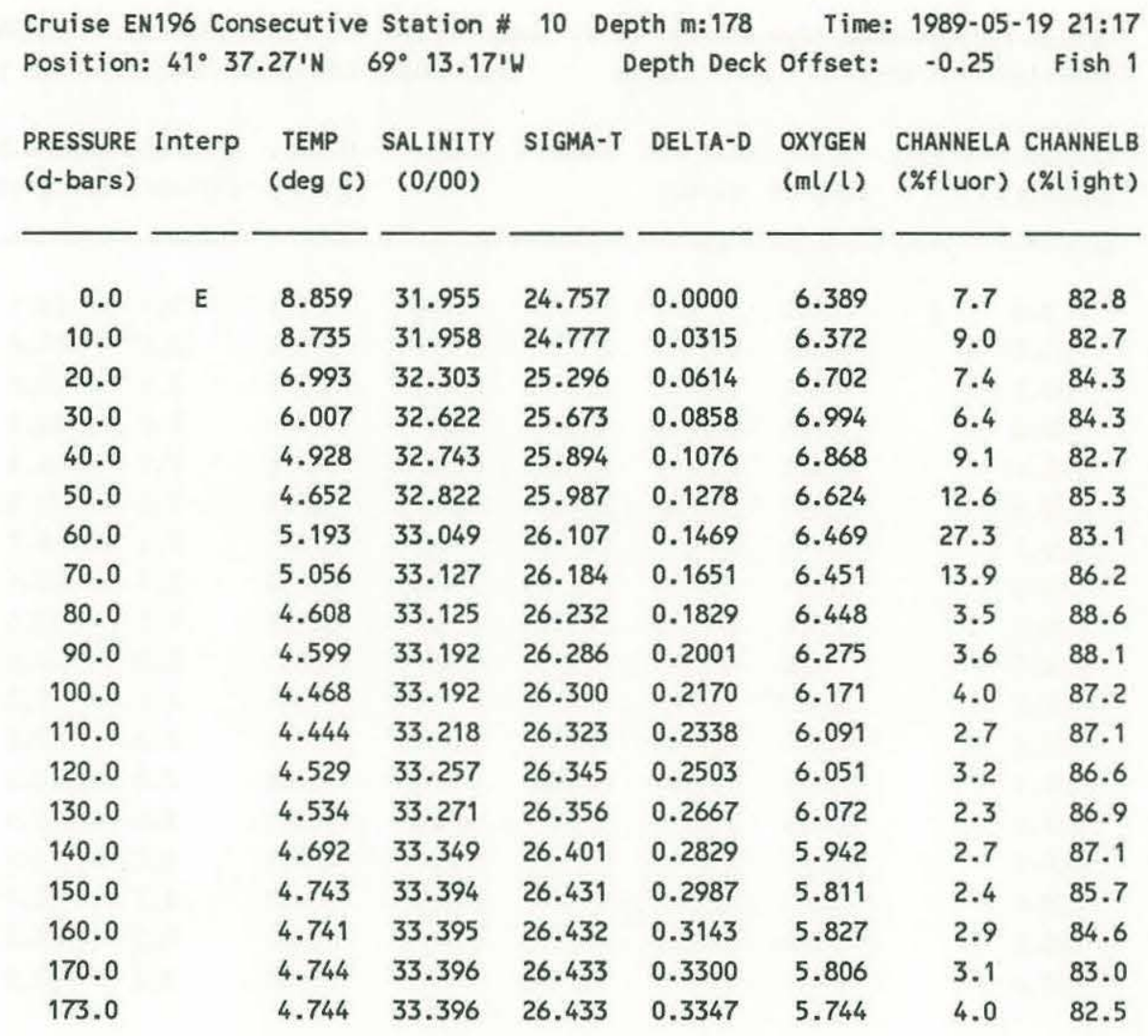
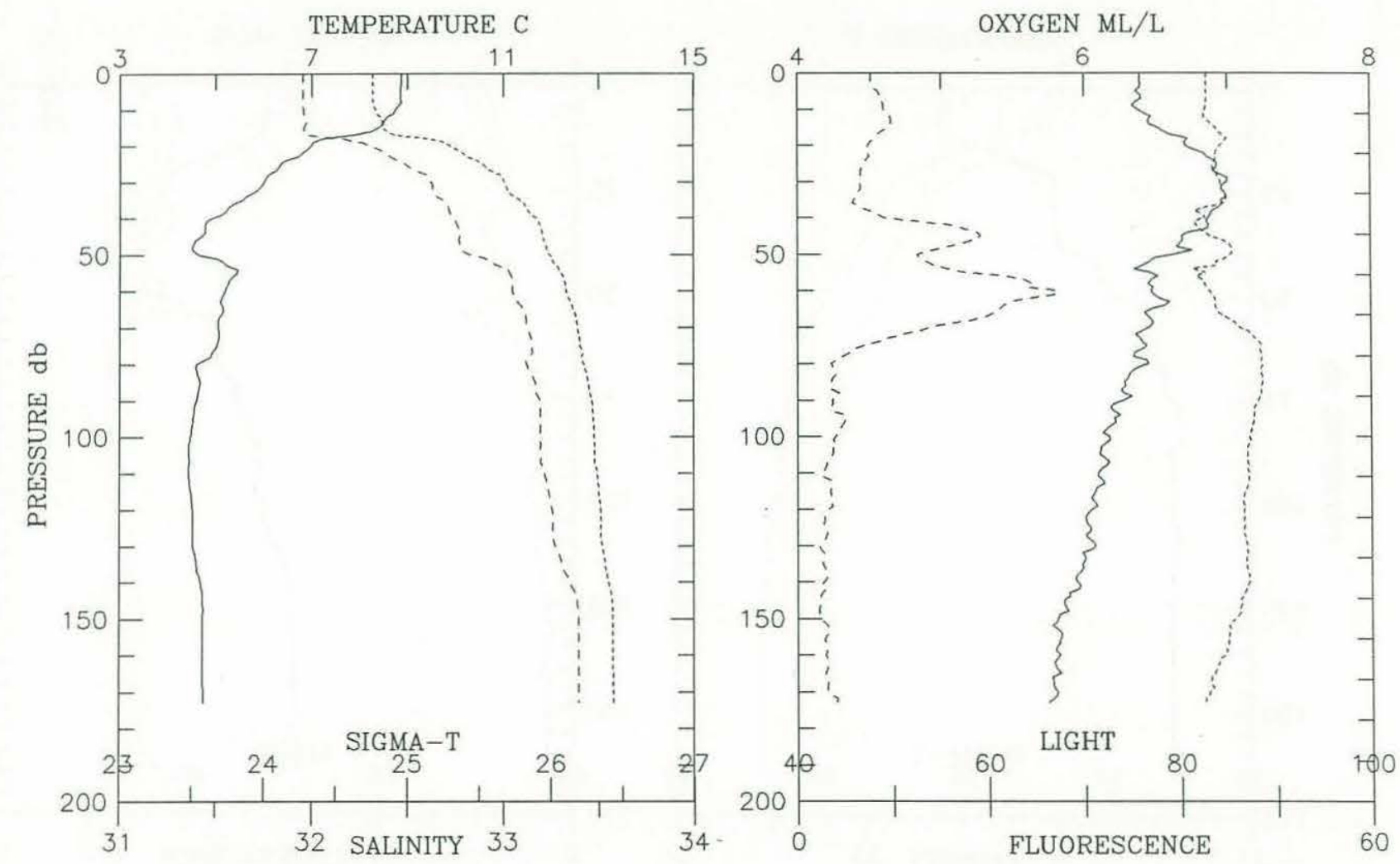


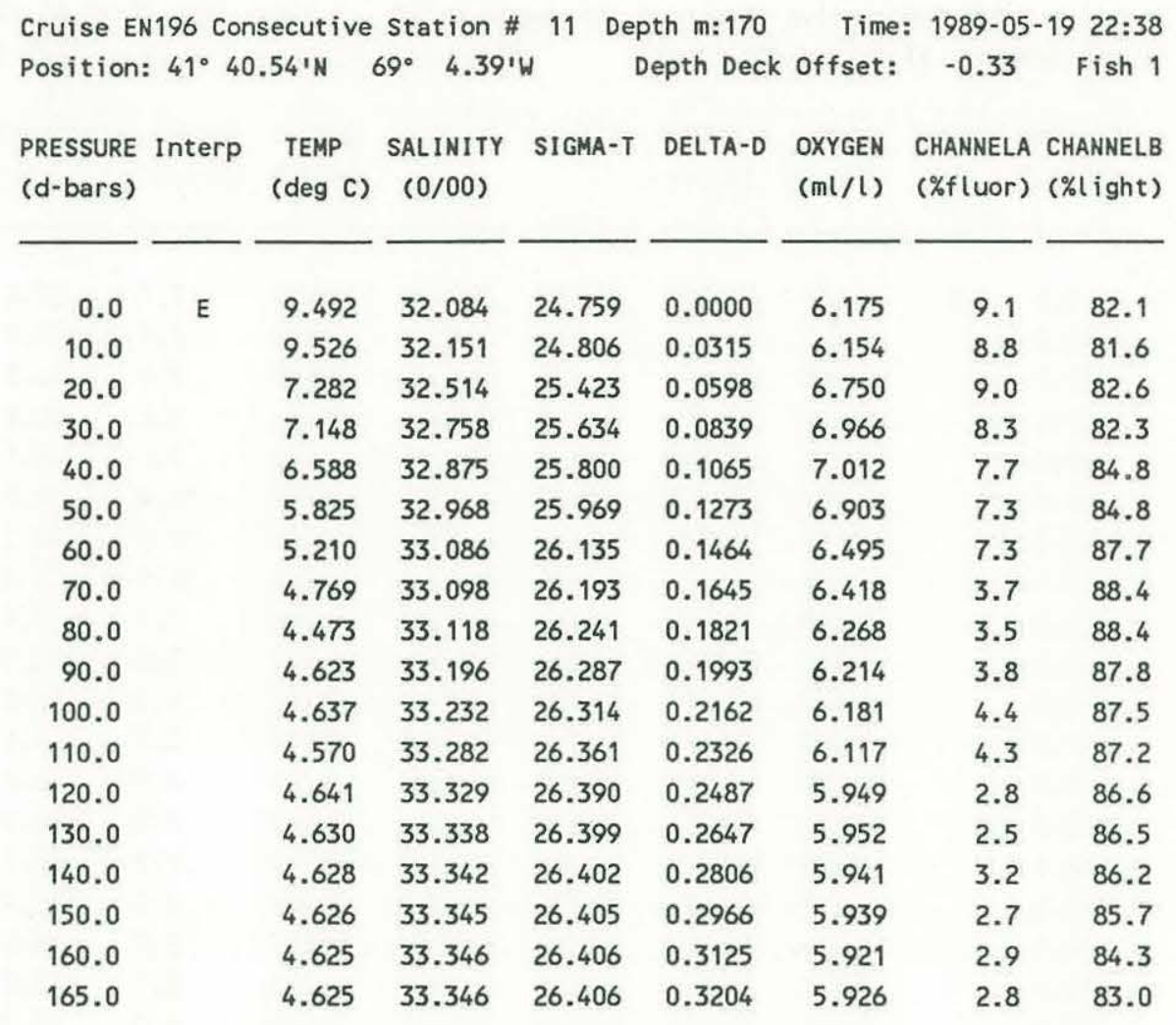
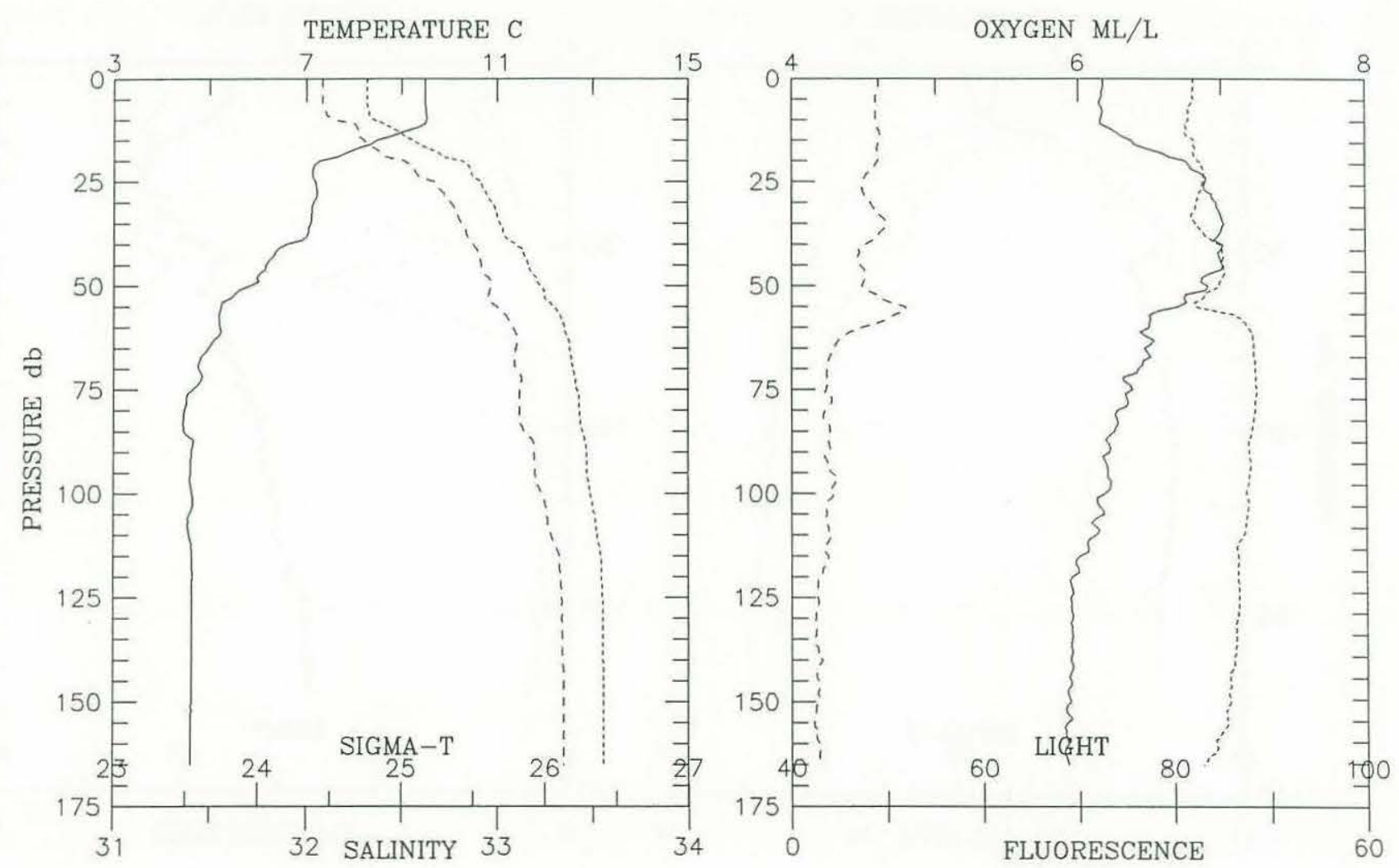
Cruise EN196 Consecutive Station \# 12 Depth m:209 Time: 1989-05-20 00:58 Position: $41^{\circ} 49.08^{\prime} \mathrm{N} 69^{\circ} 10.62^{\prime} \mathrm{W}$ Depth Deck Offset: -0.33 Fish 1

\begin{tabular}{|c|c|c|c|c|c|c|c|c|}
\hline $\begin{array}{l}\text { PRESSURE } \\
\text { (d-bars) }\end{array}$ & Interp & $\begin{array}{l}\text { TEMP } \\
(\operatorname{deg} \mathrm{C})\end{array}$ & $\begin{array}{l}\text { SALINITY } \\
(0 / 00)\end{array}$ & SI GMA-T & DELTA-D & $\begin{array}{l}\text { OXYGEN } \\
(\mathrm{ml} / \mathrm{l})\end{array}$ & $\begin{array}{l}\text { CHANNELA } \\
\text { (\%fluor) }\end{array}$ & $\begin{array}{l}\text { CHANNELB } \\
\text { (\%light) }\end{array}$ \\
\hline 0.0 & E & 9.174 & 31.869 & 24.641 & 0.0000 & 6.277 & 5.0 & 85.6 \\
\hline 10.0 & & 9.124 & 31.884 & 24.661 & 0.0326 & 6.240 & 5.2 & 85.4 \\
\hline 20.0 & & 8.346 & 32.721 & 25.433 & 0.0592 & 6.605 & 16.7 & 78.8 \\
\hline 30.0 & & 5.968 & 32.752 & 25.781 & 0.0829 & 7.127 & 13.1 & 82.0 \\
\hline 40.0 & & 5.101 & 32.757 & 25.886 & 0.1042 & 6.663 & 13.0 & 79.4 \\
\hline 50.0 & & 5.231 & 32.918 & 25.999 & 0.1245 & 6.355 & 27.4 & 81.2 \\
\hline 60.0 & & 4.843 & 33.034 & 26.134 & 0.1435 & 6.439 & 33.7 & 85.0 \\
\hline 70.0 & & 4.898 & 33.105 & 26.184 & 0.1617 & 6.262 & 20.2 & 85.2 \\
\hline 80.0 & & 4.607 & 33.135 & 26.240 & 0.1794 & 6.284 & 10.5 & 87.5 \\
\hline 90.0 & & 4.530 & 33.194 & 26.295 & 0.1965 & 6.146 & 5.1 & 87.5 \\
\hline 100.0 & & 4.572 & 33.238 & 26.325 & 0.2133 & 6.089 & 4.6 & 87.6 \\
\hline 110.0 & & 4.581 & 33.290 & 26.366 & 0.2298 & 6.052 & 3.4 & 87.7 \\
\hline 120.0 & & 4.663 & 33.346 & 26.401 & 0.2459 & 6.017 & 2.8 & 87.6 \\
\hline 130.0 & & 4.664 & 33.371 & 26.421 & 0.2617 & 5.912 & 3.2 & 87.4 \\
\hline 140.0 & & 4.738 & 33.399 & 26.435 & 0.2774 & 5.831 & 2.6 & 86.6 \\
\hline 150.0 & & 4.754 & 33.407 & 26.440 & 0.2930 & 5.775 & 2.5 & 86.3 \\
\hline 160.0 & & 4.756 & 33.411 & 26.443 & 0.3086 & 5.785 & 2.5 & 86.0 \\
\hline 170.0 & & 4.757 & 33.412 & 26.444 & 0.3242 & 5.740 & 2.6 & 85.8 \\
\hline 180.0 & & 4.760 & 33.414 & 26.445 & 0.3397 & 5.730 & 3.1 & 85.8 \\
\hline 190.0 & & 4.764 & 33.415 & 26.445 & 0.3553 & 5.732 & 3.2 & 84.0 \\
\hline 200.0 & & 4.764 & 33.415 & 26.445 & 0.3709 & 5.652 & 3.6 & 83.6 \\
\hline 203.0 & & 4.763 & 33.415 & 26.445 & 0.3755 & 5.742 & 4.4 & 82.2 \\
\hline
\end{tabular}

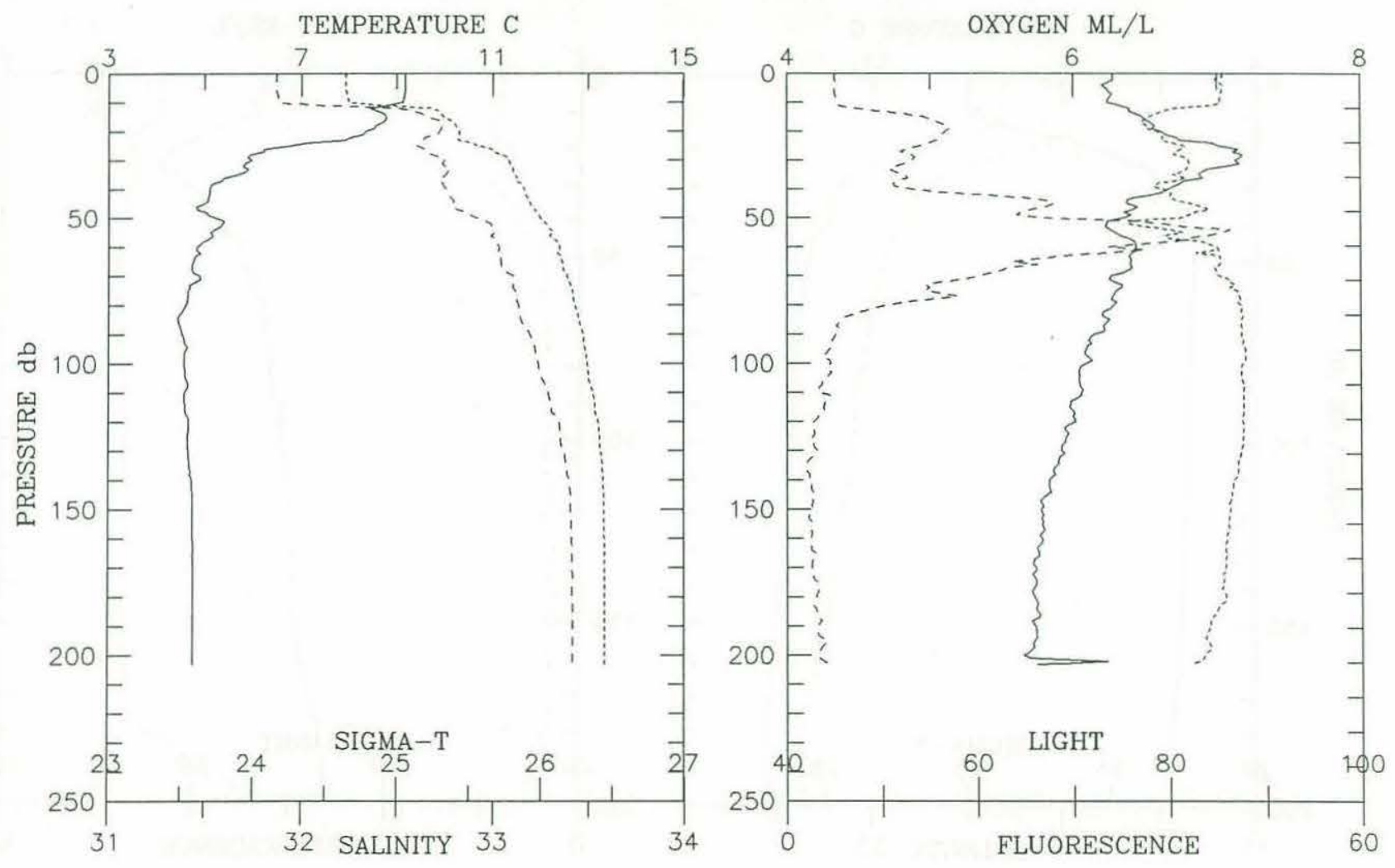




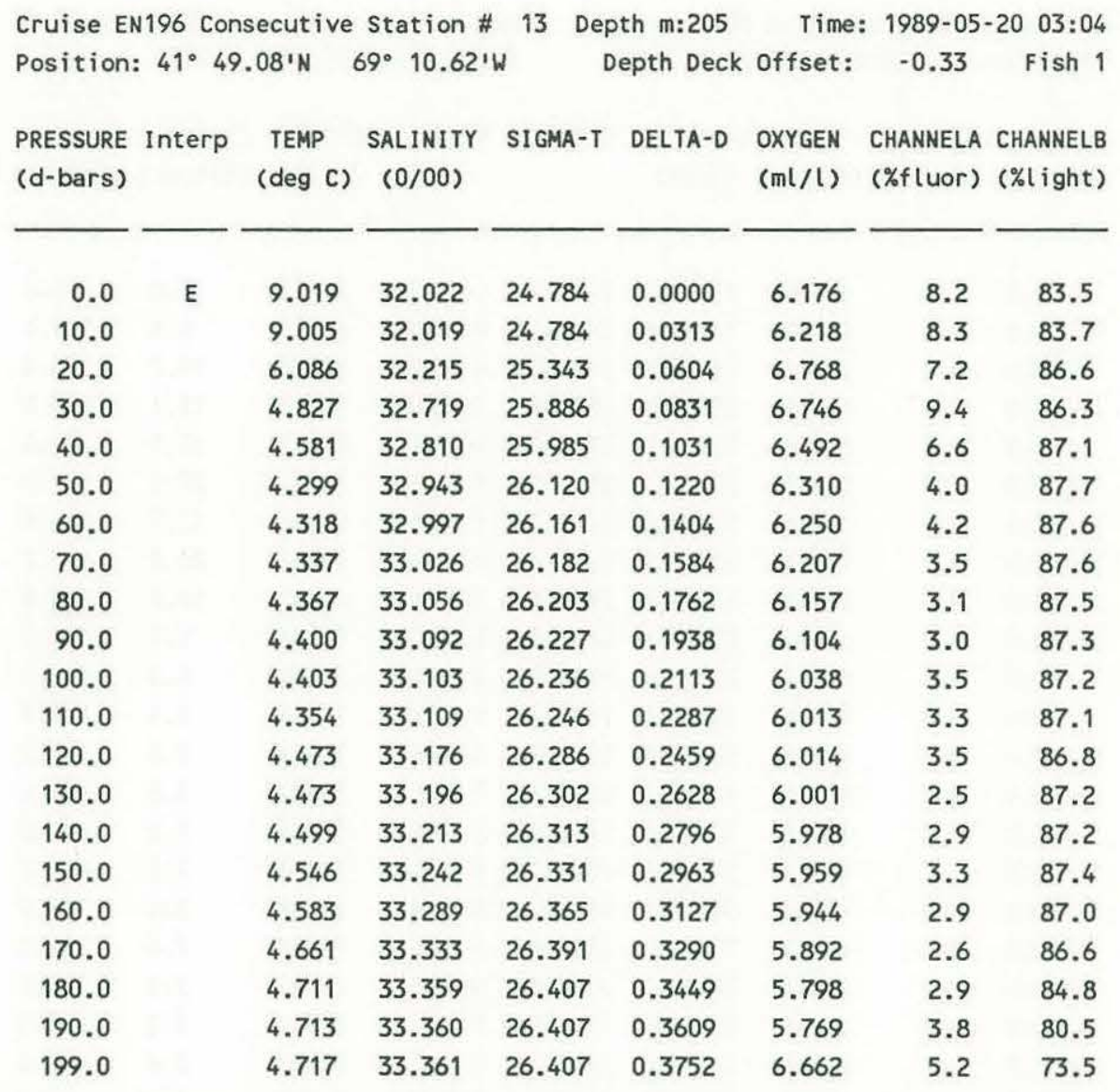
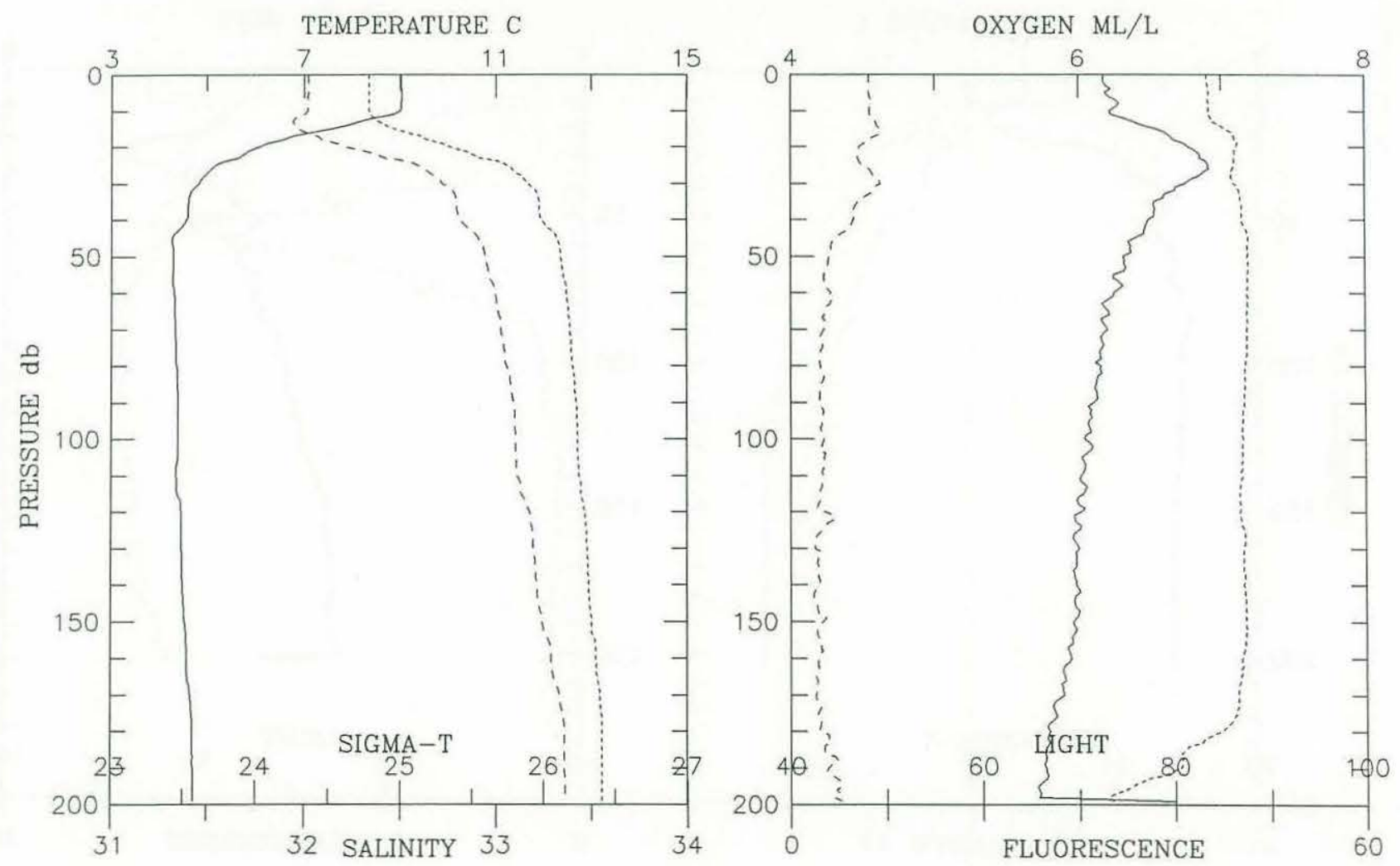


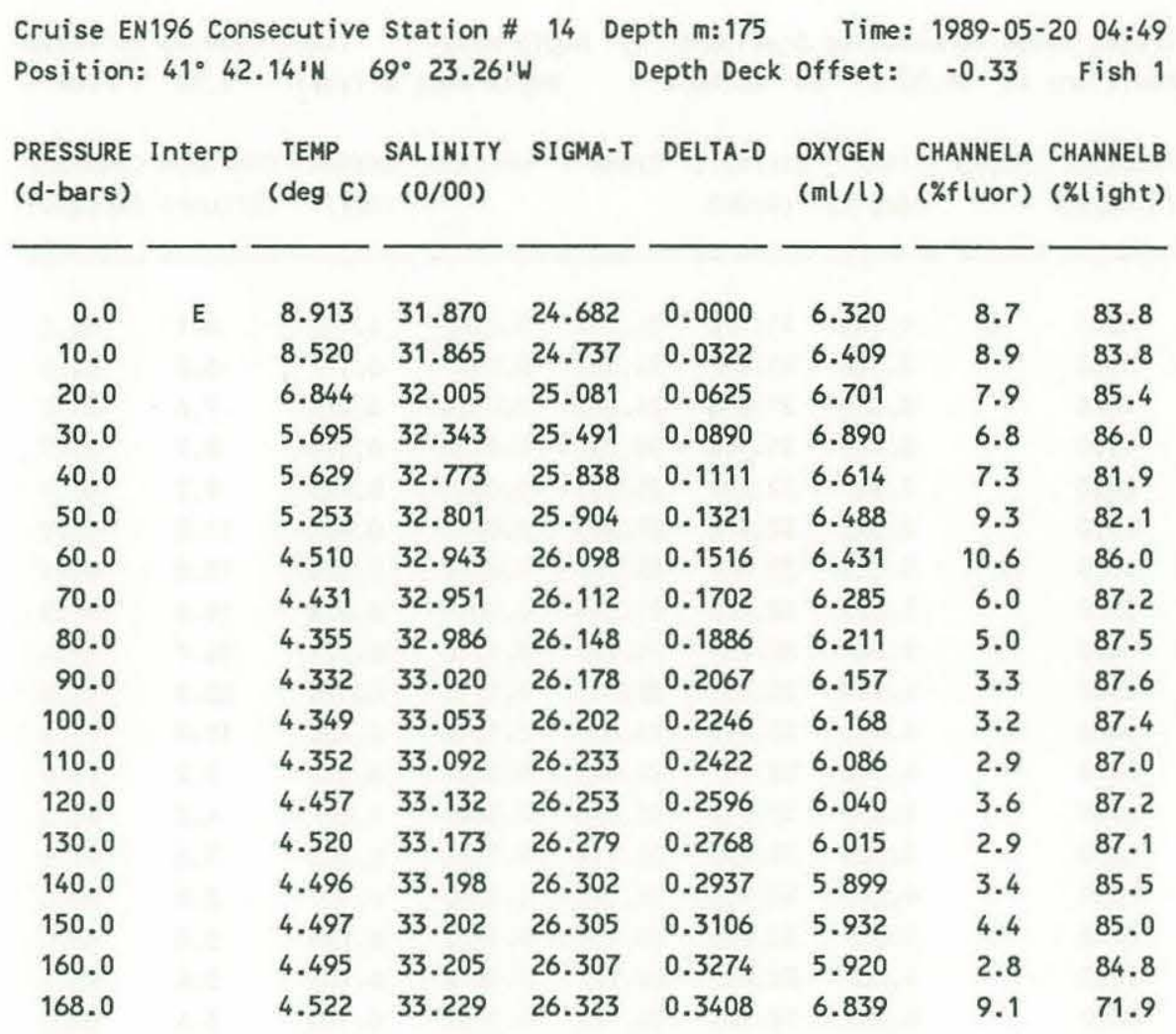
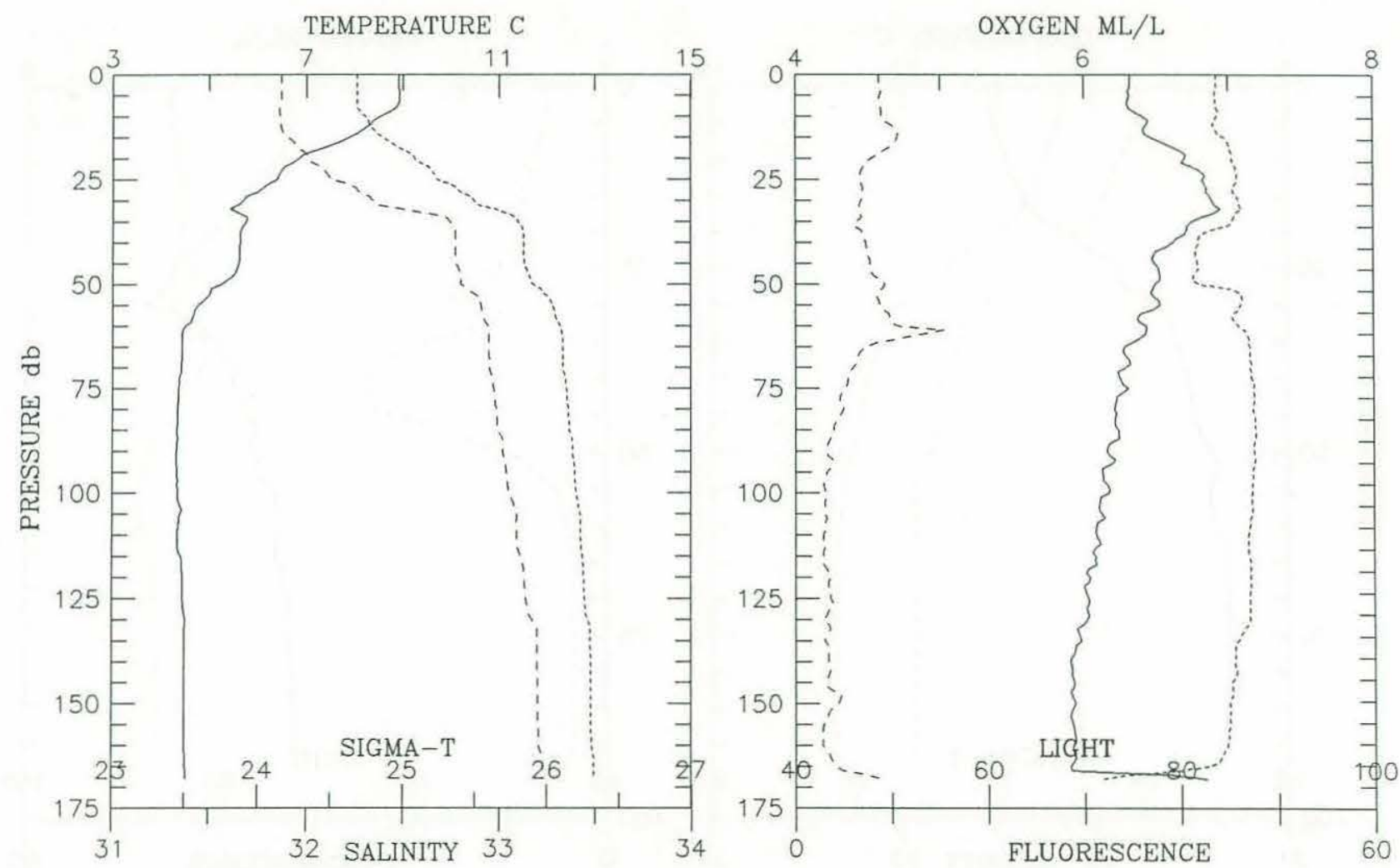
Cruise EN196 Consecutive Station \# 15 Depth m:95 Time: 1989-05-20 06:52 Position: $41^{\circ} 39.30^{\prime} \mathrm{N} 69^{\circ} 33.44^{\prime} \mathrm{W}$ Depth Deck offset: -0.33 Fish 1

PRESSURE InterP TEMP SALINITY SIGMA-T DELTA-D OXYGEN CHANNELA CHANNELB (d-bars) ( $\quad$ deg $C) \quad(0 / 00)$

(ml/l) (\%fluor) (\%light)

\begin{tabular}{|c|c|c|c|c|c|c|c|c|}
\hline 0.0 & $E$ & 9.198 & 31.772 & 24.561 & 0.0000 & 6.196 & 6.1 & 85.1 \\
\hline 5.0 & & 9.110 & 31.787 & 24.587 & 0.0167 & 6.177 & 6.2 & 84.8 \\
\hline 10.0 & & 8.831 & 31.856 & 24.683 & 0.0330 & 6.275 & 7.6 & 84.3 \\
\hline 15.0 & & 8.477 & 31.948 & 24.809 & 0.0488 & 6.499 & 8.7 & 83.9 \\
\hline 20.0 & & 7.547 & 32.087 & 25.051 & 0.0640 & 6.733 & 9.2 & 83.9 \\
\hline 25.0 & & 6.542 & 32.348 & 25.390 & 0.0775 & 6.994 & 11.5 & 82.7 \\
\hline 30.0 & & 5.413 & 32.597 & 25.724 & 0.0897 & 7.178 & 13.2 & 85.1 \\
\hline 35.0 & & 5.293 & 32.721 & 25.836 & 0.1005 & 6.662 & 18.6 & 81.3 \\
\hline 40.0 & & 5.069 & 32.787 & 25.913 & 0.1110 & 6.573 & 24.7 & 82.4 \\
\hline 45.0 & & 4.929 & 32.831 & 25.964 & 0.1211 & 6.474 & 22.1 & 84.9 \\
\hline 50.0 & & 4.591 & 32.859 & 26.022 & 0.1310 & 6.436 & 10.8 & 87.0 \\
\hline 55.0 & & 4.582 & 32.931 & 26.081 & 0.1405 & 6.292 & 5.2 & 87.9 \\
\hline 60.0 & & 4.498 & 32.943 & 26.099 & 0.1499 & 6.297 & 4.2 & 88.0 \\
\hline 65.0 & & 4.223 & 32.930 & 26.117 & 0.1592 & 6.283 & 3.6 & 87.0 \\
\hline 70.0 & & 4.227 & 32.936 & 26.122 & 0.1685 & 6.187 & 3.4 & 86.6 \\
\hline 75.0 & & 4.227 & 32.930 & 26.117 & 0.1777 & 6.195 & 3.4 & 86.4 \\
\hline 80.0 & & 4.227 & 32.939 & 26.124 & 0.1870 & 6.175 & 3.4 & 86.3 \\
\hline 85.0 & & 4.227 & 32.939 & 26.124 & 0.1962 & 6.146 & 3.4 & 86.3 \\
\hline 90.0 & & 4.226 & 32.939 & 26.124 & 0.2055 & 6.169 & 3.4 & 86.3 \\
\hline 92.0 & & 4.226 & 32.936 & 26.122 & 0.2092 & 20.148 & 7.2 & 86.1 \\
\hline
\end{tabular}
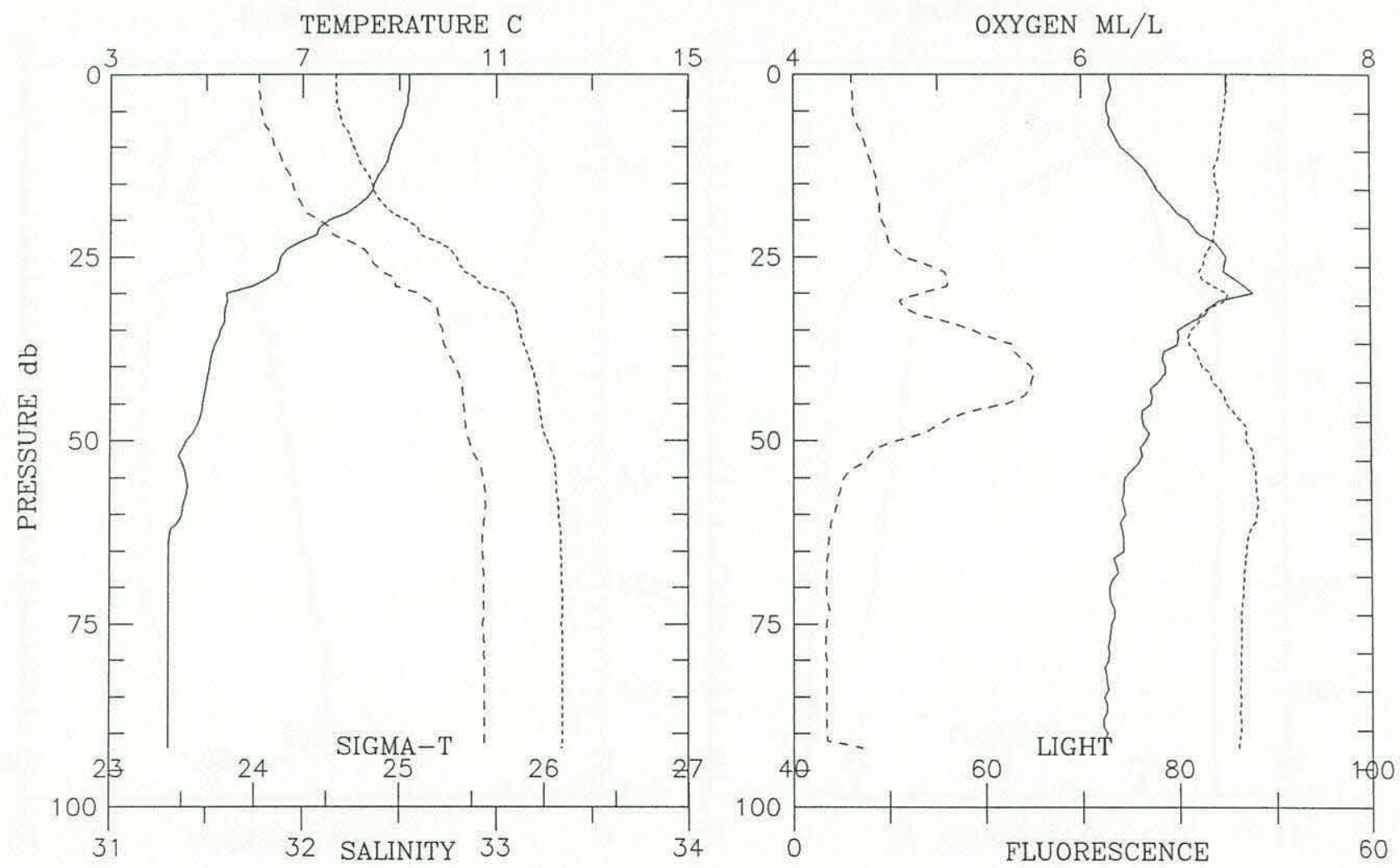
Cruise EN196 Consecutive Station \# 16 Depth m:90 Time: 1989-05-20 09:50 Position: $41^{\circ} 45.69^{\prime} \mathrm{N} 69^{\circ} 46.52^{\prime} \mathrm{W}$ Depth Deck offset: -0.25 Fish 1

\begin{tabular}{|c|c|c|c|c|c|c|c|c|}
\hline $\begin{array}{l}\text { PRESSURE } \\
\text { (d-bars) }\end{array}$ & Interp & $\begin{array}{c}\text { TEMP } \\
(\operatorname{deg} C)\end{array}$ & $\begin{array}{l}\text { SALINITY } \\
(0 / 00)\end{array}$ & SIGMA-T & DELTA-D & $\begin{array}{l}\text { OXYGEN } \\
(\mathrm{ml} / \mathrm{l})\end{array}$ & $\begin{array}{l}\text { CHANNELA } \\
\text { ( } \% \text { fluor) }\end{array}$ & $\begin{array}{l}\text { CHANNELB } \\
\text { (\%light) }\end{array}$ \\
\hline 0.0 & $E$ & 9.133 & 31.429 & 24.303 & 0.0000 & 6.252 & 3.9 & 87.2 \\
\hline 5.0 & & 9.092 & 31.457 & 24.331 & 0.0179 & 6.267 & 3.9 & 87.2 \\
\hline 10.0 & & 8.498 & 31.509 & 24.461 & 0.0354 & 6.411 & 5.3 & 86.9 \\
\hline 15.0 & & 6.007 & 31.927 & 25.124 & 0.0508 & 6.805 & 4.0 & 88.9 \\
\hline 20.0 & & 5.132 & 32.313 & 25.531 & 0.0638 & 6.750 & 4.5 & 88.8 \\
\hline 25.0 & & 4.676 & 32.463 & 25.699 & 0.0753 & 6.550 & 3.9 & 88.3 \\
\hline 30.0 & & 4.593 & 32.516 & 25.750 & 0.0865 & 6.361 & 5.0 & 87.9 \\
\hline 35.0 & & 4.486 & 32.569 & 25.803 & 0.0973 & 6.237 & 5.7 & 87.1 \\
\hline 40.0 & & 4.482 & 32.574 & 25.808 & 0.1080 & 6.057 & 5.7 & 86.9 \\
\hline 45.0 & & 4.468 & 32.592 & 25.823 & 0.1187 & 6.039 & 6.1 & 86.8 \\
\hline 50.0 & & 4.435 & 32.599 & 25.833 & 0.1294 & 5.939 & 6.4 & 86.7 \\
\hline 55.0 & & 4.448 & 32.629 & 25.855 & 0.1399 & 5.911 & 7.6 & 86.2 \\
\hline 60.0 & & 4.448 & 32.625 & 25.852 & 0.1504 & 5.906 & 7.6 & 86.2 \\
\hline 65.0 & & 4.449 & 32.648 & 25.871 & 0.1609 & 5.913 & 8.0 & 85.7 \\
\hline 70.0 & & 4.454 & 32.657 & 25.876 & 0.1714 & 5.955 & 9.3 & 85.6 \\
\hline 75.0 & & 4.449 & 32.686 & 25.901 & 0.1817 & 5.944 & 9.9 & 84.5 \\
\hline 80.0 & & 4.449 & 32.708 & 25.918 & 0.1920 & 5.926 & 10.9 & 84.0 \\
\hline 85.0 & & 4.436 & 32.740 & 25.945 & 0.2021 & 5.923 & 9.5 & 83.7 \\
\hline
\end{tabular}
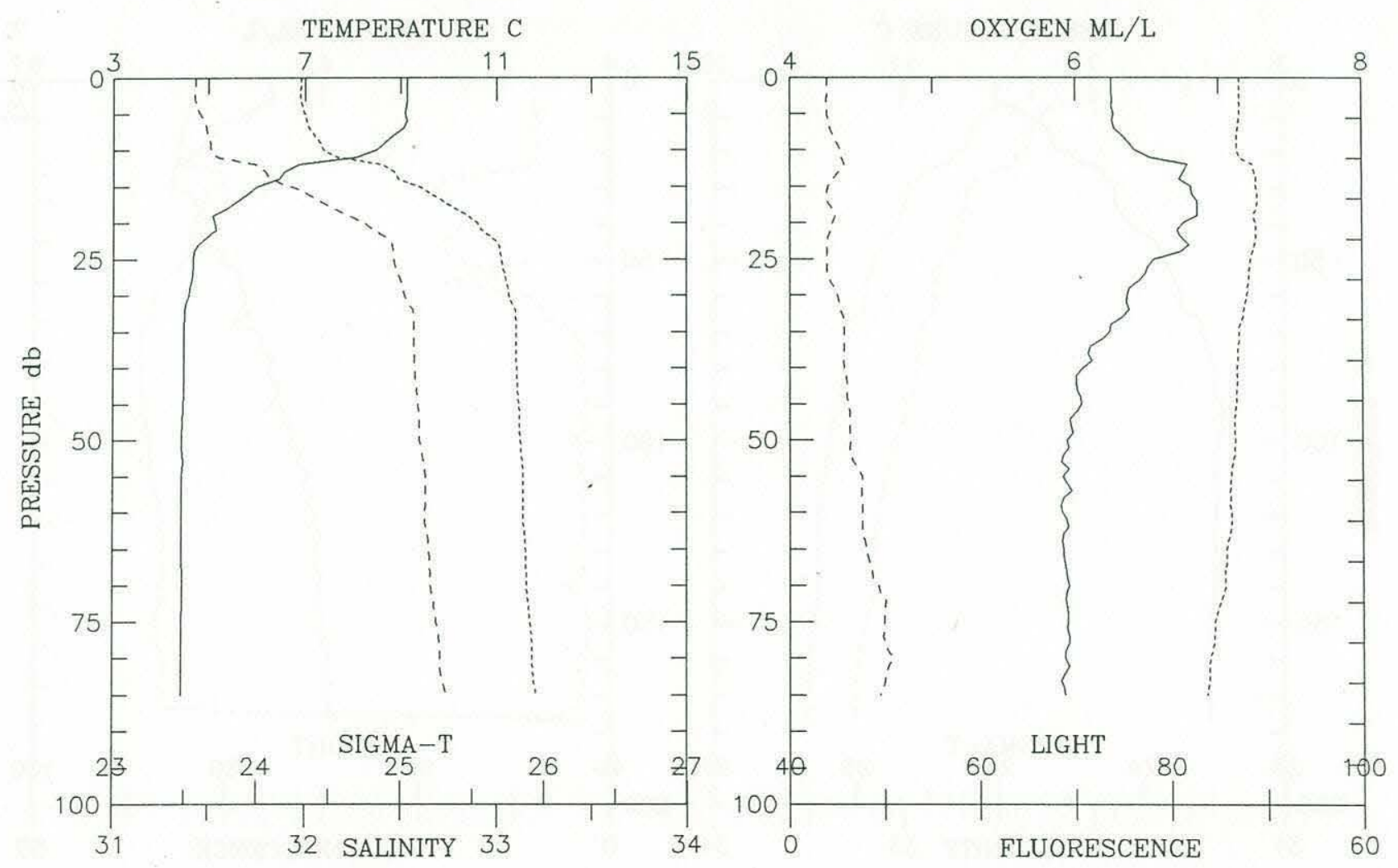


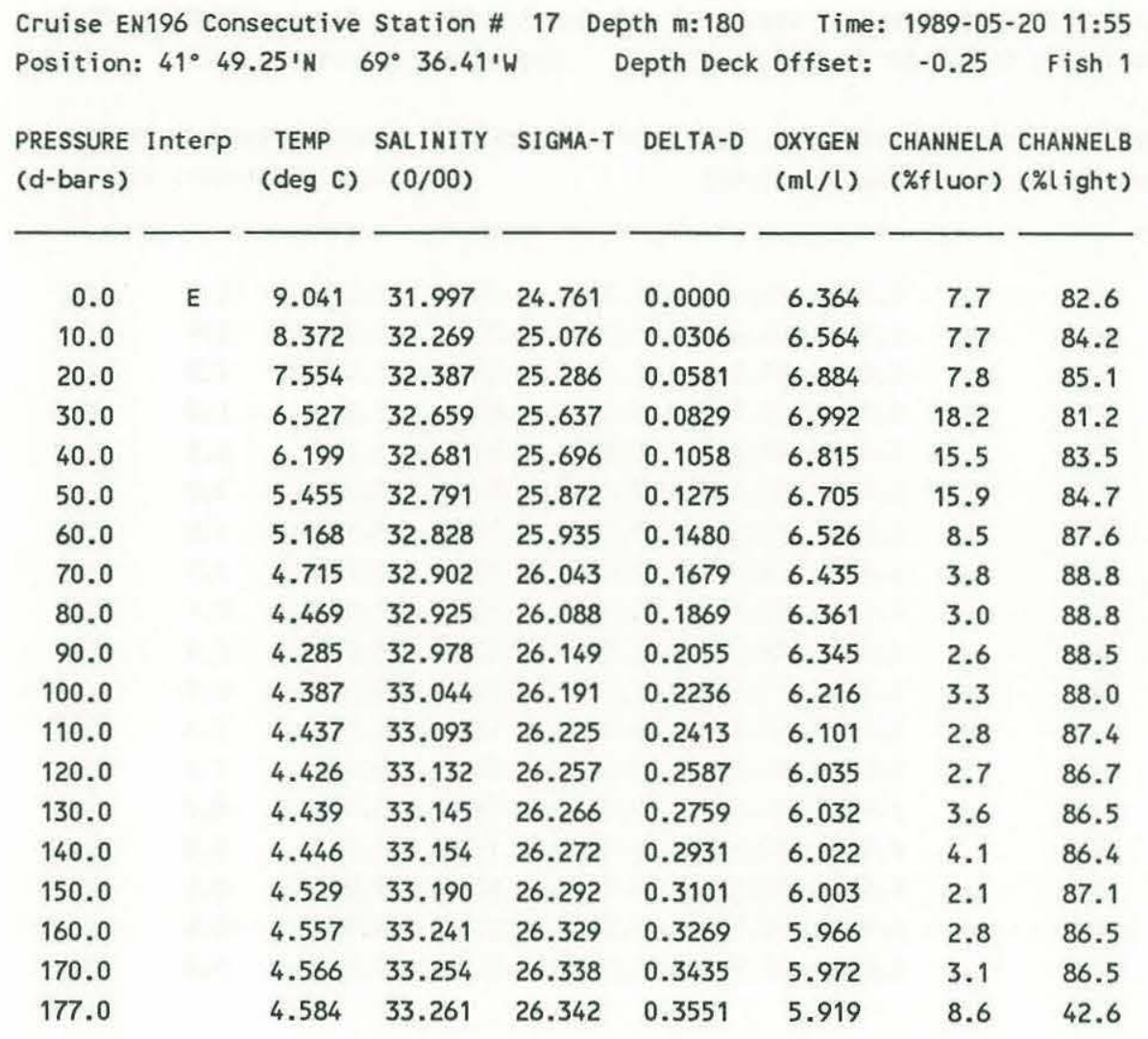
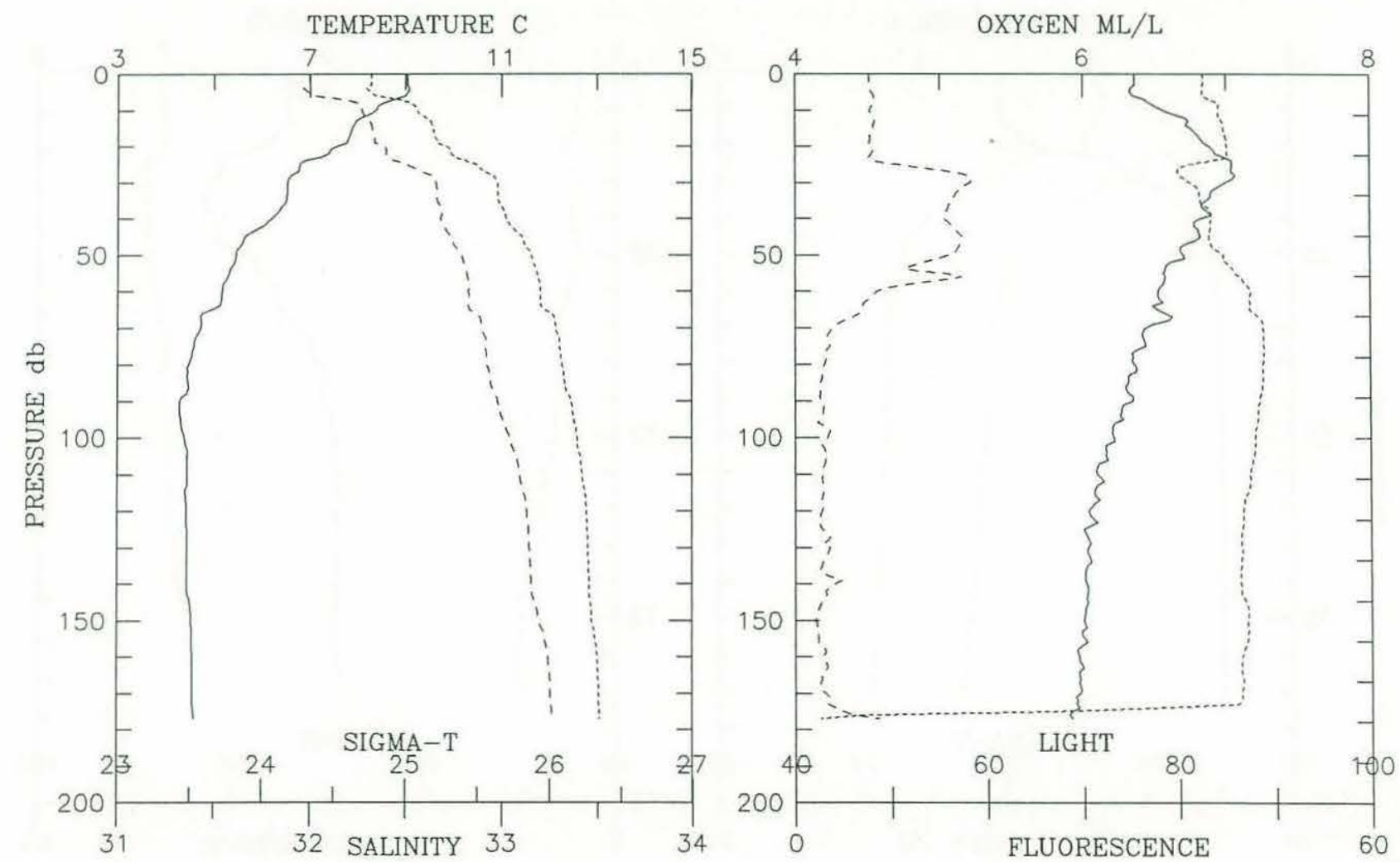
Cruise EN196 Consecutive Station \# 18 Depth m:208 Time: 1989-05-20 14:08 Position: $41^{\circ} 53.10^{\prime} \mathrm{N} 69^{\circ} 25.86 \mathrm{~W}$ W Depth Deck offset: -0.25 Fish 1

\begin{tabular}{|c|c|c|c|c|c|c|c|c|}
\hline $\begin{array}{l}\text { PRESSURE } \\
\text { (d-bars) }\end{array}$ & Interp & $\begin{array}{c}\text { TEMP } \\
(\operatorname{deg} \mathrm{C})\end{array}$ & $\begin{array}{c}\text { SALINITY } \\
(0 / 00)\end{array}$ & SIGMA-T & DELTA-D & $\begin{array}{l}\text { OXYGEN } \\
(\mathrm{ml} / \mathrm{l})\end{array}$ & $\begin{array}{l}\text { CHANNELA } \\
\text { (\%fluor) }\end{array}$ & $\begin{array}{l}\text { CHANNELB } \\
\text { (\%light) }\end{array}$ \\
\hline 0.0 & $E$ & 9.820 & 31.865 & 24.535 & 0.0000 & 6.271 & 5.3 & 82.5 \\
\hline 10.0 & & 9.207 & 31.939 & 24.690 & 0.0334 & 6.558 & 6.9 & 82.2 \\
\hline 20.0 & & 6.654 & 32.608 & 25.581 & 0.0604 & 7.497 & 9.8 & 83.4 \\
\hline 30.0 & & 6.161 & 32.849 & 25.833 & 0.0830 & 7.397 & 18.5 & 80.5 \\
\hline 40.0 & & 5.687 & 32.910 & 25.939 & 0.1038 & 6.930 & 29.6 & 81.7 \\
\hline 50.0 & & 5.167 & 33.021 & 26.088 & 0.1235 & 6.609 & 48.6 & 75.3 \\
\hline 60.0 & & 4.632 & 33.071 & 26.186 & 0.1419 & 6.440 & 22.4 & 87.1 \\
\hline 70.0 & & 4.584 & 33.149 & 26.254 & 0.1594 & 6.312 & 3.3 & 88.1 \\
\hline 80.0 & & 4.475 & 33.178 & 26.288 & 0.1765 & 6.193 & 4.2 & 87.7 \\
\hline 90.0 & & 4.477 & 33.209 & 26.313 & 0.1933 & 6.183 & 5.0 & 87.6 \\
\hline 100.0 & & 4.544 & 33.278 & 26.360 & 0.2098 & 6.192 & 3.9 & 87.2 \\
\hline 110.0 & & 4.654 & 33.351 & 26.406 & 0.2259 & 6.065 & 3.0 & 87.3 \\
\hline 120.0 & & 4.701 & 33.375 & 26.420 & 0.2417 & 5.958 & 2.3 & 87.1 \\
\hline 130.0 & & 4.737 & 33.389 & 26.427 & 0.2574 & 5.915 & 2.8 & 87.0 \\
\hline 140.0 & & 4.787 & 33.411 & 26.439 & 0.2730 & 5.862 & 2.2 & 86.8 \\
\hline 150.0 & & 4.820 & 33.422 & 26.444 & 0.2886 & 5.816 & 2.0 & 86.5 \\
\hline 160.0 & & 4.852 & 33.431 & 26.448 & 0.3041 & 5.793 & 1.7 & 86.3 \\
\hline 170.0 & & 4.865 & 33.441 & 26.455 & 0.3196 & 5.775 & 1.9 & 86.5 \\
\hline 180.0 & & 4.908 & 33.463 & 26.467 & 0.3350 & 5.685 & 1.9 & 85.9 \\
\hline 190.0 & & 5.040 & 33.500 & 26.482 & 0.3504 & 5.579 & 2.2 & 85.0 \\
\hline 197.0 & & 5.170 & 33.543 & 26.501 & 0.3610 & 5.362 & 2.8 & 80.8 \\
\hline
\end{tabular}
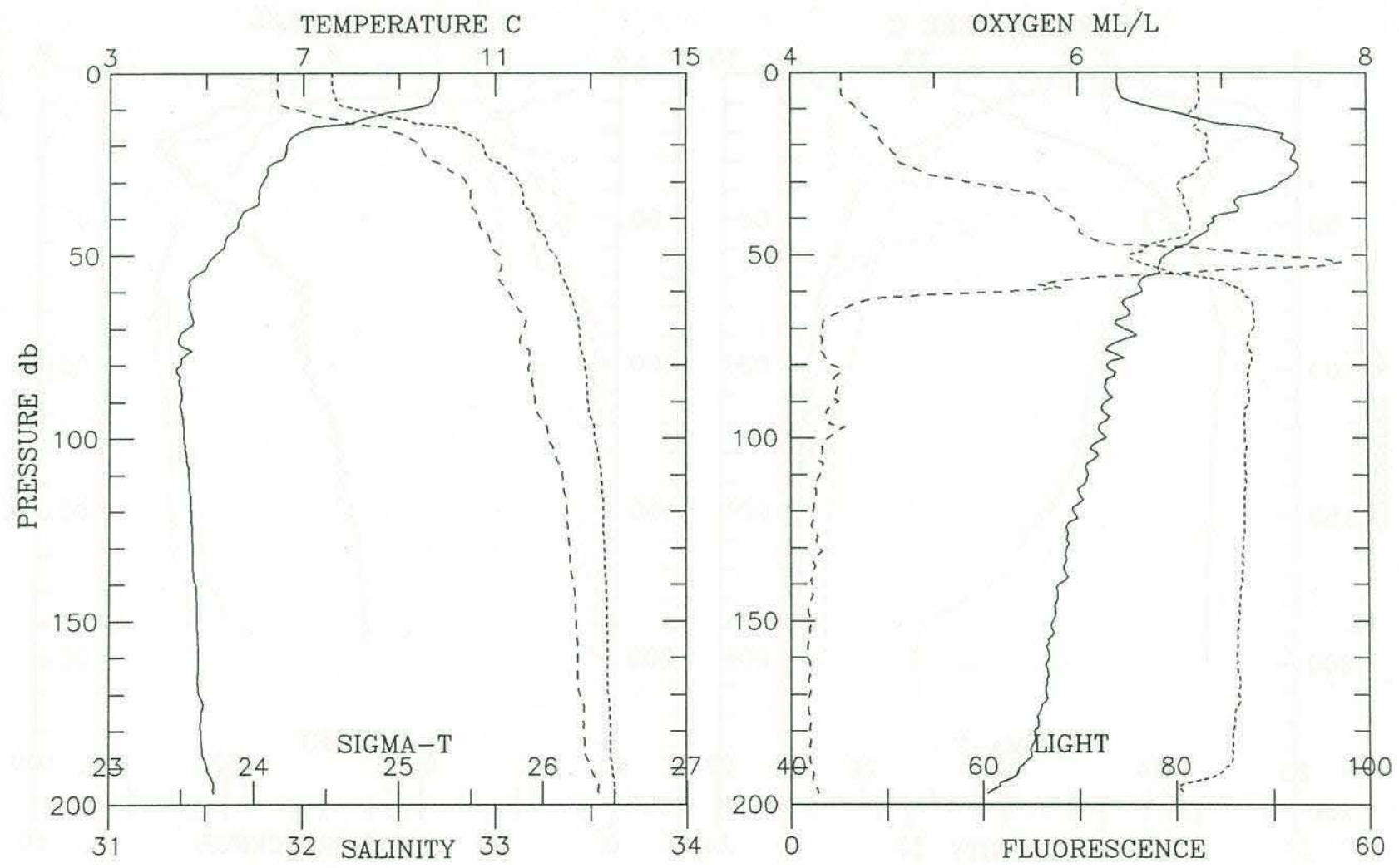
Cruise EN196 Consecutive Station \# 19 Depth m:210 Time: 1989-05-20 16:20 Position: $41^{\circ} 57.10^{\prime} \mathrm{N} 69^{\circ} 15.00^{\mathrm{W}}$ Depth Deck offset: -0.25 Fish 1

PRESSURE InterP TEMP SALINITY SIGMA-T DELTA-D OXYGEN CHANNELA CHANNELB (d-bars) (deg C) (0/00) (ml/l) (\%fluor) (\%light)

\begin{tabular}{|c|c|c|c|c|c|c|c|c|}
\hline 0.0 & E & 10.090 & 32.286 & 24.819 & 0.0000 & 6.400 & 4.2 & 79.3 \\
\hline 10.0 & & 9.496 & 32.571 & 25.139 & 0.0299 & 6.606 & 8.4 & 75.0 \\
\hline 20.0 & & 8.151 & 32.774 & 25.504 & 0.0559 & 7.037 & 15.0 & 79.6 \\
\hline 30.0 & & 6.686 & 33.006 & 25.890 & 0.0786 & 7.137 & 5.9 & 86.3 \\
\hline 40.0 & & 5.880 & 33.085 & 26.055 & 0.0985 & 6.972 & 8.5 & 85.8 \\
\hline 50.0 & & 5.668 & 33.132 & 26.117 & 0.1173 & 6.730 & 5.3 & 85.9 \\
\hline 60.0 & & 5.179 & 33.179 & 26.211 & 0.1353 & 6.478 & 8.9 & 86.3 \\
\hline 70.0 & & 4.805 & 33.227 & 26.291 & 0.1526 & 6.300 & 6.6 & 87.5 \\
\hline 80.0 & & 4.701 & 33.265 & 26.333 & 0.1693 & 6.174 & 3.4 & 88.1 \\
\hline 90.0 & & 4.540 & 33.283 & 26.365 & 0.1856 & 6.161 & 2.7 & 87.6 \\
\hline 100.0 & & 4.577 & 33.326 & 26.395 & 0.2017 & 6.137 & 2.3 & 87.8 \\
\hline 110.0 & & 4.695 & 33.382 & 26.426 & 0.2176 & 6.115 & 2.1 & 86.9 \\
\hline 120.0 & & 4.710 & 33.401 & 26.440 & 0.2332 & 5.952 & 1.8 & 86.5 \\
\hline 130.0 & & 4.740 & 33.415 & 26.448 & 0.2487 & 5.915 & 1.7 & 86.2 \\
\hline 140.0 & & 4.751 & 33.420 & 26.450 & 0.2642 & 5.926 & 1.7 & 86.2 \\
\hline 150.0 & & 4.789 & 33.432 & 26.456 & 0.2796 & 5.836 & 1.7 & 86.3 \\
\hline 160.0 & & 4.817 & 33.440 & 26.459 & 0.2951 & 5.771 & 1.9 & 86.1 \\
\hline 170.0 & & 4.839 & 33.446 & 26.461 & 0.3105 & 5.764 & 1.9 & 85.4 \\
\hline 180.0 & & 4.851 & 33.449 & 26.462 & 0.3259 & 5.746 & 2.1 & 84.2 \\
\hline 190.0 & & 4.848 & 33.448 & 26.462 & 0.3413 & 5.724 & 2.0 & 84.0 \\
\hline 200.0 & & 4.848 & 33.449 & 26.463 & 0.3567 & 5.731 & 2.1 & 83.6 \\
\hline 201.0 & & 4.848 & 33.448 & $26.462^{\prime}$ & 0.3583 & 5.736 & 2.1 & 83.4 \\
\hline
\end{tabular}
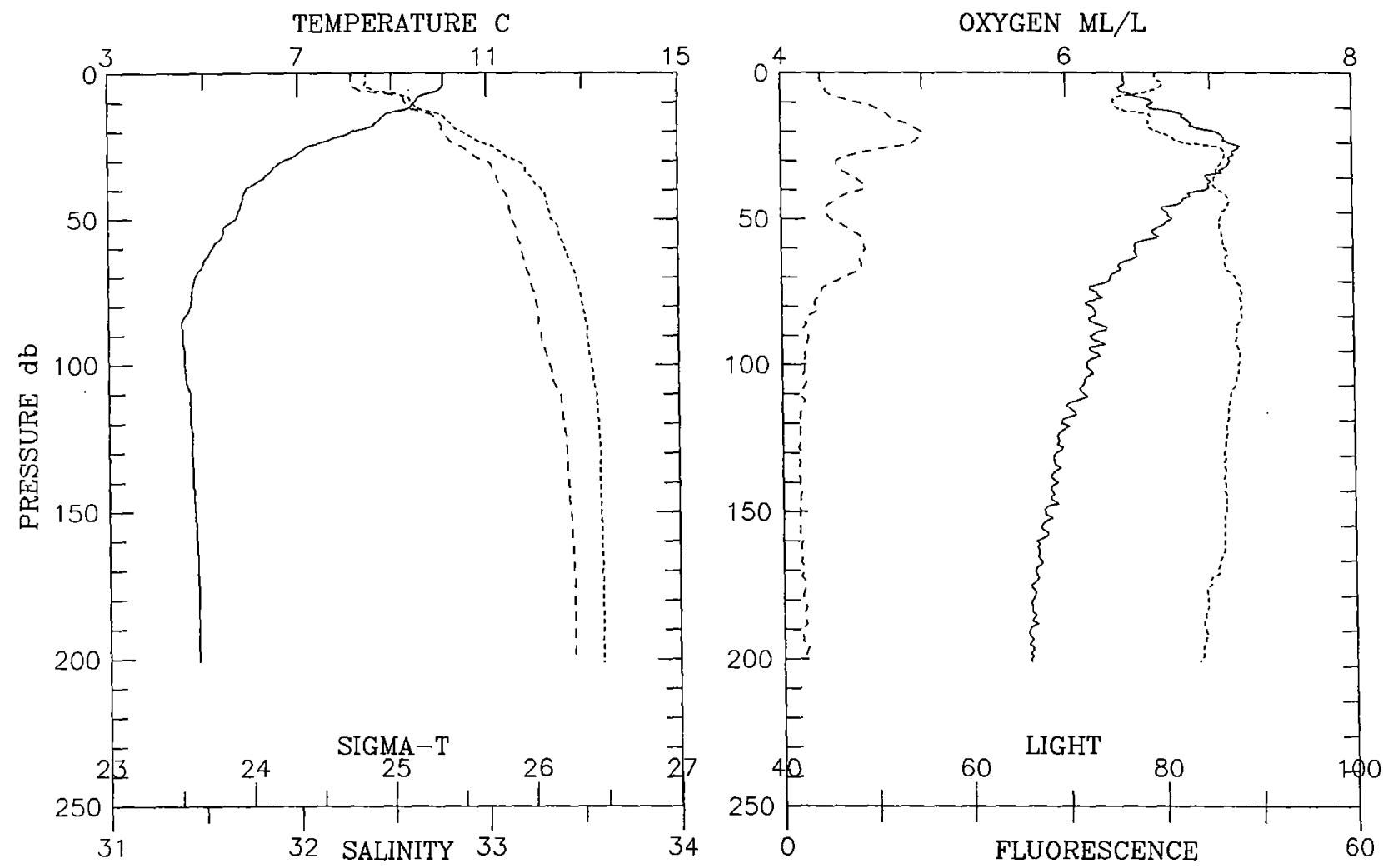
Cruise EN196 Consecutive Station \# 20 Depth m:206 Time: 1989-05-20 19:16 Position: $42^{\circ} 7.92 \mathrm{~N} 69^{\circ} 22.48 \mathrm{~W} \mathrm{~W}$ Depth Deck Offset: -0.25 Fish 1

\begin{tabular}{|c|c|c|c|c|c|c|c|c|}
\hline $\begin{array}{l}\text { PRESSURE } \\
\text { (d-bars) }\end{array}$ & Interp & $\begin{array}{l}\text { TEMP } \\
(\operatorname{deg} \mathrm{C})\end{array}$ & $\begin{array}{c}\text { SALINITY } \\
(0 / 00)\end{array}$ & SIGHA-T & DELTA-D & $\begin{array}{l}\text { OXYGEN } \\
(\mathrm{ml} / \mathrm{l})\end{array}$ & $\begin{array}{l}\text { CHANNELA } \\
\text { (\%fluor) }\end{array}$ & $\begin{array}{l}\text { CHANNELB } \\
\text { (\%light) }\end{array}$ \\
\hline 0.0 & $\mathbf{E}$ & 9.907 & 32.744 & 25.207 & 0.0000 & 6.559 & 7.3 & 73.0 \\
\hline 10.0 & & 7.390 & 32.774 & 25.613 & 0.0266 & 7.141 & 8.1 & 81.8 \\
\hline 20.0 & & 6.268 & 32.880 & 25.844 & 0.0486 & 7.273 & 4.8 & 84.5 \\
\hline 30.0 & & 5.858 & 32.939 & 25.942 & 0.0694 & 7.030 & 5.7 & 84.3 \\
\hline 40.0 & & 4.922 & 33.045 & 26.134 & 0.0889 & 6.928 & 27.9 & 78.9 \\
\hline 50.0 & & 4.698 & 33.074 & 26.181 & 0.1070 & 6.523 & 18.6 & 84.6 \\
\hline 60.0 & & 4.388 & 33.088 & 26.226 & 0.1248 & 6.425 & 7.9 & 87.0 \\
\hline 70.0 & & 4.223 & 33.098 & 26.250 & 0.1421 & 6.345 & 5.6 & 87.1 \\
\hline 80.0 & & 4.371 & 33.146 & 26.274 & 0.1593 & 6.298 & 4.5 & 87.4 \\
\hline 90.0 & & 4.459 & 33.208 & 26.314 & 0.1763 & 6.212 & 3.8 & 87.3 \\
\hline 100.0 & & 4.524 & 33.258 & 26.347 & 0.1928 & 6.185 & 4.5 & 87.2 \\
\hline 110.0 & & 4.576 & 33.296 & 26.371 & 0.2091 & 6.120 & 3.0 & 87.5 \\
\hline 120.0 & & 4.613 & 33.326 & 26.391 & 0.2251 & 6.094 & 2.8 & 87.8 \\
\hline 130.0 & & 4.629 & 33.349 & 26.408 & 0.2411 & 6.054 & 2.8 & 87.6 \\
\hline 140.0 & & 4.798 & 33.408 & 26.436 & 0.2568 & 5.968 & 1.8 & 88.0 \\
\hline 150.0 & & 4.899 & 33.444 & 26.453 & 0.2723 & 5.803 & 2.0 & 88.0 \\
\hline 160.0 & & 5.060 & 33.491 & 26.473 & 0.2877 & 5.679 & 1.7 & 87.4 \\
\hline 170.0 & & 5.164 & 33.521 & 26.484 & 0.3029 & 5.547 & 1.7 & 87.2 \\
\hline 180.0 & & 5.478 & 33.619 & 26.525 & 0.3180 & 5.253 & 1.7 & 86.0 \\
\hline 190.0 & & 5.582 & 33.650 & 26.537 & 0.3327 & 4.979 & 2.3 & 84.0 \\
\hline 200.0 & & 5.586 & 33.651 & 26.538 & 0.3475 & 4.904 & 2.4 & 82.5 \\
\hline 203.0 & & 5.585 & 33.651 & 26.538 & 0.3519 & 5.936 & 3.0 & 81.3 \\
\hline
\end{tabular}

TEMPERATURE $\mathrm{C}$
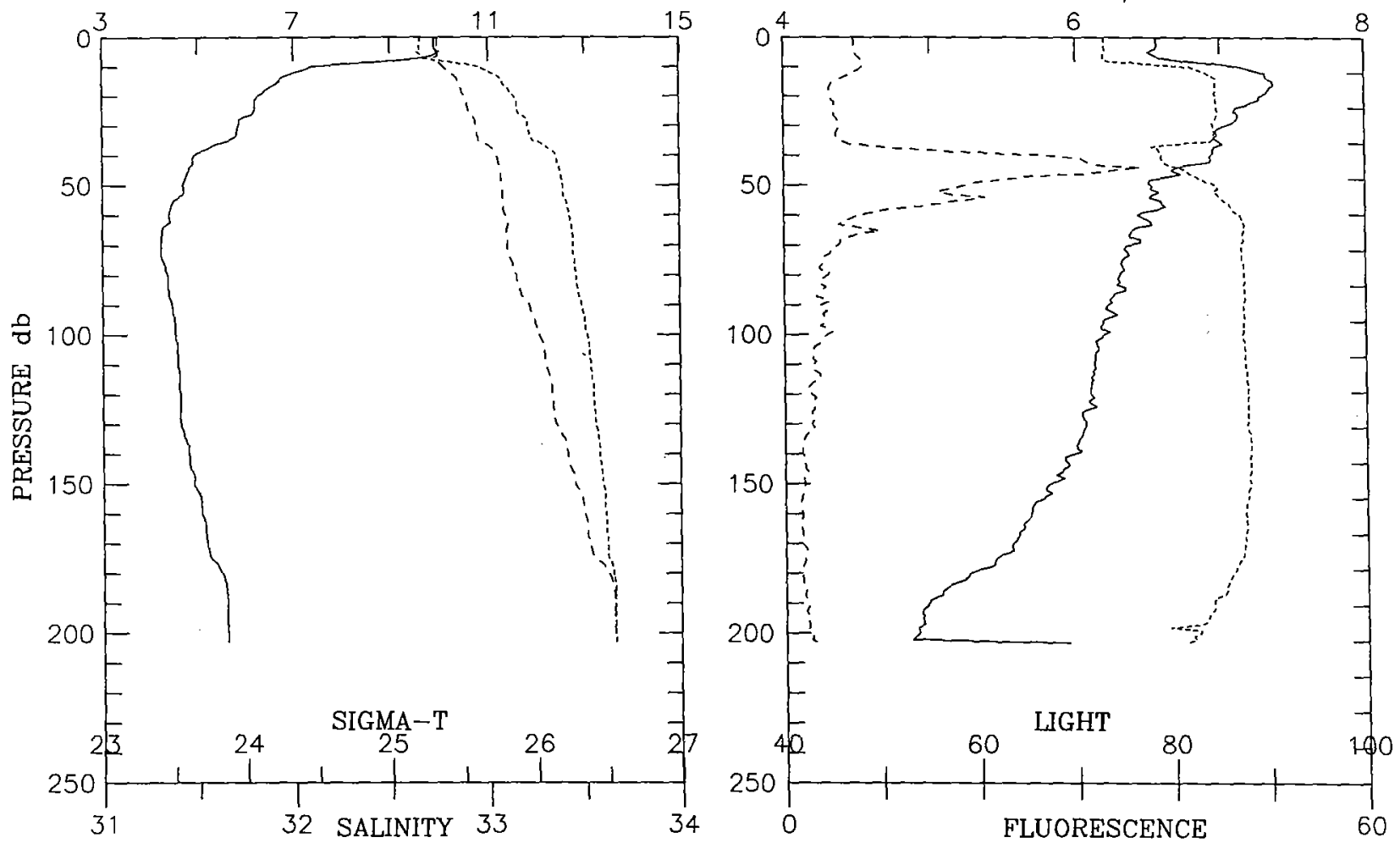


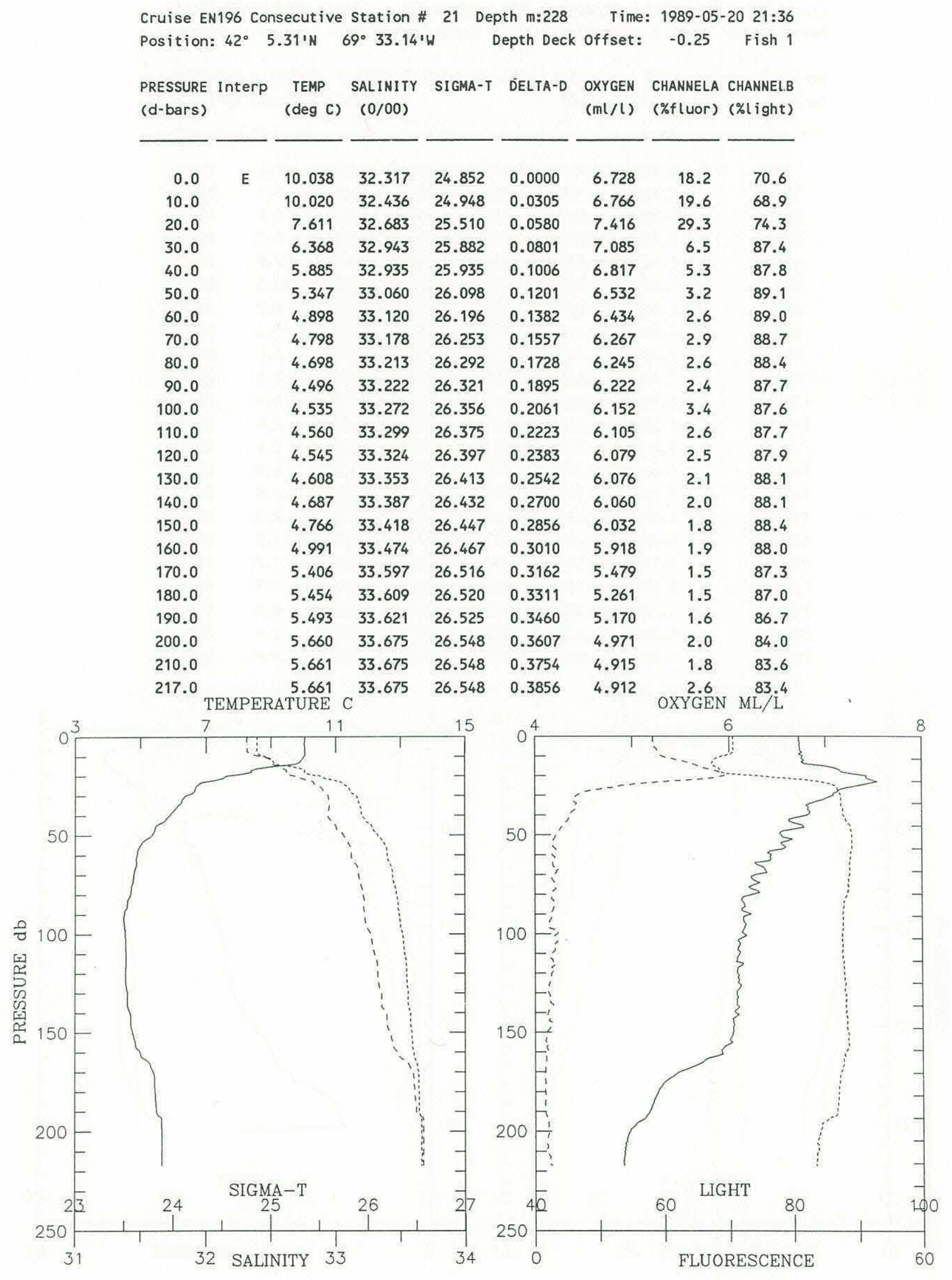




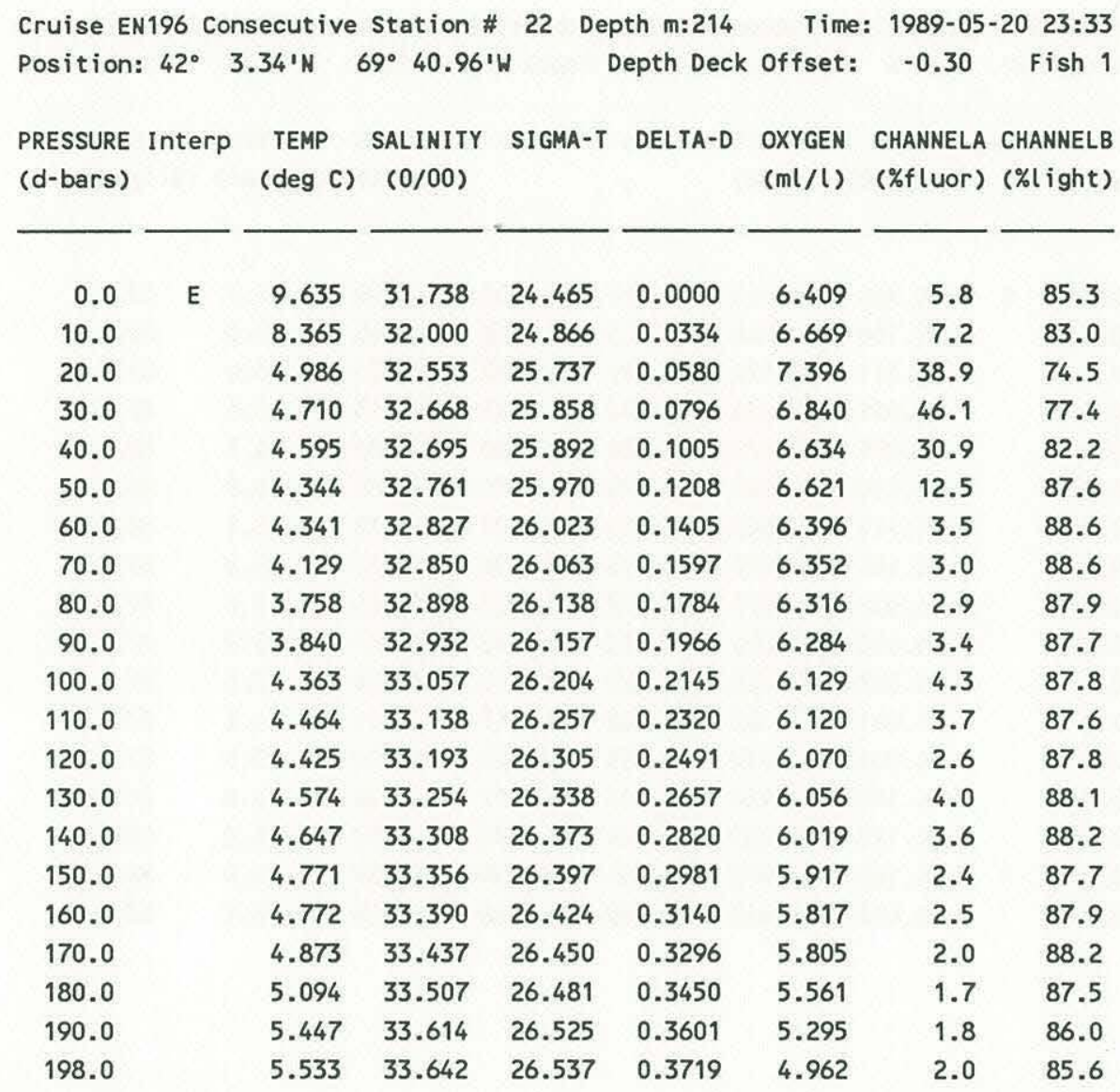
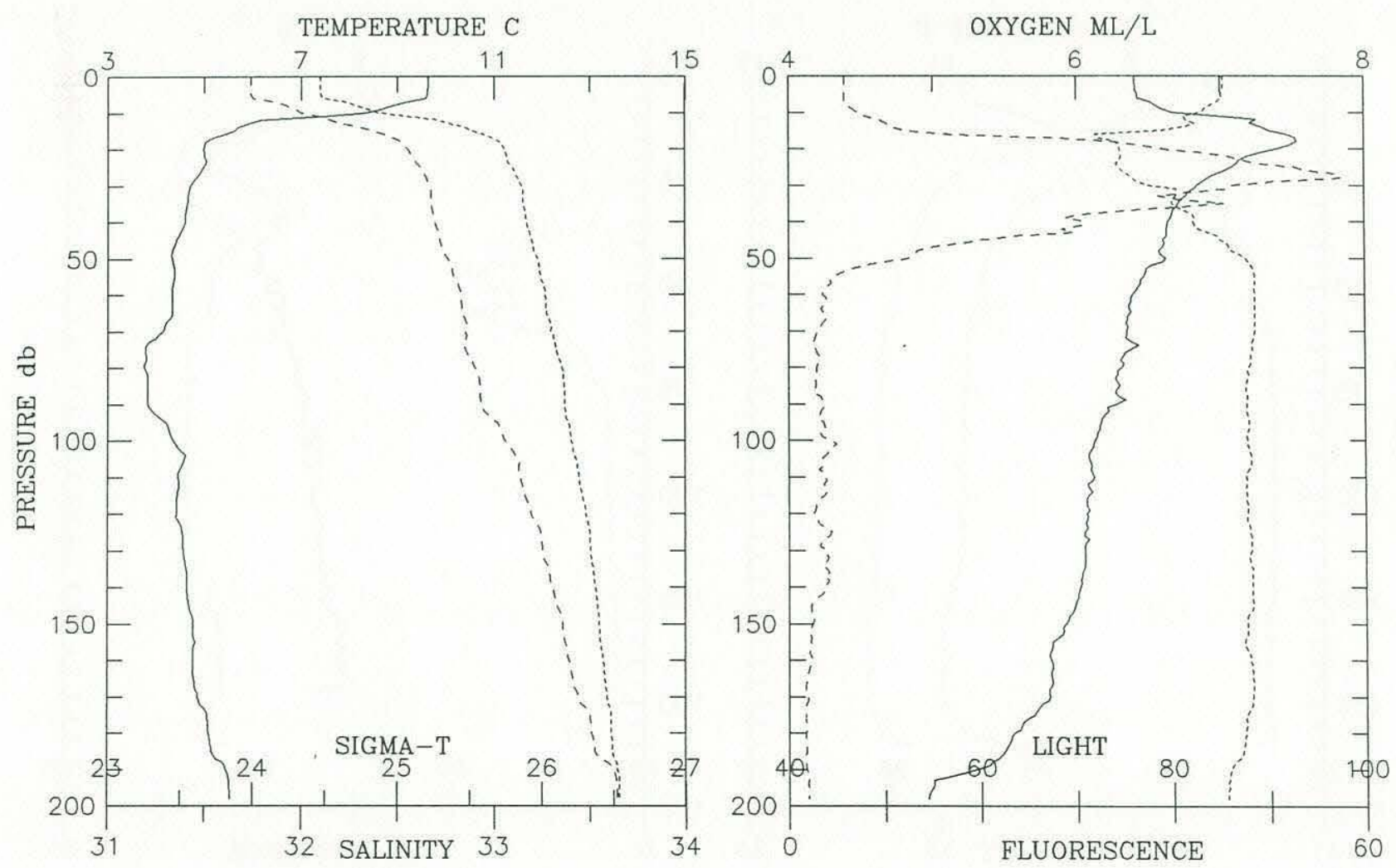


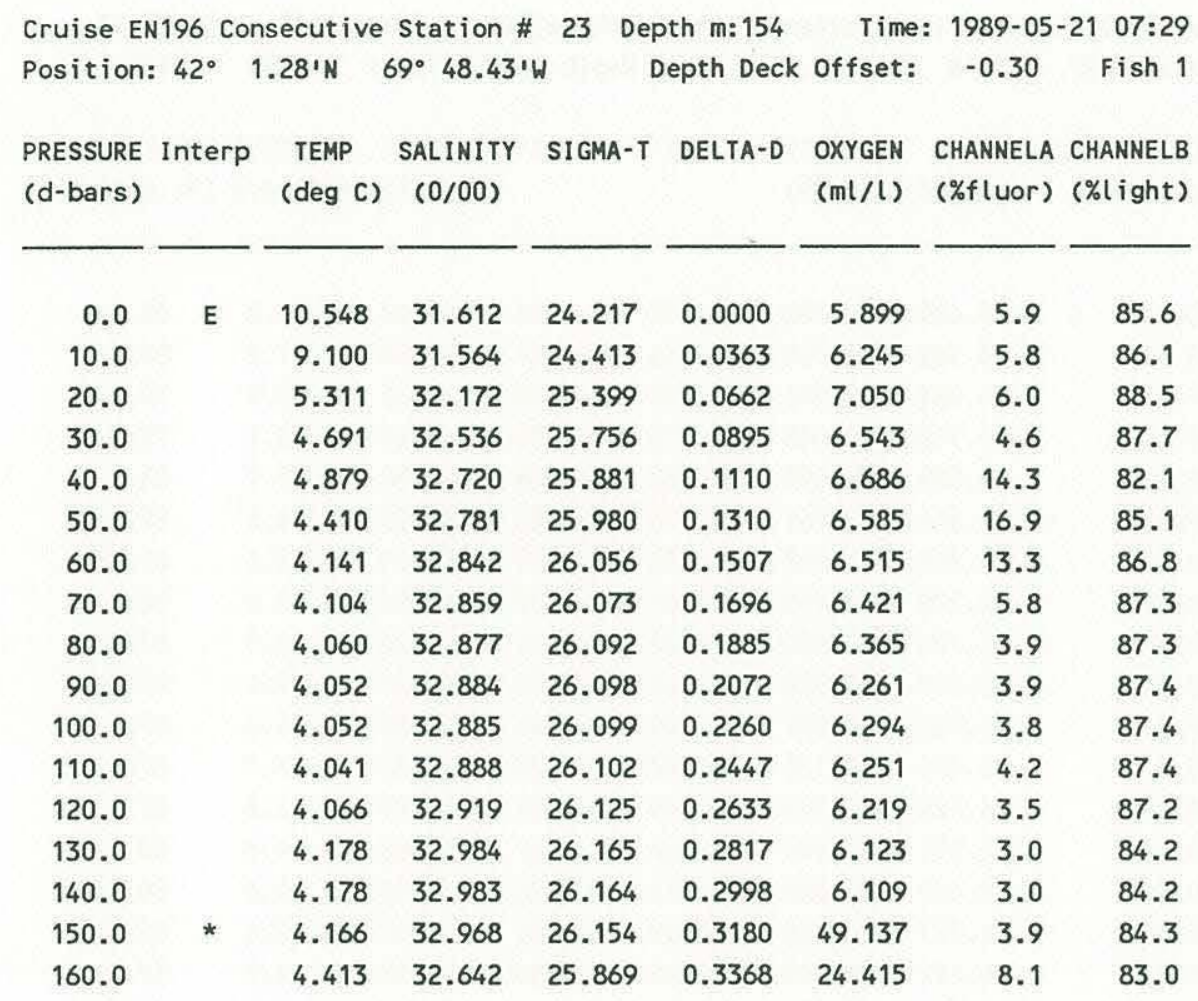
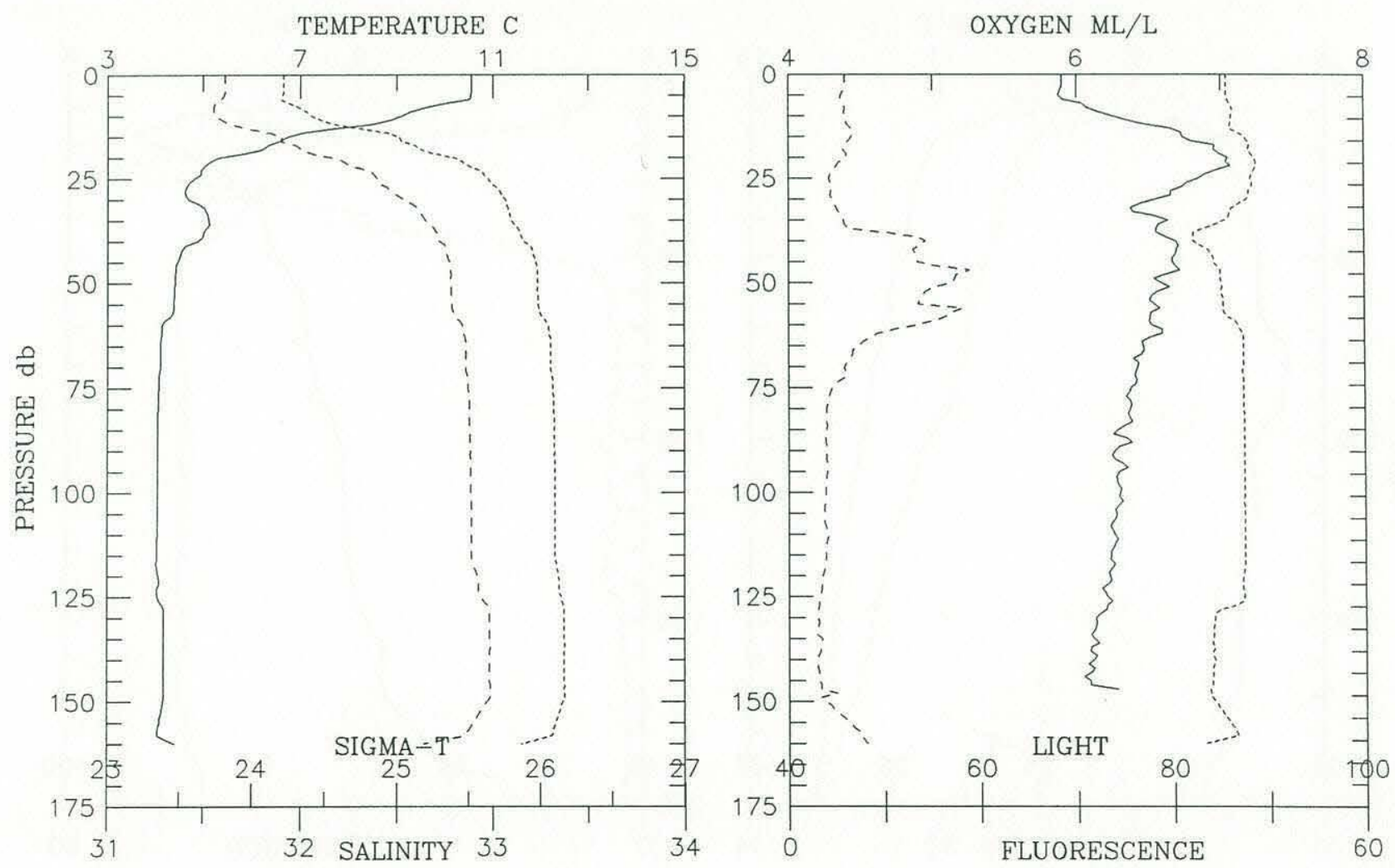
Cruise EN196 Consecutive Station \# 24 Depth m:39 Time: 1989-05-21 09:21 Position: $41^{\circ} 59.52^{\prime} \mathrm{N} 69^{\circ} 56.65^{\prime} \mathrm{W}$ Depth Deck offset: -0.25 Fish 1

\begin{tabular}{|c|c|c|c|c|c|c|c|c|}
\hline $\begin{array}{l}\text { PRESSURE } \\
\text { (d-bars) }\end{array}$ & Interp & $\begin{array}{l}\text { TEMP } \\
(\operatorname{deg} C)\end{array}$ & $\begin{array}{l}\text { SALINITY } \\
(0 / 00)\end{array}$ & SIGMA-T & DELTA-D & $\begin{array}{l}\text { OXYGEN } \\
(\mathrm{ml} / \mathrm{l})\end{array}$ & $\begin{array}{l}\text { CHANNELA } \\
\text { (\%fluor) }\end{array}$ & $\begin{array}{l}\text { CHANNELB } \\
\text { (\%light) }\end{array}$ \\
\hline 0.0 & $E$ & 9.913 & 31.610 & 24.321 & 0.0000 & 6.069 & 5.5 & 86.3 \\
\hline 2.0 & $E$ & 9.913 & 31.609 & 24.320 & 0.0071 & 6.071 & 5.5 & 86.3 \\
\hline 4.0 & & 9.911 & 31.609 & 24.320 & 0.0143 & 5.966 & 5.5 & 86.3 \\
\hline 6.0 & & 9.889 & 31.610 & 24.324 & 0.0214 & 6.018 & 5.5 & 86.3 \\
\hline 8.0 & & 9.561 & 31.637 & 24.399 & 0.0285 & 6.158 & 5.9 & 85.9 \\
\hline 10.0 & & 8.964 & 31.710 & 24.549 & 0.0353 & 6.274 & 6.7 & 85.4 \\
\hline 12.0 & & 8.762 & 31.748 & 24.609 & 0.0420 & 6.254 & 5.5 & 86.9 \\
\hline 14.0 & & 8.592 & 31.745 & 24.632 & 0.0485 & 6.283 & 5.1 & 87.0 \\
\hline 16.0 & & 6.990 & 31.991 & 25.051 & 0.0547 & 6.541 & 5.5 & 87.8 \\
\hline 18.0 & & 6.729 & 32.043 & 25.126 & 0.0604 & 6.363 & 4.7 & 87.2 \\
\hline 20.0 & & 6.462 & 32.091 & 25.198 & 0.0659 & 6.368 & 4.5 & 86.9 \\
\hline 22.0 & & 6.322 & 32.111 & 25.231 & 0.0713 & 6.308 & 4.4 & 86.5 \\
\hline 24.0 & & 5.940 & 32.204 & 25.351 & 0.0766 & 6.157 & 4.0 & 86.4 \\
\hline 26.0 & & 5.793 & 32.254 & 25.408 & 0.0817 & 6.071 & 3.6 & 86.4 \\
\hline 28.0 & & 5.715 & 32.265 & 25.426 & 0.0867 & 5.988 & 3.6 & 86.5 \\
\hline 30.0 & & 5.647 & 32.278 & 25.444 & 0.0917 & 5.925 & 3.5 & 86.7 \\
\hline 32.0 & & 5.599 & 32.283 & 25.454 & 0.0967 & 5.904 & 3.6 & 86.5 \\
\hline 34.0 & & 5.502 & 32.301 & 25.480 & 0.1016 & 5.878 & 3.5 & 86.7 \\
\hline 36.0 & & 5.454 & 32.310 & 25.492 & 0.1065 & 5.845 & 3.6 & 86.6 \\
\hline 37.0 & & 5.436 & 32.313 & 25.497 & 0.1090 & 5.842 & 3.6 & 86.6 \\
\hline
\end{tabular}
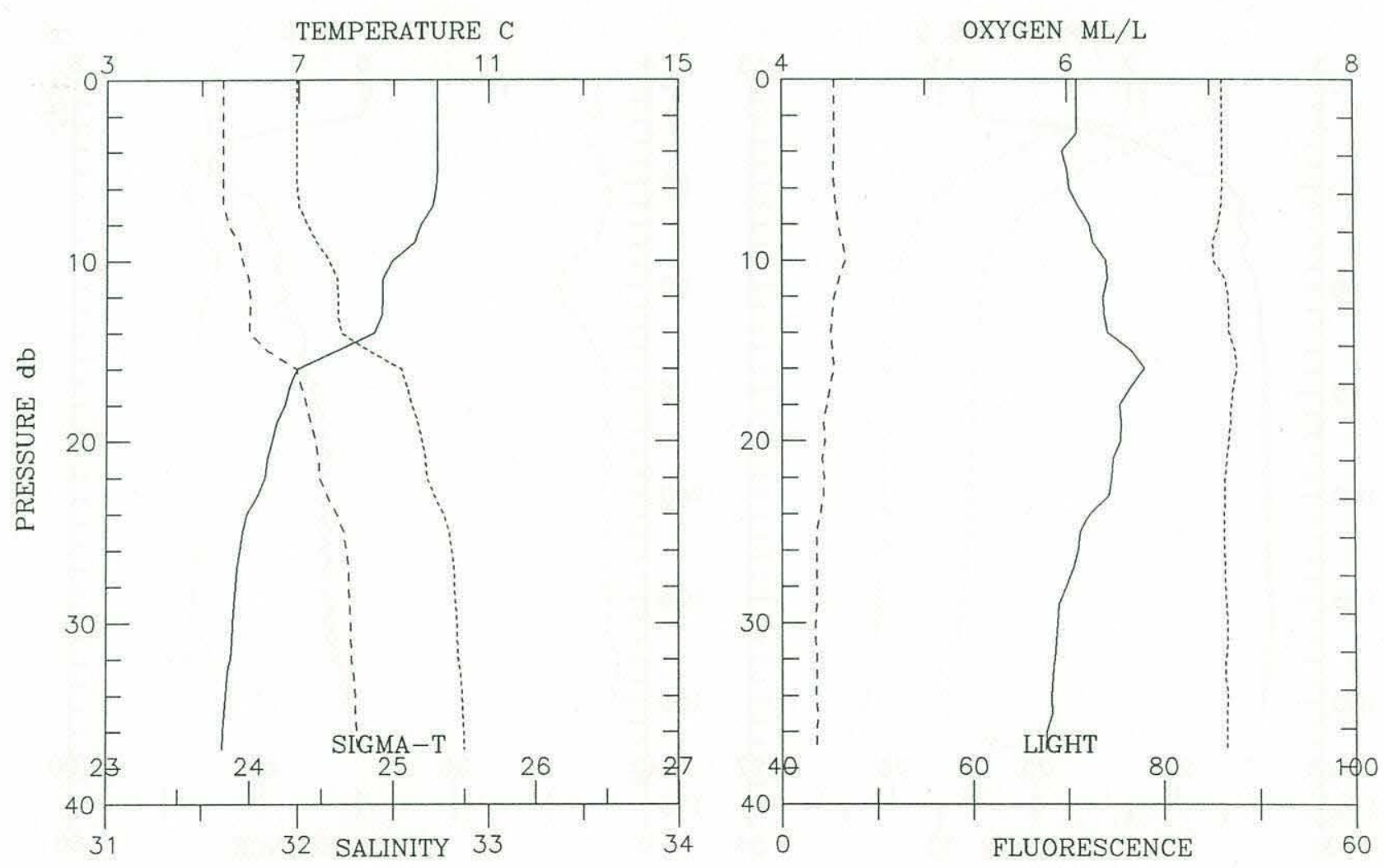


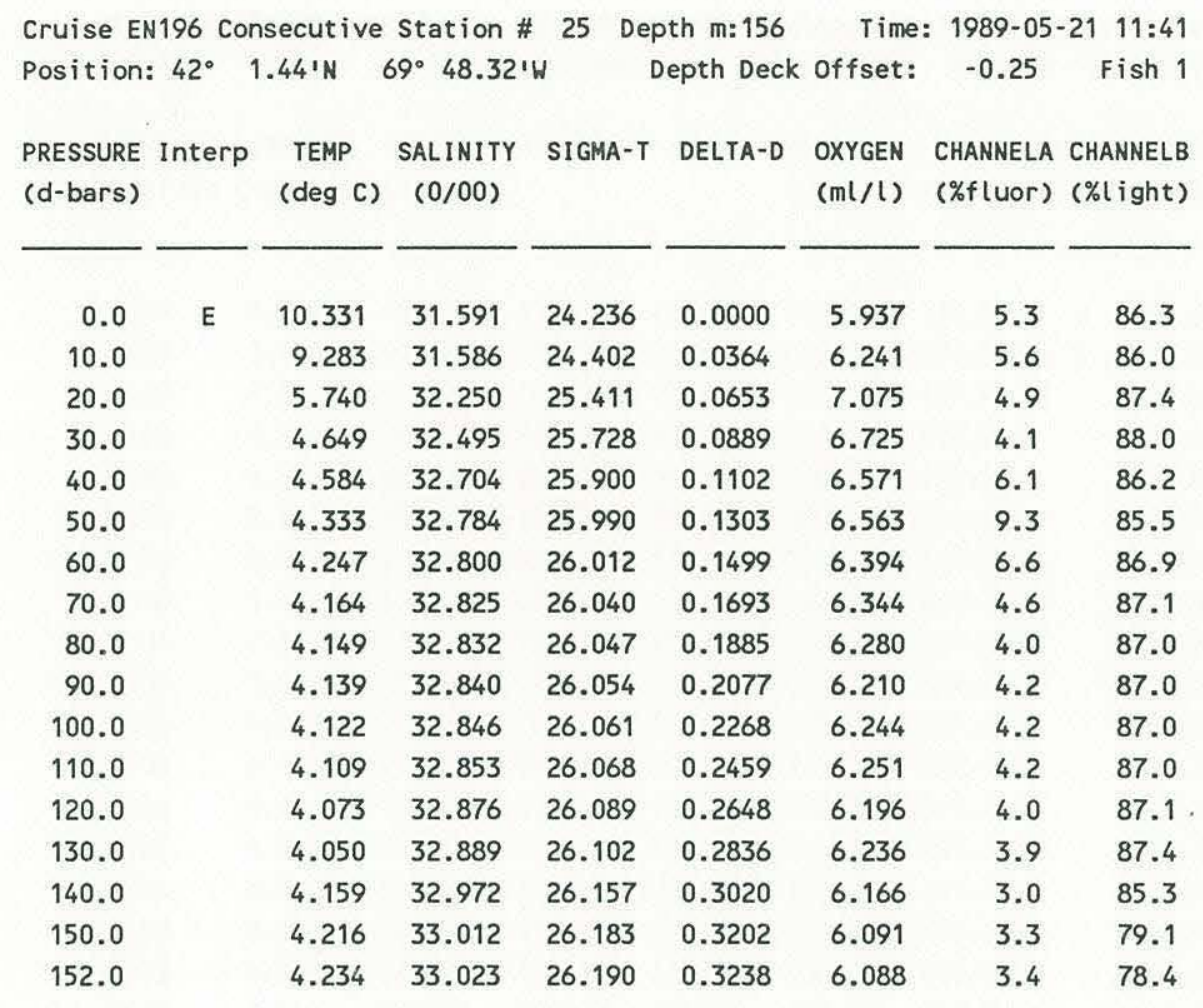
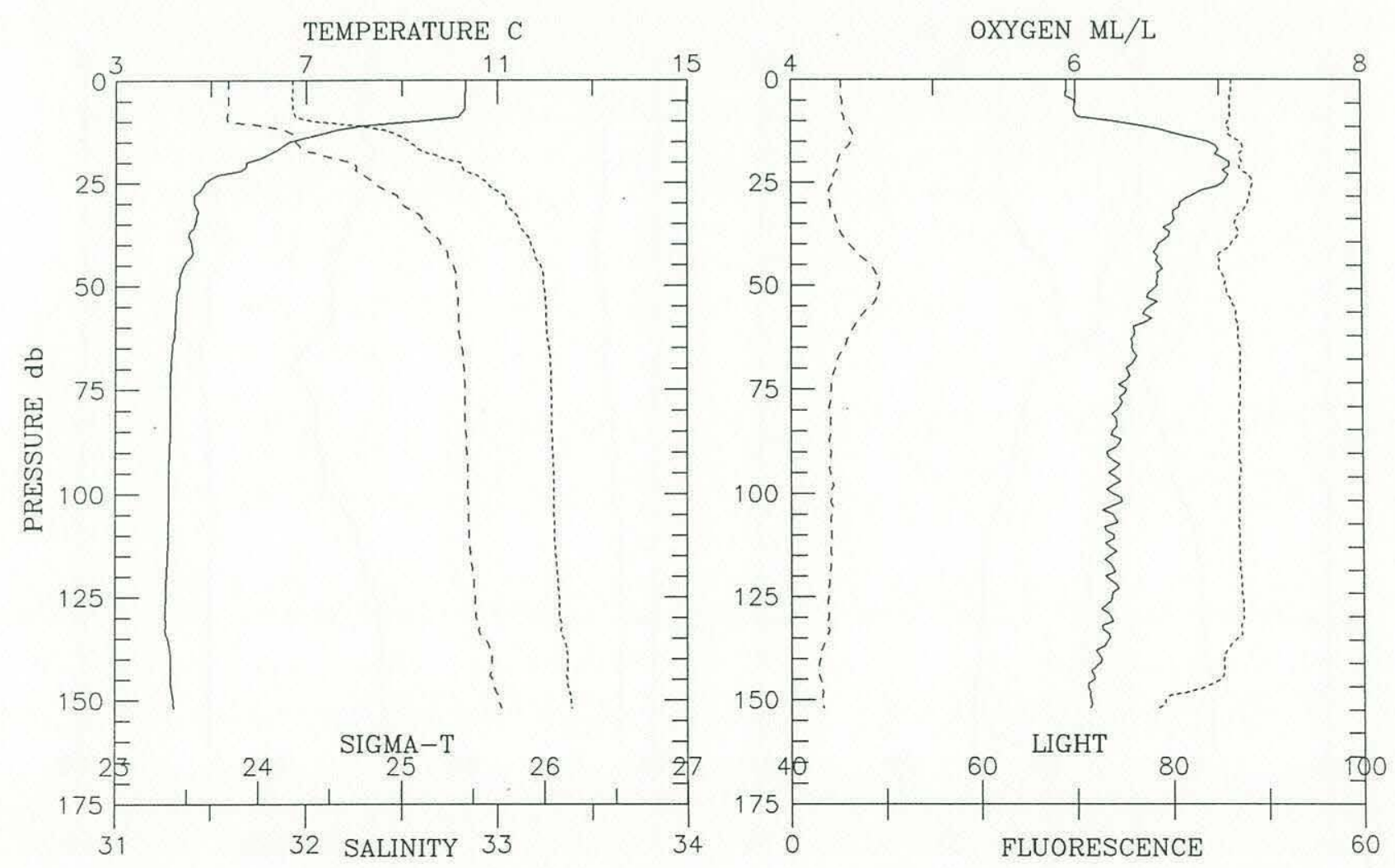
Cruise EN196 Consecutive Station \# 26 Depth m:212 Time: 1989-05-21 13:39 Position: $42^{\circ} 3.25^{\prime} \mathrm{N} 69^{\circ} 41.24^{\prime} \mathrm{W} \quad$ Depth Deck offset: -0.25 Fish 1

PRESSURE InterP TEMP SALINITY SIGMA-T DELTA-D OXYGEN CHANNELA CHANNELB (d-bars) ( $\operatorname{deg} C)(0 / 00) \quad(\mathrm{ml} / \mathrm{l})$ (\%fluor) (\%light)

\begin{tabular}{|c|c|c|c|c|c|c|c|c|}
\hline 0.0 & $E$ & 9.415 & 31.685 & 24.459 & 0.0000 & 6.684 & 3.6 & 85.0 \\
\hline 10.0 & & 8.759 & 32.070 & 24.861 & 0.0341 & 7.433 & 4.5 & 81.3 \\
\hline 20.0 & & 7.606 & 32.702 & 25.526 & 0.0605 & 8.520 & 45.1 & 68.2 \\
\hline 30.0 & & 5.076 & 32.601 & 25.766 & 0.0832 & 8.361 & 22.1 & 82.4 \\
\hline 40.0 & & 4.504 & 32.698 & 25.904 & 0.1045 & 7.380 & 46.1 & 76.2 \\
\hline 50.0 & & 4.037 & 32.745 & 25.989 & 0.1247 & 6.608 & 13.7 & 85.9 \\
\hline 60.0 & & 3.676 & 32.772 & 26.045 & 0.1442 & 6.532 & 3.5 & 88.8 \\
\hline 70.0 & & 3.549 & 32.791 & 26.072 & 0.1632 & 6.495 & 2.9 & 88.5 \\
\hline 80.0 & & 3.722 & 32.884 & 26.130 & 0.1818 & 6.367 & 2.3 & 88.0 \\
\hline 90.0 & & 3.872 & 32.923 & 26.147 & 0.2001 & 6.273 & 2.4 & 87.8 \\
\hline 100.0 & & 4.054 & 32.985 & 26.178 & 0.2183 & 6.197 & 3.3 & 87.7 \\
\hline 110.0 & & 4.130 & 33.026 & 26.203 & 0.2361 & 6.156 & 2.7 & 87.5 \\
\hline 120.0 & & 4.215 & 33.074 & 26.233 & 0.2537 & 6.124 & 2.8 & 87.4 \\
\hline 130.0 & & 4.309 & 33.124 & 26.263 & 0.2710 & 6.048 & 3.1 & 87.2 \\
\hline 140.0 & & 4.367 & 33.146 & 26.274 & 0.2882 & 6.021 & 2.4 & 86.9 \\
\hline 150.0 & & 4.432 & 33.190 & 26.302 & 0.3052 & 6.032 & 2.7 & 87.0 \\
\hline 160.0 & & 4.532 & 33.255 & 26.343 & 0.3219 & 6.051 & 3.2 & 88.0 \\
\hline 170.0 & & 4.687 & 33.335 & 26.390 & 0.3381 & 5.994 & 2.6 & 88.3 \\
\hline 180.0 & & 4.800 & 33.397 & 26.427 & 0.3540 & 5.870 & 2.2 & 87.9 \\
\hline 190.0 & & 4.923 & 33.456 & 26.460 & 0.3697 & 5.785 & 1.7 & 88.0 \\
\hline 200.0 & & 5.601 & 33.666 & 26.548 & 0.3846 & 4.999 & 2.2 & 84.2 \\
\hline 205.0 & & 5.613 & 33.668 & 26.548 & 0.3919 & 4.871 & 2.5 & 78.4 \\
\hline
\end{tabular}
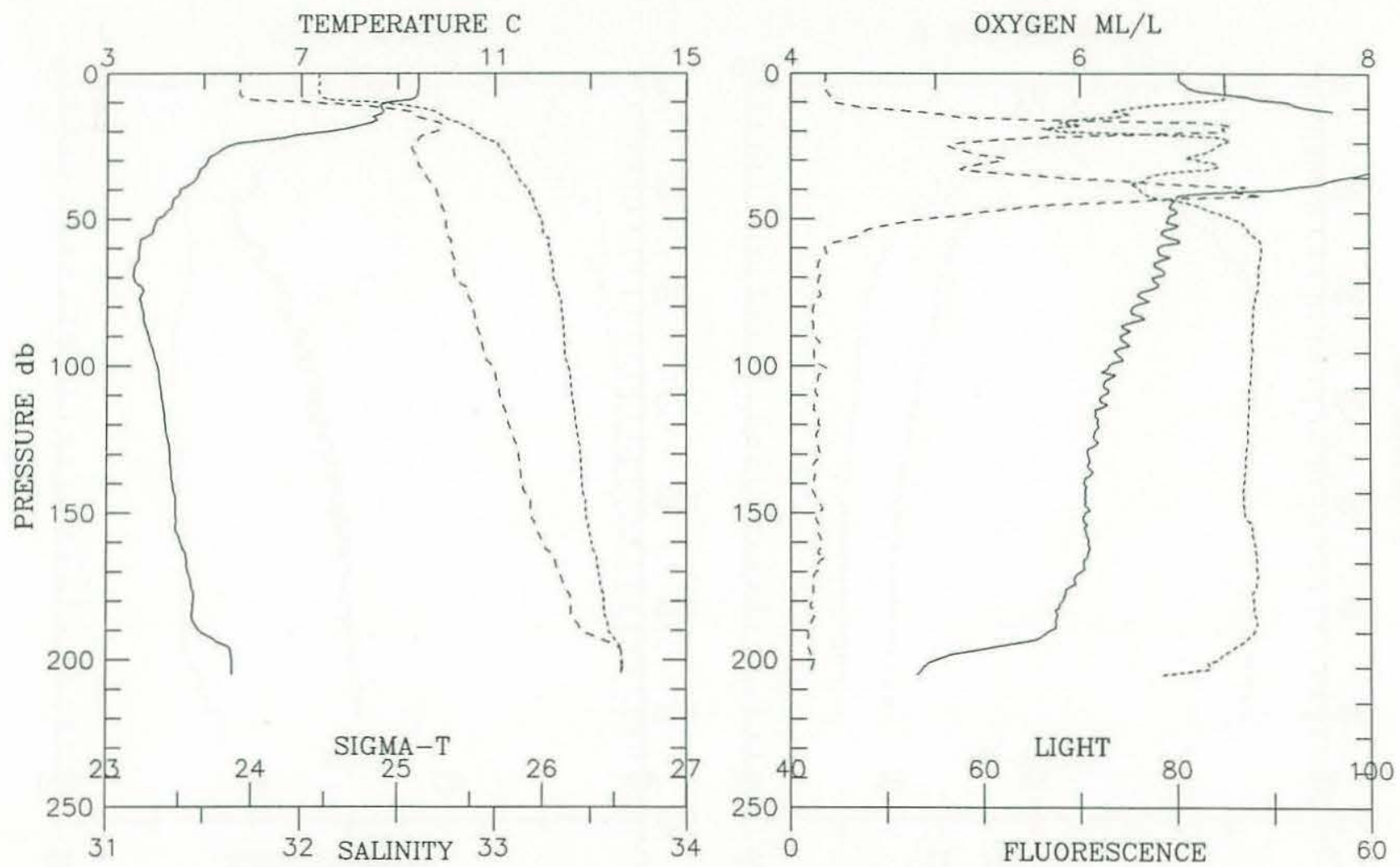


\begin{tabular}{|c|c|c|c|c|c|c|c|c|}
\hline Position: & $41^{\circ} 40$ & $81 \mathrm{~N}$ & $68^{\circ} 48.09^{\prime}$ & & epth Deck & Offset: & -0.40 & Fish 1 \\
\hline $\begin{array}{l}\text { PRESSURE } \\
\text { (d-bars) }\end{array}$ & Interp & $\begin{array}{c}\text { TEMP } \\
\text { (deg C) }\end{array}$ & $\begin{array}{l}\text { SALINITY } \\
(0 / 00)\end{array}$ & SIGMA-T & DELTA-D & $\begin{array}{l}\text { OXYGEN } \\
(\mathrm{ml} / \mathrm{l})\end{array}$ & $\begin{array}{l}\text { CHANNELA } \\
\text { (\%fluor) }\end{array}$ & $\begin{array}{l}\text { CHANNELB } \\
\text { (\%light) }\end{array}$ \\
\hline 0.0 & $E$ & 9.351 & 32.270 & 24.926 & 0.0000 & 6.073 & 8.4 & 61.3 \\
\hline 10.0 & & 9.329 & 32.282 & 24.940 & 0.0298 & 6.244 & 9.9 & 82.8 \\
\hline 20.0 & & 7.893 & 32.672 & 25.461 & 0.0565 & 6.605 & 9.9 & 85.1 \\
\hline 30.0 & & 5.969 & 32.795 & 25.814 & 0.0802 & 6.818 & 4.7 & 87.1 \\
\hline 40.0 & & 5.103 & 32.939 & 26.030 & 0.1005 & 6.597 & 4.3 & 86.5 \\
\hline 50.0 & & 4.518 & 33.005 & 26.146 & 0.1192 & 6.469 & 4.0 & 87.1 \\
\hline 60.0 & & 4.380 & 33.043 & 26.191 & 0.1372 & 6.303 & 3.2 & 88.1 \\
\hline 70.0 & & 4.410 & 33.093 & 26.228 & 0.1548 & 6.225 & 2.5 & 88.2 \\
\hline 80.0 & & 4.436 & 33.146 & 26.267 & 0.1722 & 6.267 & 2.4 & 88.1 \\
\hline 90.0 & & 4.514 & 33.185 & 26.289 & 0.1892 & 6.170 & 2.2 & 87.7 \\
\hline 100.0 & & 4.513 & 33.214 & 26.312 & 0.2060 & 6.065 & 2.5 & 87.6 \\
\hline 110.0 & & 4.527 & 33.225 & 26.320 & 0.2227 & 6.021 & 2.2 & 87.3 \\
\hline 120.0 & & 4.536 & 33.232 & 26.324 & 0.2394 & 6.029 & 2.4 & 87.1 \\
\hline 130.0 & & 4.540 & 33.235 & 26.326 & 0.2560 & 5.997 & 2.2 & 87.1 \\
\hline 140.0 & & 4.576 & 33.260 & 26.342 & 0.2725 & 5.982 & 2.5 & 85.7 \\
\hline 150.0 & & 4.590 & 33.268 & 26.347 & 0.2890 & 5.938 & 3.0 & 83.2 \\
\hline 151.0 & & 4.590 & 33.268 & 26.347 & 0.2907 & 5.938 & 3.0 & 82.4 \\
\hline
\end{tabular}
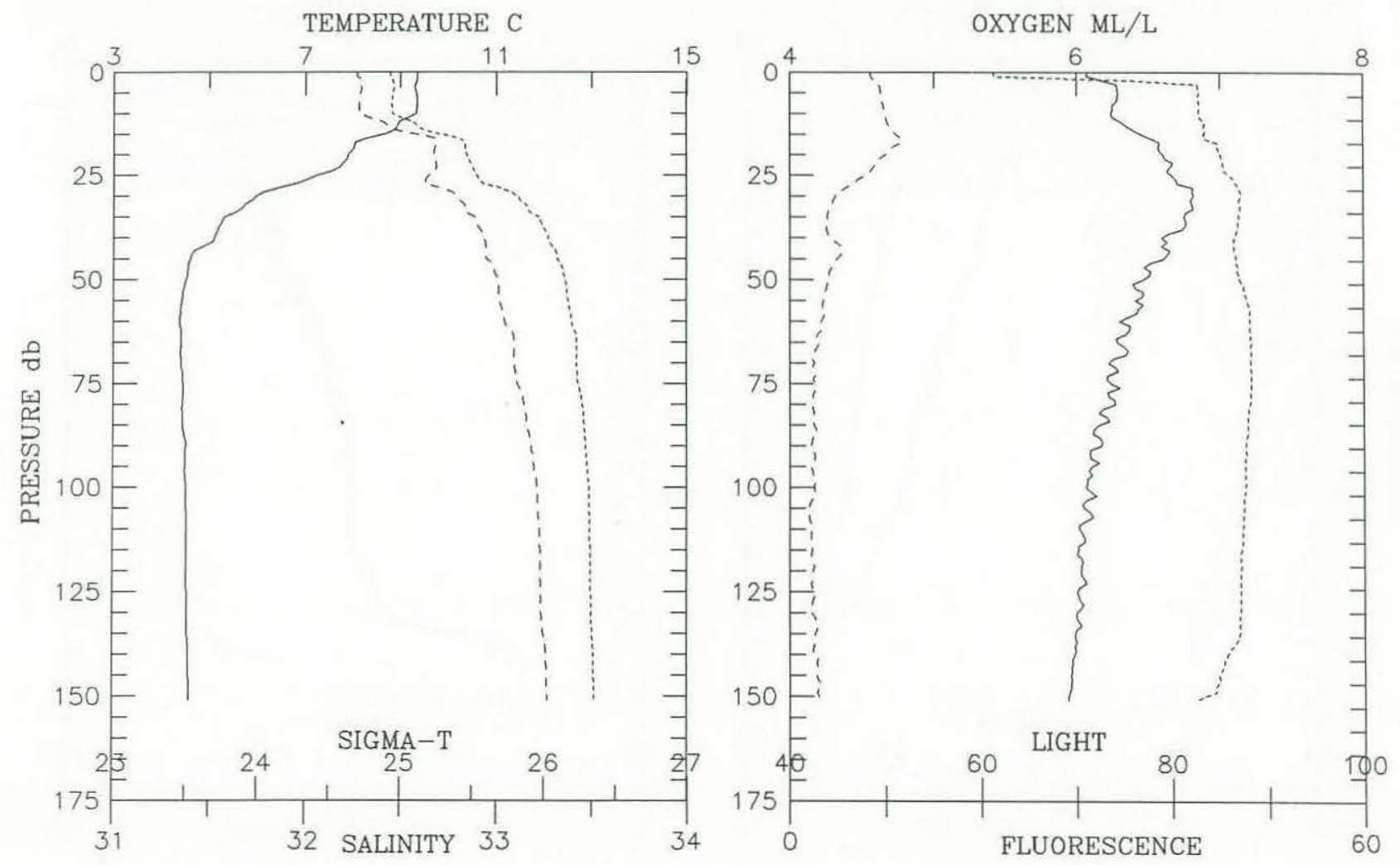


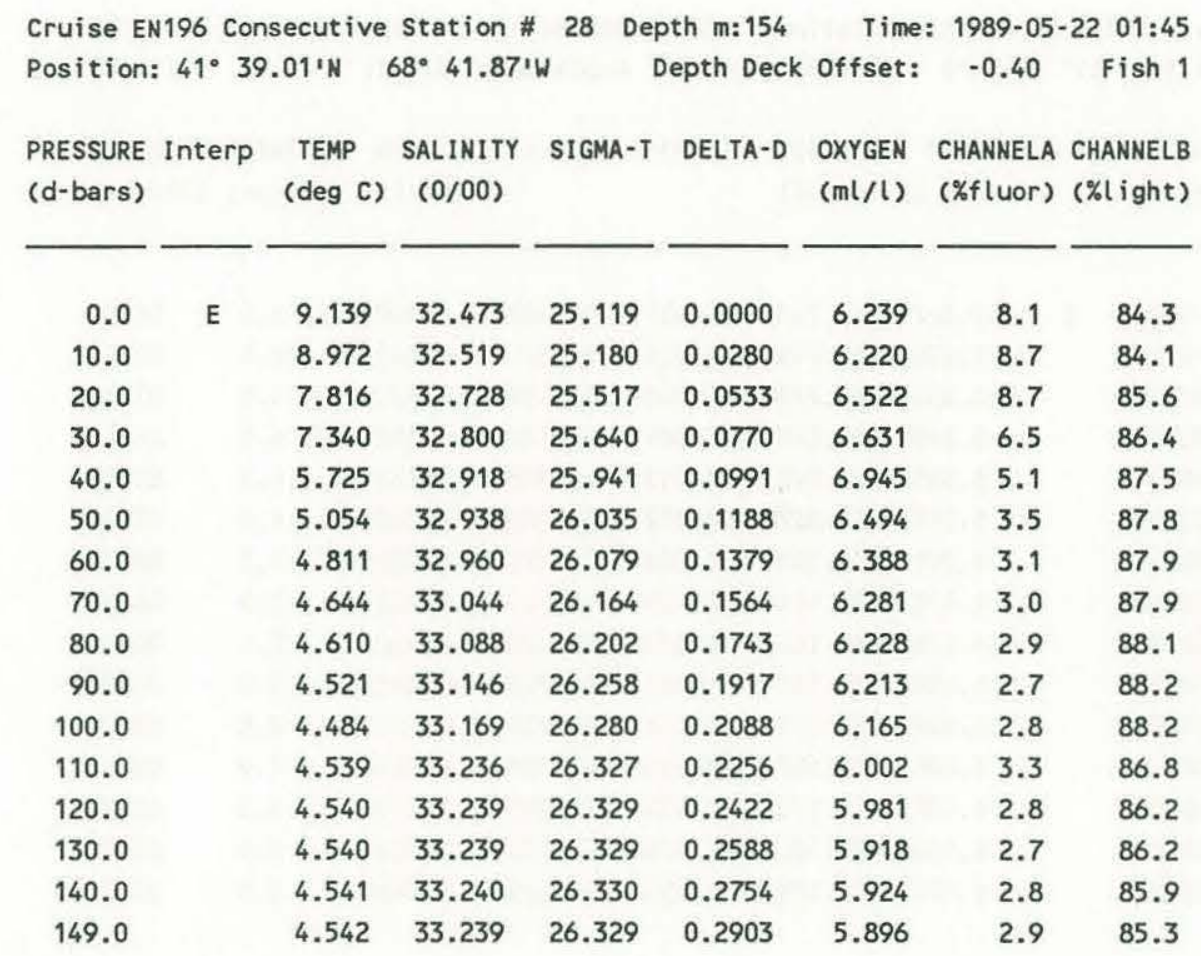
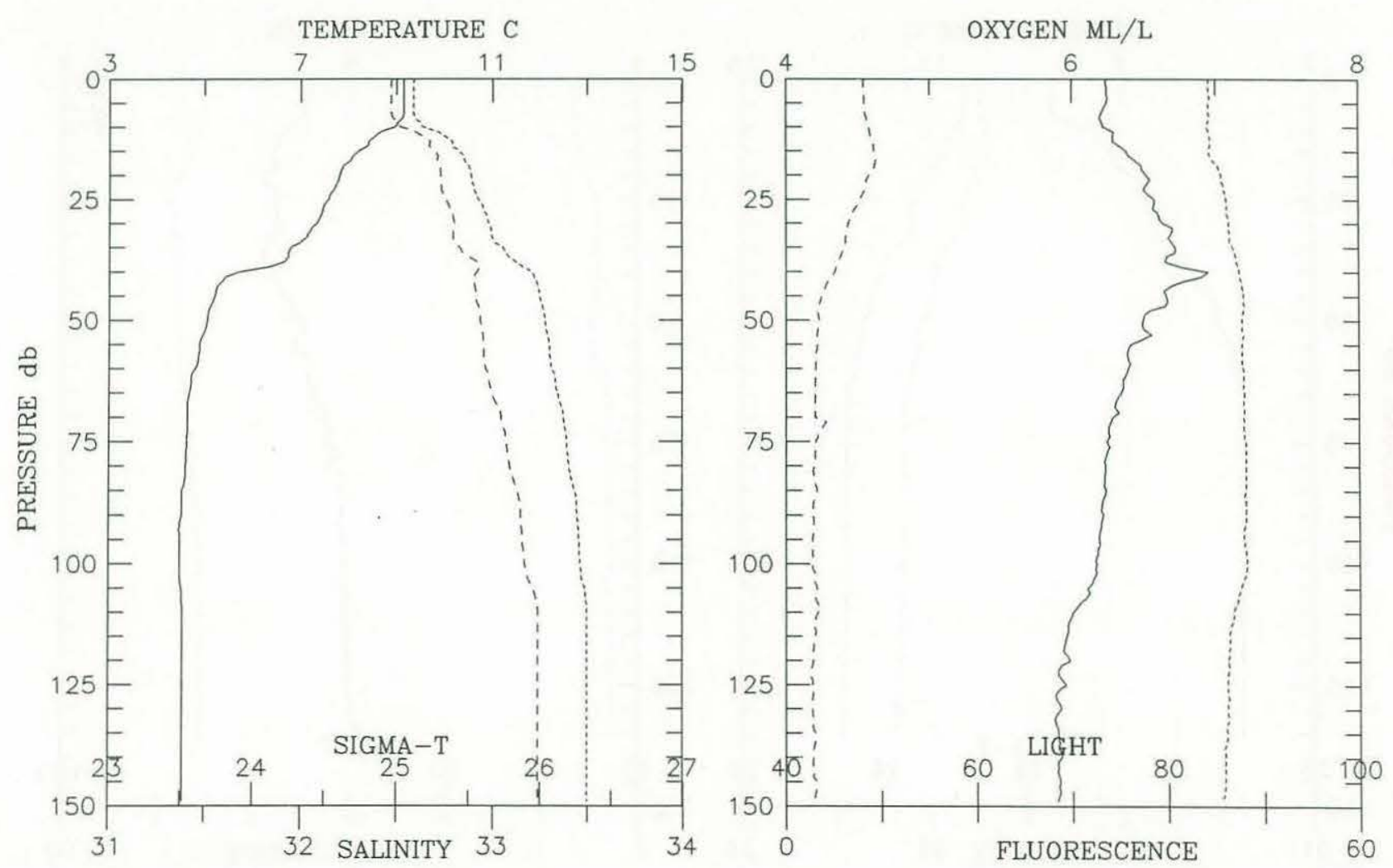


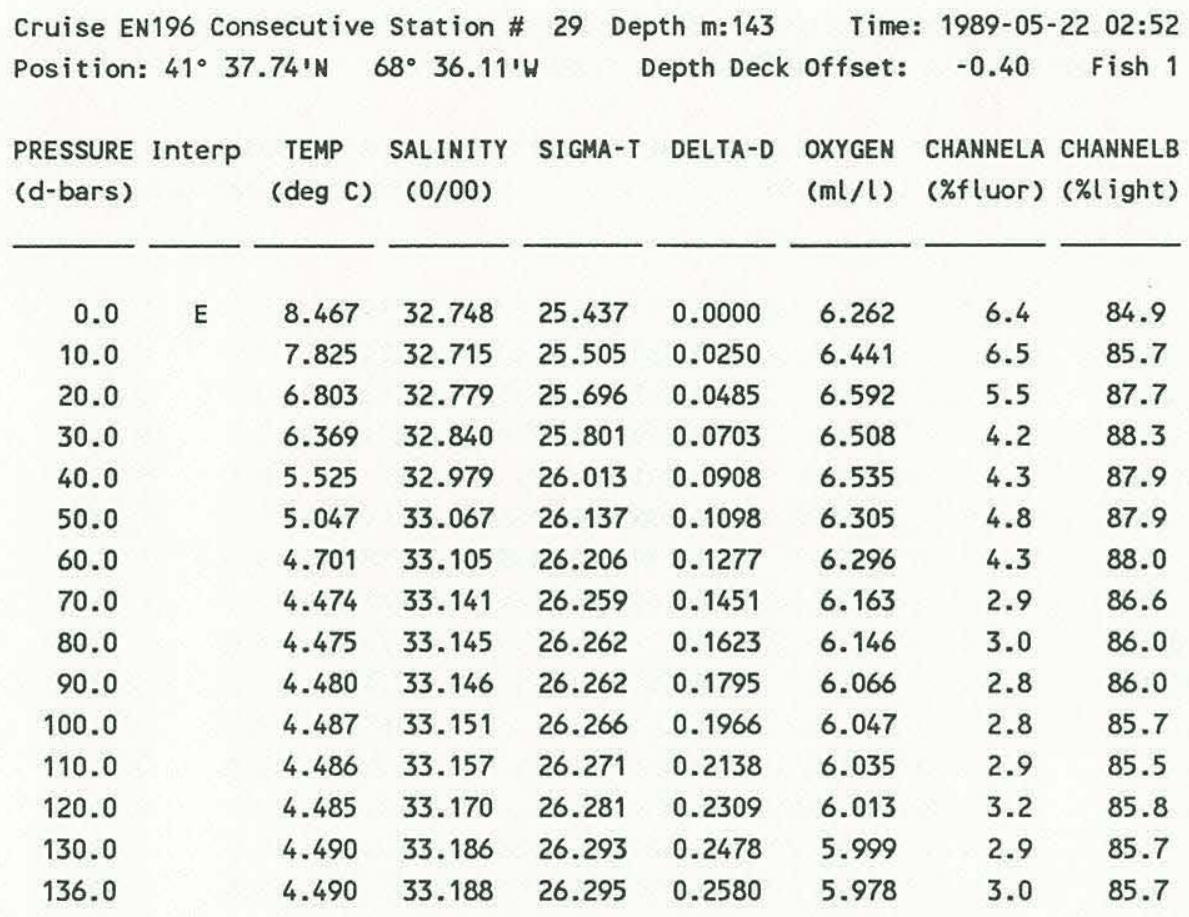
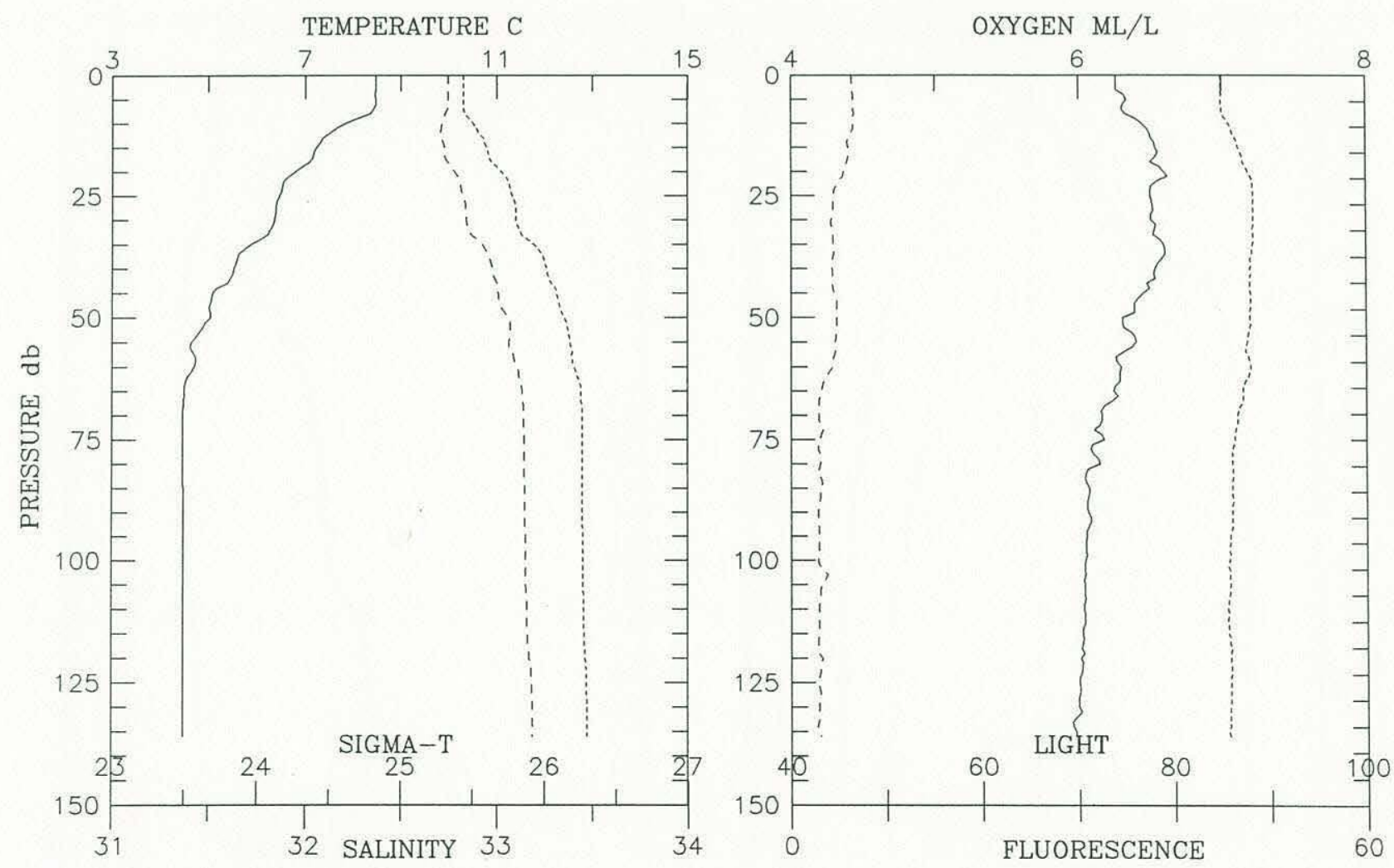


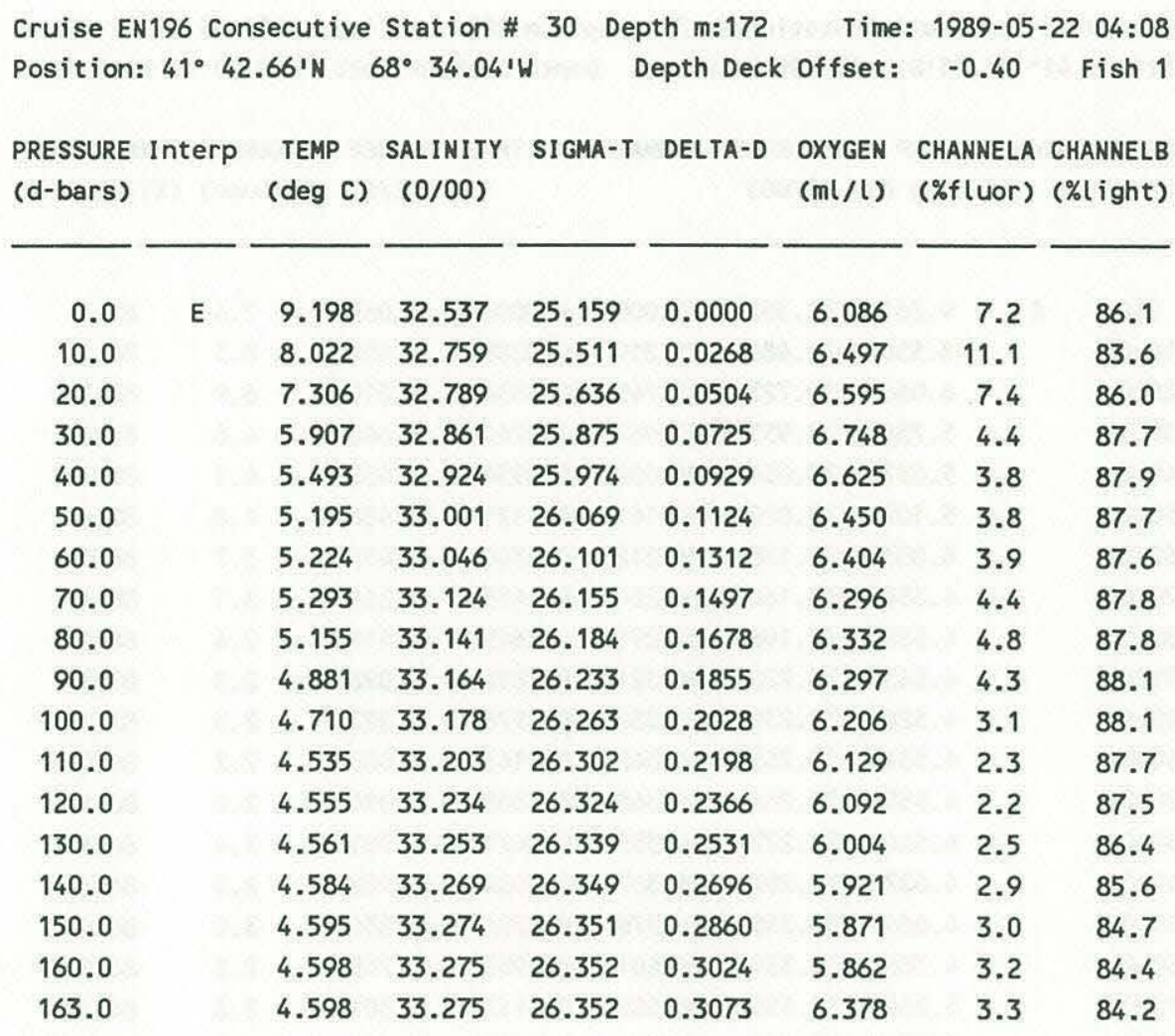
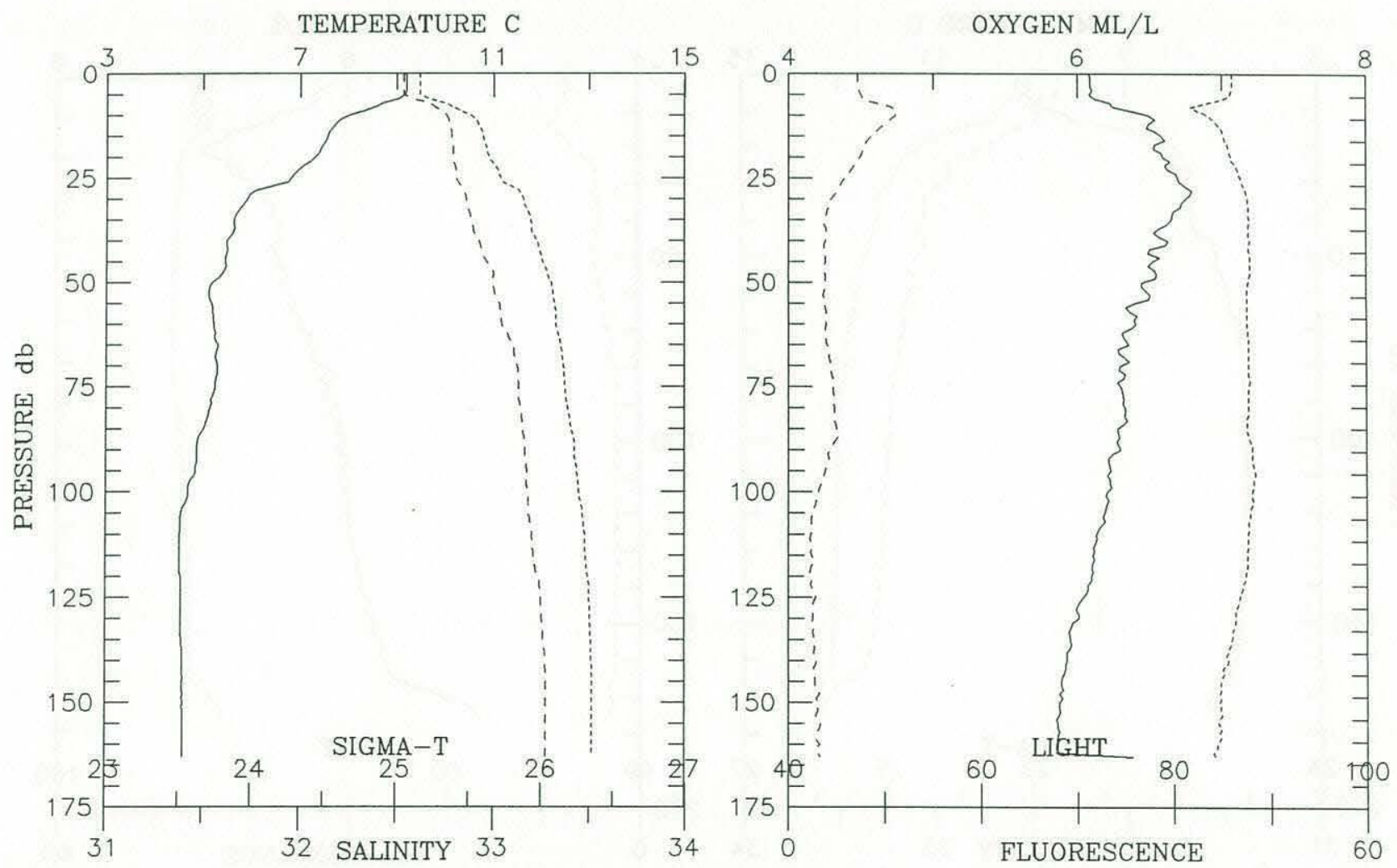
Cruise EN196 Consecutive Station \# 31 Depth m:183 Time: 1989-05-22 07:09 Position: $41^{\circ} 43.91$ ' $\mathrm{W} \quad 68^{\circ} 39.84^{\prime} \mathrm{W} \quad$ Depth Deck Offset: $-0.40 \quad$ Fish 1

\begin{tabular}{|c|c|c|c|c|c|c|c|c|}
\hline $\begin{array}{l}\text { PRESSURE } \\
\text { (d-bars) }\end{array}$ & Interp & $\begin{array}{c}\text { TEMP } \\
(\operatorname{deg} C)\end{array}$ & $\begin{array}{c}\text { SALINITY } \\
(0 / 00)\end{array}$ & SIGMA-T & DELTA-D & $\begin{array}{l}\text { OXYGEN } \\
(m l / l)\end{array}$ & $\begin{array}{l}\text { CHANNELA } \\
\text { (\%fluor) }\end{array}$ & $\begin{array}{l}\text { CHANNELB } \\
\text { (\%light) }\end{array}$ \\
\hline 0.0 & E & 9.261 & 32.352 & 25.005 & 0.0000 & 6.067 & 7.4 & 85.0 \\
\hline 10.0 & & 8.550 & 32.486 & 25.219 & 0.0285 & 6.454 & 8.5 & 84.5 \\
\hline 20.0 & & 6.066 & 32.723 & 25.745 & 0.0534 & 7.019 & 6.9 & 86.7 \\
\hline 30.0 & & 5.736 & 32.953 & 25.967 & 0.0741 & 6.640 & 4.6 & 87.3 \\
\hline 40.0 & & 5.637 & 33.054 & 26.059 & 0.0934 & 6.452 & 4.1 & 87.7 \\
\hline 50.0 & & 5.107 & 33.080 & 26.141 & 0.1121 & 6.480 & 4.8 & 87.6 \\
\hline 60.0 & & 4.883 & 33.138 & 26.212 & 0.1300 & 6.413 & 3.7 & 88.1 \\
\hline 70.0 & & 4.658 & 33.160 & 26.254 & 0.1475 & 6.266 & 3.1 & 88.4 \\
\hline 80.0 & & 4.588 & 33.196 & 26.291 & 0.1645 & 6.119 & 2.4 & 88.1 \\
\hline 90.0 & & 4.543 & 33.228 & 26.321 & 0.1813 & 6.076 & 2.3 & 87.5 \\
\hline 100.0 & & 4.520 & 33.239 & 26.332 & 0.1978 & 6.022 & 2.3 & 87.3 \\
\hline 110.0 & & 4.534 & 33.253 & 26.342 & 0.2143 & 6.000 & 2.2 & 86.9 \\
\hline 120.0 & & 4.555 & 33.264 & 26.348 & 0.2308 & 6.010 & 2.2 & 86.8 \\
\hline 130.0 & & 4.584 & 33.277 & 26.355 & 0.2471 & 5.961 & 2.4 & 86.6 \\
\hline 140.0 & & 4.627 & 33.297 & 26.367 & 0.2634 & 5.928 & 2.5 & 86.4 \\
\hline 150.0 & & 4.666 & 33.315 & 26.376 & 0.2796 & 5.836 & 3.0 & 86.4 \\
\hline 160.0 & & 4.765 & 33.359 & 26.401 & 0.2957 & 5.745 & 2.5 & 86.3 \\
\hline 170.0 & & 5.268 & 33.559 & 26.502 & 0.3113 & 5.301 & 2.8 & 84.2 \\
\hline 174.0 & & 5.330 & 33.581 & 26.512 & 0.3173 & 5.131 & 3.0 & 83.0 \\
\hline
\end{tabular}
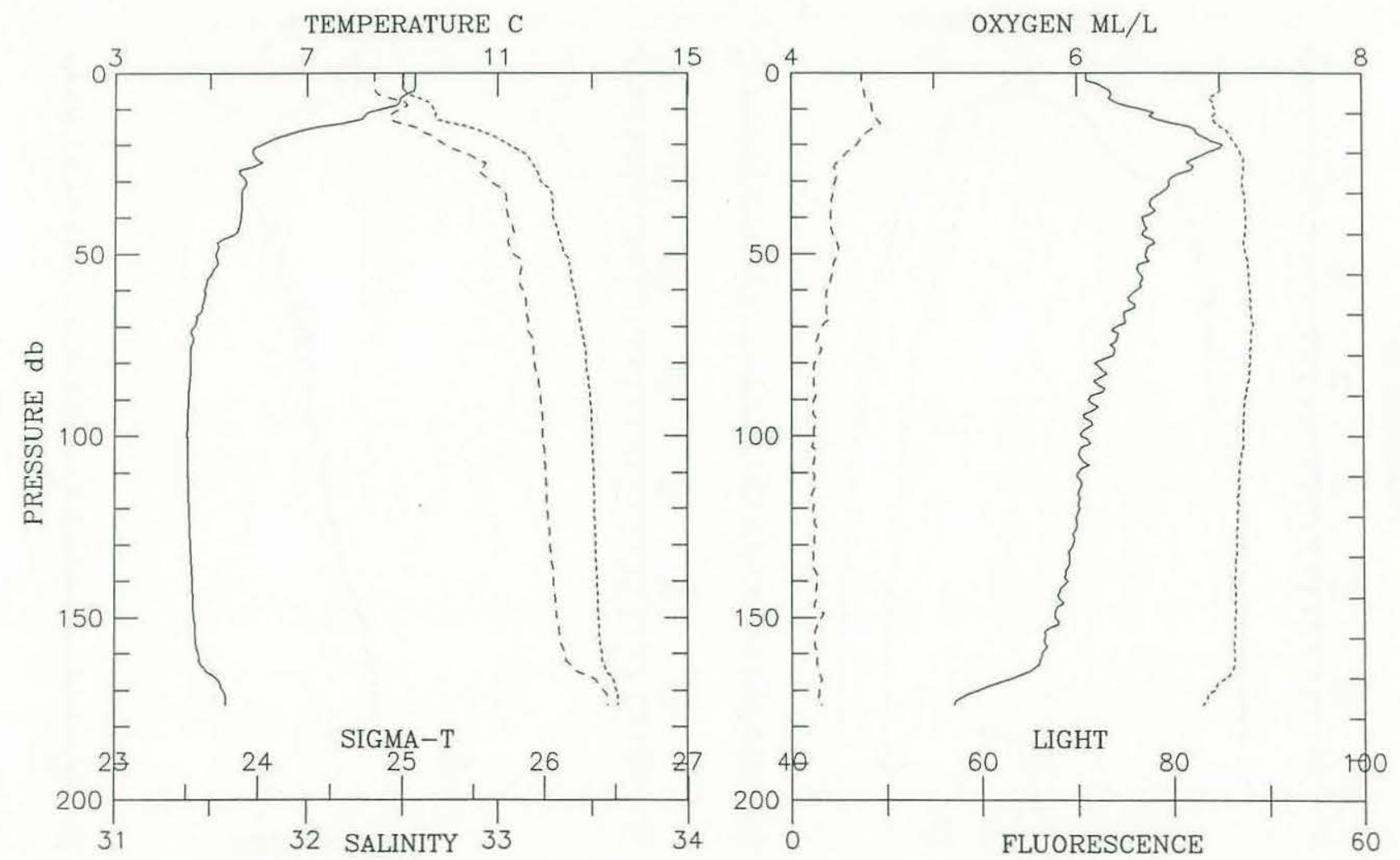


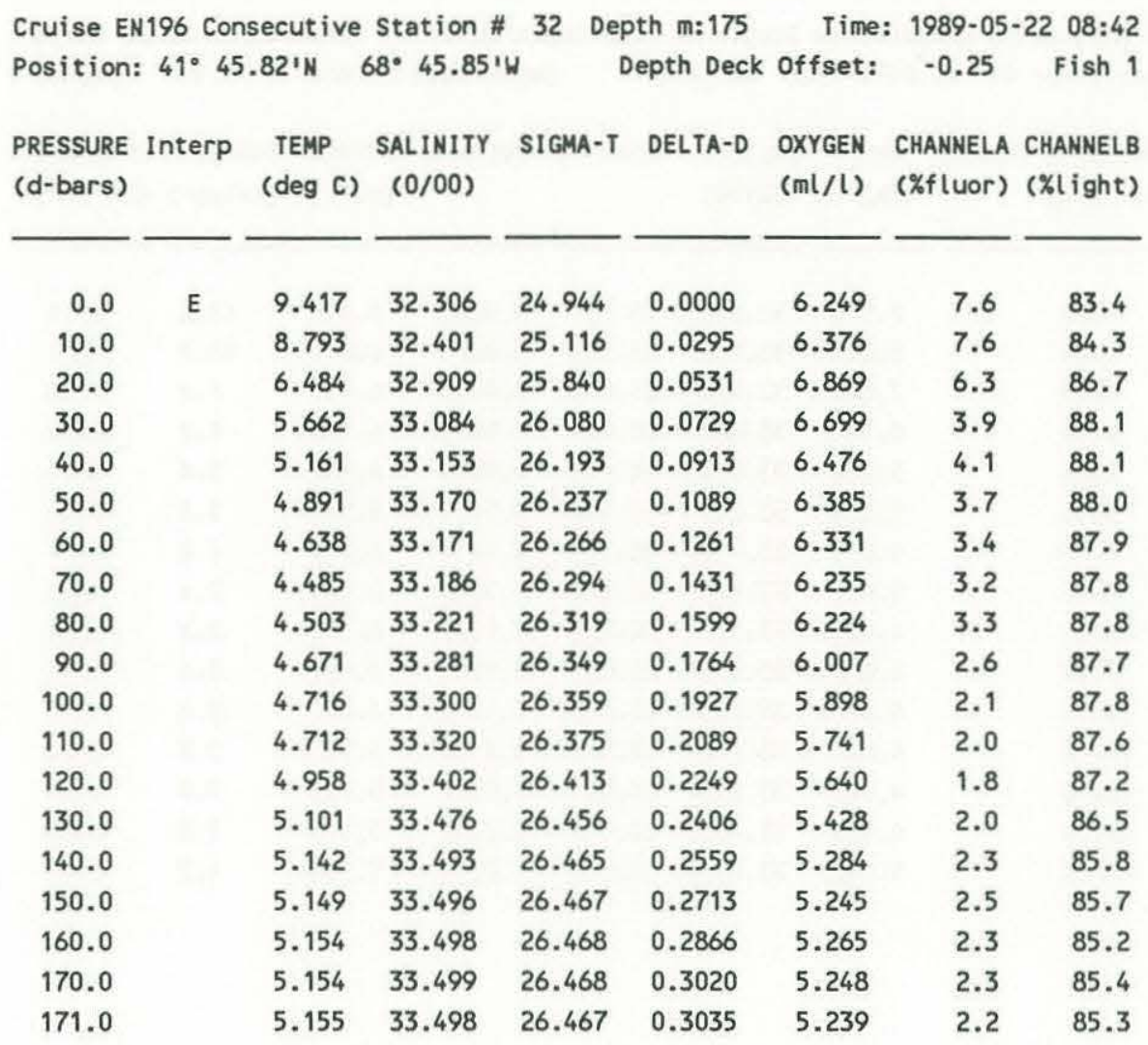
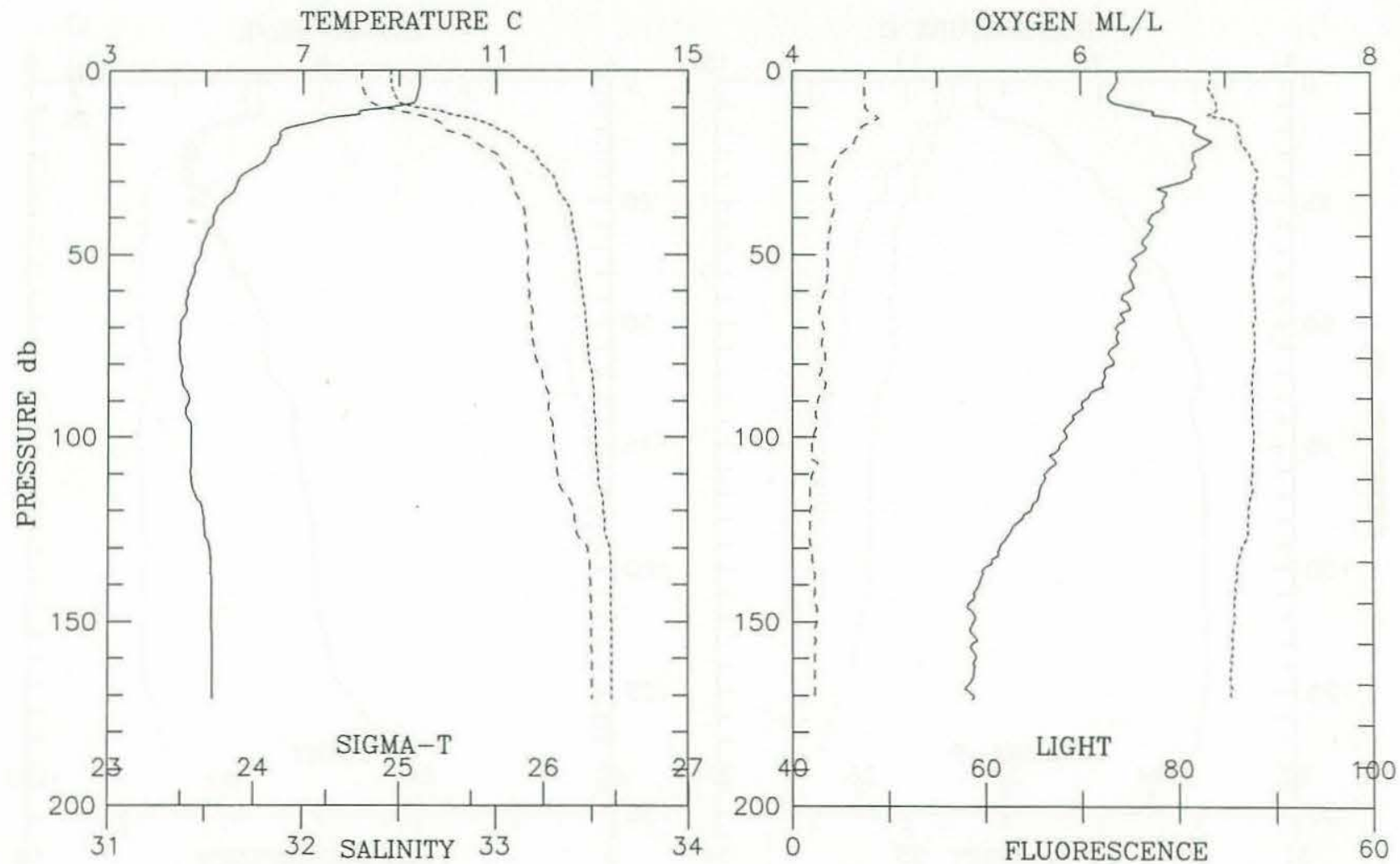


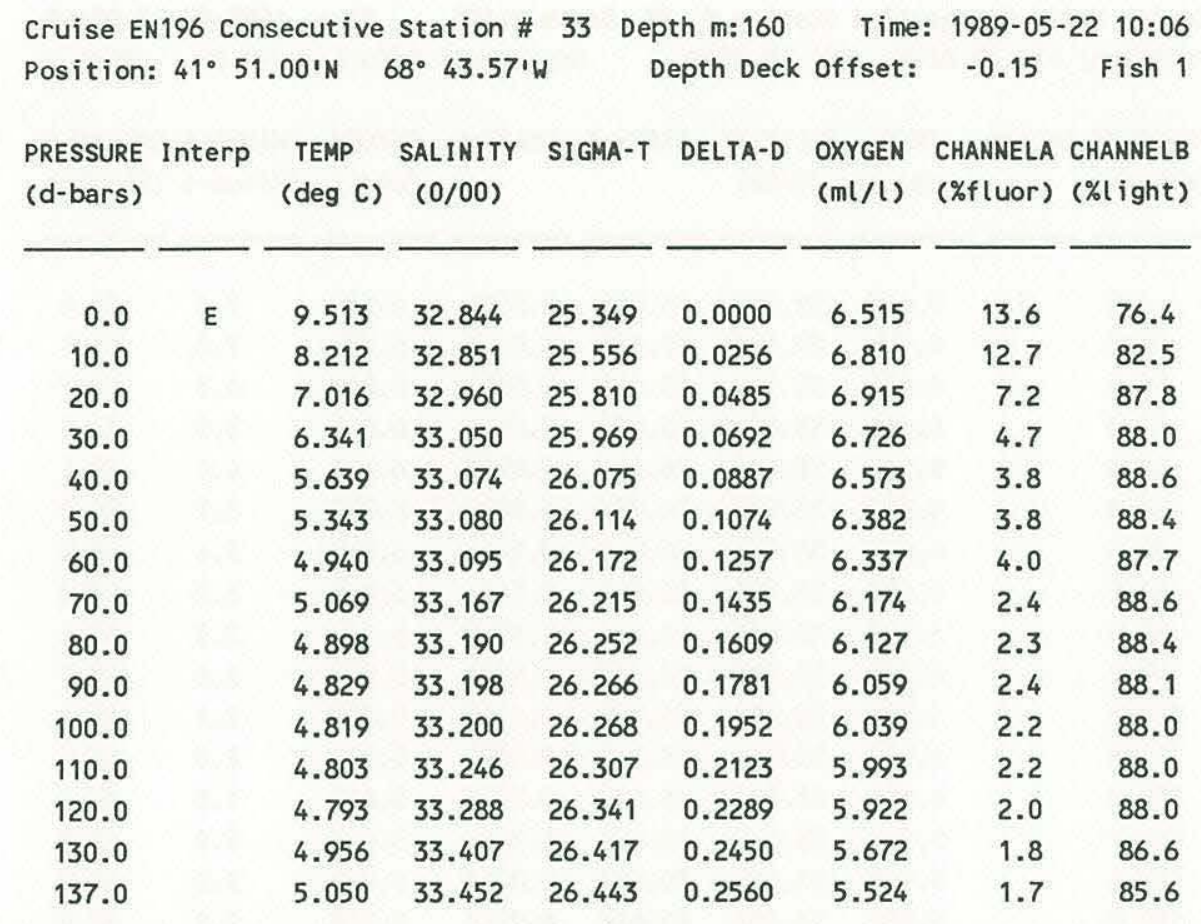
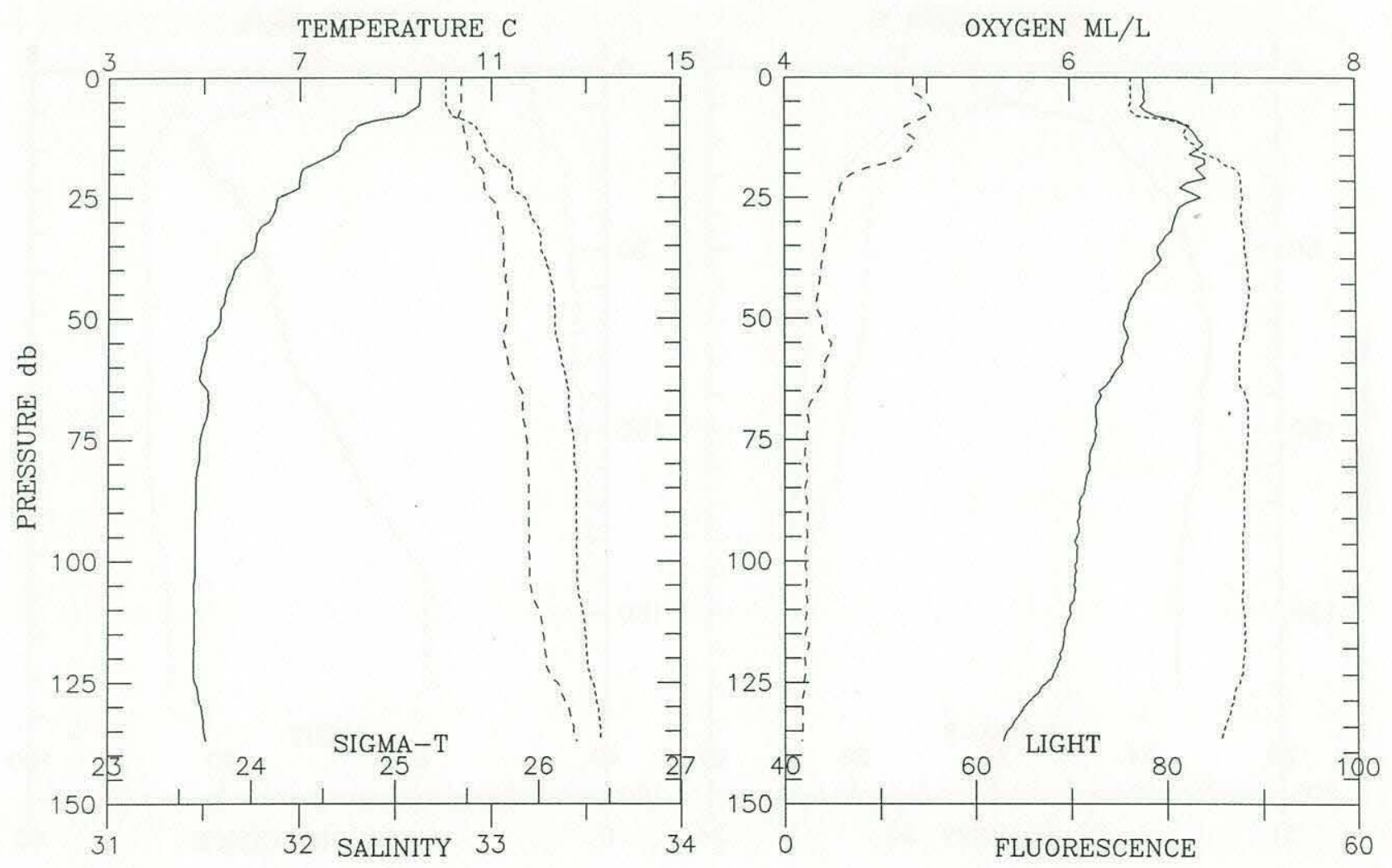


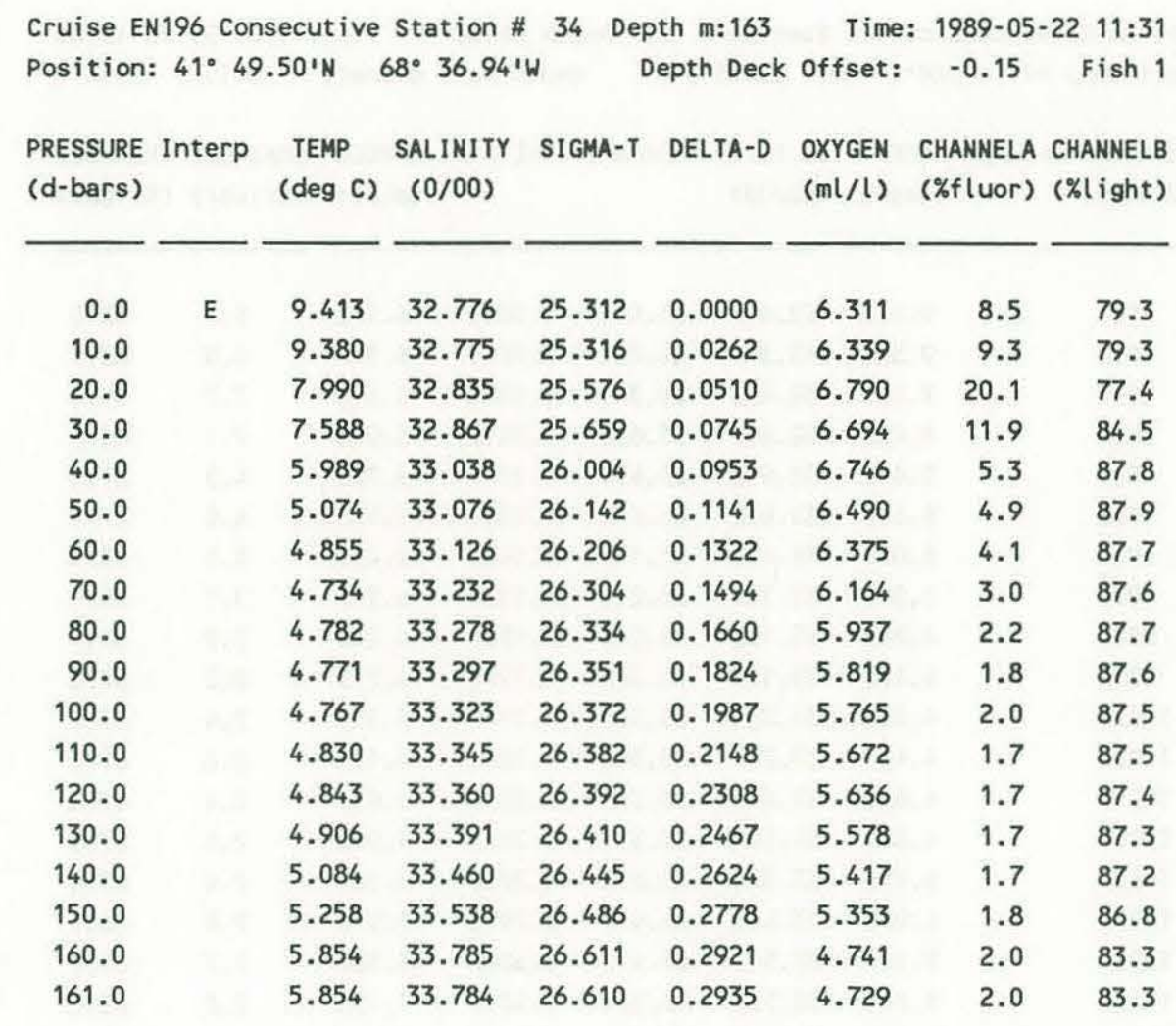
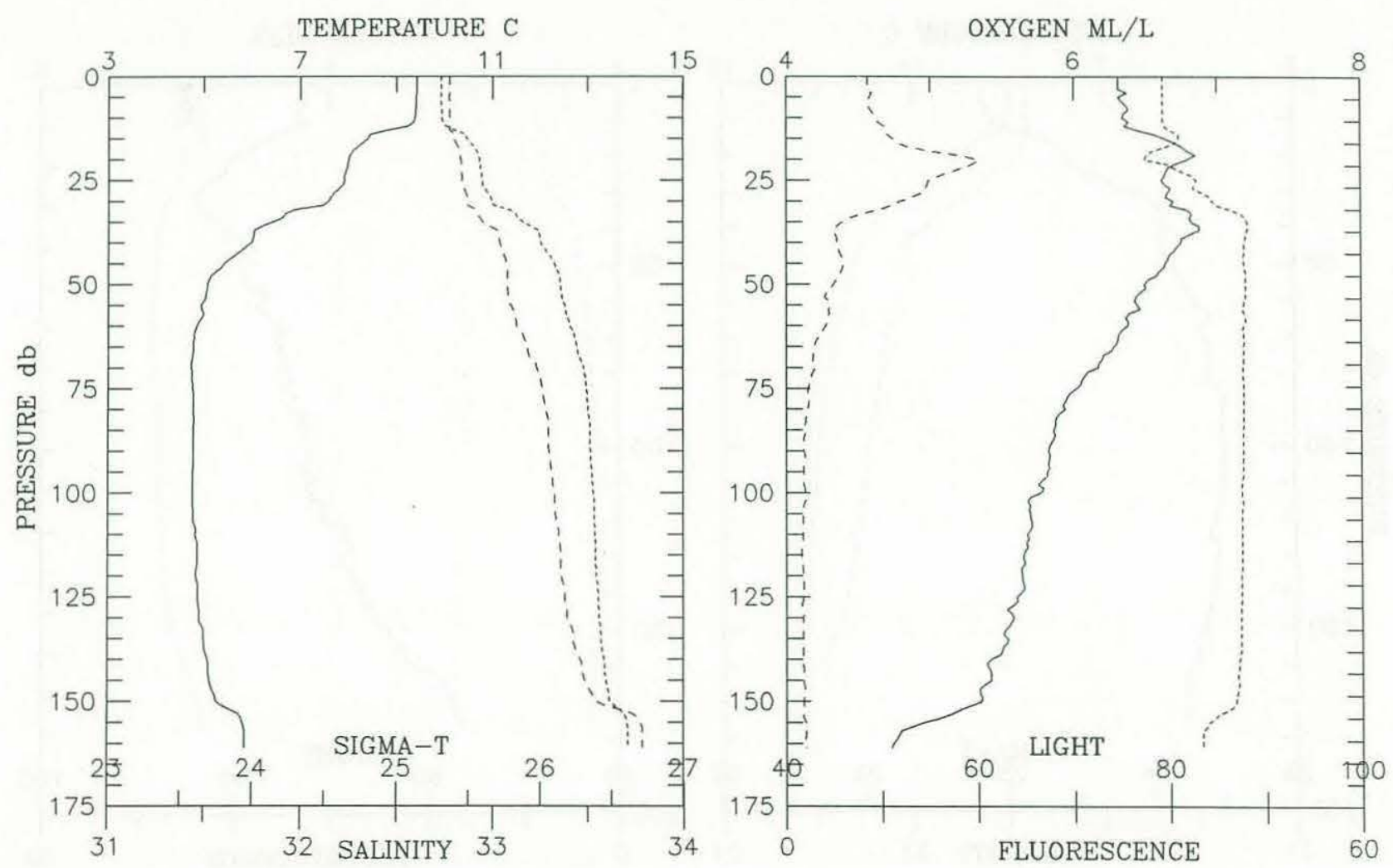


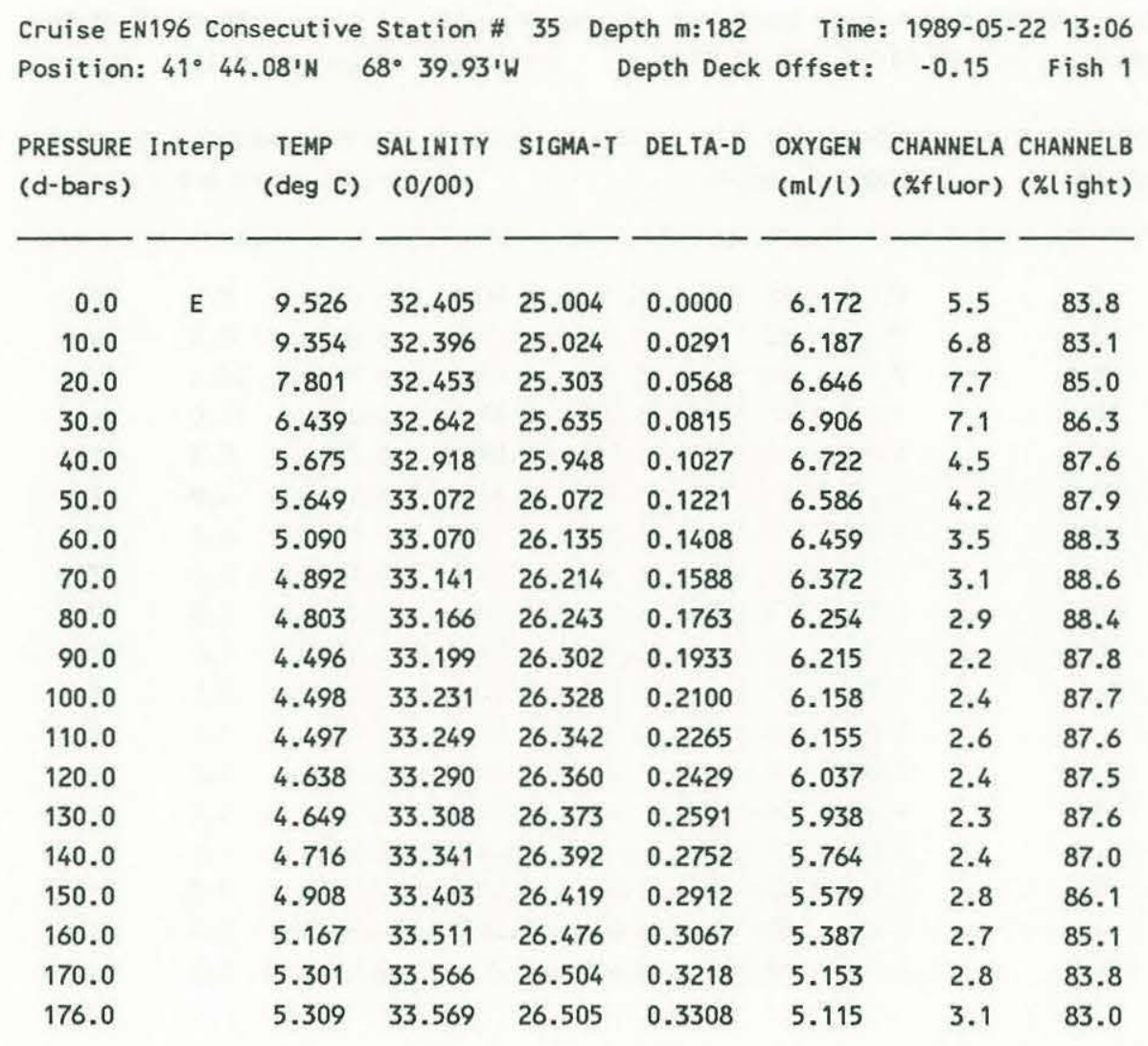
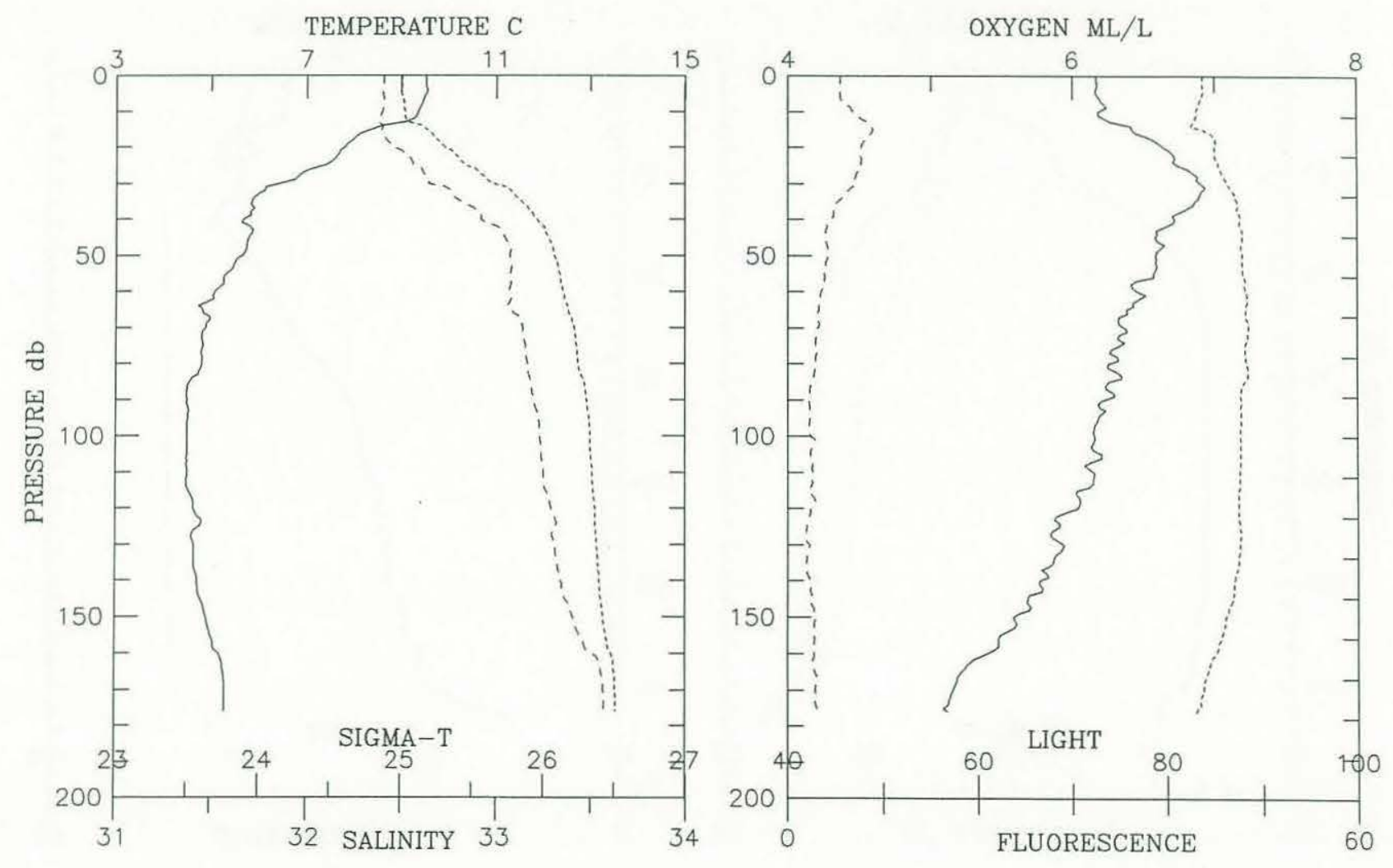


\begin{tabular}{|c|c|c|c|c|c|c|c|c|}
\hline \multirow{2}{*}{$\begin{array}{l}\text { Cruise EN } \\
\text { Position: } \\
\text { PRESSURE } \\
\text { (d-bars) }\end{array}$} & \multicolumn{2}{|c|}{$41^{\circ} 45.88^{\prime} \mathrm{N}$} & \multicolumn{2}{|c|}{$68^{\circ} 39.29^{\prime} \mathrm{W}$} & Depth Deck & Offset: & -0.15 & \multirow{2}{*}{$\begin{array}{r}\text { Fish } 1 \\
\text { CHANNELB } \\
\text { (\%) ight) }\end{array}$} \\
\hline & Interp & $\begin{array}{l}\text { TEMP } \\
(\operatorname{deg} \mathrm{C})\end{array}$ & $\begin{array}{l}\text { SALINITY } \\
(0 / 00)\end{array}$ & SIGMA-T & DELTA-D & $\begin{array}{l}\text { OXYGEN } \\
(\mathrm{ml} / \mathrm{l})\end{array}$ & $\begin{array}{l}\text { CHANNELA } \\
\text { ( } \% \text { fluor) }\end{array}$ & \\
\hline 0.0 & $E$ & 9.918 & 32.367 & 24.910 & 0.0000 & 6.436 & 3.1 & 83.9 \\
\hline 10.0 & & 8.553. & 32.543 & 25.264 & 0.0292 & 6.649 & 5.7 & 83.4 \\
\hline 20.0 & & 6.377 & 32.718 & 25.703 & 0.0538 & 7.006 & 5.1 & 86.8 \\
\hline 30.0 & & 5.659 & 32.961 & 25.983 & 0.0746 & 6.705 & 3.9 & 87.5 \\
\hline 40.0 & & 5.595 & 33.100 & 26.101 & 0.0939 & 6.559 & 3.9 & 87.9 \\
\hline 50.0 & & 5.144 & 33.106 & 26.158 & 0.1124 & 6.457 & 3.4 & 88.3 \\
\hline 60.0 & & 4.819 & 33.146 & 26.226 & 0.1301 & 6.342 & 2.9 & 88.6 \\
\hline 70.0 & & 4.757 & 33.180 & 26.260 & 0.1475 & 6.270 & 2.6 & 88.5 \\
\hline 80.0 & & 4.534 & 33.211 & 26.308 & 0.1645 & 6.220 & 2.3 & 87.9 \\
\hline 90.0 & & 4.496 & 33.231 & 26.328 & 0.1811 & 6.157 & 2.0 & 87.7 \\
\hline 100.0 & & 4.573 & 33.258 & 26.341 & 0.1976 & 6.110 & 2.0 & 87.2 \\
\hline 110.0 & & 4.532 & 33.260 & 26.347 & 0.2140 & 6.044 & 2.0 & 87.2 \\
\hline 120.0 & & 4.680 & 33.300 & 26.363 & 0.2304 & 5.980 & 2.0 & 87.4 \\
\hline 130.0 & & 4.580 & 33.297 & 26.371 & 0.2466 & 5.914 & 1.9 & 87.5 \\
\hline 140.0 & & 4.707 & 33.339 & 26.391 & 0.2627 & 5.873 & 2.2 & 87.0 \\
\hline 150.0 & & 4.885 & 33.391 & 26.412 & 0.2787 & 5.687 & 2.6 & 86.1 \\
\hline 160.0 & & 5.064 & 33.465 & 26.451 & 0.2944 & 5.498 & 2.9 & 85.1 \\
\hline 170.0 & & 5.437 & 33.627 & 26.536 & 0.3096 & 5.198 & 2.6 & 83.4 \\
\hline 171.0 & & 5.457 & 33.631 & 26.537 & 0.3110 & 5.142 & 2.6 & 83.3 \\
\hline
\end{tabular}
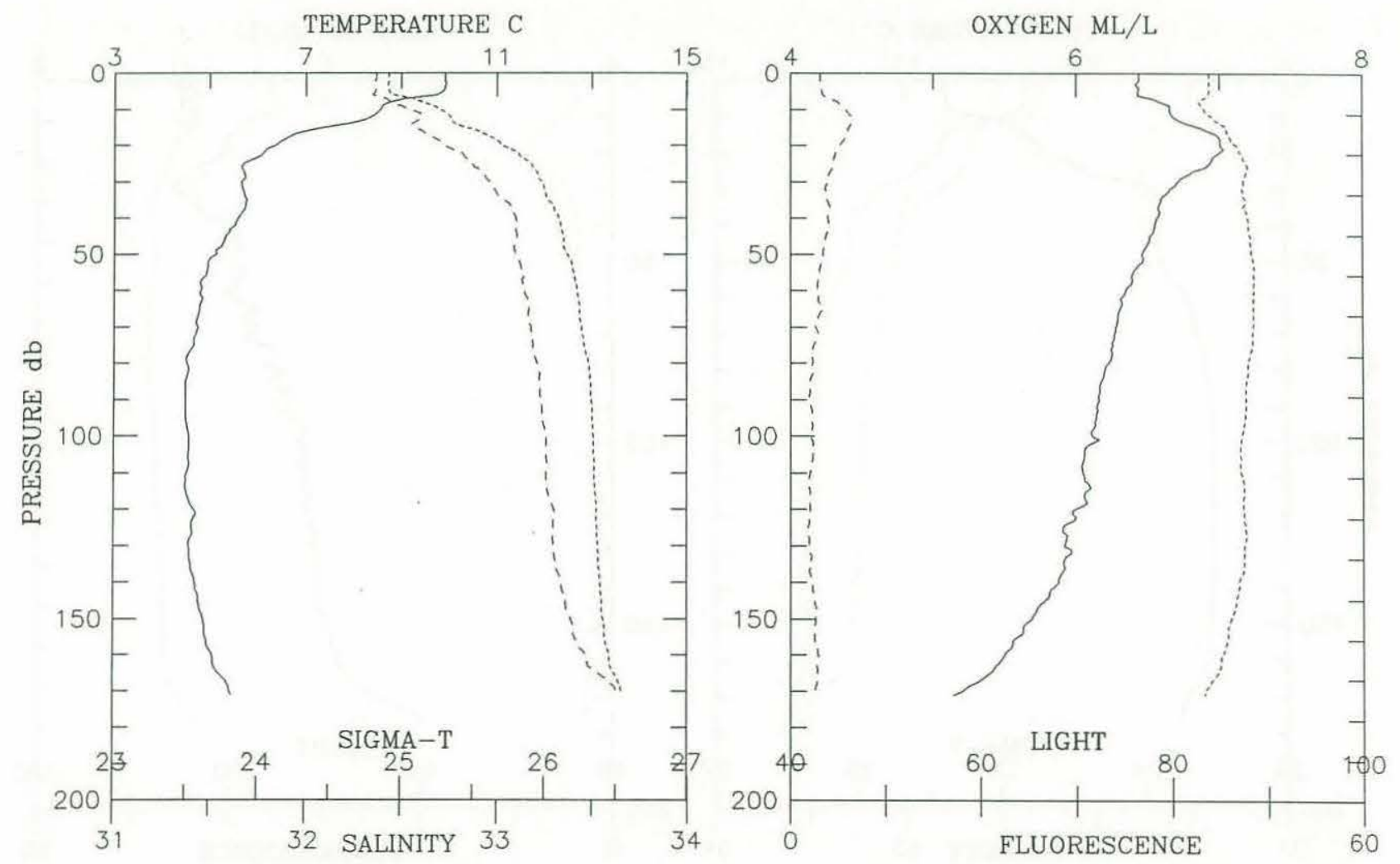


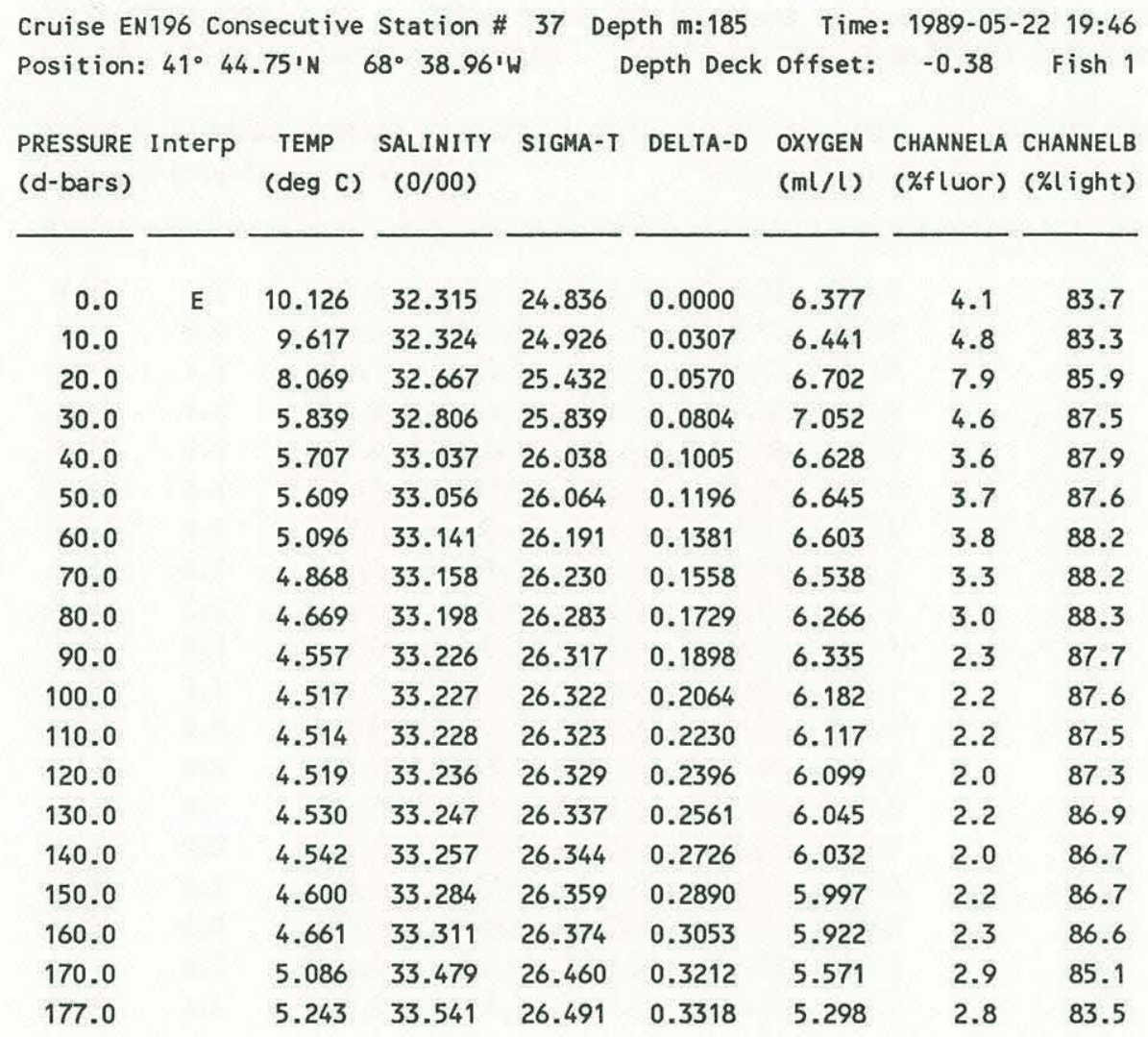
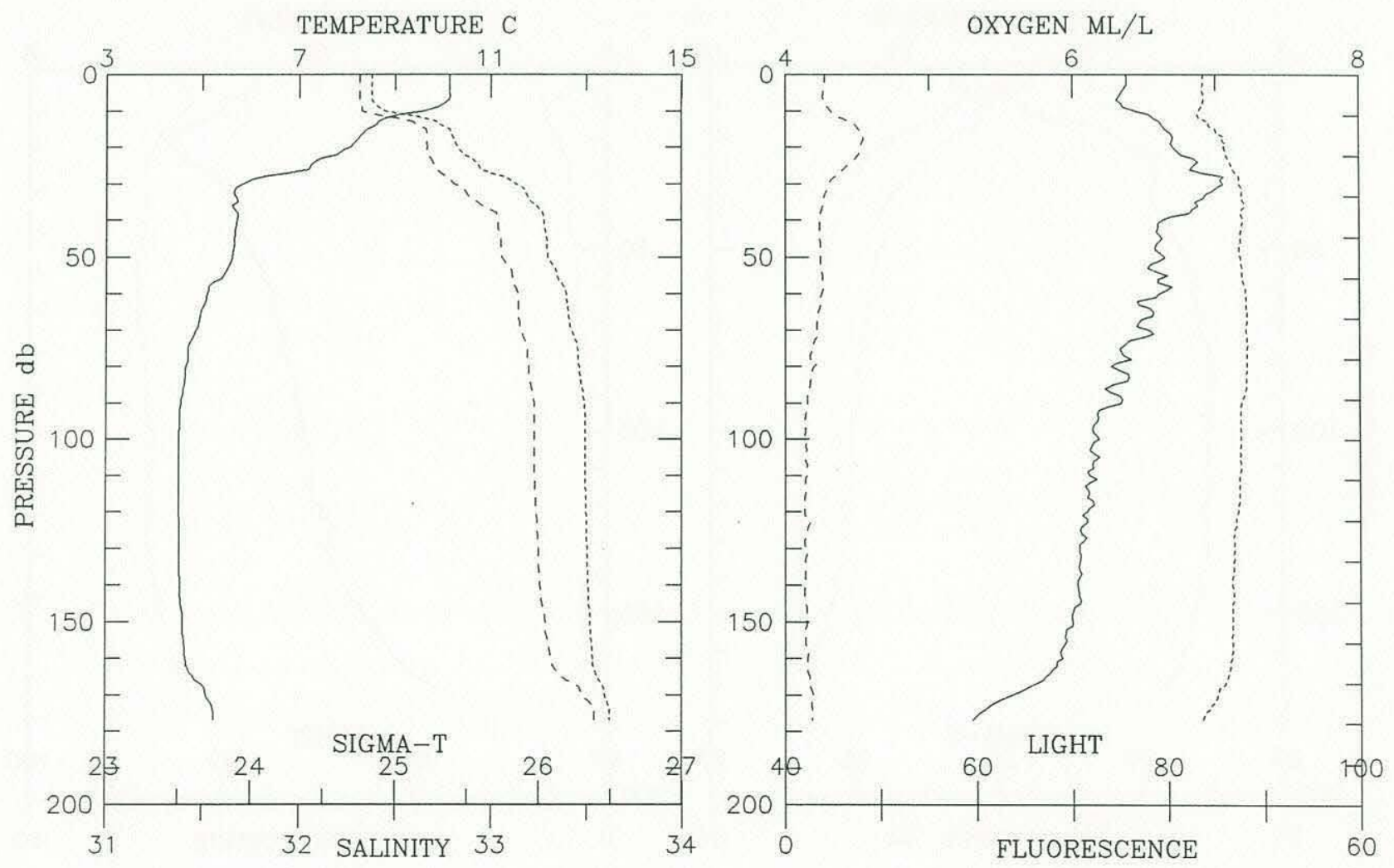


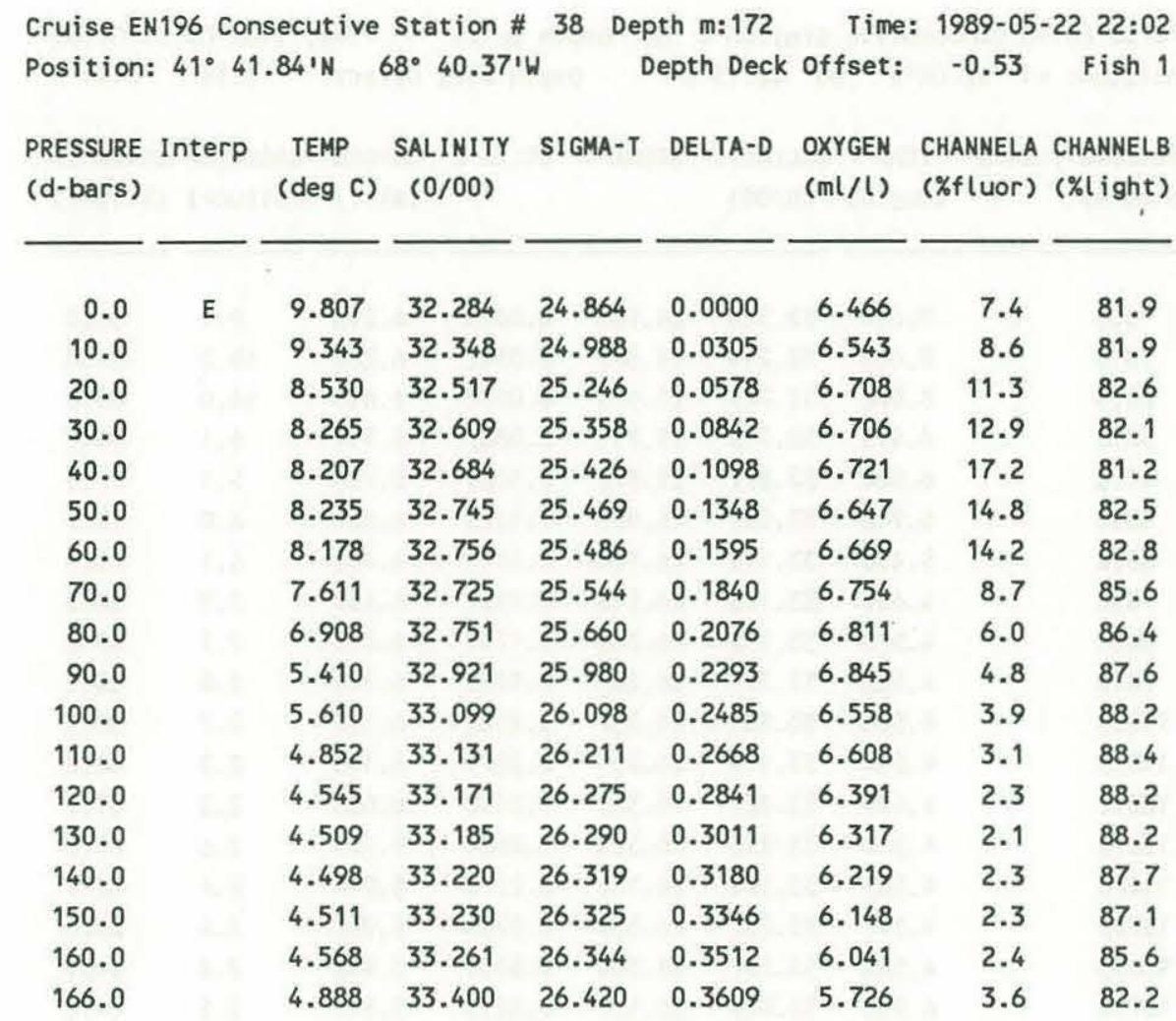
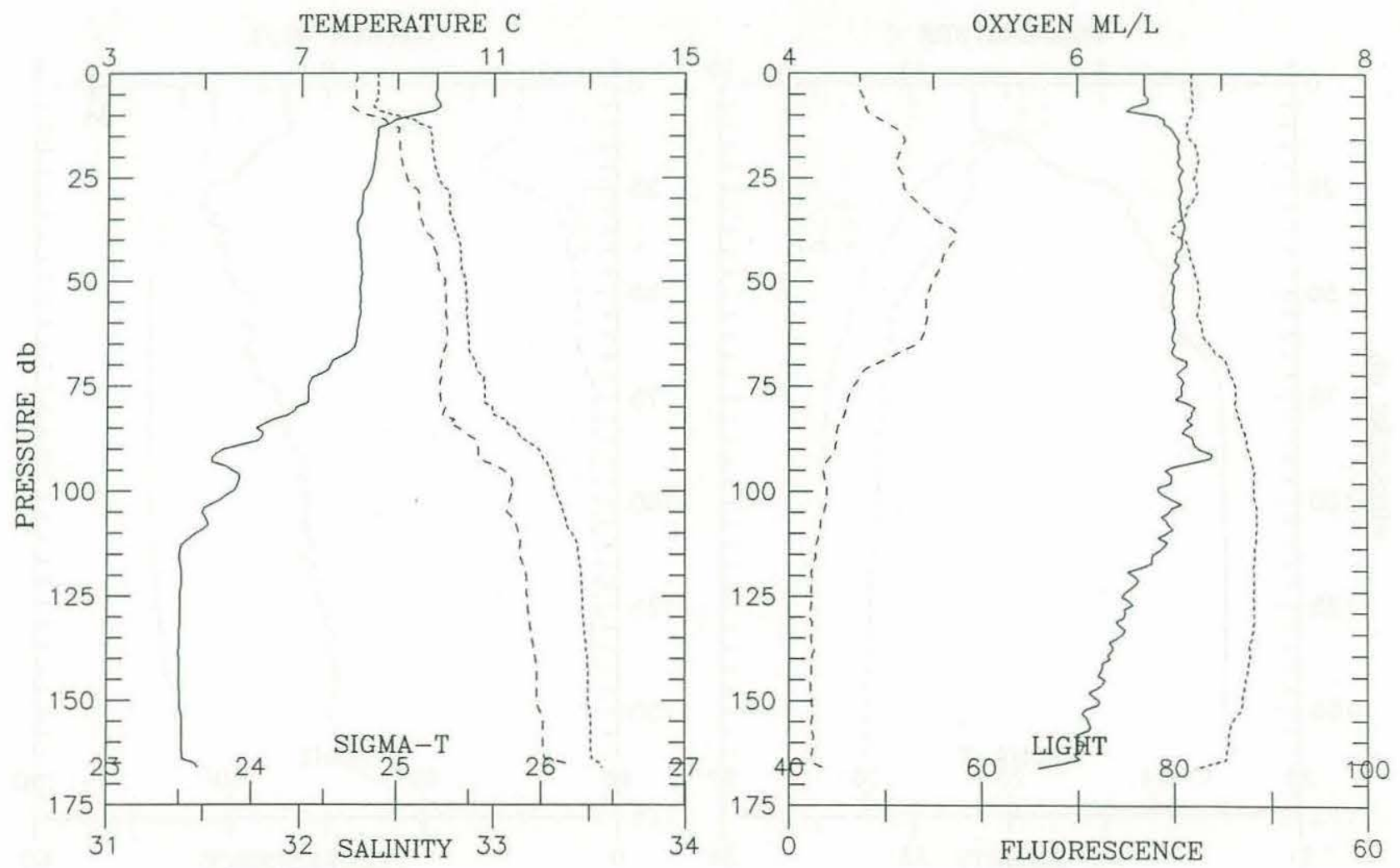


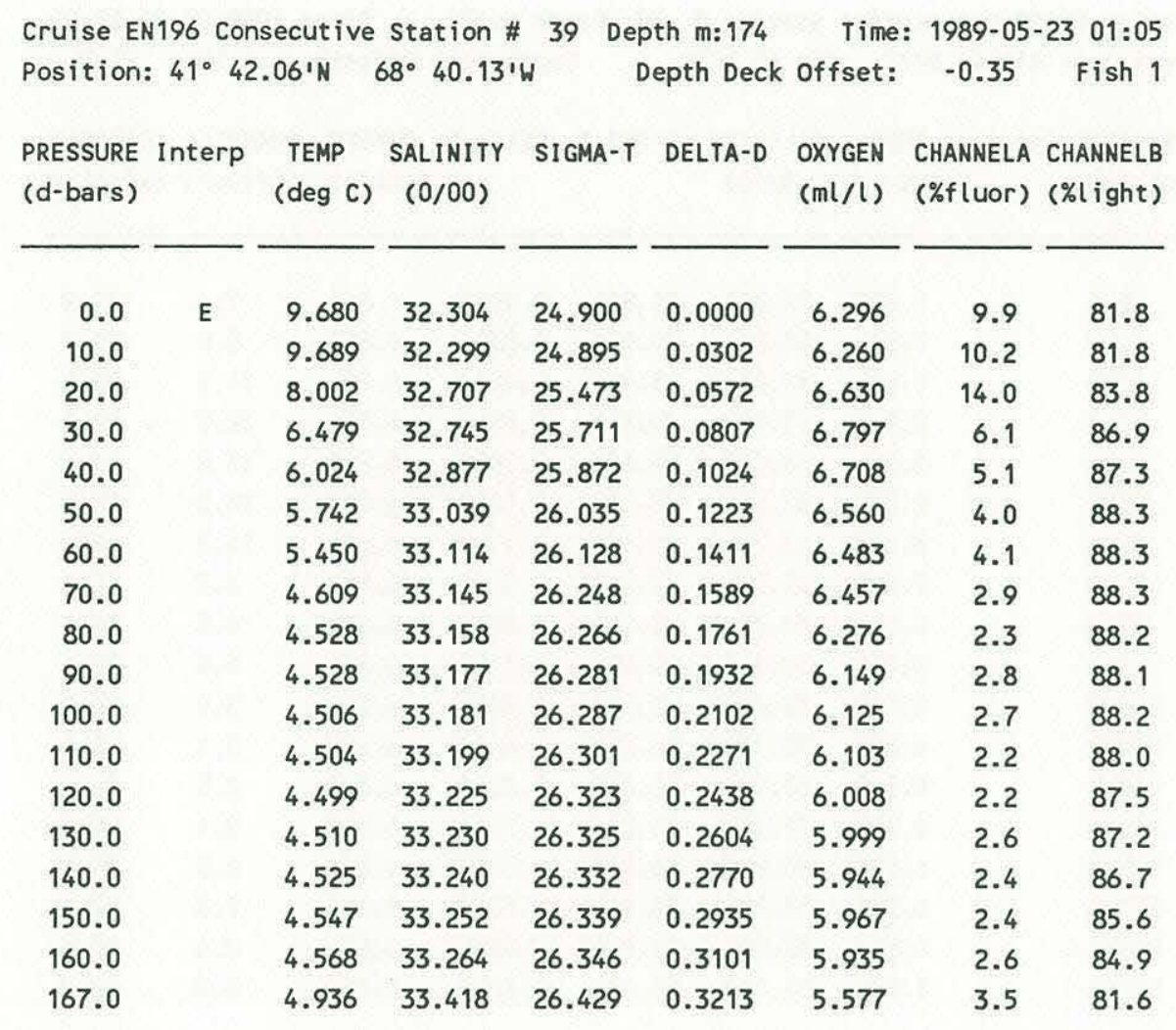
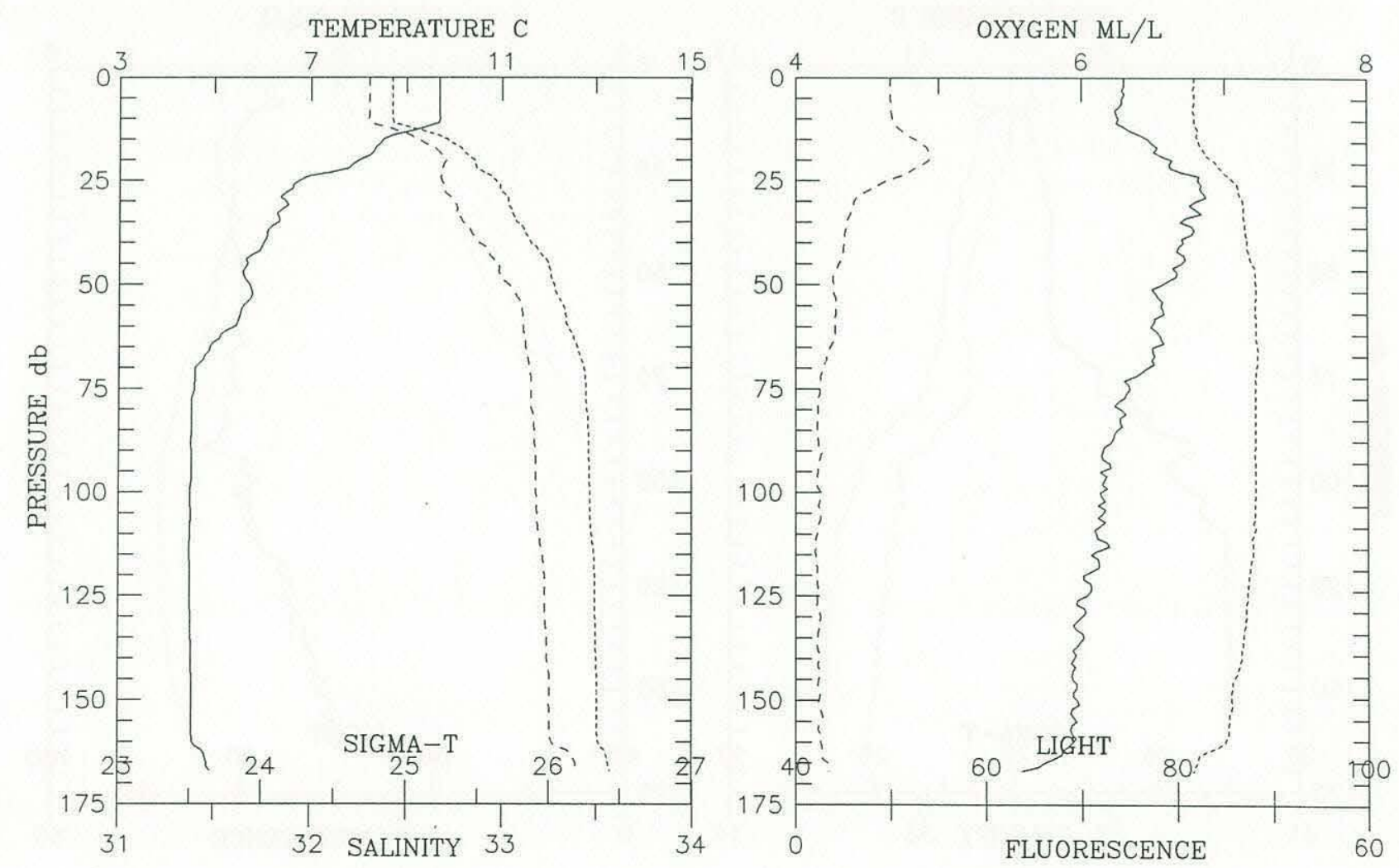


\begin{tabular}{|c|c|c|c|c|c|c|c|c|}
\hline Position: & $41^{\circ} 42$ & $.02 \cdot N$ & $68^{\circ} 40.30^{\prime}$ & & epth Deck & Offset: & -0.35 & Fish 1 \\
\hline $\begin{array}{l}\text { PRESSURE } \\
\text { (d-bars) }\end{array}$ & Interp & $\begin{array}{c}\text { TEMP } \\
\text { (deg C) }\end{array}$ & $\begin{array}{l}\text { SALINITY } \\
(0 / 00)\end{array}$ & SIGMA-T & DELTA-D & $\begin{array}{l}\text { OXYGEN } \\
(\mathrm{ml} / \mathrm{l})\end{array}$ & $\begin{array}{l}\text { CHANNELA } \\
\text { (\%fluor) }\end{array}$ & $\begin{array}{l}\text { CHANNELB } \\
\text { (\%light) }\end{array}$ \\
\hline 0.0 & E & 9.646 & 32.298 & 24.901 & 0.0000 & 6.243 & 8.3 & 83.2 \\
\hline 10.0 & & 9.646 & 32.297 & 24.900 & 0.0302 & 6.185 & 8.6 & 83.3 \\
\hline 20.0 & & 7.684 & 32.695 & 25.510 & 0.0563 & 6.602 & 13.0 & 85.6 \\
\hline 30.0 & & 5.916 & 32.915 & 25.915 & 0.0785 & 6.864 & 5.2 & 87.9 \\
\hline 40.0 & & 5.636 & 33.063 & 26.067 & 0.0982 & 6.527 & 3.6 & 88.0 \\
\hline 50.0 & & 4.994 & 33.019 & 26.105 & 0.1171 & 6.442 & 3.4 & 88.5 \\
\hline 60.0 & & 4.611 & 33.059 & 26.179 & 0.1353 & 6.373 & 2.6 & 88.5 \\
\hline 70.0 & & 4.569 & 33.114 & 26.227 & 0.1530 & 6.249 & 2.6 & 88.4 \\
\hline 80.0 & & 4.539 & 33.125 & 26.239 & 0.1704 & 6.291 & 2.8 & 88.3 \\
\hline 90.0 & & 4.552 & 33.147 & 26.255 & 0.1877 & 6.149 & 2.7 & 88.2 \\
\hline 100.0 & & 4.494 & 33.155 & 26.268 & 0.2049 & 6.159 & 2.8 & 88.3 \\
\hline 110.0 & & 4.480 & 33.161 & 26.274 & 0.2220 & 6.150 & 2.5 & 88.4 \\
\hline 120.0 & & 4.497 & 33.186 & 26.292 & 0.2390 & 6.088 & 2.6 & 88.1 \\
\hline 130.0 & & 4.568 & 33.241 & 26.328 & 0.2557 & 5.954 & 2.7 & 86.8 \\
\hline 140.0 & & 4.573 & 33.245 & 26.331 & 0.2723 & 5.921 & 3.0 & 86.4 \\
\hline 150.0 & & 4.573 & 33.245 & 26.331 & 0.2889 & 5.883 & 3.4 & 86.1 \\
\hline 160.0 & & 4.573 & 33.244 & 26.330 & 0.3055 & 5.879 & 2.9 & 85.8 \\
\hline 165.0 & & 4.572 & 33.244 & 26.330 & 0.3138 & 5.877 & 3.0 & 85.0 \\
\hline
\end{tabular}
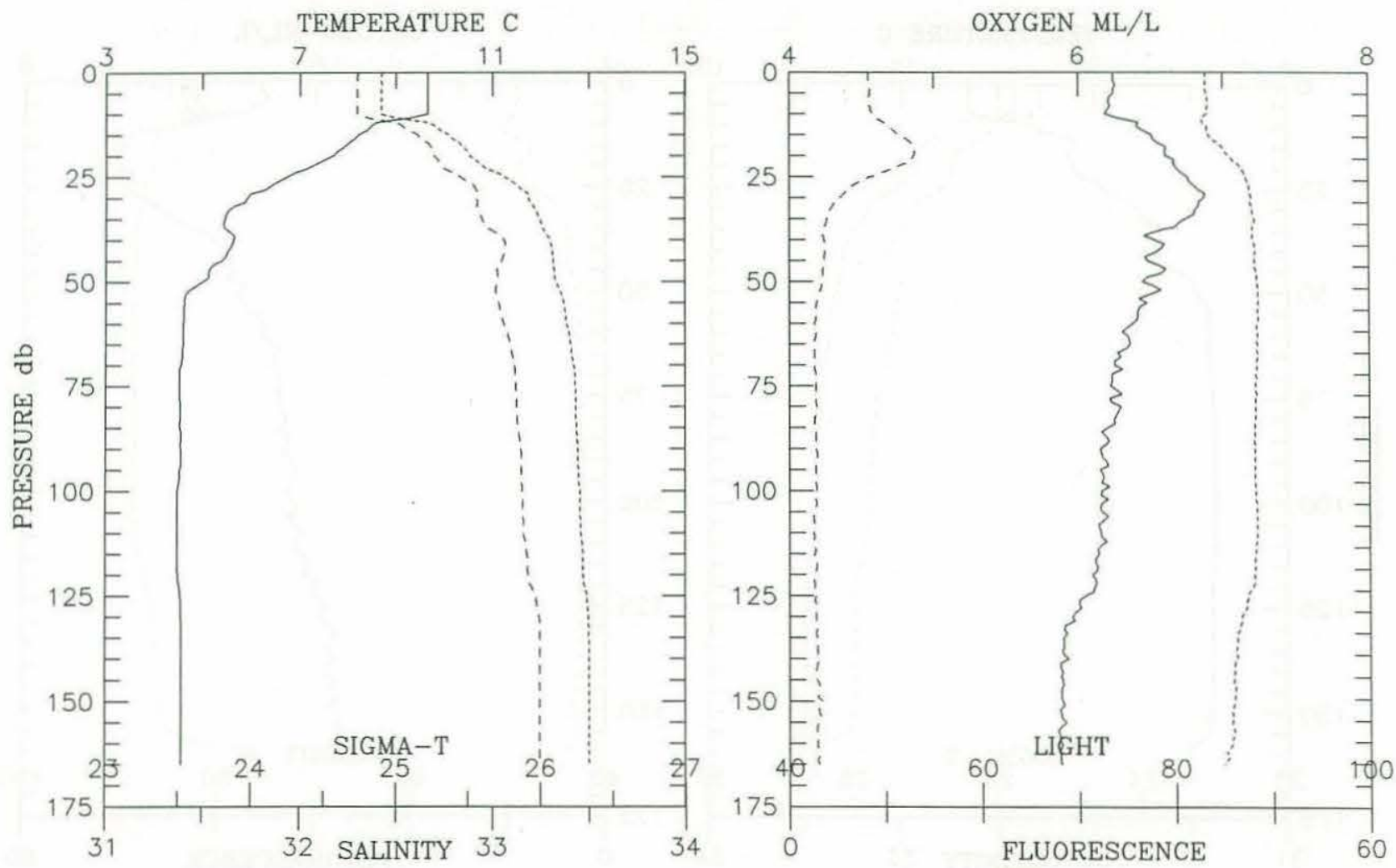


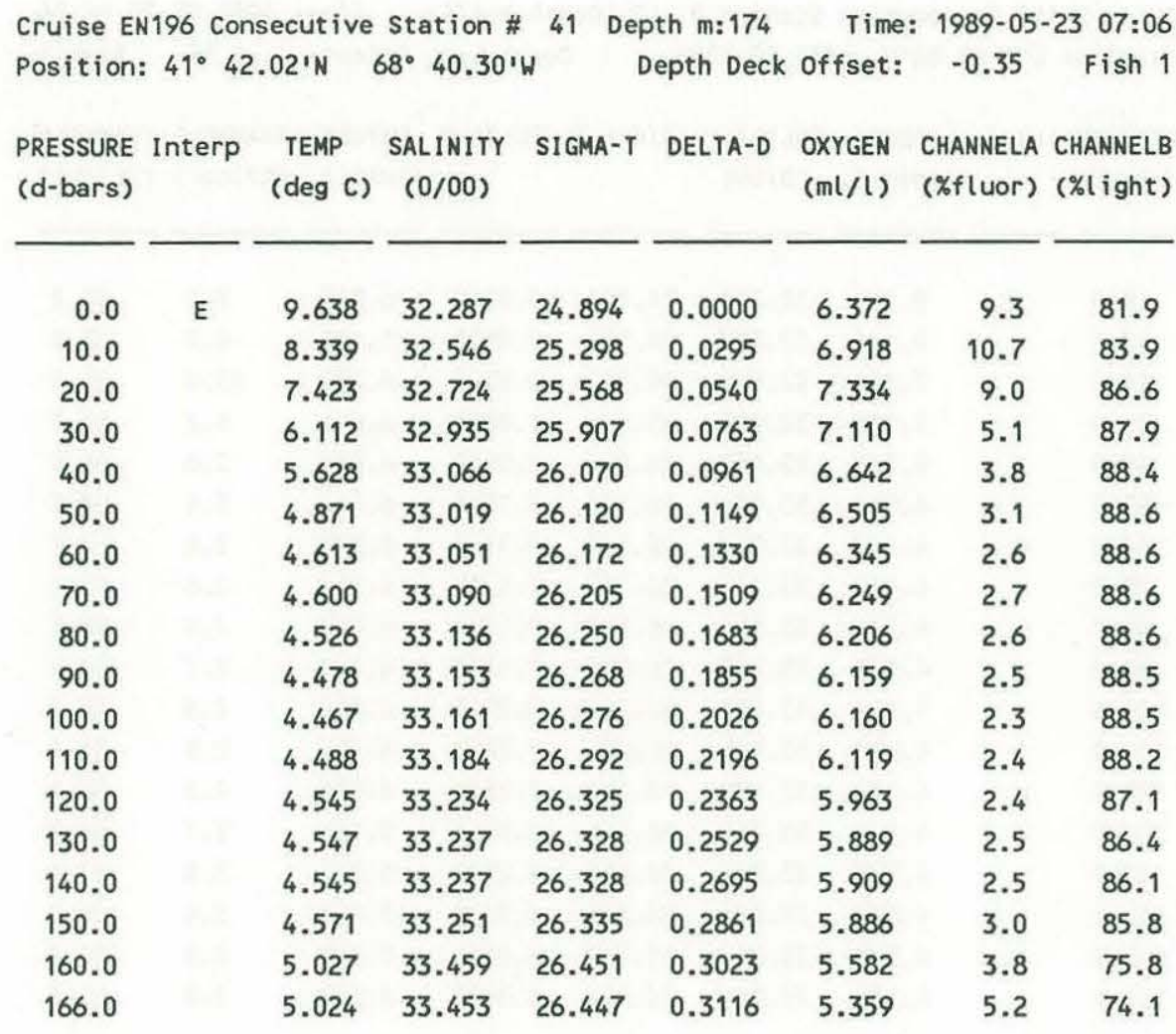
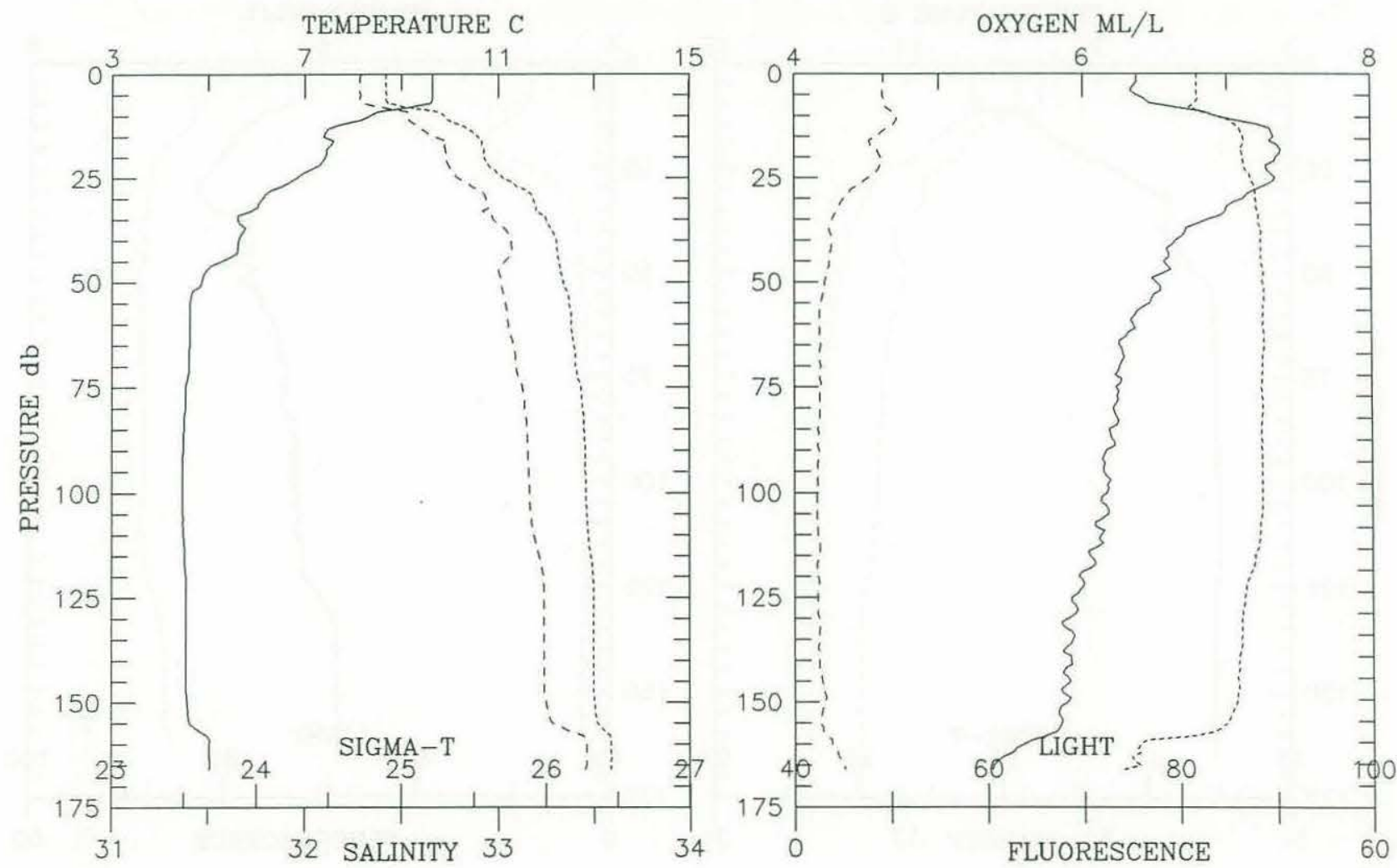
Cruise EN196 Consecutive Station \# 42 Depth m:174 Time: 1989-05-23 10:04 Position: $41^{\circ} 42.02^{\prime} \mathrm{N} \quad 68^{\circ} 40.31^{\prime} \mathrm{W}$ Depth Deck offset: -0.20 Fish 1

\begin{tabular}{|c|c|c|c|c|c|c|c|c|}
\hline $\begin{array}{l}\text { PRESSURE } \\
\text { (d-bars) }\end{array}$ & Interp & $\begin{array}{l}\text { TEMP } \\
(\operatorname{deg} C)\end{array}$ & $\begin{array}{l}\text { SALINITY } \\
(0 / 00)\end{array}$ & SIGMA-T & DELTA-D & $\begin{array}{l}\text { OXYGEN } \\
(\mathrm{ml} / \mathrm{l})\end{array}$ & $\begin{array}{l}\text { CHANNELA } \\
\text { (\%fluor) }\end{array}$ & $\begin{array}{l}\text { CHANNELB } \\
\text { (\%light) }\end{array}$ \\
\hline 0.0 & E & 9.747 & 32.254 & 24.850 & 0.0000 & 6.449 & 6.3 & 84.1 \\
\hline 10.0 & & 9.649 & 32.288 & 24.893 & 0.0306 & 6.411 & 6.8 & 83.9 \\
\hline 20.0 & & 8.394 & 32.628 & 25.353 & 0.0586 & 6.747 & 14.1 & 80.7 \\
\hline 30.0 & & 7.410 & 32.702 & 25.553 & 0.0834 & 6.858 & 8.7 & 85.5 \\
\hline 40.0 & & 6.131 & 32.874 & 25.857 & 0.1058 & 6.899 & 4.5 & 87.7 \\
\hline 50.0 & & 5.678 & 32.961 & 25.981 & 0.1263 & 6.724 & 4.3 & 88.2 \\
\hline 60.0 & & 4.718 & 32.966 & 26.094 & 0.1457 & 6.566 & 3.5 & 88.6 \\
\hline 70.0 & & 5.012 & 33.076 & 26.149 & 0.1642 & 6.441 & 3.6 & 88.4 \\
\hline 80.0 & & 4.593 & 33.096 & 26.211 & 0.1821 & 6.542 & 2.9 & 88.5 \\
\hline 90.0 & & 4.507 & 33.163 & 26.273 & 0.1994 & 6.293 & 2.2 & 88.2 \\
\hline 100.0 & & 4.491 & 33.167 & 26.278 & 0.2165 & 6.223 & 2.2 & 88.2 \\
\hline 110.0 & & 4.490 & 33.172 & 26.282 & 0.2335 & 6.216 & 2.1 & 88.1 \\
\hline 120.0 & & 4.487 & 33.212 & 26.314 & 0.2504 & 6.144 & 2.2 & 87.9 \\
\hline 130.0 & & 4.504 & 33.225 & 26.323 & 0.2671 & 6.075 & 2.4 & 87.5 \\
\hline 140.0 & & 4.569 & 33.264 & 26.347 & 0.2836 & 6.049 & 2.4 & 87.0 \\
\hline 150.0 & & 4.709 & 33.332 & 26.385 & 0.2999 & 5.949 & 2.4 & 86.7 \\
\hline 160.0 & & 5.226 & 33.540 & 26.492 & 0.3155 & 5.479 & 3.0 & 83.0 \\
\hline 170.0 & & 5.238 & 33.543 & 26.493 & 0.3306 & 5.241 & 3.2 & 82.5 \\
\hline
\end{tabular}
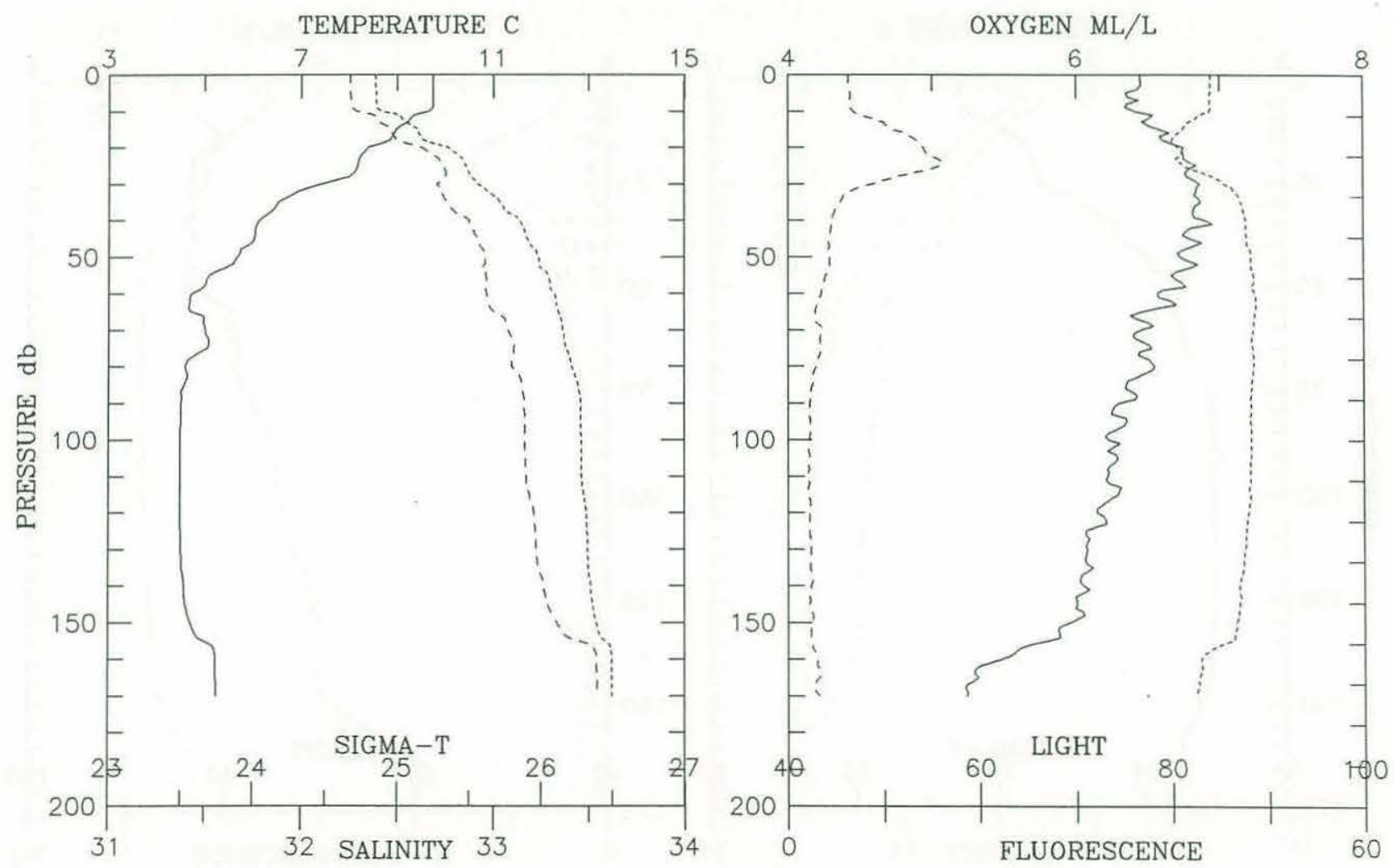


\begin{tabular}{|c|c|c|c|c|c|c|c|c|}
\hline \multicolumn{3}{|c|}{ Position: $41^{\circ} 41.991 \mathrm{~N}$} & \multicolumn{2}{|c|}{$68^{\circ} 40.23^{\prime} \mathrm{W}$} & Depth Deck & \multirow{2}{*}{$\begin{array}{l}\text { Offset: } \\
\text { OXYGEN } \\
(\mathrm{ml} / \mathrm{l})\end{array}$} & \multirow{2}{*}{$\begin{array}{r}-0.20 \\
\text { CHANNELA } \\
\text { (\%fluor) }\end{array}$} & \multirow{2}{*}{$\begin{array}{r}\text { Fish } 1 \\
\text { CHANNELB } \\
\text { (\%light) }\end{array}$} \\
\hline $\begin{array}{l}\text { PRESSURE } \\
\text { (d-bars) }\end{array}$ & Interp & $\begin{array}{l}\text { TEMP } \\
(\operatorname{deg} C)\end{array}$ & $\begin{array}{l}\text { SALINITY } \\
(0 / 00)\end{array}$ & SI GMA-T & DELTA-D & & & \\
\hline 0.0 & E & 9.796 & 32.268 & 24.853 & 0.0000 & 6.455 & 5.0 & 83.8 \\
\hline 10.0 & & 9.209 & 32.420 & 25.066 & 0.0302 & 6.499 & 7.3 & 81.8 \\
\hline 20.0 & & 8.223 & 32.644 & 25.392 & 0.0571 & 6.794 & 13.9 & 82.9 \\
\hline 30.0 & & 7.225 & 32.724 & 25.596 & 0.0816 & 6.884 & 7.8 & 86.4 \\
\hline 40.0 & & 6.156 & 32.821 & 25.812 & 0.1043 & 6.915 & 4.4 & 87.6 \\
\hline 50.0 & & 5.182 & 32.982 & 26.055 & 0.1243 & 6.833 & 3.8 & 88.3 \\
\hline 60.0 & & 5.181 & 33.091 & 26.141 & 0.1429 & 6.566 & 3.6 & 88.3 \\
\hline 70.0 & & 4.638 & 33.096 & 26.206 & 0.1609 & 6.581 & 2.9 & 88.5 \\
\hline 80.0 & & 4.497 & 33.156 & 26.269 & 0.1783 & 6.468 & 2.2 & 88.2 \\
\hline 90.0 & & 4.493 & 33.165 & 26.276 & 0.1954 & 6.322 & 2.2 & 88.2 \\
\hline 100.0 & & 4.486 & 33.175 & 26.285 & 0.2124 & 6.291 & 2.0 & 88.1 \\
\hline 110.0 & & 4.485 & 33.207 & 26.310 & 0.2292 & 6.199 & 2.2 & 88.0 \\
\hline 120.0 & & 4.503 & 33.224 & 26.322 & 0.2459 & 6.080 & 2.6 & 87.6 \\
\hline 130.0 & & 4.518 & 33.232 & 26.327 & 0.2626 & 6.012 & 2.8 & 87.4 \\
\hline 140.0 & & 4.558 & 33.262 & 26.346 & 0.2791 & 5.985 & 2.2 & 87.2 \\
\hline 150.0 & & 4.848 & 33.386 & 26.413 & 0.2954 & 5.863 & 2.8 & 85.9 \\
\hline 160.0 & & 5.205 & 33.532 & 26.488 & 0.3109 & 5.448 & 2.7 & 83.9 \\
\hline 169.0 & & 5.232 & 33.543 & 26.494 & 0.3245 & 5.239 & 2.8 & 82.8 \\
\hline
\end{tabular}
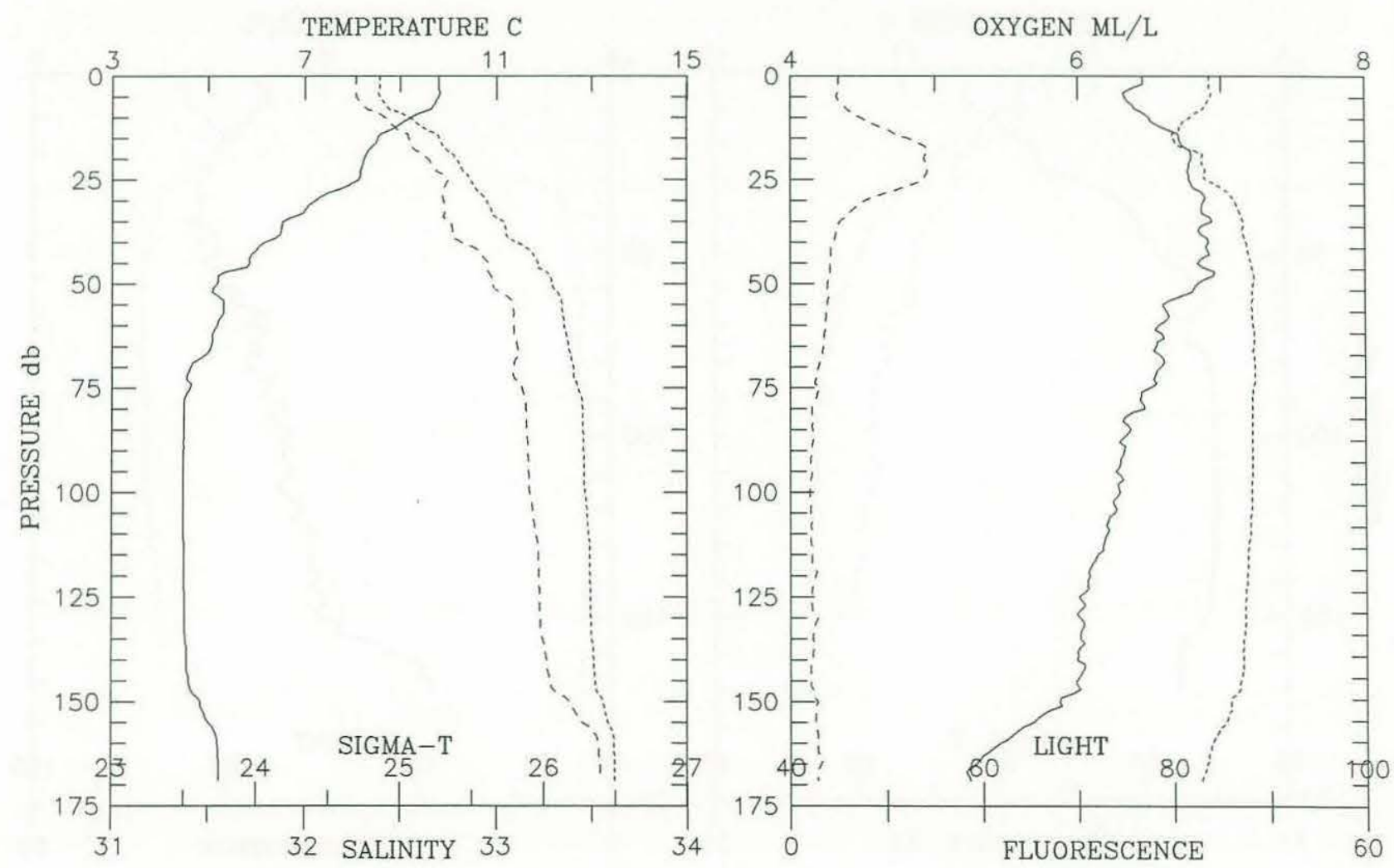


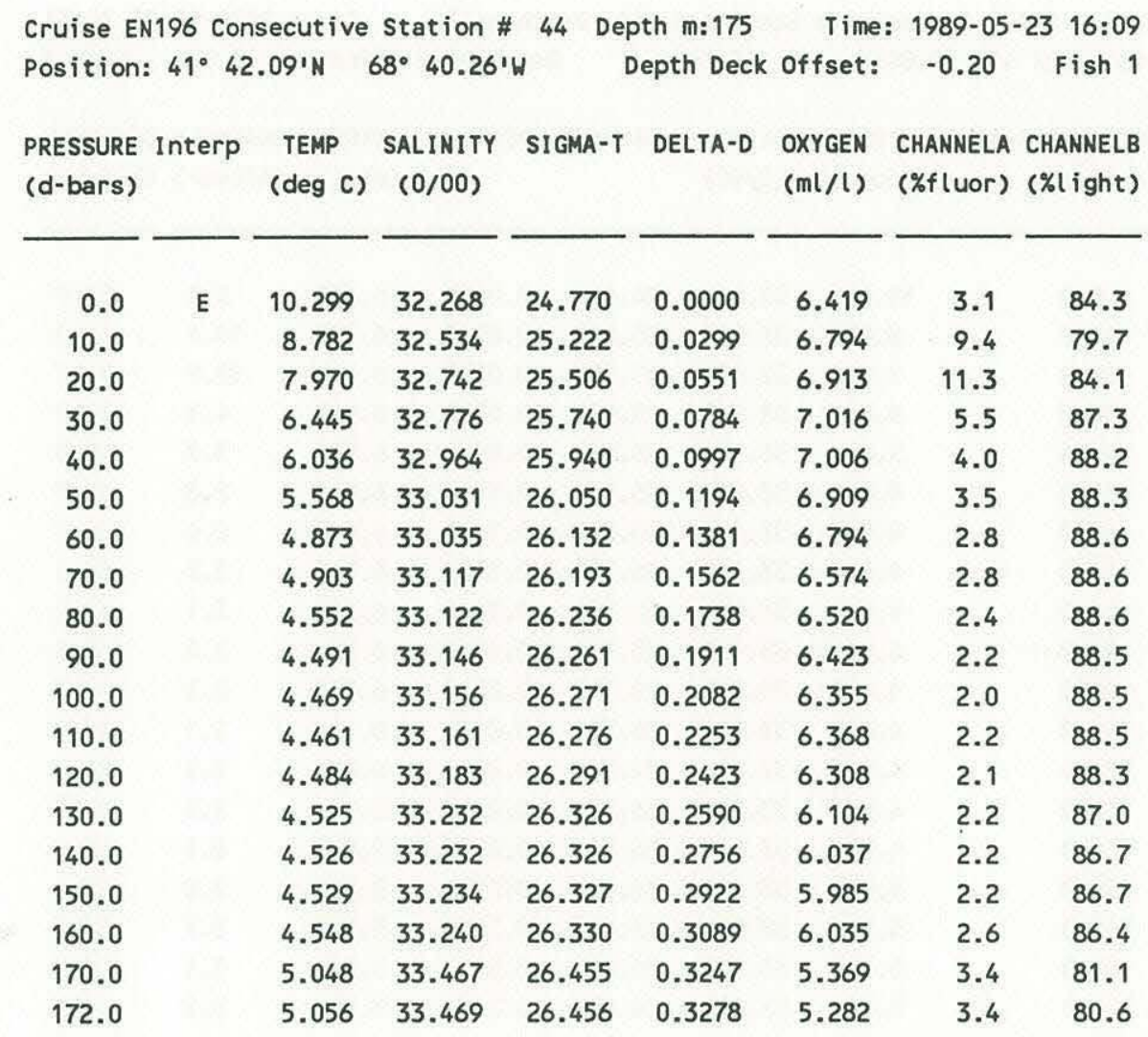
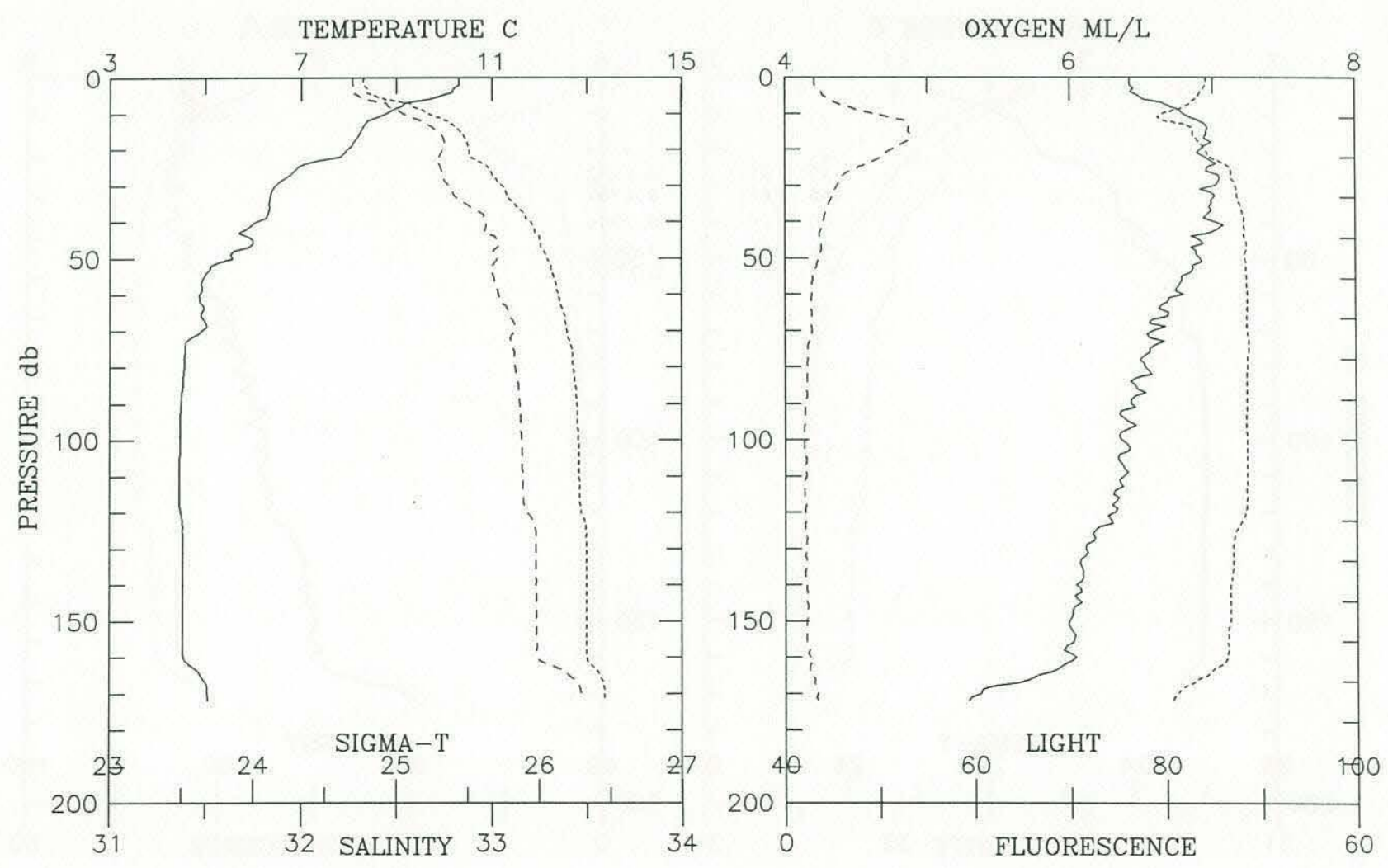


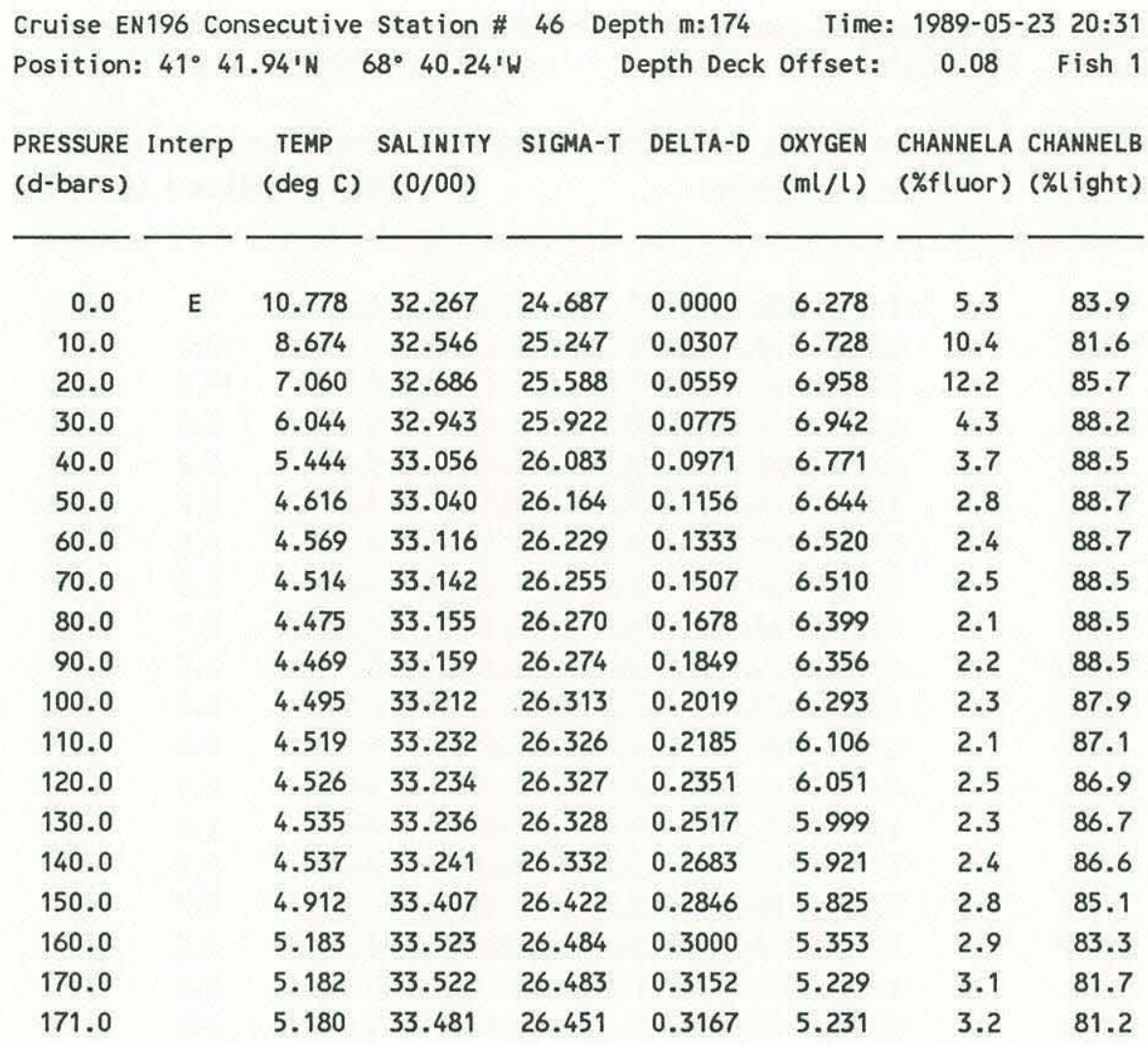
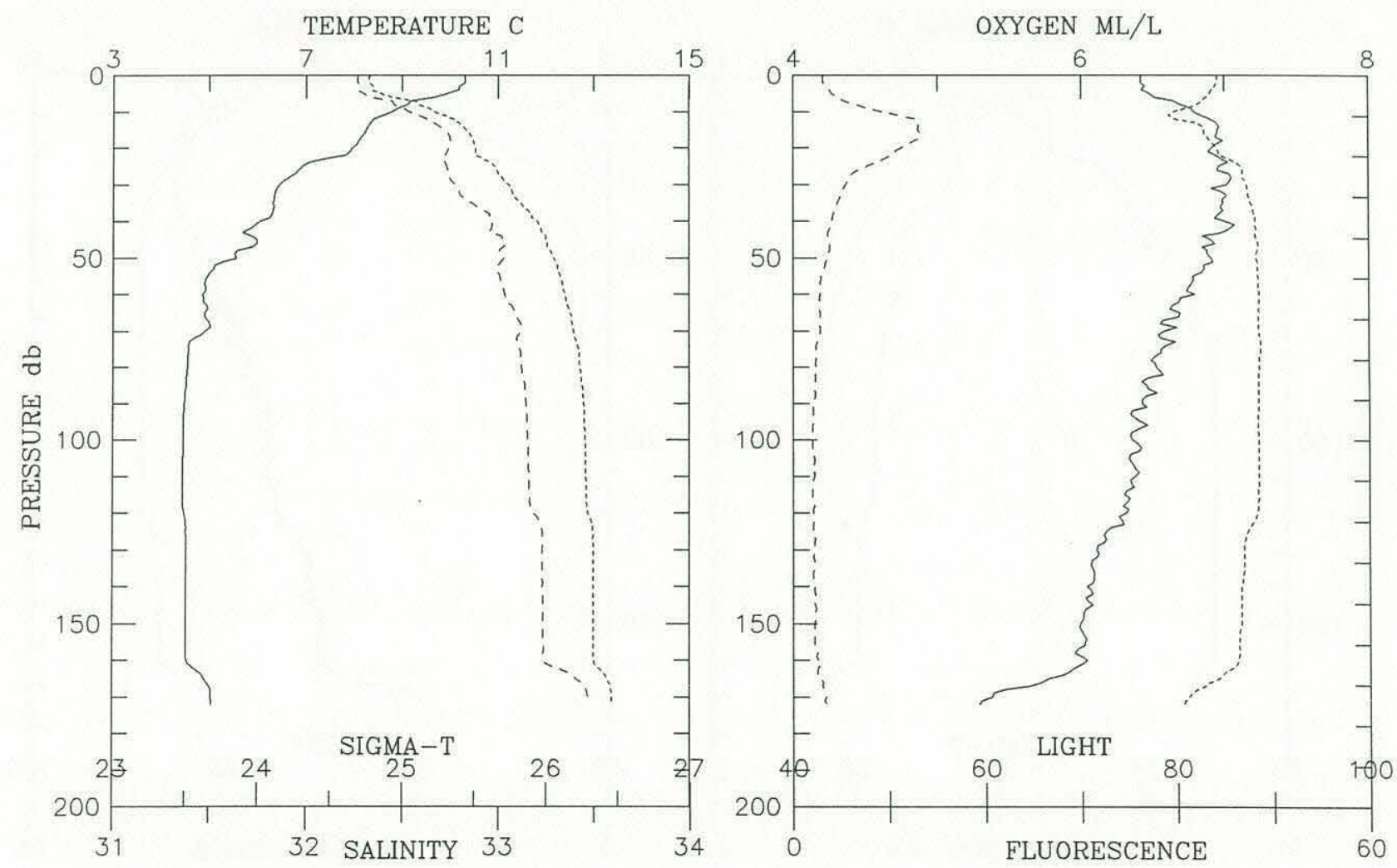
Cruise EN196 Consecutive Station \# 47 Depth m:165 Time: 1989-05-24 00:03 Position: $41^{\circ} 43.69^{\prime} \mathrm{N} \quad 68^{\circ} 48.01 \mathrm{~W} \mathrm{~W}$ Depth Deck offset: -0.38 Fish 1

\begin{tabular}{|c|c|c|c|c|c|c|c|c|}
\hline $\begin{array}{l}\text { PRESSURE } \\
\text { (d-bars) }\end{array}$ & Interp & $\begin{array}{c}\text { TEMP } \\
(\operatorname{deg} \mathrm{C})\end{array}$ & $\begin{array}{l}\text { SALINITY } \\
(0 / 00)\end{array}$ & SI GMA-T & DELTA-D & $\begin{array}{l}\text { OXYGEN } \\
(\mathrm{ml} / \mathrm{l})\end{array}$ & $\begin{array}{l}\text { CHANNELA } \\
\text { (\%fluor) }\end{array}$ & $\begin{array}{l}\text { CHANNELB } \\
\text { ( } \% \text { (ight) }\end{array}$ \\
\hline 0.0 & E & 11.023 & 32.256 & 24.636 & 0.0000 & 6.107 & 4.7 & 84.5 \\
\hline 10.0 & & 9.791 & 32.242 & 24.834 & 0.0323 & 6.261 & 5.6 & 83.7 \\
\hline 20.0 & & 6.891 & 32.793 & 25.695 & 0.0579 & 7.116 & 8.6 & 84.7 \\
\hline 30.0 & & 5.918 & 32.813 & 25.835 & 0.0797 & 6.921 & 7.1 & 86.6 \\
\hline 40.0 & & 5.184 & 32.988 & 26.060 & 0.0997 & 6.598 & 3.5 & 87.9 \\
\hline 50.0 & & 4.734 & 32.994 & 26.115 & 0.1185 & 6.498 & 3.2 & 88.3 \\
\hline 60.0 & & 4.894 & 33.119 & 26.196 & 0.1366 & 6.424 & 3.3 & 88.4 \\
\hline 70.0 & & 4.787 & 33.163 & 26.243 & 0.1543 & 6.373 & 2.7 & 88.6 \\
\hline 80.0 & & 4.647 & 33.217 & 26.301 & 0.1714 & 6.356 & 2.6 & 88.4 \\
\hline 90.0 & & 4.516 & 33.234 & 26.328 & 0.1881 & 6.226 & 2.0 & 87.8 \\
\hline 100.0 & & 4.537 & 33.259 & 26.346 & 0.2045 & 6.142 & 1.8 & 87.5 \\
\hline 110.0 & & 4.588 & 33.280 & 26.357 & 0.2209 & 6.060 & 2.0 & 87.3 \\
\hline 120.0 & & 4.702 & 33.335 & 26.389 & 0.2371 & 5.925 & 2.0 & 87.0 \\
\hline 130.0 & & 4.724 & 33.344 & 26.393 & 0.2531 & 5.809 & 2.5 & 86.6 \\
\hline 140.0 & & 4.780 & 33.367 & 26.406 & 0.2690 & 5.749 & 2.6 & 86.2 \\
\hline 150.0 & & 4.908 & 33.418 & 26.431 & 0.2848 & 5.573 & 2.7 & 84.9 \\
\hline 160.0 & & 4.910 & 33.417 & 26.430 & 0.3004 & 5.485 & 2.9 & 84.1 \\
\hline
\end{tabular}
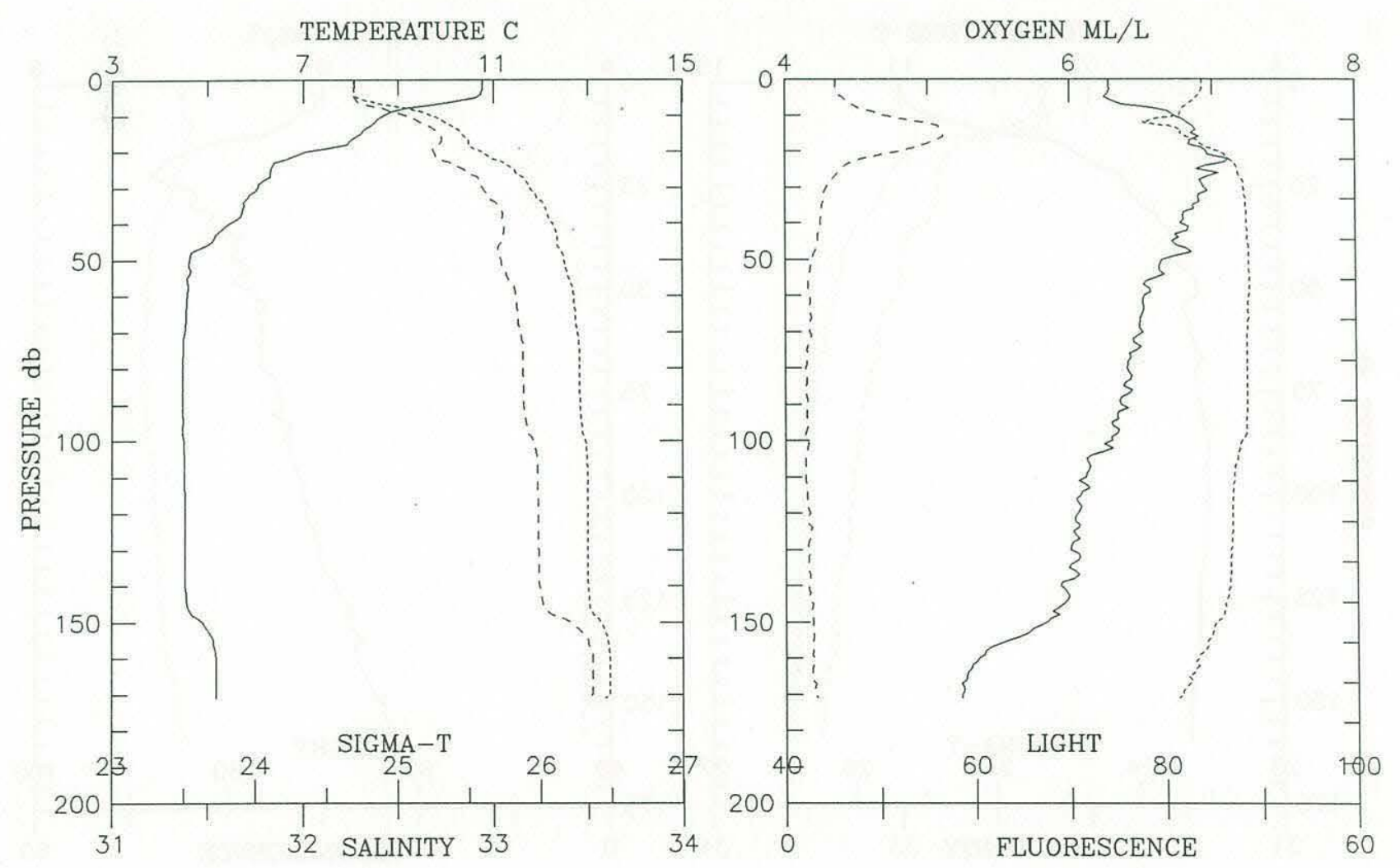


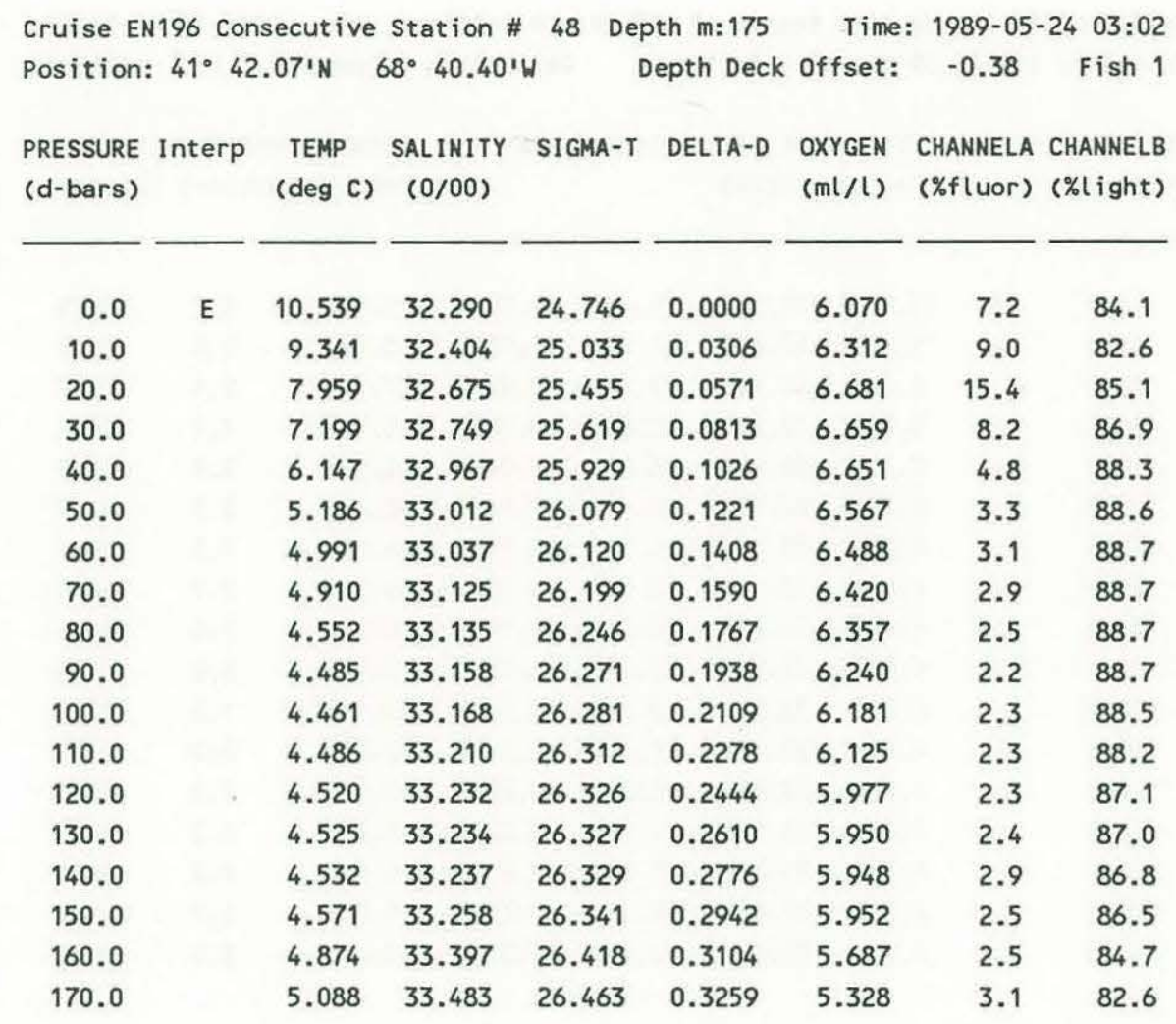
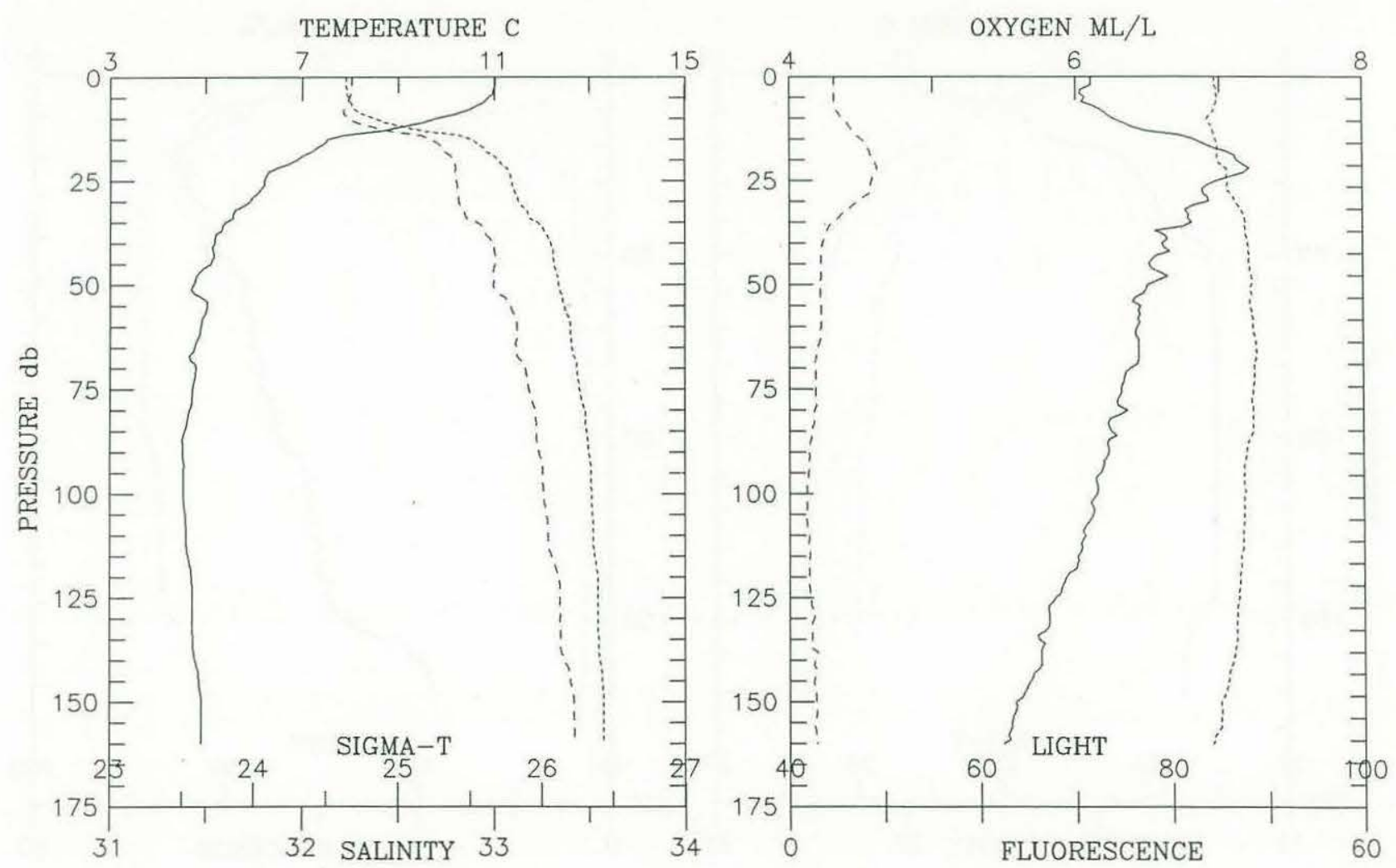


\begin{tabular}{|c|c|c|c|c|c|c|c|c|}
\hline Position: & $: 41^{\circ} 4 C$ & $.051 \mathrm{~N}$ & $68^{\circ} 33.03^{\prime}$ & & epth Deck & Offset: & -0.38 & Fish 1 \\
\hline $\begin{array}{l}\text { PRESSURE } \\
\text { (d-bars) }\end{array}$ & Interp & $\begin{array}{c}\text { TEMP } \\
(\operatorname{deg} C)\end{array}$ & $\begin{array}{l}\text { SALINITY } \\
(0 / 00)\end{array}$ & SIGMA-T & DELTA-D & $\begin{array}{l}\text { OXYGEN } \\
(\mathrm{ml} / \mathrm{l})\end{array}$ & $\begin{array}{l}\text { CHANNELA } \\
\text { (\%fluor) }\end{array}$ & $\begin{array}{l}\text { CHANNELB } \\
(\% \text { light) }\end{array}$ \\
\hline 0.0 & E & 9.347 & 32.749 & 25.301 & 0.0000 & 6.154 & 7.3 & 83.9 \\
\hline 10.0 & & 8.299 & 32.772 & 25.481 & 0.0259 & 6.375 & 7.6 & 84.3 \\
\hline 20.0 & & 8.000 & 32.797 & 25.544 & 0.0502 & 6.518 & 7.3 & 85.3 \\
\hline 30.0 & & 6.502 & 32.860 & 25.799 & 0.0728 & 6.731 & 5.7 & 87.6 \\
\hline 40.0 & & 5.529 & 32.946 & 25.987 & 0.0934 & 6.571 & 4.2 & 87.7 \\
\hline 50.0 & & 5.392 & 32.968 & 26.020 & 0.1130 & 6.403 & 4.1 & 86.9 \\
\hline 60.0 & & 5.330 & 32.985 & 26.040 & 0.1324 & 6.373 & 4.5 & 85.4 \\
\hline 70.0 & & 5.157 & 33.011 & 26.081 & 0.1515 & 6.304 & 4.4 & 85.6 \\
\hline 80.0 & & 5.016 & 33.029 & 26.111 & 0.1703 & 6.242 & 4.0 & 86.4 \\
\hline 90.0 & & 4.954 & 33.044 & 26.130 & 0.1889 & 6.185 & 3.9 & 86.5 \\
\hline 100.0 & & 4.849 & 33.067 & 26.160 & 0.2072 & 6.186 & 3.9 & 86.4 \\
\hline 110.0 & & 4.752 & 33.086 & 26.186 & 0.2252 & 6.111 & 3.8 & 86.1 \\
\hline 120.0 & & 4.700 & 33.099 & 26.202 & 0.2431 & 6.133 & 3.4 & 85.6 \\
\hline 130.0 & & 4.698 & 33.102 & 26.204 & 0.2608 & 6.037 & 3.8 & 85.4 \\
\hline 140.0 & & 4.694 & 33.109 & 26.210 & 0.2786 & 6.000 & 3.5 & 84.8 \\
\hline 150.0 & & 4.598 & 33.139 & 26.244 & 0.2962 & 6.003 & 3.8 & 84.1 \\
\hline 160.0 & & 4.584 & 33.147 & 26.252 & 0.3136 & 5.943 & 3.7 & 83.4 \\
\hline 164.0 & & 4.584 & 33.147 & 26.252 & 0.3205 & 5.988 & 3.3 & 83.2 \\
\hline
\end{tabular}
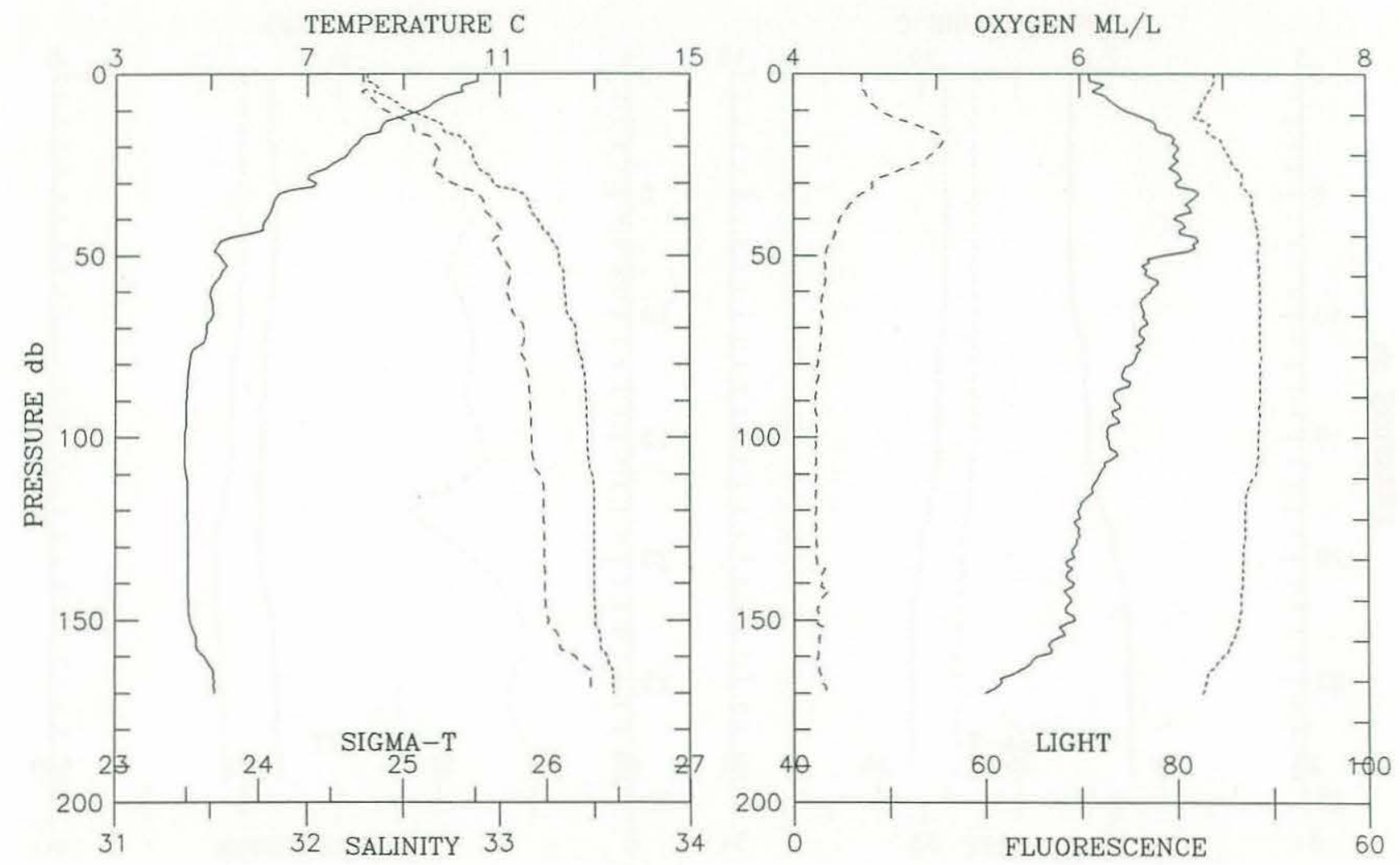


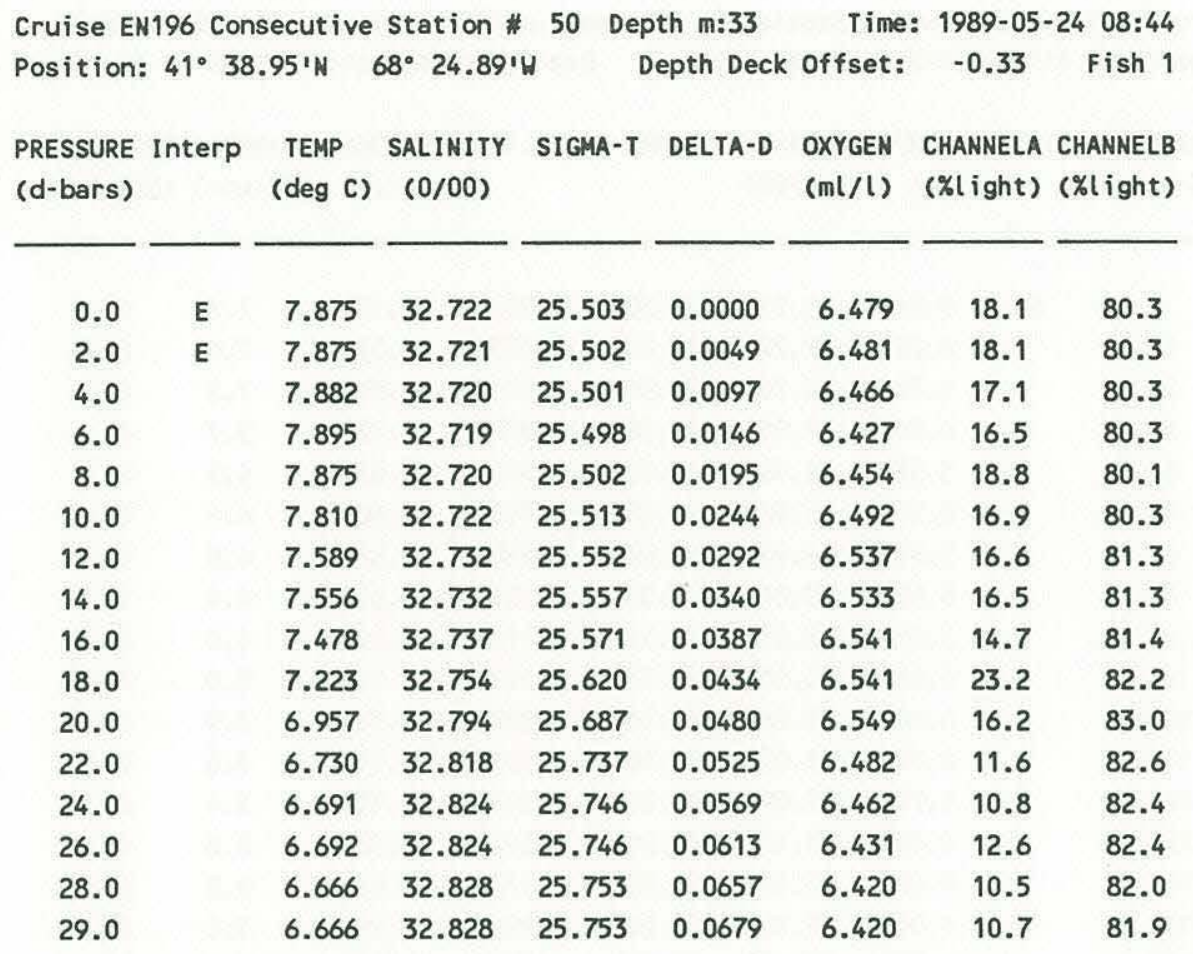
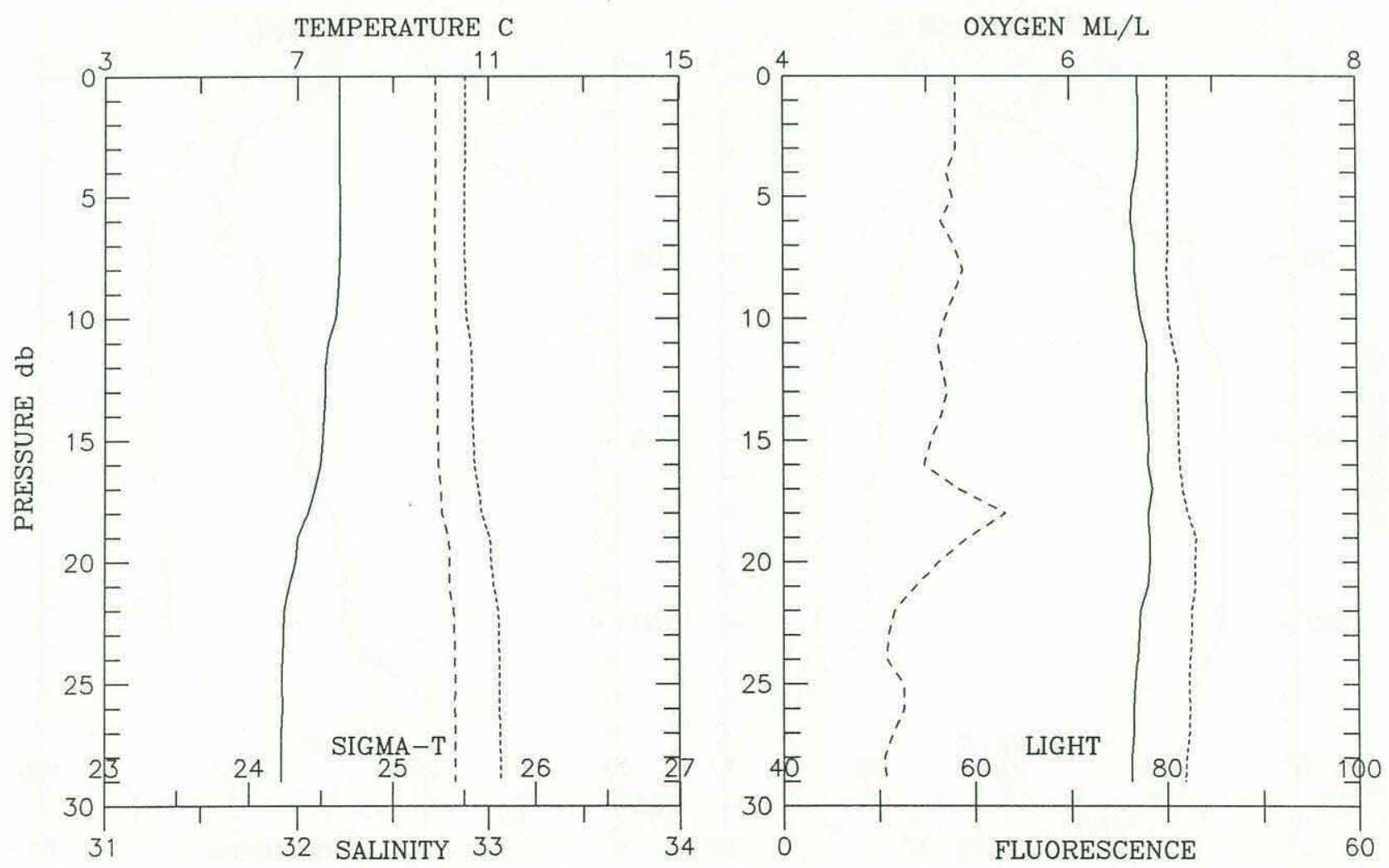
Cruise EN196 Consecutive Station \# 51 Depth m:93 Time: 1989-05-24 09:21 Position: $41^{\circ} 39.27 \mathrm{I}^{\mathrm{N}} 68^{\circ} 26.49 \mathrm{~W} \mathrm{~W}$ Depth Deck offset: -0.33 Fish 1

\begin{tabular}{|c|c|c|c|c|c|c|c|c|}
\hline $\begin{array}{l}\text { PRESSURE } \\
\text { (d-bars) }\end{array}$ & Interp & $\begin{array}{l}\text { TEMP } \\
\text { (deg C) }\end{array}$ & $\begin{array}{l}\text { SALINITY } \\
(0 / 00)\end{array}$ & SIGMA-T & DELTA-D & $\begin{array}{l}\text { OXYGEN } \\
(\mathrm{ml} / \mathrm{l})\end{array}$ & $\begin{array}{l}\text { CHANNELA } \\
\text { (\%fluor) }\end{array}$ & $\begin{array}{l}\text { CHANNELB } \\
\text { (\%light) }\end{array}$ \\
\hline 0.0 & E & 7.839 & 32.742 & 25.524 & 0.0000 & 6.614 & 13.5 & 83.0 \\
\hline 5.0 & & 7.532 & 32.718 & 25.549 & 0.0121 & 6.683 & 11.9 & 83.2 \\
\hline 10.0 & & 7.406 & 32.734 & 25.579 & 0.0239 & 6.609 & 10.7 & 83.8 \\
\hline 15.0 & & 7.409 & 32.747 & 25.589 & 0.0357 & 6.585 & 11.3 & 83.8 \\
\hline 20.0 & & 6.989 & 32.767 & 25.662 & 0.0474 & 6.617 & 10.7 & 84.5 \\
\hline 25.0 & & 6.531 & 32.832 & 25.773 & 0.0586 & 6.506 & 9.8 & 84.9 \\
\hline 30.0 & & 6.354 & 32.853 & 25.812 & 0.0694 & 6.448 & 9.7 & 84.7 \\
\hline 35.0 & & 6.271 & 32.866 & 25.833 & 0.0800 & 6.405 & 8.3 & 84.7 \\
\hline 40.0 & & 6.235 & 32.871 & 25.842 & 0.0906 & 6.389 & 9.3 & 84.7 \\
\hline 45.0 & & 6.046 & 32.898 & 25.886 & 0.1011 & 6.386 & 6.7 & 84.4 \\
\hline 50.0 & & 5.984 & 32.906 & 25.900 & 0.1115 & 6.377 & 9.5 & 83.9 \\
\hline 55.0 & & 5.880 & 32.918 & 25.922 & 0.1217 & 6.327 & 7.4 & 82.6 \\
\hline 60.0 & & 5.744 & 32.936 & 25.953 & 0.1318 & 6.286 & 8.7 & 82.7 \\
\hline 65.0 & & 5.639 & 32.951 & 25.978 & 0.1418 & 6.201 & 5.8 & 82.9 \\
\hline 70.0 & & 5.611 & 32.956 & 25.985 & 0.1517 & 6.188 & 6.0 & 83.0 \\
\hline 75.0 & & 5.586 & 32.959 & 25.990 & 0.1616 & 6.089 & 5.5 & 83.1 \\
\hline 80.0 & & 5.502 & 32.972 & 26.010 & 0.1715 & 6.143 & 6.7 & 82.5 \\
\hline 85.0 & & 5.298 & 33.003 & 26.059 & 0.1812 & 6.127 & 6.2 & 82.2 \\
\hline 90.0 & & 5.253 & 33.010 & 26.069 & 0.1907 & 6.186 & 5.6 & 80.9 \\
\hline 92.0 & & 5.251 & 33.011 & 26.071 & 0.1945 & 6.189 & 6.9 & 80.5 \\
\hline
\end{tabular}
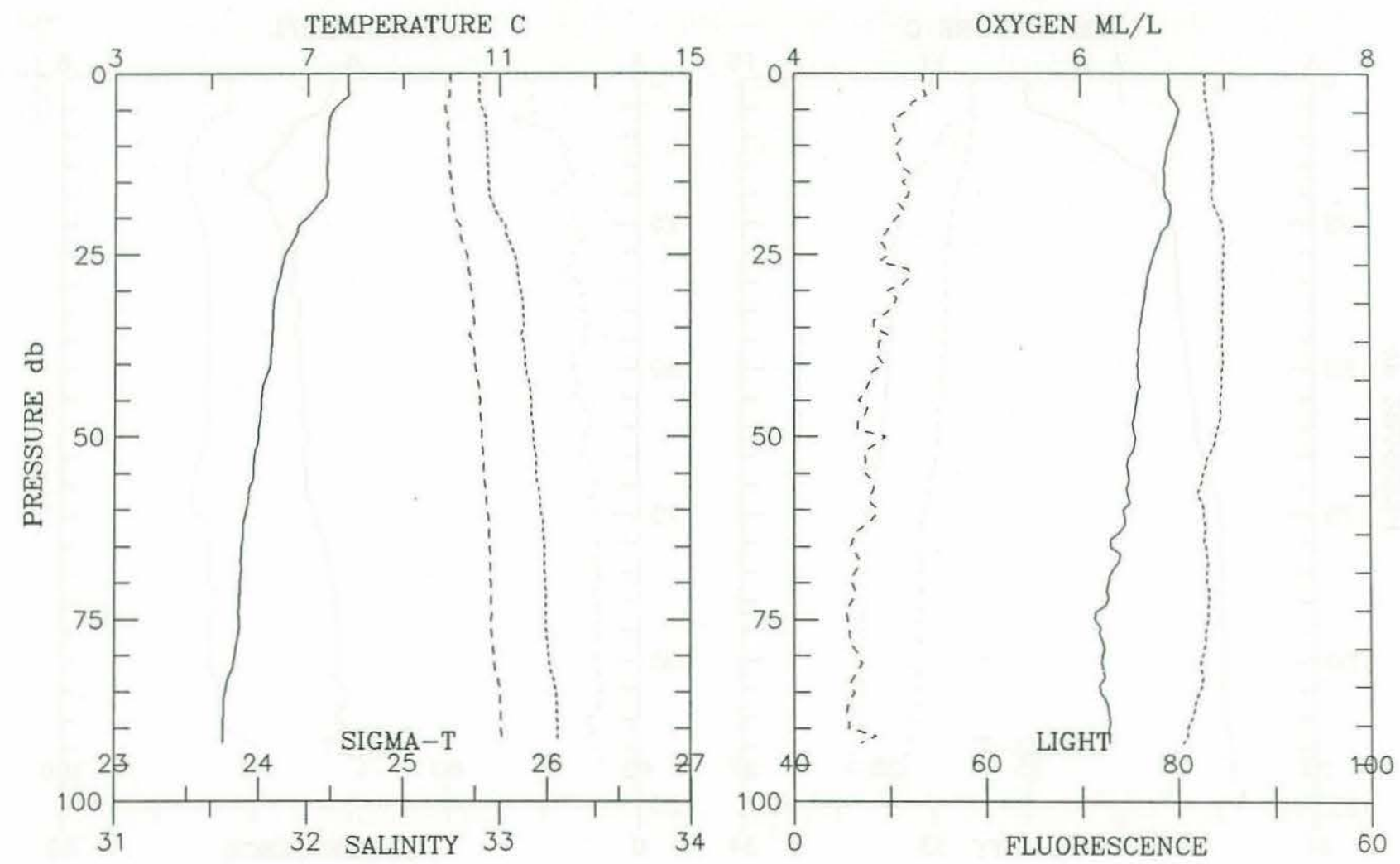


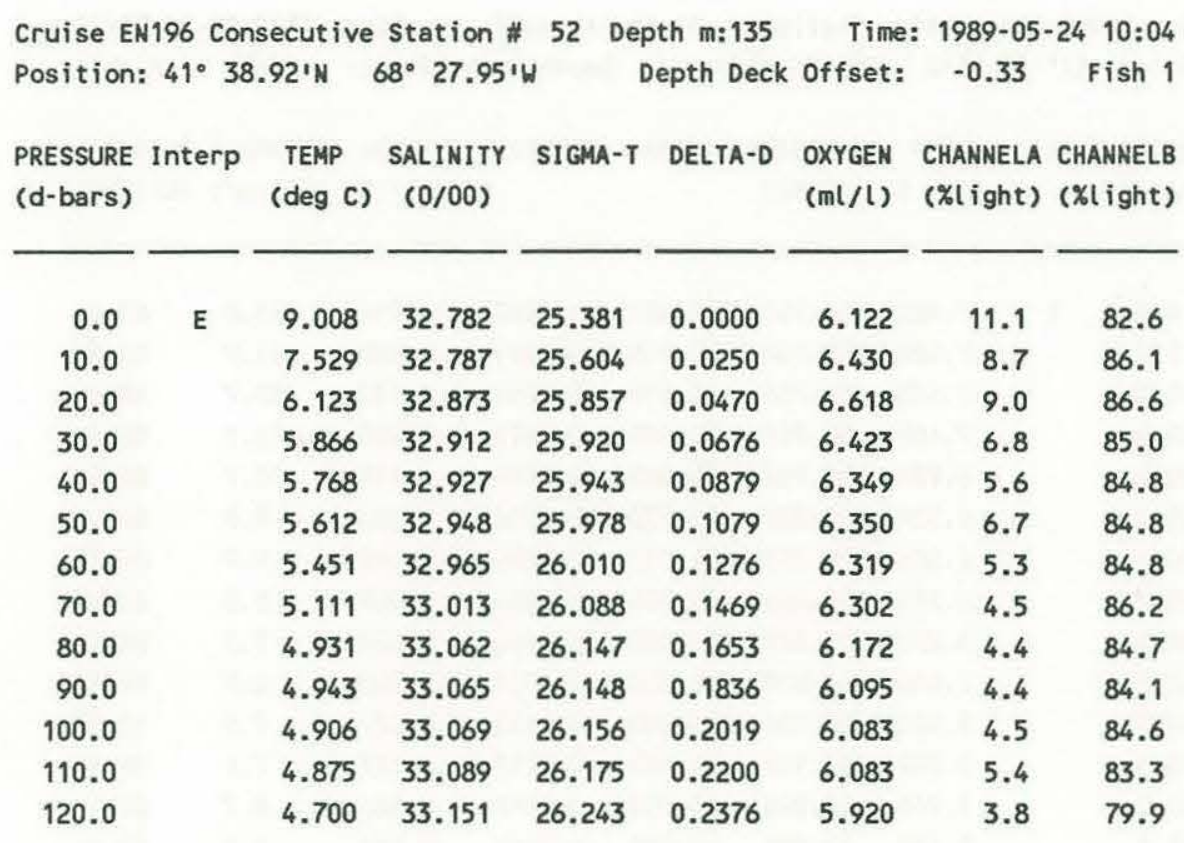
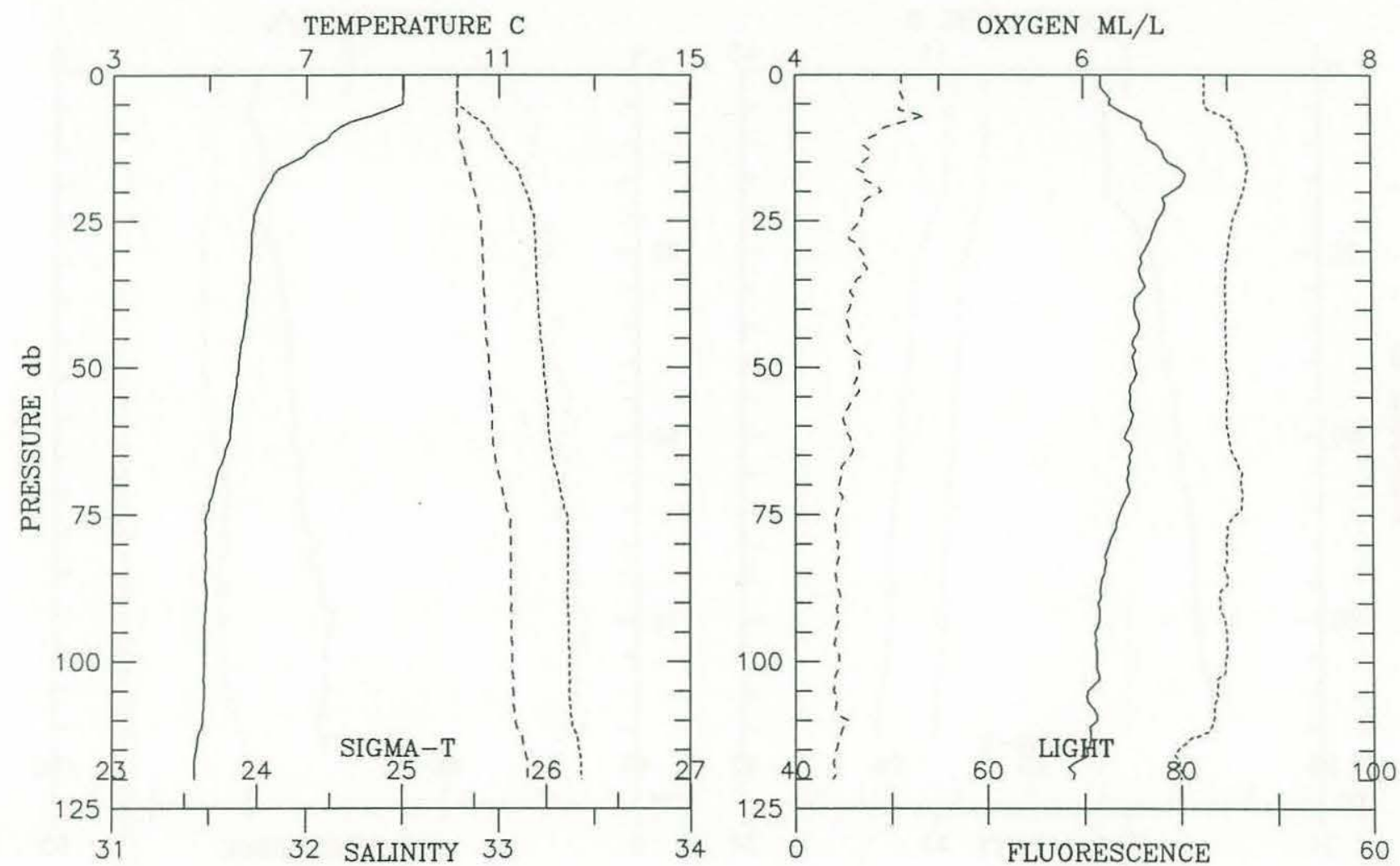


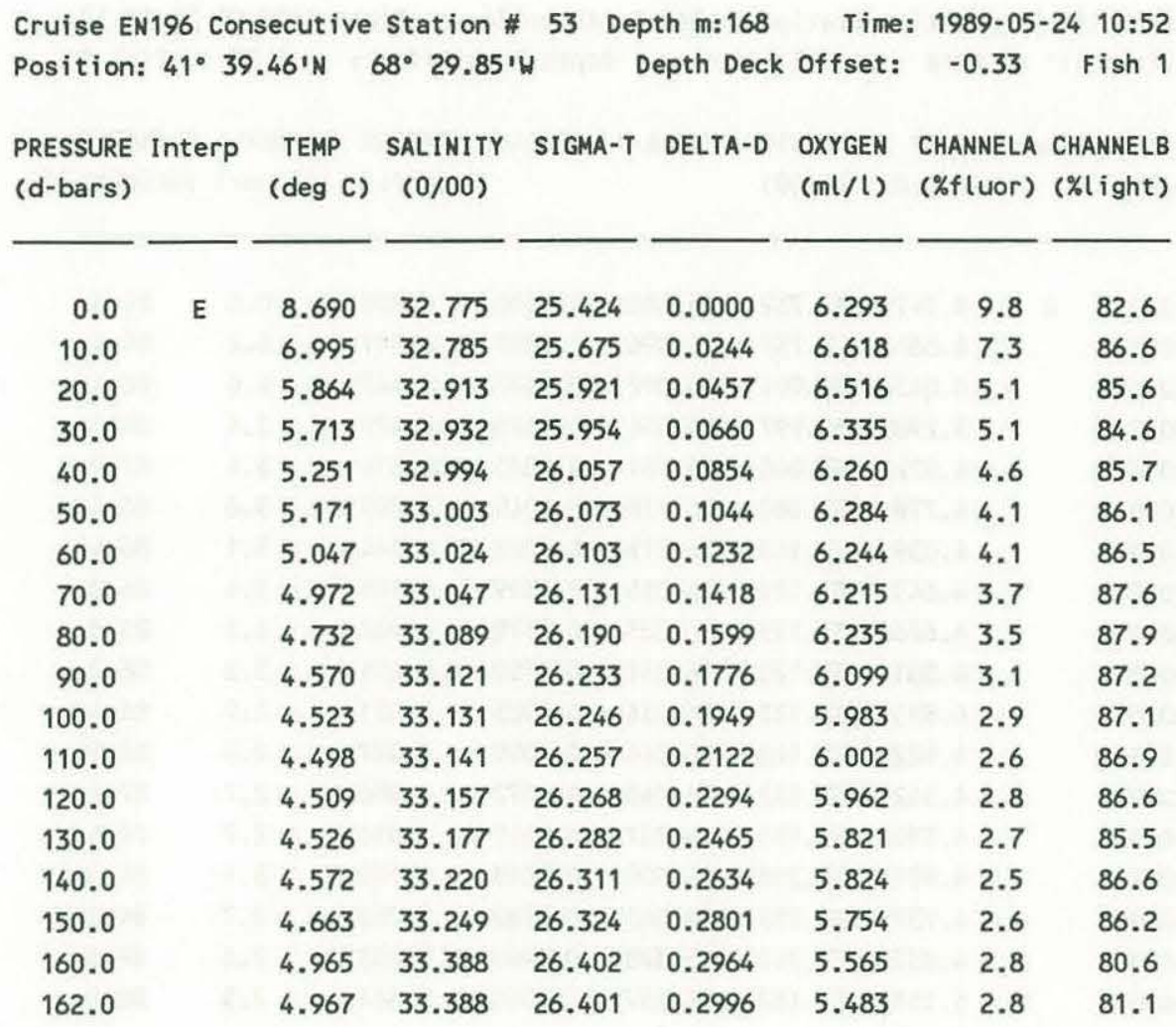
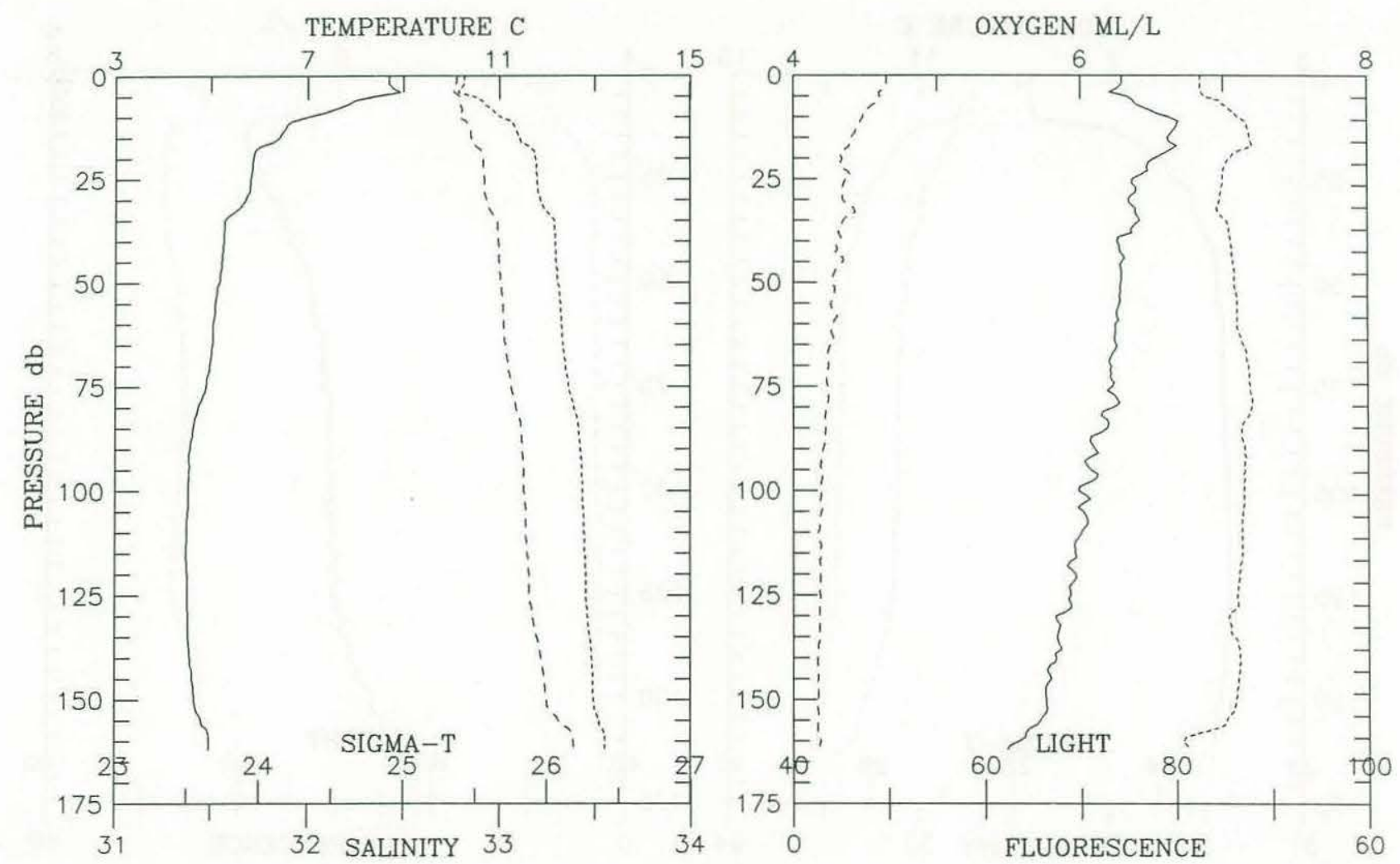


\begin{tabular}{|c|c|c|c|c|c|c|c|c|}
\hline \multicolumn{9}{|c|}{ Cruise EN196 Consecutive Station \# 54 Depth m:176 } \\
\hline $\begin{array}{l}\text { PRESSURE } \\
\text { (d-bars) }\end{array}$ & Interp & $\begin{array}{c}\text { TEMP } \\
(\operatorname{deg} \mathrm{C})\end{array}$ & $\begin{array}{c}\text { SALINITY } \\
(0 / 00)\end{array}$ & SIGMA-T & DELTA-D & $\begin{array}{l}\text { OXYGEN } \\
(\mathrm{ml} / \mathrm{l})\end{array}$ & $\begin{array}{l}\text { CHANNELA } \\
\text { (\%fluor) }\end{array}$ & $\begin{array}{l}\text { CHANNELB } \\
\text { (\%light) }\end{array}$ \\
\hline 0.0 & E & 8.747 & 32.732 & 25.382 & 0.0000 & 6.289 & 8.6 & 84.3 \\
\hline 10.0 & & 8.684 & 32.737 & 25.396 & 0.0255 & 6.301 & 8.2 & 84.4 \\
\hline 20.0 & & 6.013 & 32.901 & 25.892 & 0.0478 & 6.643 & 4.6 & 88.1 \\
\hline 30.0 & & 5.298 & 32.997 & 26.054 & 0.0676 & 6.479 & 3.4 & 88.1 \\
\hline 40.0 & & 4.972 & 33.048 & 26.131 & 0.0865 & 6.314 & 3.4 & 87.9 \\
\hline 50.0 & & 4.778 & 33.080 & 26.178 & 0.1045 & 6.205 & 3.6 & 86.4 \\
\hline 60.0 & & 4.639 & 33.108 & 26.215 & 0.1223 & 6.144 & 3.1 & 86.4 \\
\hline 70.0 & & 4.643 & 33.109 & 26.215 & 0.1399 & 6.105 & 3.4 & 86.2 \\
\hline 80.0 & & 4.626 & 33.117 & 26.223 & 0.1575 & 6.062 & 3.3 & 85.8 \\
\hline 90.0 & & 4.581 & 33.120 & 26.231 & 0.1750 & 6.051 & 3.2 & 86.2 \\
\hline 100.0 & & 4.551 & 33.122 & 26.236 & 0.1925 & 6.051 & 2.9 & 86.4 \\
\hline 110.0 & & 4.522 & 33.128 & 26.244 & 0.2099 & 6.027 & 2.8 & 86.9 \\
\hline 120.0 & & 4.512 & 33.132 & 26.248 & 0.2272 & 6.056 & 2.7 & 87.0 \\
\hline 130.0 & & 4.530 & 33.158 & 26.267 & 0.2445 & 6.016 & 2.7 & 86.4 \\
\hline 140.0 & & 4.591 & 33.208 & 26.300 & 0.2615 & 5.900 & 3.1 & 84.4 \\
\hline 150.0 & & 4.737 & 33.285 & 26.345 & 0.2782 & 5.758 & 2.7 & 84.1 \\
\hline 160.0 & & 4.832 & 33.340 & 26.378 & 0.2945 & 5.633 & 2.6 & 84.6 \\
\hline 166.0 & & 5.151 & 33.485 & 26.457 & 0.3040 & 5.364 & 2.5 & 82.0 \\
\hline
\end{tabular}
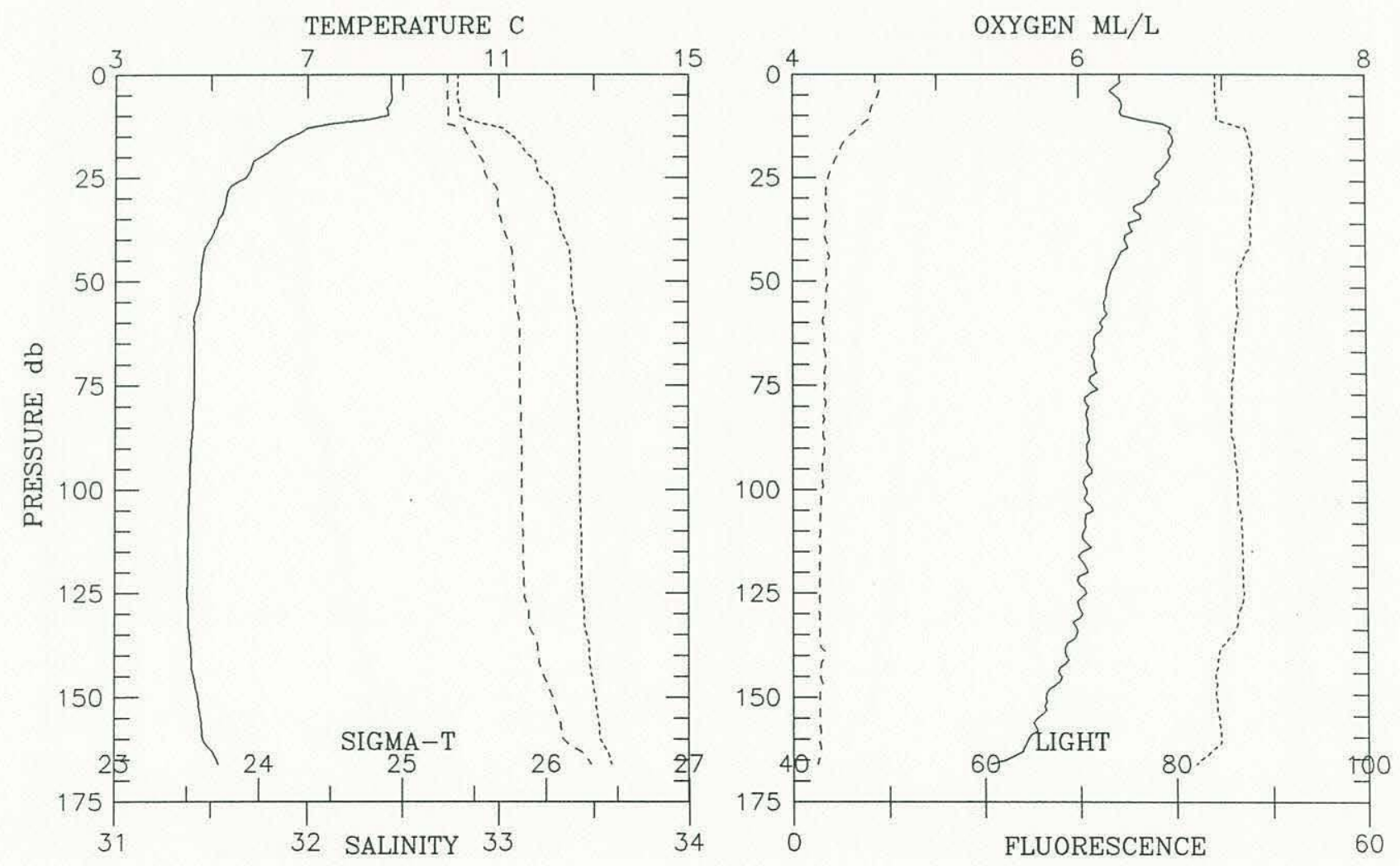
Cruise EN196 Consecutive Station \# 55 Depth m:175 Time: 1989-05-24 14:37 Position: $41^{\circ} 42.00^{\prime} \mathrm{N} 68^{\circ} 40.07^{\prime} \mathrm{W}$ Depth Deck offset: -0.33 Fish 1

\begin{tabular}{|c|c|c|c|c|c|c|c|c|}
\hline $\begin{array}{l}\text { PRESSURE } \\
\text { (d-bars) }\end{array}$ & Interp & $\begin{array}{l}\text { TEMP } \\
(\operatorname{deg} C)\end{array}$ & $\begin{array}{l}\text { SALINITY } \\
(0 / 00)\end{array}$ & SIGMA-T & DELTA-D & $\begin{array}{l}\text { OXYGEN } \\
(m l / l)\end{array}$ & $\begin{array}{l}\text { CHANNELA } \\
\text { (\%floro) }\end{array}$ & $\begin{array}{l}\text { CHANNELB } \\
\text { (\%tight) }\end{array}$ \\
\hline 0.0 & E & 9.908 & 32.425 & 24.958 & 0.0000 & 6.270 & 8.8 & 83.4 \\
\hline 10.0 & & 9.886 & 32.426 & 24.962 & 0.0296 & 6.262 & 11.9 & 83.3 \\
\hline 20.0 & & 8.285 & 32.685 & 25.415 & 0.0573 & 6.662 & 16.6 & 82.0 \\
\hline 30.0 & & 6.721 & 32.725 & 25.664 & 0.0815 & 6.805 & 7.5 & 86.8 \\
\hline 40.0 & & 5.161 & 32.927 & 26.014 & 0.1027 & 6.685 & 3.8 & 88.3 \\
\hline 50.0 & & 5.035 & 33.034 & 26.113 & 0.1218 & 6.421 & 2.8 & 88.8 \\
\hline 60.0 & & 4.916 & 33.102 & 26.180 & 0.1401 & 6.392 & 2.7 & 88.8 \\
\hline 70.0 & & 4.689 & 33.126 & 26.224 & 0.1578 & 6.288 & 2.4 & 88.9 \\
\hline 80.0 & & 4.517 & 33.153 & 26.264 & 0.1750 & 6.217 & 2.2 & 88.8 \\
\hline 90.0 & & 4.480 & 33.163 & 26.276 & 0.1921 & 6.190 & 2.1 & 88.7 \\
\hline 100.0 & & 4.462 & 33.176 & 26.288 & 0.2091 & 6.166 & 2.1 & 88.5 \\
\hline 110.0 & & 4.512 & 33.231 & 26.326 & 0.2259 & 6.035 & 2.1 & 87.4 \\
\hline 120.0 & & 4.519 & 33.235 & 26.329 & 0.2424 & 5.964 & 2.0 & 87.3 \\
\hline 130.0 & & 4.523 & 33.236 & 26.329 & 0.2590 & 5.925 & 2.2 & 87.3 \\
\hline 140.0 & & 4.576 & 33.268 & 26.349 & 0.2755 & 5.950 & 2.0 & 87.3 \\
\hline 150.0 & & 4.727 & 33.346 & 26.394 & 0.2918 & 5.888 & 2.1 & 87.4 \\
\hline 160.0 & & 5.245 & 33.547 & 26.495 & 0.3073 & 5.374 & 2.7 & 83.9 \\
\hline 166.0 & & 5.287 & 33.563 & 26.503 & 0.3163 & 5.184 & 2.6 & 82.7 \\
\hline
\end{tabular}
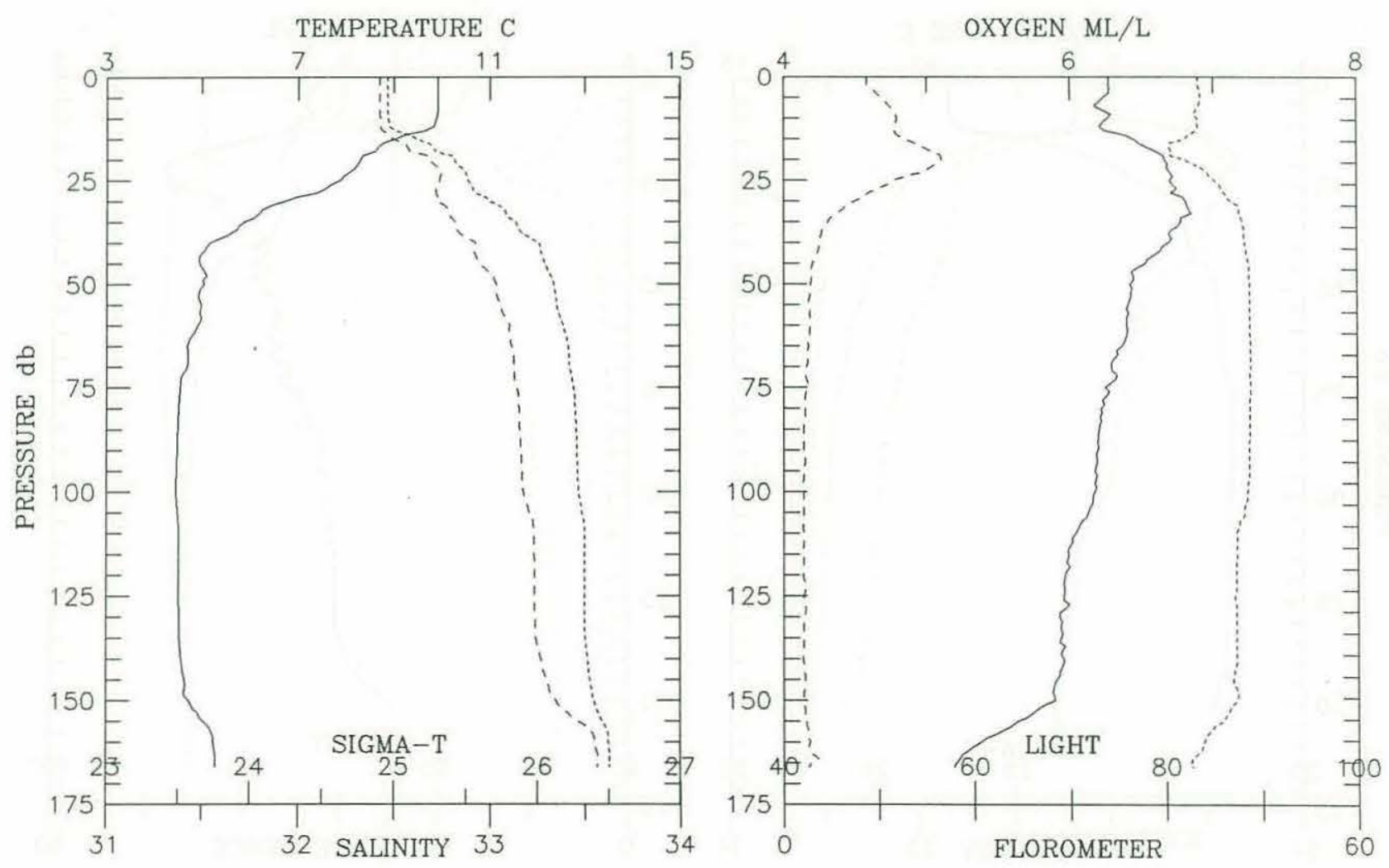


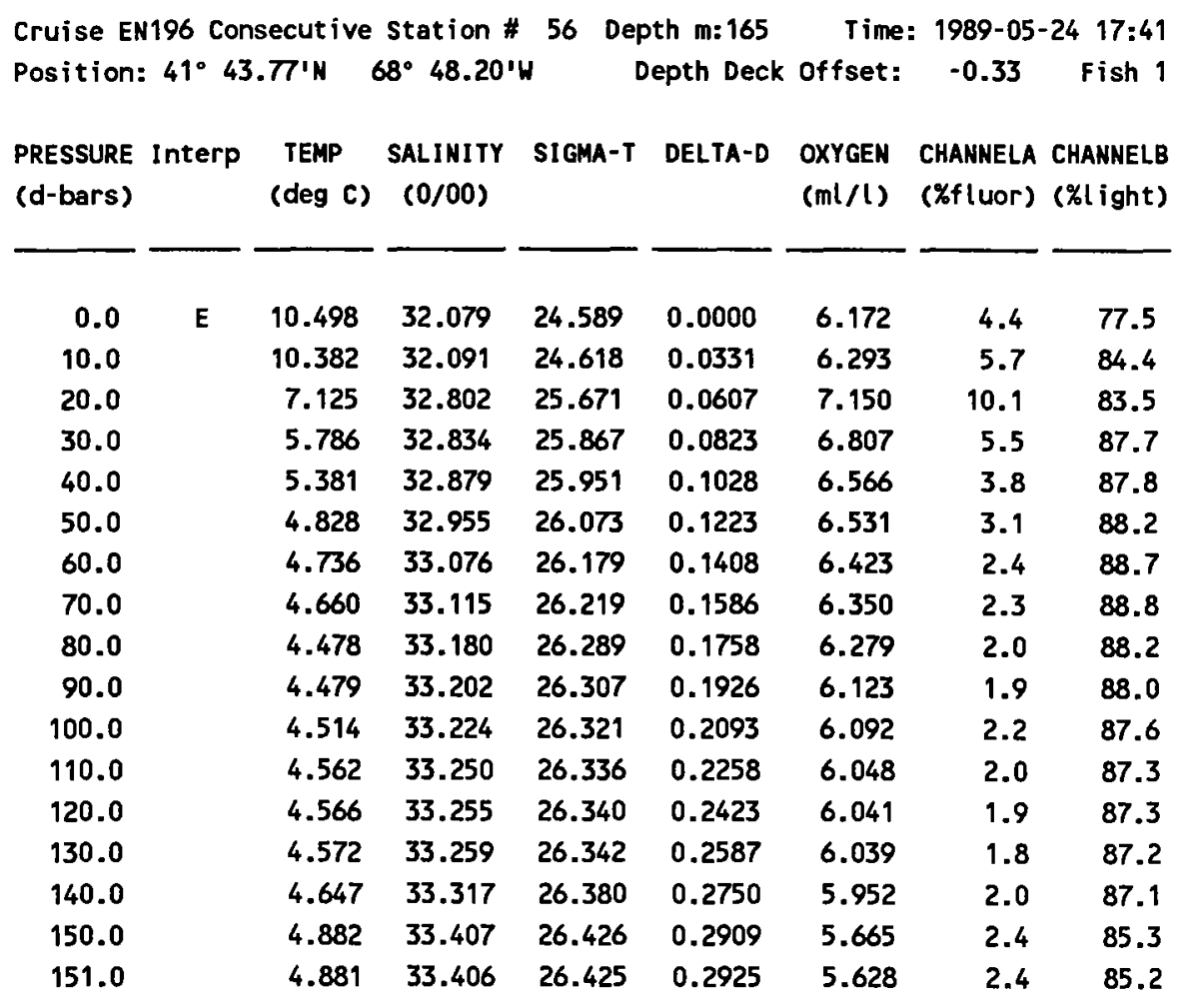
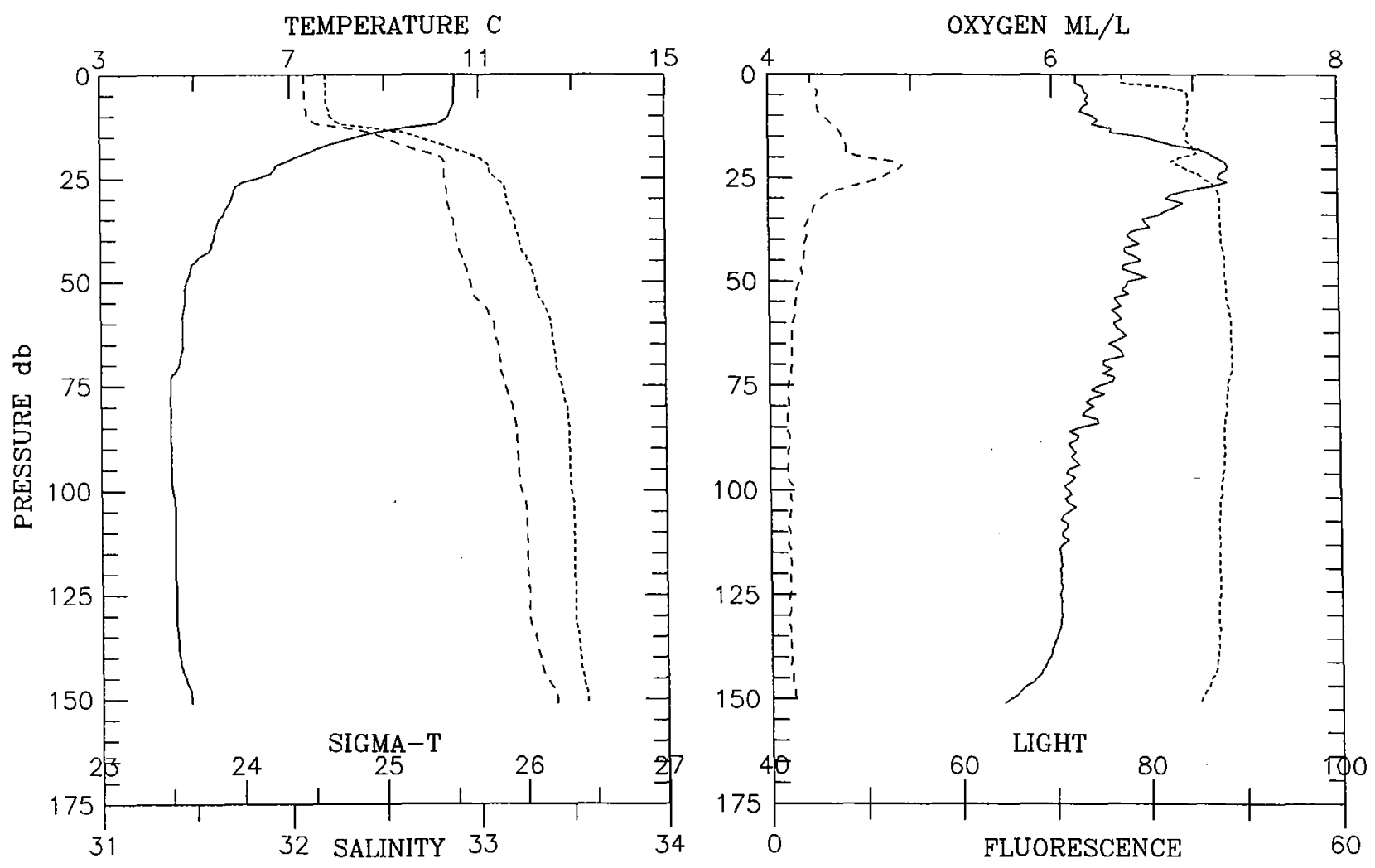
Cruise EN196 Consecutive Station \# 57 Depth m: 172 Time: 1989-05-24 22:15 Position: $41^{\circ} 43.00$ 'N $68^{\circ} 54.75 \mathrm{~W}$ Depth Deck offset: -0.30 Fish 1

\begin{tabular}{|c|c|c|c|c|c|c|c|c|}
\hline $\begin{array}{l}\text { PRESSURE } \\
\text { (d-bars) }\end{array}$ & Interp & $\begin{array}{c}\text { TEMP } \\
\text { (deg C) }\end{array}$ & $\begin{array}{c}\text { SALINITY } \\
(0 / 00)\end{array}$ & SIGMA-T & DELTA-D & $\begin{array}{l}\text { OXYGEN } \\
(\mathrm{ml} / \mathrm{l})\end{array}$ & $\begin{array}{l}\text { CHANNELA } \\
\text { (\%fluor) }\end{array}$ & $\begin{array}{l}\text { CHANNELB } \\
\text { ( } \% \text { tight) }\end{array}$ \\
\hline 0.0 & $\mathbf{E}$ & 10.389 & 31.892 & 24.462 & 0.0000 & 6.193 & 8.9 & 82.7 \\
\hline 10.0 & & 10.195 & 31.901 & 24.501 & 0.0342 & 6.130 & 9.3 & 82.3 \\
\hline 20.0 & & 6.512 & 32.483 & 25.500 & 0.0624 & 7.083 & 11.1 & 84.3 \\
\hline 30.0 & & 6.678 & 32.857 & 25.774 & 0.0852 & 6.999 & 11.5 & 86.0 \\
\hline 40.0 & & 6.317 & 32.954 & 25.897 & 0.1064 & 6.846 & 4.9 & 88.0 \\
\hline 50.0 & & 5.080 & 32.970 & 26.057 & 0.1263 & 6.585 & 3.4 & 88.3 \\
\hline 60.0 & & 4.908 & 33.030 & 26.124 & 0.1451 & 6.388 & 2.8 & 88.7 \\
\hline 70.0 & & 4.681 & 33.099 & 26.204 & 0.1632 & 6.452 & 2.7 & 88.6 \\
\hline 80.0 & & 4.717 & 33.157 & 26.246 & 0.1808 & 6.353 & 2.4 & 88.6 \\
\hline 90.0 & & 4.626 & 33.180 & 26.274 & 0.1980 & 6.228 & 2.3 & 88.6 \\
\hline 100.0 & & 4.530 & 33.213 & 26.310 & 0.2149 & 6.208 & 2.5 & 88.4 \\
\hline 110.0 & & 4.568 & 33.280 & 26.359 & 0.2313 & 6.084 & 2.3 & 87.7 \\
\hline 120.0 & & 4.570 & 33.289 & 26.366 & 0.2476 & 5.958 & 1.9 & 87.5 \\
\hline 130.0 & & 4.580 & 33.297 & 26.371 & 0.2638 & 5.935 & 1.9 & 87.3 \\
\hline 140.0 & & 4.596 & 33.307 & 26.378 & 0.2799 & 5.882 & 1.9 & 87.0 \\
\hline 150.0 & & 4.625 & 33.320 & 26.385 & 0.2960 & 5.853 & 2.1 & 85.7 \\
\hline 160.0 & & 4.627 & 33.320 & 26.385 & 0.3121 & 5.842 & 2.4 & 84.2 \\
\hline 165.0 & & 4.626 & 33.319 & 26.384 & 0.3201 & 5.857 & 2.4 & 83.1 \\
\hline
\end{tabular}
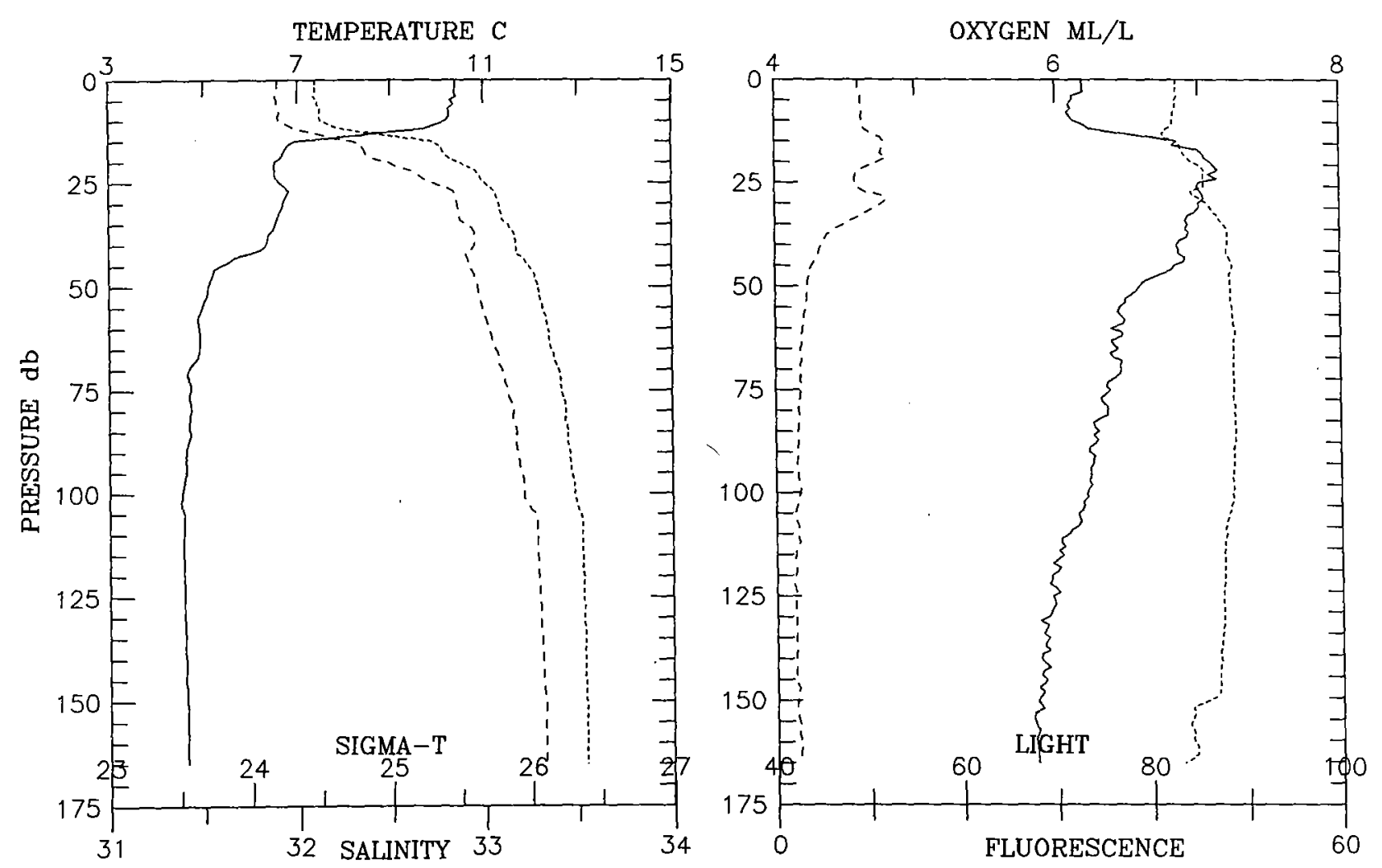


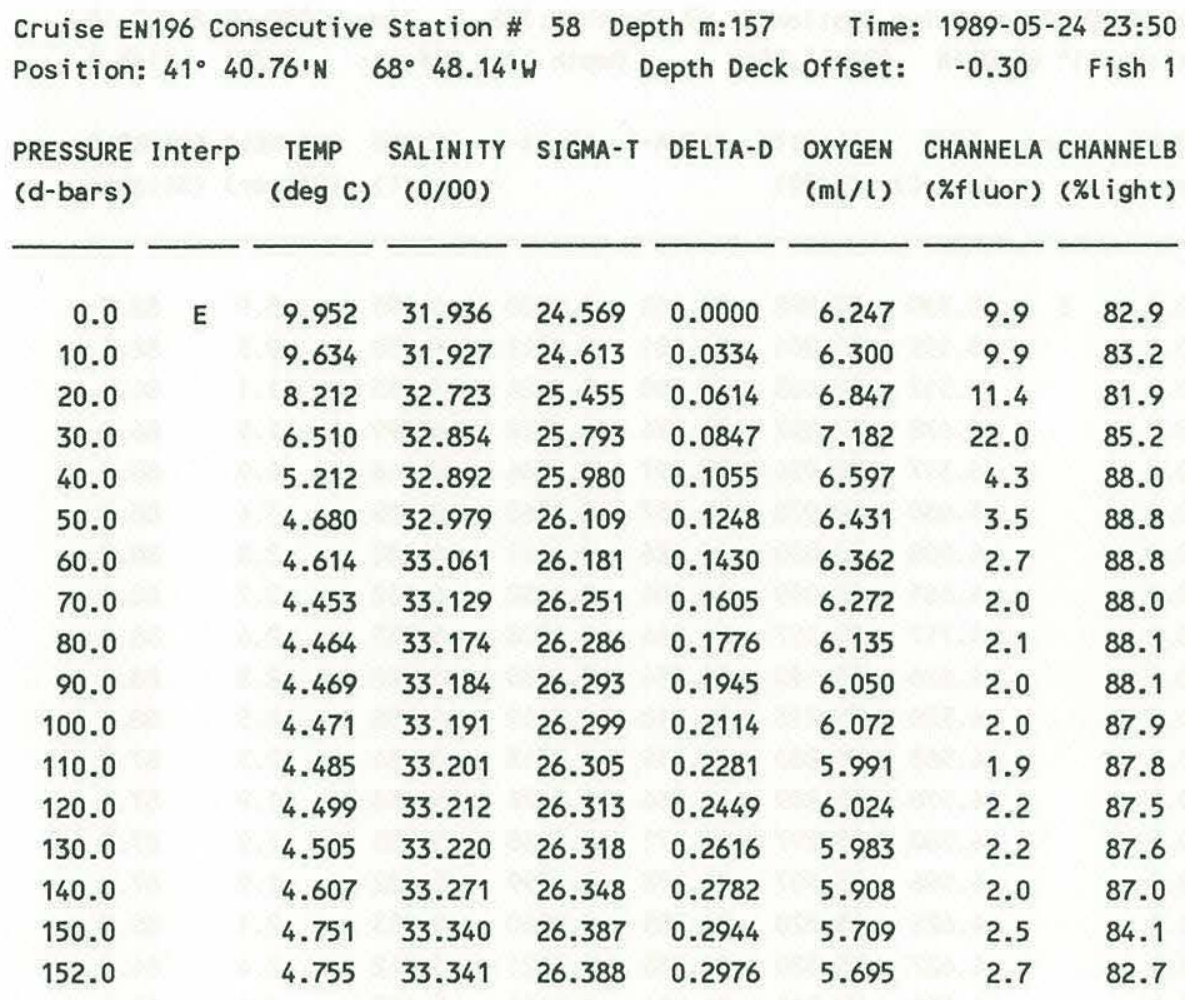
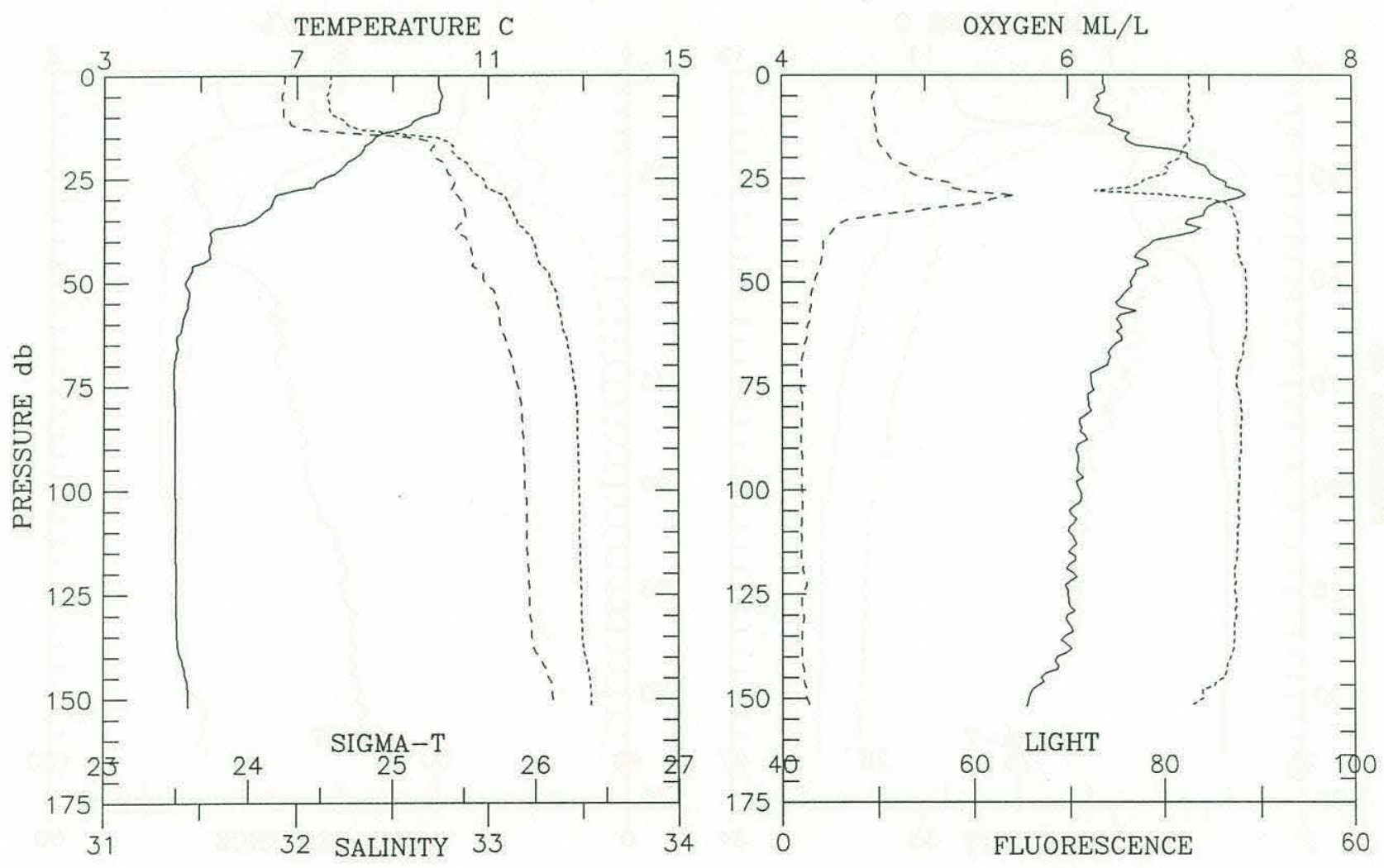


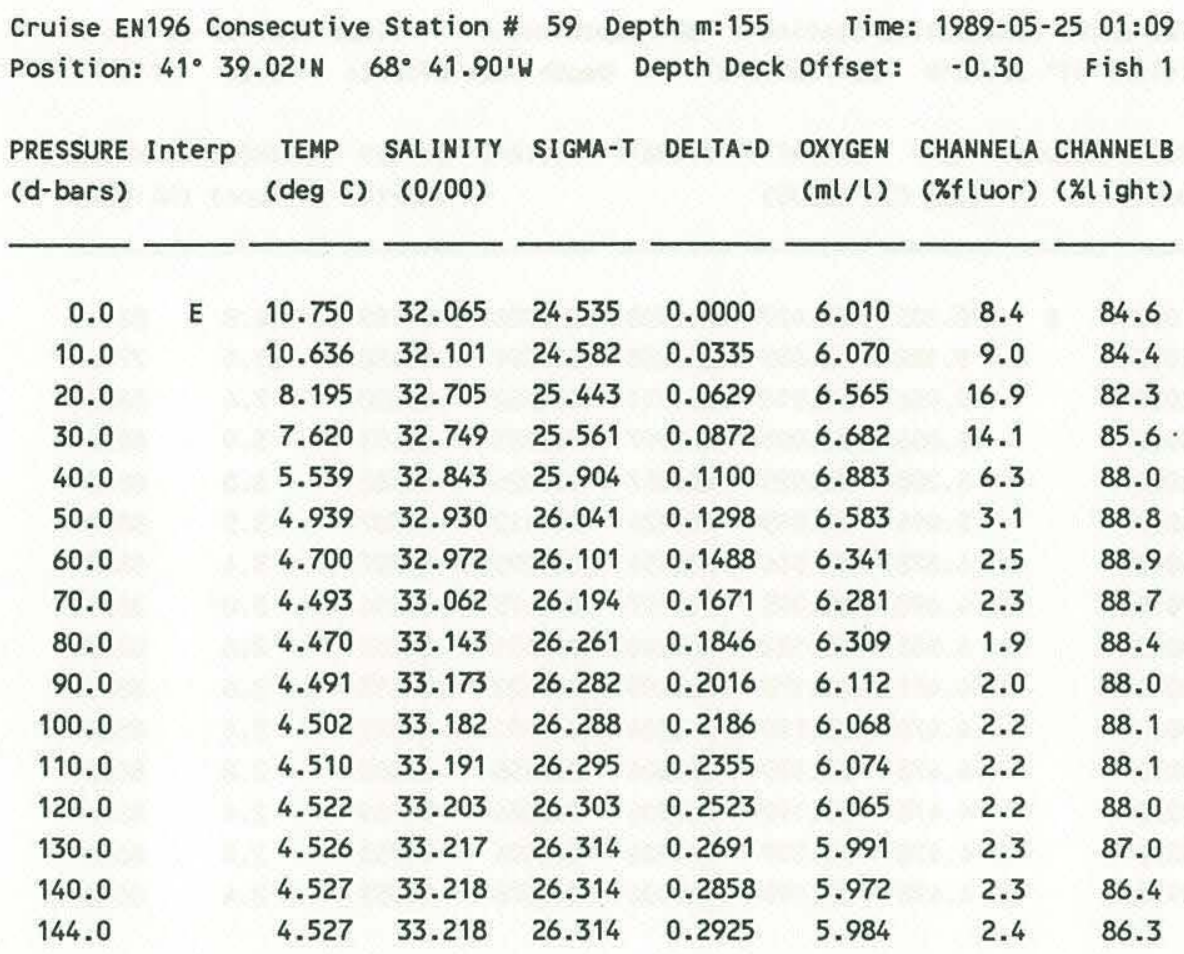
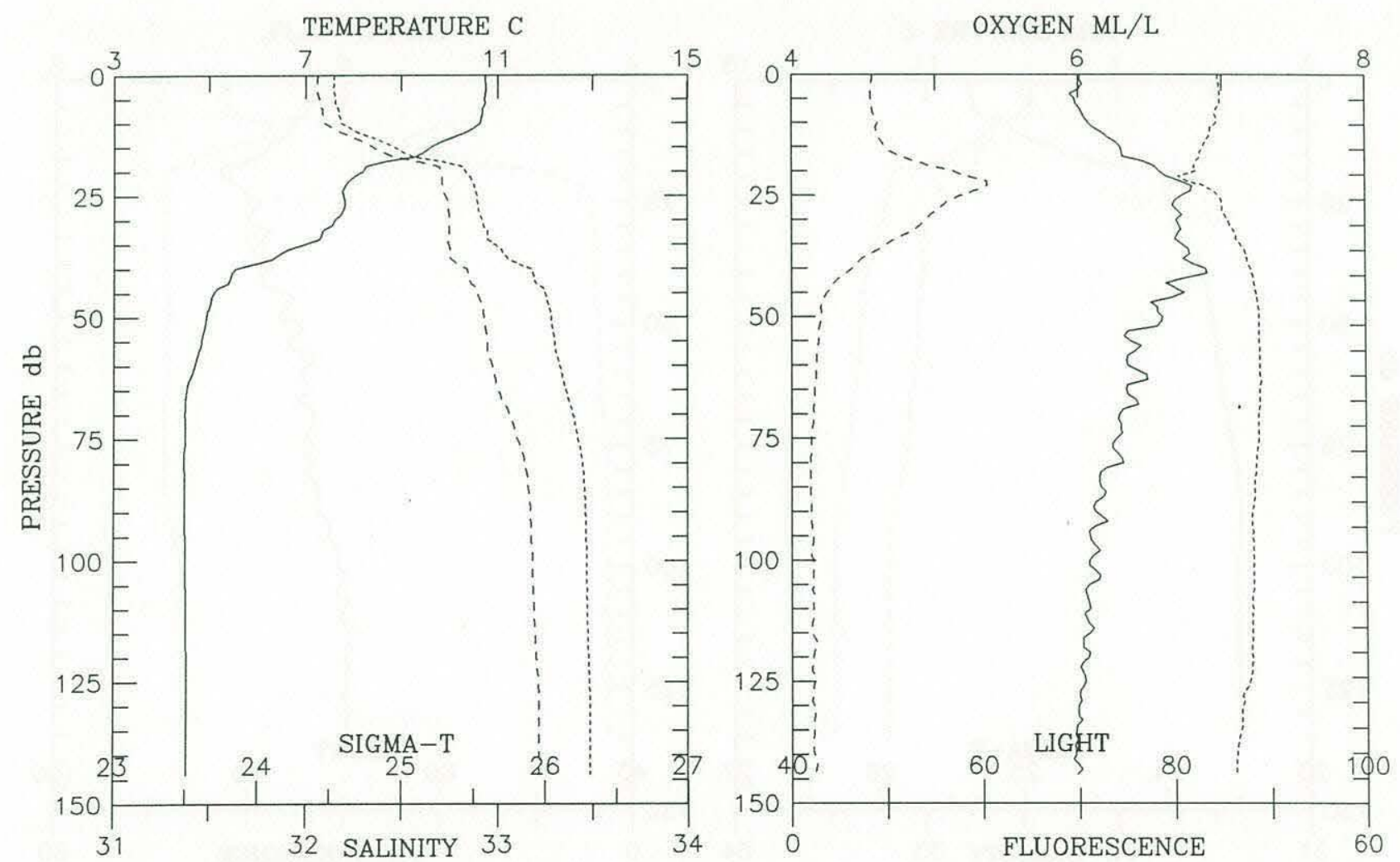


\begin{tabular}{|c|c|c|c|c|c|c|c|c|}
\hline Position: & $41^{\circ} 37$ & $.72^{\prime} \mathrm{N}$ & $68^{\circ} 36.13^{\prime}$ & & epth Deck & Offset: & -0.20 & Fish 1 \\
\hline $\begin{array}{l}\text { PRESSURE } \\
\text { (d-bars) }\end{array}$ & Interp & $\begin{array}{c}\text { TEMP } \\
(\operatorname{deg} C)\end{array}$ & $\begin{array}{c}\text { SALINITY } \\
(0 / 00)\end{array}$ & SIGMA-T & DELTA-D & $\begin{array}{l}\text { OXYGEN } \\
(\mathrm{ml} / \mathrm{l})\end{array}$ & $\begin{array}{l}\text { CHANNELA } \\
\text { ( } \% \text { fluor) }\end{array}$ & $\begin{array}{l}\text { CHANNELB } \\
\text { (\%light) }\end{array}$ \\
\hline 0.0 & $E$ & 10.105 & 32.429 & 24.928 & 0.0000 & 6.149 & 14.8 & 82.0 \\
\hline 10.0 & & 9.182 & 32.635 & 25.238 & 0.0291 & 6.388 & 21.1 & 77.6 \\
\hline 20.0 & & 5.966 & 32.916 & 25.911 & 0.0532 & 6.820 & 7.4 & 88.3 \\
\hline 30.0 & & 5.806 & 33.001 & 25.997 & 0.0733 & 6.593 & 3.9 & 88.6 \\
\hline 40.0 & & 5.208 & 33.025 & 26.087 & 0.0926 & 6.582 & 3.8 & 88.3 \\
\hline 50.0 & & 5.014 & 33.045 & 26.124 & 0.1112 & 6.307 & 3.5 & 88.1 \\
\hline 60.0 & & 4.878 & 33.064 & 26.154 & 0.1295 & 6.297 & 3.4 & 88.3 \\
\hline 70.0 & & 4.698 & 33.093 & 26.197 & 0.1475 & 6.214 & 3.0 & 88.6 \\
\hline 80.0 & & 4.505 & 33.122 & 26.240 & 0.1651 & 6.255 & 2.6 & 88.5 \\
\hline 90.0 & & 4.461 & 33.173 & 26.285 & 0.1822 & 6.115 & 2.6 & 88.0 \\
\hline 100.0 & & 4.478 & 33.199 & 26.304 & 0.1991 & 6.042 & 2.4 & 86.8 \\
\hline 110.0 & & 4.478 & 33.199 & 26.304 & 0.2158 & 6.002 & 2.8 & 86.6 \\
\hline 120.0 & & 4.478 & 33.199 & 26.304 & 0.2326 & 5.969 & 2.4 & 86.6 \\
\hline 130.0 & & 4.478 & 33.199 & 26.304 & 0.2494 & 5.953 & 2.3 & 86.6 \\
\hline 135.0 & & 4.478 & 33.199 & 26.304 & 0.2578 & 5.953 & 2.4 & 86.3 \\
\hline
\end{tabular}
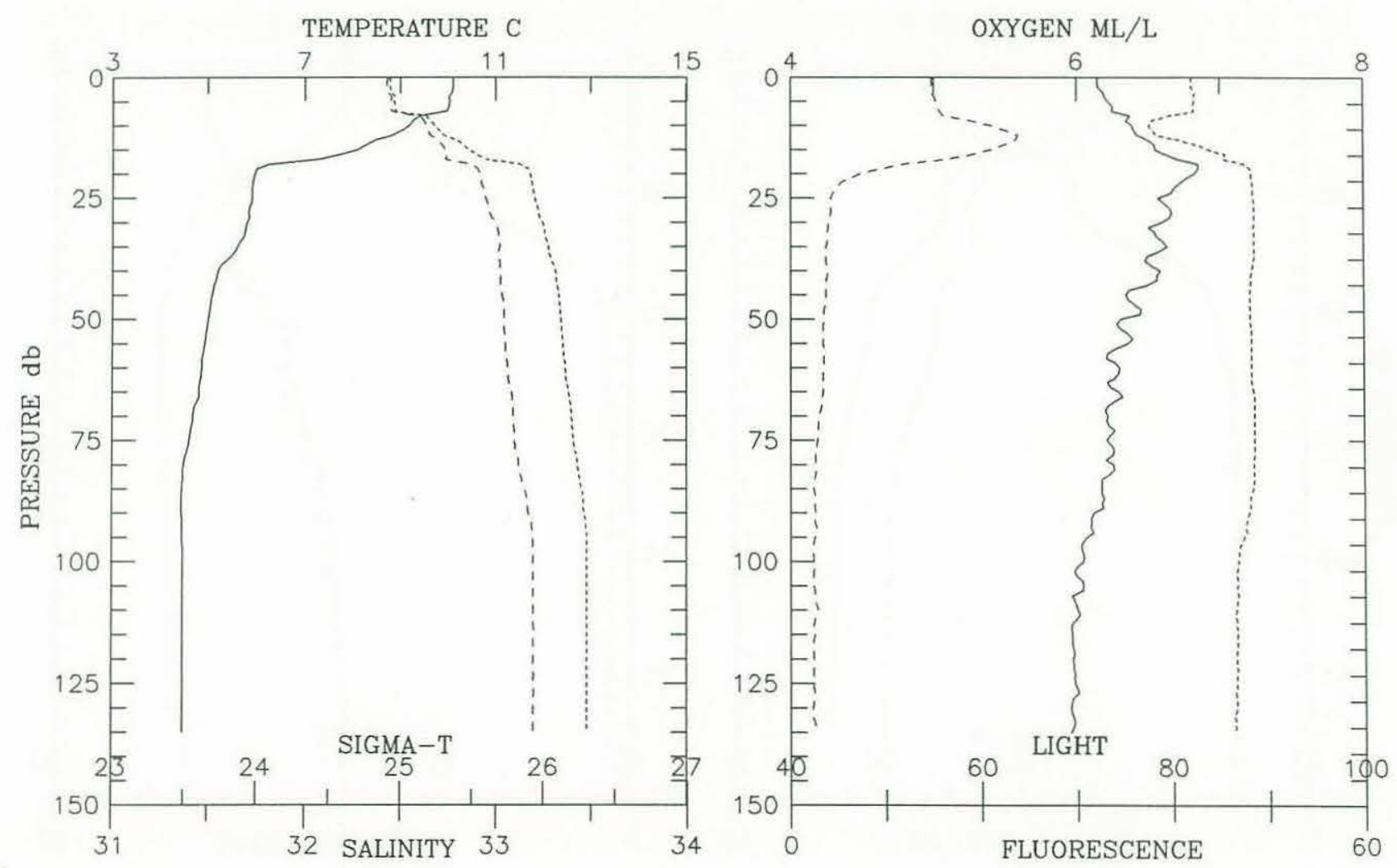
Cruise EN196 Consecutive Station \# 61 Depth m:173 Time: 1989-05-25 03:44 Position: $41^{\circ} 42.64^{\prime} \mathrm{N} \quad 68^{\circ} 34.06^{\prime} \mathrm{W}$ Depth Deck offset: -0.20 Fish 1

\begin{tabular}{|c|c|c|c|c|c|c|c|c|}
\hline $\begin{array}{l}\text { PRESSURE } \\
\text { (d-bars) }\end{array}$ & Interp & $\begin{array}{c}\text { TEMP } \\
(\operatorname{deg} \mathrm{C})\end{array}$ & $\begin{array}{l}\text { SALINITY } \\
(0 / 00)\end{array}$ & SIGMA-T & DELTA-D & $\begin{array}{l}\text { OXYGEN } \\
(\mathrm{ml} / \mathrm{l})\end{array}$ & $\begin{array}{l}\text { CHANNELA } \\
\text { ( } \% \text { fluor) }\end{array}$ & $\begin{array}{l}\text { CHANNELB } \\
\text { (\%light) }\end{array}$ \\
\hline 0.0 & E & 10.097 & 32.390 & 24.899 & 0.0000 & 6.025 & 12.7 & 82.9 \\
\hline 10.0 & & 8.558 & 32.667 & 25.360 & 0.0294 & 6.329 & 16.3 & 82.7 \\
\hline 20.0 & & 7.209 & 32.812 & 25.667 & 0.0534 & 6.486 & 7.2 & 87.2 \\
\hline 30.0 & & 5.477 & 32.967 & 26.009 & 0.0742 & 6.557 & 4.0 & 88.2 \\
\hline 40.0 & & 5.245 & 32.992 & 26.056 & 0.0935 & 6.398 & 3.7 & 87.6 \\
\hline 50.0 & & 5.141 & 33.008 & 26.081 & 0.1124 & 6.322 & 3.8 & 87.2 \\
\hline 60.0 & & 4.786 & 33.070 & 26.169 & 0.1309 & 6.296 & 3.5 & 87.8 \\
\hline 70.0 & & 4.658 & 33.107 & 26.212 & 0.1486 & 6.114 & 3.2 & 86.1 \\
\hline 80.0 & & 4.634 & 33.130 & 26.233 & 0.1662 & 6.089 & 3.5 & 85.2 \\
\hline 90.0 & & 4.586 & 33.145 & 26.250 & 0.1835 & 6.018 & 3.0 & 85.3 \\
\hline 100.0 & & 4.582 & 33.159 & 26.262 & 0.2008 & 5.991 & 2.9 & 84.7 \\
\hline 110.0 & & 4.577 & 33.171 & 26.272 & 0.2179 & 5.963 & 3.0 & 83.9 \\
\hline 120.0 & & 4.567 & 33.176 & 26.277 & 0.2350 & 5.951 & 2.8 & 84.6 \\
\hline 130.0 & & 4.548 & 33.183 & 26.284 & 0.2520 & 5.964 & 2.7 & 85.5 \\
\hline 140.0 & & 4.542 & 33.193 & 26.293 & 0.2690 & 5.922 & 2.8 & 85.5 \\
\hline 150.0 & & 4.563 & 33.214 & 26.307 & 0.2859 & 5.905 & 2.7 & 85.8 \\
\hline 160.0 & & 4.980 & 33.408 & 26.415 & 0.3023 & 5.676 & 2.7 & 83.2 \\
\hline 162.0 & & 5.101 & 33.459 & 26.442 & 0.3055 & 5.614 & 2.7 & 82.9 \\
\hline
\end{tabular}
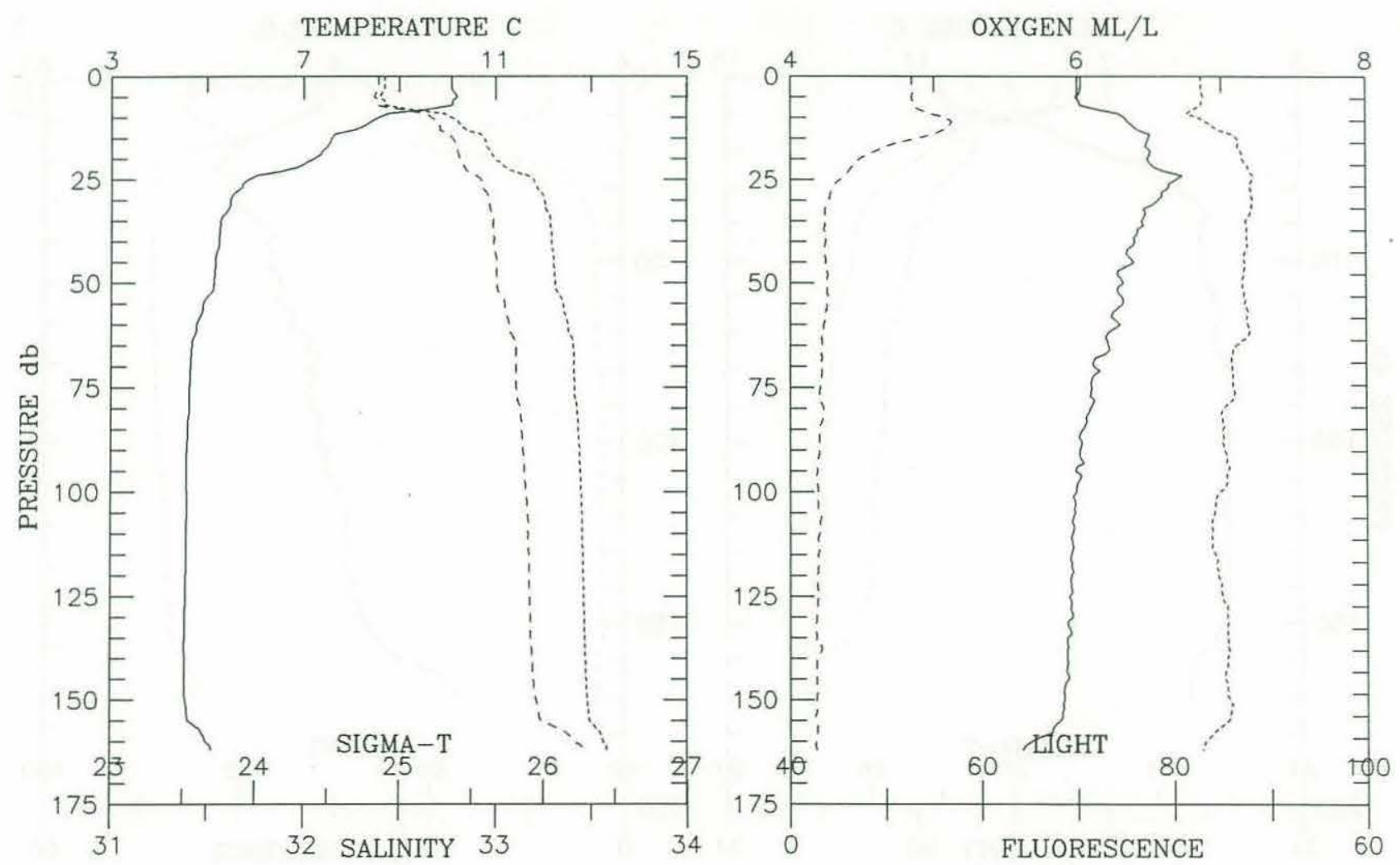


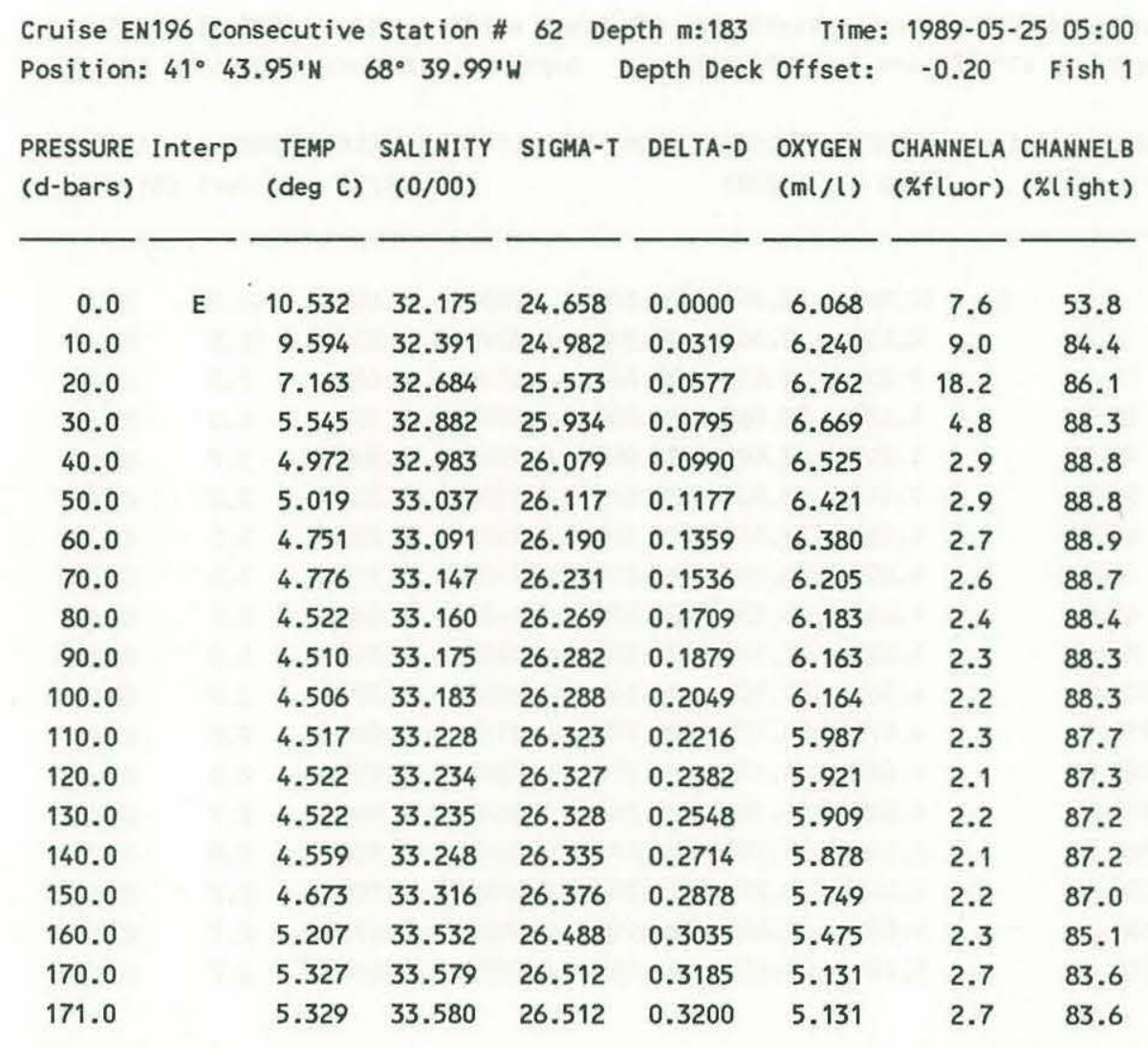
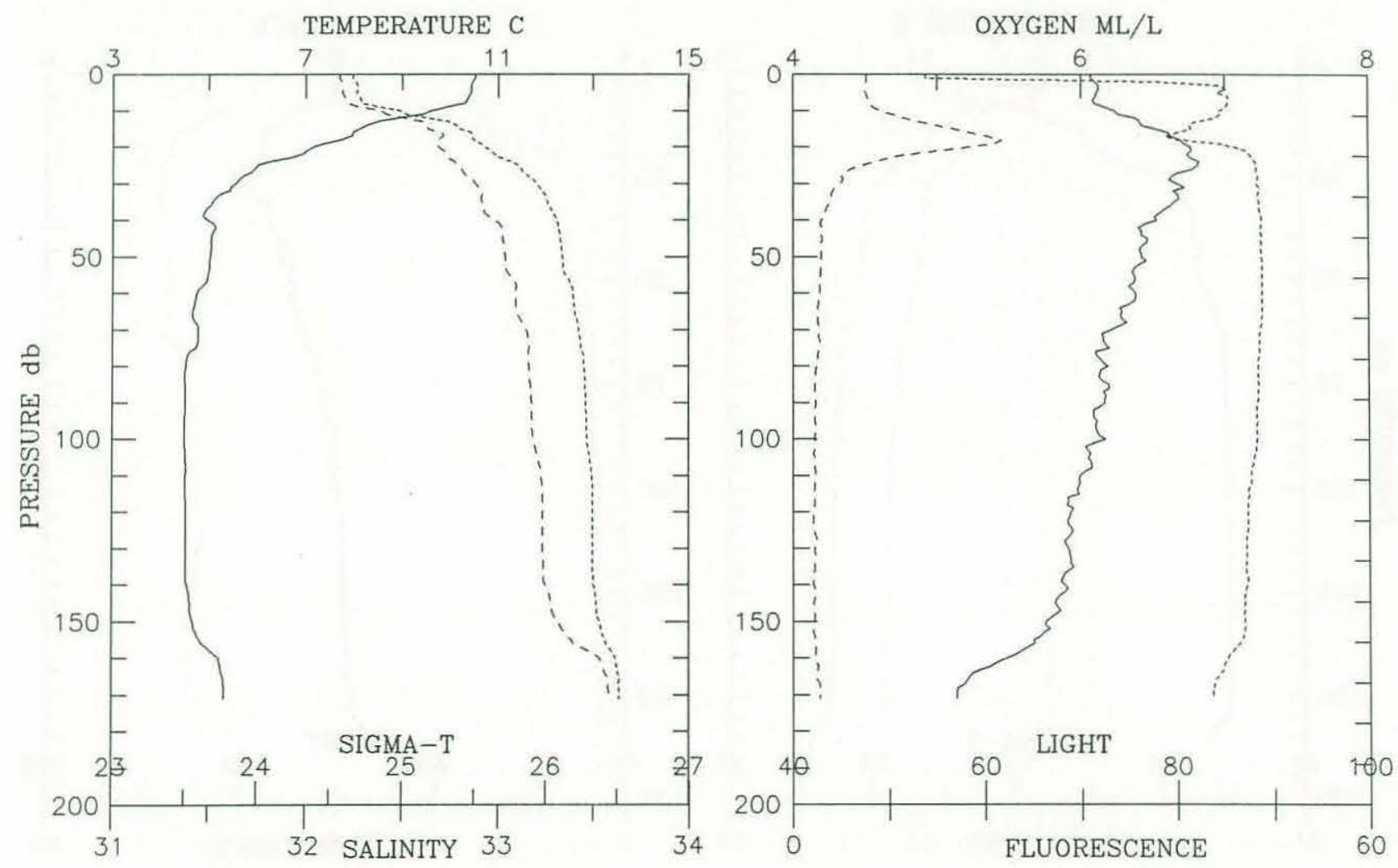


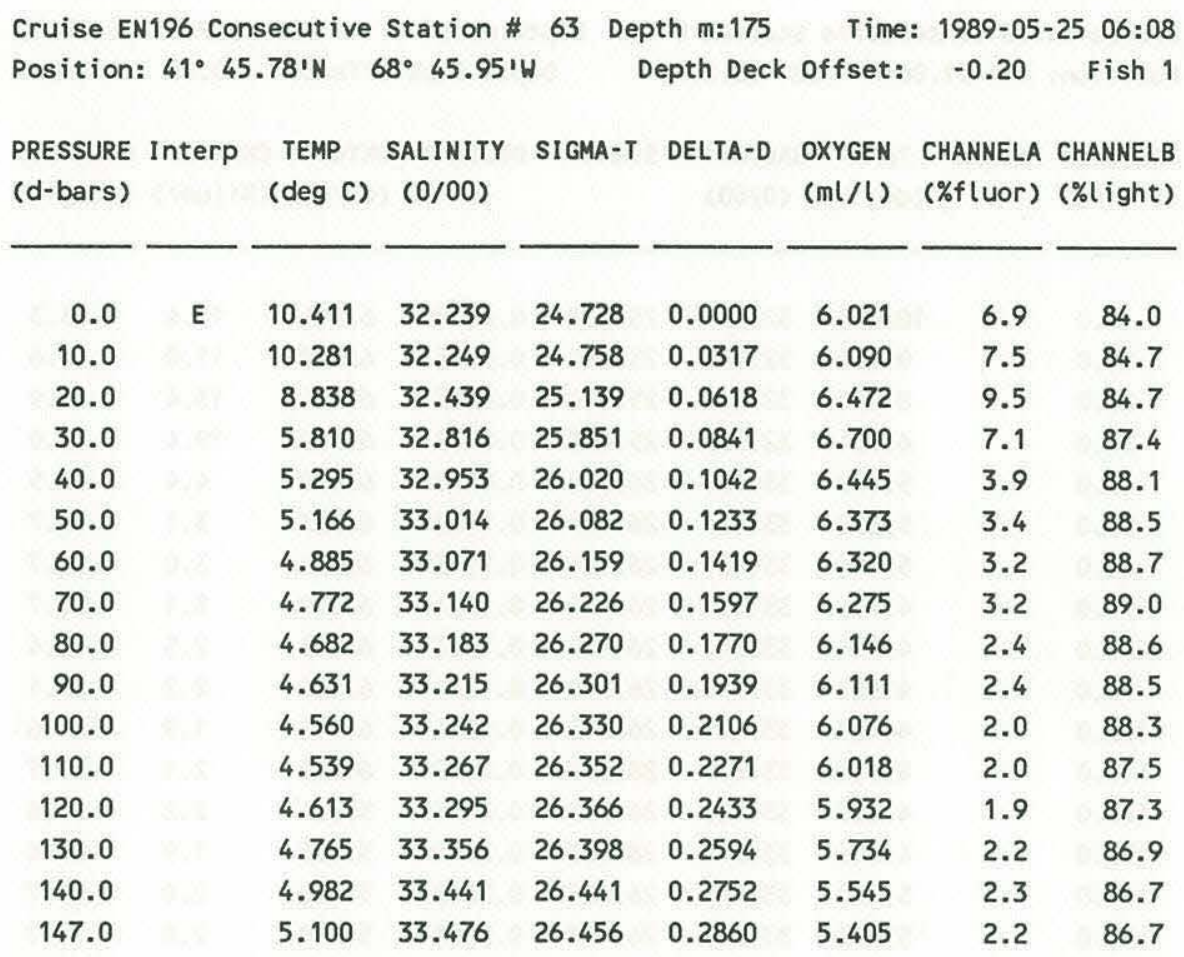
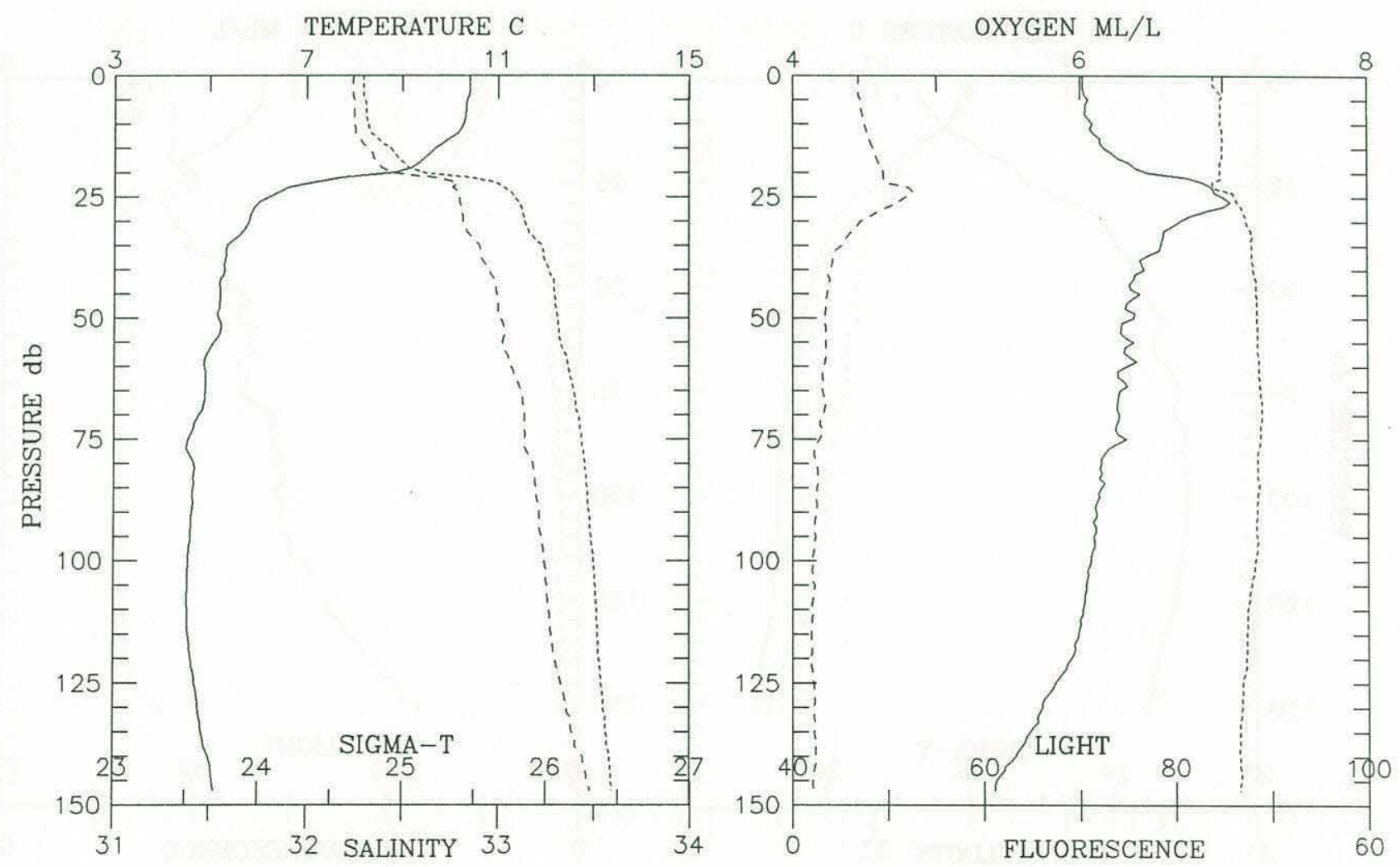


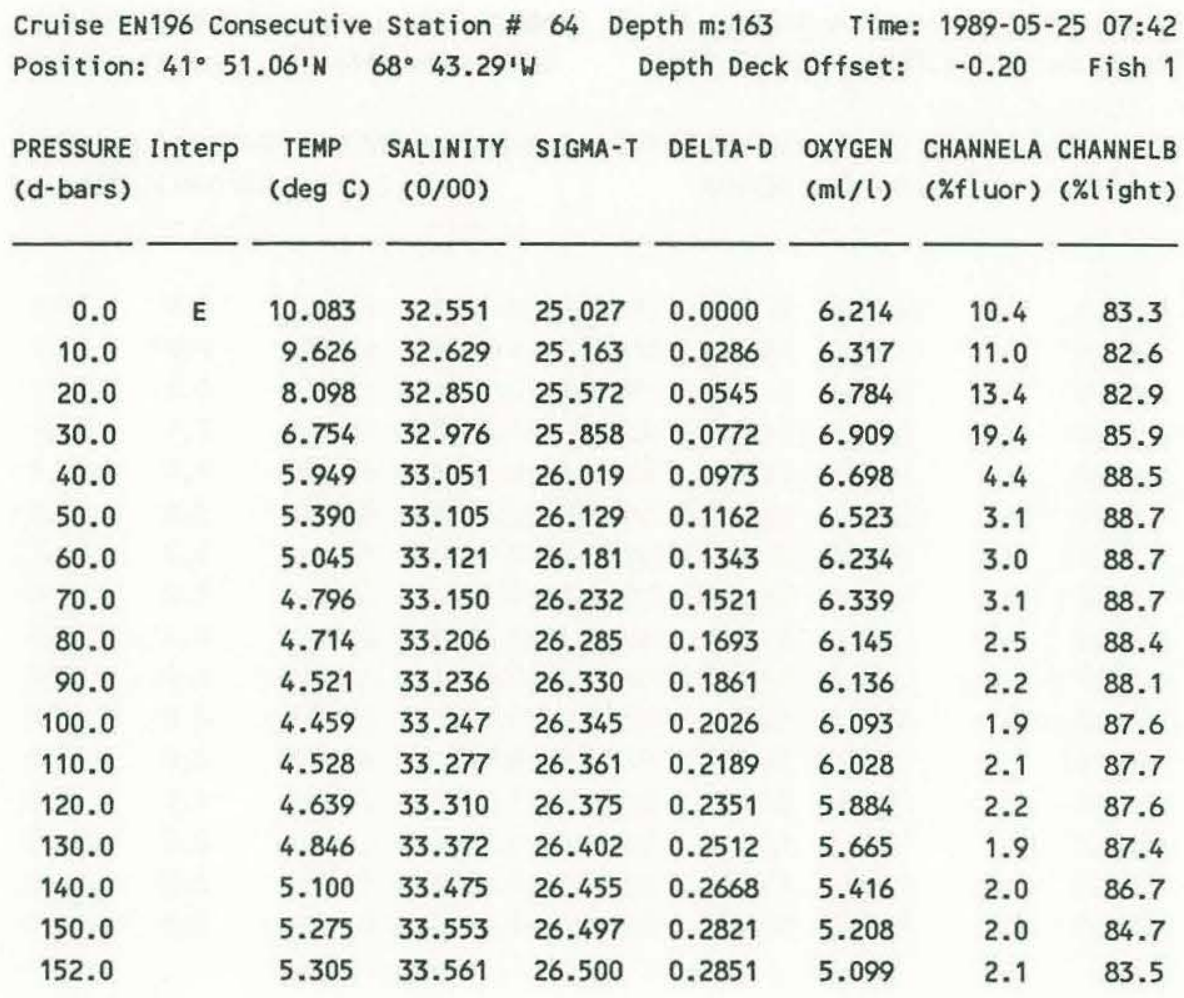
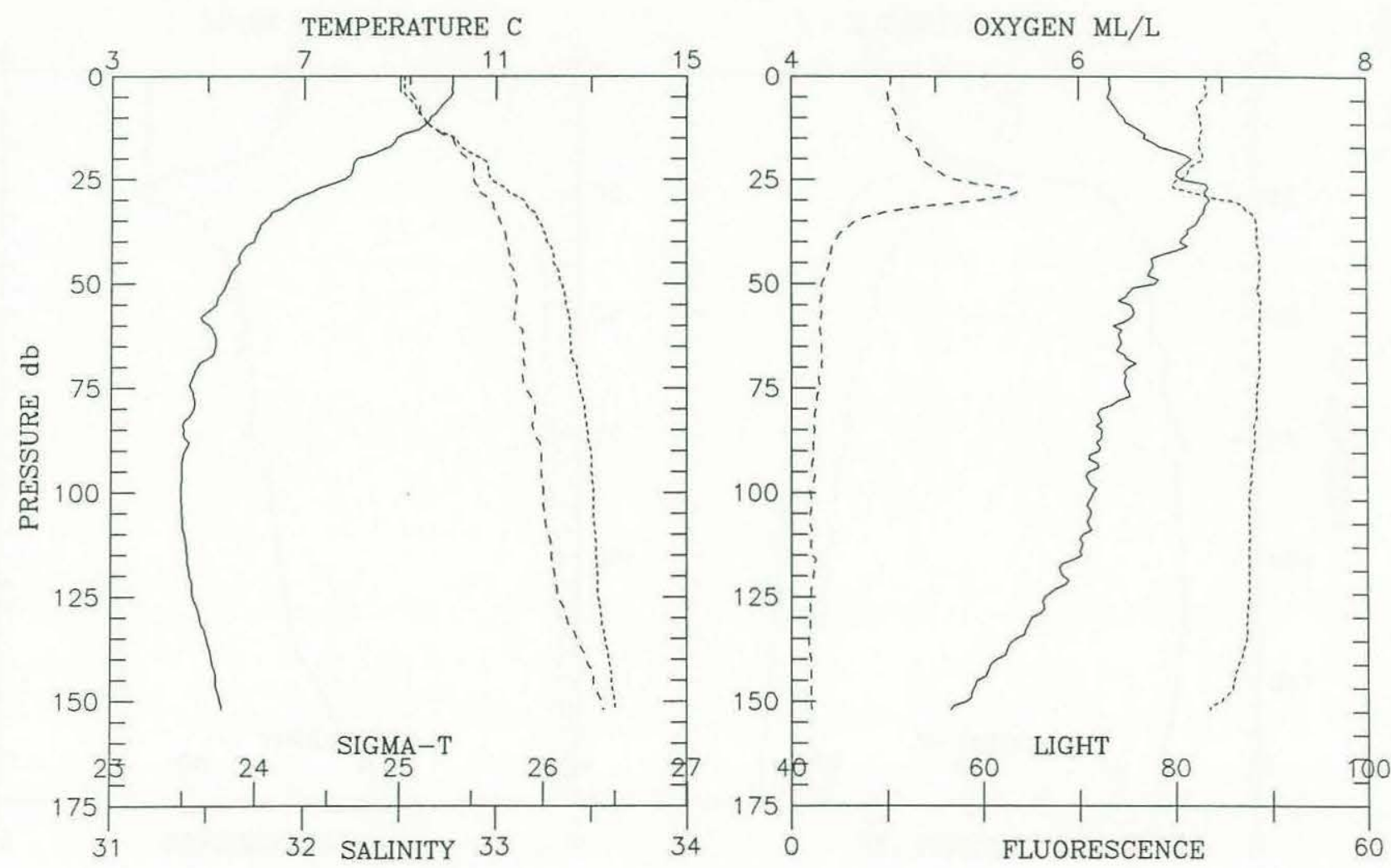
Cruise EN196 Consecutive Station \# 65 Depth m:165 Time: 1989-05-25 08:53 Position: $41^{\circ} 49.47$ ' $\mathrm{N} 68^{\circ} 37.07 \cdot \mathrm{W}$ Depth Deck offset: $-0.13 \quad$ Fish 1

\begin{tabular}{|c|c|c|c|c|c|c|c|c|}
\hline $\begin{array}{l}\text { PRESSURE } \\
\text { (d-bars) }\end{array}$ & Interp & $\begin{array}{c}\text { TEMP } \\
(\operatorname{deg} C)\end{array}$ & $\begin{array}{c}\text { SALINITY } \\
(0 / 00)\end{array}$ & SIGMA-T & DELTA-D & $\begin{array}{l}\text { OXYGEN } \\
(\mathrm{ml} / \mathrm{l})\end{array}$ & $\begin{array}{l}\text { CHANNELA } \\
\text { ( } \% \text { fluor) }\end{array}$ & $\begin{array}{l}\text { CHANNELB } \\
\text { (\%light) }\end{array}$ \\
\hline 0.0 & E & 10.119 & 32.413 & 24.913 & 0.0000 & 6.101 & 9.4 & 83.7 \\
\hline 10.0 & & 9.700 & 32.608 & 25.135 & 0.0295 & 6.251 & 10.7 & 81.5 \\
\hline 20.0 & & 7.608 & 32.894 & 25.677 & 0.0550 & 6.719 & 17.9 & 81.1 \\
\hline 30.0 & & 5.817 & 32.987 & 25.985 & 0.0763 & 6.740 & 5.4 & 87.8 \\
\hline 40.0 & & 5.550 & 33.087 & 26.095 & 0.0955 & 6.460 & 3.5 & 89.0 \\
\hline 50.0 & & 4.998 & 33.118 & 26.184 & 0.1138 & 6.277 & 2.9 & 88.8 \\
\hline 60.0 & & 4.592 & 33.143 & 26.248 & 0.1314 & 6.252 & 2.3 & 88.6 \\
\hline 70.0 & & 4.623 & 33.197 & 26.287 & 0.1485 & 6.170 & 2.3 & 88.1 \\
\hline 80.0 & & 4.788 & 33.255 & 26.315 & 0.1653 & 5.852 & 2.1 & 88.0 \\
\hline 90.0 & & 4.765 & 33.286 & 26.343 & 0.1819 & 5.840 & 1.9 & 88.1 \\
\hline 100.0 & & 4.737 & 33.302 & 26.358 & 0.1982 & 5.766 & 1.9 & 87.9 \\
\hline 110.0 & & 4.761 & 33.330 & 26.378 & 0.2144 & 5.750 & 2.1 & 87.7 \\
\hline 120.0 & & 4.803 & 33.352 & 26.391 & 0.2304 & 5.675 & 1.8 & 87.6 \\
\hline 130.0 & & 4.944 & 33.396 & 26.410 & 0.2463 & 5.530 & 1.7 & 86.6 \\
\hline 140.0 & & 5.053 & 33.452 & 26.442 & 0.2620 & 5.456 & 1.7 & 86.9 \\
\hline 150.0 & & 5.367 & 33.590 & 26.515 & 0.2772 & 5.174 & 1.9 & 84.5 \\
\hline 160.0 & & 5.640 & 33.703 & 26.572 & 0.2918 & 4.845 & 2.2 & 79.4 \\
\hline 161.0 & & 5.646 & 33.705 & 26.573 & 0.2932 & 4.827 & 2.1 & 79.3 \\
\hline
\end{tabular}
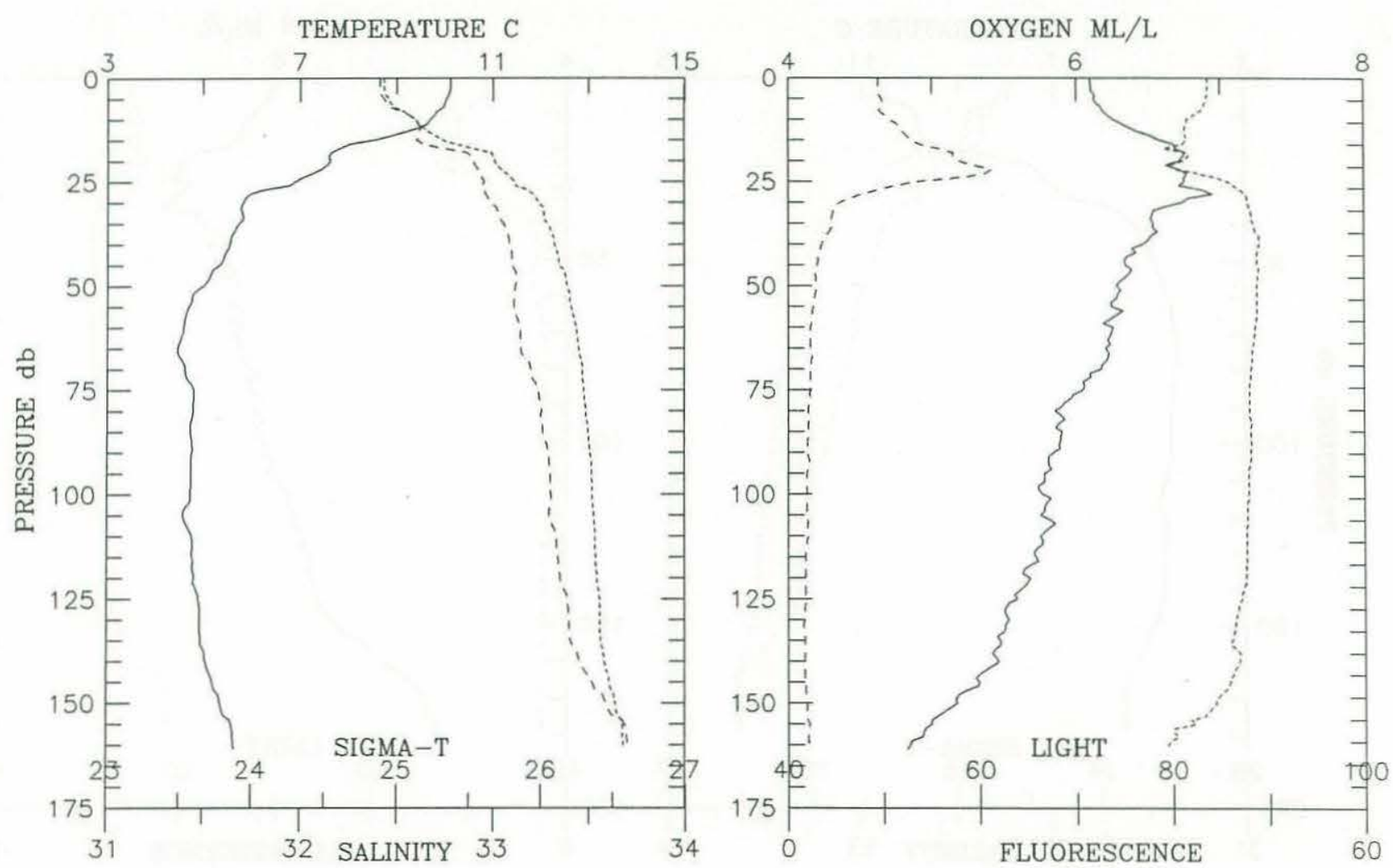


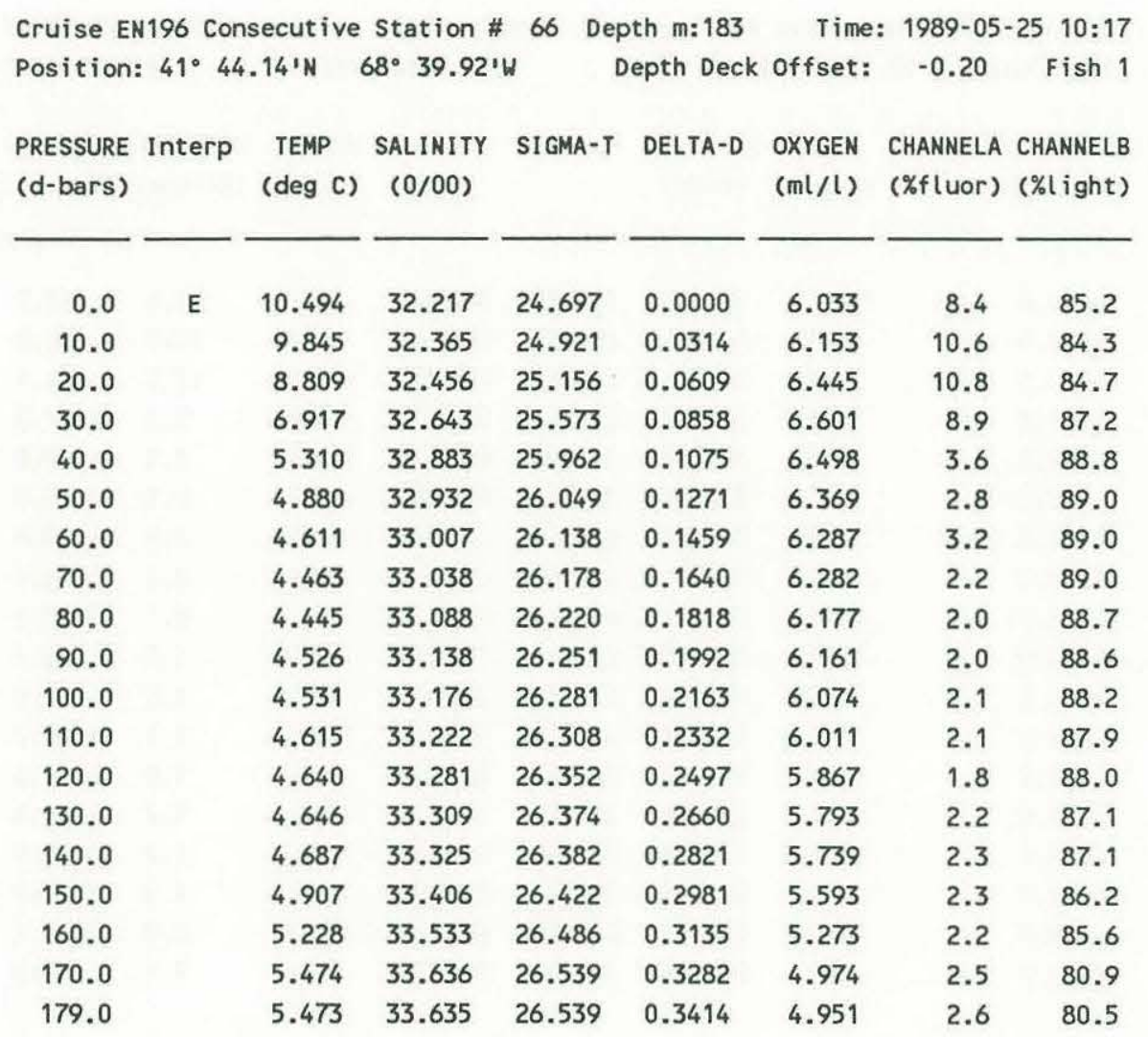
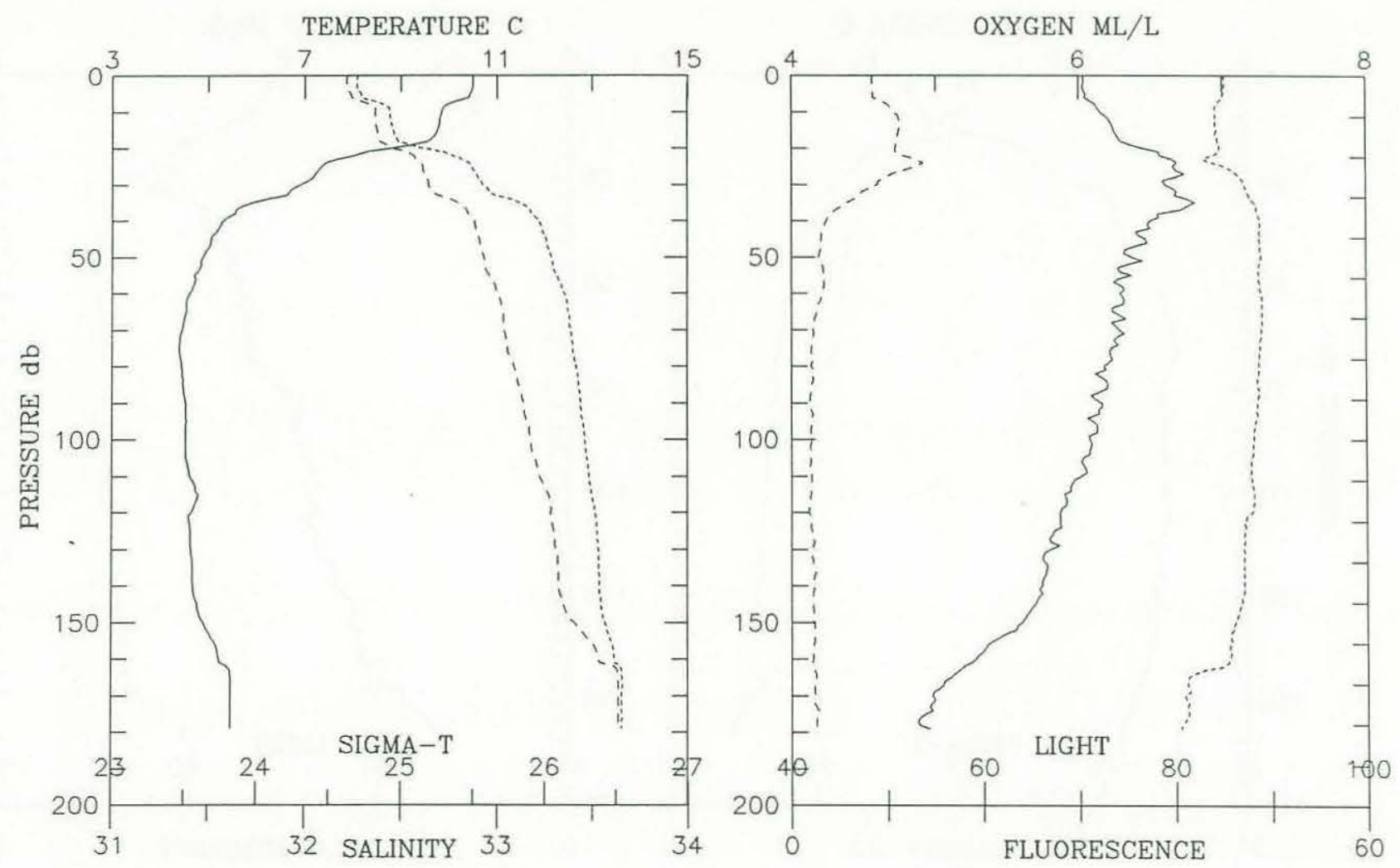


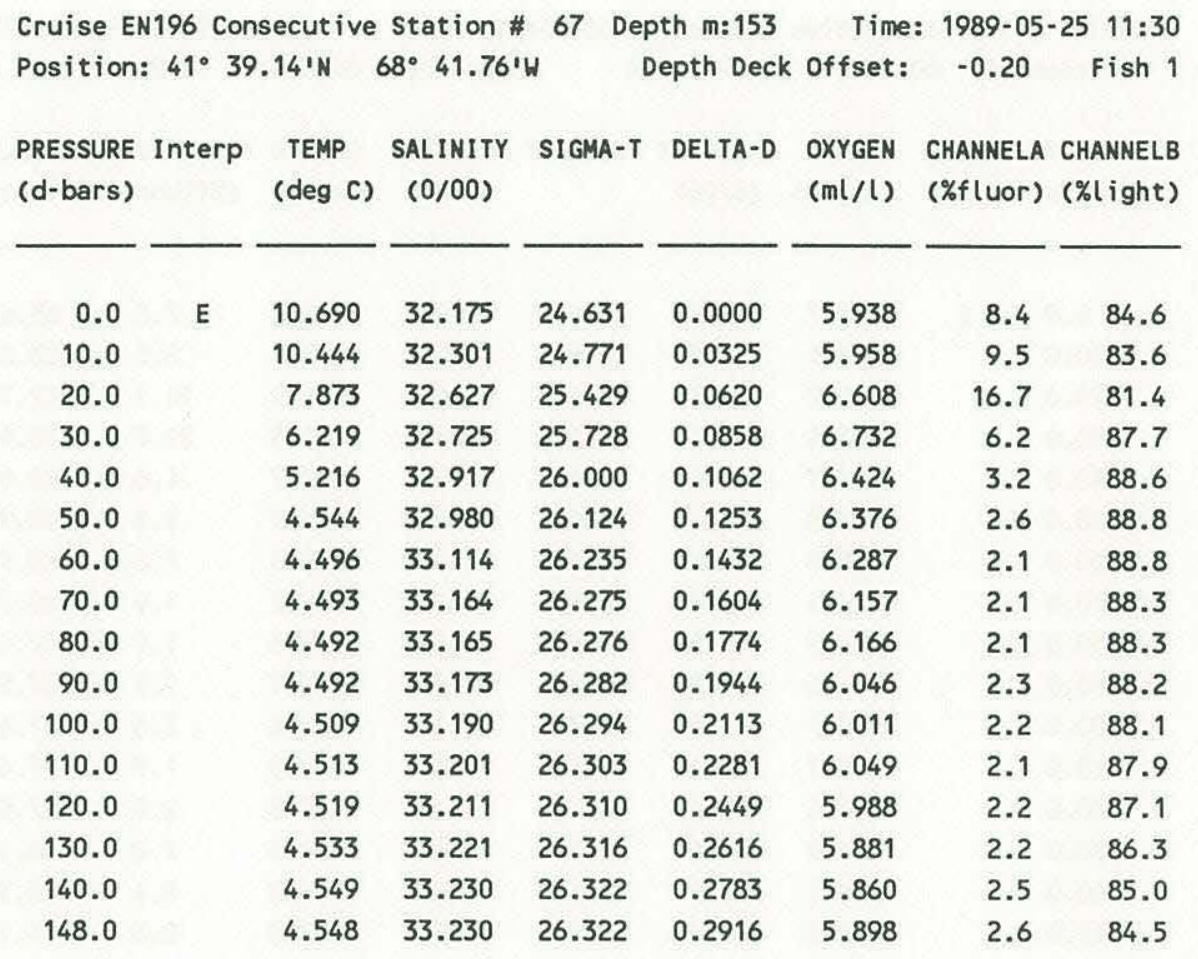
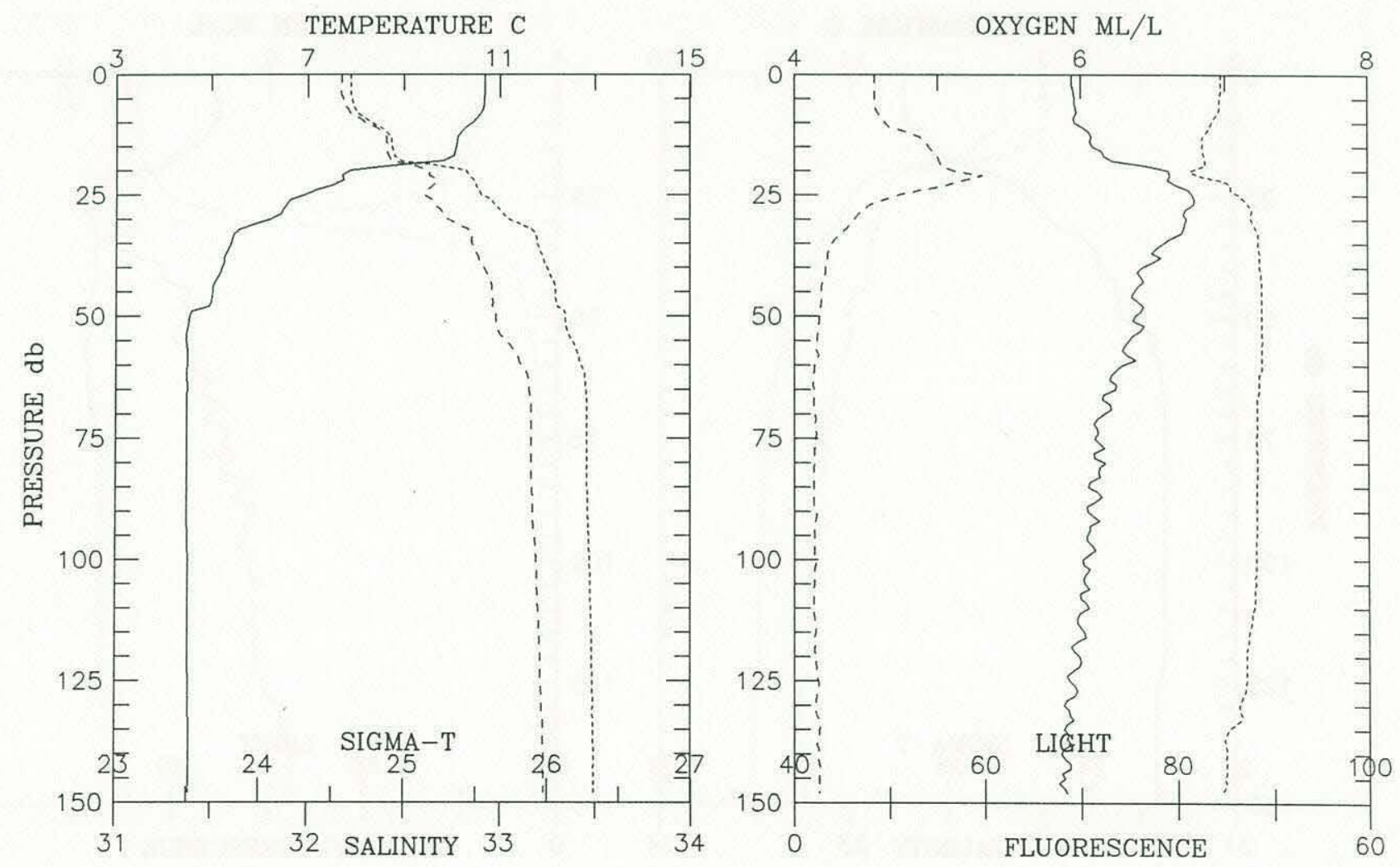


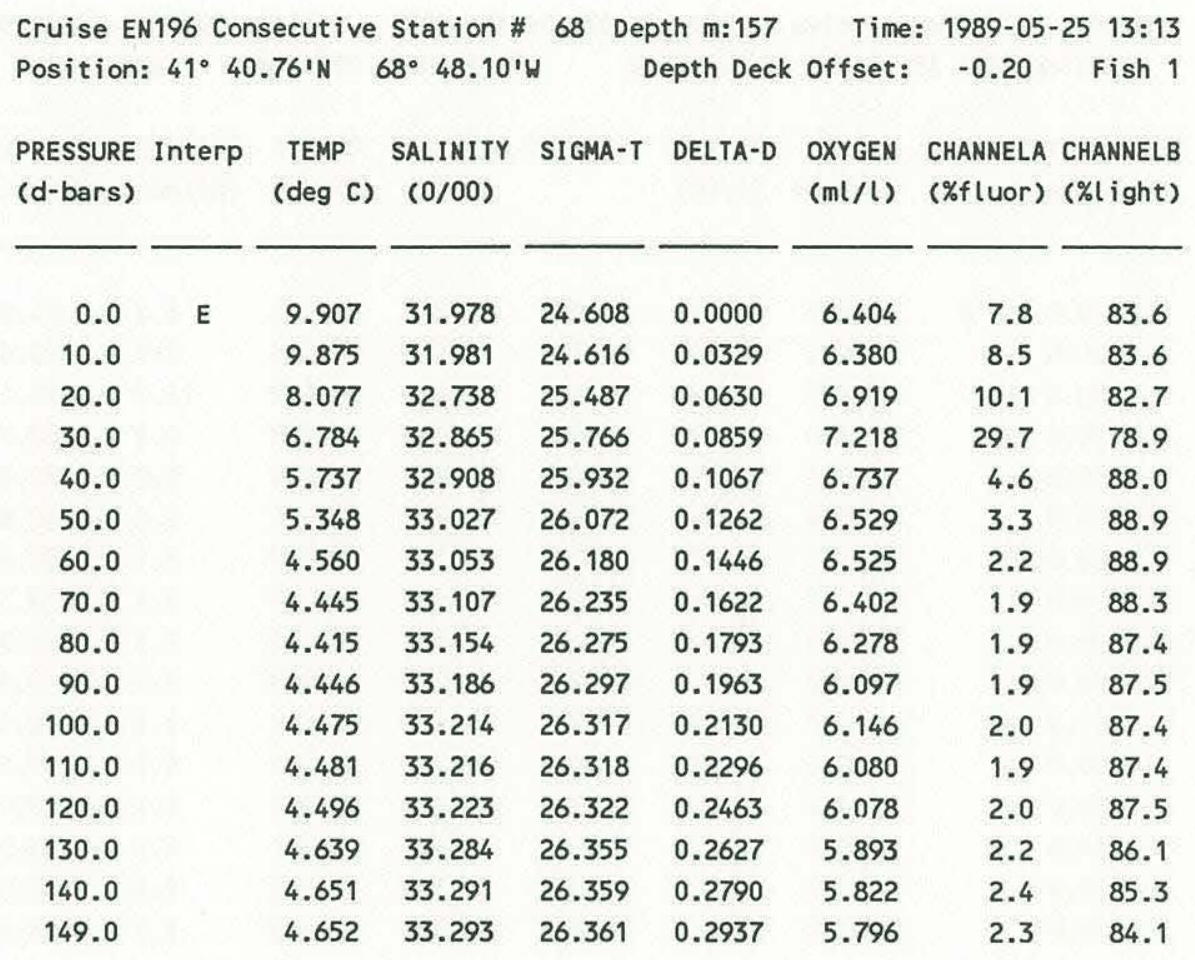
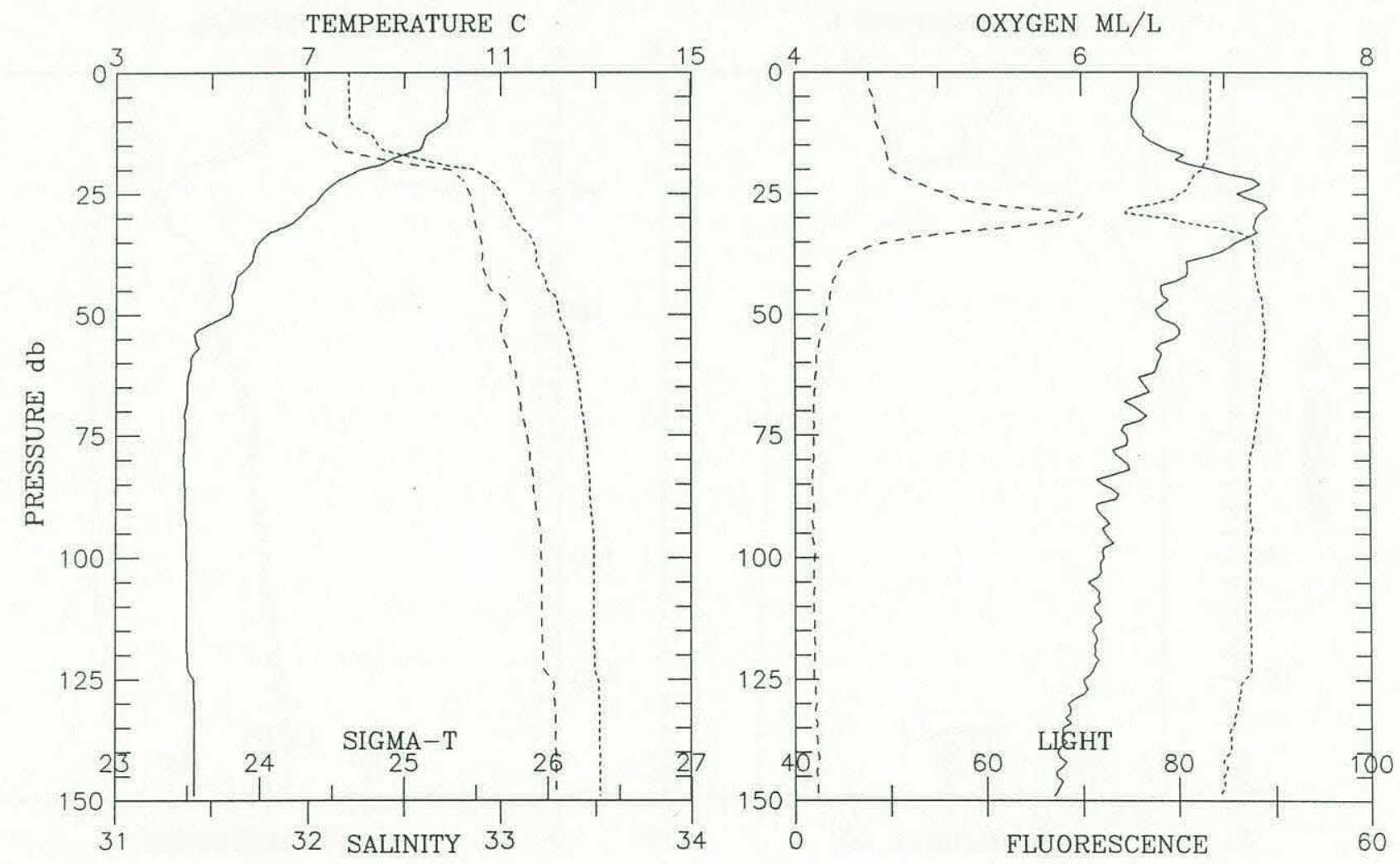


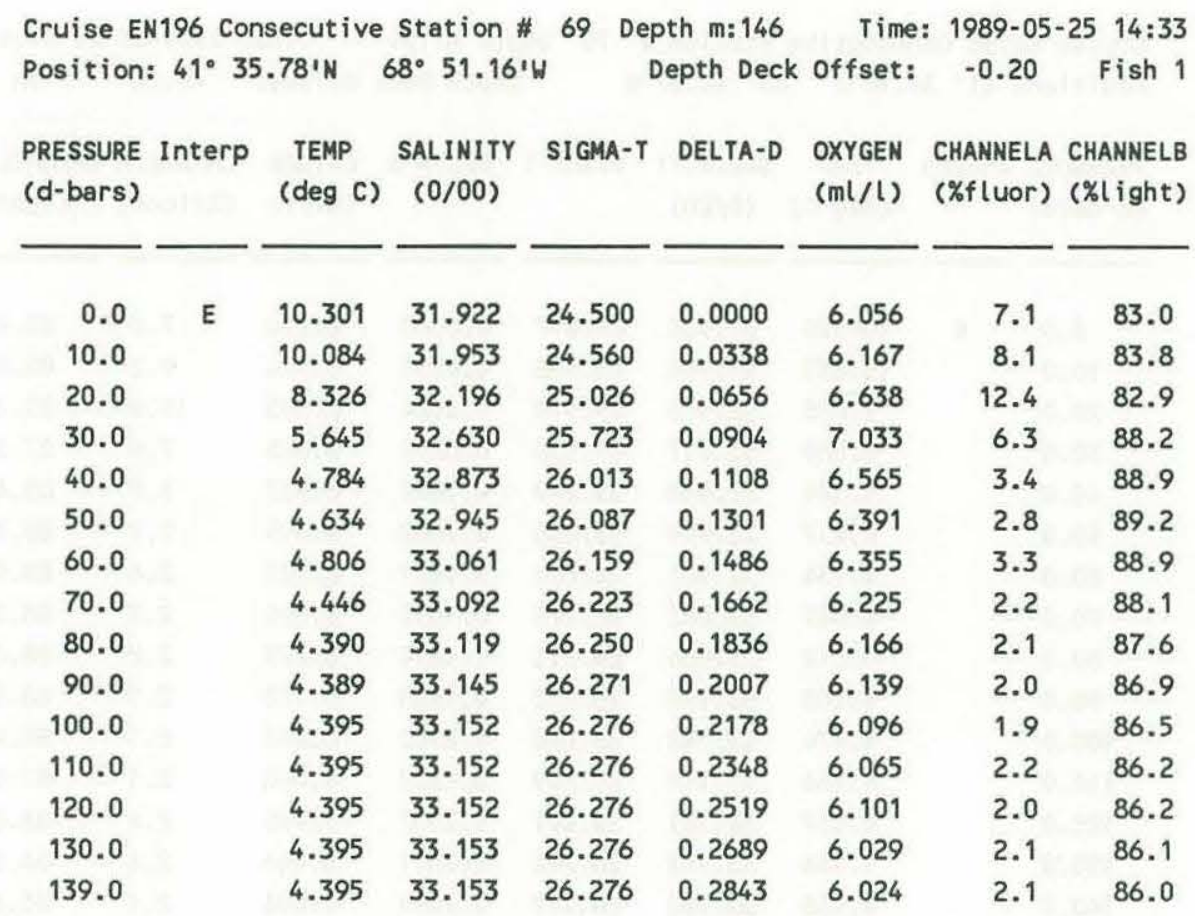
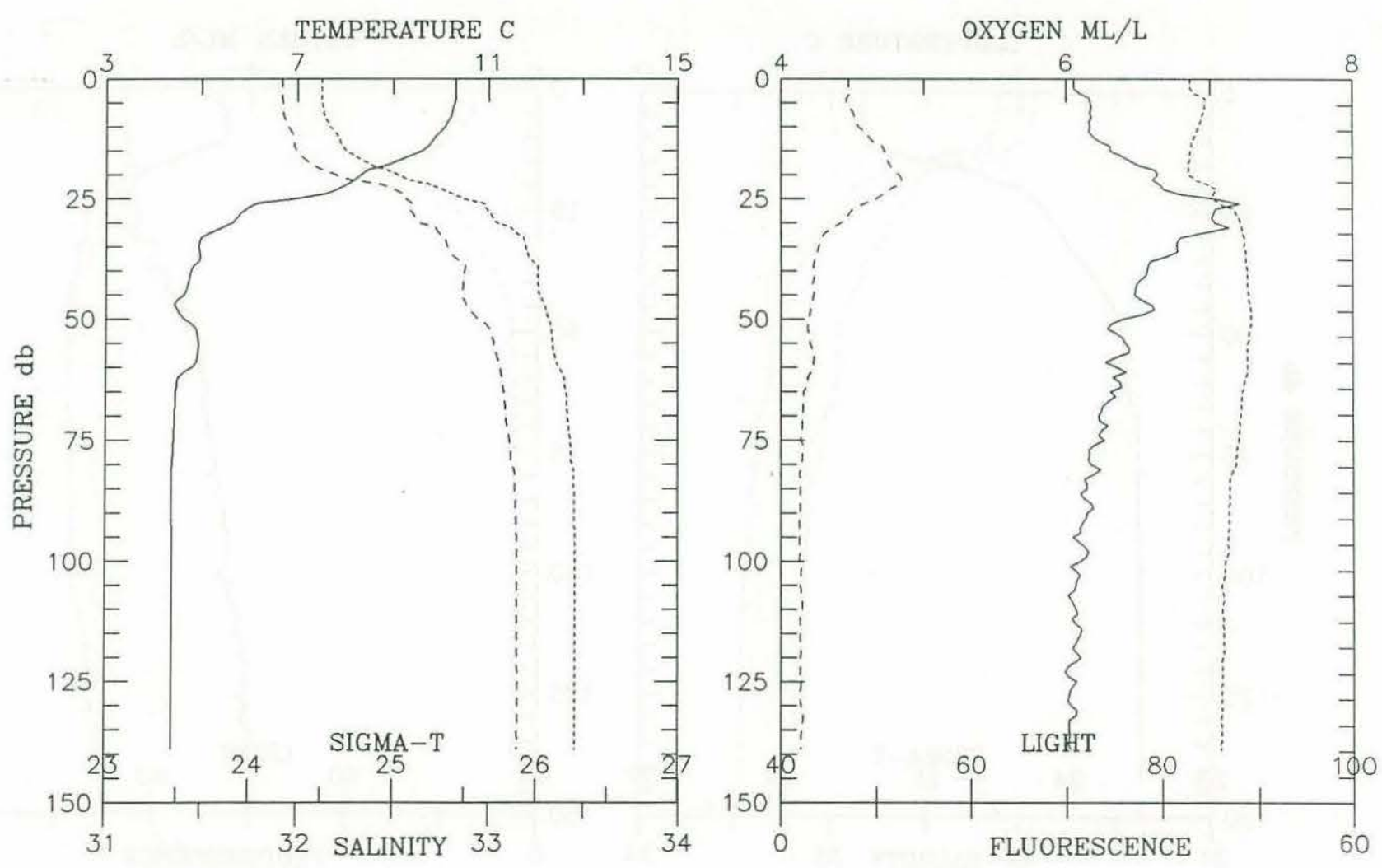


\begin{tabular}{|c|c|c|c|c|c|c|c|c|}
\hline $\begin{array}{l}\text { Cruise EN } \\
\text { Position: }\end{array}$ & $\begin{array}{l}\text { N196 Con } \\
=41^{\circ} 34\end{array}$ & $\begin{array}{l}.18^{\prime} \mathrm{N} \\
6\end{array}$ & $\begin{array}{l}\text { Station \# } \\
68^{\circ} 44.67^{\prime}\end{array}$ & $\begin{array}{r}\text { Dep } \\
\text { D }\end{array}$ & $\begin{array}{l}\text { th } \mathrm{m}: 154 \\
\text { epth Deck }\end{array}$ & $\begin{array}{r}\text { Time } \\
\text { offset: }\end{array}$ & $\begin{array}{c}1989-05- \\
-0.20\end{array}$ & $\begin{array}{r}-25 \text { 15:41 } \\
\text { Fish } 1\end{array}$ \\
\hline $\begin{array}{l}\text { PRESSURE } \\
\text { (d-bars) }\end{array}$ & Interp & $\begin{array}{l}\text { TEMP } \\
(\operatorname{deg} C)\end{array}$ & $\begin{array}{l}\text { SALINITY } \\
(0 / 00)\end{array}$ & SIGMA-T & DELTA-D & $\begin{array}{l}\text { OXYGEN } \\
(m l / l)\end{array}$ & $\begin{array}{l}\text { CHANNELA } \\
\text { (\%fluor) }\end{array}$ & $\begin{array}{l}\text { CHANNELB } \\
\text { (\%tight) }\end{array}$ \\
\hline 0.0 & E & 10.326 & 31.923 & 24.497 & 0.0000 & 6.210 & 7.0 & 83.2 \\
\hline 10.0 & & 10.233 & 31.992 & 24.566 & 0.0338 & 6.174 & 9.2 & 83.0 \\
\hline 20.0 & & 7.005 & 32.578 & 25.510 & 0.0624 & 6.803 & 15.9 & 85.2 \\
\hline 30.0 & & 6.089 & 32.717 & 25.738 & 0.0856 & 6.865 & 7.0 & 87.3 \\
\hline 40.0 & & 5.324 & 32.868 & 25.949 & 0.1067 & 6.557 & 3.9 & 88.4 \\
\hline 50.0 & & 4.857 & 32.950 & 26.066 & 0.1262 & 6.395 & 2.7 & 88.7 \\
\hline 60.0 & & 4.734 & 32.962 & 26.089 & 0.1451 & 6.327 & 2.4 & 88.9 \\
\hline 70.0 & & 4.549 & 33.043 & 26.173 & 0.1635 & 6.295 & 2.3 & 88.7 \\
\hline 80.0 & & 4.512 & 33.086 & 26.211 & 0.1814 & 6.293 & 2.2 & 88.6 \\
\hline 90.0 & & 4.508 & 33.112 & 26.232 & 0.1988 & 6.172 & 2.1 & 88.5 \\
\hline 100.0 & & 4.474 & 33.142 & 26.260 & 0.2162 & 6.201 & 2.1 & 88.4 \\
\hline 110.0 & & 4.466 & 33.178 & 26.289 & 0.2332 & 6.068 & 2.1 & 87.5 \\
\hline 120.0 & & 4.467 & 33.181 & 26.291 & 0.2502 & 5.995 & 2.4 & 86.8 \\
\hline 130.0 & & 4.468 & 33.182 & 26.292 & 0.2671 & 6.046 & 2.4 & 86.3 \\
\hline 140.0 & & 4.468 & 33.182 & 26.292 & 0.2840 & 6.004 & 2.4 & 85.6 \\
\hline 142.0 & & 4.468 & 33.182 & 26.292 & 0.2874 & 6.003 & 2.4 & 85.7 \\
\hline
\end{tabular}
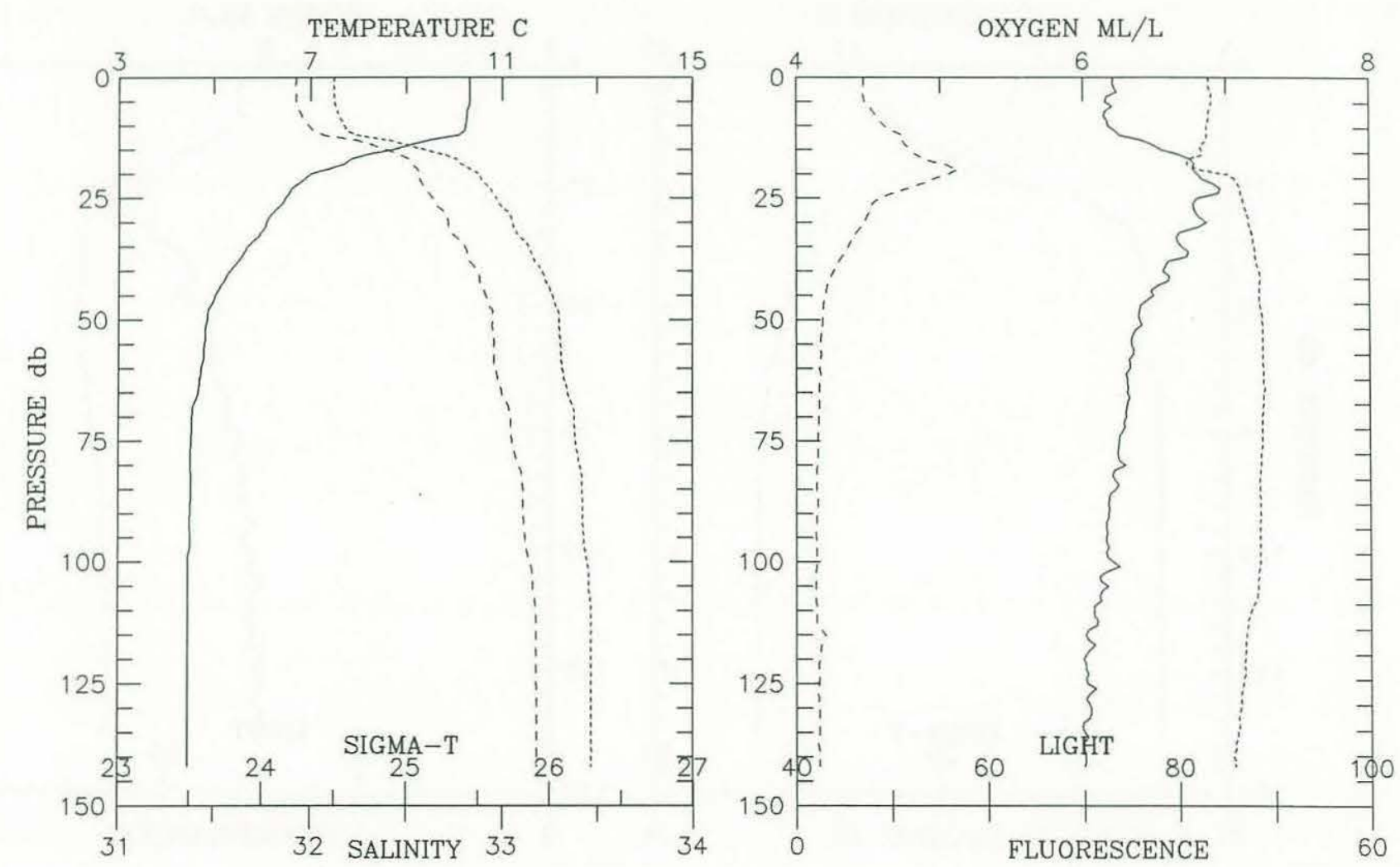


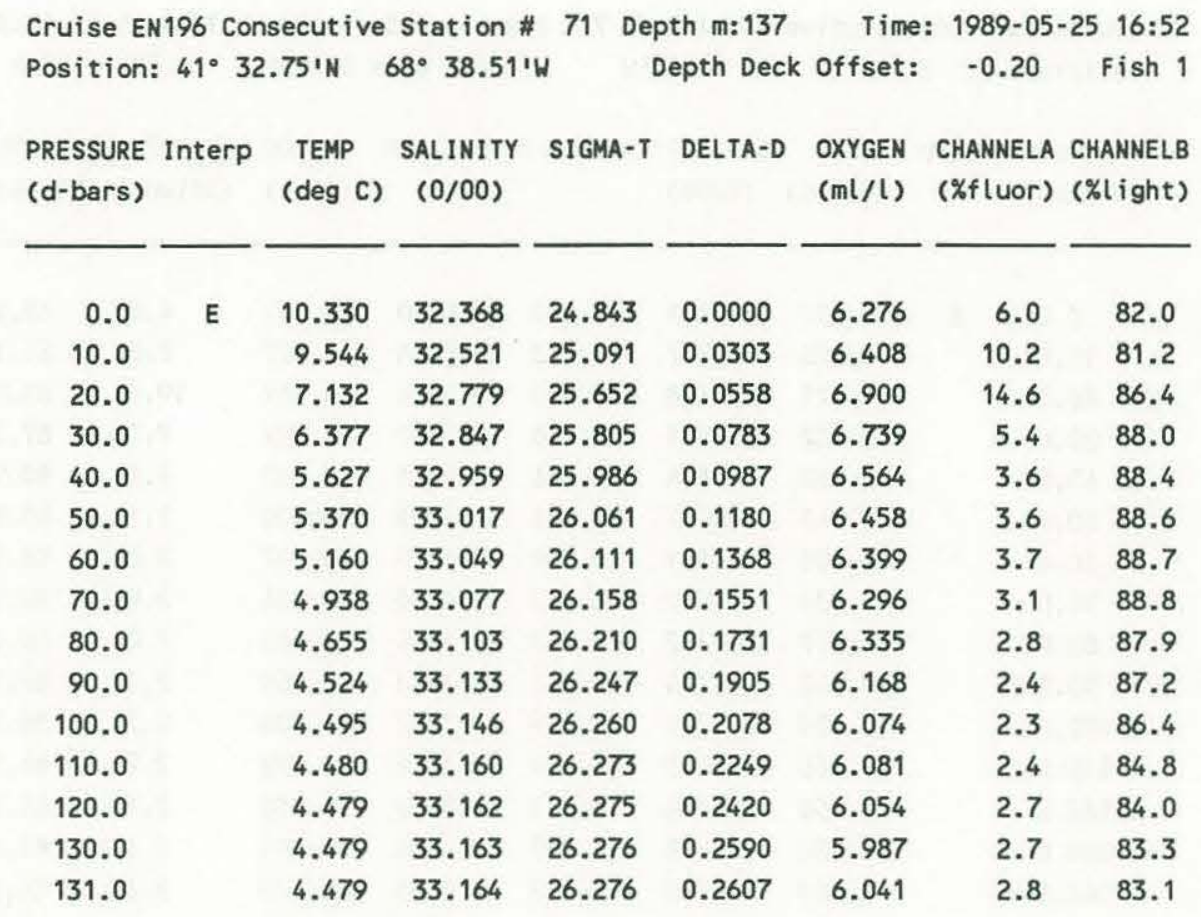
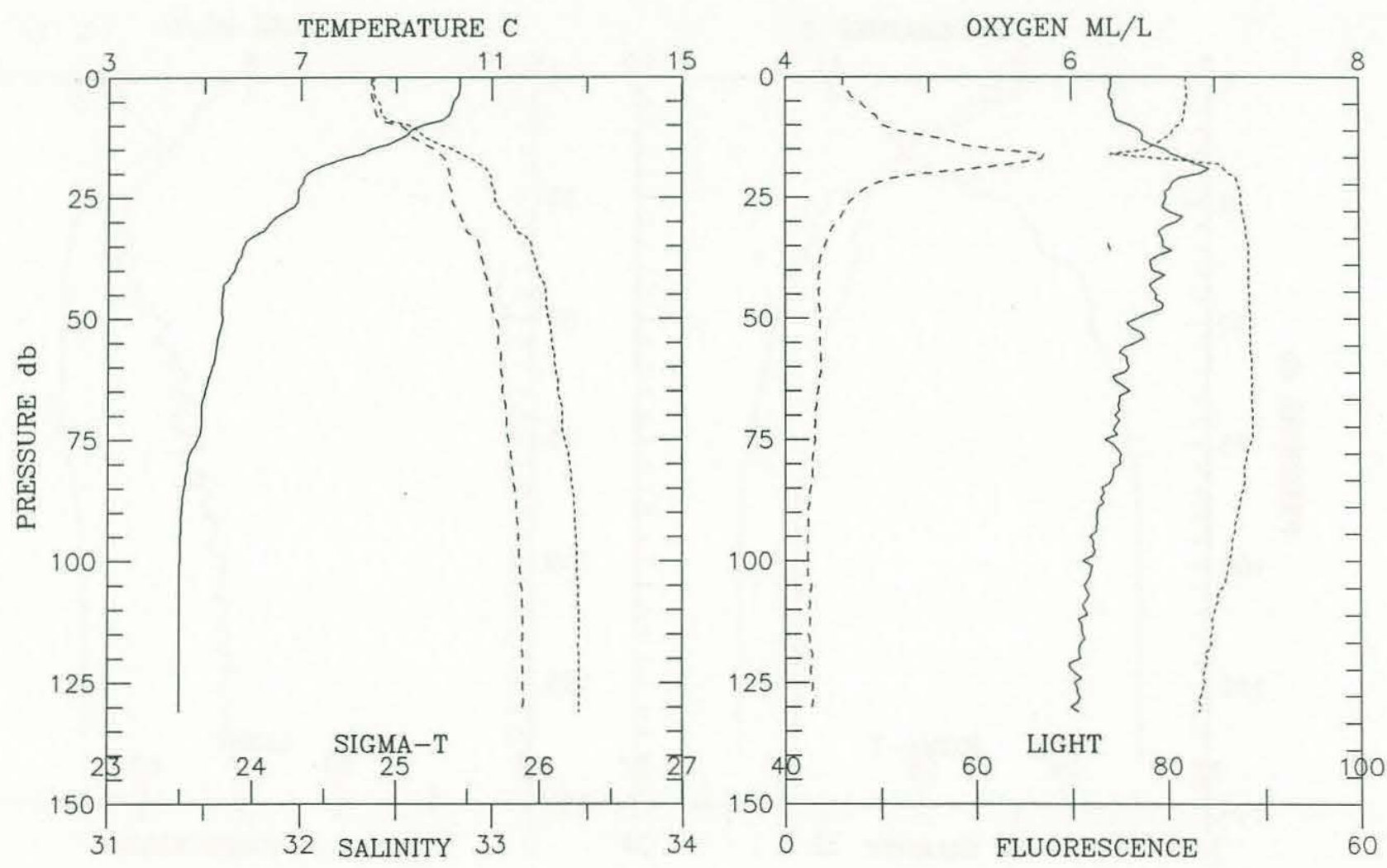


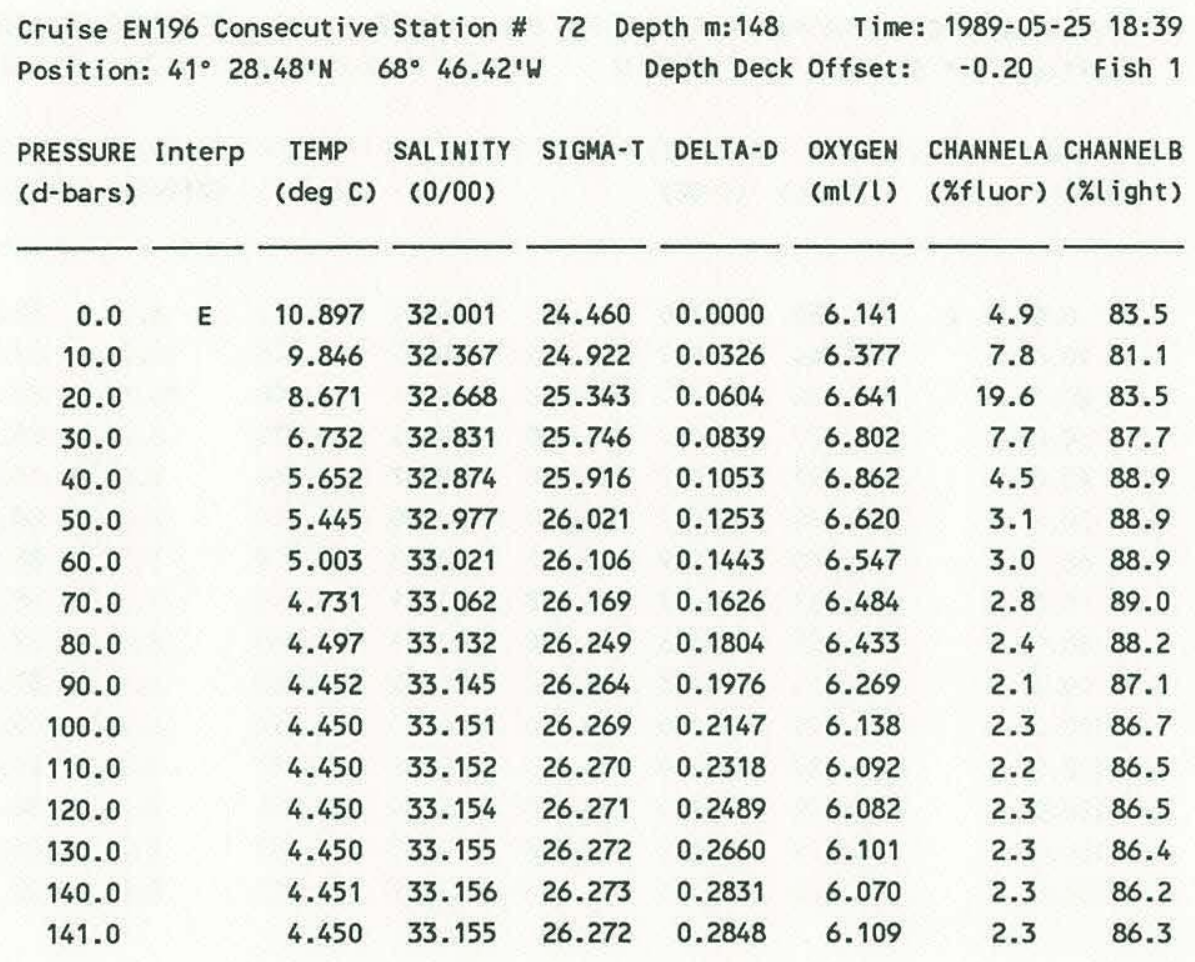
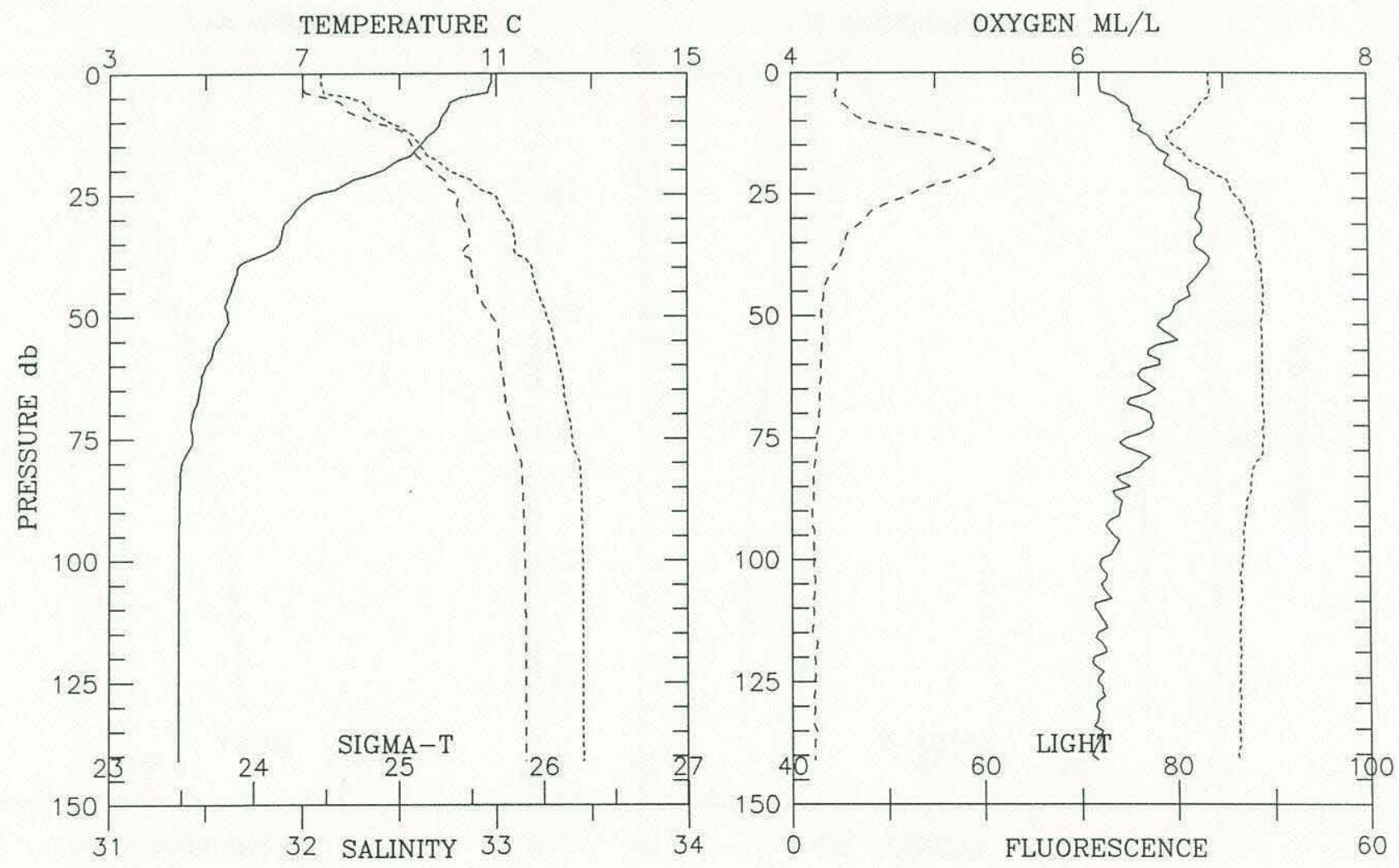


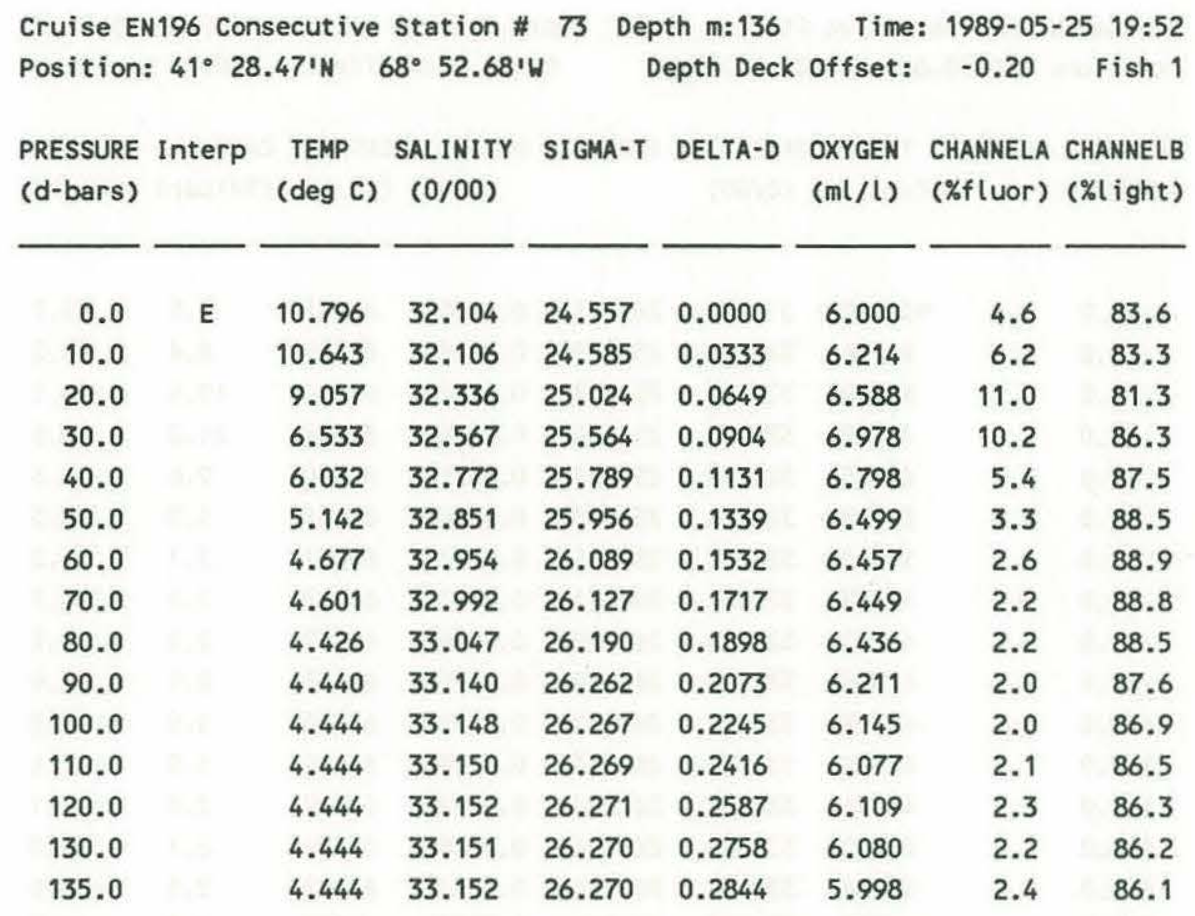
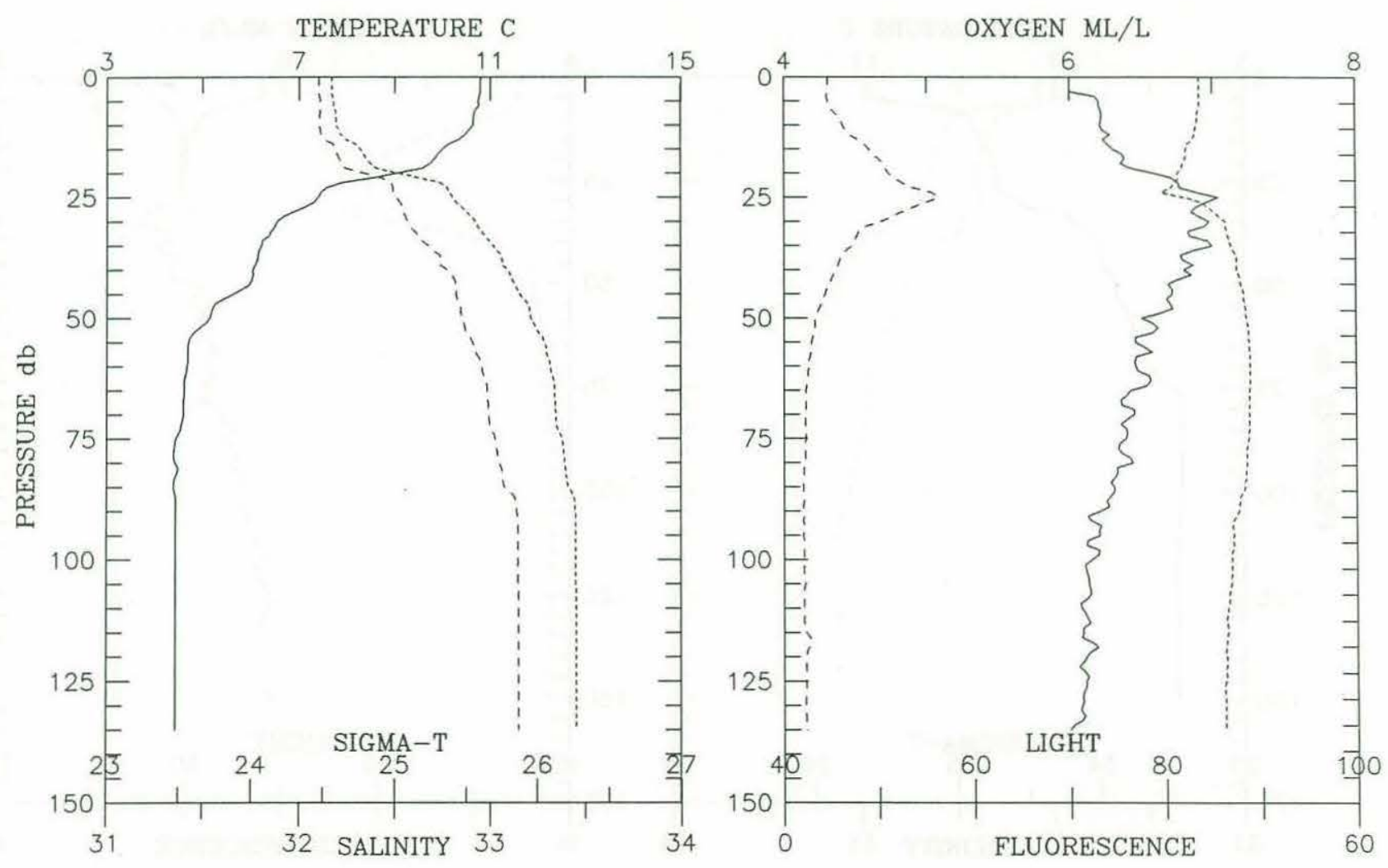


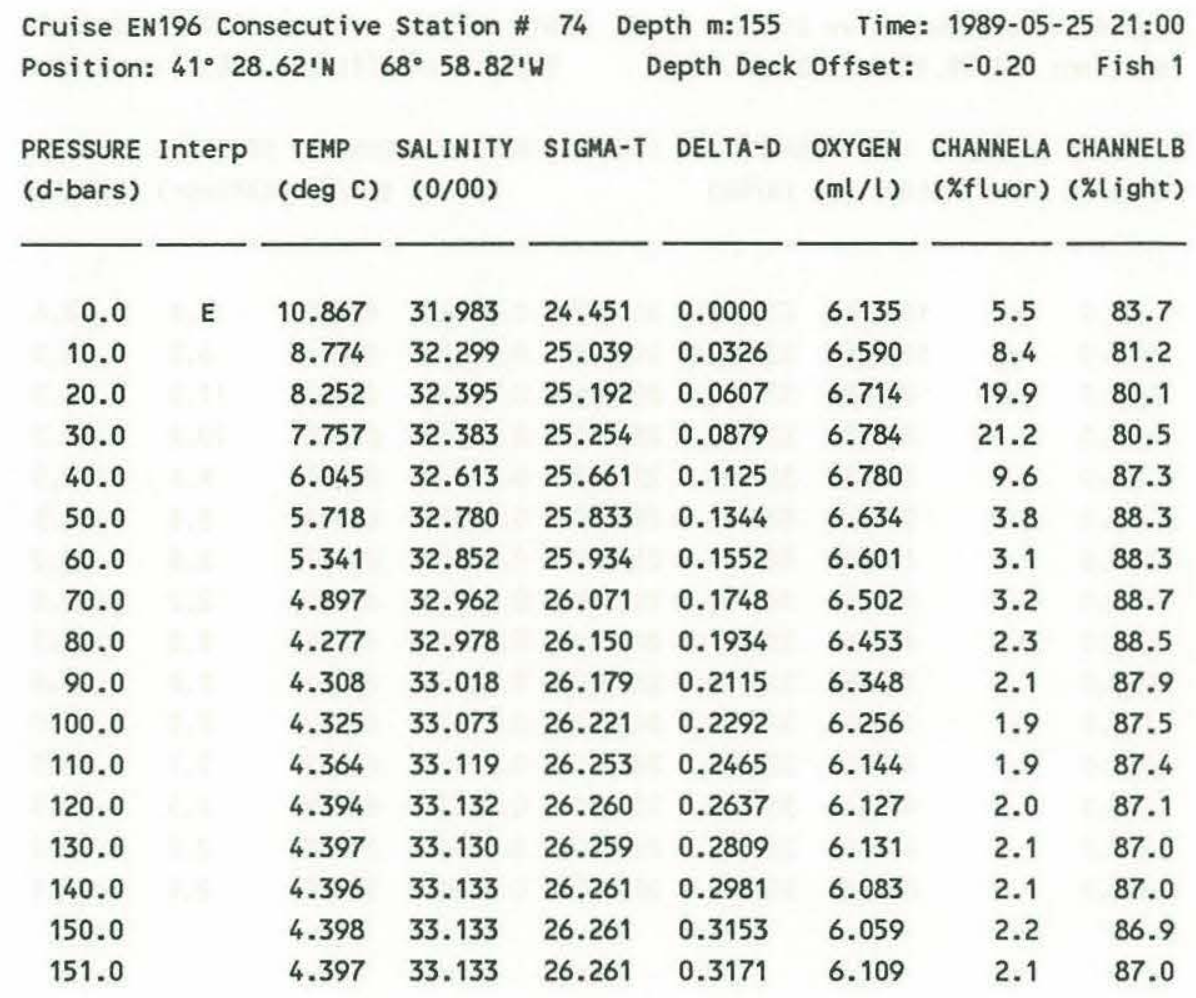
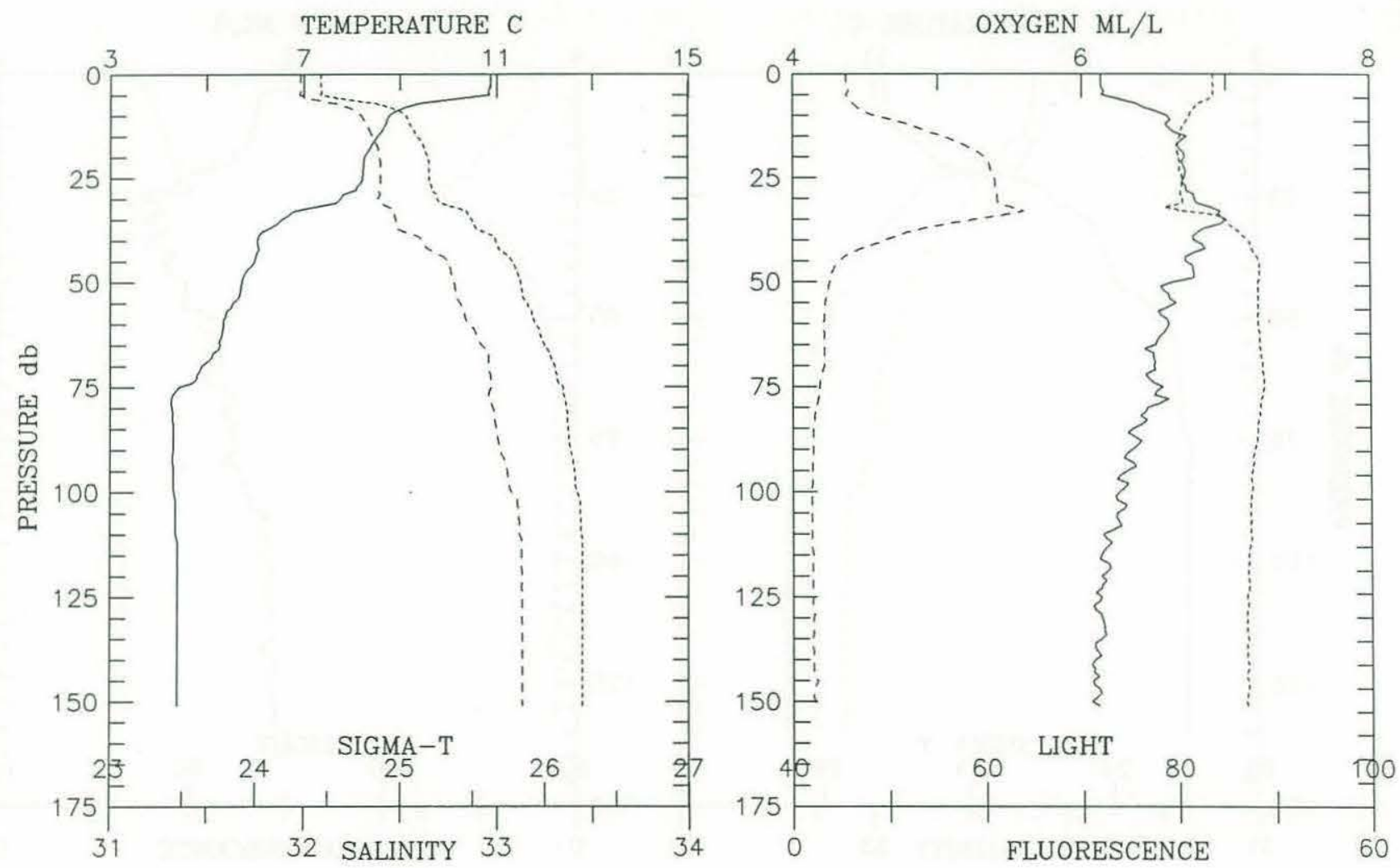


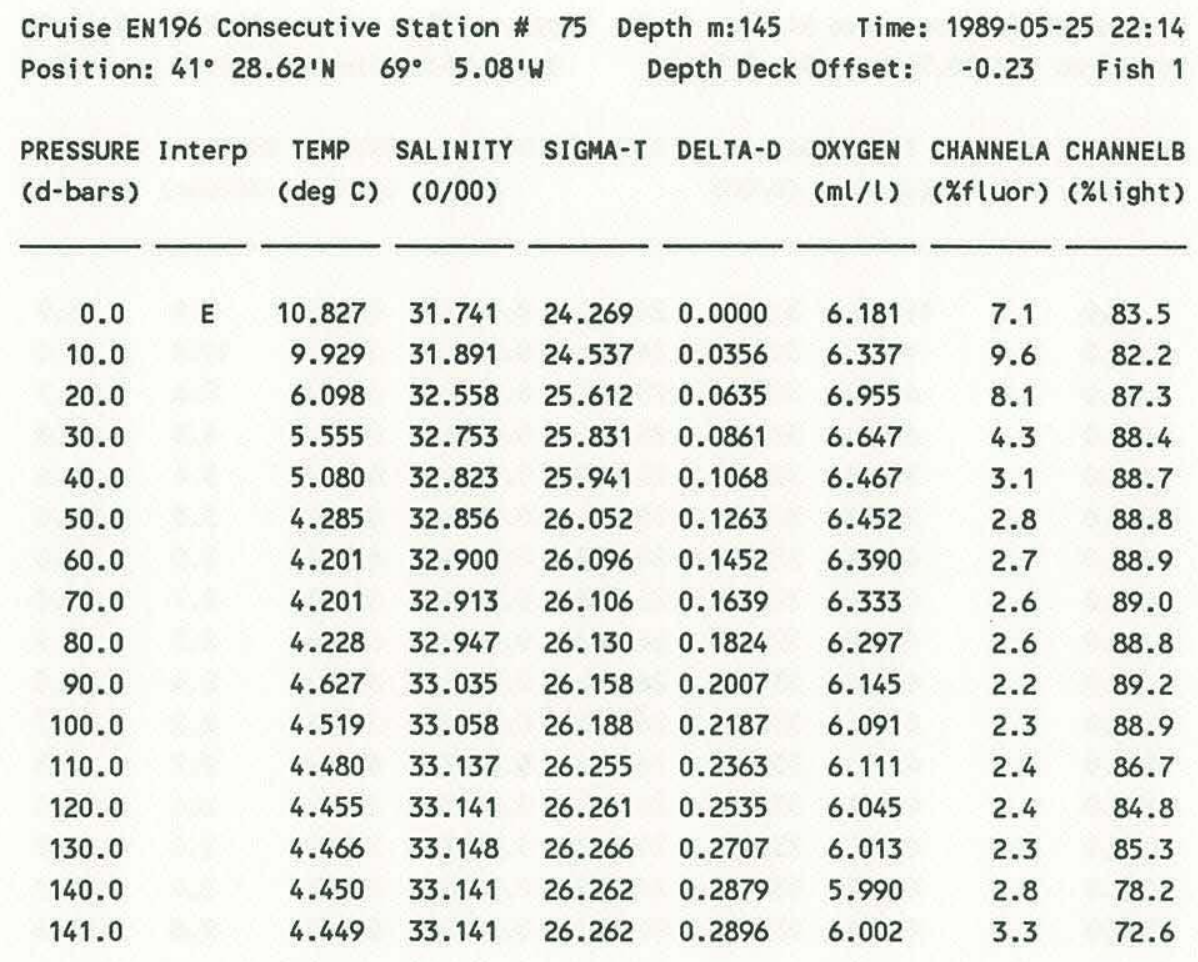
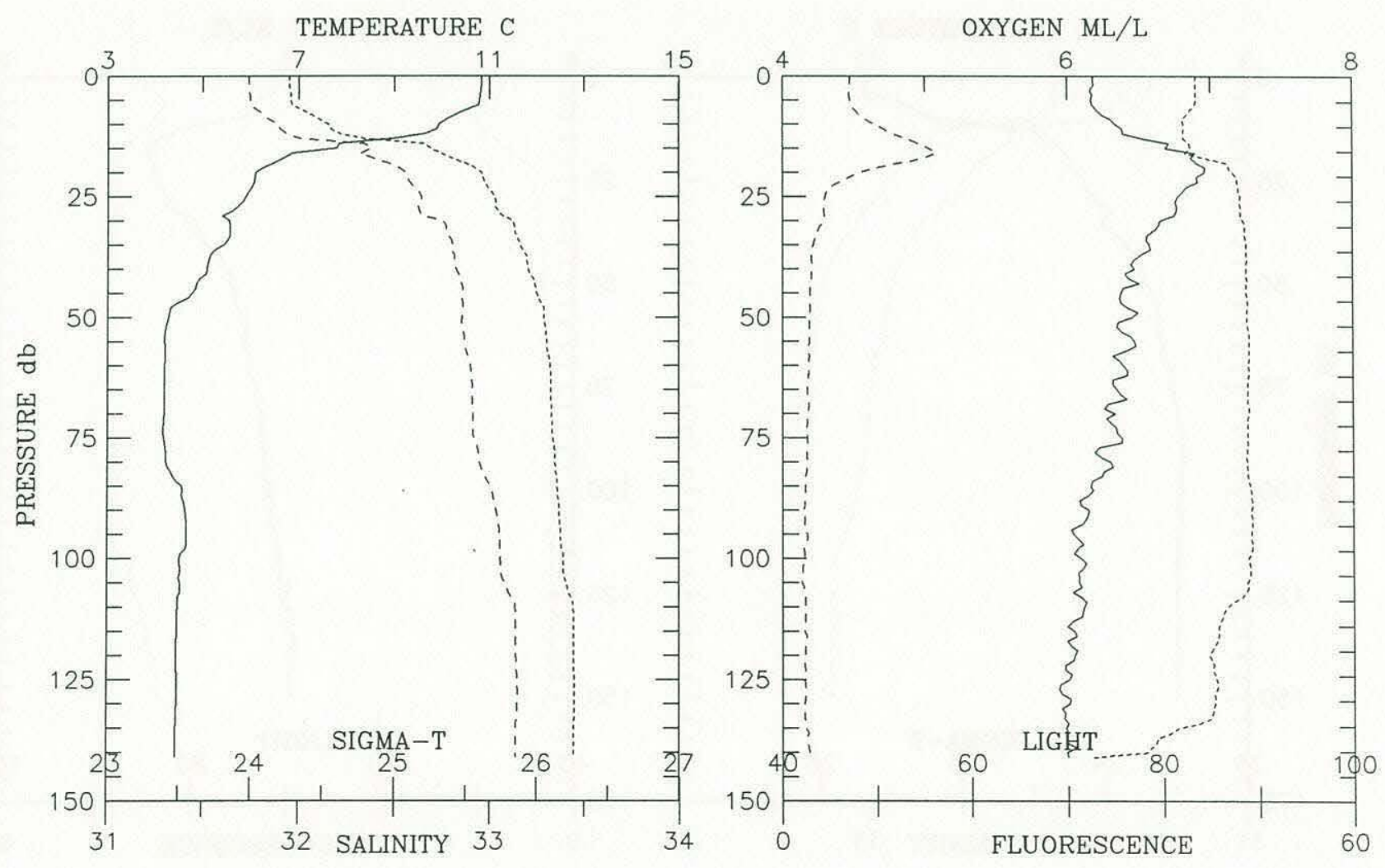


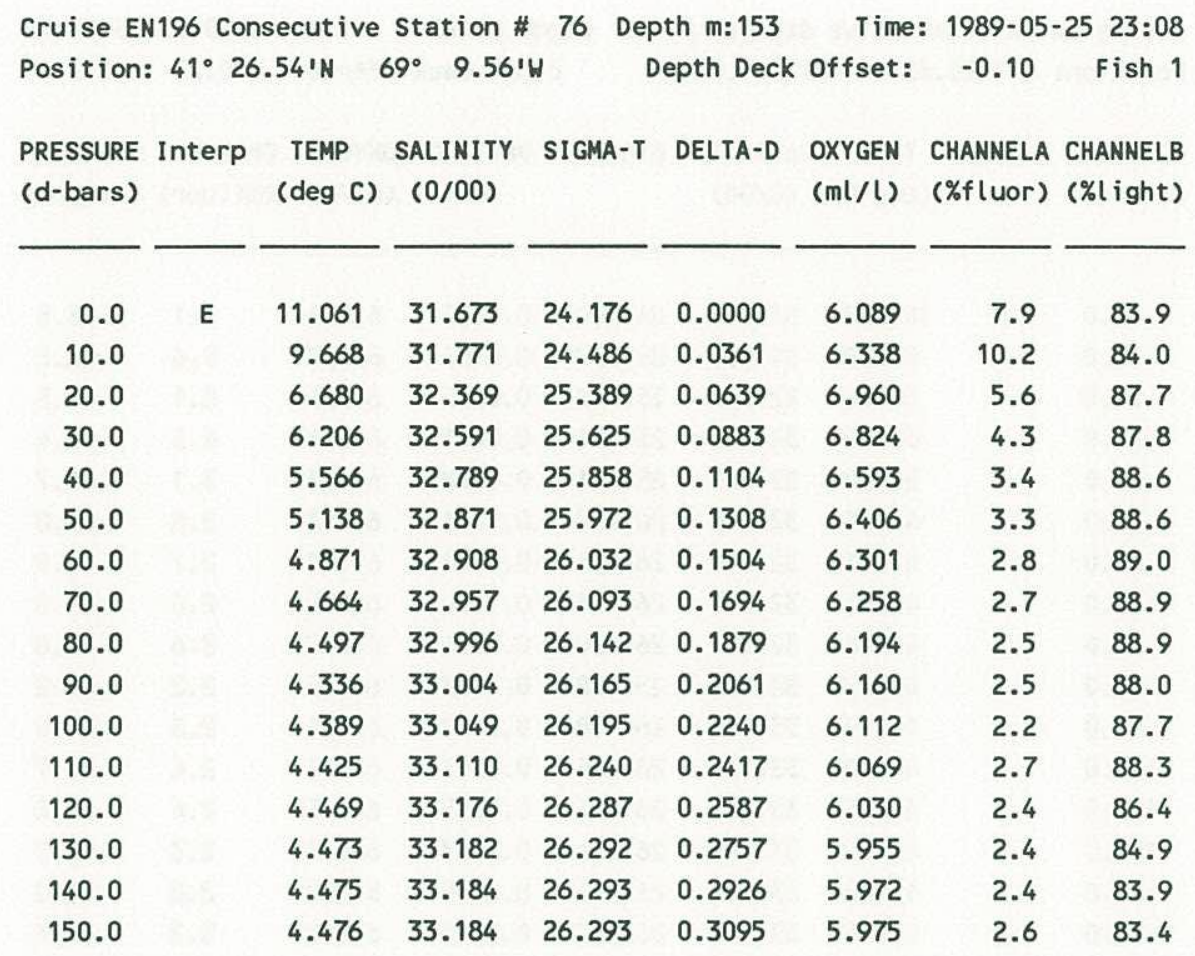
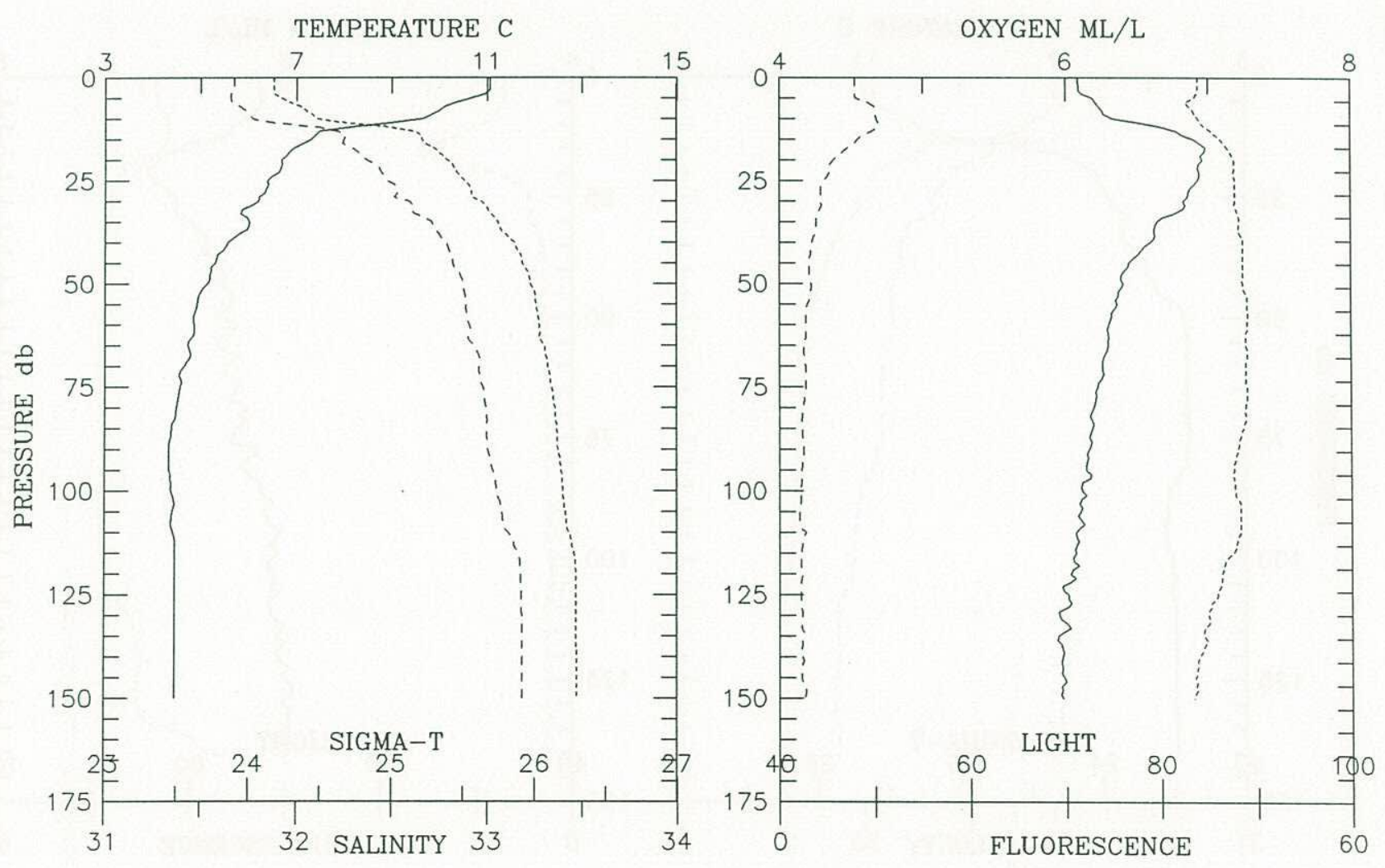


\begin{tabular}{|c|c|c|c|c|c|c|c|c|}
\hline osition: & $: 41^{\circ} 24$ & $.22^{\prime} \mathrm{N}$ & $69^{\circ} 14.29^{\prime}$ & & epth Deck & Offset: & -0.10 & Fish 1 \\
\hline $\begin{array}{l}\text { PRESSURE } \\
\text { (d-bars) }\end{array}$ & Interp & $\begin{array}{l}\text { TEMP } \\
(\operatorname{deg} C)\end{array}$ & $\begin{array}{c}\text { SALINITY } \\
(0 / 00)\end{array}$ & SIGMA-T & DELTA-D & $\begin{array}{l}\text { OXYGEN } \\
(\mathrm{ml} / \mathrm{l})\end{array}$ & $\begin{array}{l}\text { CHANNELA } \\
\text { (\%fluor) }\end{array}$ & $\begin{array}{l}\text { CHANNELB } \\
\text { (\%tight) }\end{array}$ \\
\hline 0.0 & $E$ & 7.552 & 32.097 & 25.058 & 0.0000 & 6.703 & 15.0 & 82.8 \\
\hline 10.0 & $E$ & 7.551 & 32.093 & 25.055 & 0.0286 & 6.711 & 15.0 & 82.8 \\
\hline 20.0 & & 6.357 & 32.504 & 25.536 & 0.0563 & 6.940 & 8.5 & 88.0 \\
\hline 30.0 & & 5.673 & 32.669 & 25.751 & 0.0789 & 6.794 & 3.2 & 88.8 \\
\hline 40.0 & & 5.108 & 32.772 & 25.897 & 0.0999 & 6.480 & 3.1 & 89.0 \\
\hline 50.0 & & 4.672 & 32.834 & 25.994 & 0.1202 & 6.487 & 3.3 & 88.4 \\
\hline 60.0 & & 4.522 & 32.854 & 26.026 & 0.1397 & 6.275 & 3.4 & 88.1 \\
\hline 70.0 & & 4.450 & 32.871 & 26.047 & 0.1589 & 6.281 & 3.3 & 87.9 \\
\hline 80.0 & & 4.431 & 32.876 & 26.053 & 0.1781 & 6.166 & 3.3 & 87.7 \\
\hline 90.0 & & 4.414 & 32.887 & 26.064 & 0.1972 & 6.108 & 3.3 & 87.5 \\
\hline 100.0 & & 4.376 & 32.952 & 26.119 & 0.2160 & 6.162 & 3.2 & 87.2 \\
\hline 110.0 & & 4.363 & 32.985 & 26.147 & 0.2344 & 6.102 & 3.0 & 85.5 \\
\hline 120.0 & & 4.365 & 33.000 & 26.158 & 0.2526 & 6.022 & 3.0 & 84.5 \\
\hline 130.0 & & 4.364 & 33.009 & 26.166 & 0.2707 & 6.065 & 2.9 & 83.9 \\
\hline 131.0 & & 4.364 & 33.009 & 26.166 & 0.2725 & 6.026 & 3.1 & 84.0 \\
\hline
\end{tabular}
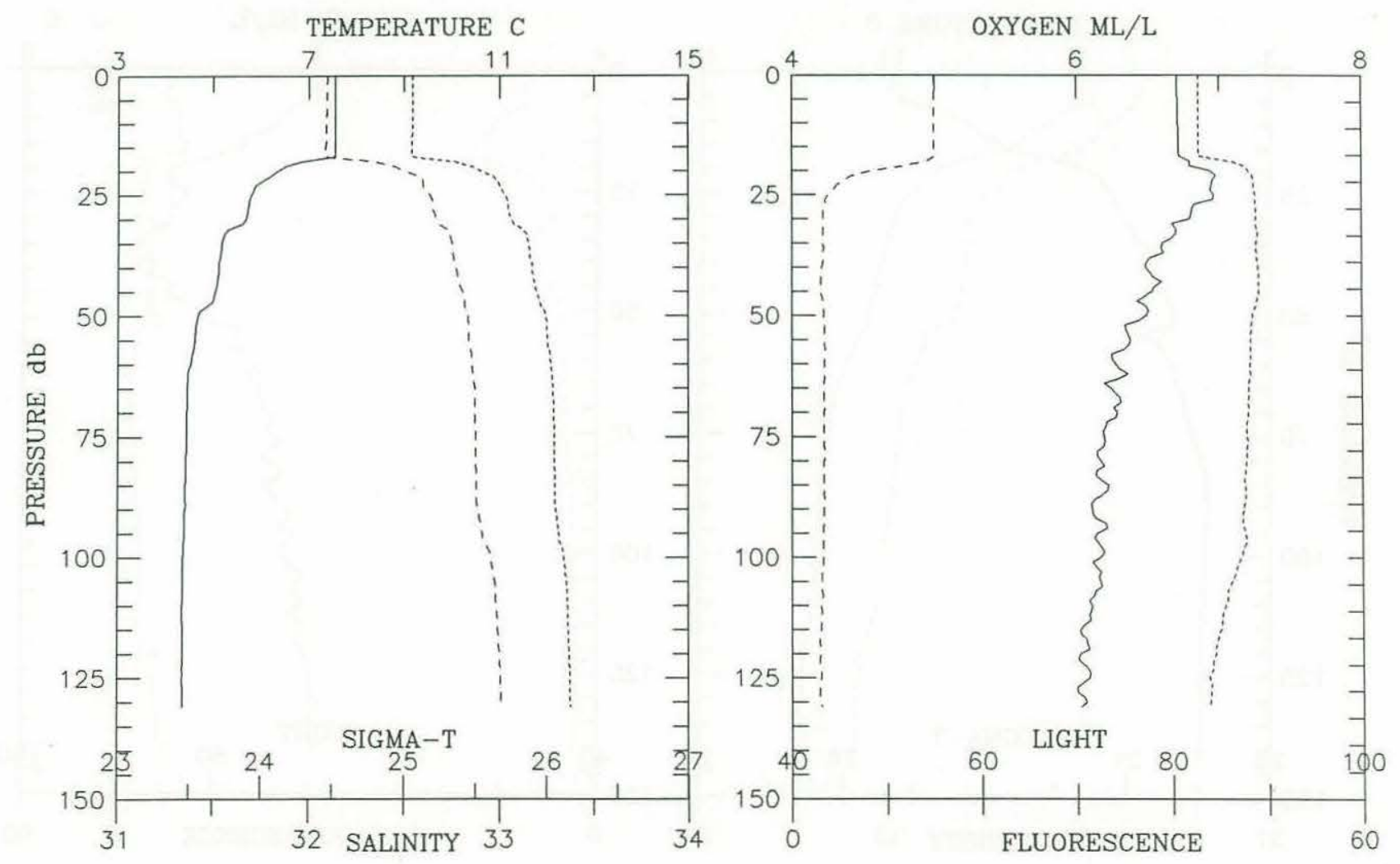


\begin{tabular}{|c|c|c|c|c|c|c|c|c|}
\hline Position: & $41^{\circ} 26$ & $.44^{\prime} \mathrm{N}$ & $69^{\circ} 9.68^{\prime}$ & & epth Deck & Offset: & -0.10 & Fish 1 \\
\hline $\begin{array}{l}\text { PRESSURE } \\
\text { (d-bars) }\end{array}$ & Interp & $\begin{array}{c}\text { TEMP } \\
(\operatorname{deg} \mathrm{C})\end{array}$ & $\begin{array}{l}\text { SALINITY } \\
(0 / 00)\end{array}$ & SIGMA-T & DELTA-D & $\begin{array}{l}\text { OXYGEN } \\
(\mathrm{ml} / \mathrm{l})\end{array}$ & $\begin{array}{l}\text { CHANNELA } \\
\text { (\%fluor) }\end{array}$ & $\begin{array}{l}\text { CHANNELB } \\
\text { (\%light) }\end{array}$ \\
\hline 0.0 & E & 10.859 & 31.696 & 24.229 & 0.0000 & 5.930 & 7.7 & 85.1 \\
\hline 10.0 & & 9.510 & 31.820 & 24.550 & 0.0357 & 6.224 & 10.3 & 84.0 \\
\hline 20.0 & & 6.729 & 32.358 & 25.374 & 0.0660 & 6.899 & 9.8 & 86.7 \\
\hline 30.0 & & 6.060 & 32.577 & 25.631 & 0.0903 & 7.005 & 4.9 & 87.2 \\
\hline 40.0 & & 5.428 & 32.604 & 25.728 & 0.1129 & 7.135 & 6.0 & 86.7 \\
\hline 50.0 & & 5.030 & 32.655 & 25.813 & 0.1347 & 6.798 & 3.8 & 86.9 \\
\hline 60.0 & & 5.095 & 32.853 & 25.963 & 0.1554 & 6.397 & 3.5 & 88.3 \\
\hline 70.0 & & 4.757 & 32.937 & 26.067 & 0.1749 & 6.325 & 3.2 & 88.8 \\
\hline 80.0 & & 4.410 & 32.962 & 26.124 & 0.1936 & 6.315 & 2.9 & 88.4 \\
\hline 90.0 & & 4.305 & 32.997 & 26.162 & 0.2119 & 6.221 & 2.6 & 88.1 \\
\hline 100.0 & & 4.346 & 33.041 & 26.193 & 0.2298 & 6.212 & 2.3 & 87.7 \\
\hline 110.0 & & 4.390 & 33.071 & 26.212 & 0.2476 & 6.034 & 2.2 & 87.8 \\
\hline 120.0 & & 4.437 & 33.144 & 26.265 & 0.2650 & 6.047 & 2.1 & 87.5 \\
\hline 130.0 & & 4.465 & 33.174 & 26.286 & 0.2820 & 5.988 & 2.1 & 86.1 \\
\hline 140.0 & & 4.470 & 33.178 & 26.289 & 0.2990 & 5.967 & 2.4 & 85.2 \\
\hline 141.0 & & 4.469 & 33.178 & 26.289 & 0.3007 & 5.957 & 2.4 & 85.2 \\
\hline
\end{tabular}
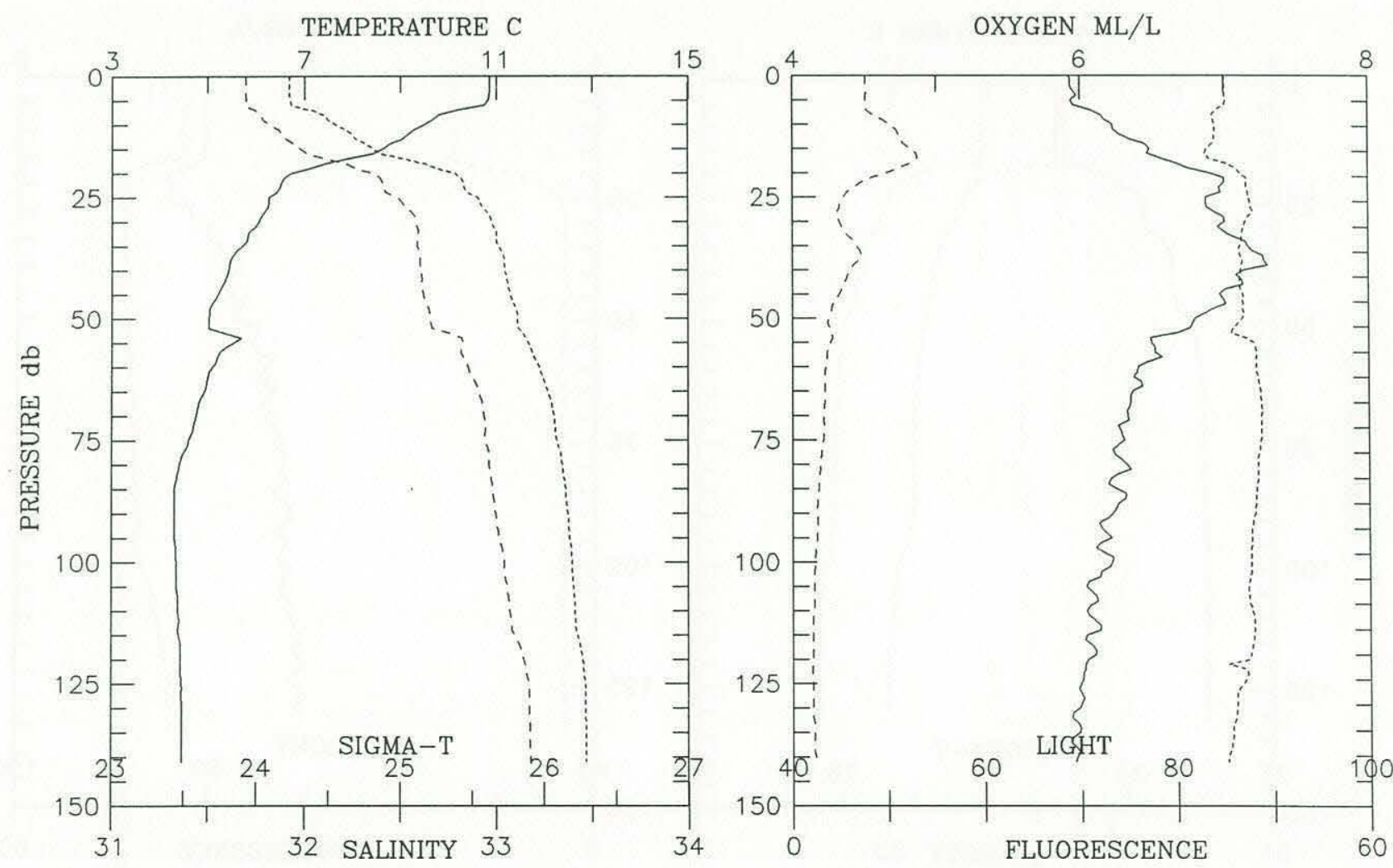


\begin{tabular}{|c|c|c|c|c|c|c|c|c|}
\hline $\begin{array}{l}\text { Cruise EN } \\
\text { Position: }\end{array}$ & $=41^{\circ} 28$ & $\begin{array}{l}\text { secutive } \\
.73 \text { N }\end{array}$ & $\begin{array}{l}\text { Station \# } \\
69^{\circ} \quad 5.21^{1}\end{array}$ & 79 & $\begin{array}{l}\text { th } \mathrm{m}: 145 \\
\text { epth Deck }\end{array}$ & $\begin{array}{r}\text { Time: } \\
\text { offset: }\end{array}$ & $\begin{array}{c}=1989-05- \\
-0.10\end{array}$ & $\begin{array}{r}2606: 13 \\
\text { Fish } 1\end{array}$ \\
\hline $\begin{array}{l}\text { PRESSURE } \\
\text { (d-bars) }\end{array}$ & Interp & $\begin{array}{c}\text { TEMP } \\
(\operatorname{deg} C)\end{array}$ & $\begin{array}{l}\text { SALINITY } \\
(0 / 00)\end{array}$ & SIGMA-T & DELTA-D & $\begin{array}{l}\text { OXYGEN } \\
(\mathrm{ml} / \mathrm{l})\end{array}$ & $\begin{array}{l}\text { CHANNELA } \\
\text { (\%fluor) }\end{array}$ & $\begin{array}{l}\text { CHANNELB } \\
\text { (\%light) }\end{array}$ \\
\hline 0.0 & E & 10.780 & 31.935 & 24.429 & 0.0000 & 5.931 & 8.0 & 83.8 \\
\hline 10.0 & & 9.787 & 32.134 & 24.750 & 0.0334 & 6.152 & 10.2 & 82.9 \\
\hline 20.0 & & 7.969 & 32.108 & 25.008 & 0.0642 & 6.626 & 13.7 & 83.0 \\
\hline 30.0 & & 6.224 & 32.590 & 25.621 & 0.0896 & 7.054 & 11.4 & 86.9 \\
\hline 40.0 & & 5.889 & 32.662 & 25.719 & 0.1124 & 6.695 & 4.6 & 88.0 \\
\hline 50.0 & & 5.332 & 32.764 & 25.865 & 0.1339 & 6.494 & 3.3 & 88.5 \\
\hline 60.0 & & 4.509 & 32.841 & 26.017 & 0.1539 & 6.390 & 3.4 & 88.9 \\
\hline 70.0 & & 4.235 & 32.868 & 26.067 & 0.1731 & 6.321 & 2.8 & 89.1 \\
\hline 80.0 & & 4.257 & 32.910 & 26.098 & 0.1919 & 6.280 & 2.6 & 89.0 \\
\hline 90.0 & & 4.377 & 32.978 & 26.140 & 0.2105 & 6.172 & 3.0 & 88.8 \\
\hline 100.0 & & 4.333 & 33.022 & 26.179 & 0.2286 & 6.152 & 2.5 & 88.6 \\
\hline 110.0 & & 4.342 & 33.053 & 26.203 & 0.2464 & 6.083 & 2.2 & 88.4 \\
\hline 120.0 & & 4.396 & 33.107 & 26.240 & 0.2639 & 6.002 & 2.2 & 87.2 \\
\hline 130.0 & & 4.516 & 33.213 & 26.312 & 0.2809 & 5.938 & 2.1 & 85.9 \\
\hline 133.0 & & 4.517 & 33.215 & 26.313 & 0.2859 & 6.065 & 2.3 & 84.4 \\
\hline
\end{tabular}
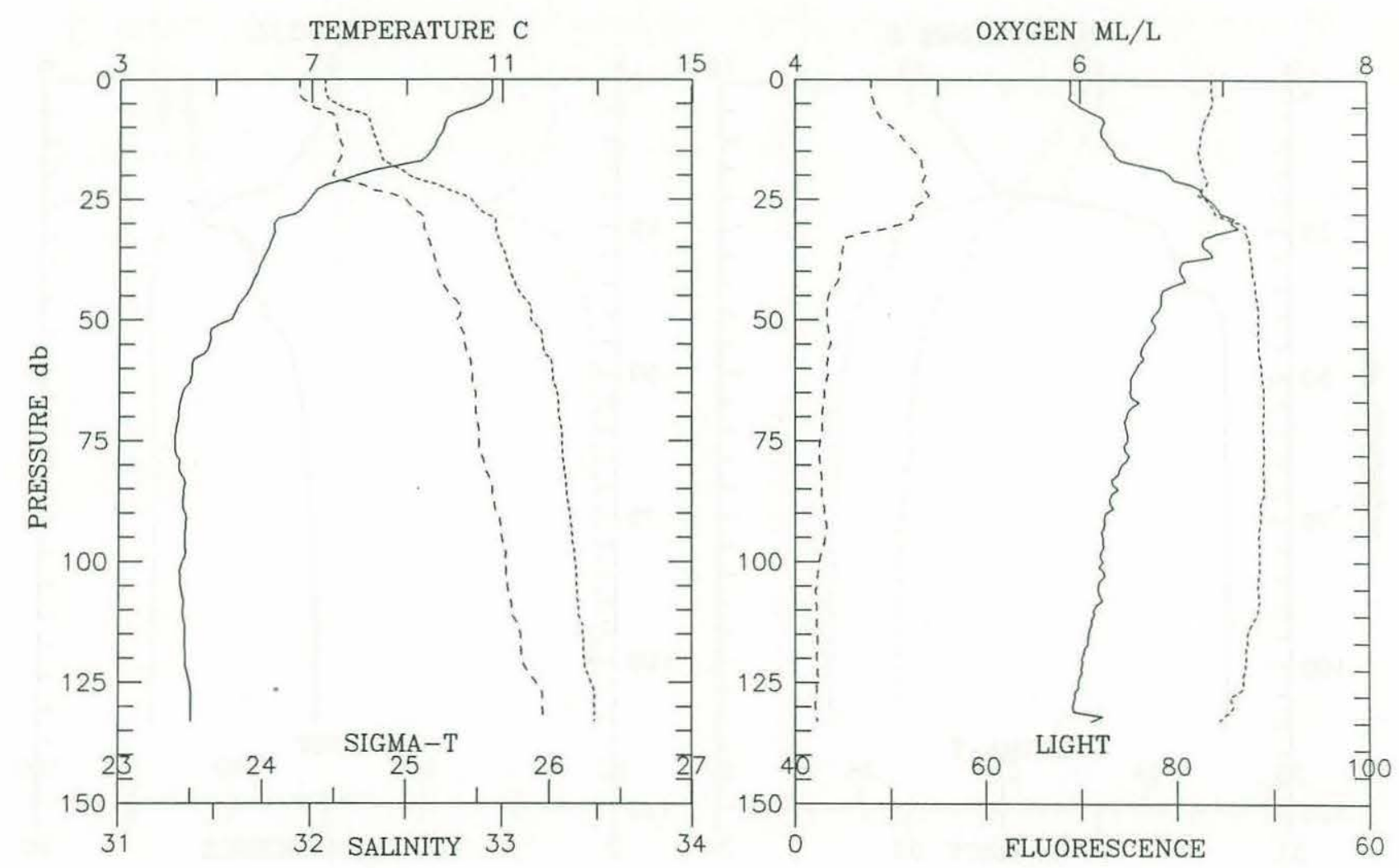


\begin{tabular}{|c|c|c|c|c|c|c|c|c|}
\hline $\begin{array}{l}\text { Cruise EN } \\
\text { Position: }\end{array}$ & $\begin{array}{l}196 \text { Con } \\
41^{\circ} 36\end{array}$ & $\begin{array}{l}\text { secutive } \\
.001 \mathrm{~N}\end{array}$ & $\begin{array}{l}\text { Station } \\
68^{\circ} 56.25^{\prime}\end{array}$ & 80 & epth Deck & offset: & -0.40 & $\begin{array}{r}20 \text { fish } 1 \\
\text { Fis }\end{array}$ \\
\hline $\begin{array}{l}\text { PRESSURE } \\
\text { (d-bars) }\end{array}$ & Interp & $\begin{array}{c}\text { TEMP } \\
(\operatorname{deg} C)\end{array}$ & $\begin{array}{l}\text { SALINITY } \\
(0 / 00)\end{array}$ & SIGMA-T & DELTA-D & $\begin{array}{l}\text { OXYGEN } \\
(\mathrm{ml} / \mathrm{l})\end{array}$ & $\begin{array}{l}\text { CHANNELA } \\
\text { (\%fluor) }\end{array}$ & $\begin{array}{l}\text { CHANNELB } \\
\text { (\%light) }\end{array}$ \\
\hline 0.0 & E & 10.425 & 32.061 & 24.587 & 0.0000 & 6.060 & 6.8 & 85.1 \\
\hline 10.0 & & 9.933 & 32.098 & 24.698 & 0.0329 & 6.165 & 7.5 & 84.7 \\
\hline 20.0 & & 7.878 & 32.362 & 25.220 & 0.0636 & 6.679 & 12.2 & 82.7 \\
\hline 30.0 & & 5.478 & 32.692 & 25.792 & 0.0867 & 6.536 & 5.0 & 88.1 \\
\hline 40.0 & & 4.413 & 32.902 & 26.075 & 0.1069 & 6.424 & 3.1 & 88.7 \\
\hline 50.0 & & 4.349 & 32.971 & 26.137 & 0.1255 & 6.248 & 2.4 & 88.5 \\
\hline 60.0 & & 4.335 & 32.993 & 26.156 & 0.1436 & 6.175 & 2.3 & 88.3 \\
\hline 70.0 & & 4.312 & 33.031 & 26.188 & 0.1616 & 6.137 & 2.1 & 88.0 \\
\hline 80.0 & & 4.313 & 33.049 & 26.202 & 0.1794 & 6.093 & 2.0 & 88.0 \\
\hline 90.0 & & 4.319 & 33.063 & 26.213 & 0.1970 & 6.080 & 2.0 & 88.1 \\
\hline 100.0 & & 4.320 & 33.063 & 26.213 & 0.2147 & 6.075 & 2.0 & 88.1 \\
\hline 110.0 & & 4.321 & 33.064 & 26.214 & 0.2323 & 6.069 & 2.1 & 88.2 \\
\hline 111.0 & & 4.321 & 33.064 & 26.214 & 0.2341 & 6.081 & 2.2 & 88.1 \\
\hline
\end{tabular}
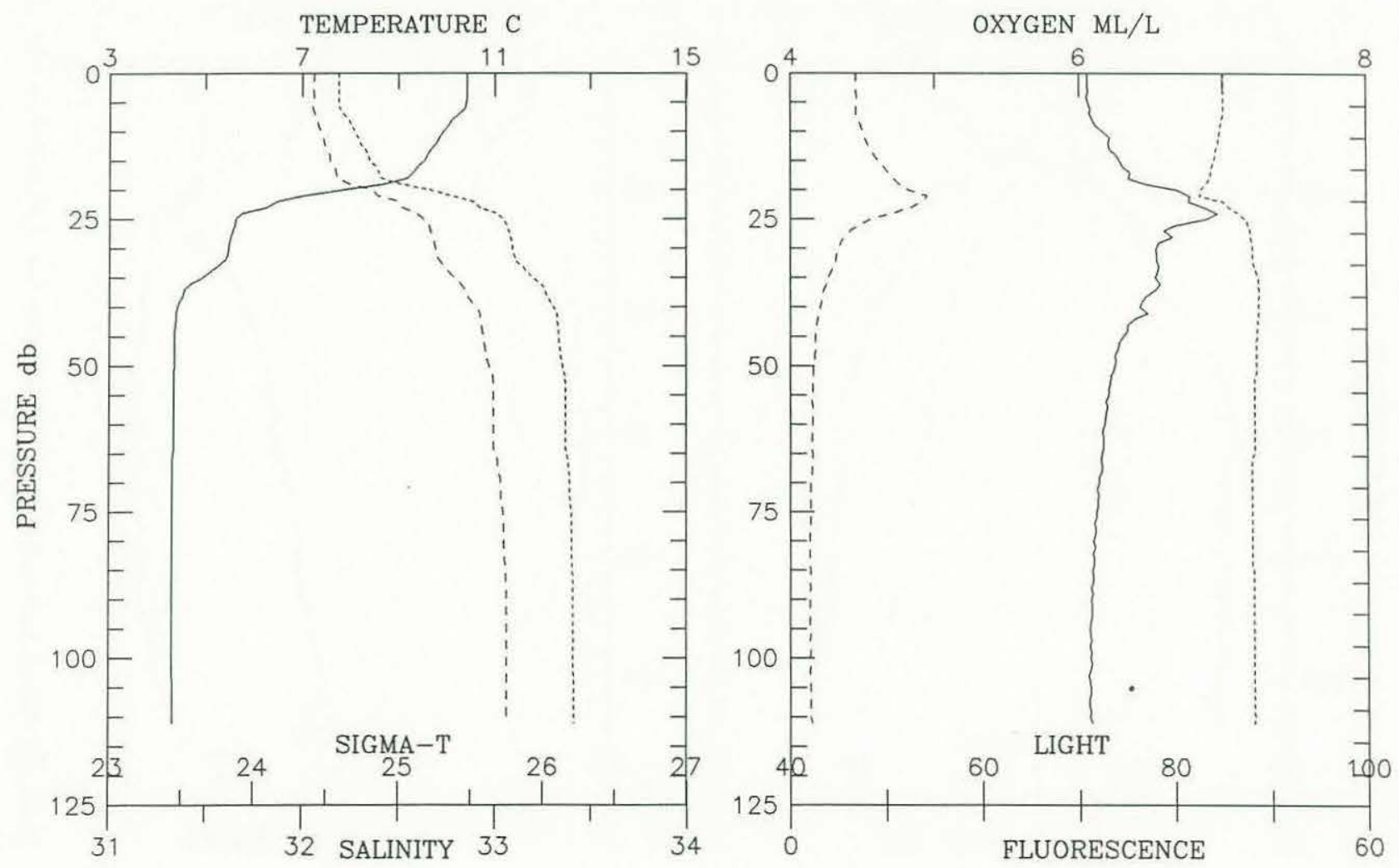


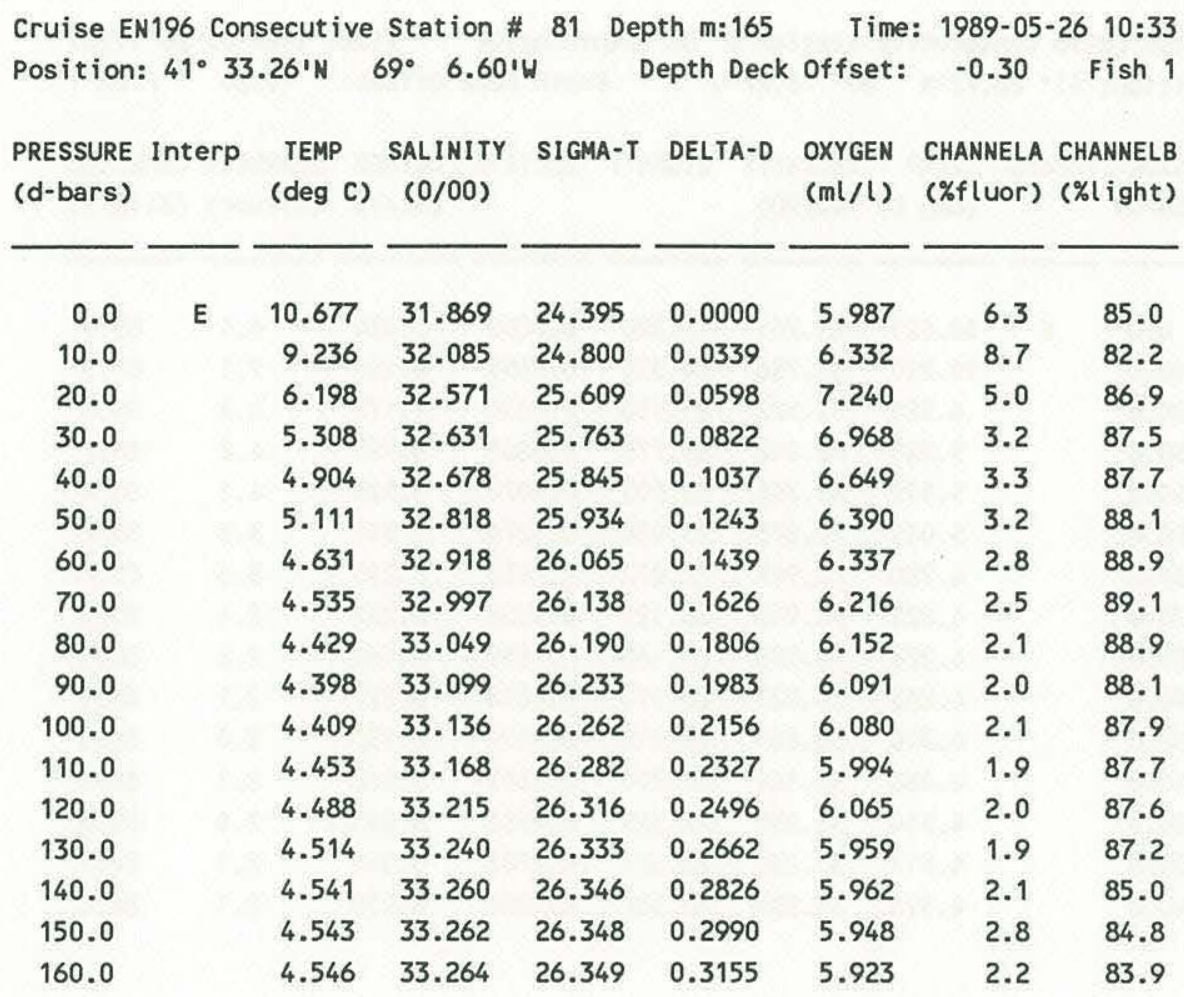
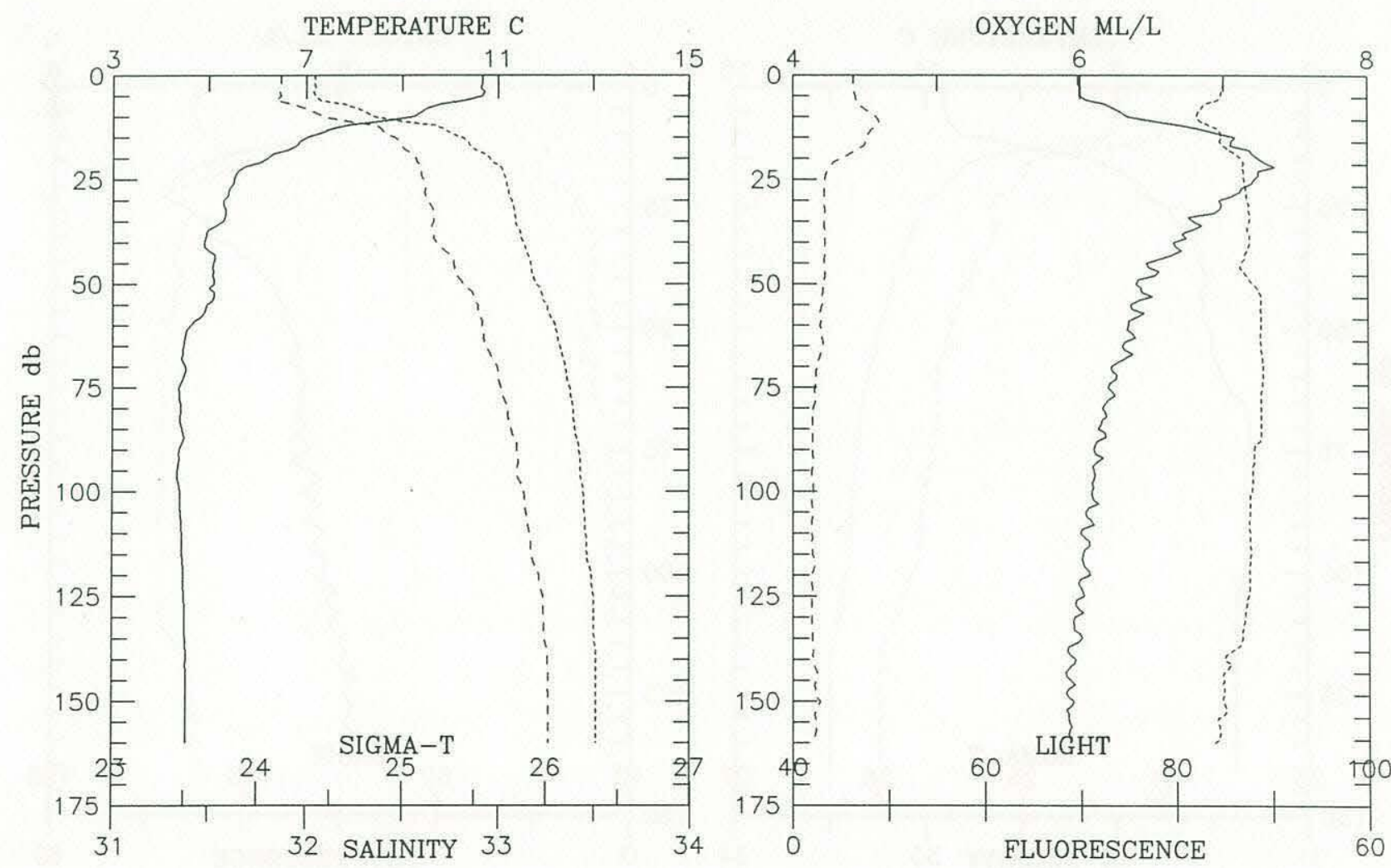


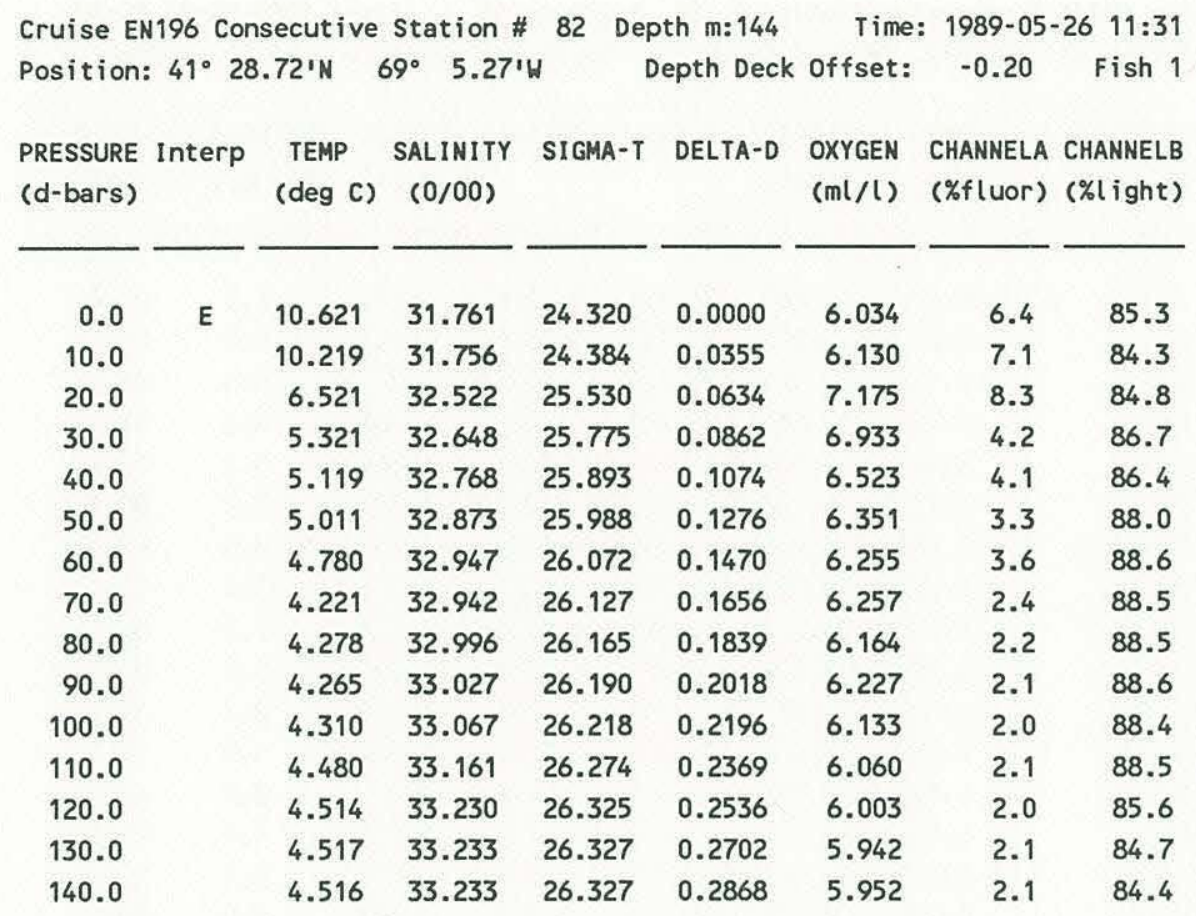
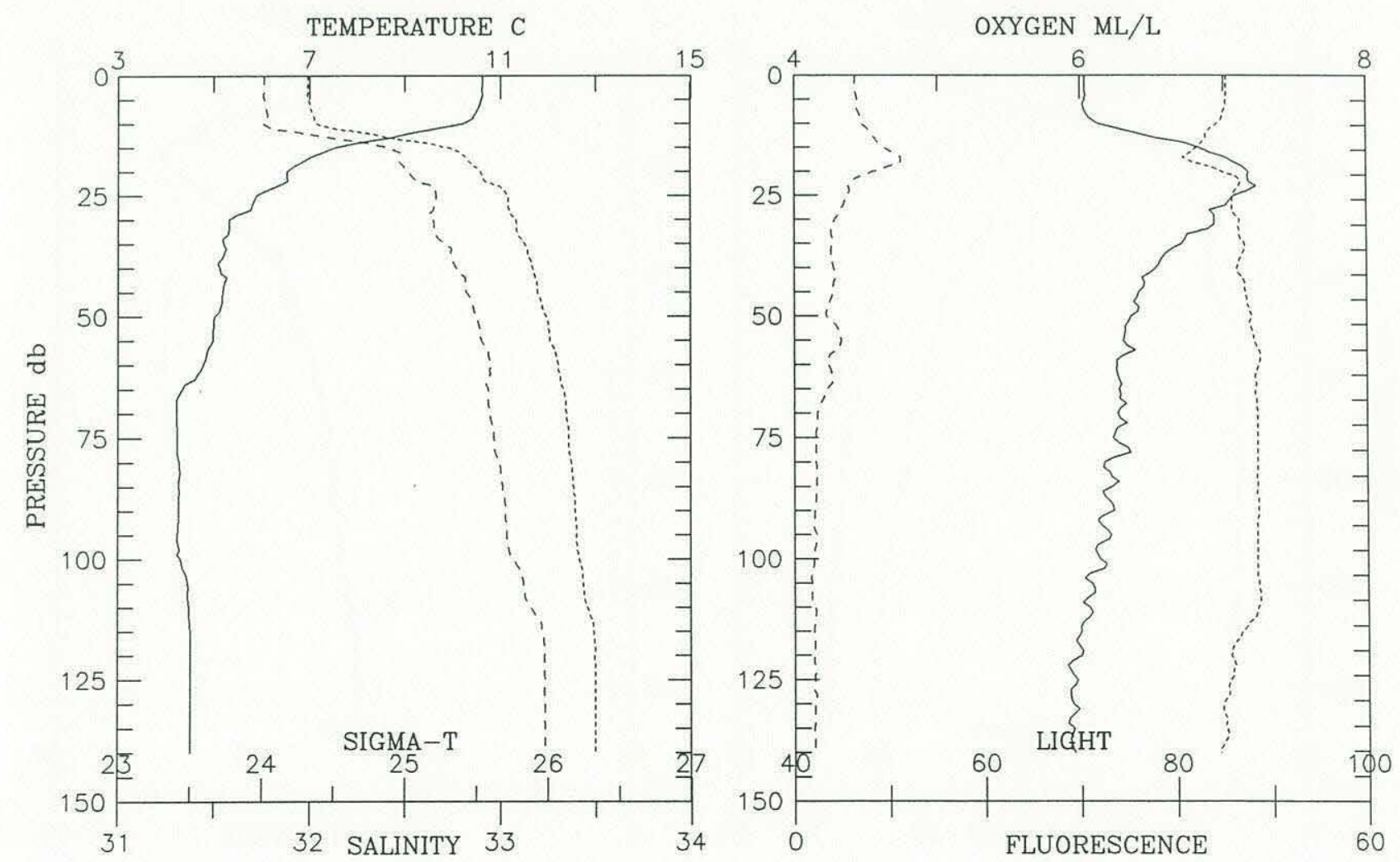


\begin{tabular}{|c|c|c|c|c|c|c|c|c|}
\hline $\begin{array}{l}\text { Cruise EN } \\
\text { Position: }\end{array}$ & $\begin{array}{l}196 \text { Con } \\
41^{\circ} 26\end{array}$ & $\begin{array}{l}\text { secutive } \\
.491 \mathrm{~N}\end{array}$ & $\begin{array}{l}\text { Station } \\
69^{\circ} \quad 9.62^{\prime}\end{array}$ & 83 & epth Deck & offset: & -0.20 & Fish 1 \\
\hline $\begin{array}{l}\text { PRESSURE } \\
\text { (d-bars) }\end{array}$ & Interp & $\begin{array}{c}\text { TEMP } \\
(\operatorname{deg} C)\end{array}$ & $\begin{array}{l}\text { SALINITY } \\
(0 / 00)\end{array}$ & SIGMA-T & DELTA-D & $\begin{array}{l}\text { OXYGEN } \\
(\mathrm{ml} / \mathrm{l})\end{array}$ & $\begin{array}{l}\text { CHANNELA } \\
\text { (\%fluor) }\end{array}$ & $\begin{array}{l}\text { CHANNELB } \\
\text { (\%tight) }\end{array}$ \\
\hline 0.0 & $E$ & 10.573 & 31.772 & 24.337 & 0.0000 & 6.116 & 7.5 & 84.5 \\
\hline 10.0 & & 9.633 & 31.852 & 24.555 & 0.0350 & 6.243 & 7.9 & 84.0 \\
\hline 20.0 & & 6.485 & 32.580 & 25.581 & 0.0635 & 7.023 & 6.0 & 88.5 \\
\hline 30.0 & & 5.884 & 32.720 & 25.766 & 0.0861 & 6.954 & 4.5 & 88.1 \\
\hline 40.0 & & 5.199 & 32.764 & 25.881 & 0.1073 & 6.771 & 3.6 & 88.6 \\
\hline 50.0 & & 5.101 & 32.854 & 25.963 & 0.1276 & 6.637 & 3.3 & 88.2 \\
\hline 60.0 & & 4.845 & 32.887 & 26.017 & 0.1473 & 6.536 & 4.5 & 88.4 \\
\hline 70.0 & & 4.388 & 32.966 & 26.129 & 0.1663 & 6.467 & 3.2 & 88.4 \\
\hline 80.0 & & 4.371 & 32.994 & 26.153 & 0.1846 & 6.314 & 2.4 & 87.9 \\
\hline 90.0 & & 4.343 & 33.025 & 26.181 & 0.2026 & 6.312 & 2.2 & 87.4 \\
\hline 100.0 & & 4.352 & 33.043 & 26.194 & 0.2205 & 6.223 & 2.2 & 87.1 \\
\hline 110.0 & & 4.384 & 33.084 & 26.223 & 0.2382 & 6.155 & 2.2 & 86.0 \\
\hline 120.0 & & 4.398 & 33.099 & 26.233 & 0.2557 & 6.131 & 2.4 & 84.0 \\
\hline 130.0 & & 4.399 & 33.101 & 26.235 & 0.2731 & 6.057 & 2.5 & 83.7 \\
\hline 140.0 & & 4.402 & 33.103 & 26.236 & 0.2906 & 6.040 & 2.6 & 83.2 \\
\hline 150.0 & & 4.404 & 33.105 & 26.237 & 0.3080 & 6.038 & 2.6 & 82.5 \\
\hline
\end{tabular}
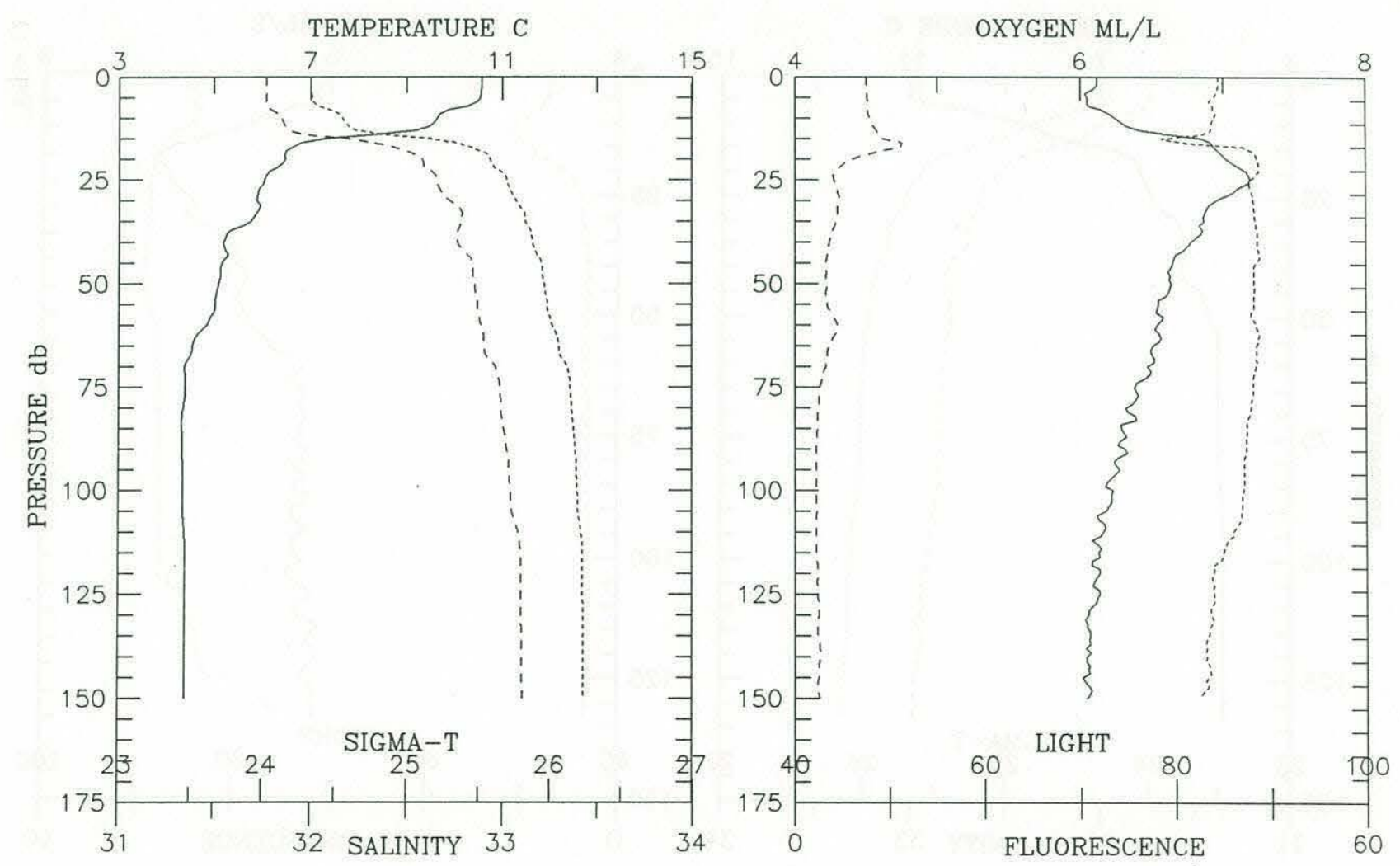


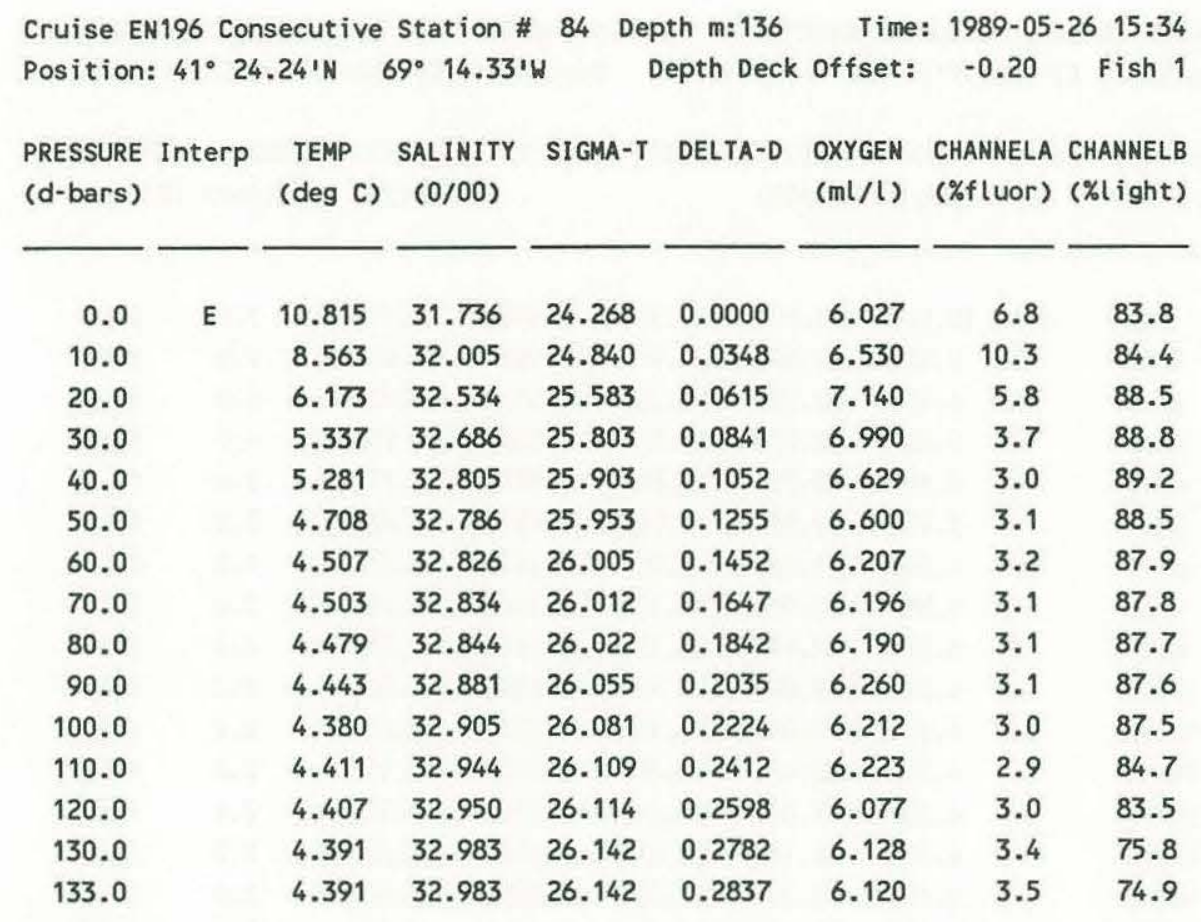
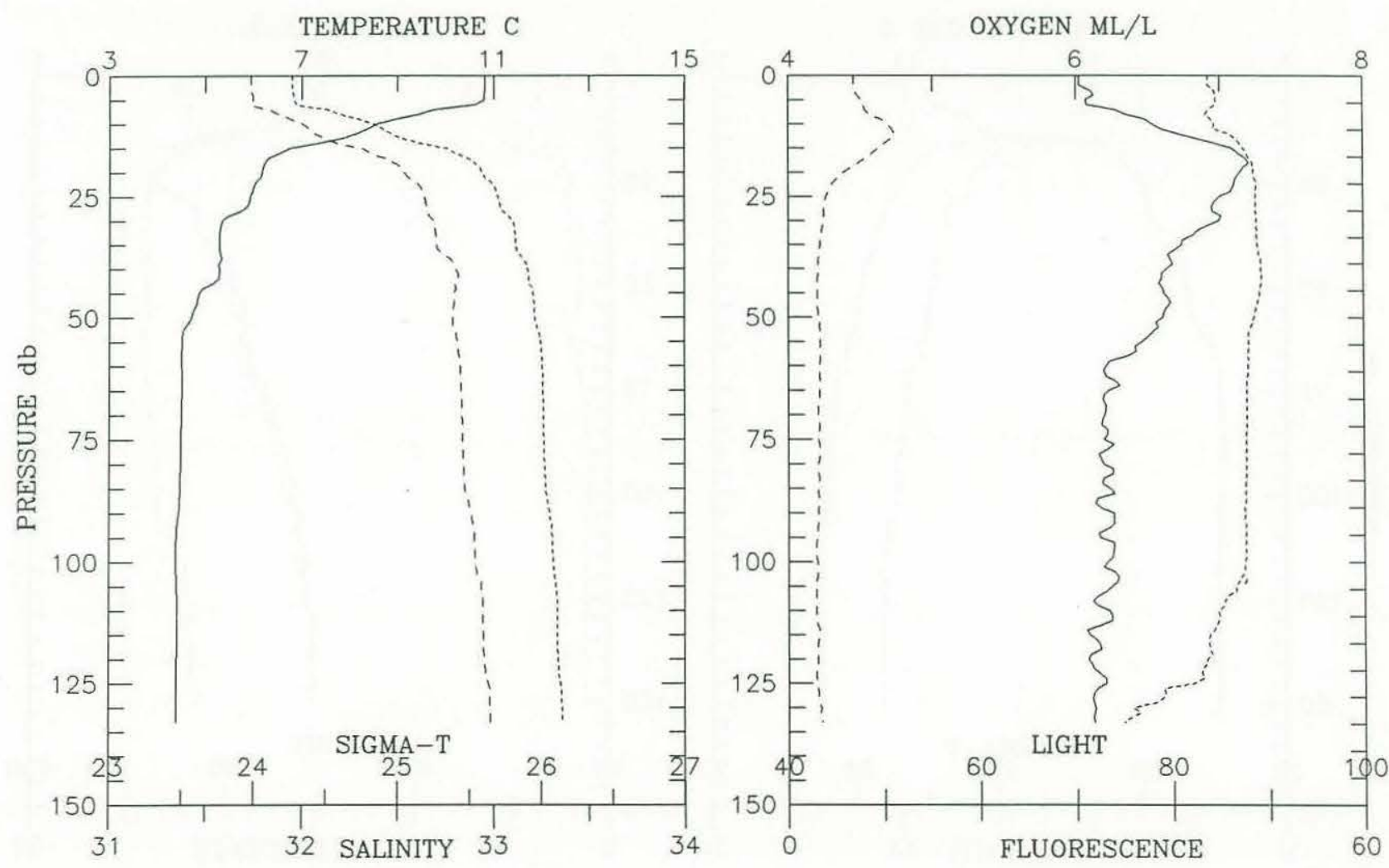
Cruise EN196 Consecutive Station \# 85 Depth m:73 Time: 1989-05-26 17:47 Position: $41^{\circ} 23.67 \mathrm{~N} 69^{\circ} 19.75^{\prime} \mathrm{W}$ Depth Deck offset: -0.20 Fish 1

\begin{tabular}{|c|c|c|c|c|c|c|c|c|}
\hline $\begin{array}{l}\text { PRESSURE } \\
\text { (d-bars) }\end{array}$ & Interp & $\begin{array}{c}\text { TEMP } \\
\text { (deg C) }\end{array}$ & $\begin{array}{c}\text { SALINITY } \\
(0 / 00)\end{array}$ & SIGMA-T & DELTA-D & $\begin{array}{l}\text { OXYGEN } \\
(\mathrm{ml} / \mathrm{l})\end{array}$ & $\begin{array}{l}\text { CHANNELA } \\
\text { ( } \% \text { fluor) }\end{array}$ & $\begin{array}{l}\text { CHANNELB } \\
\text { (\%light) }\end{array}$ \\
\hline 0.0 & E & 10.682 & 31.818 & 24.354 & 0.0000 & 6.277 & 4.8 & 64.7 \\
\hline 5.0 & & 10.652 & 31.823 & 24.363 & 0.0176 & 6.306 & 5.9 & 83.4 \\
\hline 10.0 & & 9.288 & 31.959 & 24.693 & 0.0350 & 6.646 & 8.4 & 82.6 \\
\hline 15.0 & & 6.301 & 32.510 & 25.548 & 0.0491 & 7.175 & 11.0 & 87.5 \\
\hline 20.0 & & 5.405 & 32.602 & 25.729 & 0.0607 & 7.327 & 5.4 & 88.1 \\
\hline 25.0 & & 5.100 & 32.665 & 25.813 & 0.0717 & 6.876 & 4.0 & 88.3 \\
\hline 30.0 & & 5.021 & 32.700 & 25.850 & 0.0822 & 6.650 & 3.9 & 88.3 \\
\hline 35.0 & & 4.993 & 32.715 & 25.865 & 0.0927 & 6.589 & 3.7 & 88.2 \\
\hline 40.0 & & 4.968 & 32.728 & 25.878 & 0.1031 & 6.508 & 3.5 & 88.2 \\
\hline 45.0 & & 4.936 & 32.742 & 25.892 & 0.1135 & 6.611 & 3.6 & 88.1 \\
\hline 50.0 & & 4.925 & 32.747 & 25.898 & 0.1238 & 6.440 & 3.5 & 88.0 \\
\hline 55.0 & & 4.924 & 32.748 & 25.898 & 0.1341 & 6.383 & 3.5 & 88.0 \\
\hline 60.0 & & 4.915 & 32.752 & 25.903 & 0.1443 & 6.280 & 3.4 & 87.9 \\
\hline 65.0 & & 4.914 & 32.752 & 25.903 & 0.1546 & 6.258 & 3.5 & 87.9 \\
\hline 70.0 & & 4.915 & 32.752 & 25.903 & 0.1649 & 6.161 & 3.5 & 88.0 \\
\hline
\end{tabular}
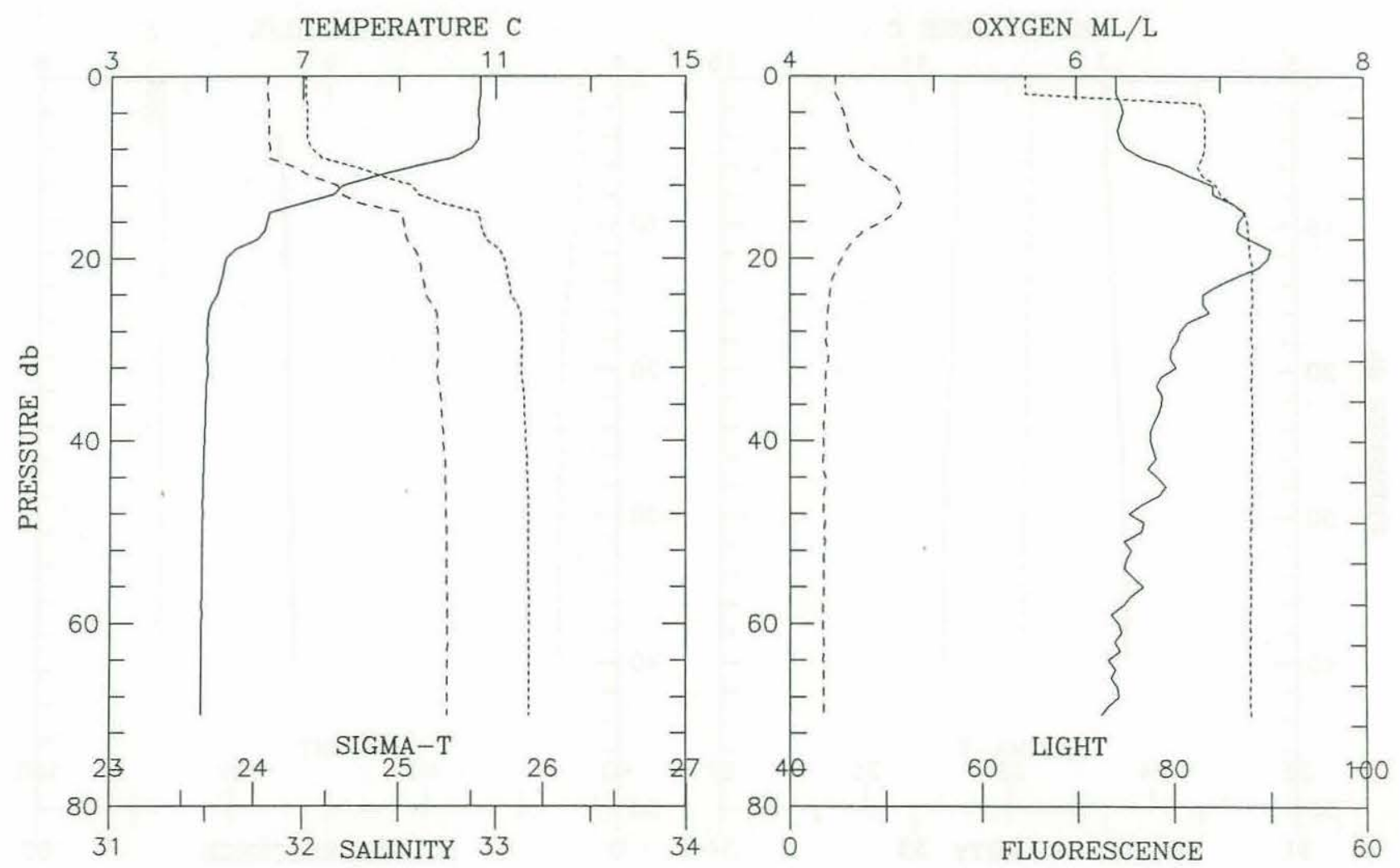


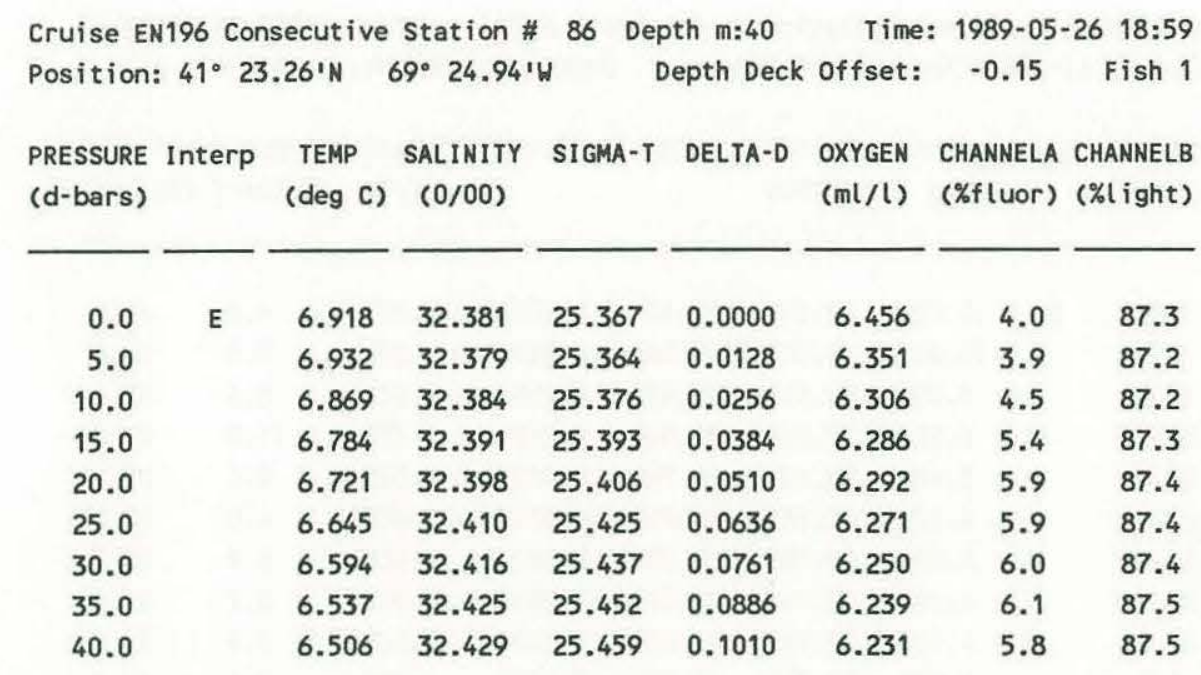
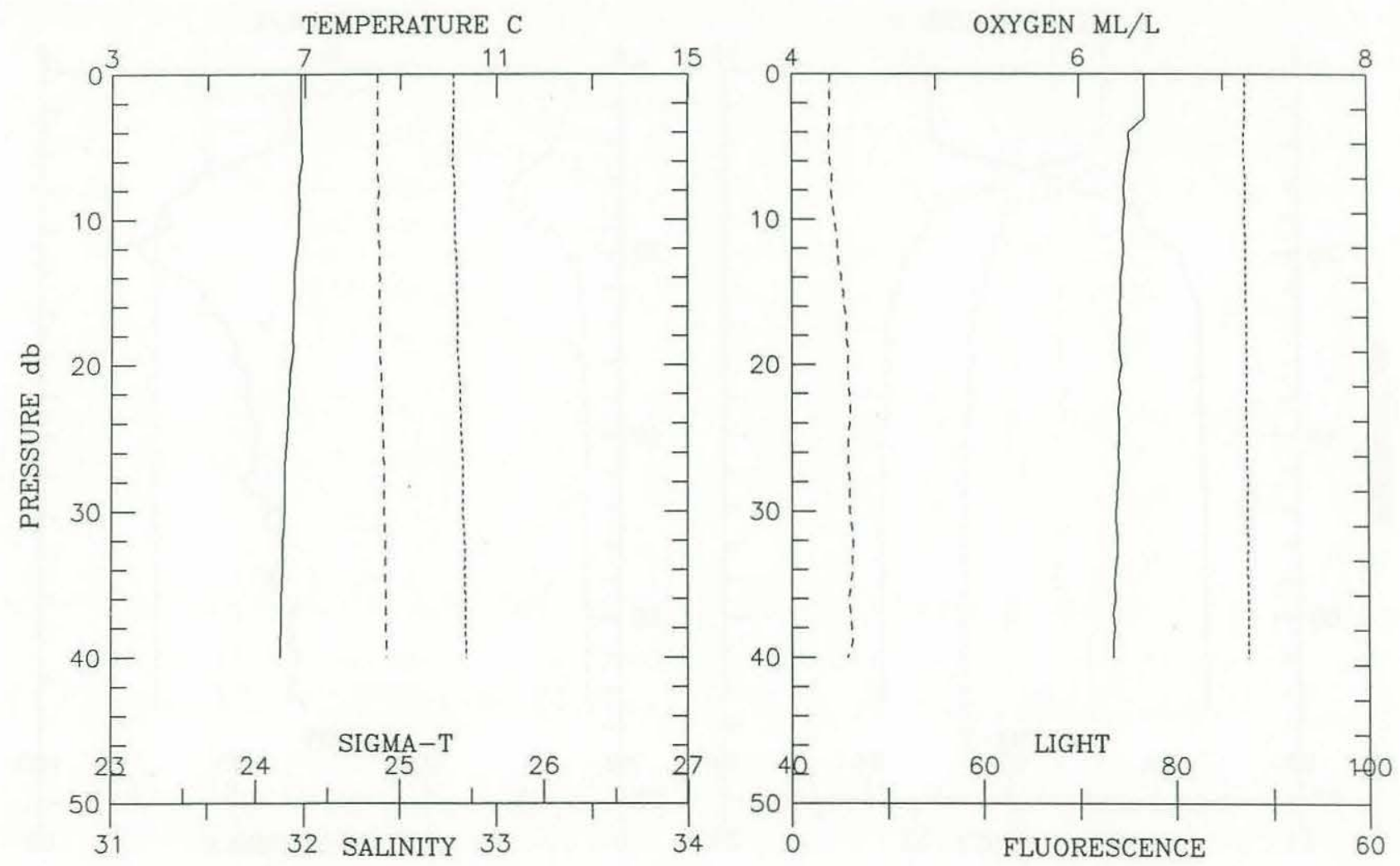


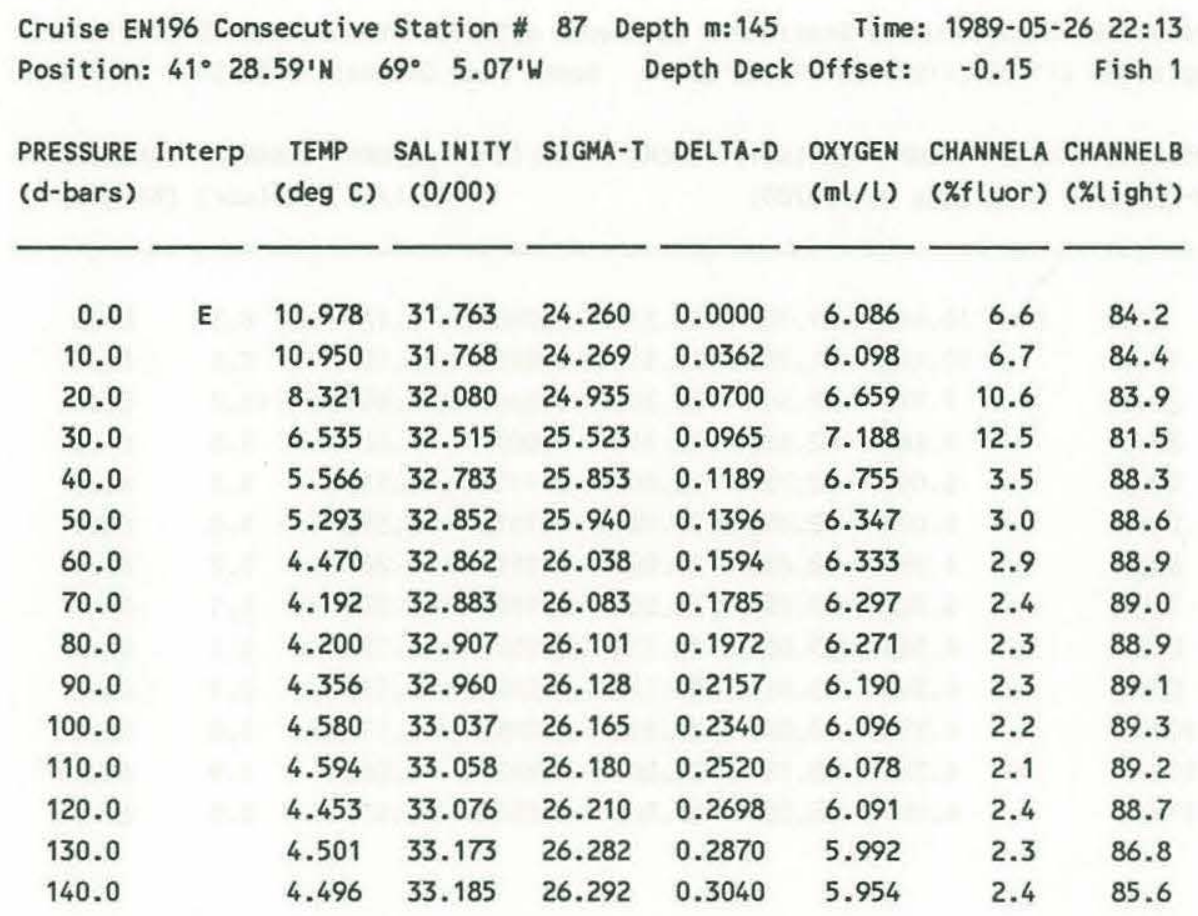
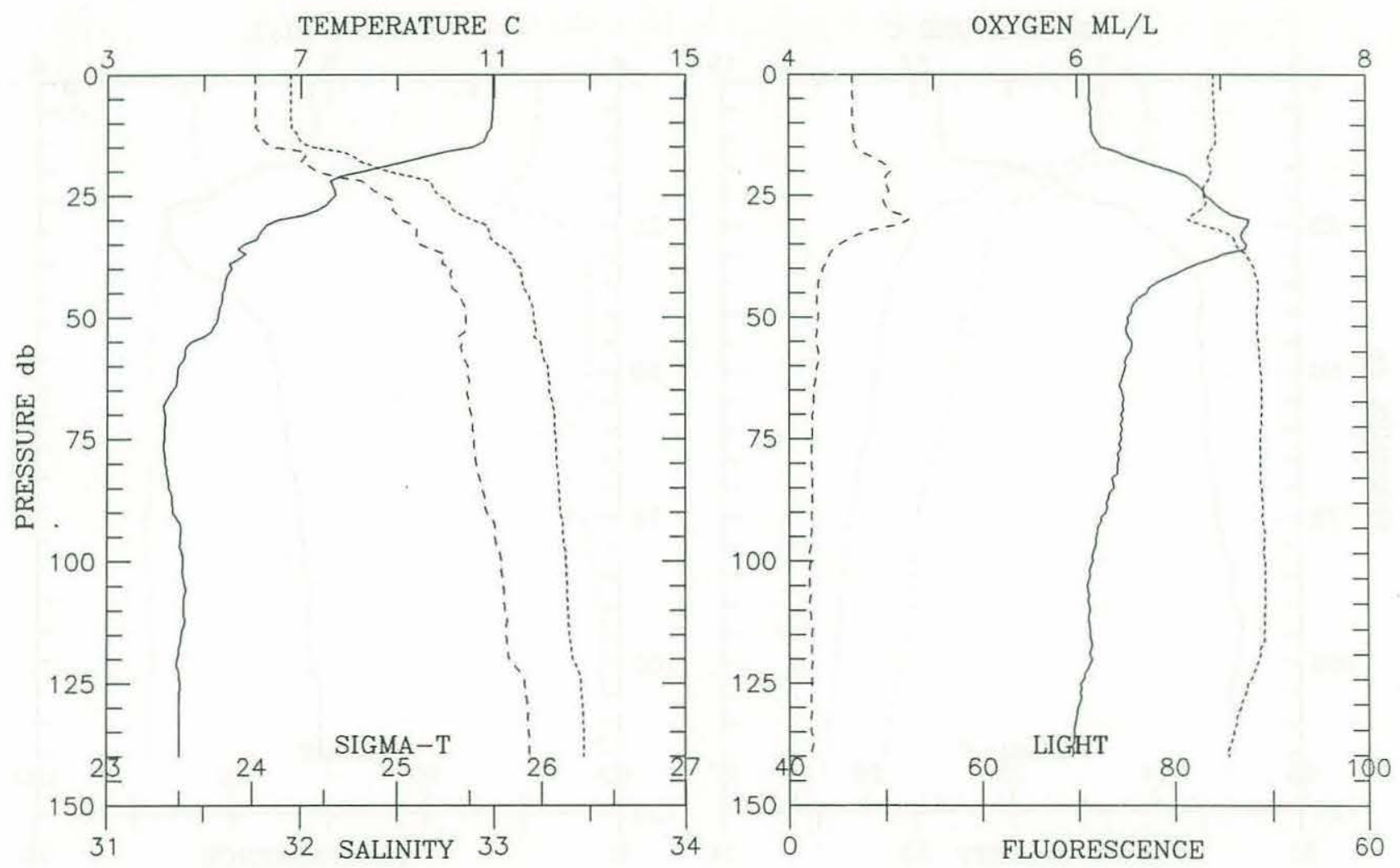


\begin{tabular}{|c|c|c|c|c|c|c|c|c|}
\hline $\begin{array}{l}\text { Cruise EN } \\
\text { Position: }\end{array}$ & $\begin{array}{l}\text { N196 Con } \\
: 41^{\circ} 28\end{array}$ & $\begin{array}{l}\text { secutive } \\
.711 \mathrm{~N}\end{array}$ & $\begin{array}{l}\text { Station } \\
69^{\circ} \quad 5.32\end{array}$ & Def & $\begin{array}{l}\text { th } \mathrm{m}: 143 \\
\text { epth Deck }\end{array}$ & $\begin{array}{r}\text { Time } \\
\text { offset: }\end{array}$ & $\begin{array}{c}1989-05- \\
-0.15\end{array}$ & $\begin{array}{r}701: 06 \\
\text { Fish } 1\end{array}$ \\
\hline $\begin{array}{l}\text { PRESSURE } \\
\text { (d-bars) }\end{array}$ & Interp & $\begin{array}{c}\text { TEMP } \\
\text { (deg C) }\end{array}$ & $\begin{array}{l}\text { SALINITY } \\
(0 / 00)\end{array}$ & SIGMA-T & DELTA-D & $\begin{array}{l}\text { OXYGEN } \\
(m l / l)\end{array}$ & $\begin{array}{l}\text { CHANNELA } \\
\text { (\%fluor) }\end{array}$ & $\begin{array}{l}\text { CHANNELB } \\
\text { (\%light) }\end{array}$ \\
\hline 0.0 & E & 10.443 & 31.787 & 24.371 & 0.0000 & 6.121 & 8.3 & 83.8 \\
\hline 10.0 & & 10.442 & 31.785 & 24.370 & 0.0352 & 6.108 & 8.6 & 84.1 \\
\hline 20.0 & & 7.715 & 32.363 & 25.244 & 0.0663 & 6.851 & 12.2 & 82.4 \\
\hline 30.0 & & 5.466 & 32.626 & 25.741 & 0.0897 & 7.024 & 3.6 & 87.6 \\
\hline 40.0 & & 5.045 & 32.724 & 25.866 & 0.1111 & 6.517 & 3.3 & 88.2 \\
\hline 50.0 & & 5.029 & 32.803 & 25.930 & 0.1316 & 6.338 & 3.0 & 88.4 \\
\hline 60.0 & & 4.953 & 32.858 & 25.983 & 0.1517 & 6.284 & 5.7 & 86.0 \\
\hline 70.0 & & 4.742 & 32.951 & 26.080 & 0.1709 & 6.220 & 5.1 & 88.6 \\
\hline 80.0 & & 4.563 & 33.001 & 26.138 & 0.1895 & 6.153 & 3.1 & 88.8 \\
\hline 90.0 & & 4.343 & 33.014 & 26.172 & 0.2076 & 6.139 & 2.1 & 88.6 \\
\hline 100.0 & & 4.315 & 33.061 & 26.212 & 0.2253 & 6.112 & 1.8 & 88.3 \\
\hline 110.0 & & 4.371 & 33.134 & 26.264 & 0.2427 & 6.060 & 1.9 & 88.4 \\
\hline 119.0 & & 4.494 & 33.203 & 26.306 & 0.2580 & 5.974 & 2.0 & 87.5 \\
\hline
\end{tabular}
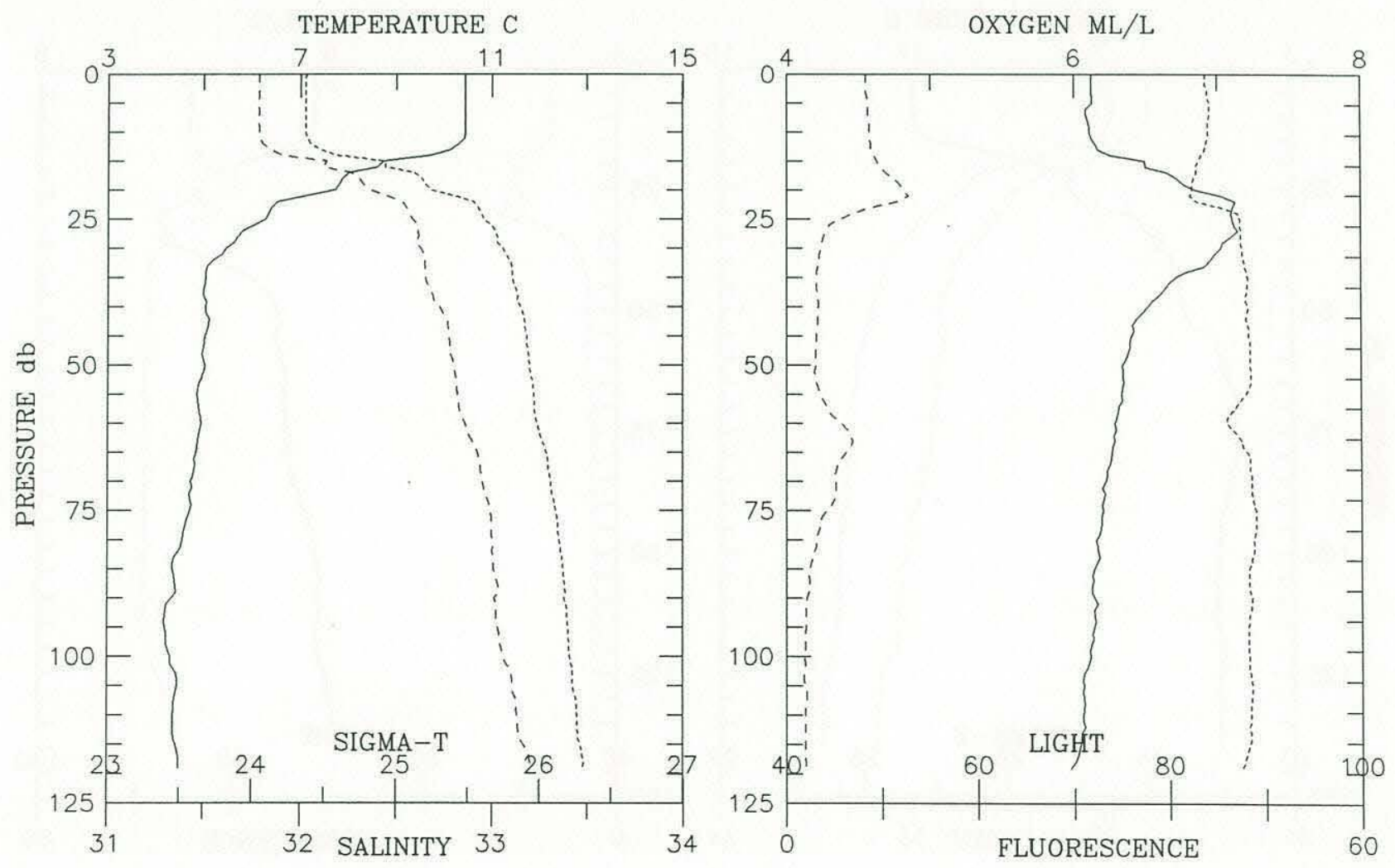


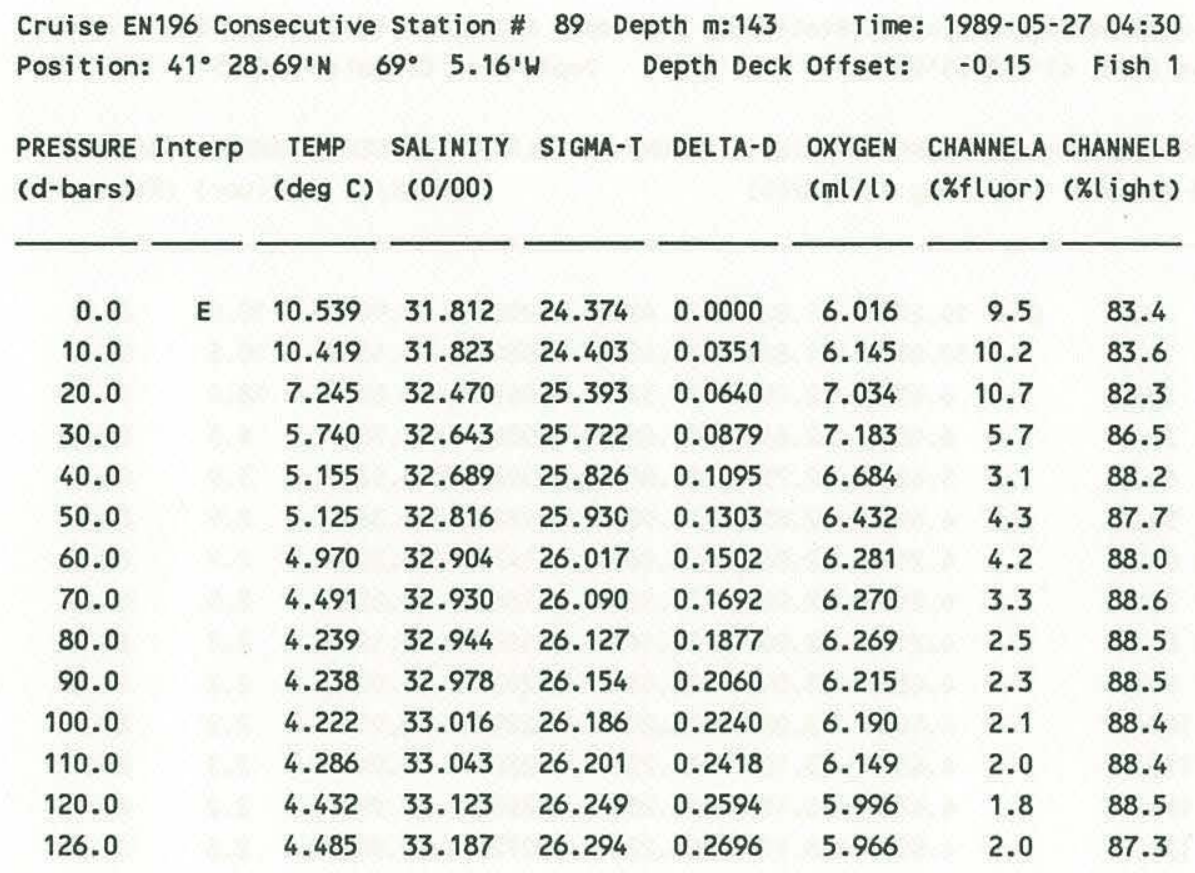
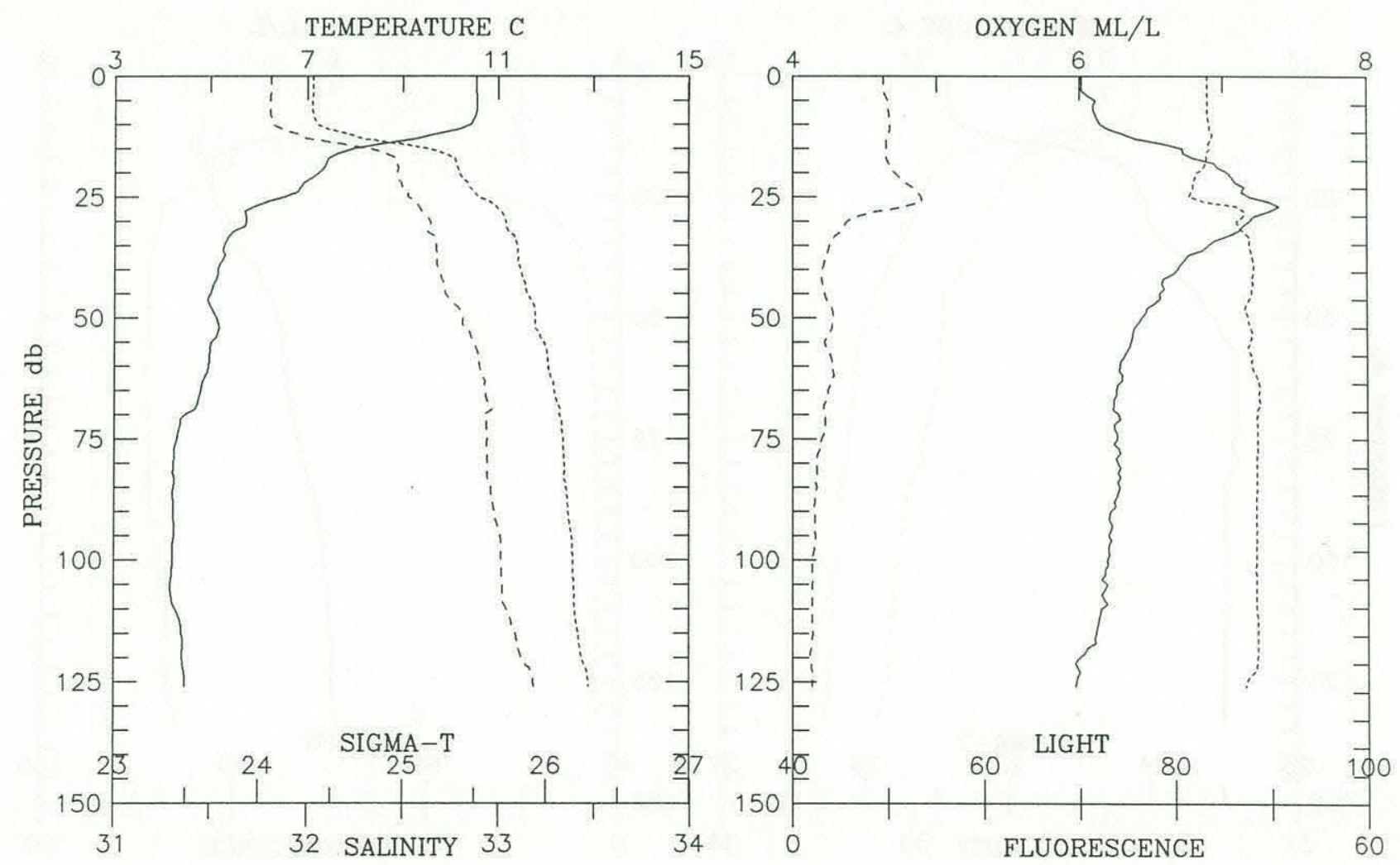


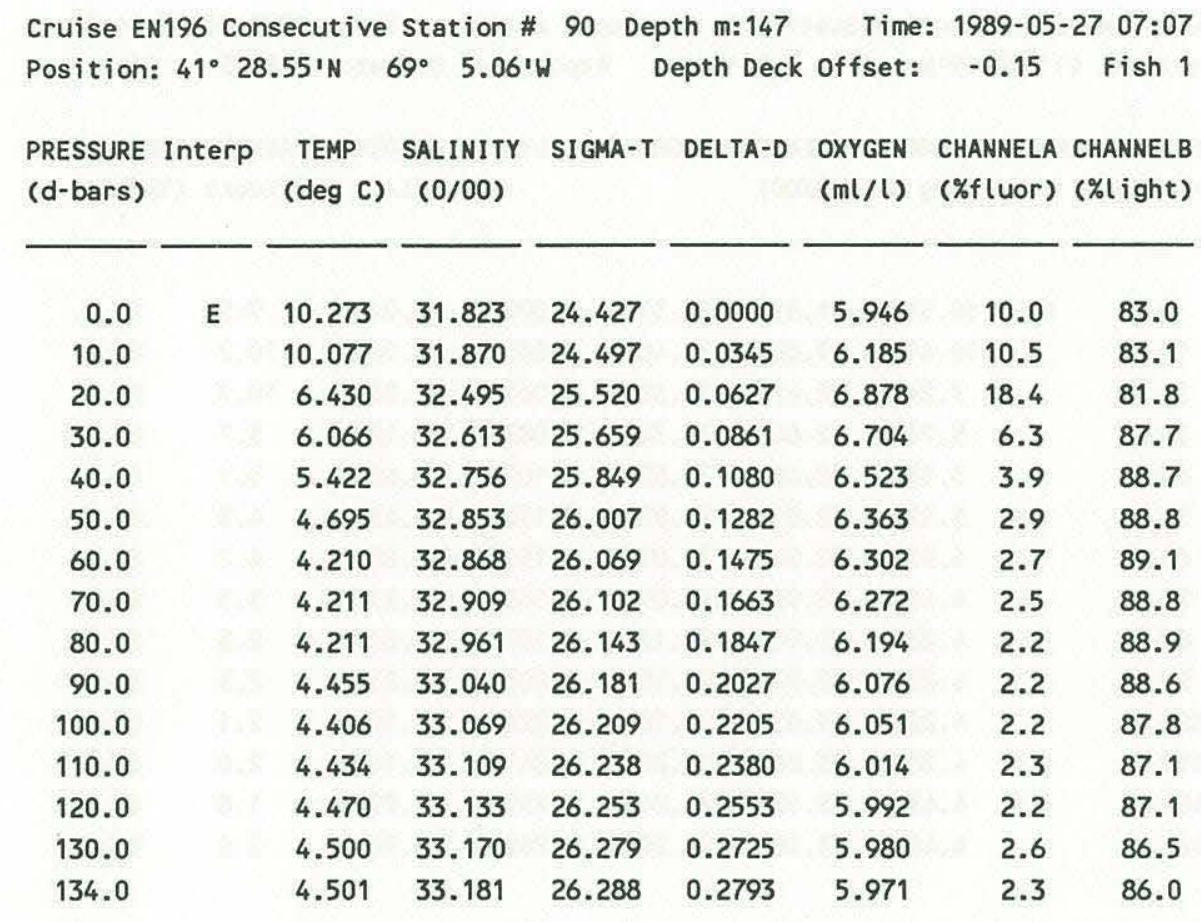
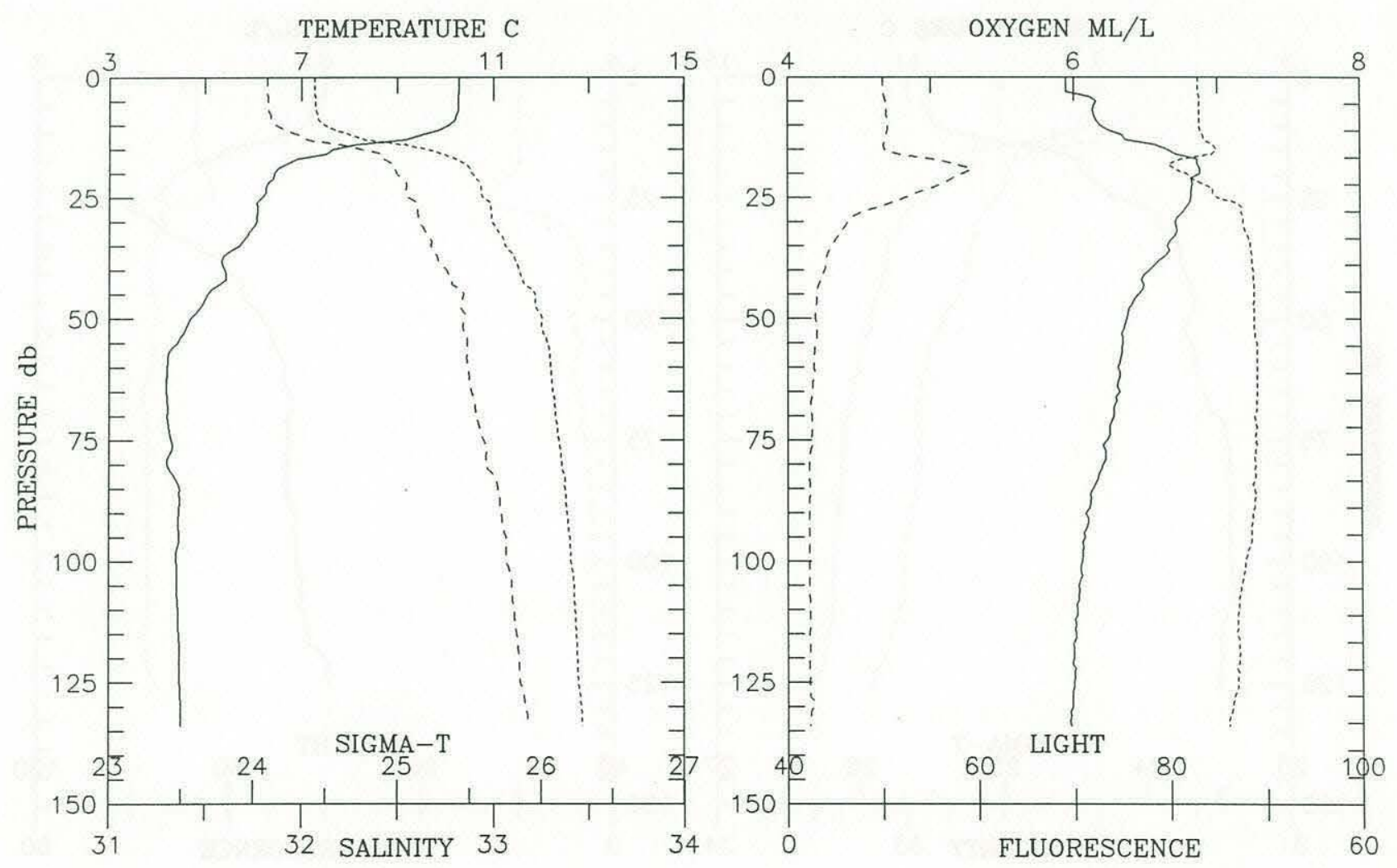


\begin{tabular}{|c|c|c|c|c|c|c|c|c|}
\hline Position: & $=41^{\circ} 28$ & $.45^{\prime} \mathrm{N}$ & $69^{\circ} \quad 5.21^{\prime}$ & & epth Deck & offset: & -0.30 & Fish 1 \\
\hline $\begin{array}{l}\text { PRESSURE } \\
\text { (d-bars) }\end{array}$ & Interp & $\begin{array}{c}\text { TEMP } \\
\text { (deg } \mathrm{C} \text { ) }\end{array}$ & $\begin{array}{c}\text { SALINITY } \\
(0 / 00)\end{array}$ & SIGMA-T & DELTA-D & $\begin{array}{l}\text { OXYGEN } \\
(\mathrm{ml} / \mathrm{l})\end{array}$ & $\begin{array}{l}\text { CHANNELA } \\
\text { ( } \% \text { fluor) }\end{array}$ & $\begin{array}{l}\text { CHANNELB } \\
\text { (\%light) }\end{array}$ \\
\hline 0.0 & E & 10.208 & 31.871 & 24.475 & 0.0000 & 5.929 & 7.5 & 85.0 \\
\hline 10.0 & & 10.216 & 31.875 & 24.477 & 0.0342 & 5.964 & 8.0 & 85.3 \\
\hline 20.0 & & 8.415 & 32.212 & 25.025 & 0.0674 & 6.415 & 9.0 & 83.9 \\
\hline 30.0 & & 5.754 & 32.665 & 25.738 & 0.0910 & 7.035 & 4.6 & 88.0 \\
\hline 40.0 & & 5.438 & 32.831 & 25.907 & 0.1123 & 6.616 & 3.5 & 88.7 \\
\hline 50.0 & & 5.003 & 32.867 & 25.984 & 0.1323 & 6.381 & 2.8 & 88.9 \\
\hline 60.0 & & 4.390 & 32.871 & 26.053 & 0.1517 & 6.411 & 2.7 & 89.0 \\
\hline 70.0 & & 4.191 & 32.893 & 26.092 & 0.1706 & 6.309 & 2.3 & 89.0 \\
\hline 80.0 & & 4.302 & 32.931 & 26.110 & 0.1892 & 6.287 & 2.4 & 89.0 \\
\hline 90.0 & & 4.395 & 32.984 & 26.142 & 0.2076 & 6.204 & 2.3 & 88.9 \\
\hline 100.0 & & 4.462 & 33.032 & 26.174 & 0.2258 & 6.134 & 2.3 & 89.0 \\
\hline 110.0 & & 4.415 & 33.065 & 26.205 & 0.2436 & 6.131 & 2.0 & 88.6 \\
\hline 120.0 & & 4.490 & 33.169 & 26.279 & 0.2610 & 6.023 & 2.1 & 87.9 \\
\hline 130.0 & & 4.496 & 33.193 & 26.298 & 0.2779 & 5.965 & 2.1 & 87.0 \\
\hline 140.0 & & 4.501 & 33.195 & 26.299 & 0.2947 & 5.950 & 2.1 & 84.7 \\
\hline 143.0 & & 4.501 & 33.195 & 26.299 & 0.2997 & 5.948 & 2.2 & 84.6 \\
\hline
\end{tabular}
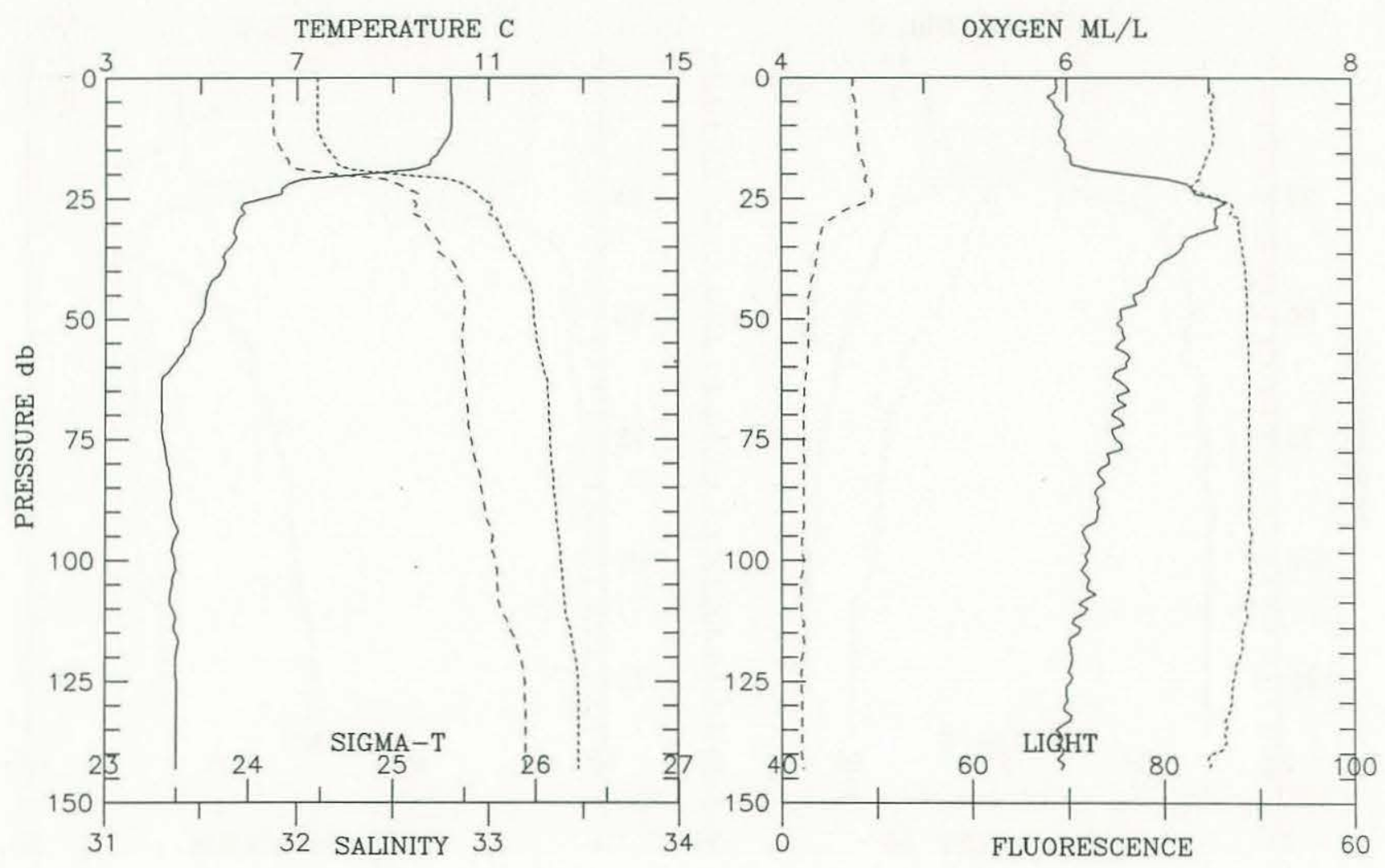


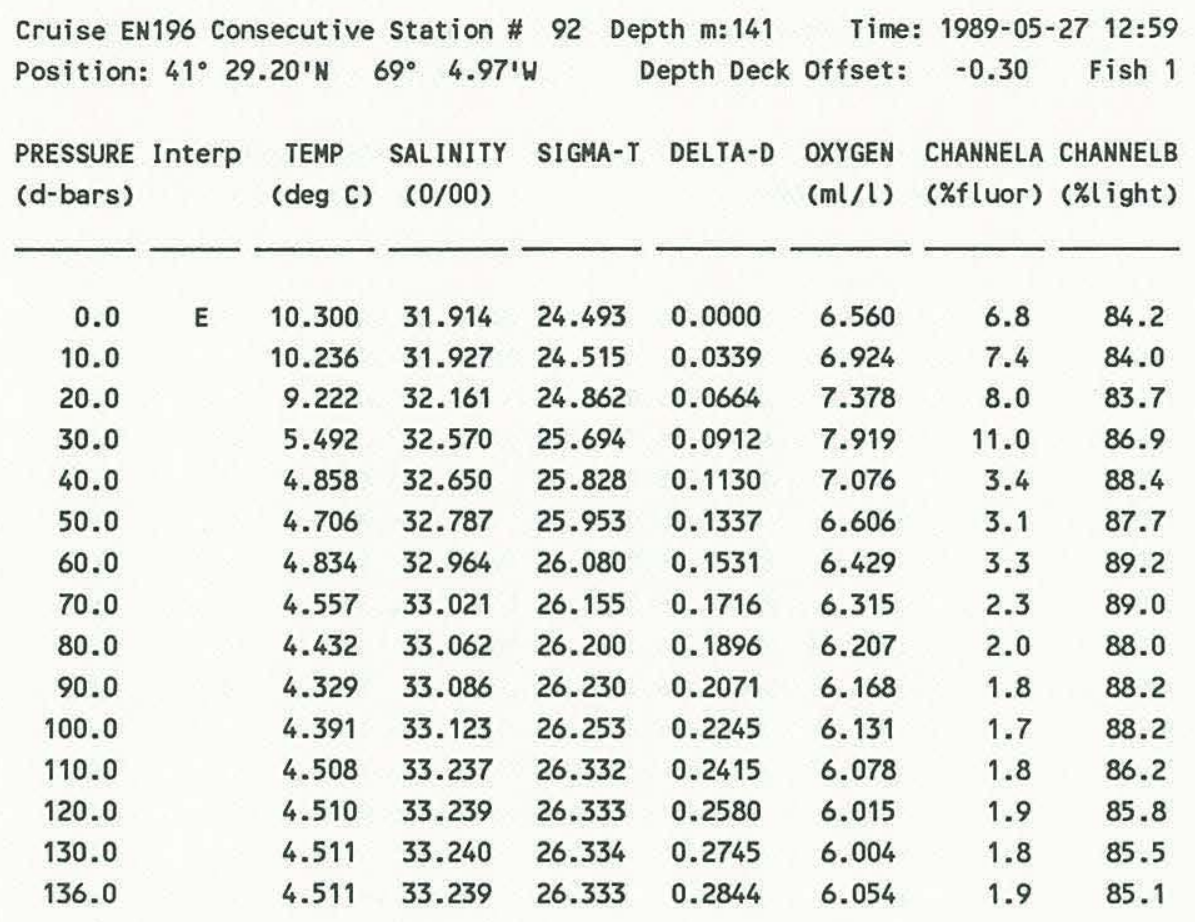
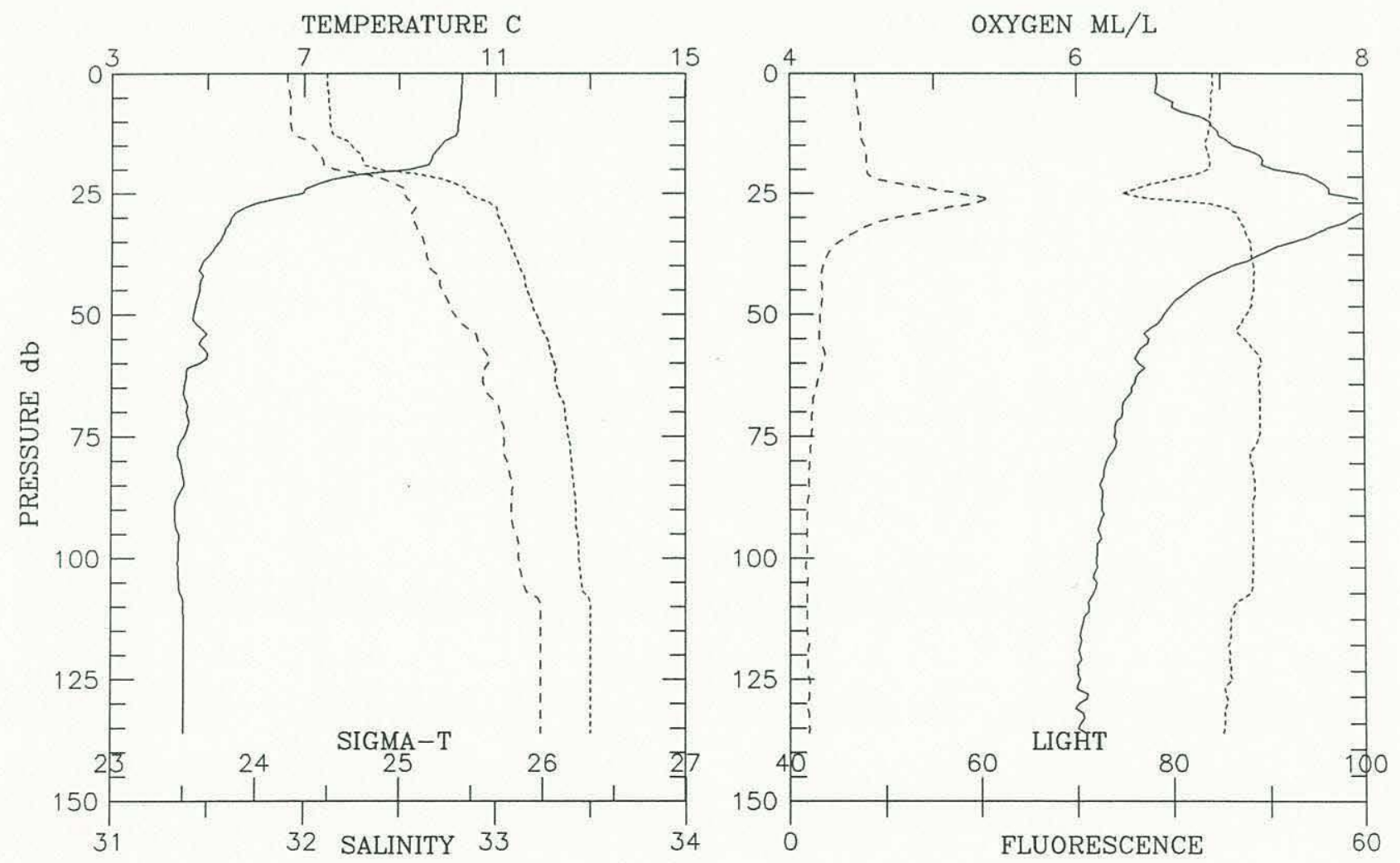


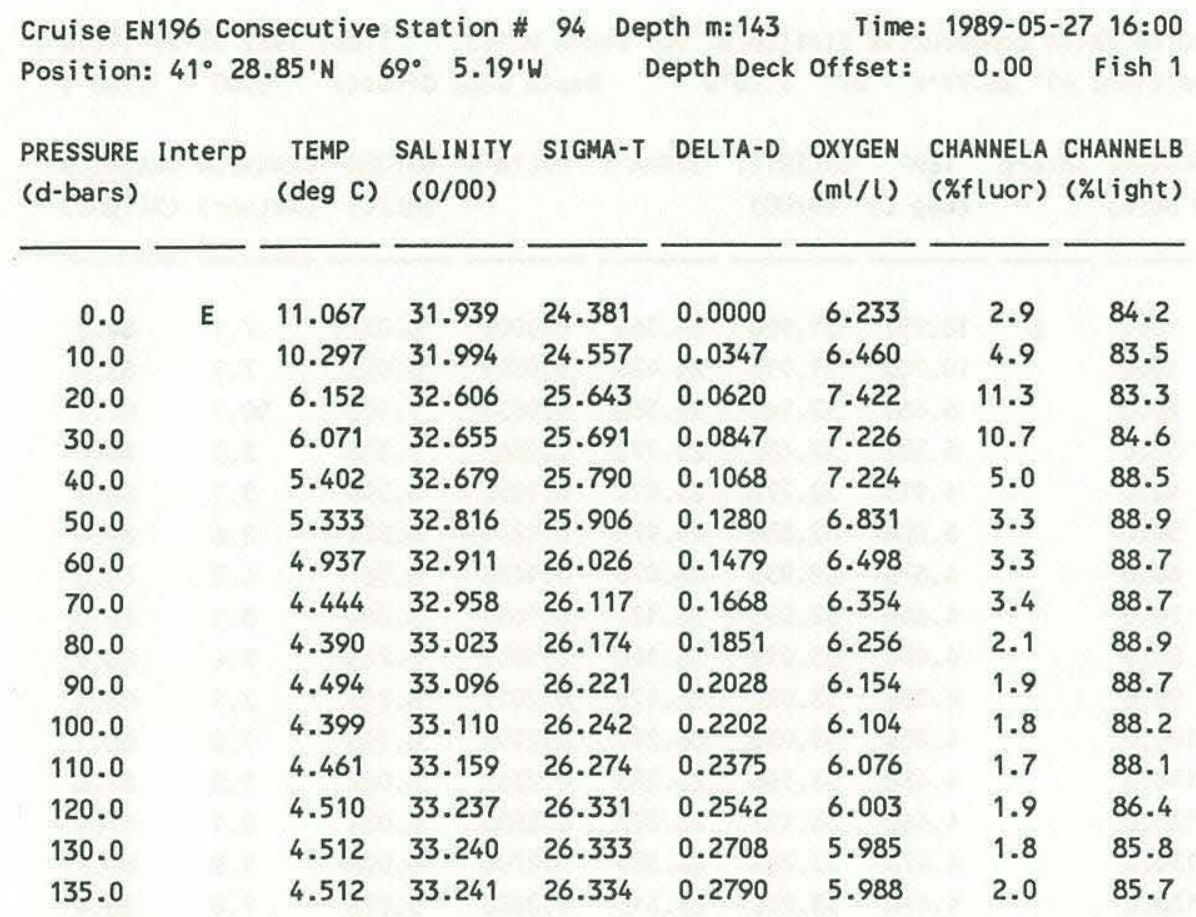
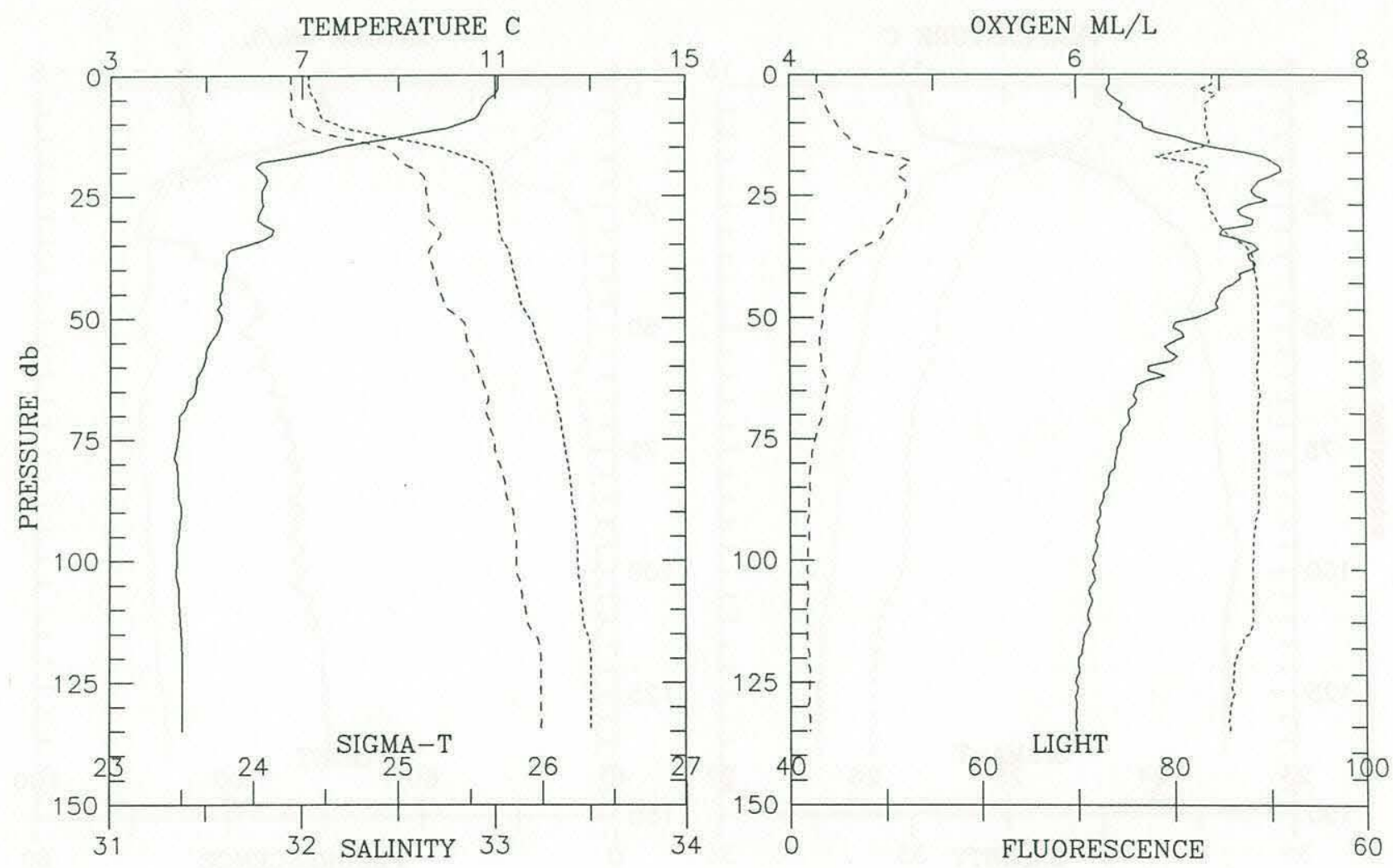


\begin{tabular}{|c|c|c|c|c|c|c|c|c|}
\hline Position: & $=41^{\circ} 28$ & Nי & $69^{\circ} \quad 5.20^{\circ}$ & & epth Deck & offset: & 0.00 & Fish 1 \\
\hline $\begin{array}{l}\text { PRESSURE } \\
\text { (d-bars) }\end{array}$ & Interp & $\begin{array}{c}\text { TEMP } \\
(\operatorname{deg} \mathrm{C})\end{array}$ & $\begin{array}{l}\text { SALINITY } \\
(0 / 00)\end{array}$ & SIGMA-T & DELTA-D & $\begin{array}{l}\text { OXYGEN } \\
(\mathrm{ml} / \mathrm{l})\end{array}$ & $\begin{array}{l}\text { CHANNELA } \\
\text { (\%fluor) }\end{array}$ & $\begin{array}{l}\text { CHANNELB } \\
\text { (\%light) }\end{array}$ \\
\hline 0.0 & E & 10.997 & 31.900 & 24.363 & 0.0000 & 6.032 & 7.1 & 84.8 \\
\hline 10.0 & & 10.705 & 31.915 & 24.426 & 0.0351 & 6.055 & 7.8 & 83.6 \\
\hline 20.0 & & 6.461 & 32.560 & 25.568 & 0.0635 & 7.196 & 10.1 & 87.1 \\
\hline 30.0 & & 5.307 & 32.650 & 25.778 & 0.0863 & 7.336 & 3.3 & 88.4 \\
\hline 40.0 & & 4.913 & 32.770 & 25.917 & 0.1073 & 6.599 & 3.1 & 88.3 \\
\hline 50.0 & & 5.008 & 32.856 & 25.975 & 0.1274 & 6.552 & 2.6 & 89.0 \\
\hline 60.0 & & 4.675 & 32.933 & 26.073 & 0.1468 & 6.369 & 4.3 & 88.6 \\
\hline 70.0 & & 4.609 & 32.993 & 26.127 & 0.1654 & 6.264 & 3.1 & 89.0 \\
\hline 80.0 & & 4.496 & 33.019 & 26.160 & 0.1837 & 6.246 & 2.4 & 88.9 \\
\hline 90.0 & & 4.386 & 33.028 & 26.179 & 0.2017 & 6.213 & 2.1 & 88.5 \\
\hline 100.0 & & 4.256 & 33.050 & 26.209 & 0.2194 & 6.130 & 1.8 & 88.1 \\
\hline 110.0 & & 4.432 & 33.166 & 26.283 & 0.2367 & 6.062 & 1.8 & 87.6 \\
\hline 120.0 & & 4.460 & 33.191 & 26.300 & 0.2536 & 6.021 & 2.1 & 87.3 \\
\hline 130.0 & & 4.473 & 33.204 & 26.309 & 0.2704 & 6.006 & 1.9 & 86.7 \\
\hline 138.0 & & 4.478 & 33.210 & 26.313 & 0.2838 & 5.976 & 2.0 & 86.4 \\
\hline
\end{tabular}
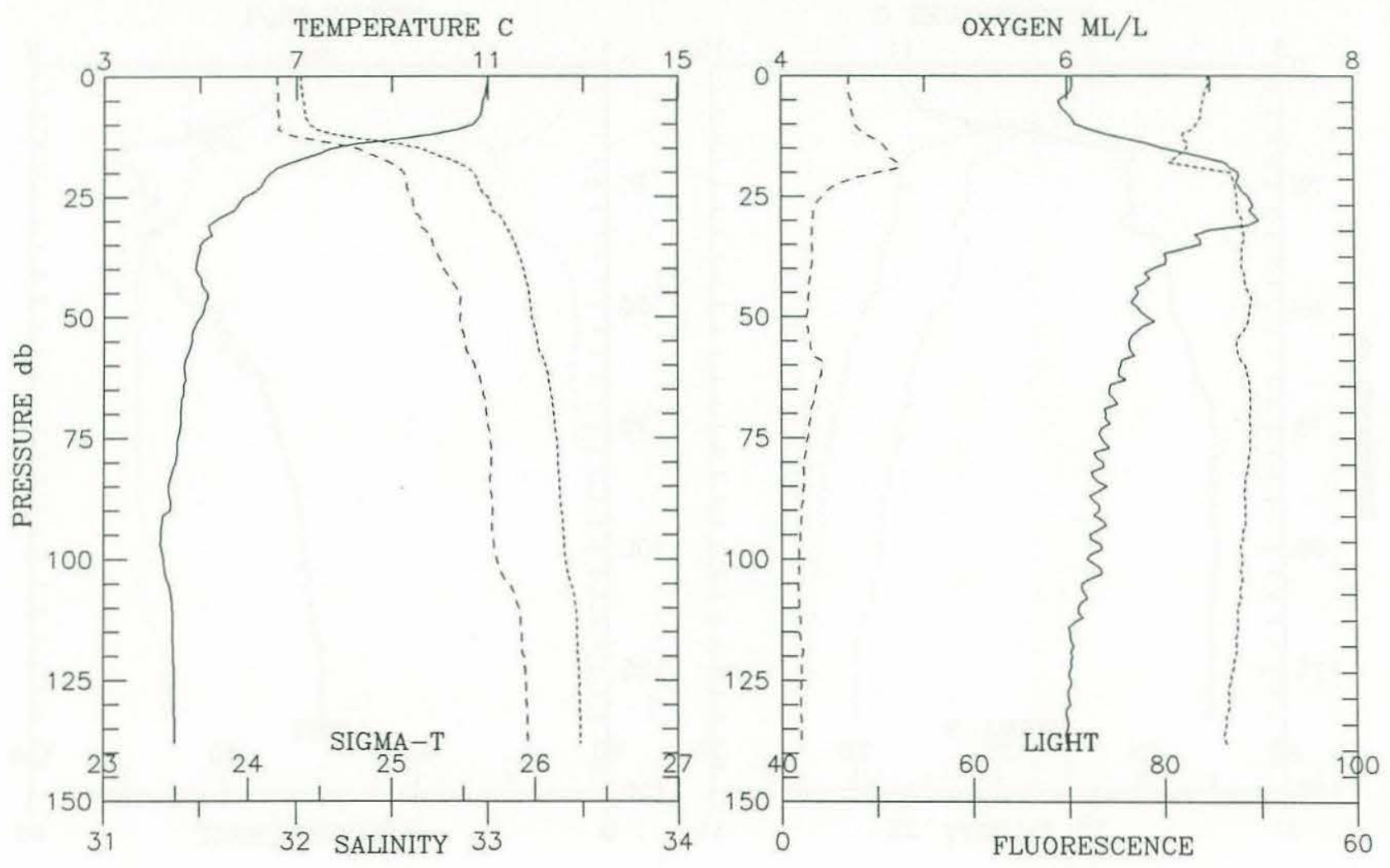


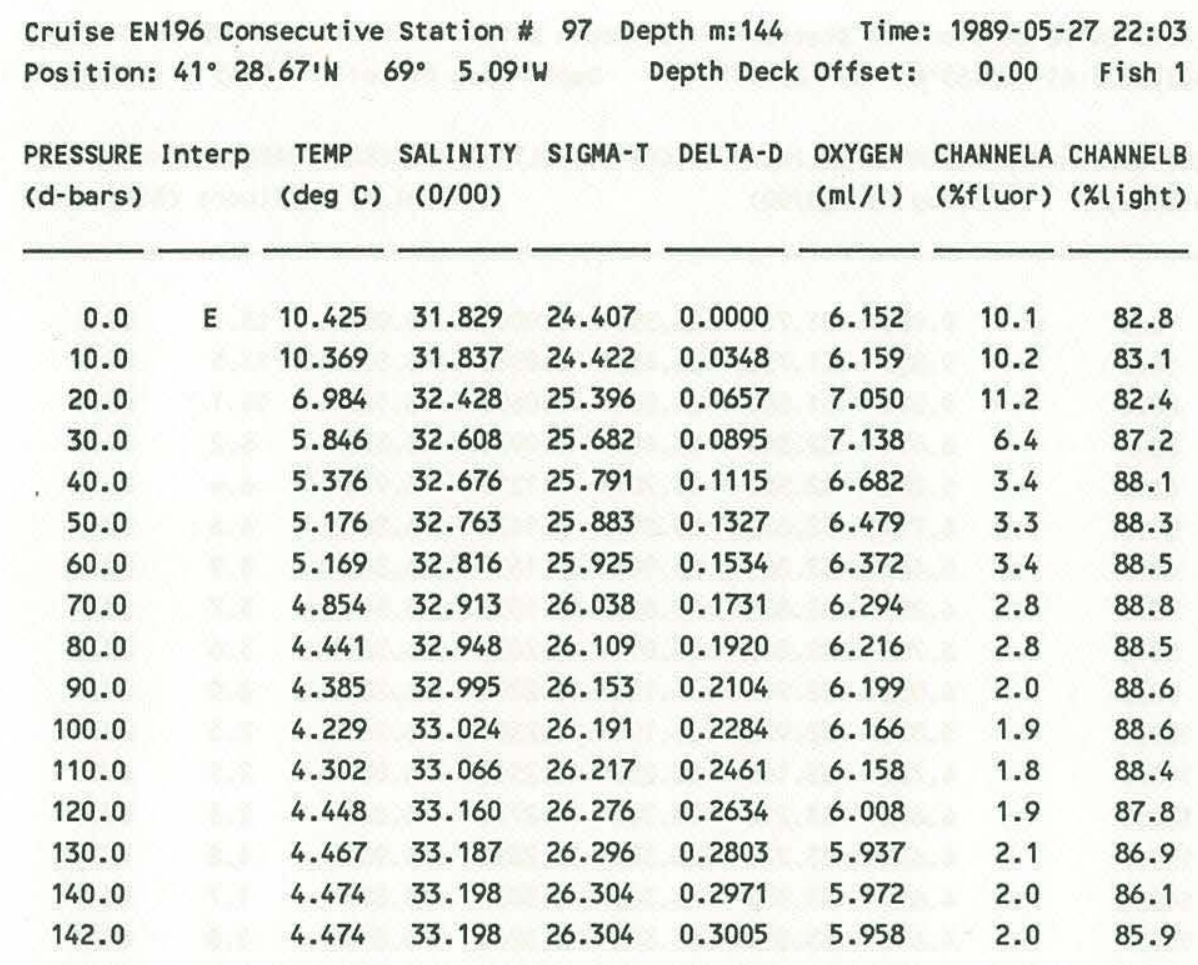
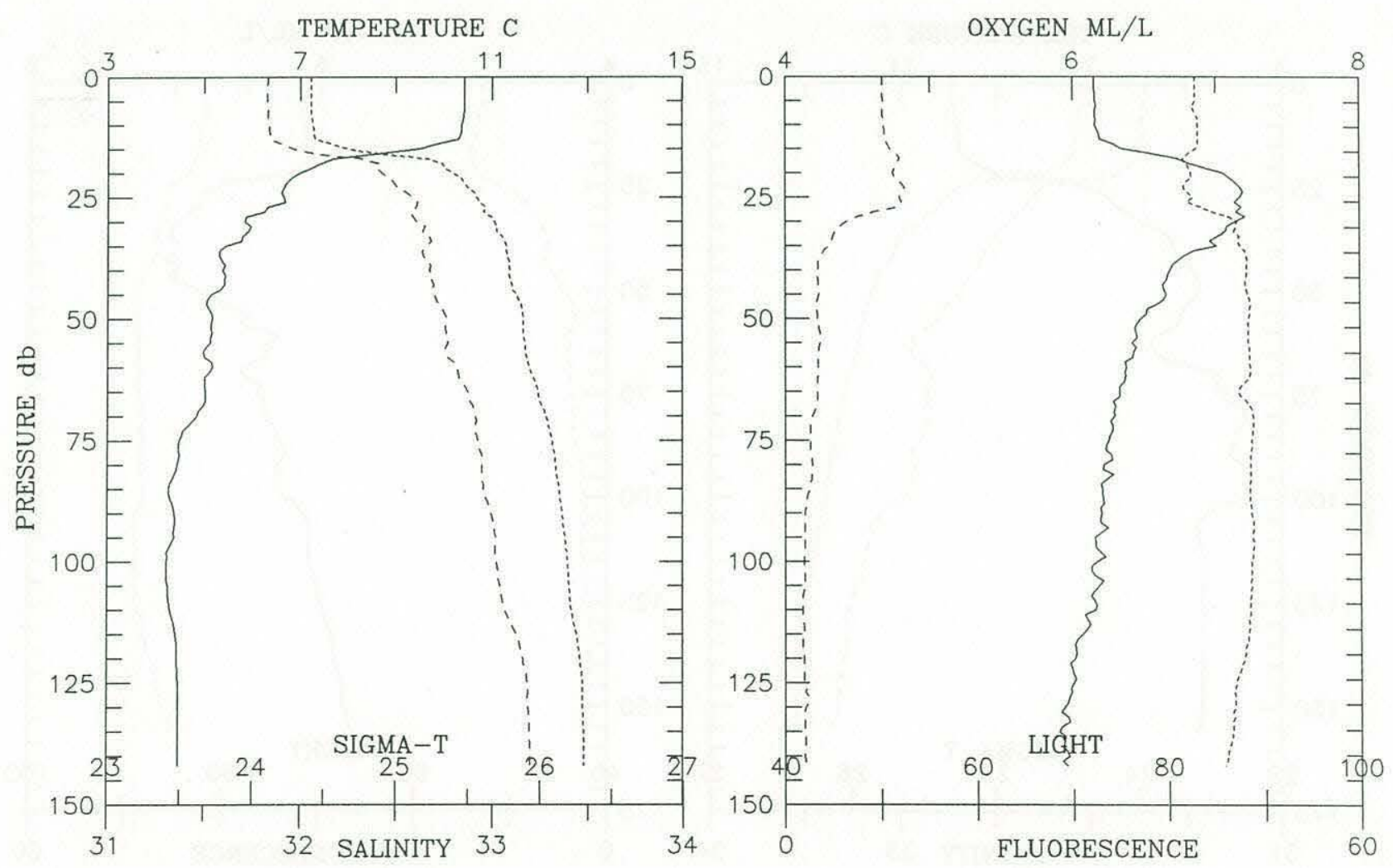


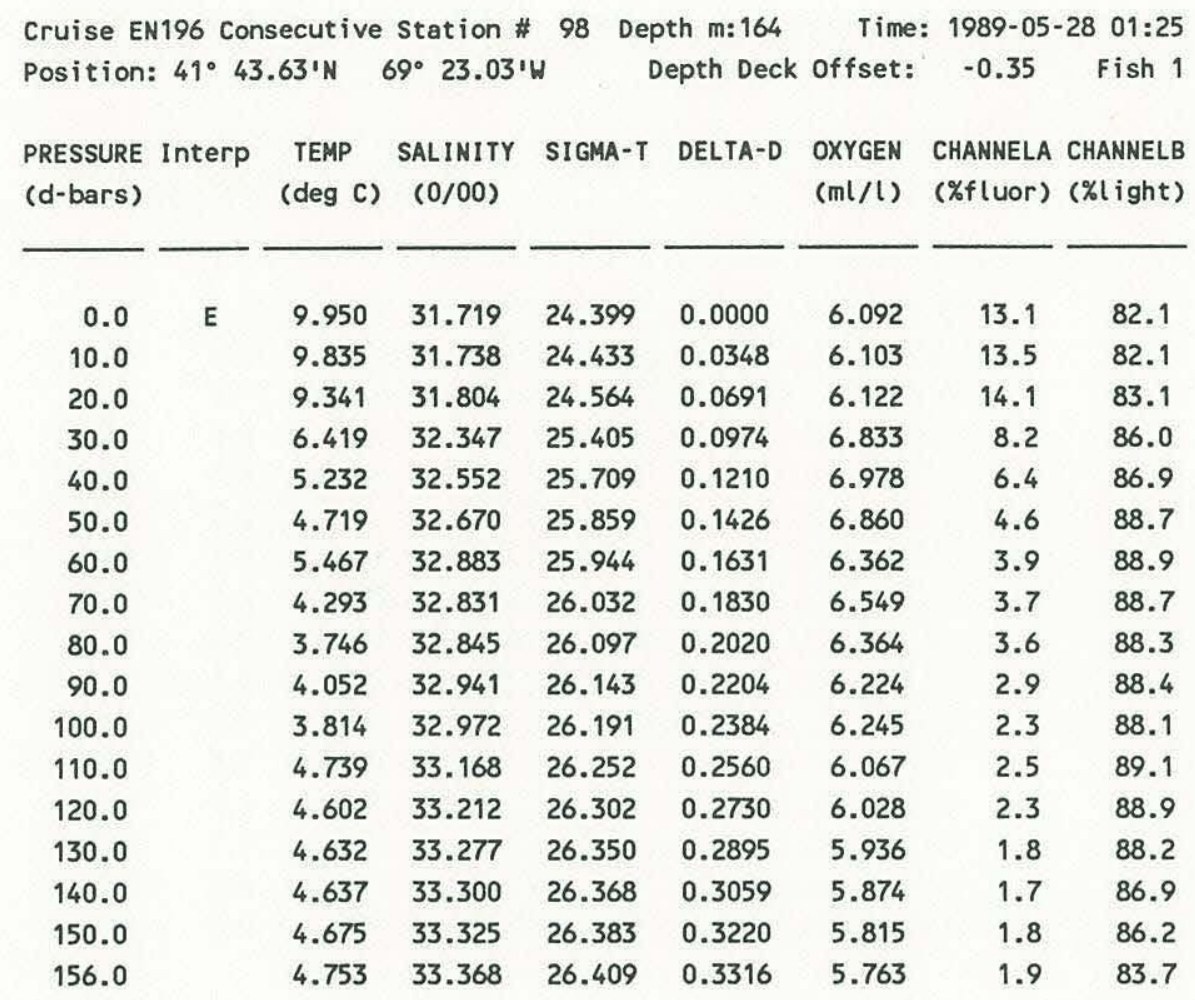
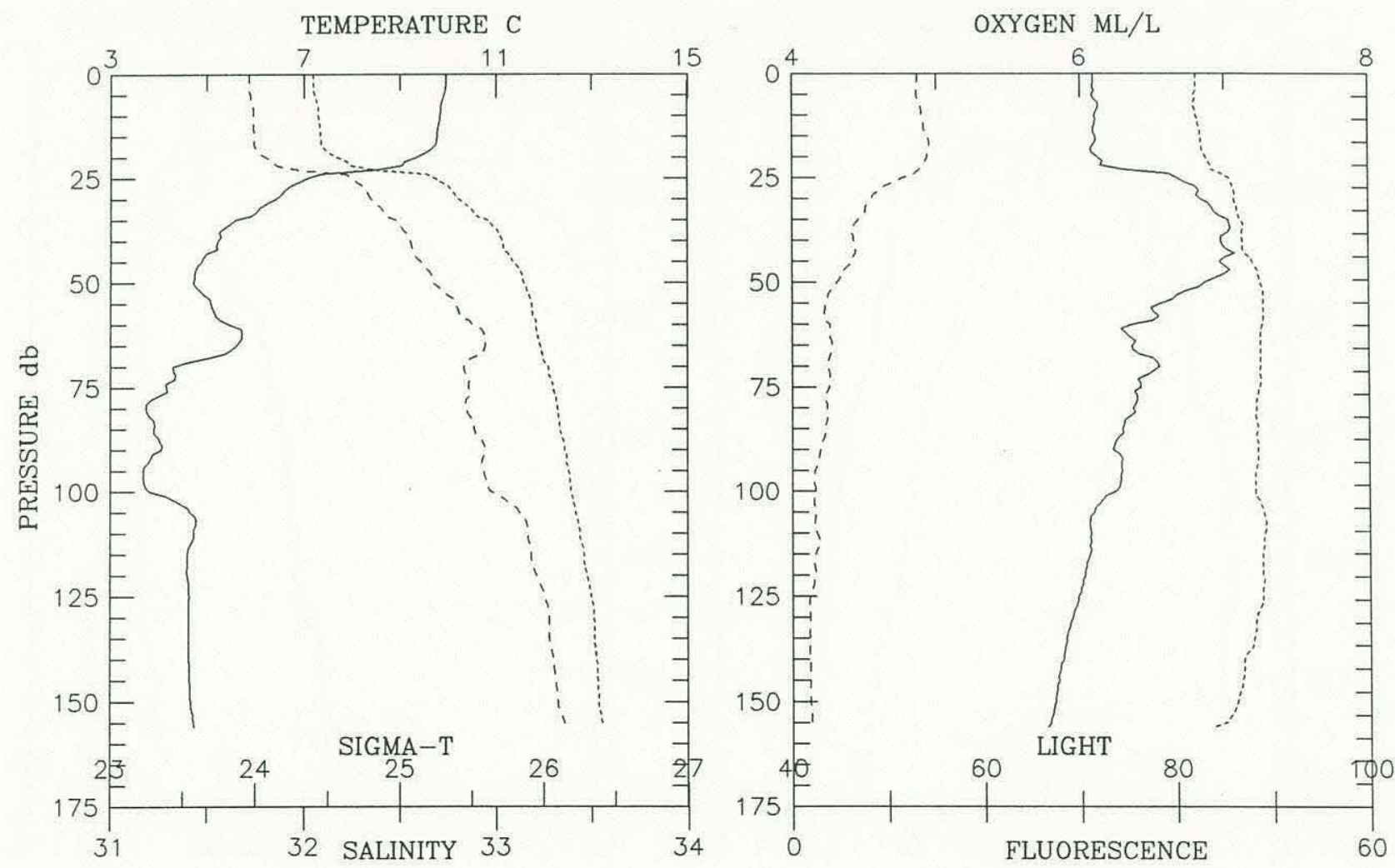


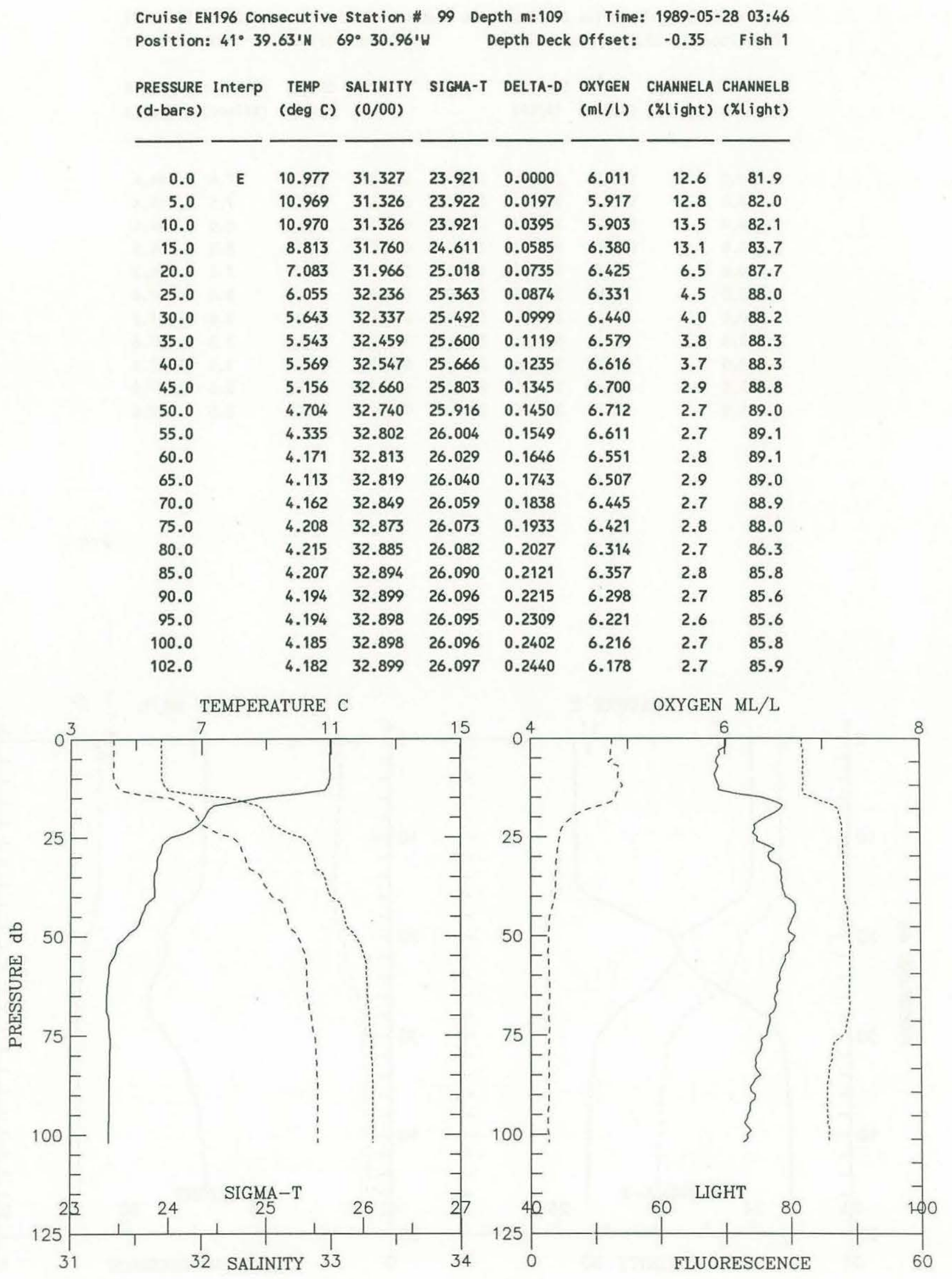




\begin{tabular}{|c|c|c|c|c|c|c|c|c|}
\hline $\begin{array}{l}\text { Cruise EN } \\
\text { Position: }\end{array}$ & \multicolumn{2}{|c|}{$\begin{array}{l}\text { N196 Consecutive } \\
=41^{\circ} 35.38^{\prime} \mathrm{N}\end{array}$} & $\begin{array}{l}\text { Station \# } 1 \\
69^{\circ} 37.80 \text { W }\end{array}$ & \multicolumn{2}{|c|}{ Depth Deck } & Time: & $\begin{array}{l}1989-05- \\
-0.35\end{array}$ & $\begin{array}{r}-2806: 01 \\
\text { Fish } 1\end{array}$ \\
\hline $\begin{array}{l}\text { PRESSURE } \\
\text { (d-bars) }\end{array}$ & Interp & $\begin{array}{c}\text { TEMP } \\
\text { (deg C) }\end{array}$ & $\begin{array}{c}\text { SALINITY } \\
(0 / 00)\end{array}$ & SIGMA-T & DELTA-D & $\begin{array}{l}\text { OXYGEN } \\
(\mathrm{ml} / \mathrm{l})\end{array}$ & $\begin{array}{l}\text { CHANNELA } \\
\text { (\%fluor) }\end{array}$ & $\begin{array}{l}\text { CHANNELB } \\
\text { (\%light) }\end{array}$ \\
\hline 0.0 & E & 11.433 & 31.530 & 23.999 & 0.0000 & 5.879 & 7.6 & 84.4 \\
\hline 5.0 & & 11.430 & 31.525 & 23.996 & 0.0194 & 5.887 & 7.5 & 84.4 \\
\hline 10.0 & & 11.426 & 31.525 & 23.996 & 0.0388 & 5.905 & 8.0 & 84.4 \\
\hline 15.0 & & 11.338 & 31.528 & 24.014 & 0.0581 & 5.939 & 8.3 & 84.5 \\
\hline 20.0 & & 8.432 & 31.830 & 24.723 & 0.0759 & 6.319 & 7.4 & 86.3 \\
\hline 25.0 & & 7.054 & 31.943 & 25.004 & 0.0913 & 6.443 & 5.4 & 87.4 \\
\hline 30.0 & & 5.155 & 32.380 & 25.582 & 0.1042 & 6.285 & 3.8 & 87.8 \\
\hline 35.0 & & 4.911 & 32.452 & 25.665 & 0.1158 & 6.029 & 3.8 & 87.6 \\
\hline 40.0 & & 4.873 & 32.464 & 25.679 & 0.1271 & 5.936 & 3.6 & 87.4 \\
\hline 45.0 & & 4.822 & 32.485 & 25.702 & 0.1384 & 5.881 & 3.6 & 87.4 \\
\hline 46.0 & & 4.816 & 32.487 & 25.704 & 0.1406 & 5.855 & 3.5 & 87.4 \\
\hline
\end{tabular}
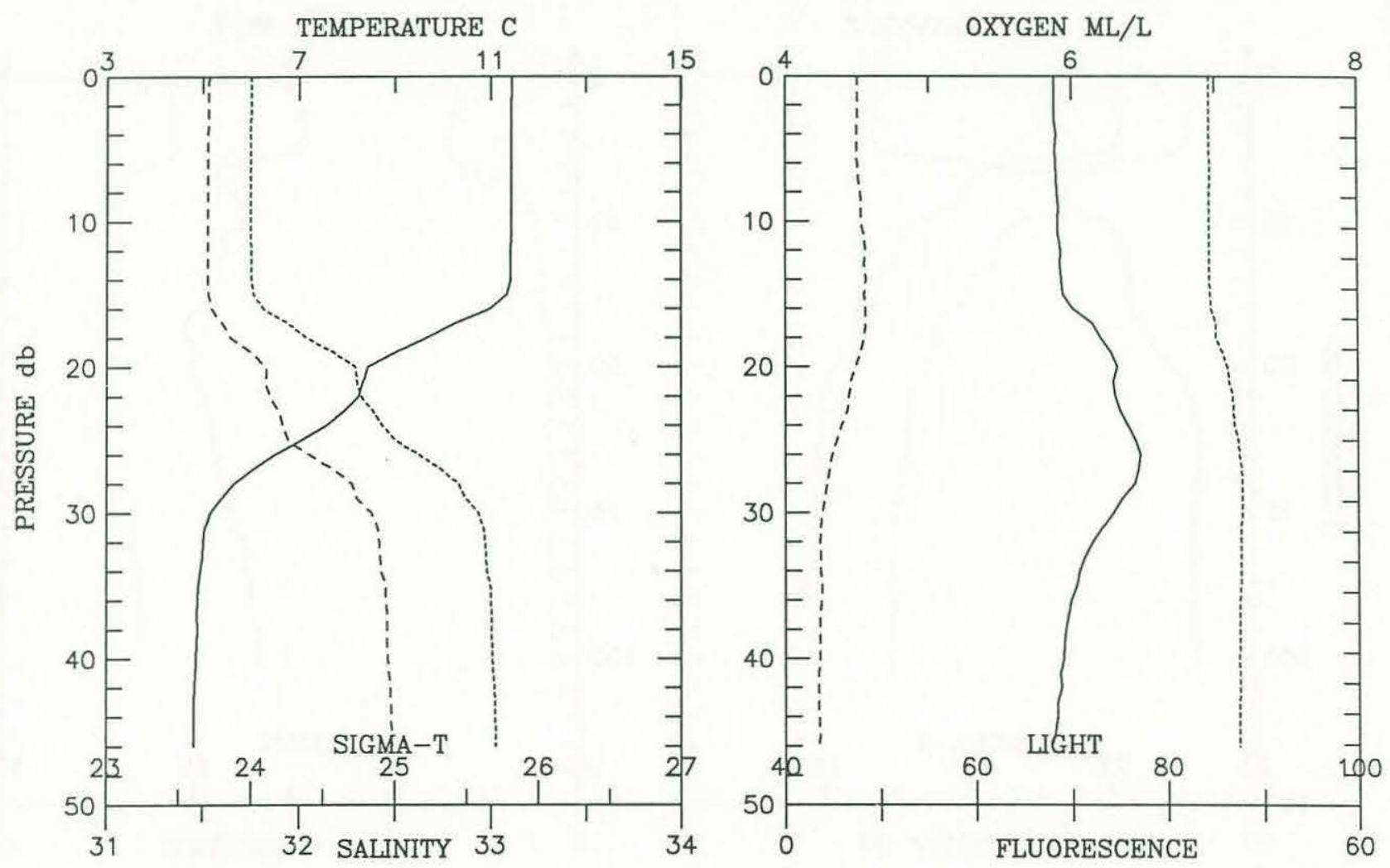


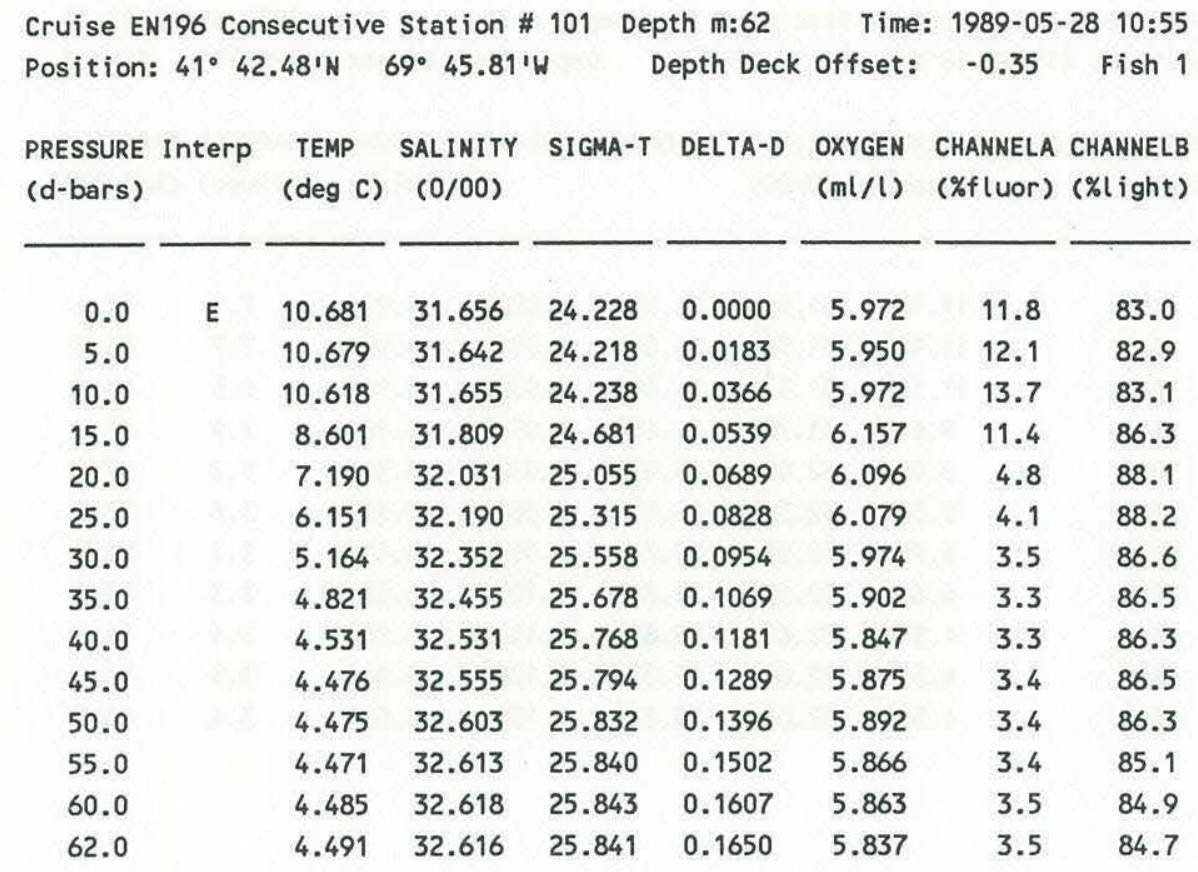
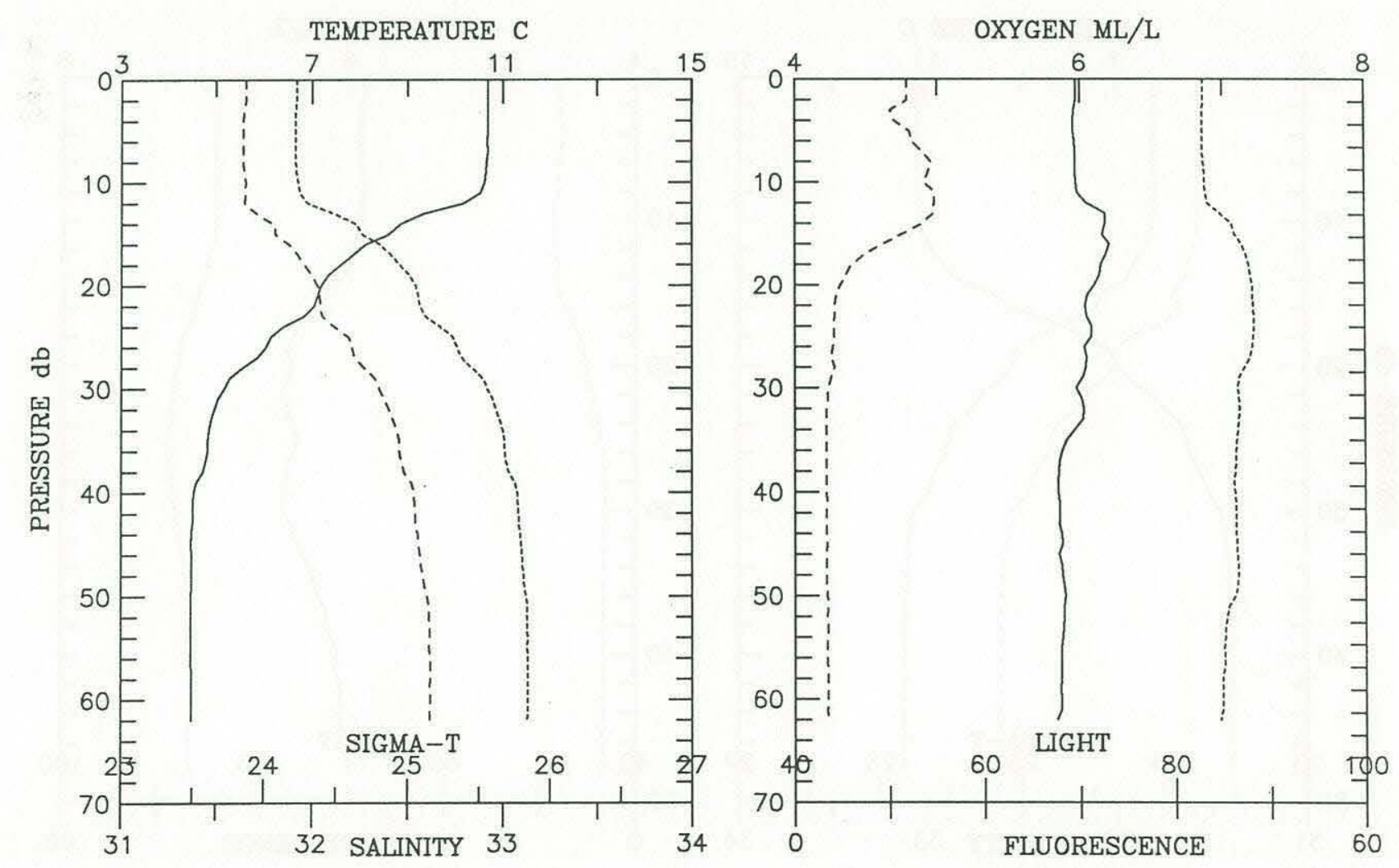


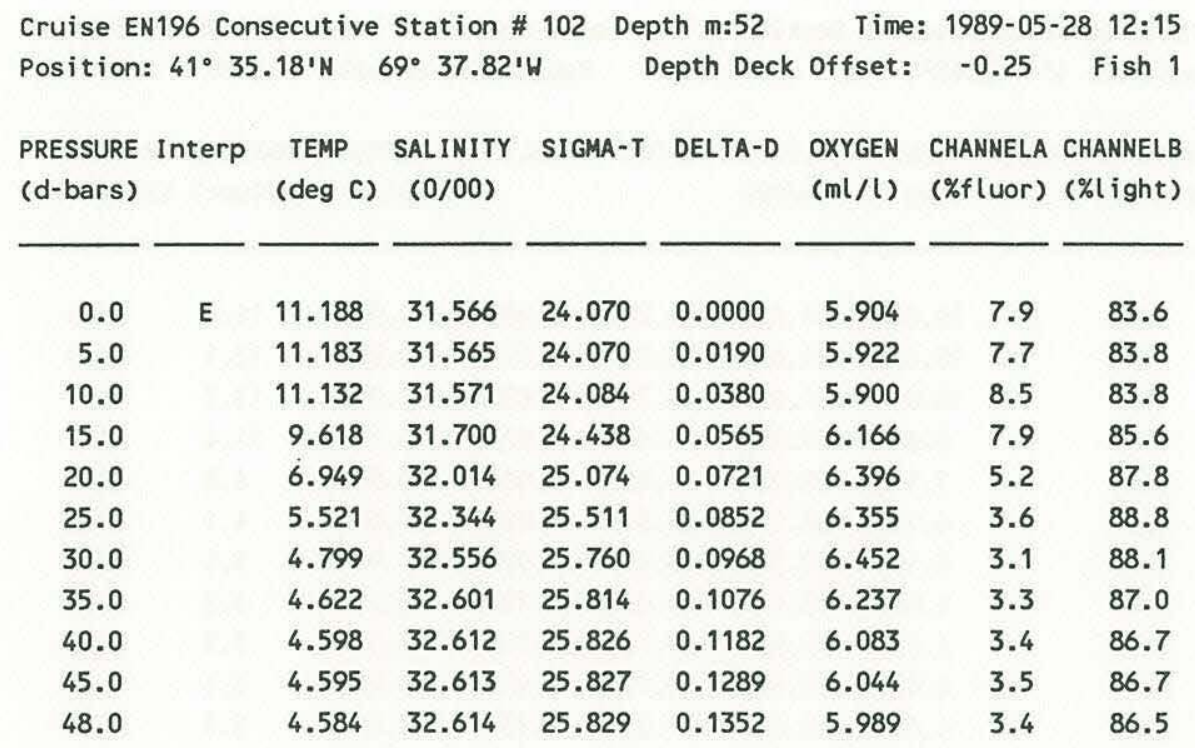
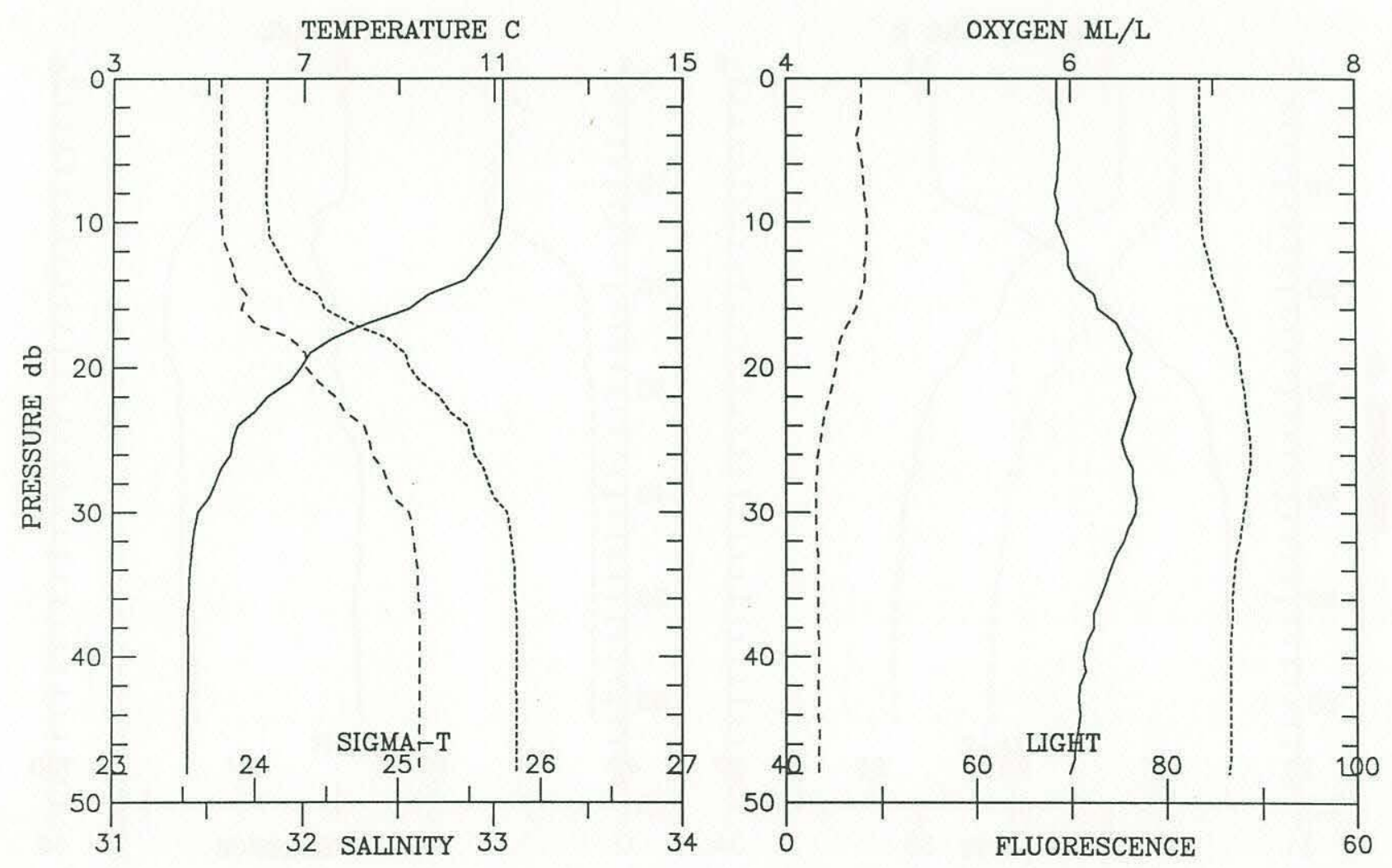


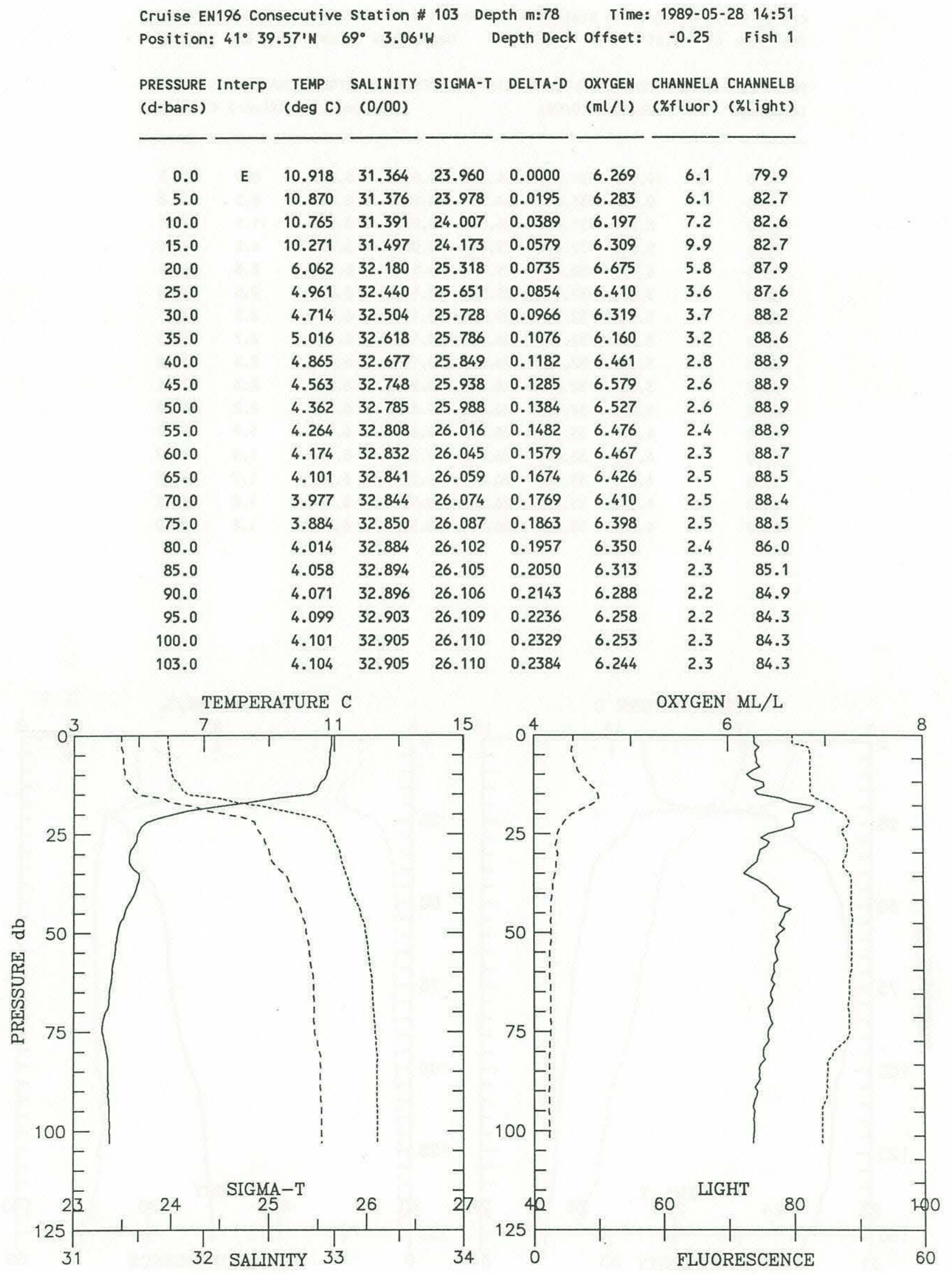




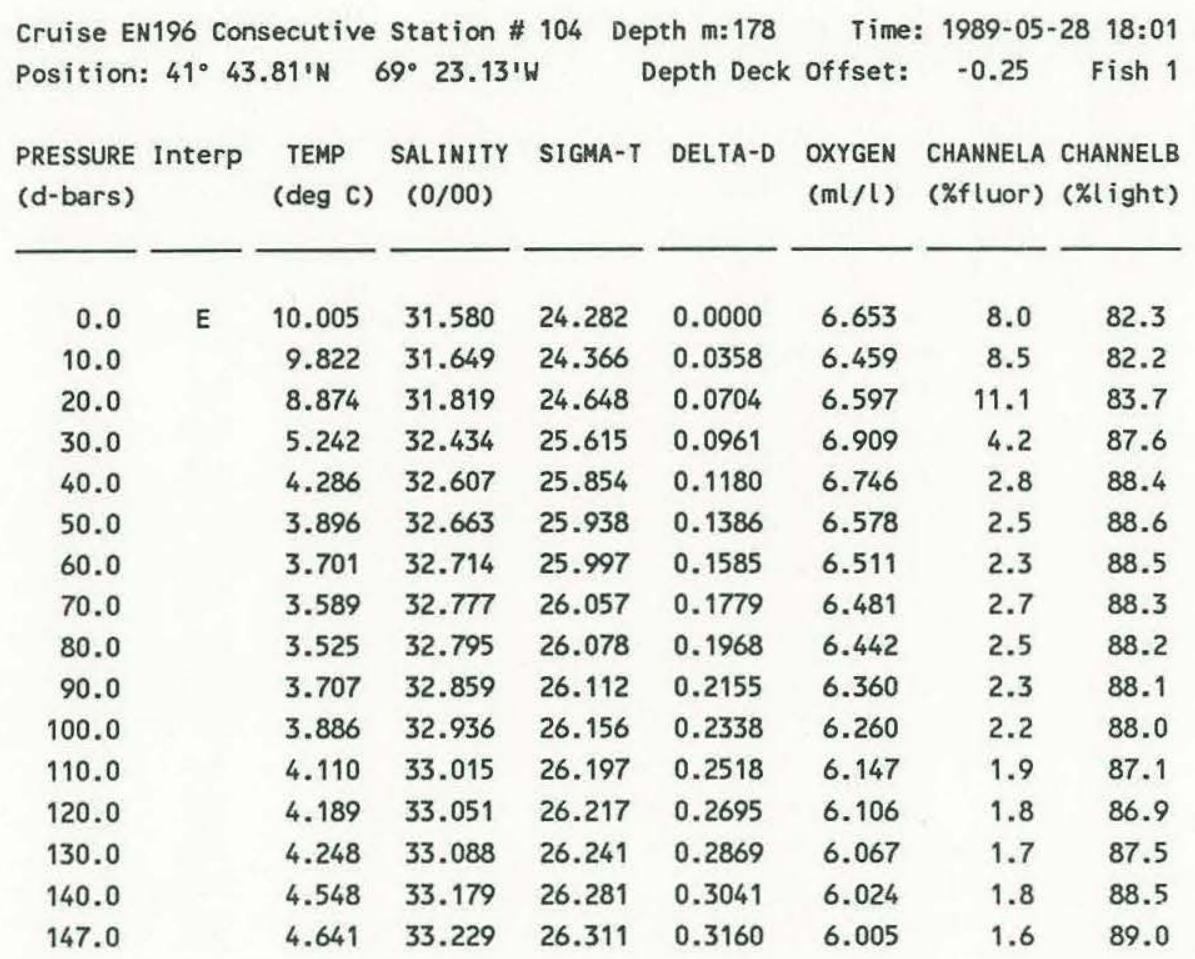
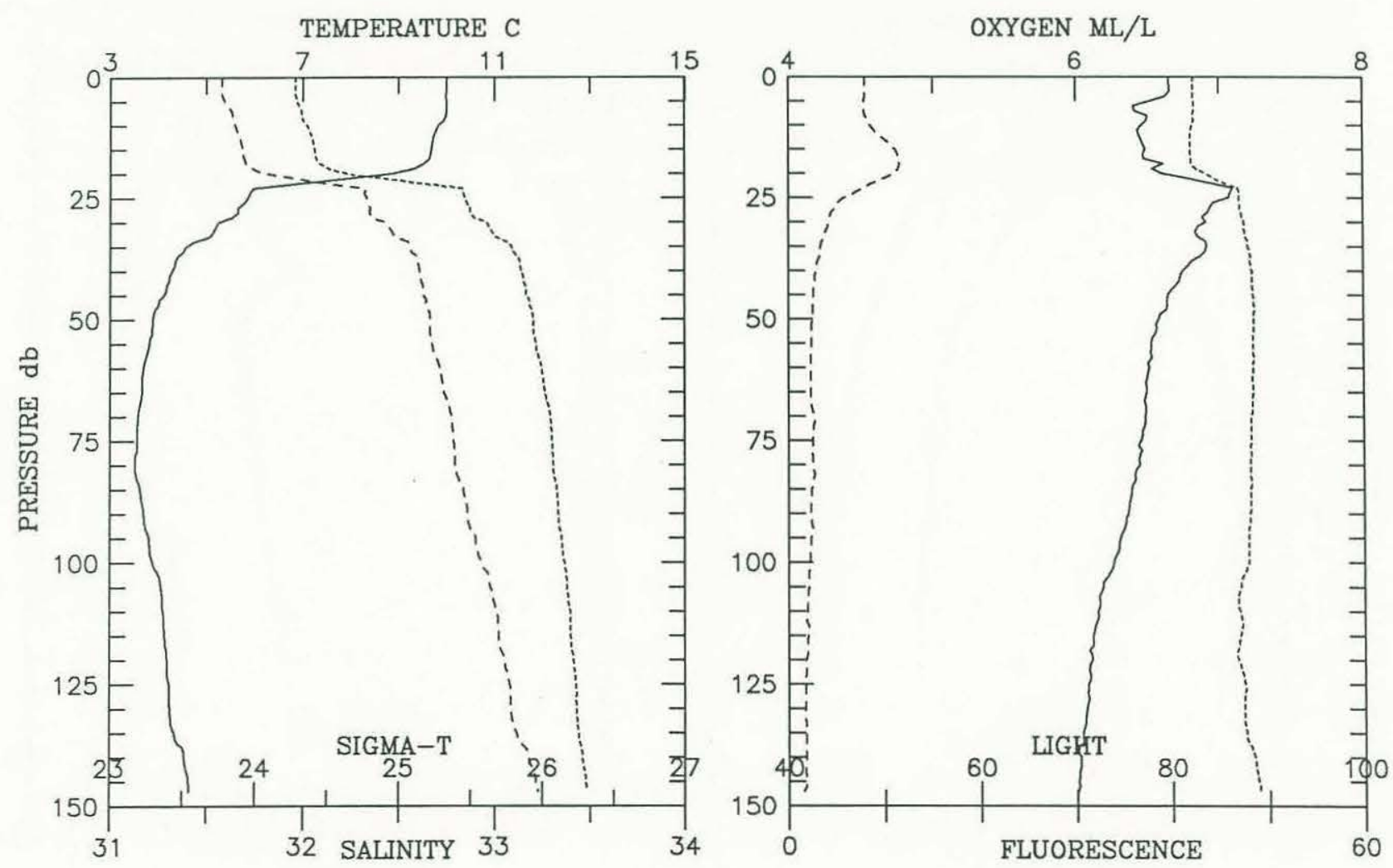


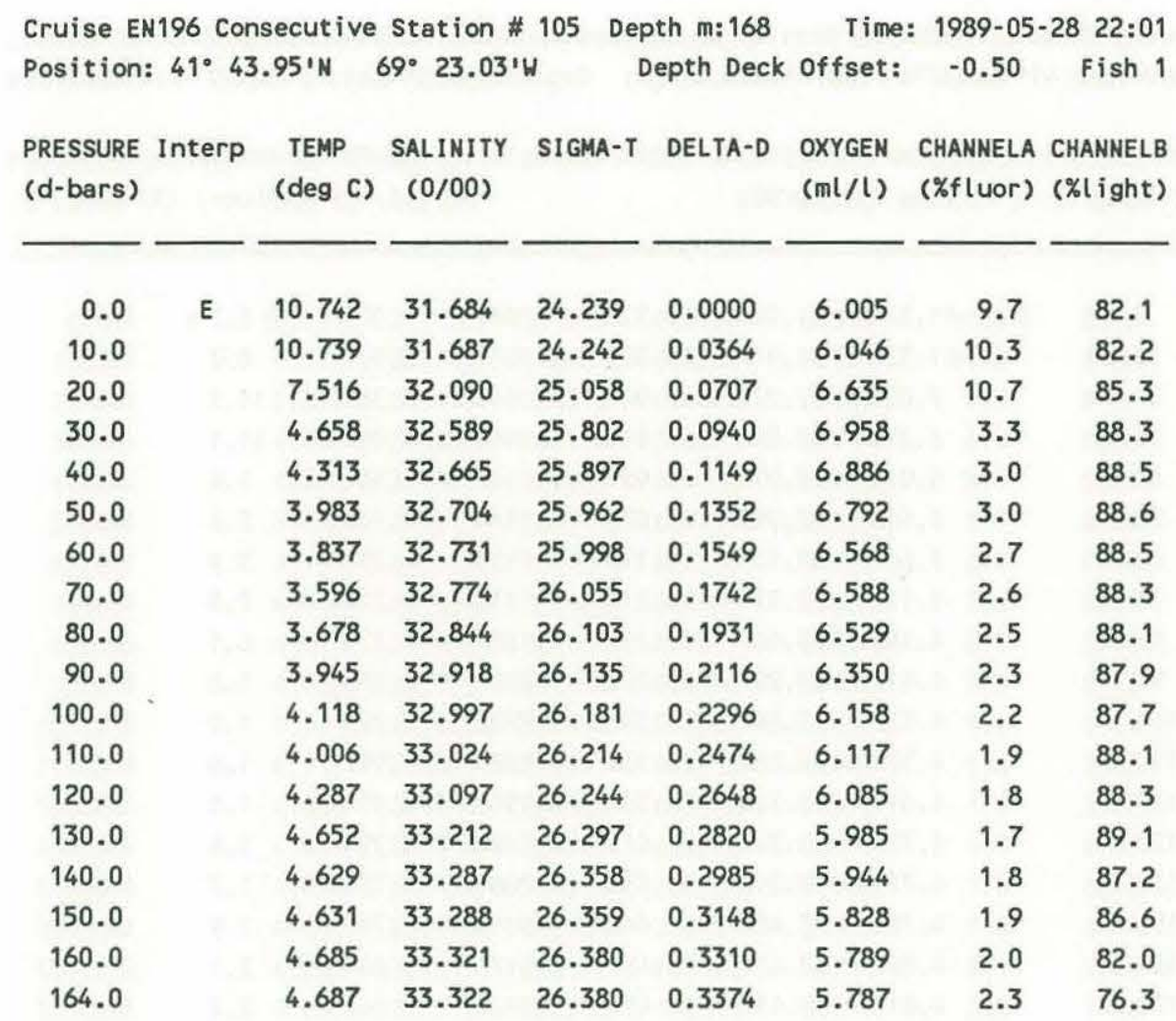
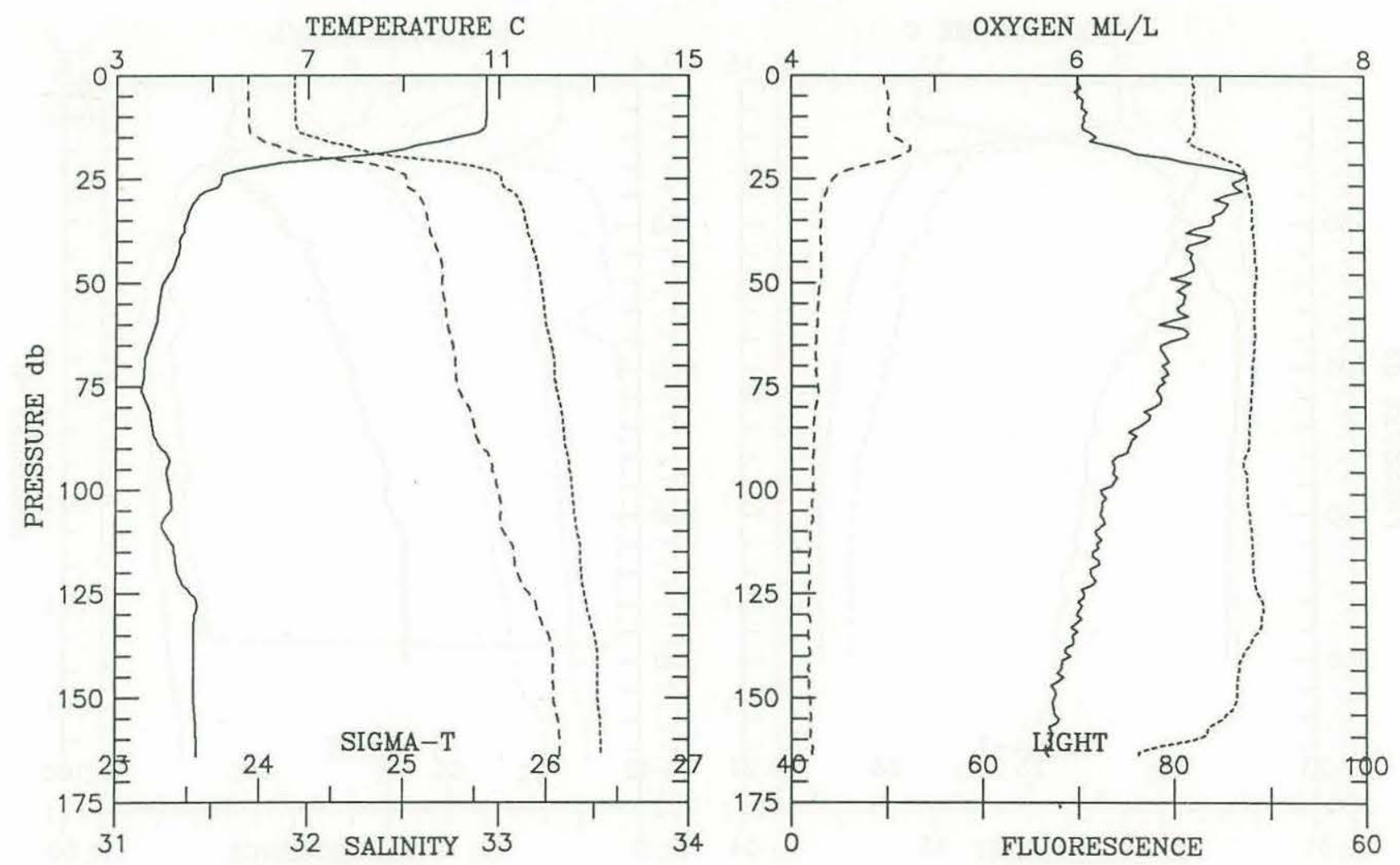
Cruise EN196 Consecutive Station \# 106 Depth m:205 Time: 1989-05-29 00:27 Position: $41^{\circ} 45.87^{\prime} \mathrm{W} 69^{\circ} 15.73 \mathrm{~W} \quad$ Depth Deck offset: -0.40 Fish 1

PRESSURE InTETP TEMP SALINITY SIGMA-T DELTA-D OXYGEN CHANNELA CHANNELB (d-bars) ( $\operatorname{deg} C)(0 / 00)$ (ml/l) (\%fluor) (\%light)

\begin{tabular}{|c|c|c|c|c|c|c|c|c|}
\hline 0.0 & $E$ & 11.344 & 31.926 & 24.322 & 0.0000 & 5.812 & 8.3 & 82.3 \\
\hline 10.0 & & 11.330 & 31.919 & 24.320 & 0.0357 & 5.901 & 8.2 & 82.2 \\
\hline 20.0 & & 9.024 & 32.227 & 24.944 & 0.0697 & 6.357 & 10.5 & 82.1 \\
\hline 30.0 & & 6.279 & 32.846 & 25.816 & 0.0940 & 7.005 & 11.1 & 86.6 \\
\hline 40.0 & & 6.015 & 32.974 & 25.951 & 0.1147 & 6.551 & 4.4 & 88.4 \\
\hline 50.0 & & 5.498 & 32.999 & 26.032 & 0.1344 & 6.500 & 3.6 & 88.7 \\
\hline 60.0 & & 5.667 & 33.123 & 26.110 & 0.1533 & 6.297 & 3.9 & 88.8 \\
\hline 70.0 & & 5.110 & 33.171 & 26.213 & 0.1713 & 6.222 & 2.8 & 89.1 \\
\hline 80.0 & & 4.583 & 33.162 & 26.264 & 0.1887 & 6.179 & 6.1 & 87.7 \\
\hline 90.0 & & 4.616 & 33.207 & 26.296 & 0.2056 & 6.058 & 3.3 & 88.9 \\
\hline 100.0 & & 4.522 & 33.246 & 26.337 & 0.2222 & 6.025 & 1.9 & 88.6 \\
\hline 110.0 & & 4.572 & 33.288 & 26.365 & 0.2385 & 5.987 & 1.6 & 88.2 \\
\hline 120.0 & & 4.617 & 33.322 & 26.387 & 0.2546 & 5.894 & 1.8 & 88.1 \\
\hline 130.0 & & 4.730 & 33.377 & 26.419 & 0.2704 & 5.791 & 1.6 & 87.5 \\
\hline 140.0 & & 4.719 & 33.396 & 26.435 & 0.2861 & 5.753 & 1.7 & 87.7 \\
\hline 150.0 & & 4.795 & 33.423 & 26.448 & 0.3015 & 5.707 & 1.9 & 87.3 \\
\hline 160.0 & & 4.807 & 33.432 & 26.453 & 0.3170 & 5.641 & 2.1 & 87.1 \\
\hline 170.0 & & 4.811 & 33.435 & 26.456 & 0.3324 & 5.640 & 2.2 & 86.2 \\
\hline 180.0 & & 4.812 & 33.435 & 26.455 & 0.3478 & 5.636 & 2.2 & 85.6 \\
\hline 190.0 & & 4.812 & 33.435 & 26.455 & 0.3632 & 5.599 & 2.2 & 85.1 \\
\hline 200.0 & & 4.816 & 33.433 & 26.453 & 0.3787 & 5.623 & 5.7 & 23.6 \\
\hline 201.0 & & 4.816 & 33.433 & 26.453 & 0.3802 & 5.604 & 6.3 & 22.6 \\
\hline
\end{tabular}
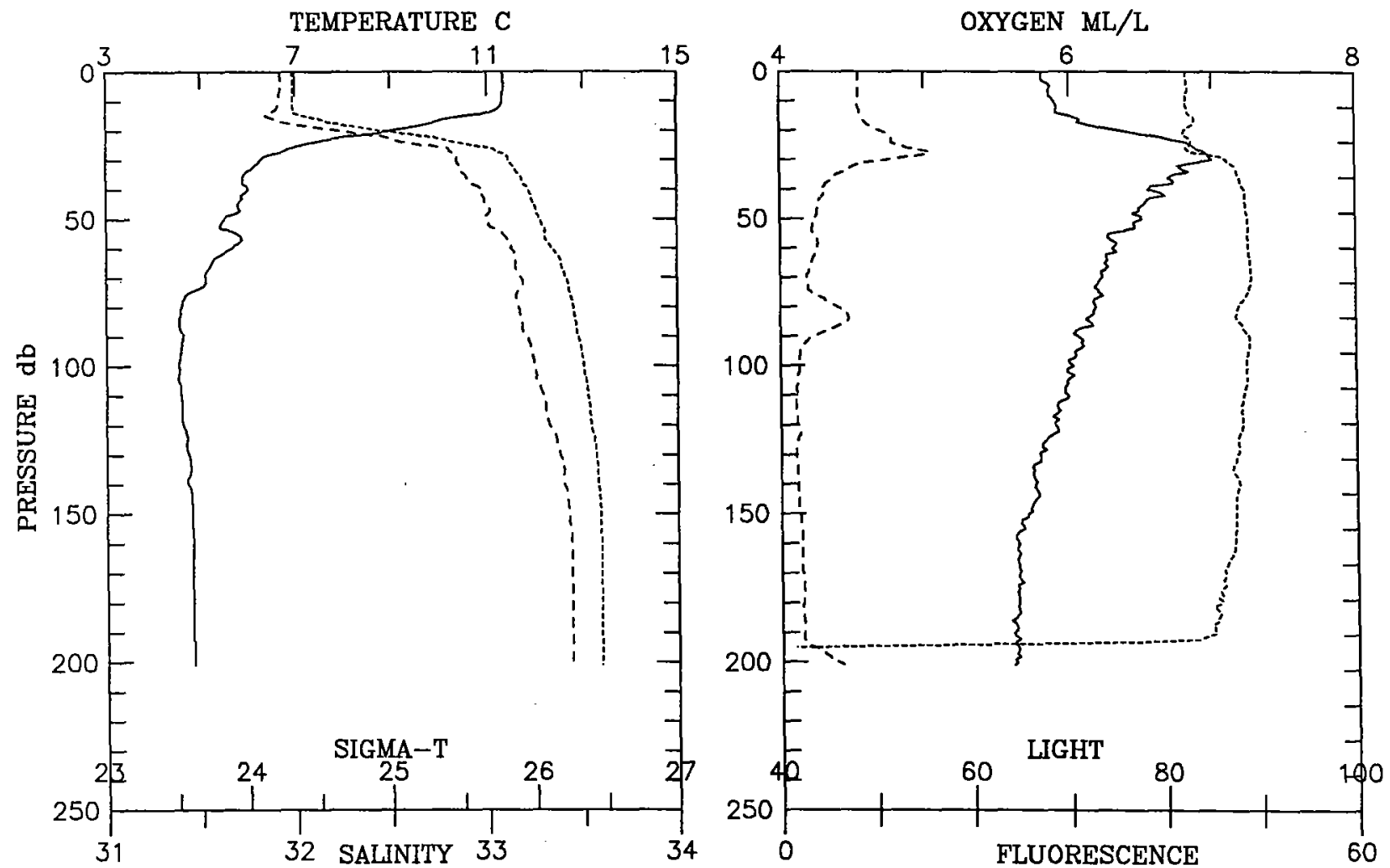


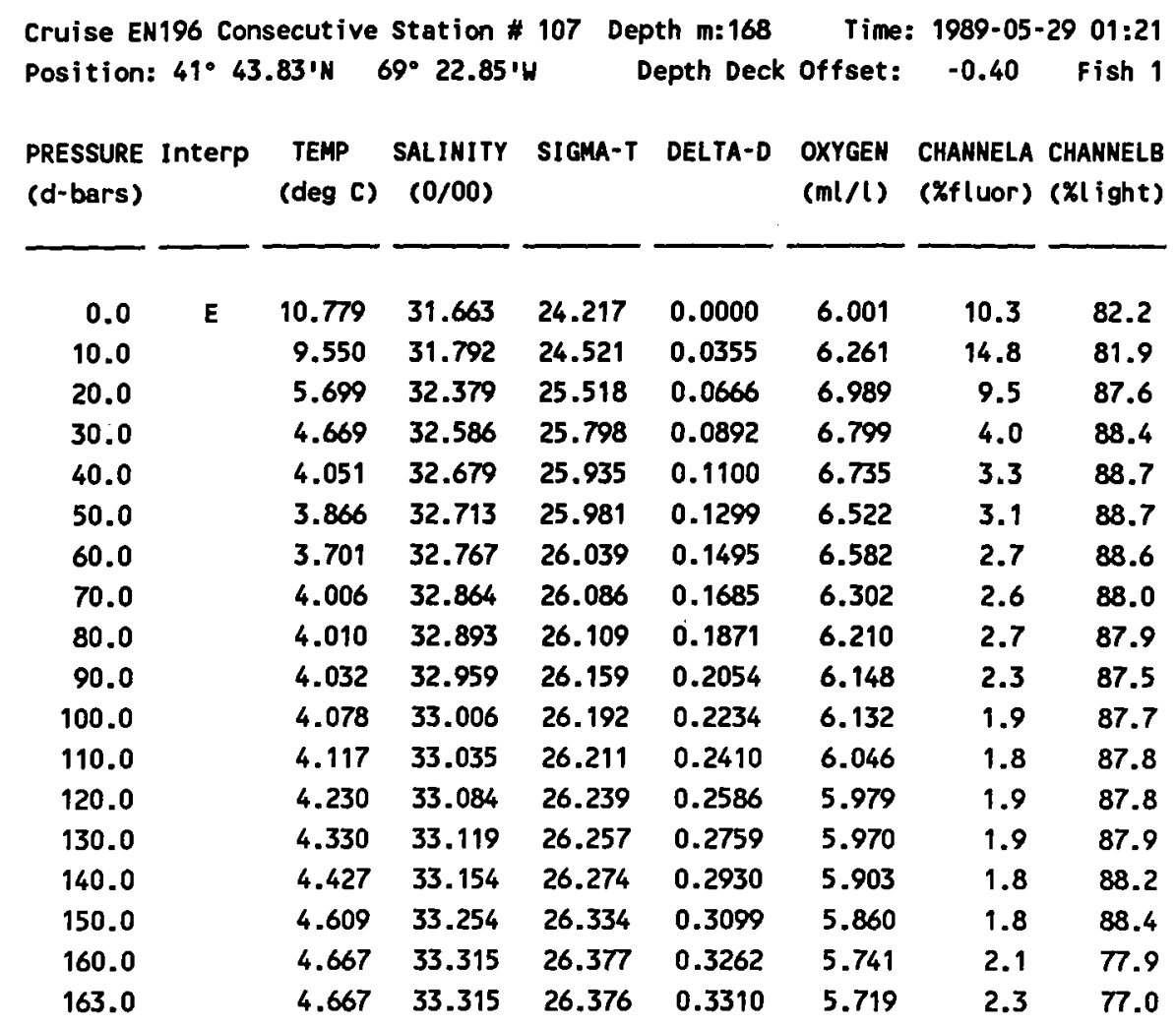
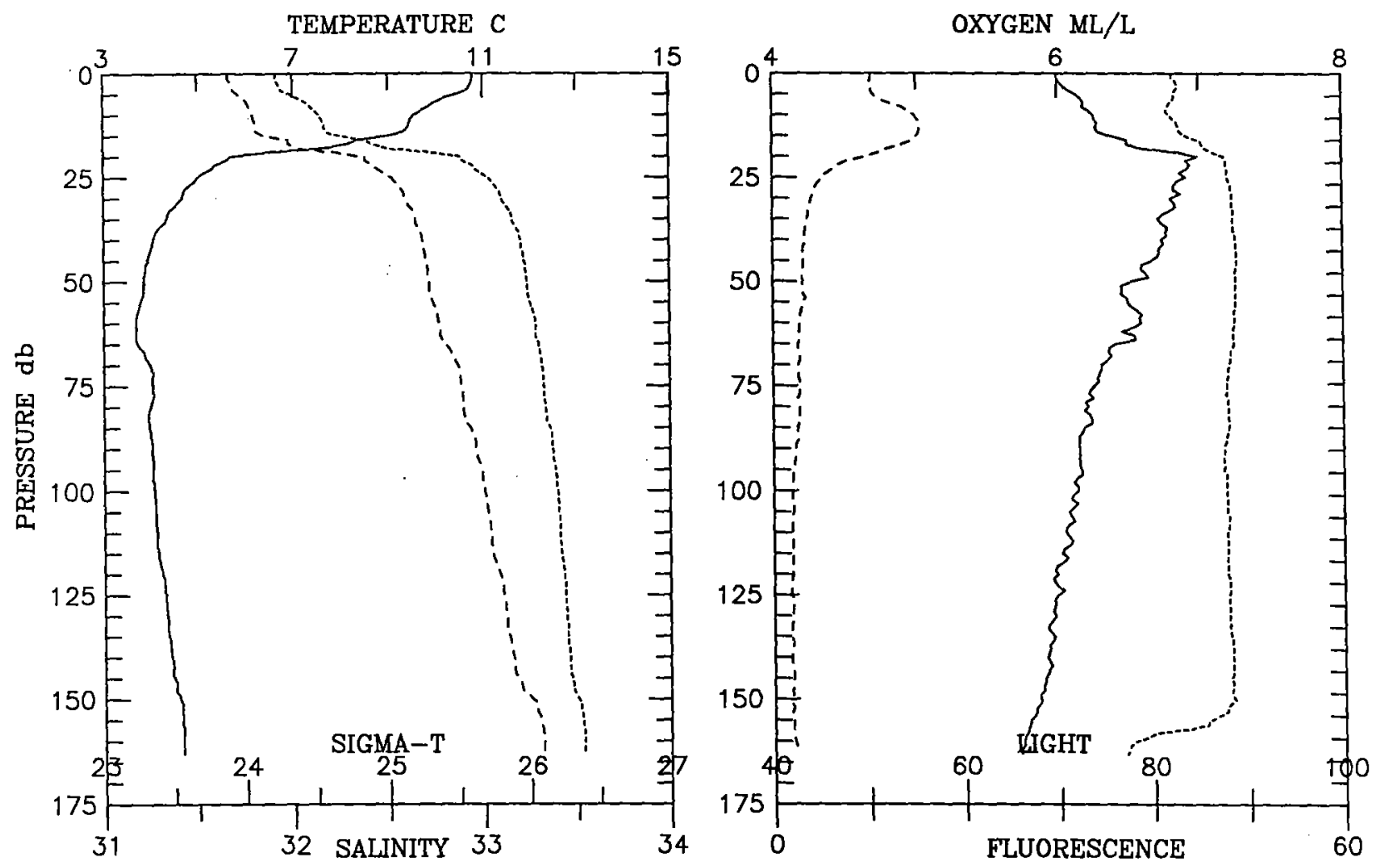


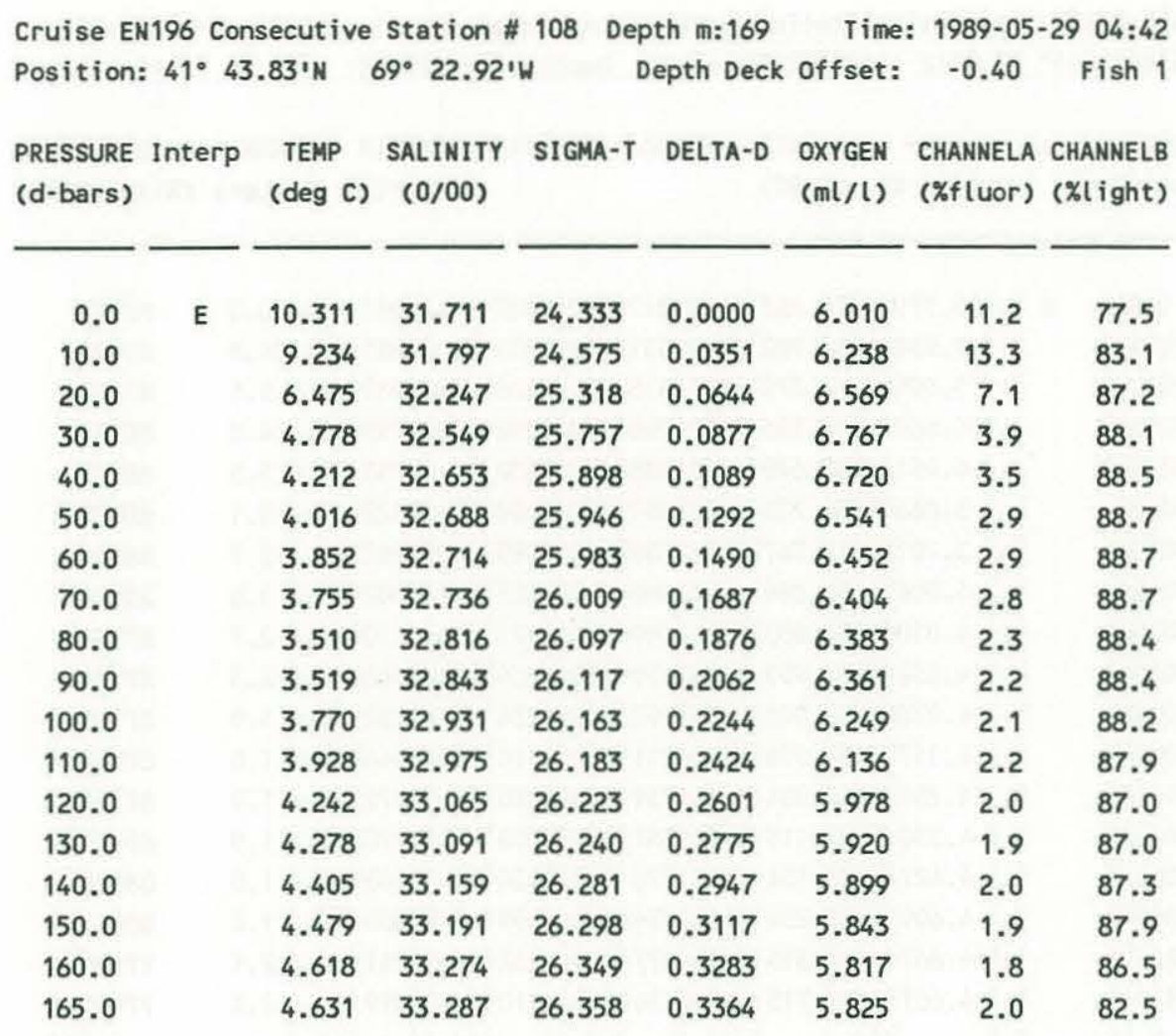
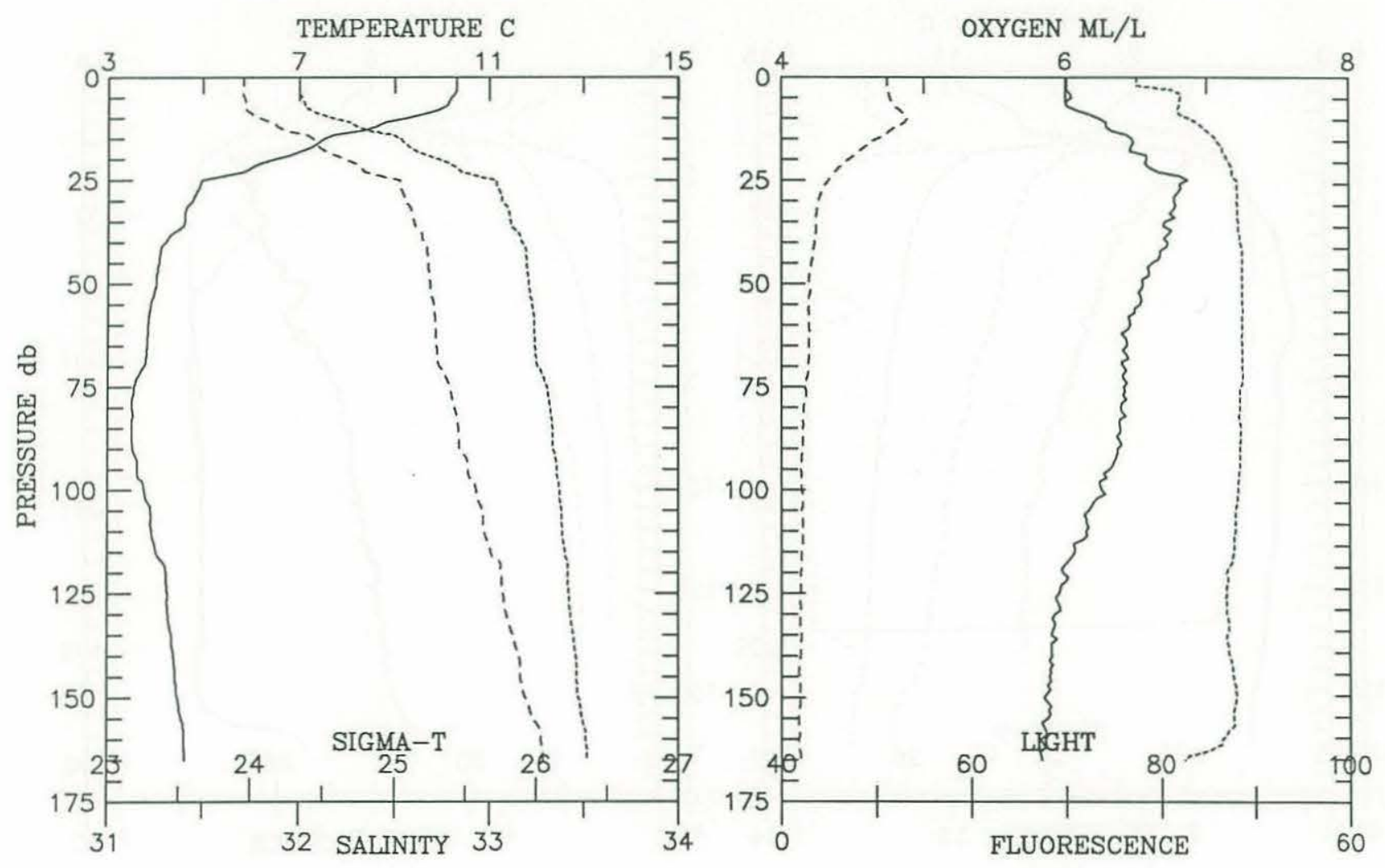


\begin{tabular}{|c|c|c|c|c|c|c|c|c|}
\hline \multirow{2}{*}{$\begin{array}{l}\text { Position: } \\
\text { PRESSURE } \\
\text { (d-bars) }\end{array}$} & \multicolumn{2}{|c|}{$41^{\circ} 43.781 \mathrm{~N}$} & \multicolumn{2}{|c|}{$69^{\circ} 22.83^{\prime} \mathrm{W}$} & Depth Deck & Offset: & \multirow{2}{*}{$\begin{array}{r}-0.40 \\
\text { CHANNELA } \\
\text { (\%fluor) }\end{array}$} & \multirow{2}{*}{$\begin{array}{l}\text { Fish } \\
\text { CHANNELB } \\
\text { (\%light) }\end{array}$} \\
\hline & Interp & $\begin{array}{c}\text { TEMP } \\
(\operatorname{deg} C)\end{array}$ & $\begin{array}{c}\text { SALINITY } \\
(0 / 00)\end{array}$ & SI GMA-T & DELTA-D & $\begin{array}{l}\text { OXYGEN } \\
(\mathrm{ml} / \mathrm{l})\end{array}$ & & \\
\hline 0.0 & E & 10.141 & 31.685 & 24.342 & 0.0000 & 6.087 & 12.1 & 82.5 \\
\hline 10.0 & & 10.049 & 31.697 & 24.366 & 0.0354 & 6.071 & 12.7 & 82.6 \\
\hline 20.0 & & 5.671 & 32.378 & 25.520 & 0.0651 & 6.715 & 6.1 & 87.7 \\
\hline 30.0 & & 4.061 & 32.673 & 25.929 & 0.0867 & 6.691 & 3.2 & 88.6 \\
\hline 40.0 & & 3.951 & 32.697 & 25.960 & 0.1068 & 6.610 & 2.9 & 88.8 \\
\hline 50.0 & & 3.812 & 32.729 & 25.998 & 0.1266 & 6.475 & 2.9 & 88.7 \\
\hline 60.0 & & 3.597 & 32.780 & 26.060 & 0.1458 & 6.409 & 2.5 & 88.6 \\
\hline 70.0 & & 3.516 & 32.807 & 26.089 & 0.1647 & 6.435 & 2.3 & 88.5 \\
\hline 80.0 & & 3.503 & 32.841 & 26.117 & 0.1833 & 6.391 & 2.2 & 88.5 \\
\hline 90.0 & & 3.858 & 32.935 & 26.158 & 0.2016 & 6.255 & 2.2 & 88.1 \\
\hline 100.0 & & 4.039 & 32.995 & 26.187 & 0.2196 & 6.069 & 2.3 & 87.6 \\
\hline 110.0 & & 4.191 & 33.042 & 26.210 & 0.2373 & 5.988 & 2.0 & 87.2 \\
\hline 120.0 & & 4.192 & 33.055 & 26.220 & 0.2549 & 5.962 & 2.0 & 87.5 \\
\hline 130.0 & & 4.290 & 33.103 & 26.248 & 0.2724 & 5.945 & 1.9 & 87.9 \\
\hline 140.0 & & 4.596 & 33.239 & 26.324 & 0.2893 & 5.905 & 1.8 & 88.3 \\
\hline 150.0 & & 4.615 & 33.267 & 26.344 & 0.3058 & 5.849 & 1.9 & 82.8 \\
\hline 160.0 & & 4.623 & 33.276 & 26.350 & 0.3222 & 5.770 & 3.3 & 67.9 \\
\hline 166.0 & & 4.624 & 33.276 & 26.350 & 0.3321 & 6.803 & 4.6 & 59.4 \\
\hline
\end{tabular}
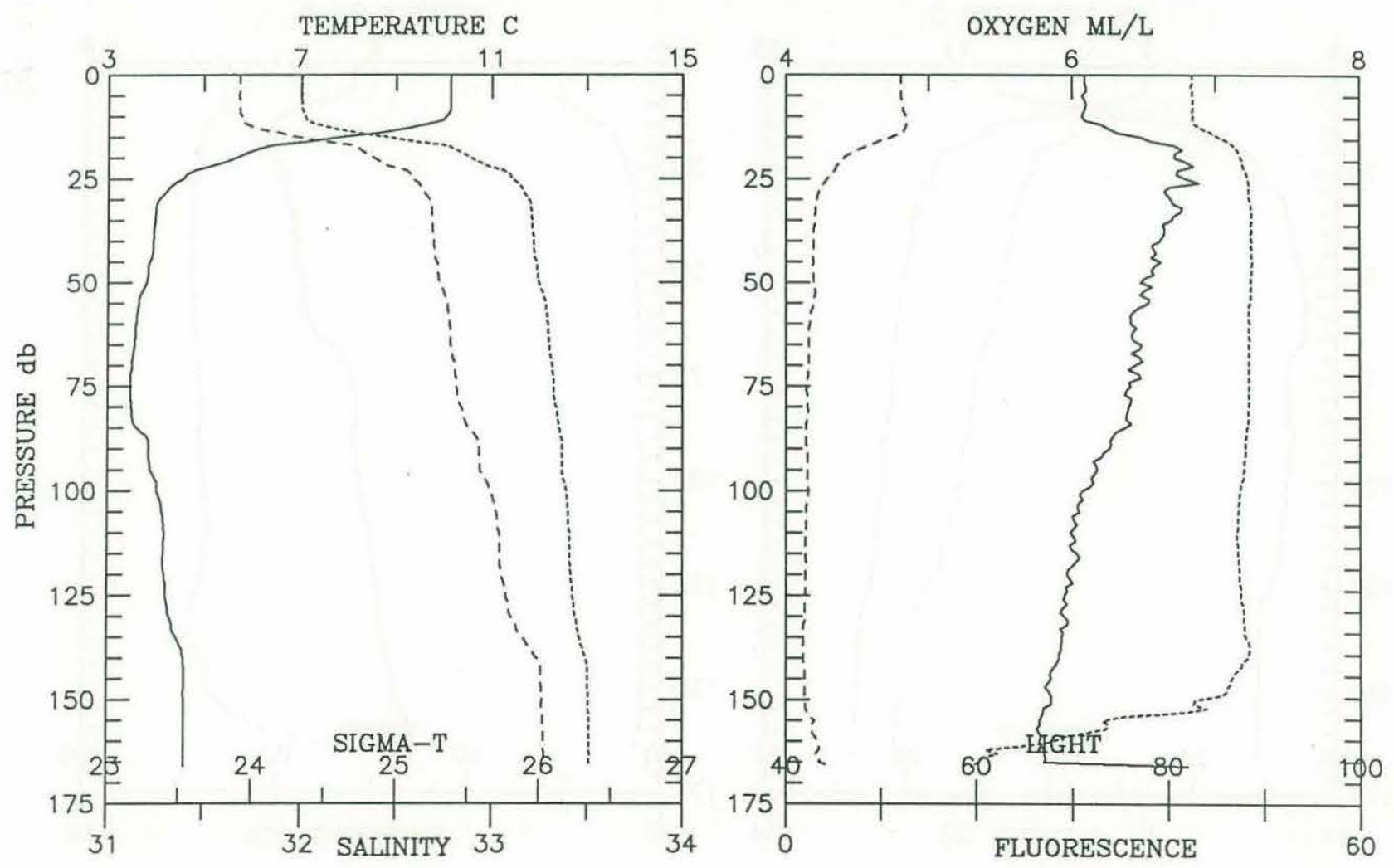
Cruise EN196 Consecutive Station \# 110 Depth m:166 Time: 1989-05-29 10:03 Position: $41^{\circ} 43.70^{\prime} \mathrm{N} 69^{\circ} 23.04^{\prime} \mathrm{W}$ Depth Deck offset: -0.30 Fish 1

\begin{tabular}{|c|c|c|c|c|c|c|c|c|}
\hline $\begin{array}{l}\text { PRESSURE } \\
\text { (d-bars) }\end{array}$ & Interp & $\begin{array}{c}\text { TEMP } \\
(\operatorname{deg} C)\end{array}$ & $\begin{array}{l}\text { SALINITY } \\
(0 / 00)\end{array}$ & SIGMA-T & DELTA-D & $\begin{array}{l}\text { OXYGEN } \\
(\mathrm{ml} / \mathrm{l})\end{array}$ & $\begin{array}{l}\text { CHANNELA } \\
\text { (\%fluor) }\end{array}$ & $\begin{array}{l}\text { CHANNELB } \\
\text { (\%light) }\end{array}$ \\
\hline 0.0 & E & 10.104 & 31.670 & 24.336 & 0.0000 & 6.137 & 10.4 & 83.2 \\
\hline 10.0 & & 8.481 & 31.890 & 24.762 & 0.0348 & 6.421 & 10.1 & 85.2 \\
\hline 20.0 & & 5.109 & 32.532 & 25.707 & 0.0608 & 6.835 & 4.7 & 88.0 \\
\hline 30.0 & & 4.252 & 32.652 & 25.894 & 0.0822 & 6.623 & 3.2 & 88.6 \\
\hline 40.0 & & 3.931 & 32.699 & 25.963 & 0.1024 & 6.556 & 2.8 & 88.8 \\
\hline 50.0 & & 3.726 & 32.748 & 26.021 & 0.1221 & 6.502 & 2.6 & 88.8 \\
\hline 60.0 & & 3.636 & 32.793 & 26.066 & 0.1412 & 6.464 & 2.4 & 88.5 \\
\hline 70.0 & & 3.942 & 32.862 & 26.092 & 0.1600 & 6.240 & 2.3 & 88.1 \\
\hline 80.0 & & 4.005 & 32.893 & 26.110 & 0.1786 & 6.125 & 2.5 & 87.8 \\
\hline 90.0 & & 3.900 & 32.940 & 26.158 & 0.1970 & 6.144 & 2.4 & 88.1 \\
\hline 100.0 & & 4.054 & 32.976 & 26.171 & 0.2150 & 6.096 & 2.1 & 87.5 \\
\hline 110.0 & & 4.136 & 33.030 & 26.205 & 0.2329 & 6.055 & 2.0 & 87.1 \\
\hline 120.0 & & 4.206 & 33.066 & 26.227 & 0.2504 & 6.012 & 1.9 & 87.3 \\
\hline 130.0 & & 4.602 & 33.205 & 26.296 & 0.2676 & 5.965 & 1.8 & 88.9 \\
\hline 140.0 & & 4.626 & 33.260 & 26.337 & 0.2842 & 5.919 & 1.8 & 88.3 \\
\hline 150.0 & & 4.608 & 33.270 & 26.347 & 0.3006 & 5.853 & 1.9 & 86.9 \\
\hline 160.0 & & 4.673 & 33.306 & 26.368 & 0.3169 & 5.746 & 2.3 & 80.9 \\
\hline 166.0 & & 4.686 & 33.310 & 26.370 & 0.3266 & 5.713 & 2.8 & 70.7 \\
\hline
\end{tabular}
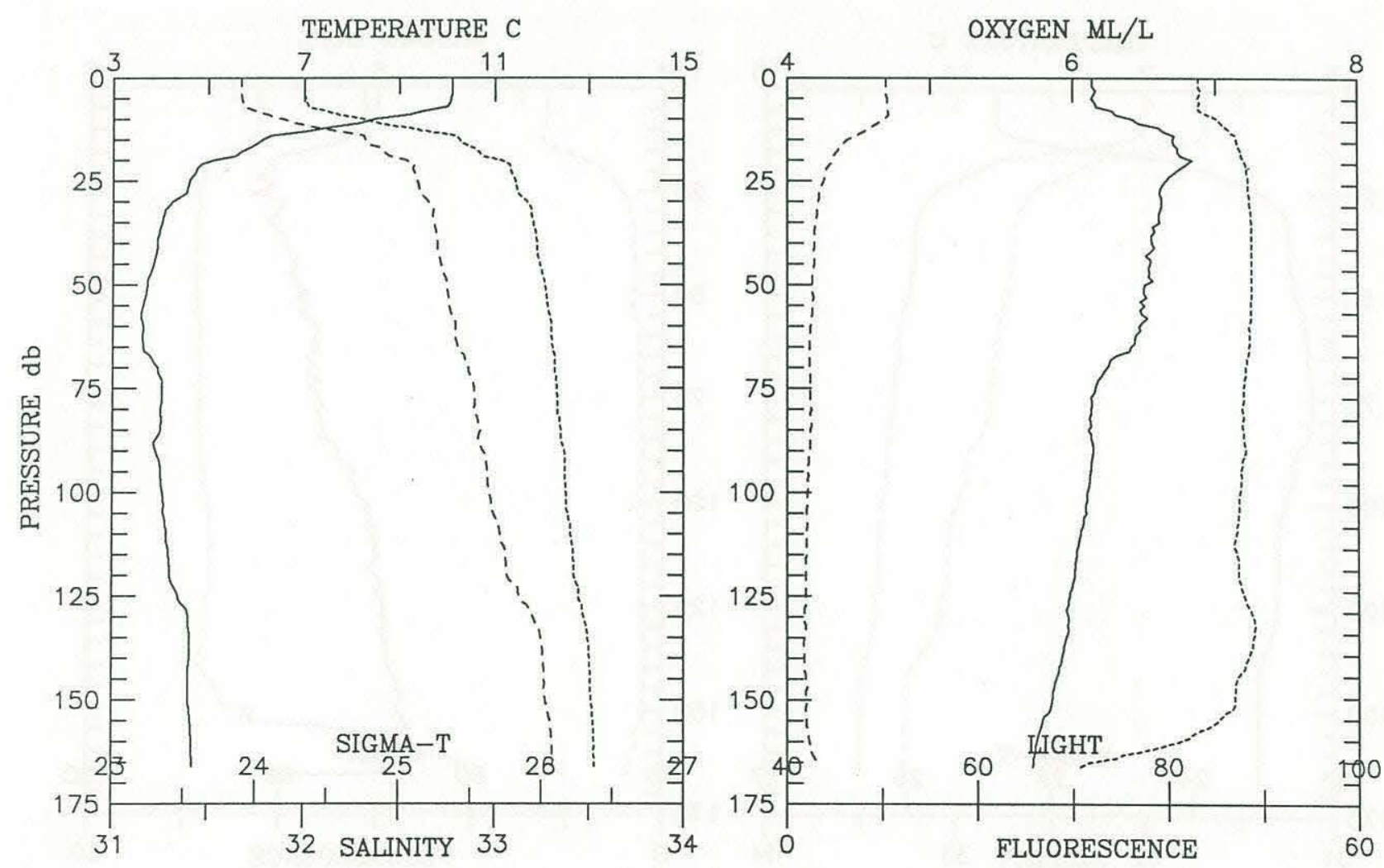


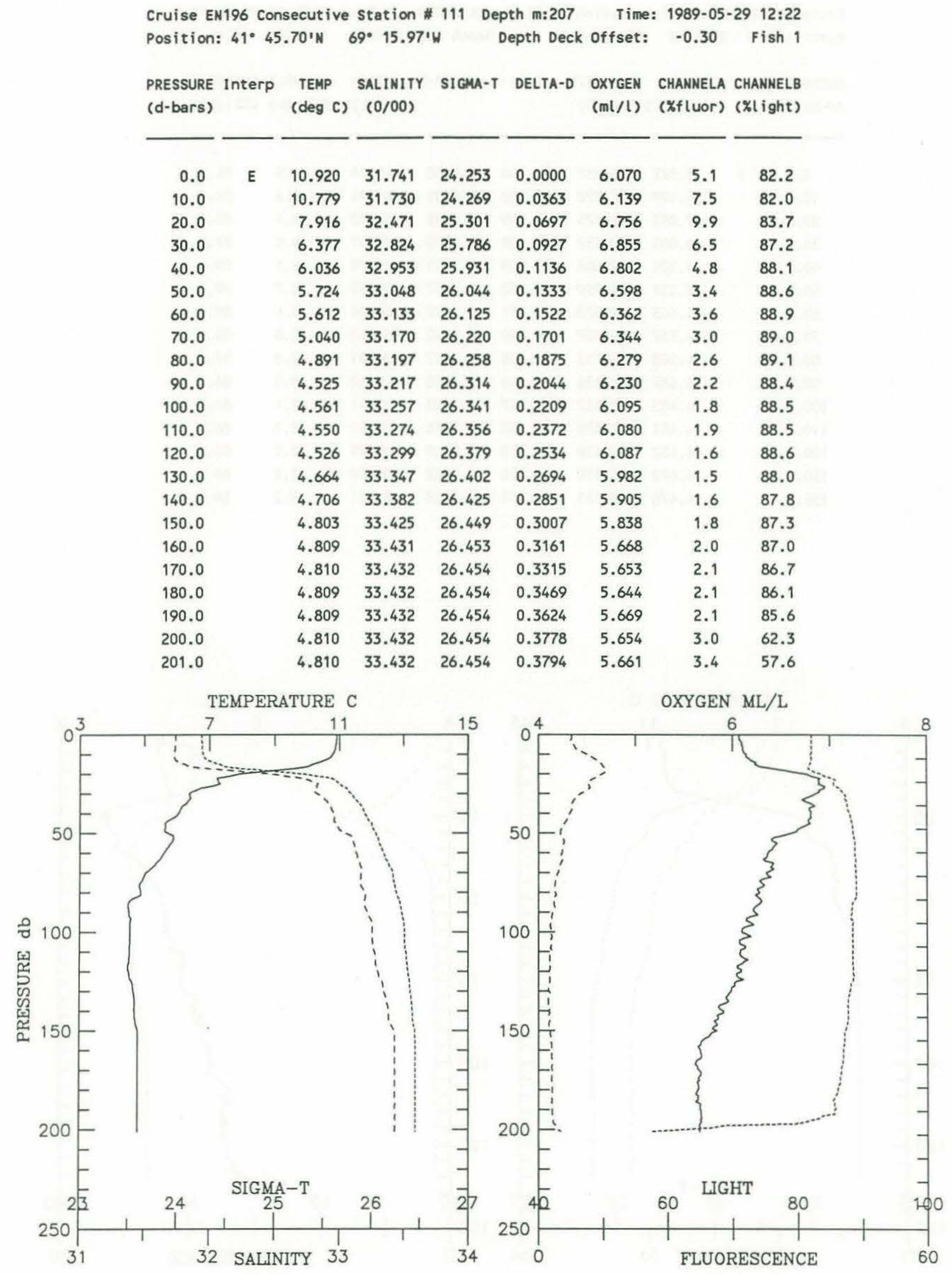




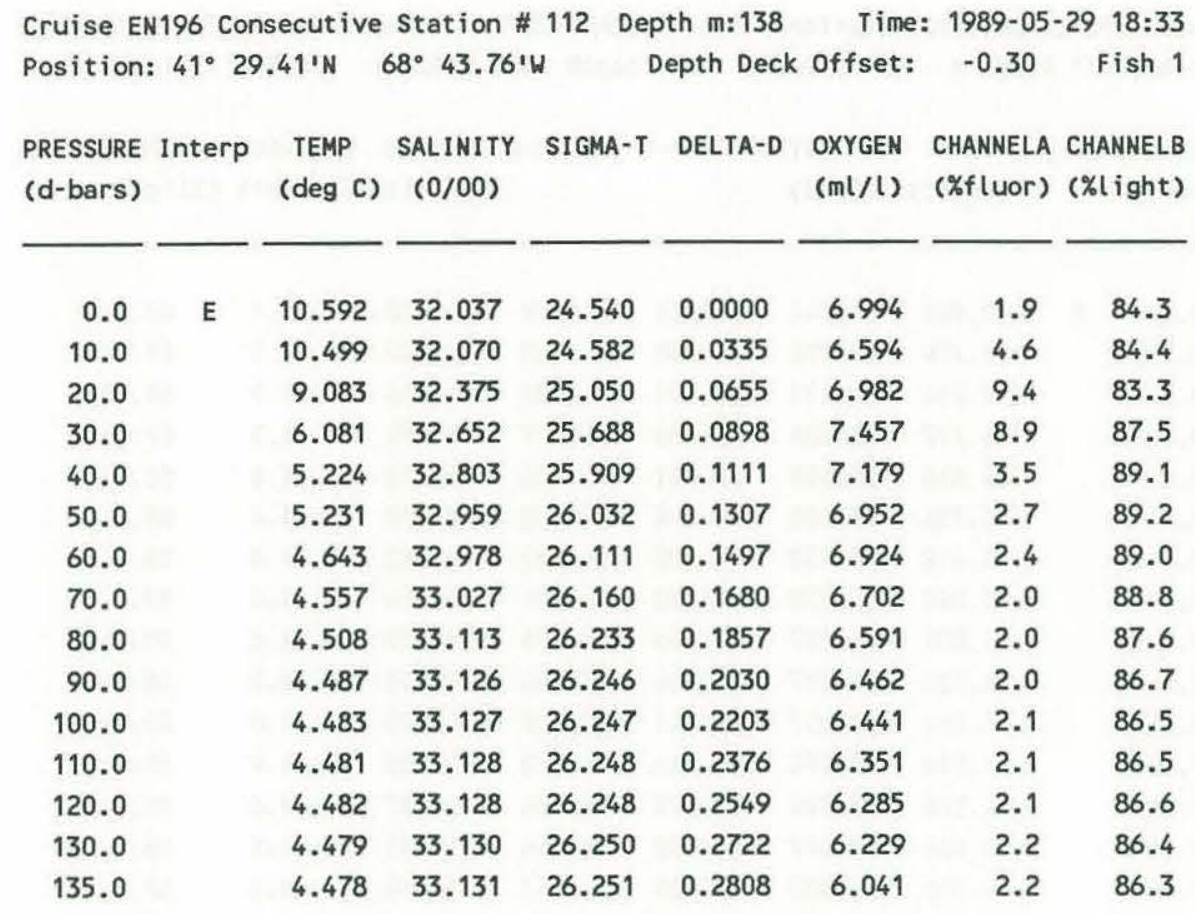
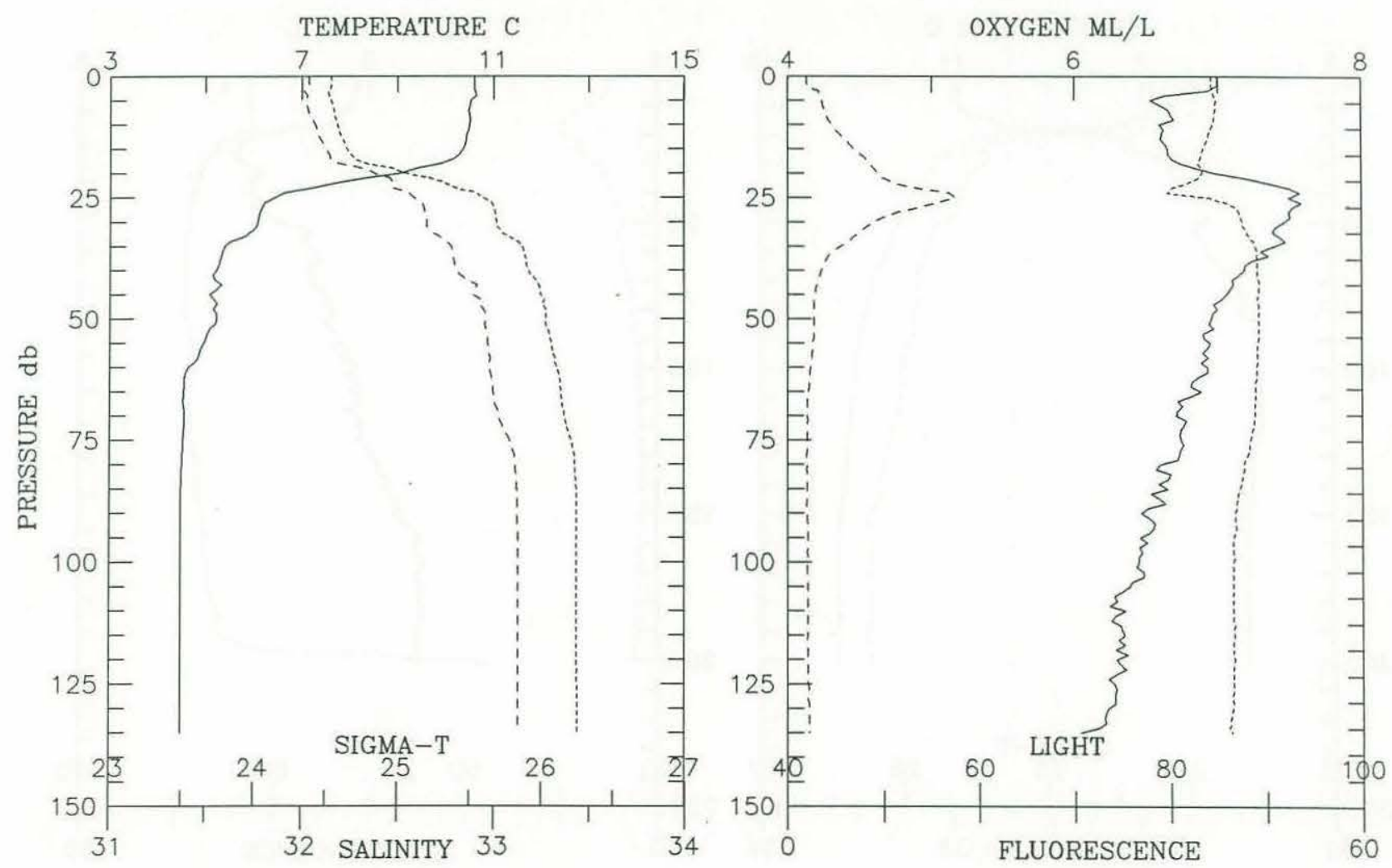


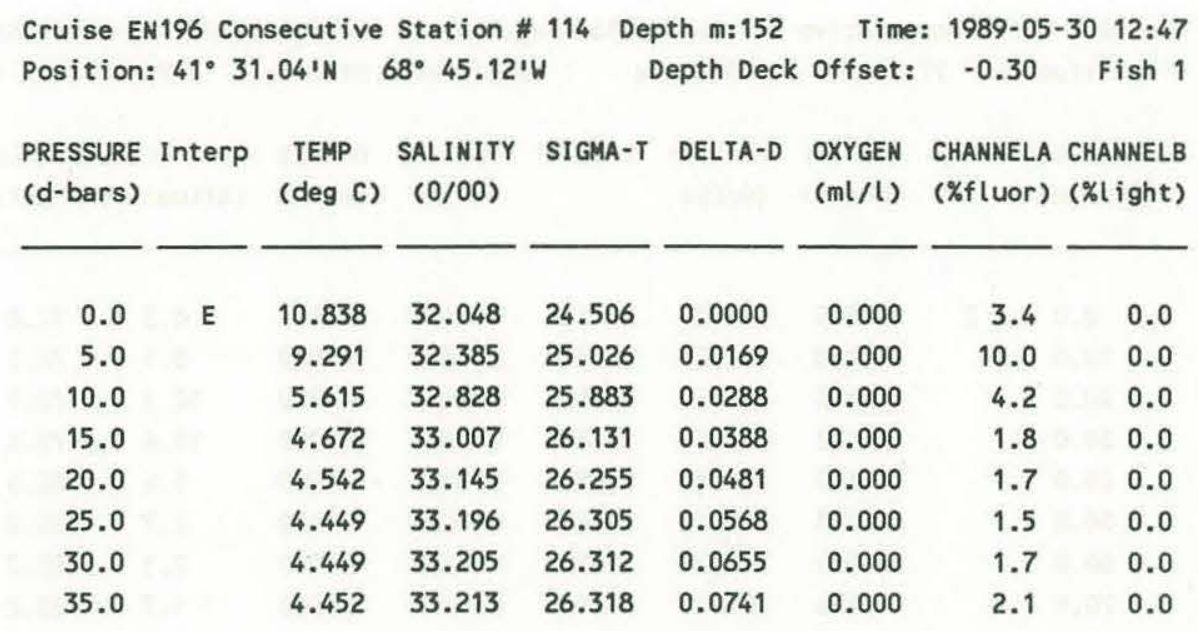
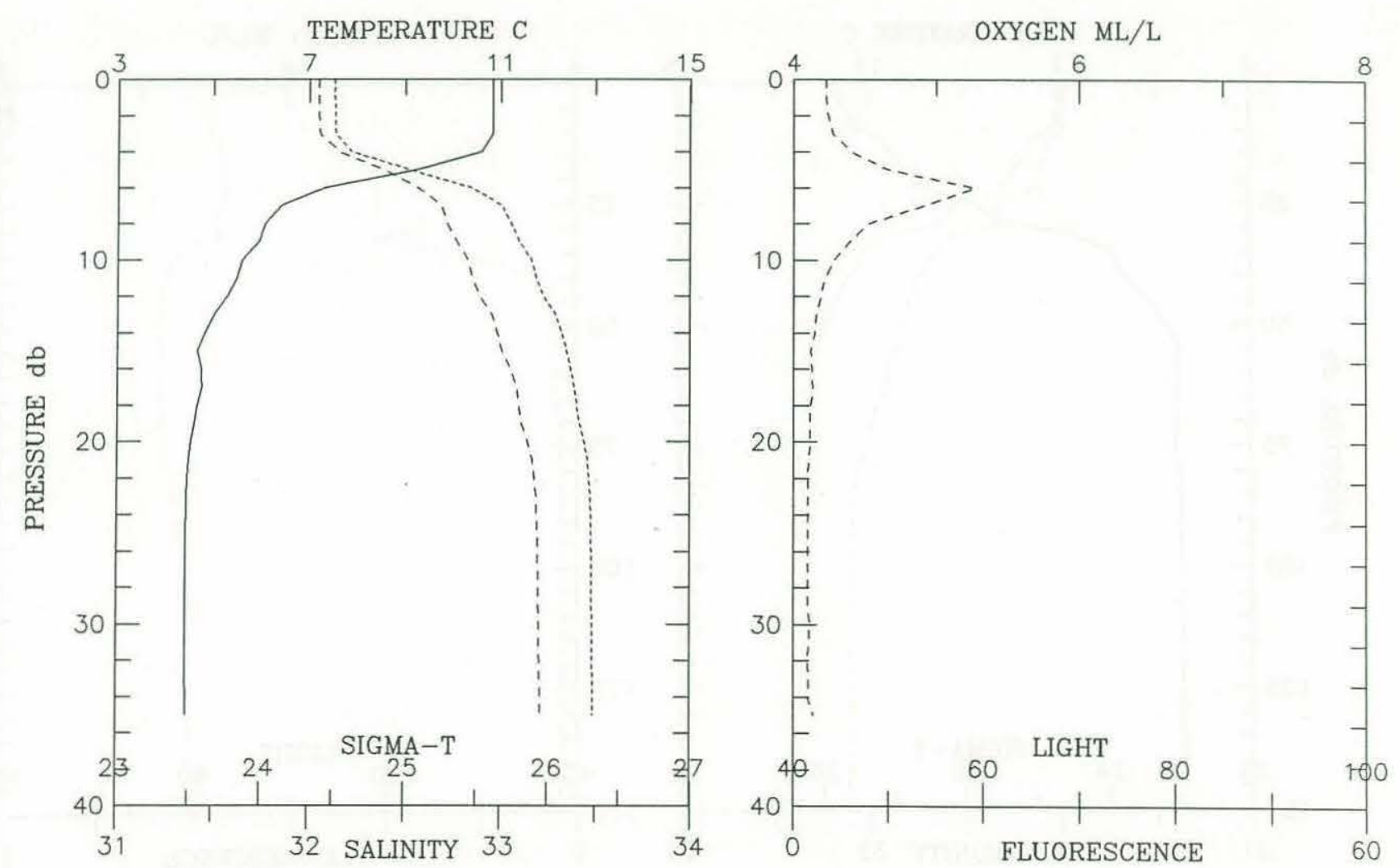


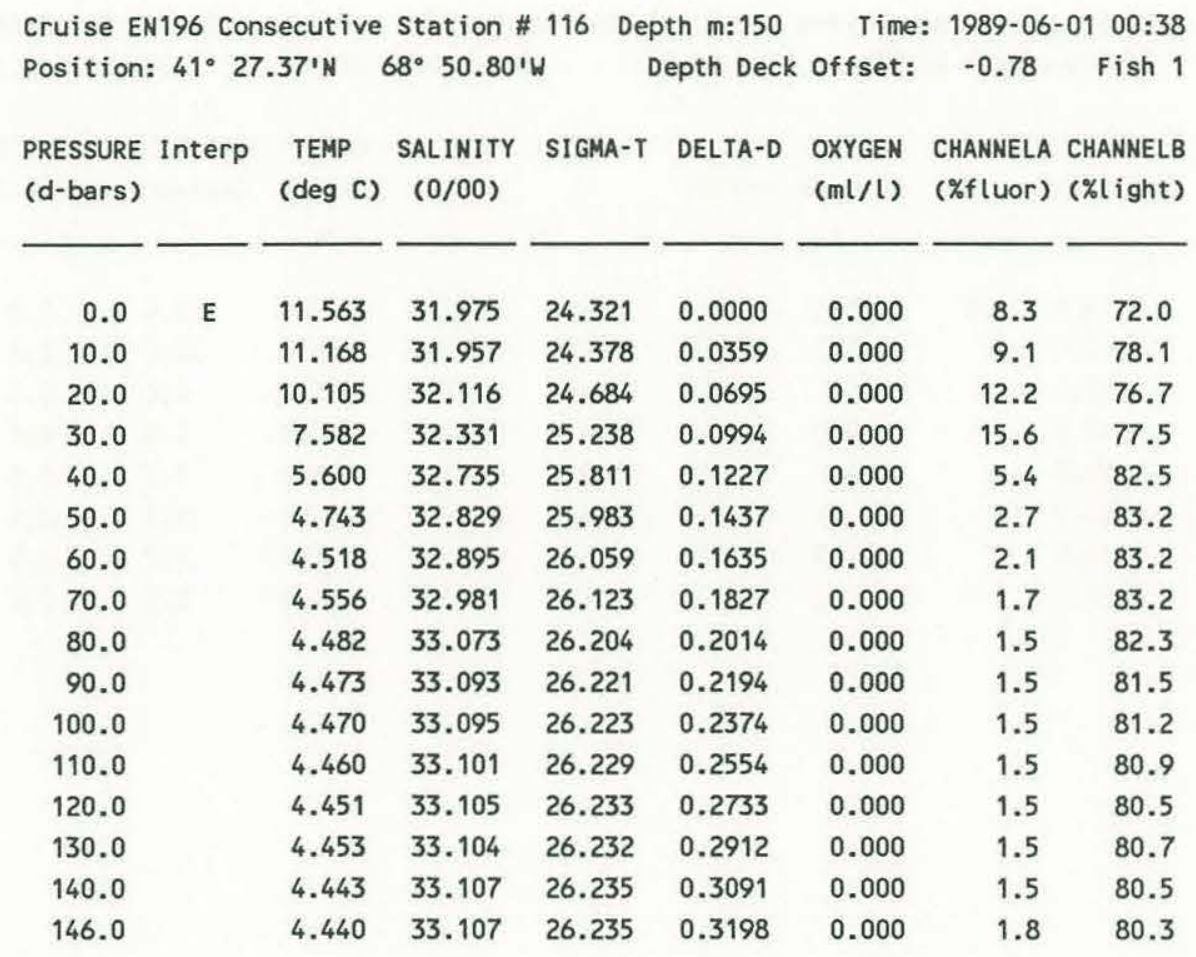
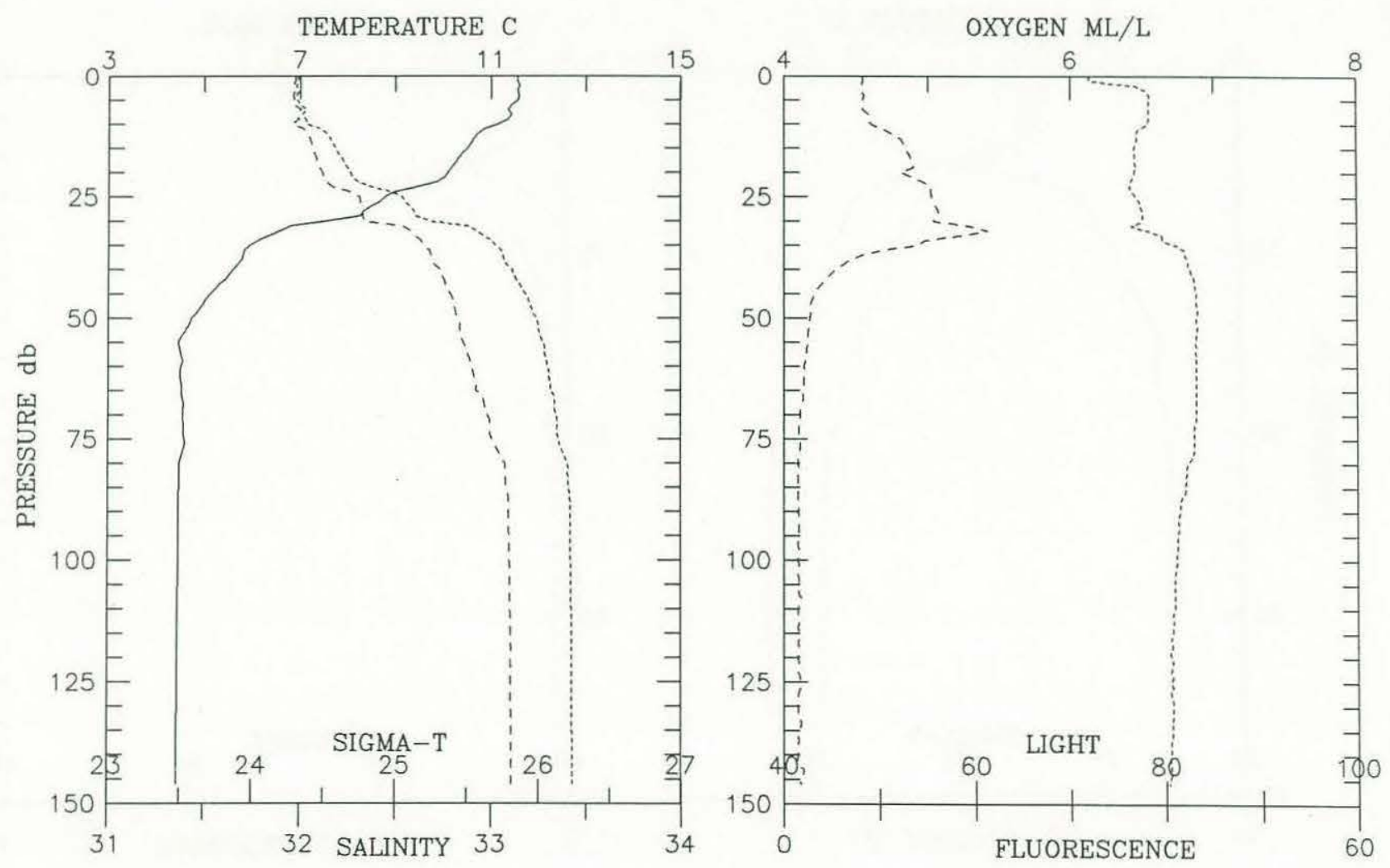
Cruise EN196 Consecutive Station \# 117 Depth m:169 Time: 1989-06-01 06:44 Position: $41^{\circ} 41.49^{\prime} \mathrm{N} 68^{\circ} 40.32^{\prime} \mathrm{W}$ Depth Deck Offset: -0.78 Fish 1

\begin{tabular}{|c|c|c|c|c|c|c|c|c|}
\hline $\begin{array}{l}\text { PRESSURE } \\
\text { (d-bars) }\end{array}$ & Interp & $\begin{array}{l}\text { TEMP } \\
\text { (deg C) }\end{array}$ & $\begin{array}{l}\text { SALINITY } \\
(0 / 00)\end{array}$ & SI GMA-T & DELTA-D & $\begin{array}{l}\text { OXYGEN } \\
(\mathrm{ml} / \mathrm{ll})\end{array}$ & $\begin{array}{l}\text { CHANNELA } \\
\text { ( } \% \text { fluor) }\end{array}$ & $\begin{array}{l}\text { CHANNELB } \\
\text { (\%tight) }\end{array}$ \\
\hline 0.0 & $\mathbf{E}$ & 11.778 & 32.055 & 24.344 & 0.0000 & 0.000 & 7.0 & 78.1 \\
\hline 10.0 & & 11.206 & 32.157 & 24.527 & 0.0351 & 0.000 & 7.6 & 77.5 \\
\hline 20.0 & & 10.761 & 32.170 & 24.615 & 0.0685 & 0.000 & 13.0 & 75.5 \\
\hline 30.0 & & 6.898 & 32.451 & 25.425 & 0.0980 & 0.000 & 15.6 & 78.3 \\
\hline 40.0 & & 5.279 & 32.840 & 25.932 & 0.1204 & 0.000 & 2.9 & 83.1 \\
\hline 50.0 & & 4.592 & 32.981 & 26.119 & 0.1400 & 0.000 & 1.9 & 83.0 \\
\hline 60.0 & & 4.400 & 33.027 & 26.176 & 0.1588 & 0.000 & 1.6 & 82.8 \\
\hline 70.0 & & 4.409 & 33.067 & 26.207 & 0.1769 & 0.000 & 1.8 & 82.9 \\
\hline 80.0 & & 4.374 & 33.074 & 26.216 & 0.1950 & 0.000 & 1.3 & 82.7 \\
\hline 90.0 & & 4.352 & 33.081 & 26.224 & 0.2130 & 0.000 & 1.3 & 82.4 \\
\hline 100.0 & & 4.361 & 33.094 & 26.233 & 0.2309 & 0.000 & 1.4 & 82.4 \\
\hline 110.0 & & 4.383 & 33.111 & 26.245 & 0.2487 & 0.000 & 1.3 & 82.2 \\
\hline 120.0 & & 4.415 & 33.131 & 26.257 & 0.2664 & 0.000 & 1.3 & 82.2 \\
\hline 130.0 & & 4.476 & 33.173 & 26.284 & 0.2840 & 0.000 & 1.3 & 82.3 \\
\hline 140.0 & & 4.548 & 33.239 & 26.329 & 0.3012 & 0.000 & 1.2 & 82.1 \\
\hline 150.0 & & 5.029 & 33.450 & 26.443 & 0.3178 & 0.000 & 1.4 & 79.9 \\
\hline 160.0 & & 5.081 & 33.462 & 26.447 & 0.3338 & 0.000 & 1.6 & 77.5 \\
\hline 165.0 & & 5.080 & 33.461 & 26.446 & 0.3418 & 0.000 & 1.6 & 77.0 \\
\hline
\end{tabular}
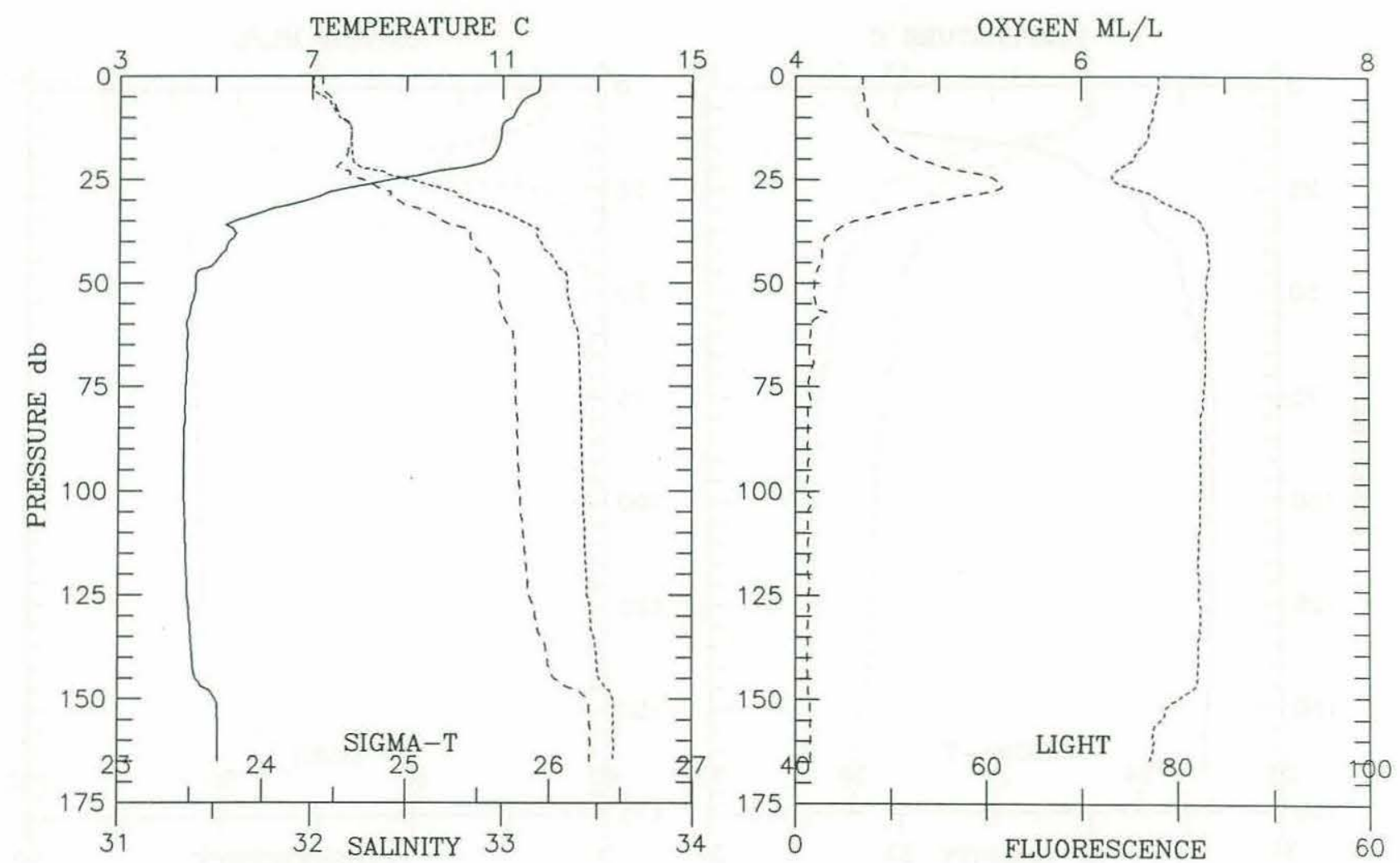


\begin{tabular}{|c|c|c|c|c|c|c|c|c|}
\hline \multirow{2}{*}{$\begin{array}{l}\text { Position: } \\
\text { PRESSURE } \\
\text { (d-bars) }\end{array}$} & \multicolumn{2}{|c|}{$=41^{\circ} 41.31 \cdot \mathrm{N}$} & \multicolumn{2}{|c|}{$68^{\circ} 39.98^{\prime} \mathrm{W}$} & Depth Deck & Offset: & -0.78 & Fish 1 \\
\hline & Interp & $\begin{array}{c}\text { TEMP } \\
(\operatorname{deg} \mathrm{C})\end{array}$ & $\begin{array}{l}\text { SALINITY } \\
(0 / 00)\end{array}$ & SIGMA-T & DELTA-D & $\begin{array}{l}\text { OXYGEN } \\
(\mathrm{ml} / \mathrm{l})\end{array}$ & $\begin{array}{l}\text { CHANNELA } \\
\text { (\%fluor) }\end{array}$ & $\begin{array}{l}\text { CHANNELB } \\
\text { (\%light) }\end{array}$ \\
\hline 0.0 & $E$ & 11.889 & 31.972 & 24.259 & 0.0000 & 0.000 & 6.2 & 78.3 \\
\hline 10.0 & & 11.133 & 32.068 & 24.470 & 0.0362 & 0.000 & 8.9 & 75.3 \\
\hline 20.0 & & 7.091 & 32.529 & 25.461 & 0.0654 & 0.000 & 15.1 & 75.9 \\
\hline 30.0 & & 5.724 & 32.820 & 25.864 & 0.0879 & 0.000 & 4.1 & 82.8 \\
\hline 40.0 & & 5.096 & 32.880 & 25.984 & 0.1087 & 0.000 & 2.3 & 83.3 \\
\hline 50.0 & & 4.929 & 32.971 & 26.075 & 0.1283 & 0.000 & 2.1 & 82.5 \\
\hline 60.0 & & 4.746 & 33.028 & 26.140 & 0.1473 & 0.000 & 1.7 & 83.3 \\
\hline 70.0 & & 4.425 & 33.049 & 26.191 & 0.1659 & 0.000 & 1.4 & 82.9 \\
\hline 80.0 & & 4.364 & 33.083 & 26.224 & 0.1840 & 0.000 & 1.3 & 82.3 \\
\hline 90.0 & & 4.416 & 33.122 & 26.250 & 0.2018 & 0.000 & 1.3 & 82.3 \\
\hline 100.0 & & 4.412 & 33.128 & 26.255 & 0.2195 & 0.000 & 1.3 & 82.2 \\
\hline 110.0 & & 4.419 & 33.136 & 26.261 & 0.2371 & 0.000 & 1.3 & 82.0 \\
\hline 120.0 & & 4.444 & 33.151 & 26.270 & 0.2547 & 0.000 & 1.3 & 81.8 \\
\hline 130.0 & & 4.454 & 33.155 & 26.272 & 0.2722 & 0.000 & 1.4 & 81.8 \\
\hline 140.0 & & 4.471 & 33.163 & 26.277 & 0.2897 & 0.000 & 1.3 & 81.6 \\
\hline 150.0 & & 4.586 & 33.224 & 26.313 & 0.3071 & 0.000 & 1.5 & 79.3 \\
\hline 160.0 & & 4.622 & 33.239 & 26.321 & 0.3242 & 0.000 & 1.6 & 74.7 \\
\hline 166.0 & & 4.642 & 33.250 & 26.328 & 0.3345 & 0.000 & 1.8 & 72.1 \\
\hline
\end{tabular}
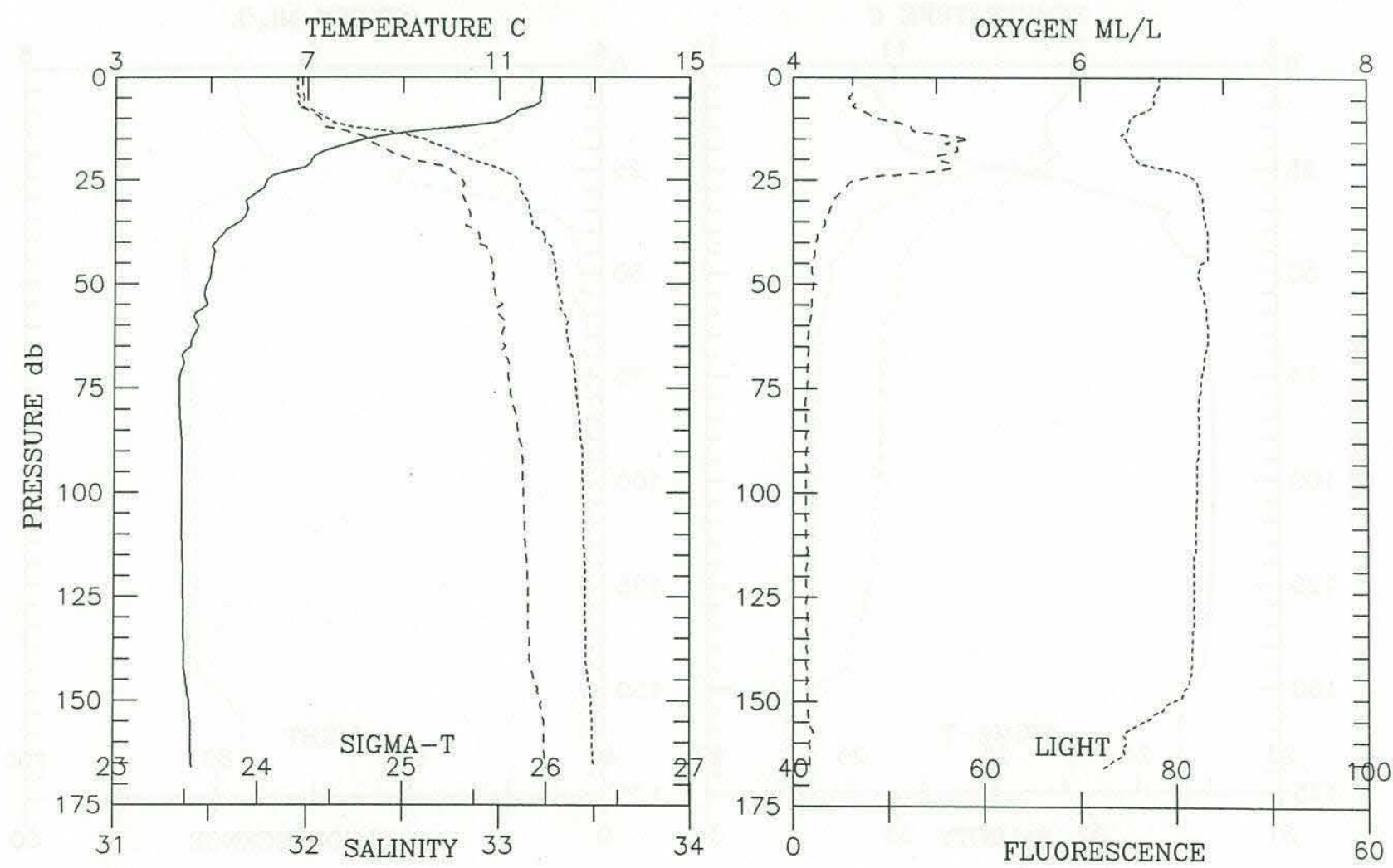


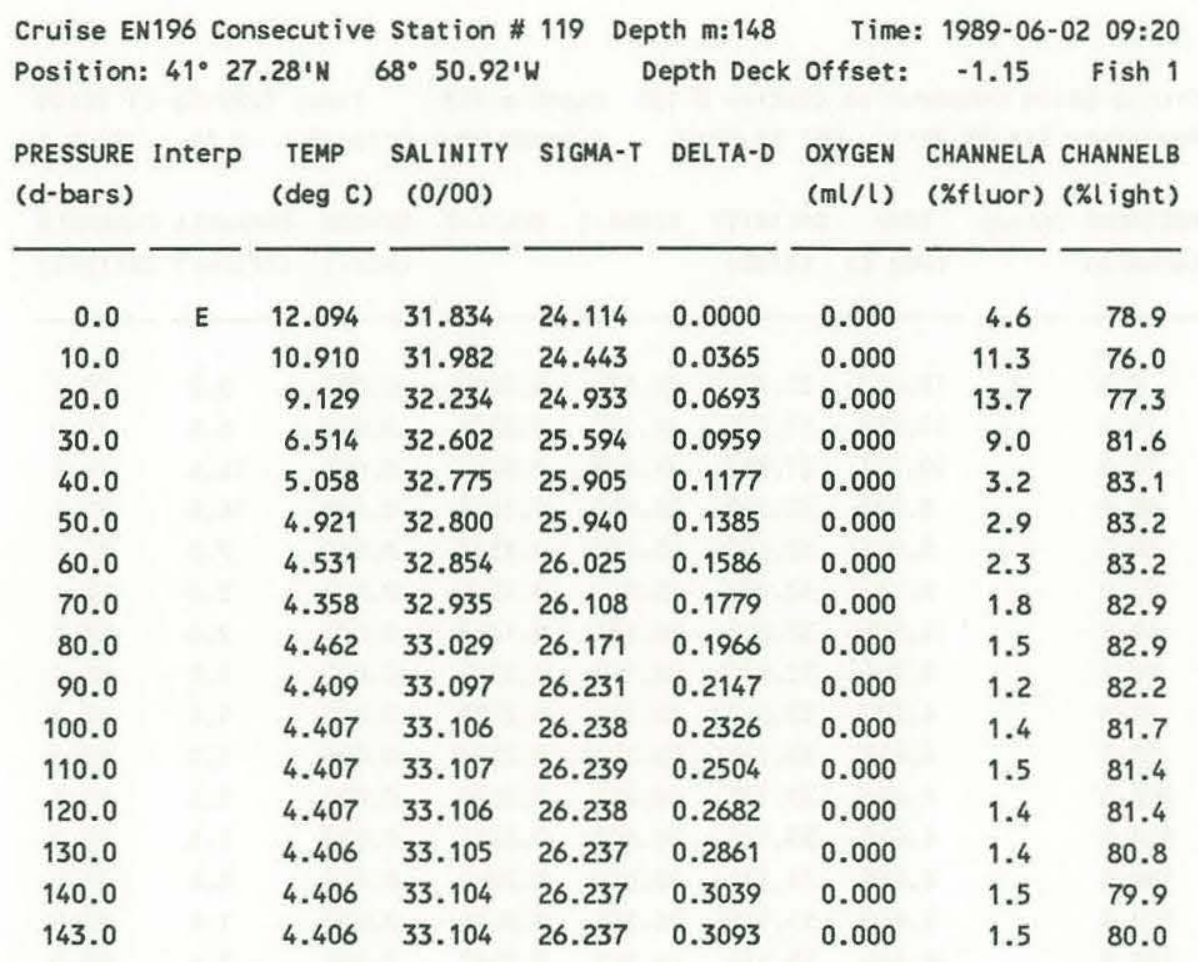
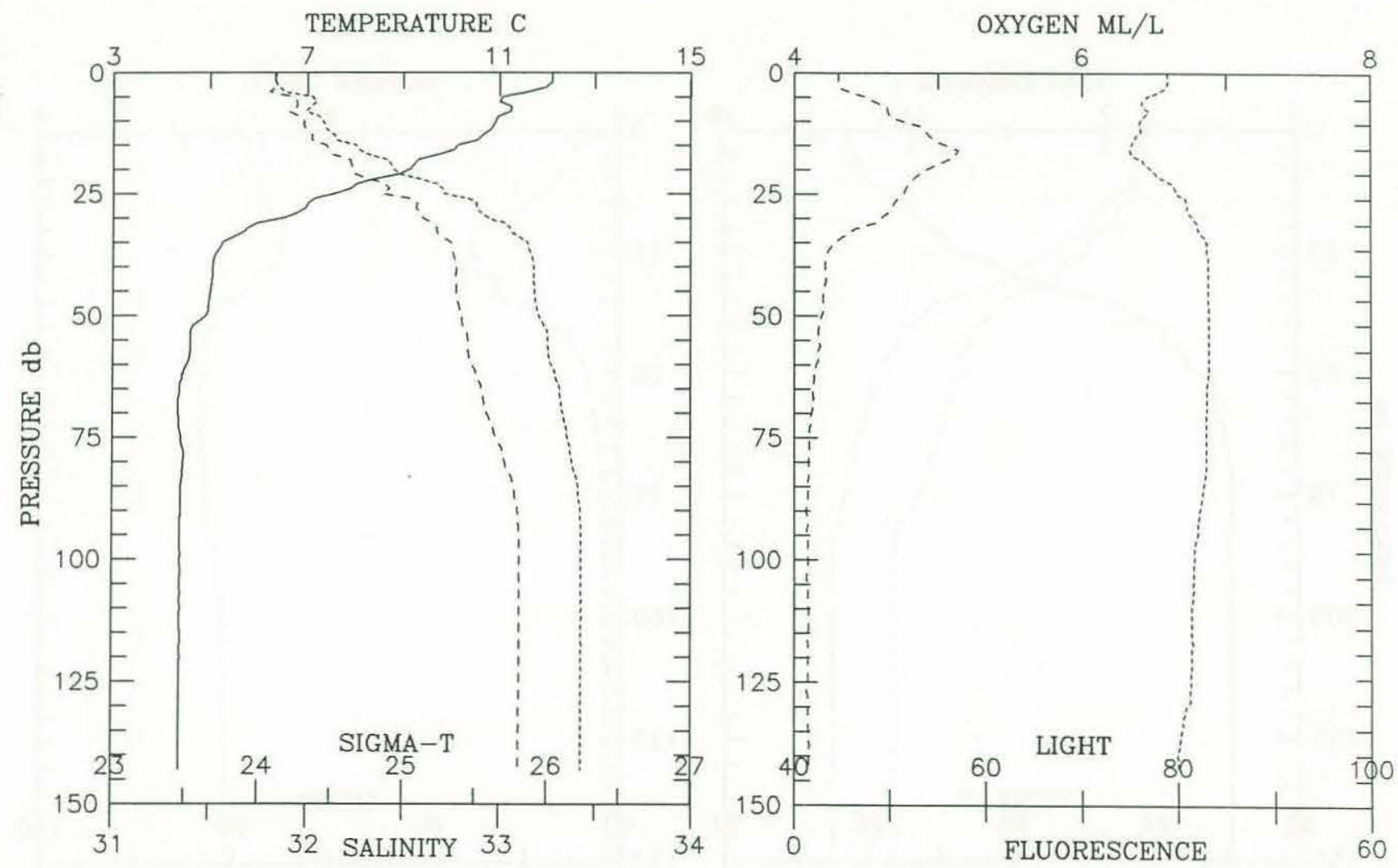


\begin{tabular}{|c|c|c|c|c|c|c|c|c|}
\hline $\begin{array}{l}\text { Cruise El } \\
\text { Position }\end{array}$ & $\begin{array}{l}\text { N196 Con } \\
\text { : } 41^{\circ} \quad 27\end{array}$ & $\begin{array}{l}\text { secutive } \\
.23^{\prime} N\end{array}$ & $\begin{array}{l}\text { Station } \\
68^{\circ} 50.80^{\prime}\end{array}$ & Def & $\begin{array}{l}\text { th } m: 158 \\
\text { epth Deck }\end{array}$ & $\begin{array}{r}\text { Time } \\
\text { offset: }\end{array}$ & $\begin{array}{c}1989-06- \\
-0.63\end{array}$ & $\begin{array}{r}01 \text { 19:00 } \\
\text { Fish } 1\end{array}$ \\
\hline $\begin{array}{l}\text { PRESSURE } \\
\text { (d-bars) }\end{array}$ & Interp & $\begin{array}{c}\text { TEMP } \\
(\operatorname{deg} C)\end{array}$ & $\begin{array}{l}\text { SALINITY } \\
(0 / 00)\end{array}$ & SIGMA-T & DELTA-D & $\begin{array}{l}\text { OXYGEN } \\
(\mathrm{ml} / \mathrm{l})\end{array}$ & $\begin{array}{l}\text { CHANNELA } \\
\text { ( } \% \text { fluor) }\end{array}$ & $\begin{array}{l}\text { CHANNELB } \\
\text { (\%light) }\end{array}$ \\
\hline 0.0 & E & 12.408 & 31.794 & 24.024 & 0.0000 & 0.000 & 3.2 & 79.1 \\
\hline 10.0 & & 11.931 & 31.835 & 24.145 & 0.0383 & 0.000 & 6.9 & 77.0 \\
\hline 20.0 & & 10.741 & 31.965 & 24.458 & 0.0744 & 0.000 & 14.4 & 74.5 \\
\hline 30.0 & & 8.922 & 32.269 & 24.993 & 0.1066 & 0.000 & 14.4 & 77.6 \\
\hline 40.0 & & 5.926 & 32.644 & 25.700 & 0.1326 & 0.000 & 7.3 & 82.1 \\
\hline 50.0 & & 5.141 & 32.732 & 25.862 & 0.1544 & 0.000 & 3.6 & 83.1 \\
\hline 60.0 & & 4.596 & 32.843 & 26.010 & 0.1748 & 0.000 & 2.6 & 83.3 \\
\hline 70.0 & & 4.364 & 32.942 & 26.112 & 0.1943 & 0.000 & 1.8 & 83.0 \\
\hline 80.0 & & 4.392 & 33.048 & 26.194 & 0.2129 & 0.000 & 1.4 & 82.8 \\
\hline 90.0 & & 4.417 & 33.111 & 26.241 & 0.2308 & 0.000 & 1.3 & 81.4 \\
\hline 100.0 & & 4.418 & 33.115 & 26.244 & 0.2486 & 0.000 & 1.4 & 81.0 \\
\hline 110.0 & & 4.418 & 33.116 & 26.245 & 0.2664 & 0.000 & 1.3 & 81.0 \\
\hline 120.0 & & 4.419 & 33.116 & 26.245 & 0.2841 & 0.000 & 1.4 & 81.0 \\
\hline 130.0 & & 4.418 & 33.116 & 26.245 & 0.3019 & 0.000 & 1.4 & 80.9 \\
\hline 140.0 & & 4.419 & 33.116 & 26.245 & 0.3197 & 0.000 & 1.4 & 80.9 \\
\hline 147.0 & & 4.419 & 33.116 & 26.245 & 0.3322 & 0.000 & 1.4 & 80.8 \\
\hline
\end{tabular}
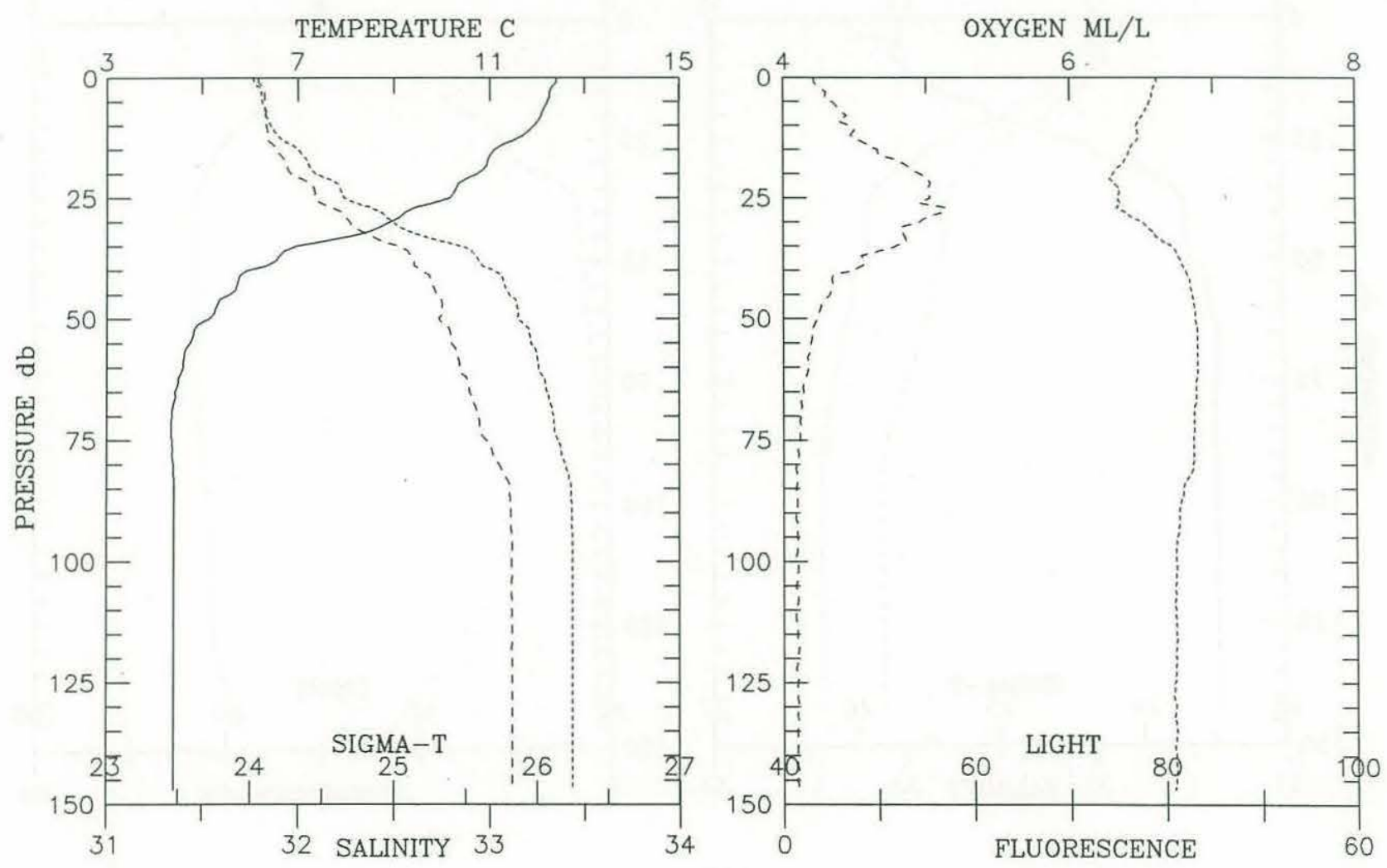


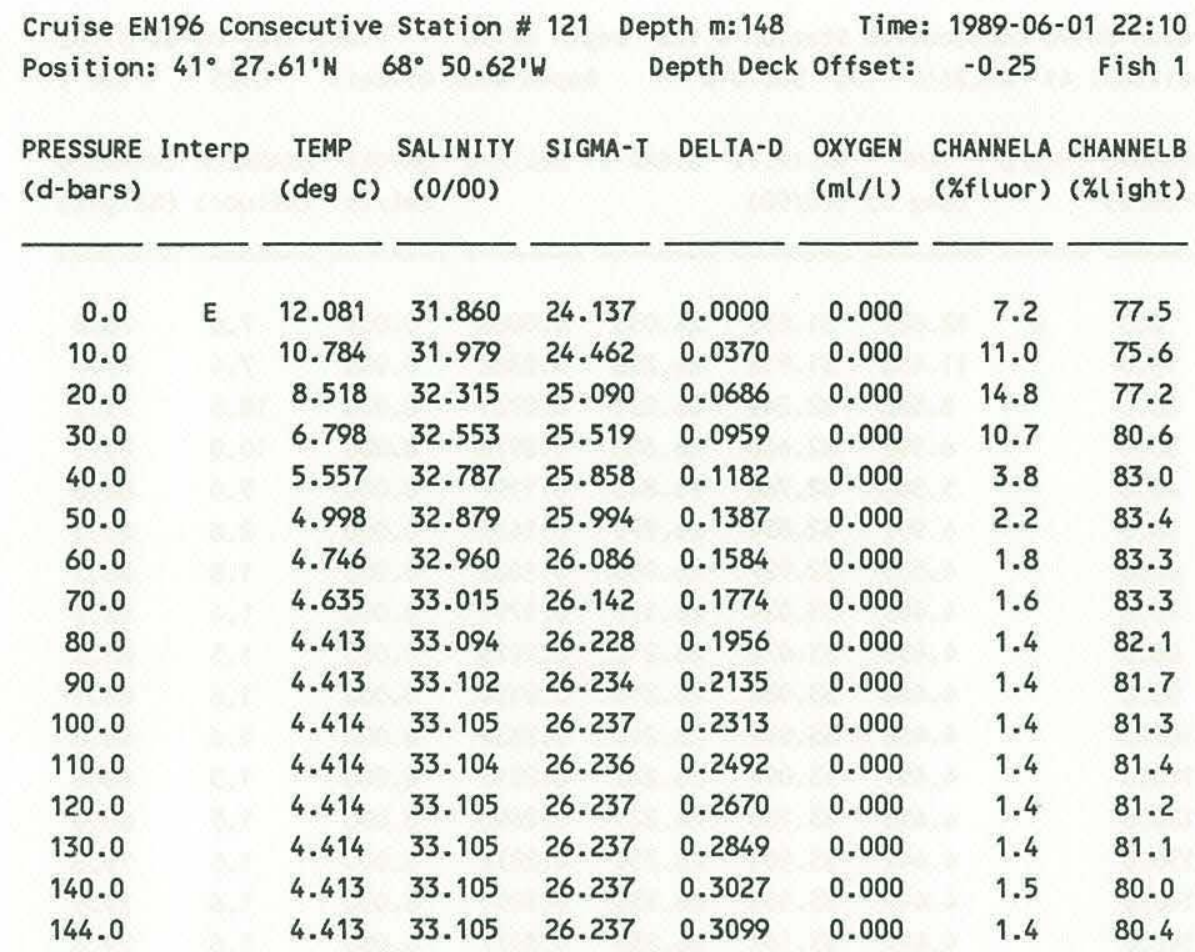
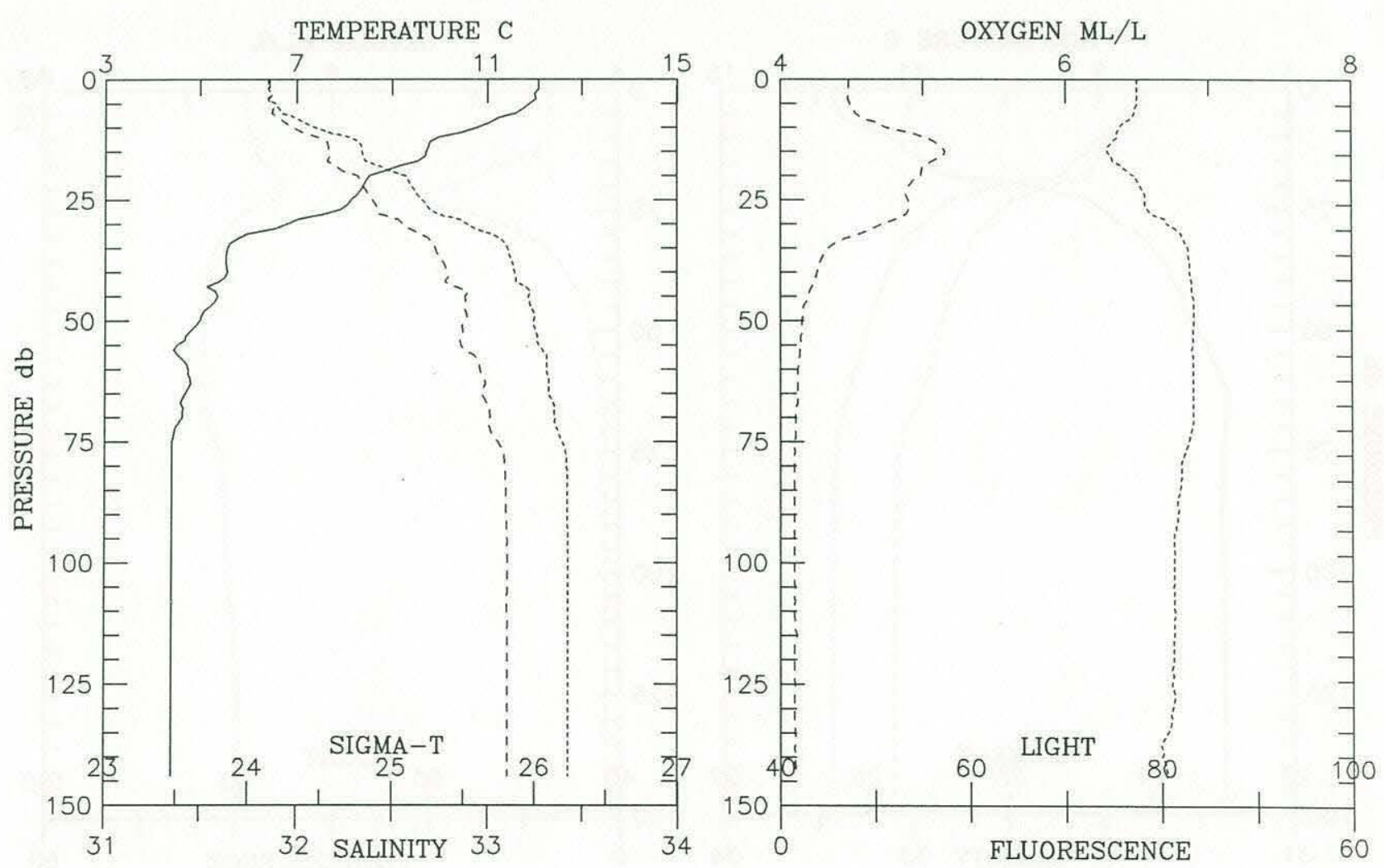


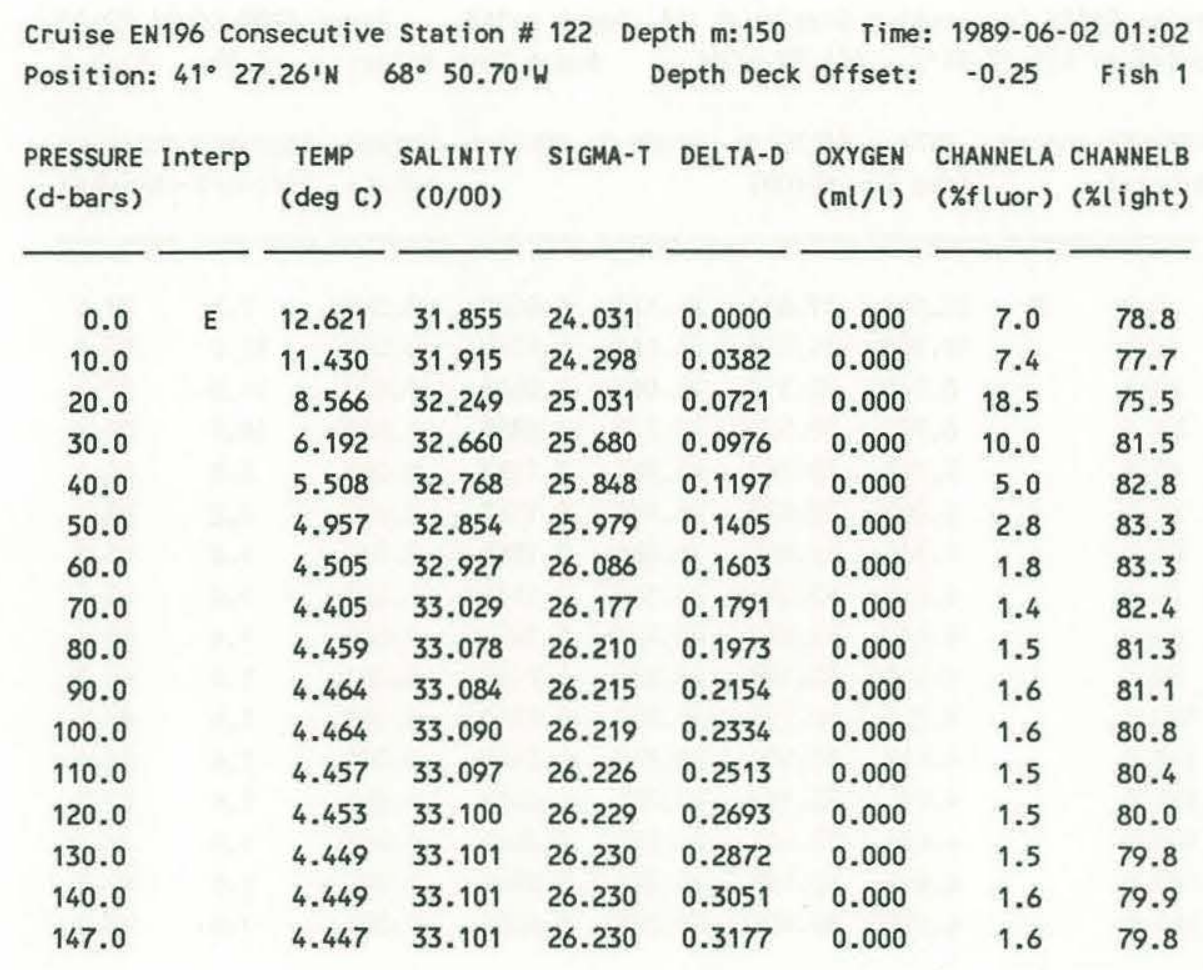
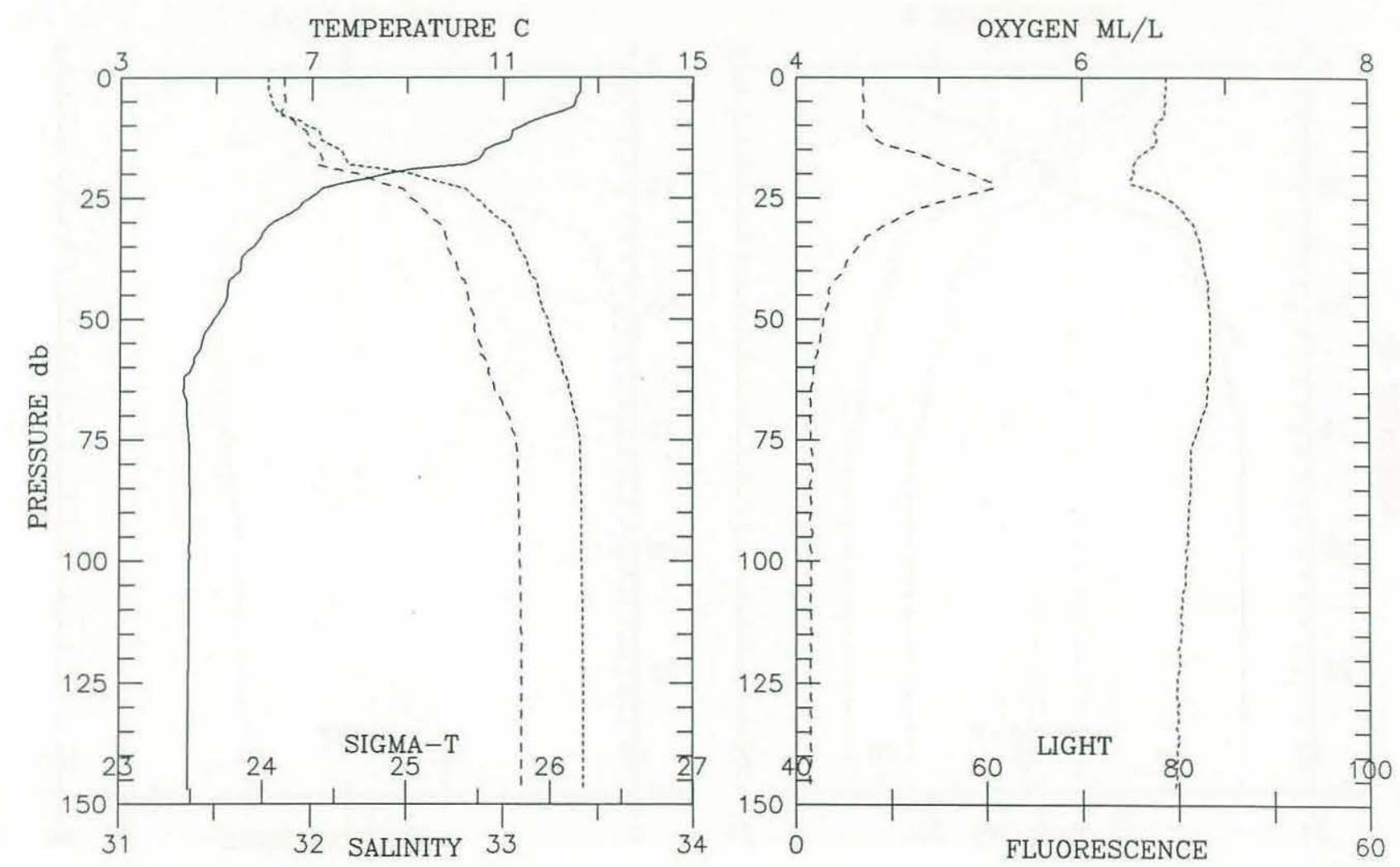


\begin{tabular}{|c|c|c|c|c|c|c|c|c|}
\hline \multicolumn{4}{|c|}{ Cruise EN196 Consecutive Station \# 123} & ${ }_{W}^{123}$ & epth m: 149 & $\begin{array}{r}\text { Time } \\
\text { offset: }\end{array}$ & $\begin{array}{c}1989-06- \\
-0.25\end{array}$ & $\begin{array}{r}-0205: 44 \\
\text { Fish } 1\end{array}$ \\
\hline $\begin{array}{l}\text { PRESSURE } \\
\text { (d-bars) }\end{array}$ & Interp & $\begin{array}{l}\text { TEMP } \\
(\operatorname{deg} C)\end{array}$ & $\begin{array}{l}\text { SALINITY } \\
(0 / 00)\end{array}$ & SIGMA-T & DELTA-D & $\begin{array}{l}\text { OXYGEN } \\
(\mathrm{ml} / \mathrm{l})\end{array}$ & $\begin{array}{l}\text { CHANNELA } \\
\text { (\%fluor) }\end{array}$ & $\begin{array}{l}\text { CHANNELB } \\
\text { (\%tight) }\end{array}$ \\
\hline 0.0 & E & 11.932 & 31.759 & 24.086 & 0.0000 & 0.000 & 5.8 & 80.1 \\
\hline 10.0 & & 11.360 & 31.806 & 24.226 & 0.0378 & 0.000 & 8.2 & 78.9 \\
\hline 20.0 & & 11.106 & 31.804 & 24.270 & 0.0746 & 0.000 & 9.6 & 78.5 \\
\hline 30.0 & & 9.980 & 32.049 & 24.652 & 0.1098 & 0.000 & 14.9 & 77.7 \\
\hline 40.0 & & 8.847 & 32.278 & 25.011 & 0.1412 & 0.000 & 17.4 & 77.0 \\
\hline 50.0 & & 5.522 & 32.714 & 25.804 & 0.1661 & 0.000 & 5.2 & 82.8 \\
\hline 60.0 & & 4.786 & 32.809 & 25.962 & 0.1873 & 0.000 & 3.1 & 83.2 \\
\hline 70.0 & & 4.404 & 32.892 & 26.068 & 0.2071 & 0.000 & 2.1 & 83.0 \\
\hline 80.0 & & 4.380 & 32.923 & 26.096 & 0.2264 & 0.000 & 1.8 & 83.0 \\
\hline 90.0 & & 4.373 & 33.045 & 26.193 & 0.2450 & 0.000 & 1.5 & 82.6 \\
\hline 100.0 & & 4.405 & 33.101 & 26.234 & 0.2630 & 0.000 & 1.3 & 81.9 \\
\hline 110.0 & & 4.411 & 33.106 & 26.238 & 0.2809 & 0.000 & 1.4 & 81.4 \\
\hline 120.0 & & 4.412 & 33.108 & 26.239 & 0.2987 & 0.000 & 1.3 & 80.2 \\
\hline 130.0 & & 4.411 & 33.108 & 26.239 & 0.3165 & 0.000 & 1.3 & 79.1 \\
\hline 140.0 & & 4.410 & 33.107 & 26.239 & 0.3343 & 0.000 & 1.5 & 79.1 \\
\hline 146.0 & & 4.411 & 33.107 & 26.239 & 0.3450 & 0.000 & 1.4 & 78.5 \\
\hline
\end{tabular}
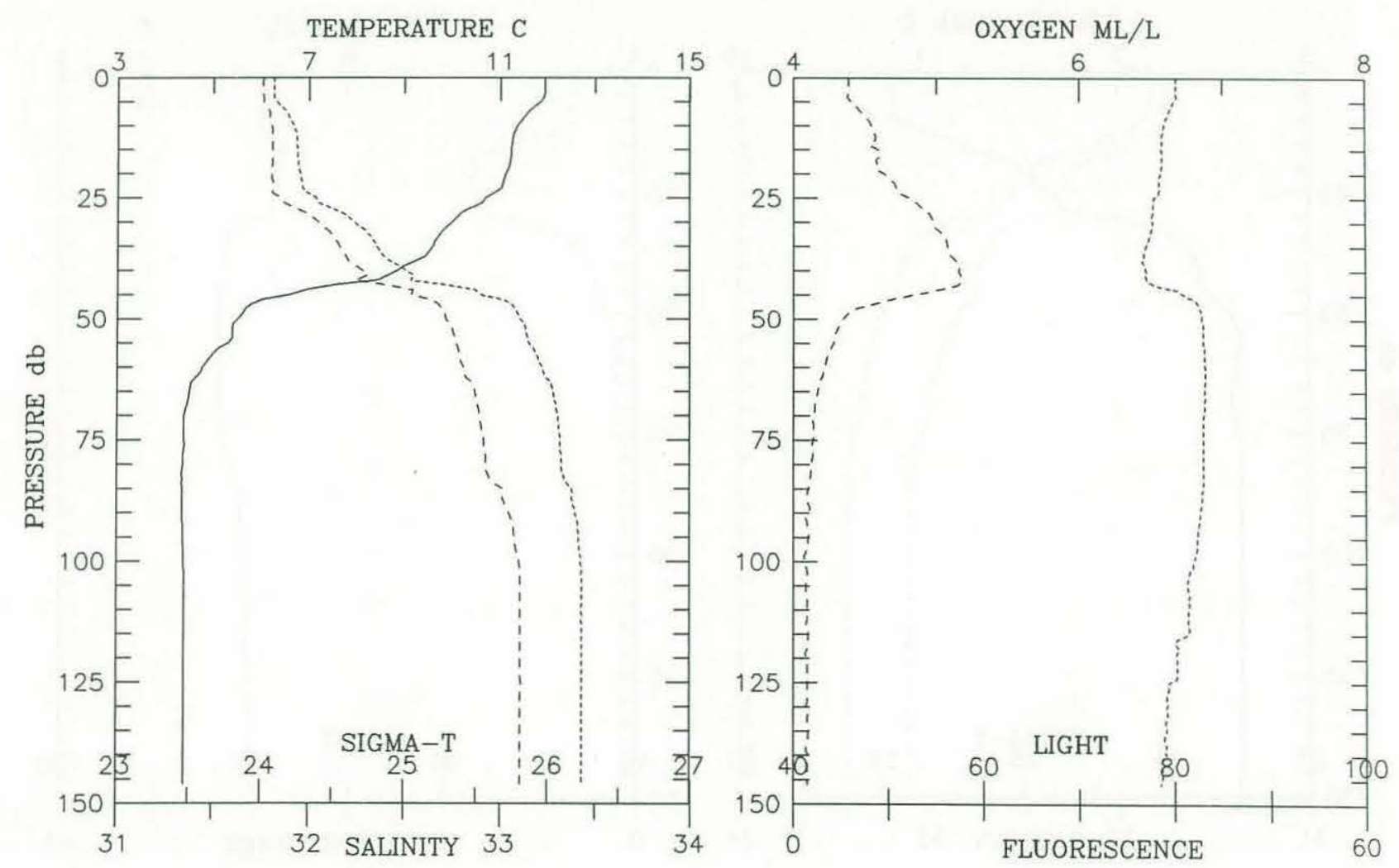


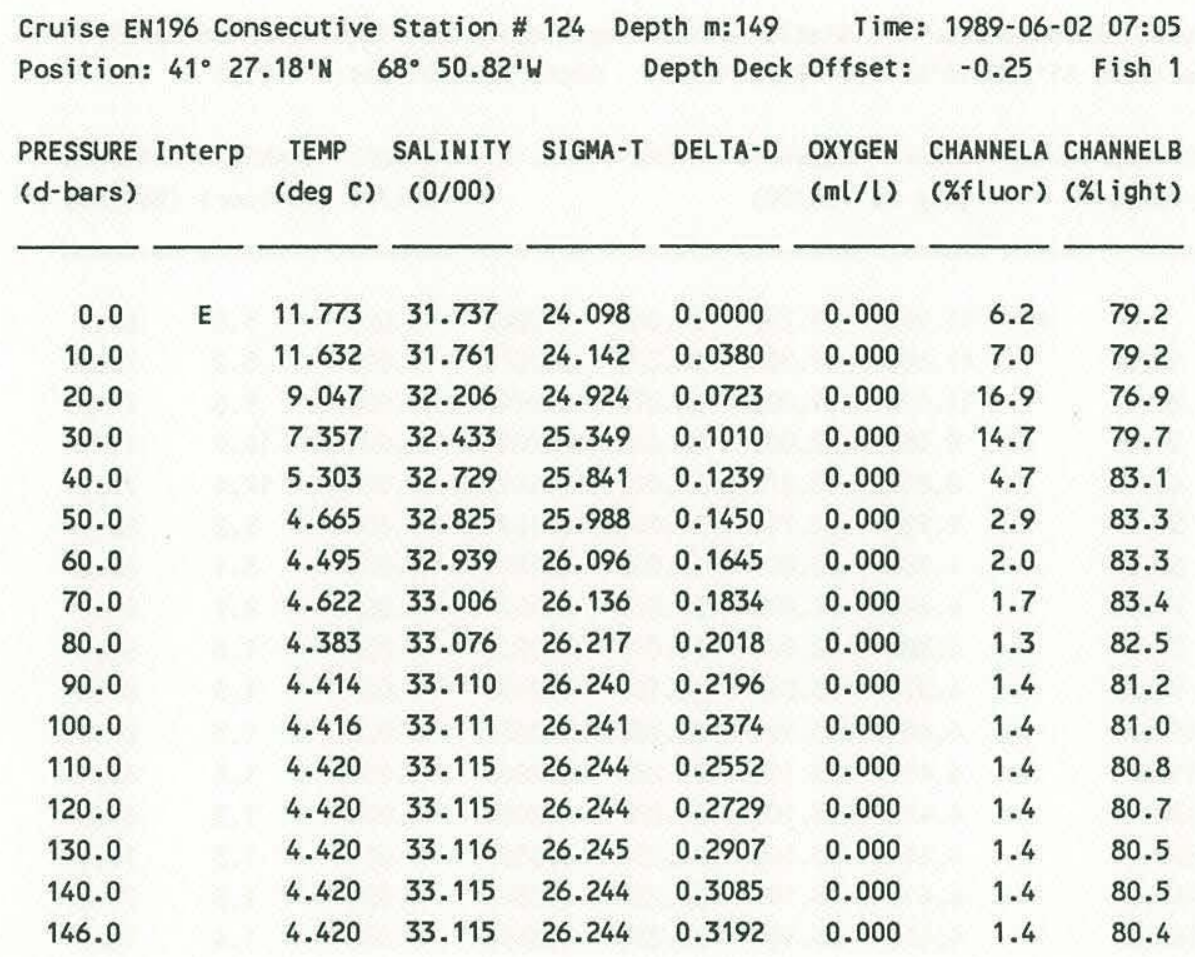
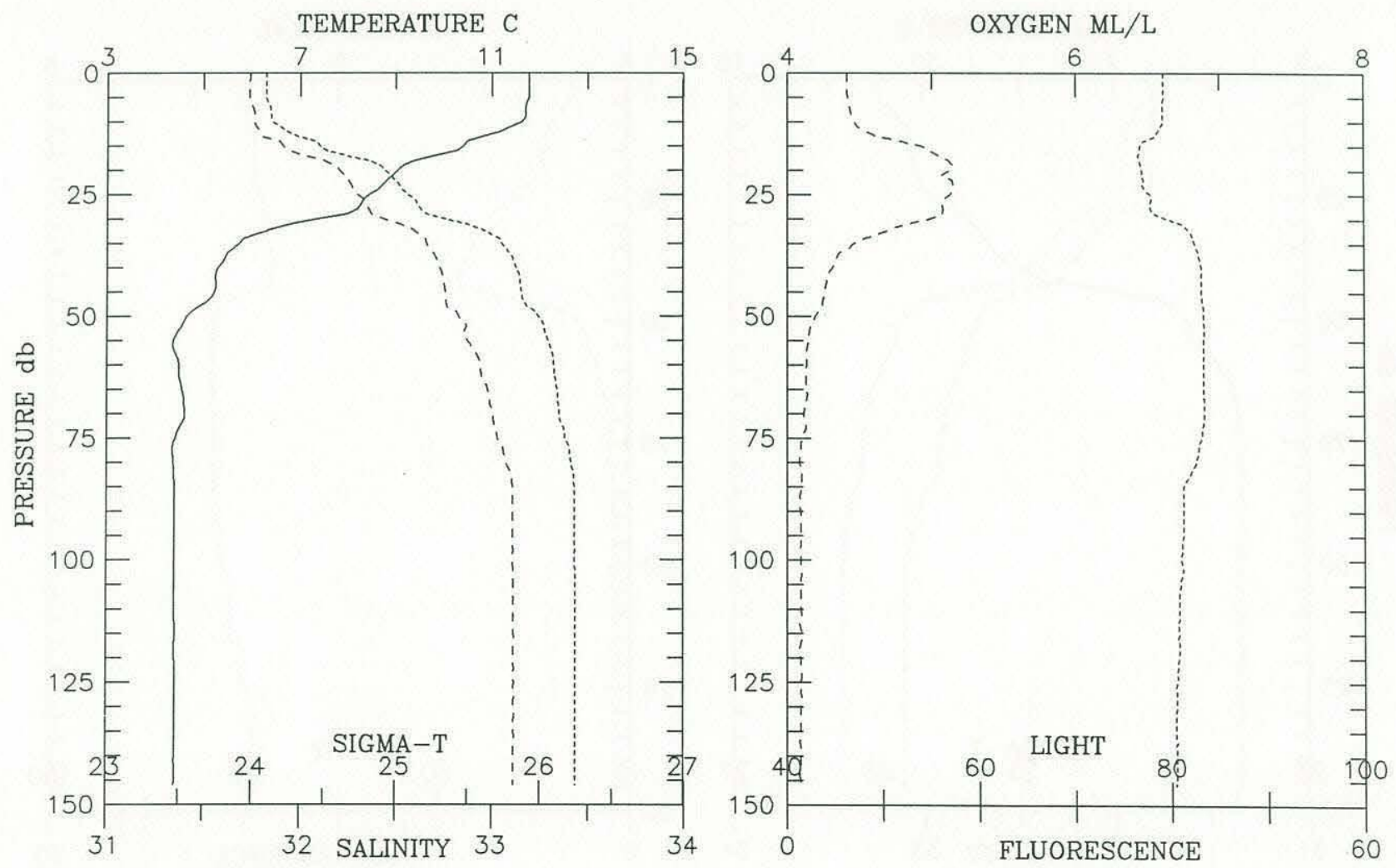


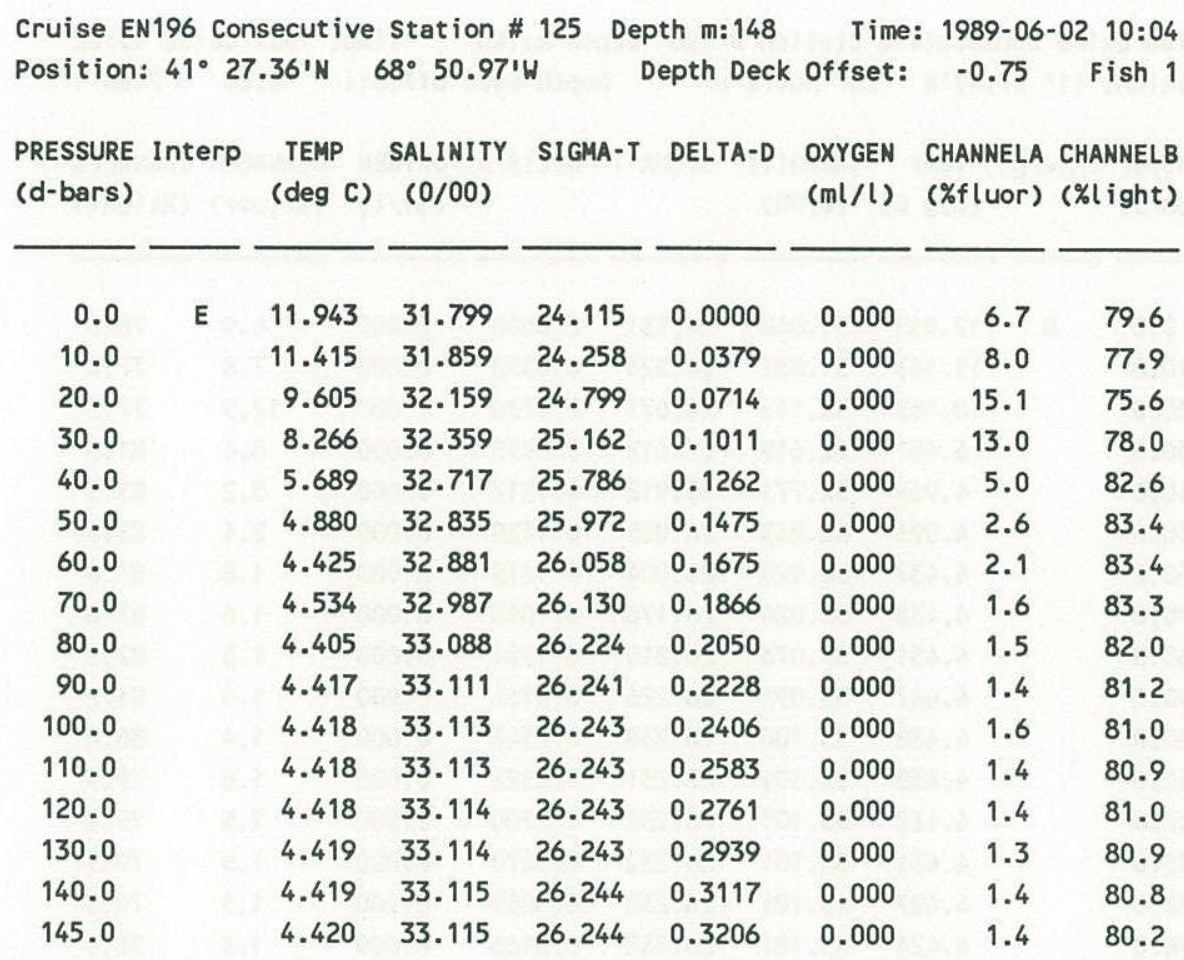
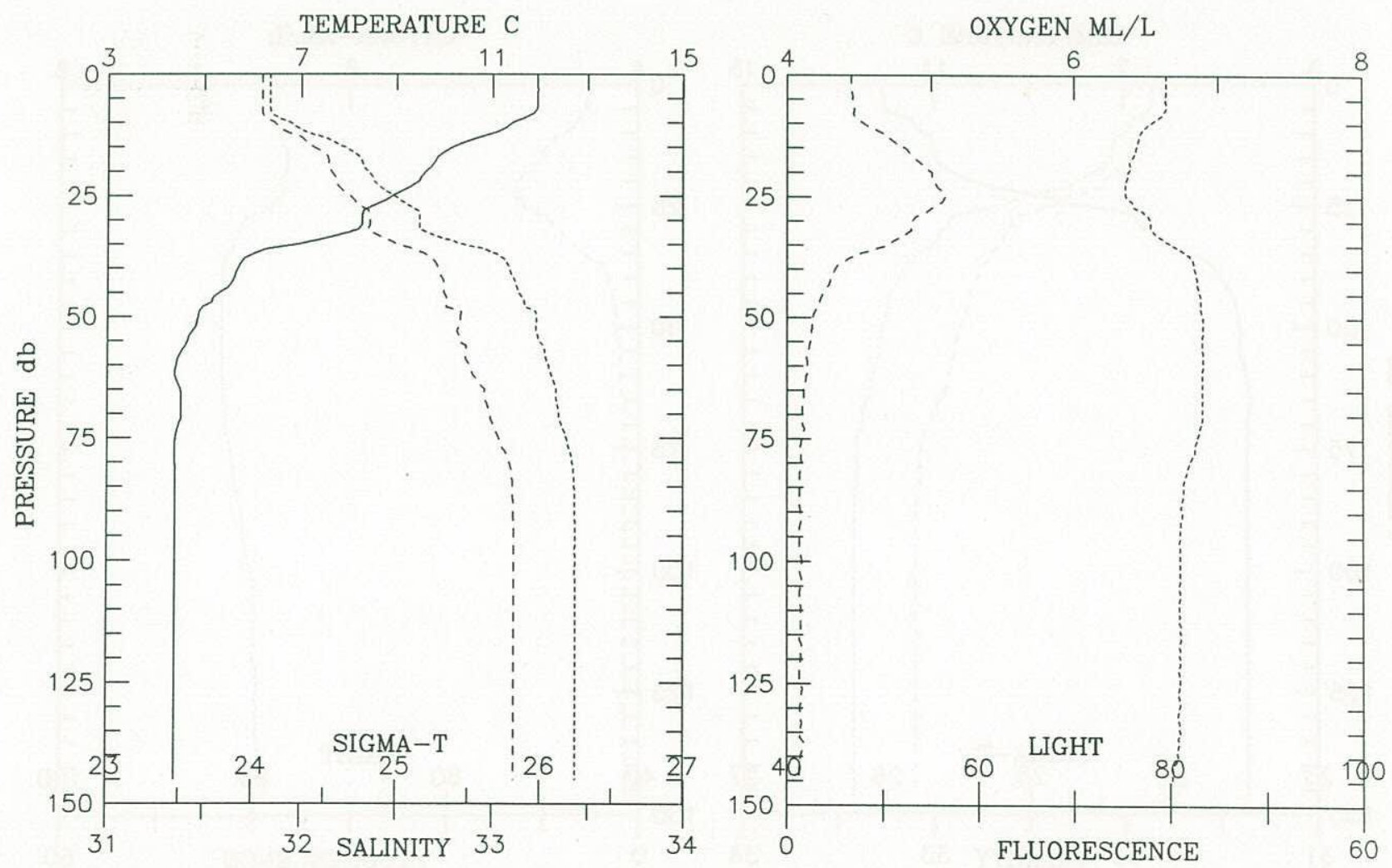


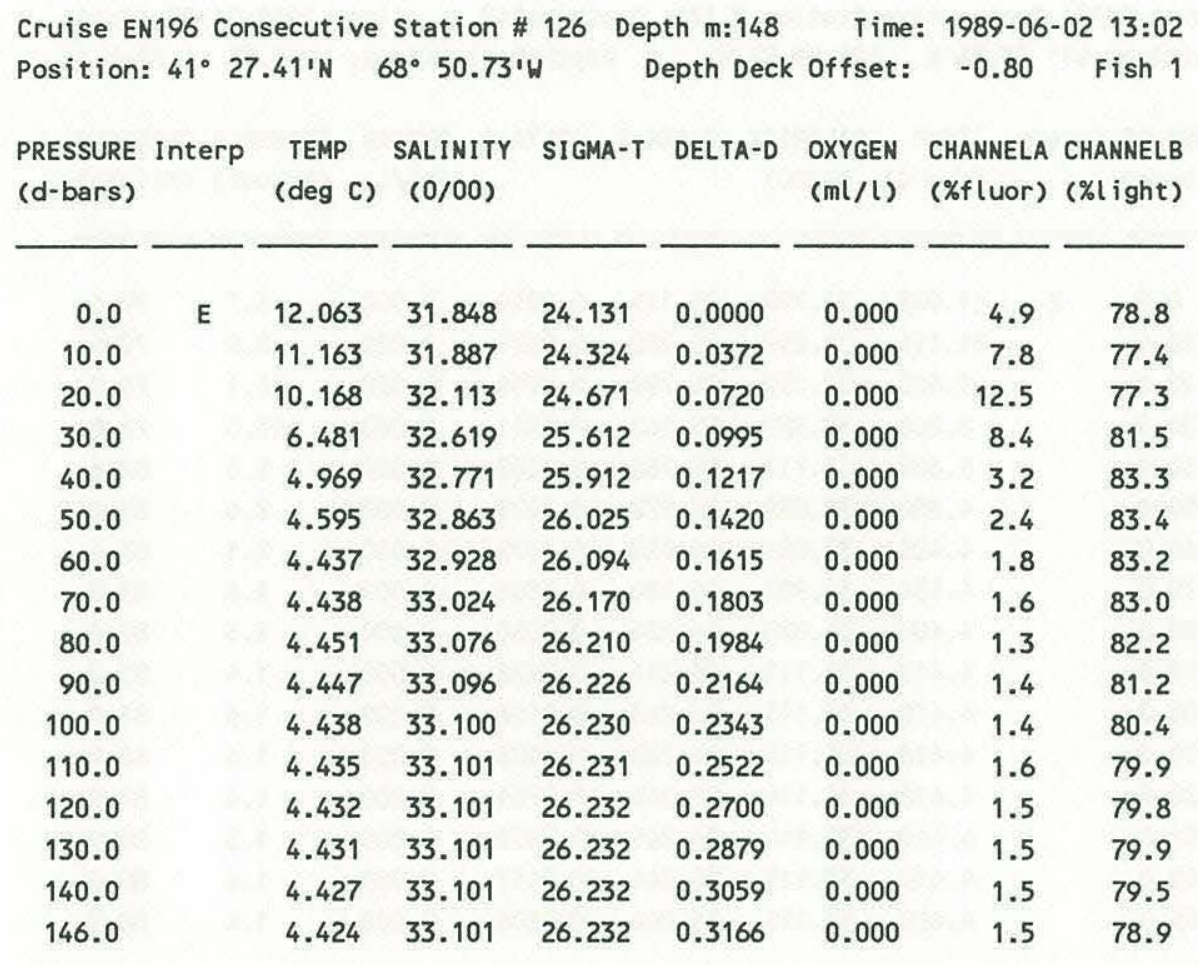
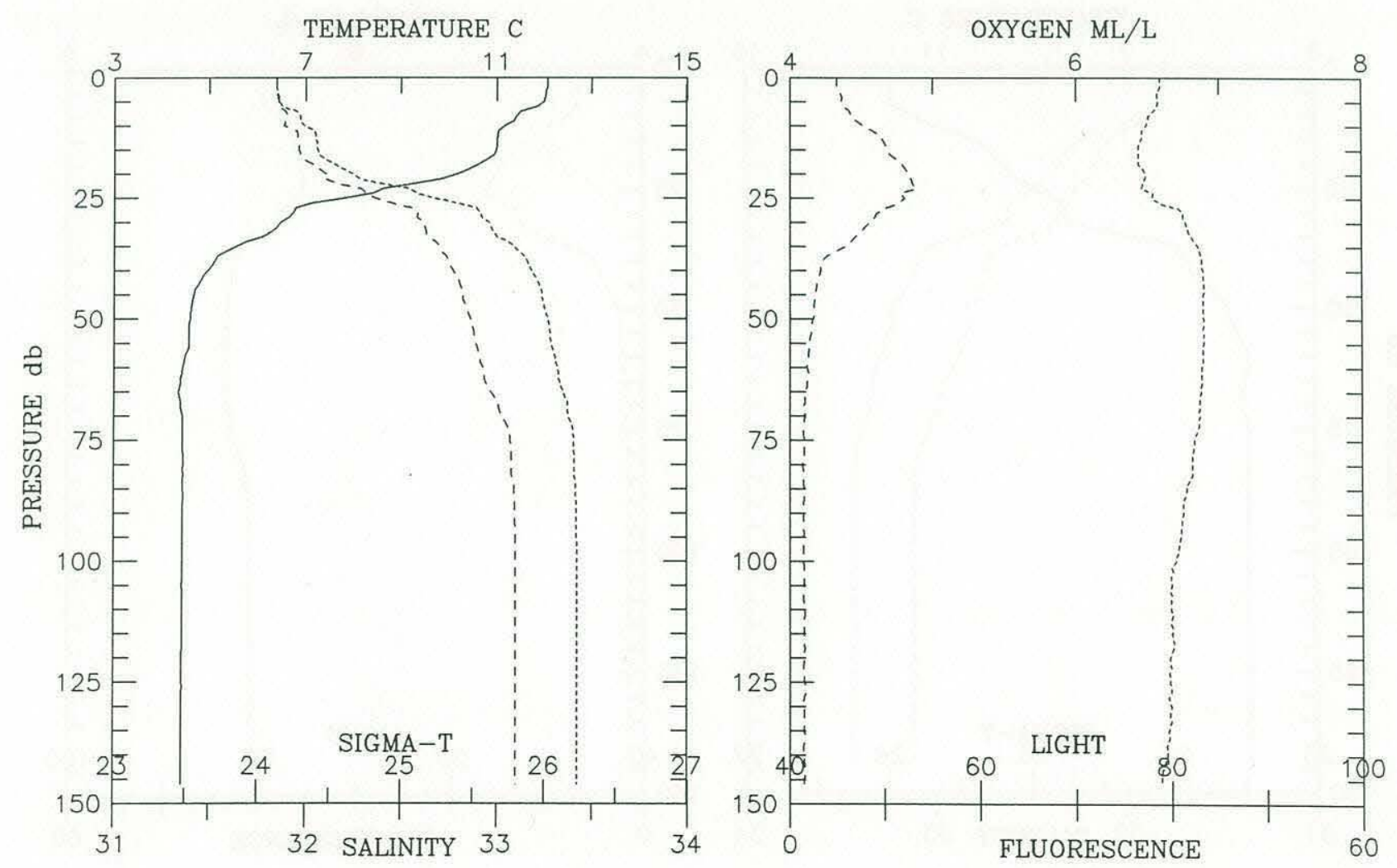


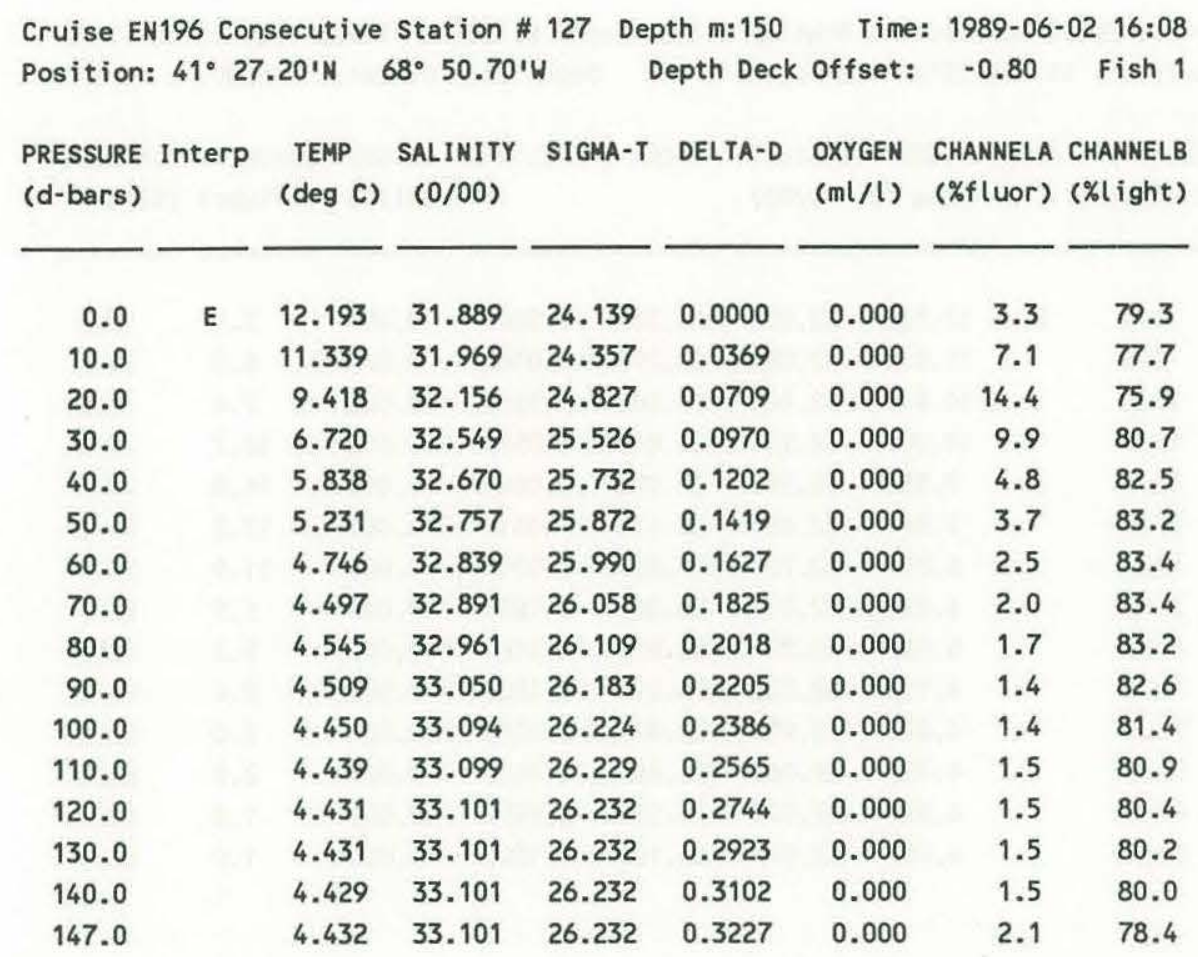
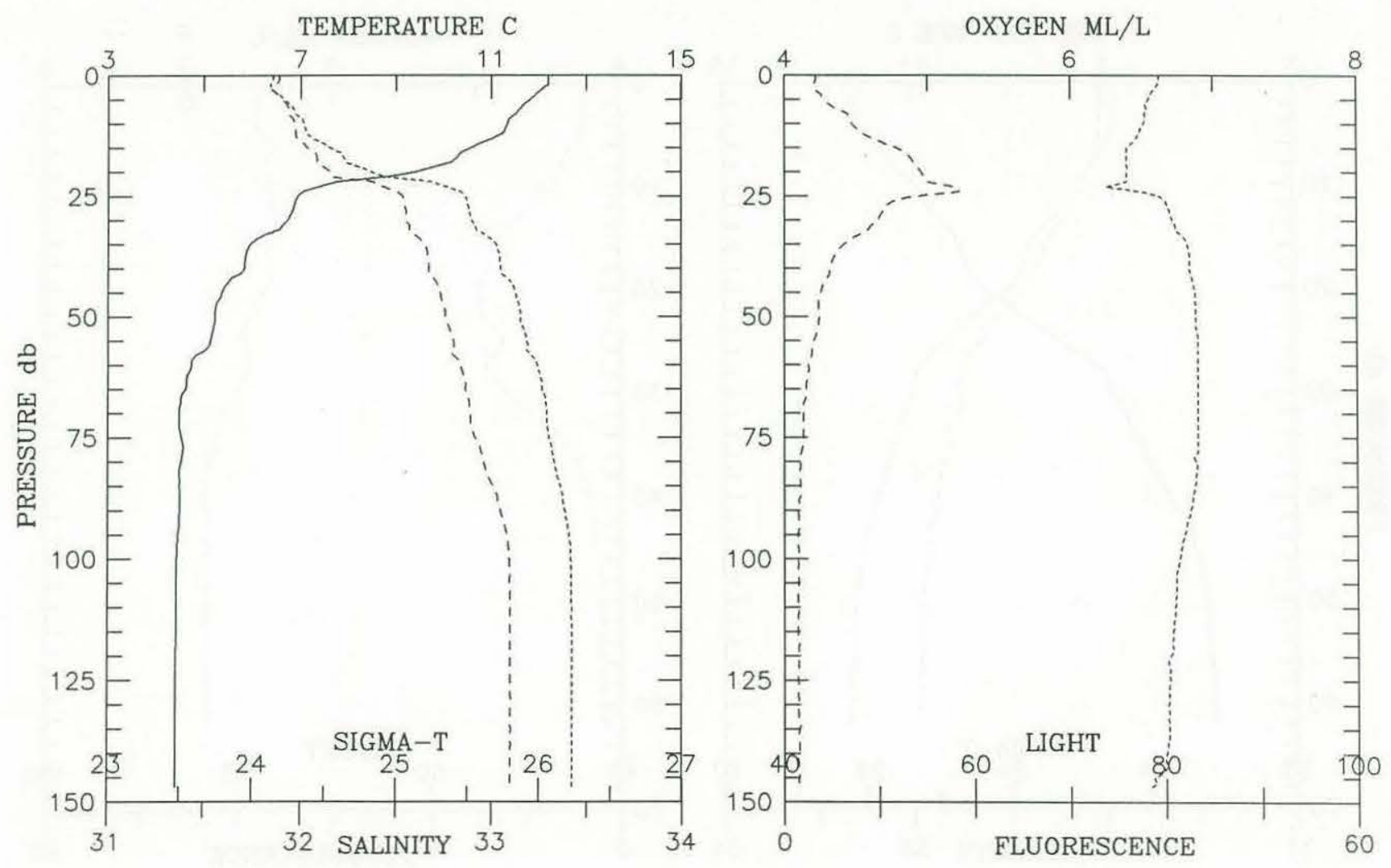


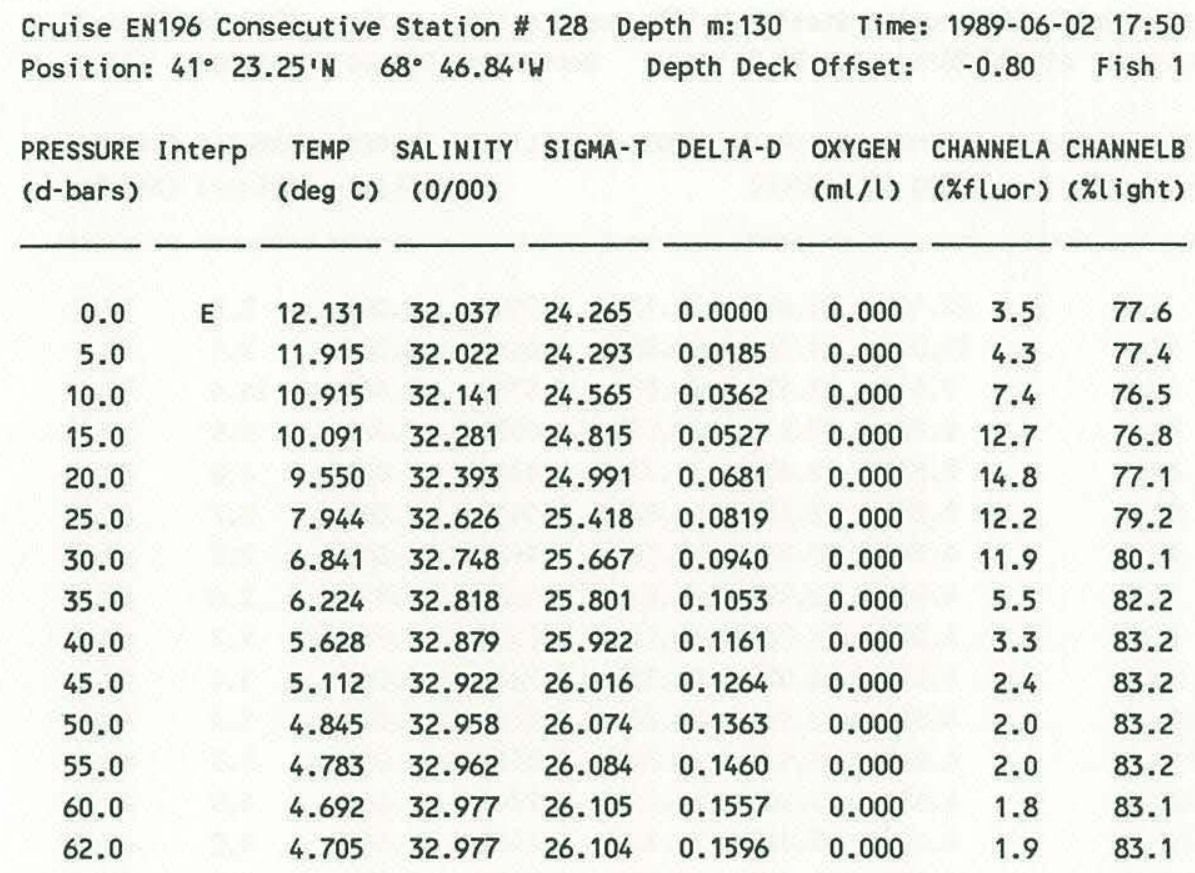
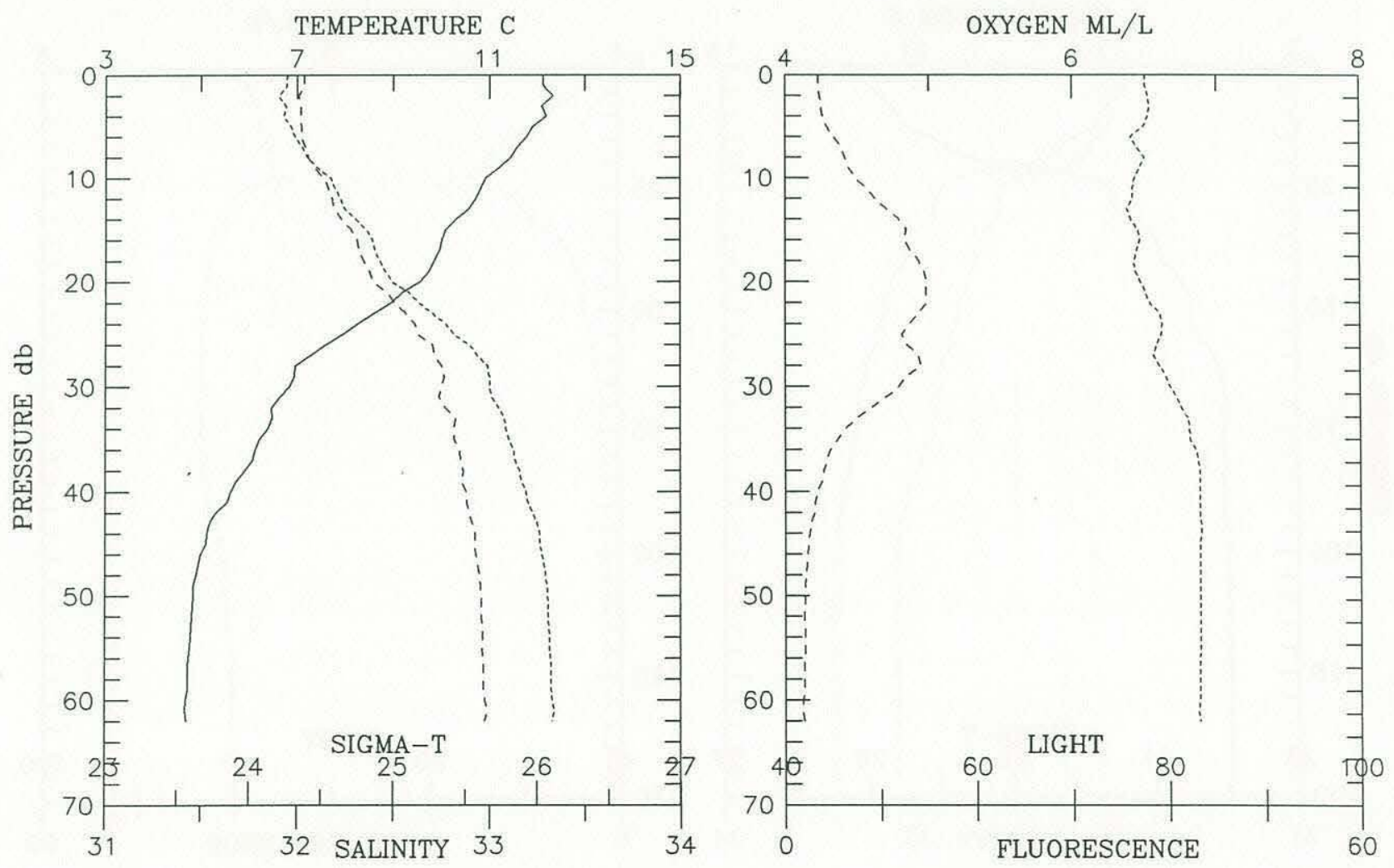


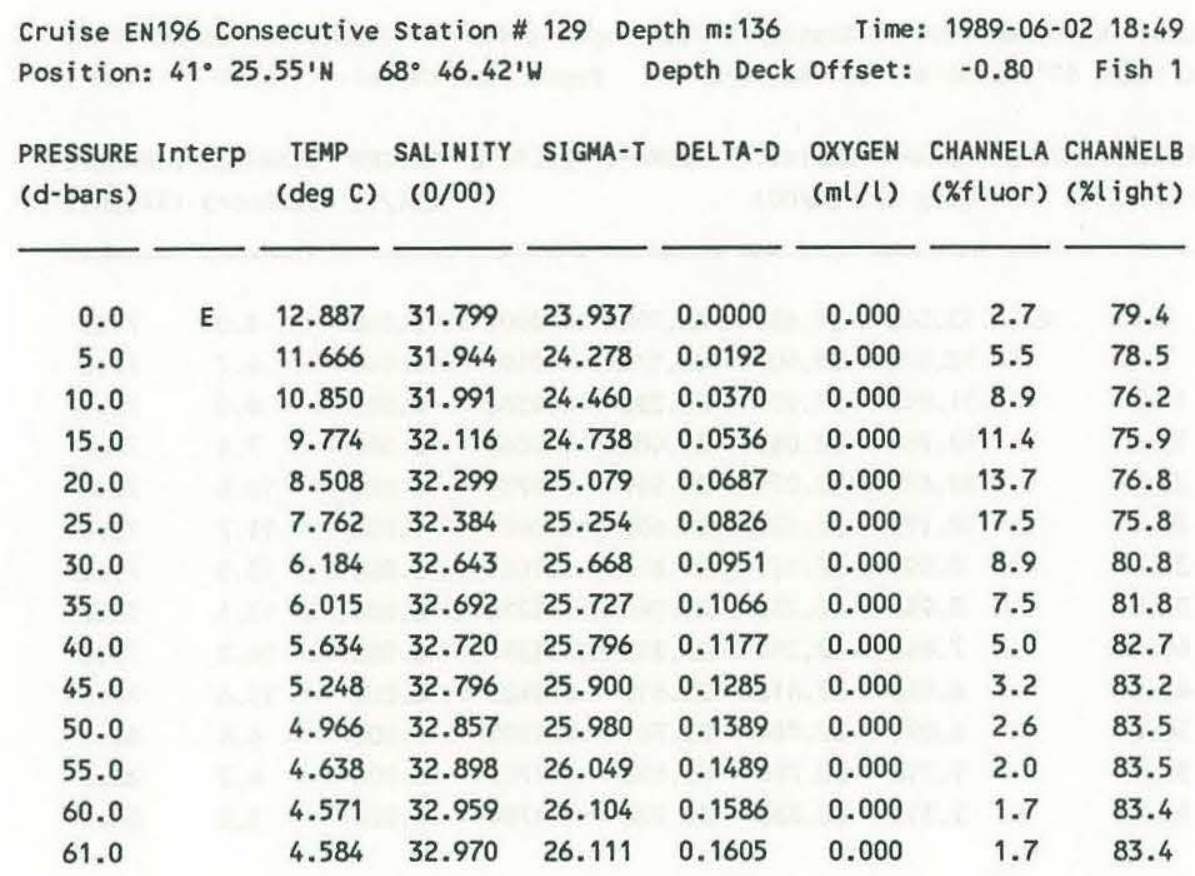
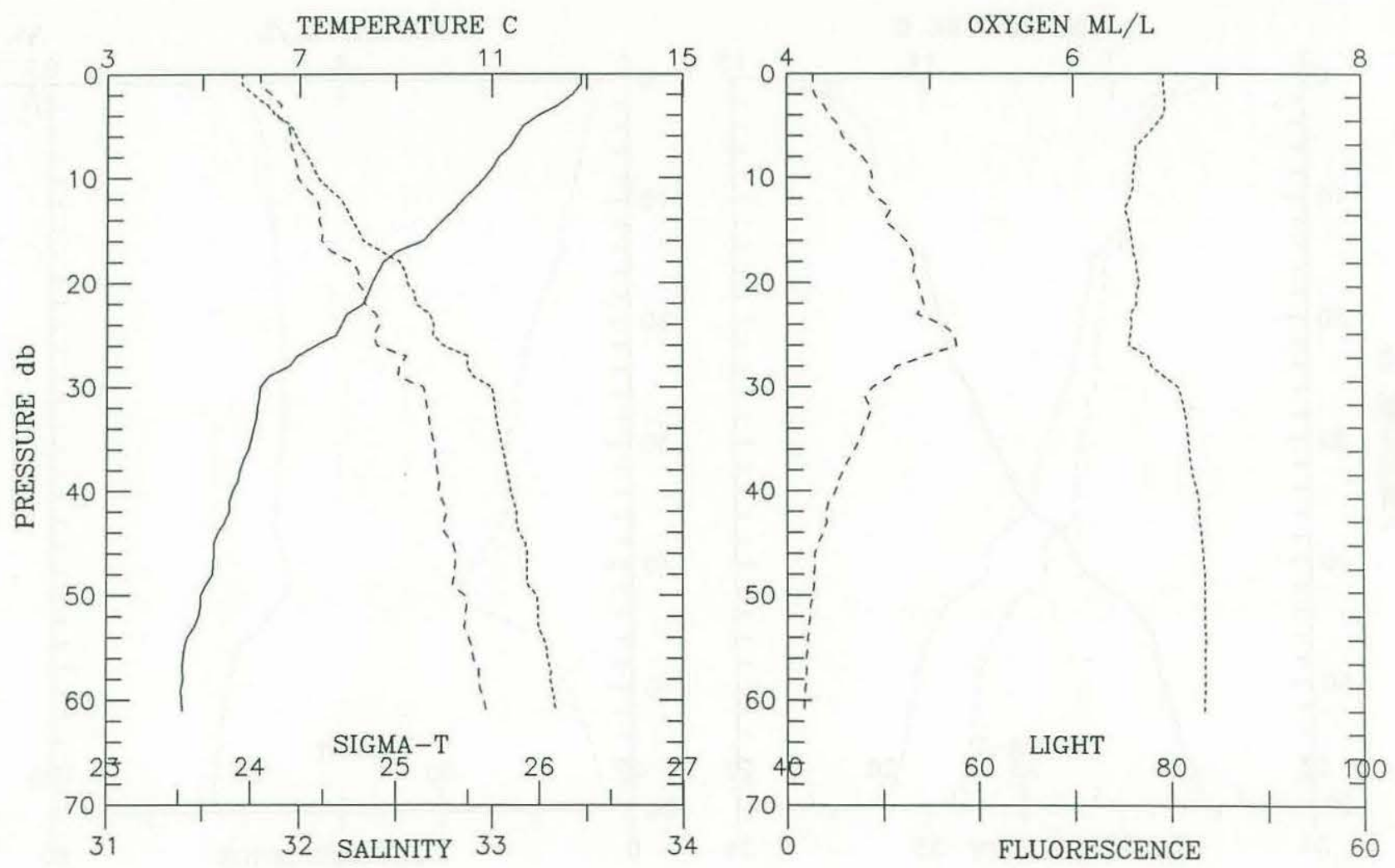


\begin{tabular}{|c|c|c|c|c|c|c|c|c|}
\hline Position: & \multicolumn{2}{|c|}{$41^{\circ} 28.04^{\prime} \mathrm{N}$} & \multicolumn{2}{|c|}{$68^{\circ} 46.22^{\prime} \mathrm{W}$} & Depth Deck & Offset: & -0.80 & \multirow{2}{*}{$\begin{array}{r}\text { Fish } 1 \\
\text { CHANNELB } \\
\text { (\%light) }\end{array}$} \\
\hline $\begin{array}{l}\text { PRESSURE } \\
\text { (d-bars) }\end{array}$ & Interp & $\begin{array}{c}\text { TEMP } \\
\text { (deg C) }\end{array}$ & $\begin{array}{l}\text { SALINITY } \\
(0 / 00)\end{array}$ & SIGMA-T & DELTA-D & $\begin{array}{l}\text { OXYGEN } \\
(\mathrm{ml} / \mathrm{l})\end{array}$ & $\begin{array}{l}\text { CHANNELA } \\
\text { (\%fluor) }\end{array}$ & \\
\hline 0.0 & E & 13.508 & 31.650 & 23.700 & 0.0000 & 0.000 & 3.0 & 79.7 \\
\hline 5.0 & & 12.079 & 31.904 & 24.172 & 0.0197 & 0.000 & 4.7 & 77.8 \\
\hline 10.0 & & 11.898 & 31.937 & 24.230 & 0.0382 & 0.000 & 6.0 & 77.4 \\
\hline 15.0 & & 10.962 & 32.045 & 24.482 & 0.0563 & 0.000 & 7.4 & 76.5 \\
\hline 20.0 & & 10.670 & 32.079 & 24.559 & 0.0734 & 0.000 & 10.0 & 76.0 \\
\hline 25.0 & & 10.191 & 32.098 & 24.655 & 0.0901 & 0.000 & 11.7 & 76.0 \\
\hline 30.0 & & 9.599 & 32.183 & 24.819 & 0.1061 & 0.000 & 13.0 & 76.2 \\
\hline 35.0 & & 8.954 & 32.234 & 24.960 & 0.1214 & 0.000 & 13.1 & 76.7 \\
\hline 40.0 & & 7.840 & 32.390 & 25.248 & 0.1357 & 0.000 & 16.8 & 75.8 \\
\hline 45.0 & & 6.453 & 32.612 & 25.610 & 0.1485 & 0.000 & 11.6 & 78.7 \\
\hline 50.0 & & 6.074 & 32.744 & 25.761 & 0.1599 & 0.000 & 6.5 & 82.0 \\
\hline 55.0 & & 5.716 & 32.786 & 25.838 & 0.1709 & 0.000 & 4.2 & 82.7 \\
\hline 59.0 & & 5.311 & 32.832 & 25.922 & 0.1794 & 0.000 & 3.0 & 83.4 \\
\hline
\end{tabular}
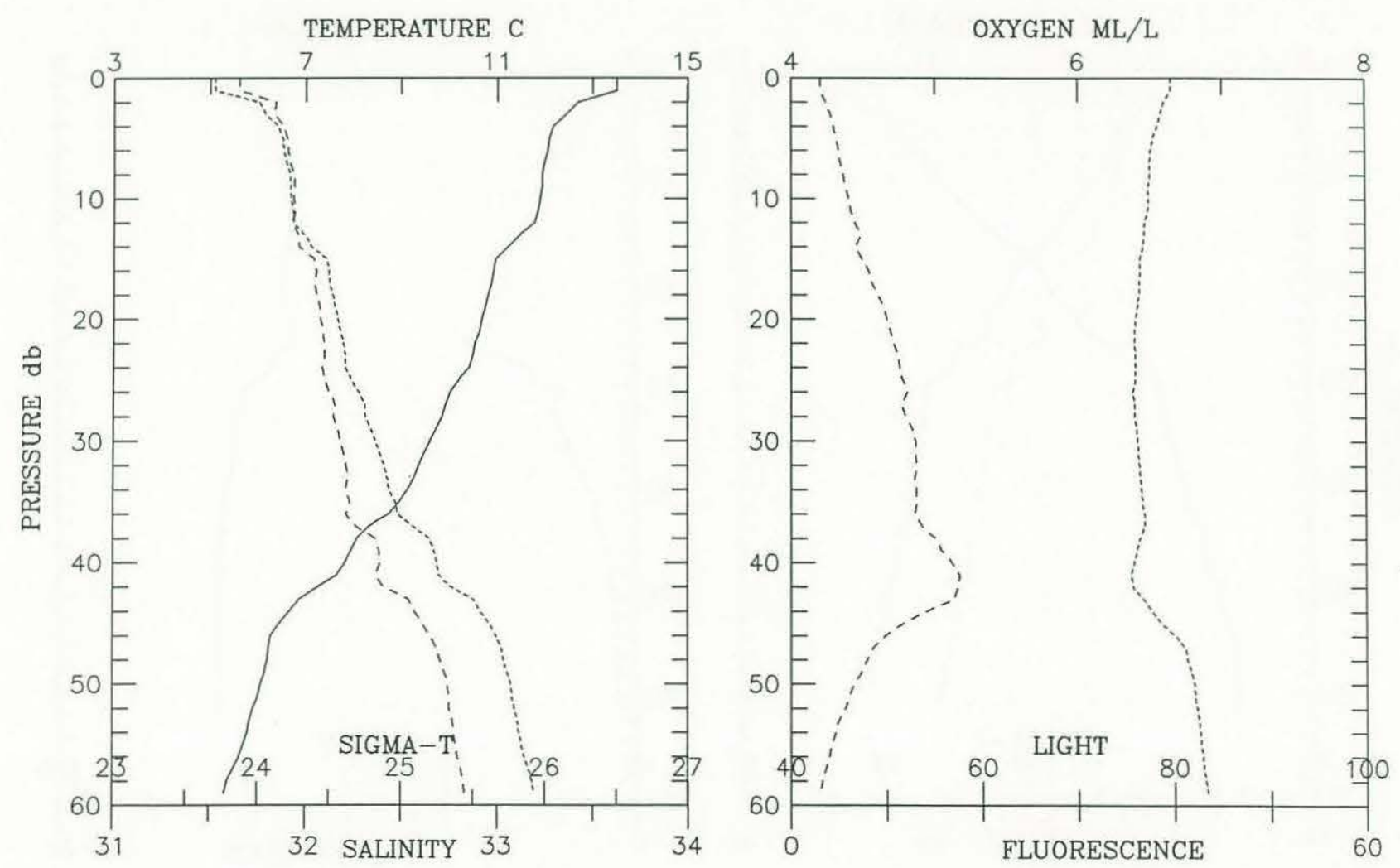
Cruise EN196 Consecutive Station \# 131 Depth m:125 Time: 1989-06-02 20:18 Position: $41^{\circ} 26.75 \mathrm{~N} 68^{\circ} 43.20^{\prime} \mathrm{W} \quad$ Depth Deck offset: -0.38 Fish 1

\begin{tabular}{|c|c|c|c|c|c|c|c|c|}
\hline $\begin{array}{l}\text { PRESSURE } \\
\text { (d-bars) }\end{array}$ & Interp & $\begin{array}{c}\text { TEMP } \\
(\operatorname{deg} \mathrm{C})\end{array}$ & $\begin{array}{l}\text { SALINITY } \\
(0 / 00)\end{array}$ & SIGMA-T & DELTA-D & $\begin{array}{l}\text { OXYGEN } \\
(\mathrm{ml} / \mathrm{l})\end{array}$ & $\begin{array}{l}\text { CHANNELA } \\
\text { (\%fluor) }\end{array}$ & $\begin{array}{l}\text { CHANNELB } \\
\text { (\%light) }\end{array}$ \\
\hline 0.0 & E & 12.118 & 32.019 & 24.253 & 0.0000 & 0.000 & 3.9 & 77.4 \\
\hline 5.0 & & 11.862 & 32.004 & 24.289 & 0.0183 & 0.000 & 4.2 & 77.3 \\
\hline 10.0 & & 11.642 & 31.991 & 24.319 & 0.0364 & 0.000 & 6.3 & 77.2 \\
\hline 15.0 & & 11.132 & 32.002 & 24.419 & 0.0543 & 0.000 & 7.3 & 77.3 \\
\hline 20.0 & & 10.646 & 32.082 & 24.566 & 0.0714 & 0.000 & 9.7 & 76.6 \\
\hline 25.0 & & 10.454 & 32.115 & 24.624 & 0.0881 & 0.000 & 10.5 & 76.5 \\
\hline 30.0 & & 9.507 & 32.186 & 24.836 & 0.1043 & 0.000 & 12.4 & 76.2 \\
\hline 35.0 & & 7.853 & 32.323 & 25.193 & 0.1191 & 0.000 & 13.6 & 77.0 \\
\hline 40.0 & & 6.827 & 32.488 & 25.464 & 0.1323 & 0.000 & 13.6 & 77.8 \\
\hline 45.0 & & 5.730 & 32.710 & 25.776 & 0.1438 & 0.000 & 6.0 & 82.1 \\
\hline 50.0 & & 5.296 & 32.799 & 25.897 & 0.1546 & 0.000 & 3.3 & 83.4 \\
\hline 55.0 & & 5.172 & 32.813 & 25.922 & 0.1651 & 0.000 & 2.7 & 83.6 \\
\hline 60.0 & & 5.016 & 32.839 & 25.961 & 0.1754 & 0.000 & 2.5 & 83.6 \\
\hline
\end{tabular}
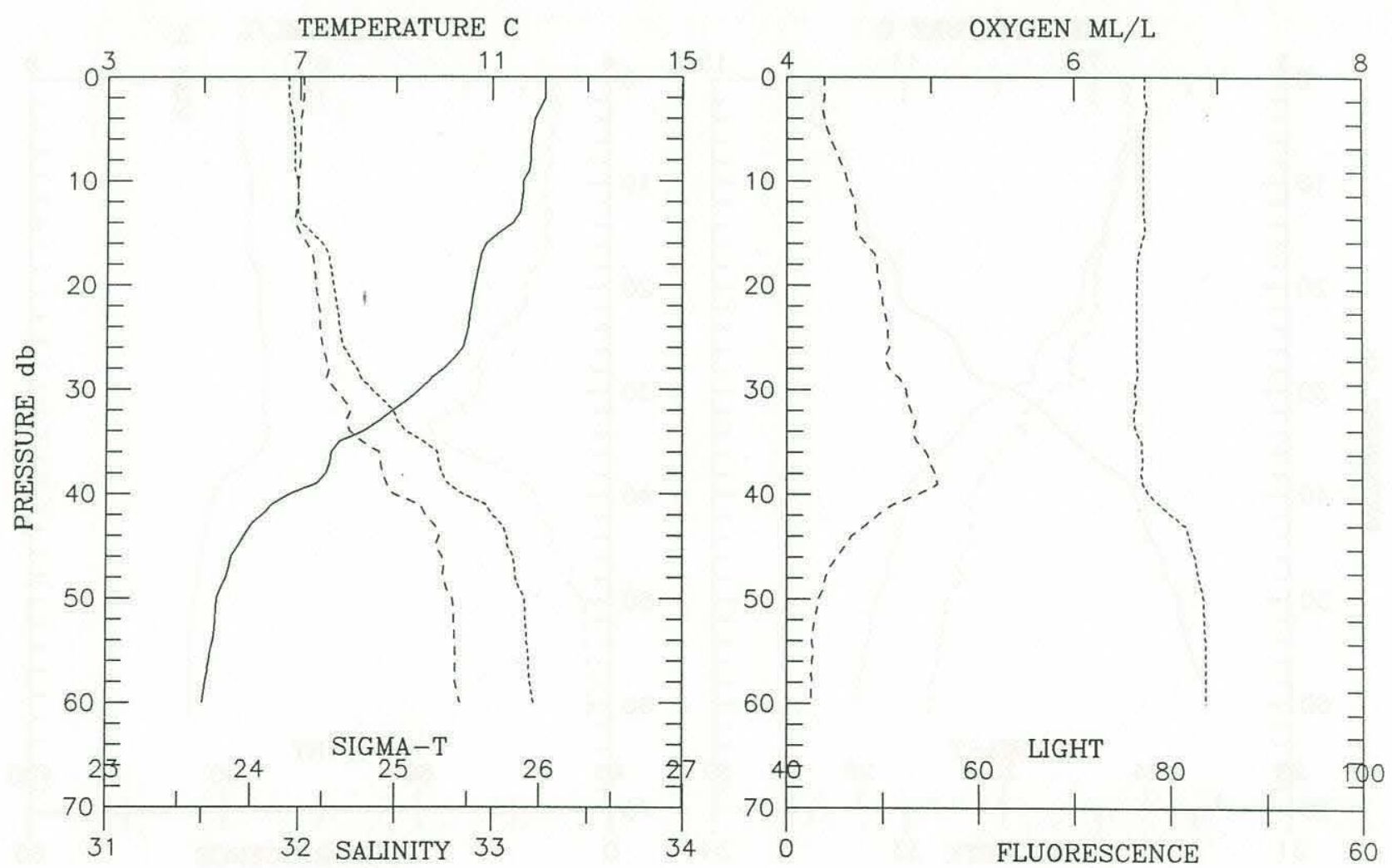


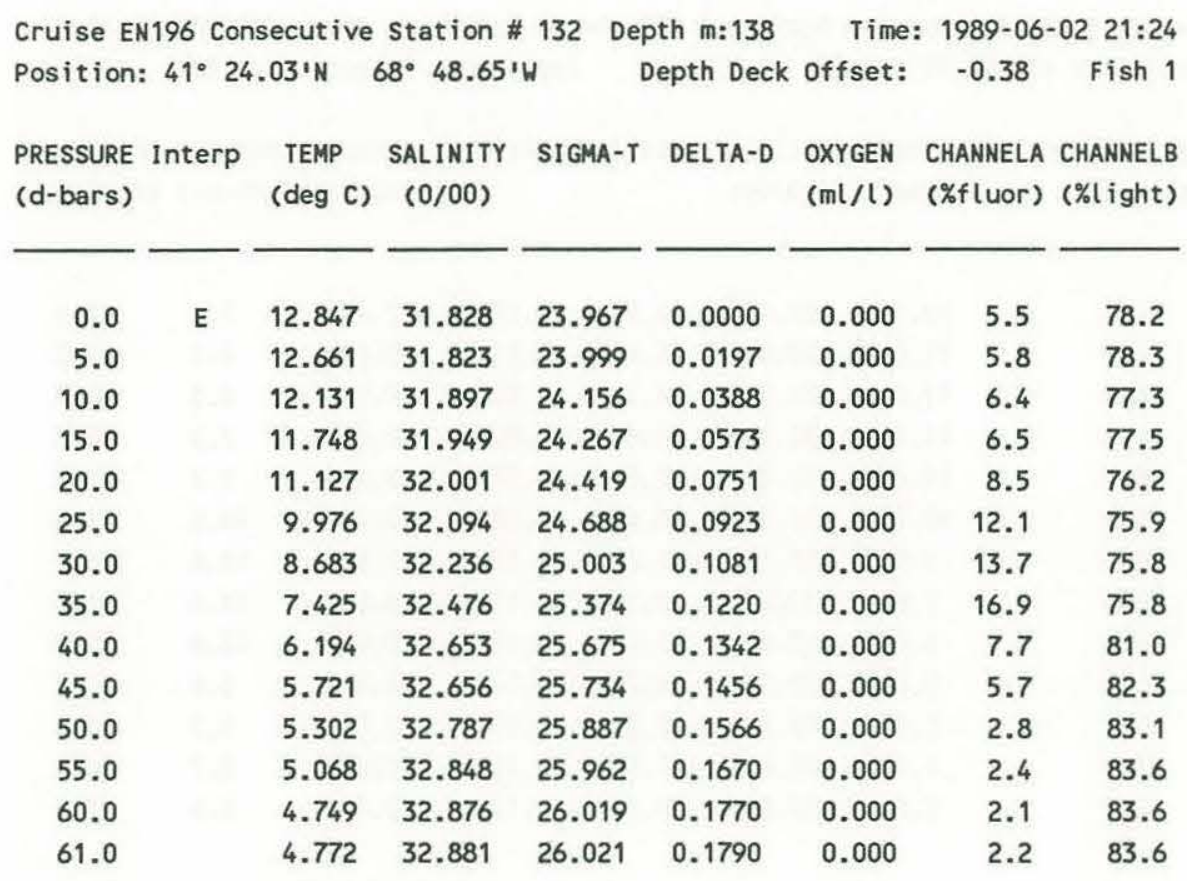
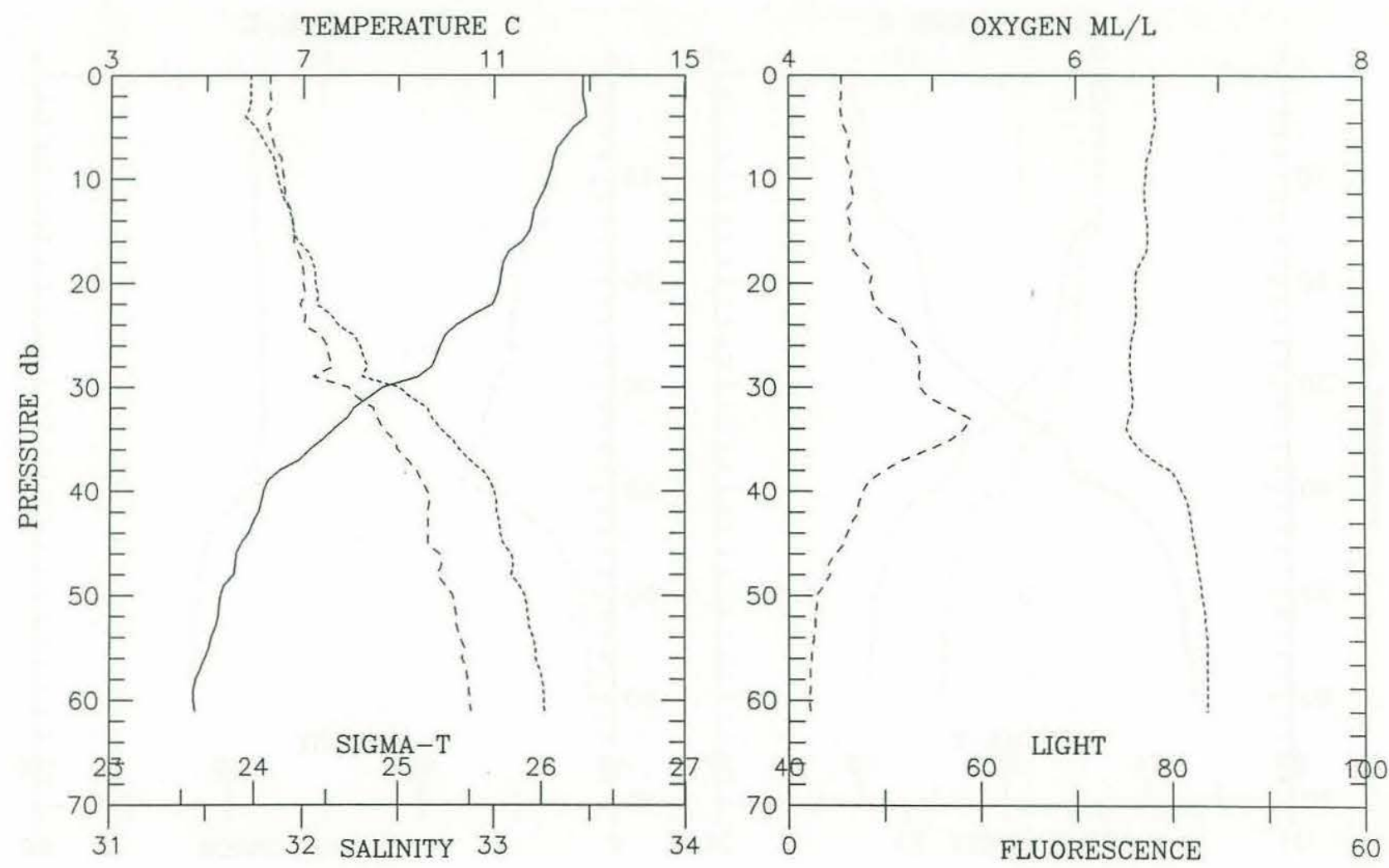
Cruise EN196 Consecutive Station \# 133 Depth m:146 Time: 1989-06-02 22:06 Position: $41^{\circ} 26.38^{\prime} \mathrm{N} 68^{\circ} 43.57^{\prime} \mathrm{H} \quad$ Depth Deck offset: -0.38 Fish 1

\begin{tabular}{|c|c|c|c|c|c|c|c|c|}
\hline $\begin{array}{l}\text { PRESSURE } \\
\text { (d-bars) }\end{array}$ & Interp & $\begin{array}{c}\text { TEMP } \\
(\operatorname{deg} C)\end{array}$ & $\begin{array}{c}\text { SALINITY } \\
(0 / 00)\end{array}$ & SIGMA-T & DELTA-D & $\begin{array}{l}\text { OXYGEN } \\
(\mathrm{ml} / \mathrm{l})\end{array}$ & $\begin{array}{l}\text { CHANNELA } \\
\text { ( } \% \text { fluor) }\end{array}$ & $\begin{array}{l}\text { CHANNELB } \\
\text { (\%light) }\end{array}$ \\
\hline 0.0 & E & 13.312 & 31.800 & 23.854 & 0.0000 & 0.000 & 5.4 & 77.9 \\
\hline 5.0 & & 12.816 & 31.795 & 23.947 & 0.0201 & 0.000 & 4.9 & 77.8 \\
\hline 10.0 & & 10.904 & 31.979 & 24.441 & 0.0387 & 0.000 & 7.7 & 76.5 \\
\hline 15.0 & & 10.191 & 32.103 & 24.659 & 0.0556 & 0.000 & 11.2 & 75.4 \\
\hline 20.0 & & 9.340 & 32.180 & 24.858 & 0.0717 & 0.000 & 13.2 & 75.3 \\
\hline 25.0 & & 7.647 & 32.455 & 25.326 & 0.0859 & 0.000 & 17.5 & 74.2 \\
\hline 30.0 & & 7.167 & 32.525 & 25.447 & 0.0988 & 0.000 & 13.9 & 77.5 \\
\hline 35.0 & & 6.036 & 32.753 & 25.773 & 0.1106 & 0.000 & 6.6 & 82.4 \\
\hline 40.0 & & 5.321 & 32.771 & 25.872 & 0.1214 & 0.000 & 3.4 & 83.3 \\
\hline 45.0 & & 4.944 & 32.825 & 25.957 & 0.1319 & 0.000 & 2.8 & 83.4 \\
\hline 50.0 & & 5.056 & 32.922 & 26.022 & 0.1419 & 0.000 & 2.2 & 83.6 \\
\hline 55.0 & & 4.959 & 32.957 & 26.060 & 0.1517 & 0.000 & 1.9 & 83.6 \\
\hline 60.0 & & 4.792 & 32.992 & 26.106 & 0.1613 & 0.000 & 1.8 & 83.6 \\
\hline 61.0 & & 4.794 & 32.995 & 26.109 & 0.1632 & 0.000 & 1.7 & 83.5 \\
\hline
\end{tabular}
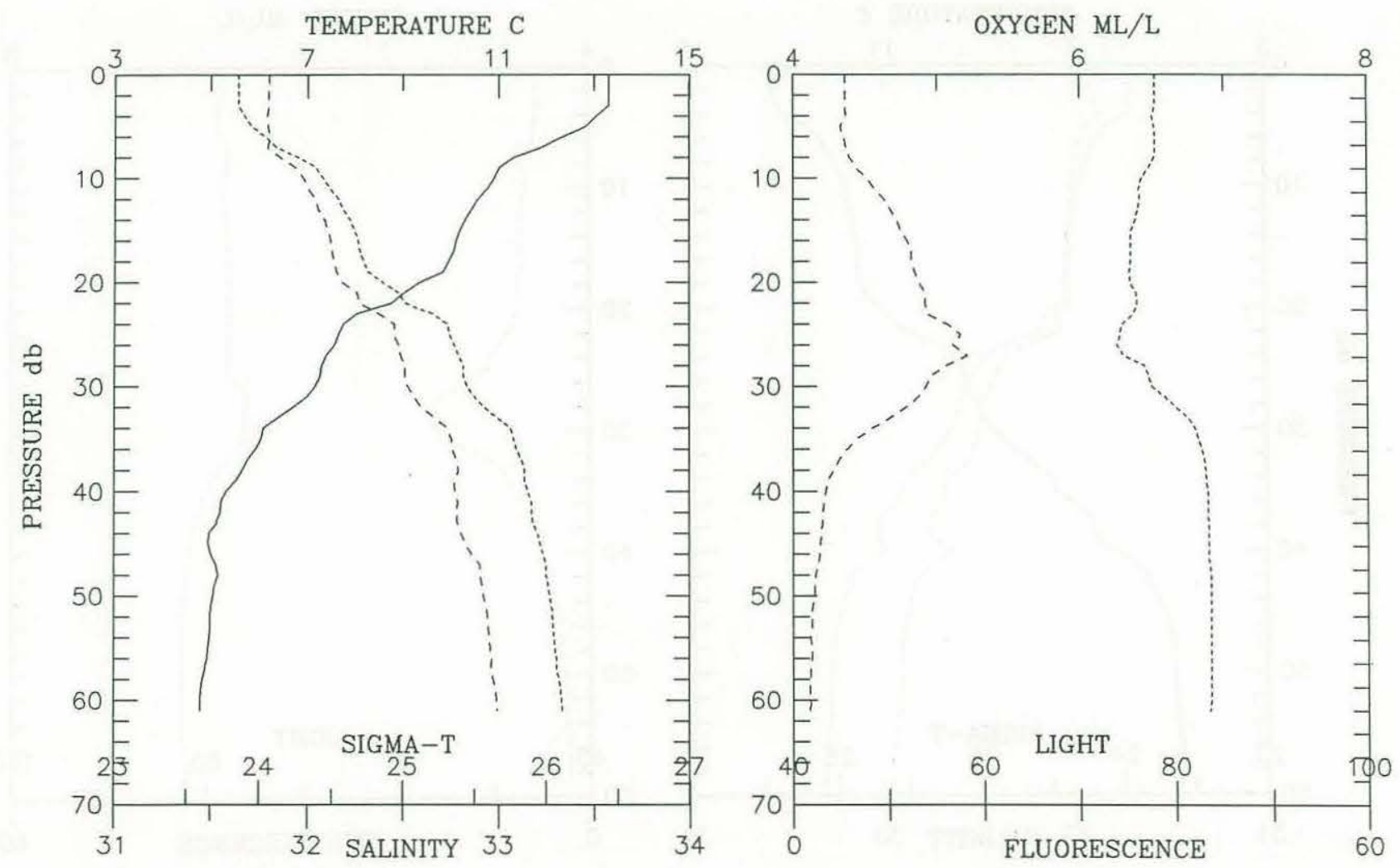


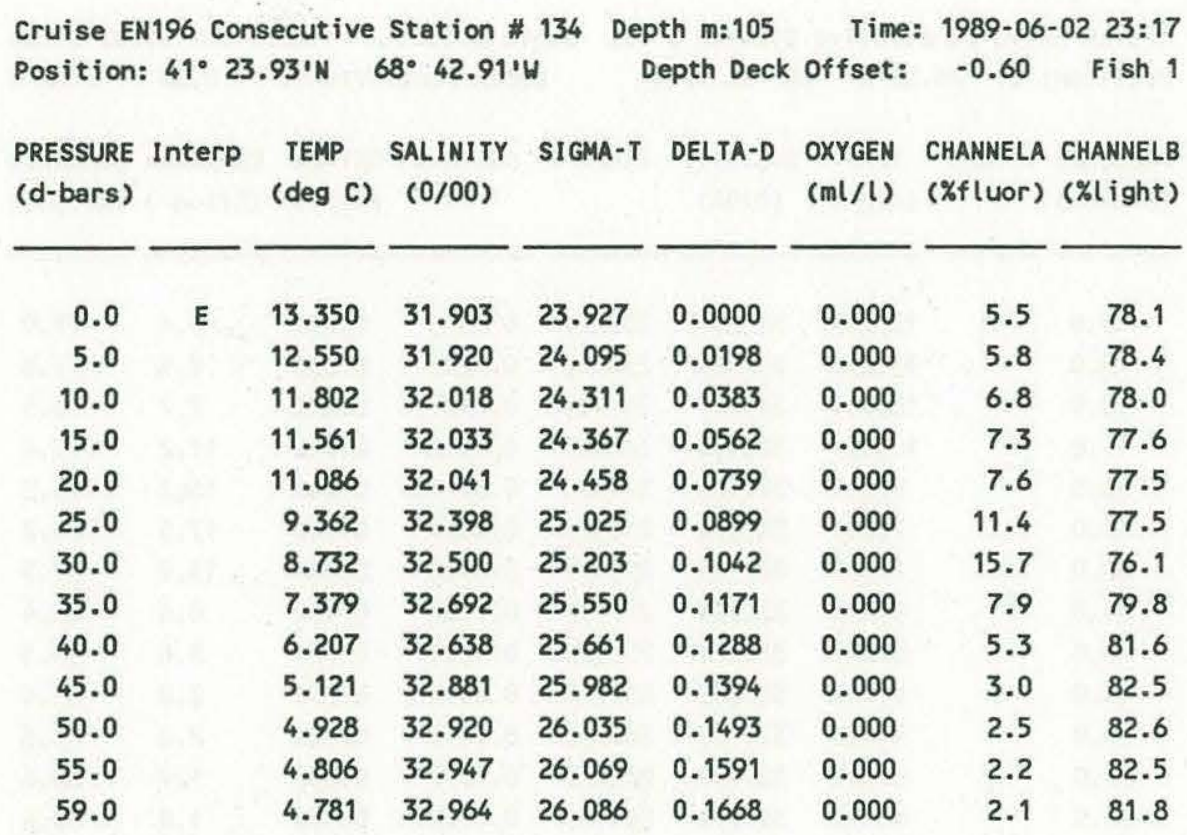
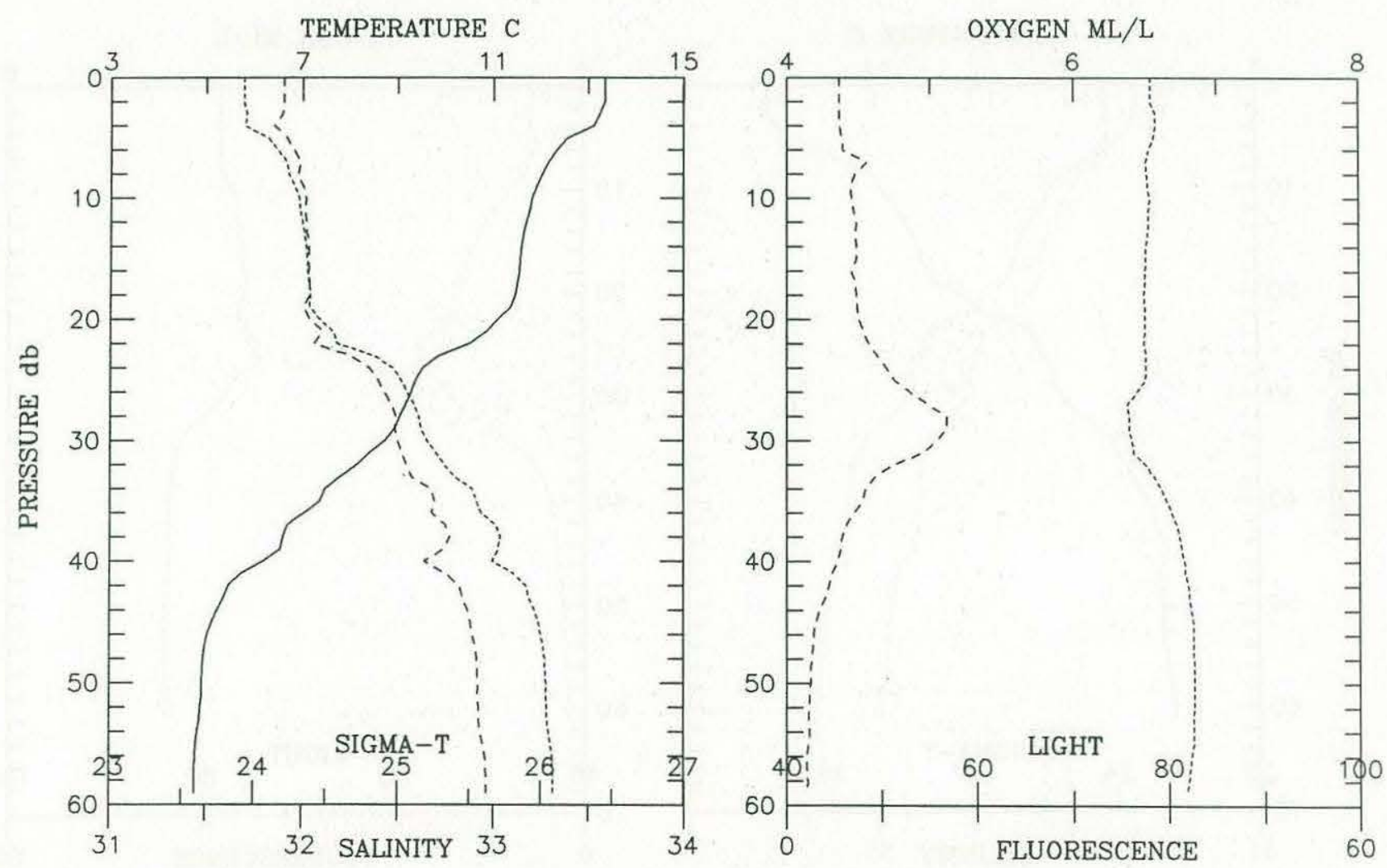
Cruise EN196 Consecutive Station \# 135 Depth m:120 Time: 1989-06-03 00:32 Position: $41^{\circ} 22.70^{\prime} \mathrm{N} \quad 68^{\circ} 45.77 \cdot \mathrm{H}$ Depth Deck offset: -0.60 Fish 1

\begin{tabular}{|c|c|c|c|c|c|c|c|c|}
\hline $\begin{array}{l}\text { PRESSURE } \\
\text { (d-bars) }\end{array}$ & Interp & $\begin{array}{c}\text { TEMP } \\
(\operatorname{deg} \mathrm{C})\end{array}$ & $\begin{array}{l}\text { SALINITY } \\
(0 / 00)\end{array}$ & SIGMA-T & DELTA-D & $\begin{array}{l}\text { OXYGEN } \\
(\mathrm{ml} / \mathrm{l})\end{array}$ & $\begin{array}{l}\text { CHANNELA } \\
\text { (\%fluor) }\end{array}$ & $\begin{array}{l}\text { CHANNELB } \\
\text { (\%light) }\end{array}$ \\
\hline 0.0 & $E$ & 12.700 & 31.963 & 24.100 & 0.0000 & 0.000 & 6.9 & 77.7 \\
\hline 5.0 & & 12.651 & 31.962 & 24.108 & 0.0190 & 0.000 & 6.9 & 77.6 \\
\hline 10.0 & & 12.356 & 31.972 & 24.172 & 0.0379 & 0.000 & 7.5 & 77.2 \\
\hline 15.0 & & 12.155 & 31.996 & 24.229 & 0.0565 & 0.000 & 8.1 & 76.9 \\
\hline 20.0 & & 11.520 & 32.013 & 24.358 & 0.0746 & 0.000 & 8.9 & 77.0 \\
\hline 25.0 & & 10.847 & 32.090 & 24.537 & 0.0921 & 0.000 & 9.6 & 77.5 \\
\hline 30.0 & & 9.989 & 32.316 & 24.859 & 0.1083 & 0.000 & 13.4 & 77.0 \\
\hline 35.0 & & 8.648 & 32.525 & 25.235 & 0.1231 & 0.000 & 17.0 & 76.4 \\
\hline 40.0 & & 7.624 & 32.653 & 25.485 & 0.1360 & 0.000 & 20.5 & 75.9 \\
\hline 45.0 & & 6.735 & 32.615 & 25.576 & 0.1480 & 0.000 & 5.9 & 81.2 \\
\hline 50.0 & & 5.211 & 32.838 & 25.938 & 0.1589 & 0.000 & 3.8 & 82.3 \\
\hline 55.0 & & 5.051 & 32.892 & 25.999 & 0.1691 & 0.000 & 3.4 & 81.7 \\
\hline 60.0 & & 5.004 & 32.901 & 26.011 & 0.1790 & 0.000 & 2.9 & 81.3 \\
\hline 61.0 & & 4.990 & 32.905 & 26.016 & 0.1810 & 0.000 & 2.9 & 81.2 \\
\hline
\end{tabular}
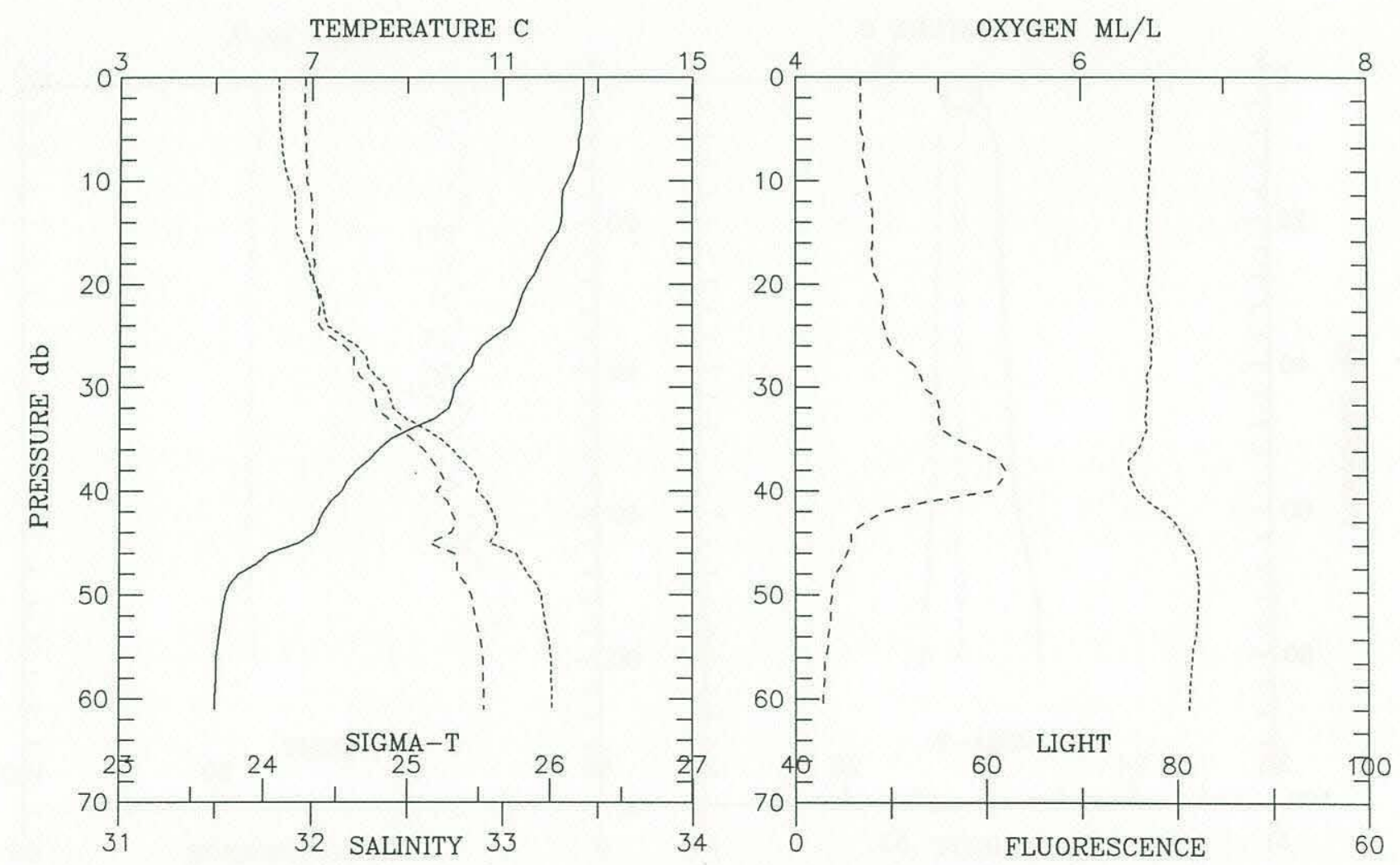


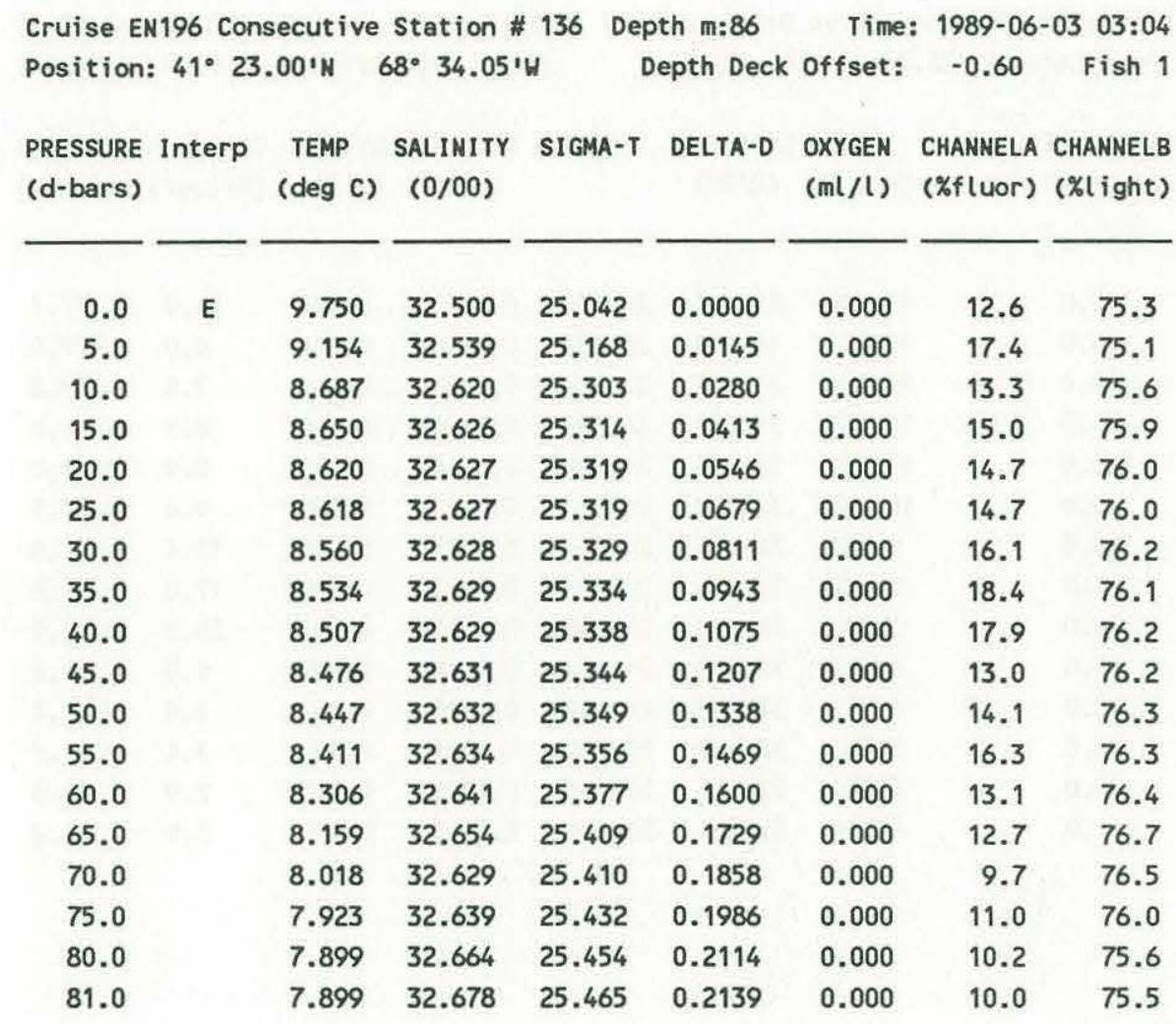
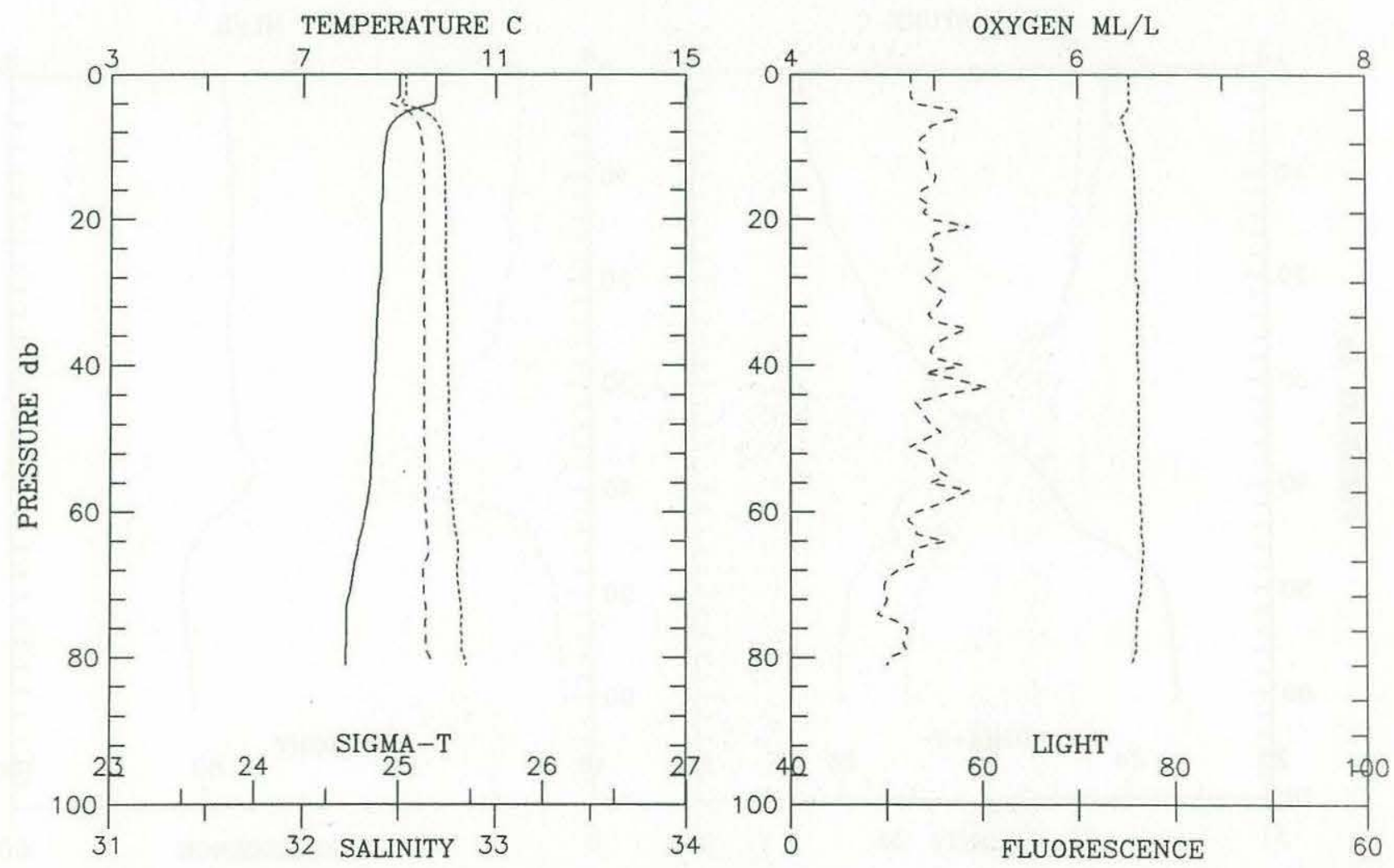


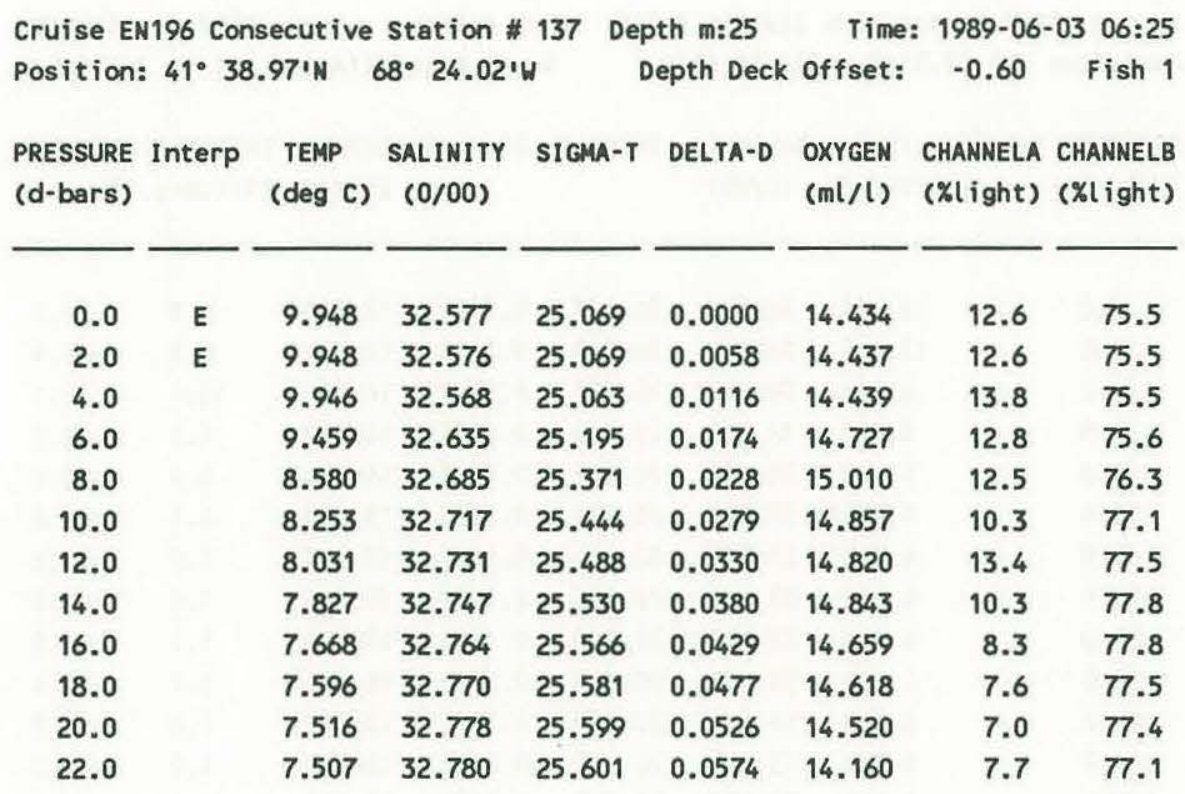
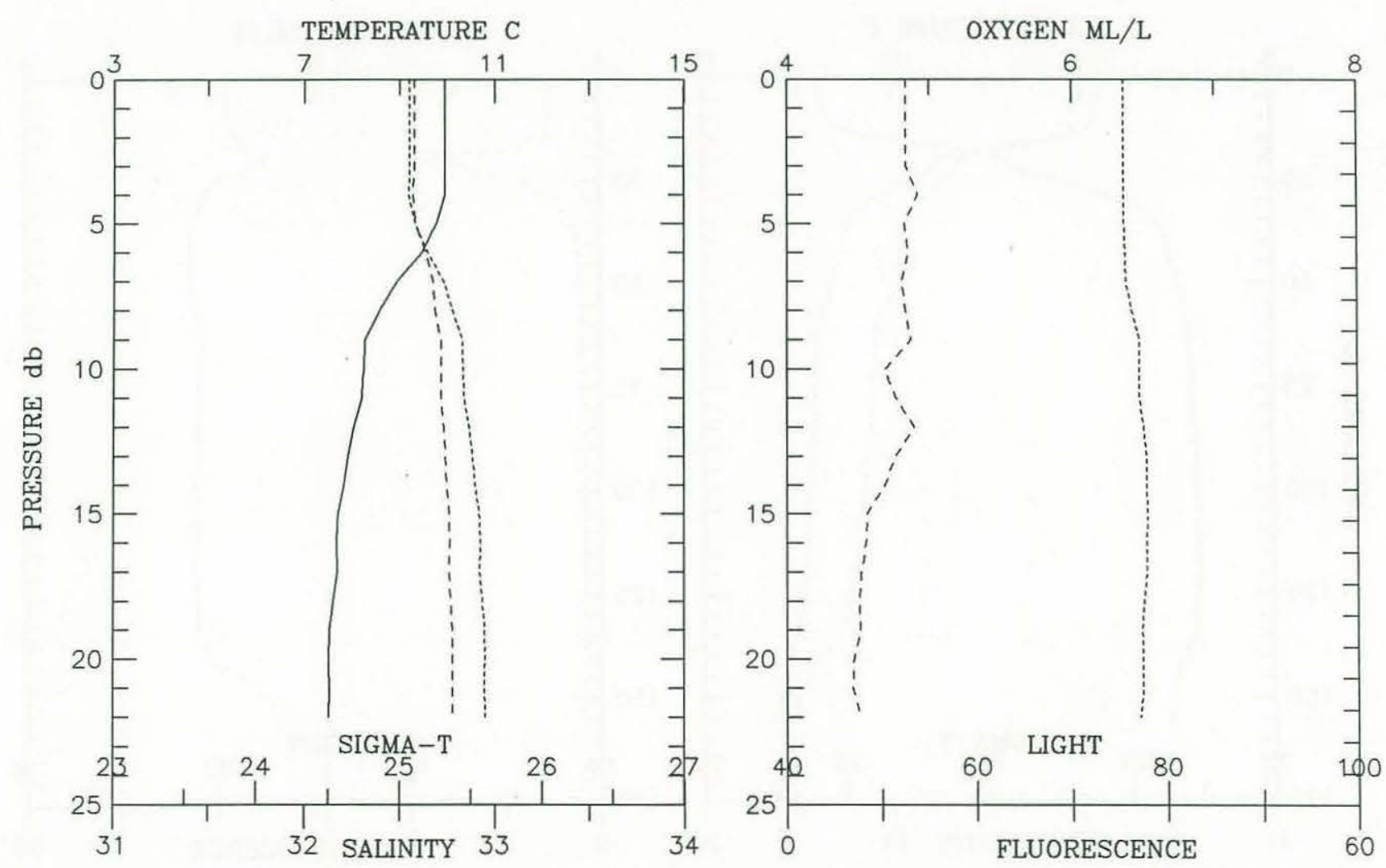


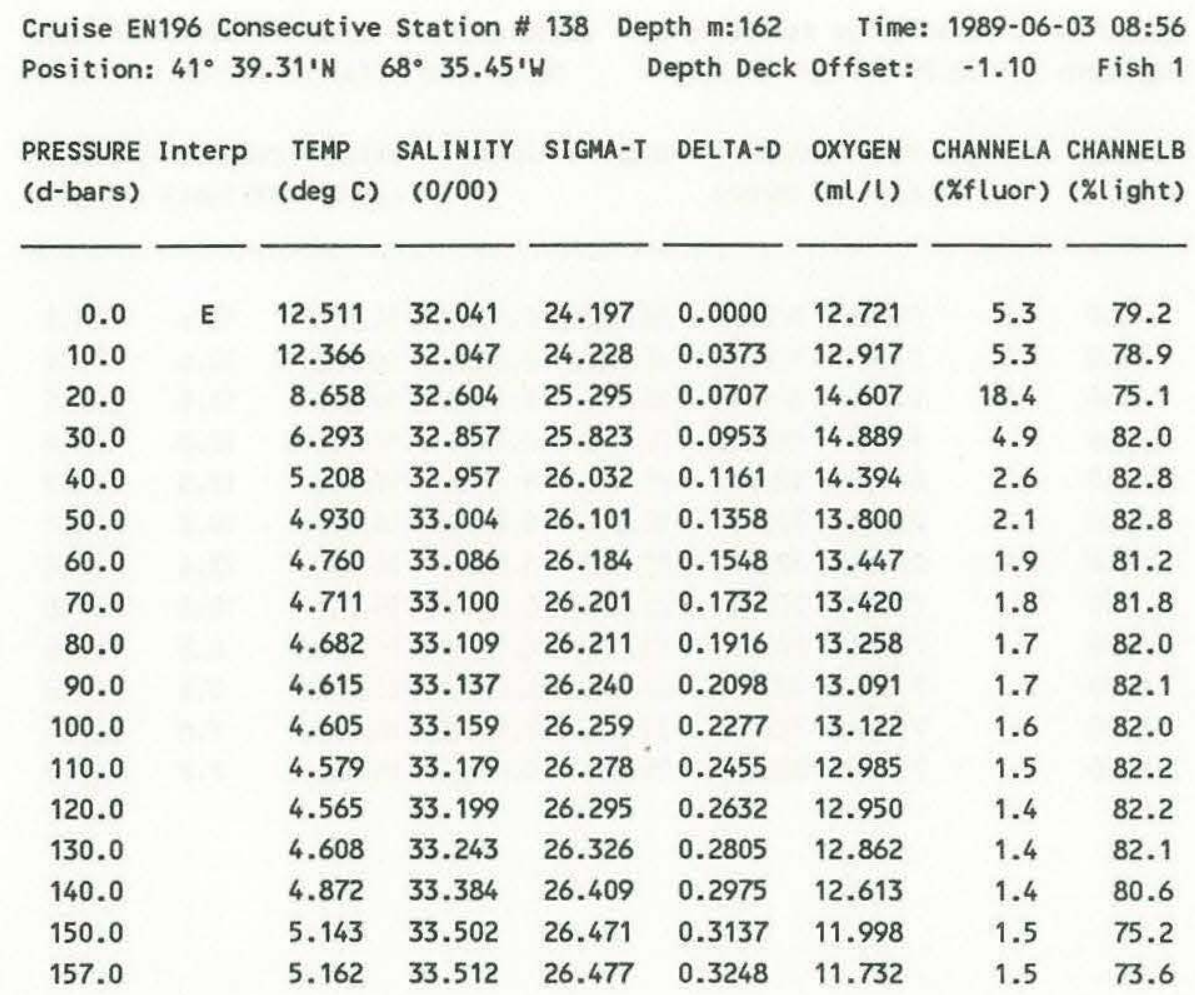
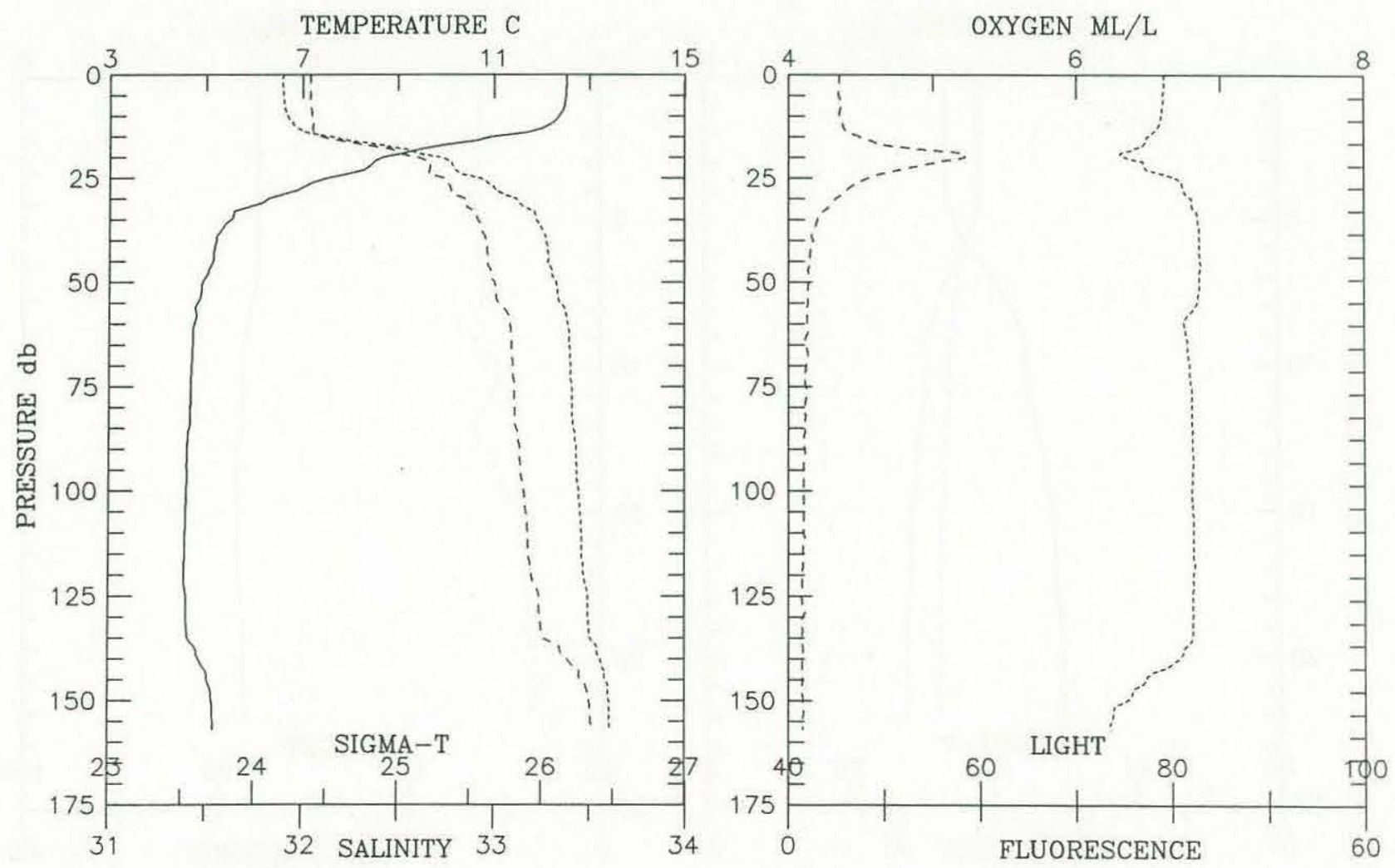


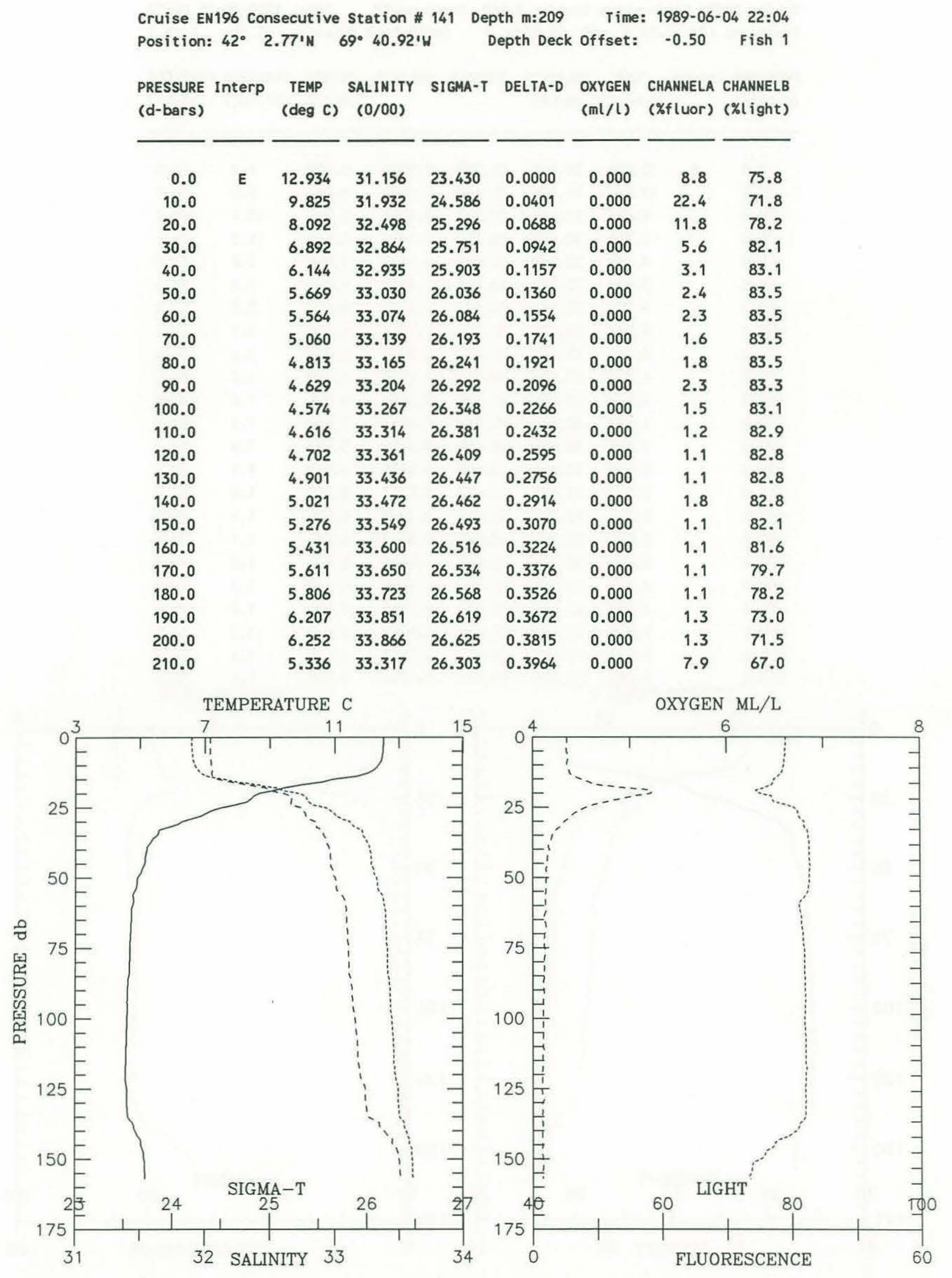




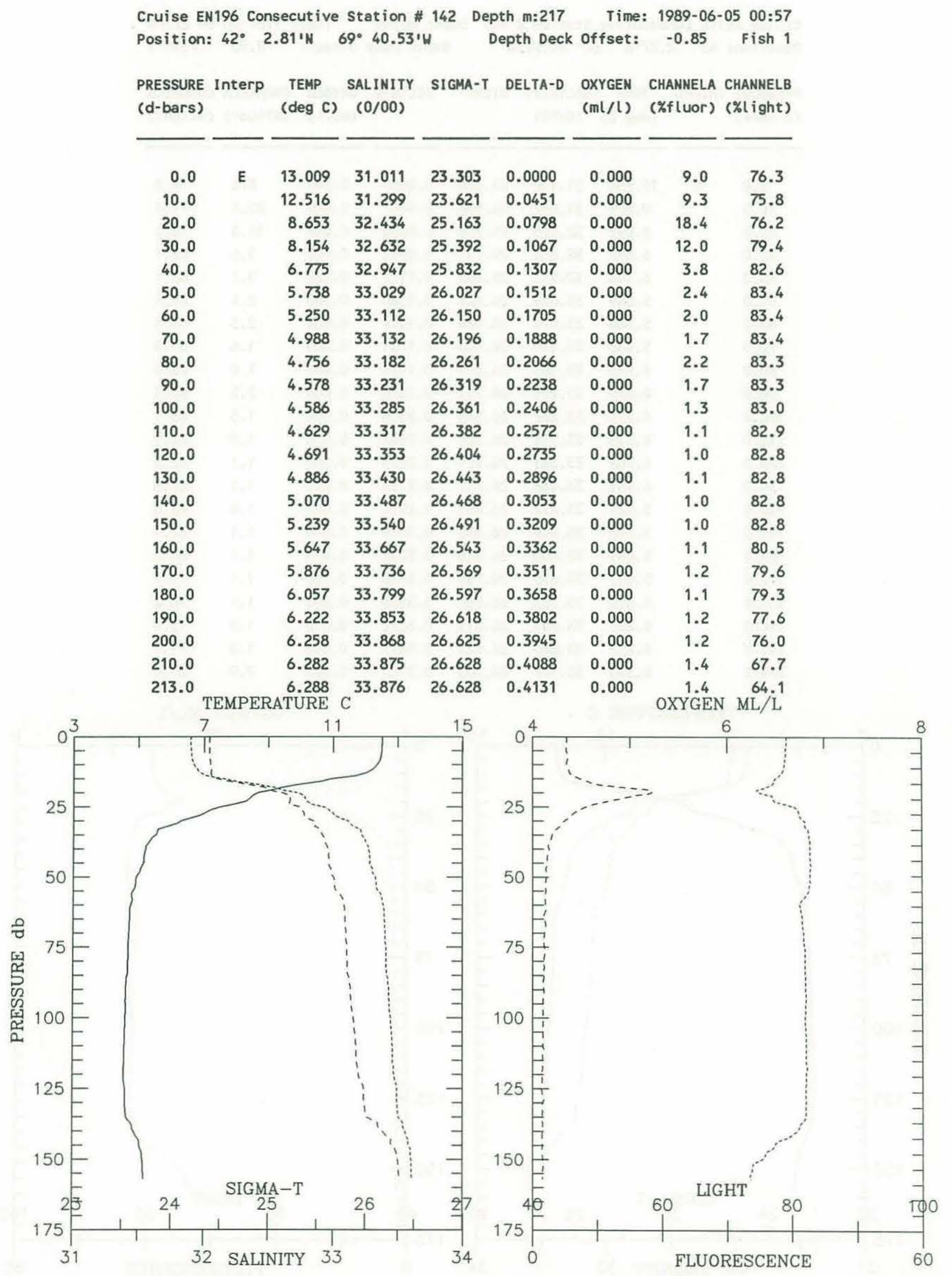




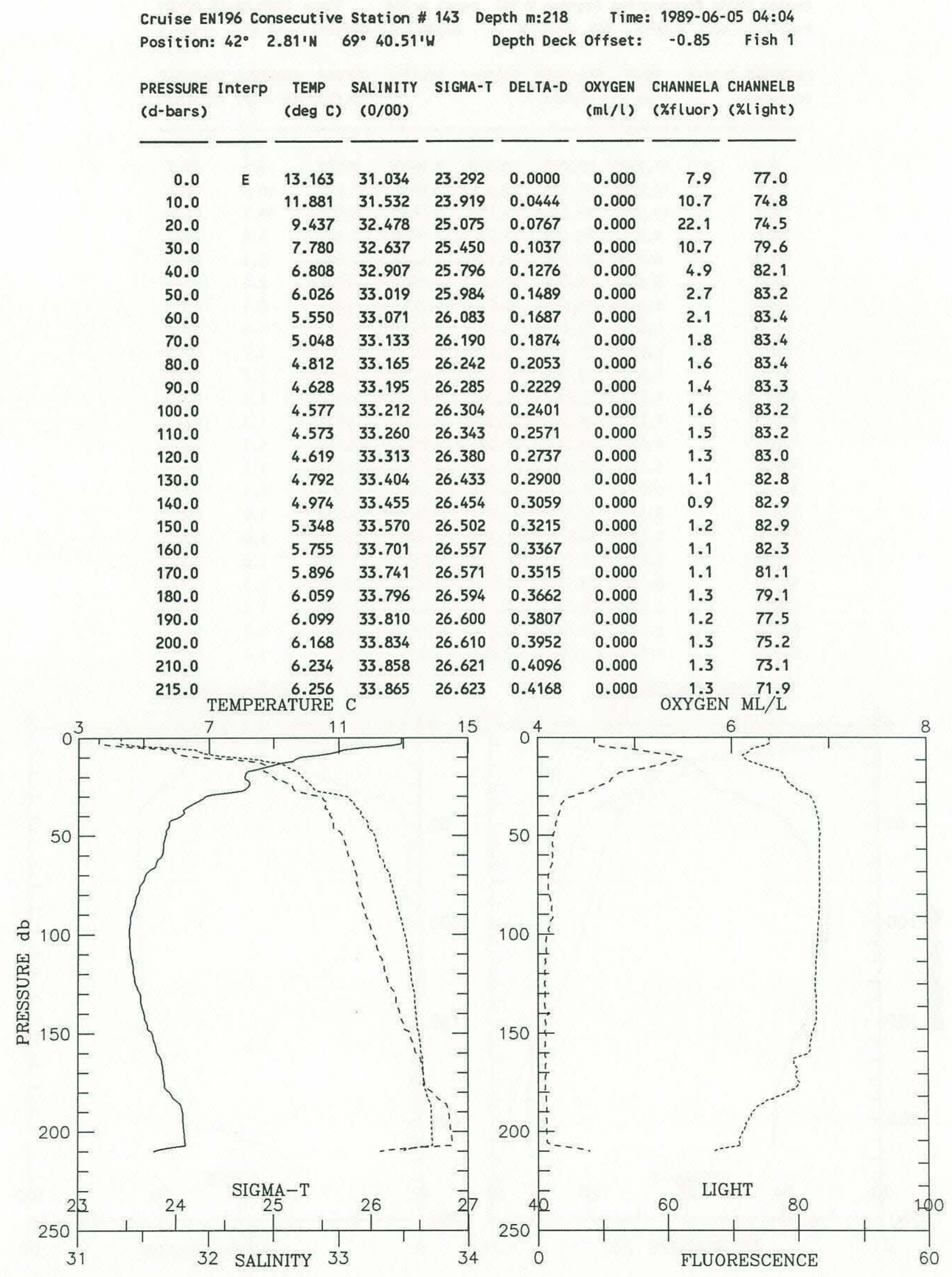


Cruise EN196 Consecutive Station \# 144 Depth m:216 Time: 1989-06-05 07:09 Position: $42^{\circ} 2.60^{\prime} \mathrm{W} 69^{\circ} 40.57^{\prime} \mathrm{W}$ Depth Deck offset: -0.85 Fish 1

\begin{tabular}{|c|c|c|c|c|c|c|c|c|}
\hline $\begin{array}{l}\text { PRESSURE } \\
\text { (d-bars) }\end{array}$ & Interp & $\begin{array}{c}\text { TEMP } \\
(\operatorname{deg} C)\end{array}$ & $\begin{array}{l}\text { SALINITY } \\
(0 / 00)\end{array}$ & SI GMA-T & DELTA-D & $\begin{array}{l}\text { OXYGEN } \\
(\mathrm{ml} / \mathrm{l})\end{array}$ & $\begin{array}{l}\text { CHANNELA } \\
\text { (\%fluor) }\end{array}$ & $\begin{array}{l}\text { CHANNELB } \\
\text { (\%light) }\end{array}$ \\
\hline 0.0 & E & 13.294 & 30.920 & 23.178 & 0.0000 & 0.000 & 7.6 & 76.5 \\
\hline 10.0 & & 12.272 & 31.125 & 23.532 & 0.0462 & 0.000 & 10.6 & 75.3 \\
\hline 20.0 & & 10.292 & 32.296 & 24.793 & 0.0823 & 0.000 & 19.7 & 74.4 \\
\hline 30.0 & & 7.963 & 32.838 & 25.582 & 0.1091 & 0.000 & 5.8 & 81.2 \\
\hline 40.0 & & 6.273 & 32.986 & 25.927 & 0.1314 & 0.000 & 3.1 & 82.9 \\
\hline 50.0 & & 5.647 & 33.015 & 26.027 & 0.1517 & 0.000 & 2.4 & 83.4 \\
\hline 60.0 & & 5.396 & 33.054 & 26.088 & 0.1711 & 0.000 & 2.1 & 83.6 \\
\hline 70.0 & & 5.087 & 33.118 & 26.174 & 0.1898 & 0.000 & 1.8 & 83.6 \\
\hline 80.0 & & 4.903 & 33.145 & 26.216 & 0.2080 & 0.000 & 1.7 & 83.5 \\
\hline 90.0 & & 4.676 & 33.196 & 26.281 & 0.2258 & 0.000 & 1.7 & 83.5 \\
\hline 100.0 & & 4.559 & 33.256 & 26.341 & 0.2428 & 0.000 & 1.4 & 83.2 \\
\hline 110.0 & & 4.570 & 33.293 & 26.369 & 0.2595 & 0.000 & 1.3 & 83.1 \\
\hline 120.0 & & 4.657 & 33.331 & 26.390 & 0.2760 & 0.000 & 1.1 & 82.7 \\
\hline 130.0 & & 4.752 & 33.384 & 26.422 & 0.2923 & 0.000 & 1.1 & 82.7 \\
\hline 140.0 & & 4.887 & 33.429 & 26.443 & 0.3083 & 0.000 & 1.1 & 82.7 \\
\hline 150.0 & & 5.130 & 33.504 & 26.475 & 0.3240 & 0.000 & 1.0 & 82.8 \\
\hline 160.0 & & 5.529 & 33.625 & 26.524 & 0.3394 & 0.000 & 1.0 & 82.7 \\
\hline 170.0 & & 5.911 & 33.748 & 26.575 & 0.3544 & 0.000 & 1.0 & 81.6 \\
\hline 180.0 & & 6.003 & 33.777 & 26.586 & 0.3691 & 0.000 & 1.1 & 79.4 \\
\hline 190.0 & & 6.023 & 33.785 & 26.590 & 0.3838 & 0.000 & 1.1 & 78.6 \\
\hline 200.0 & & 6.227 & 33.861 & 26.624 & 0.3983 & 0.000 & 1.2 & 75.4 \\
\hline 210.0 & & 6.339 & 33.894 & 26.636 & 0.4126 & 0.000 & 1.4 & 67.4 \\
\hline
\end{tabular}
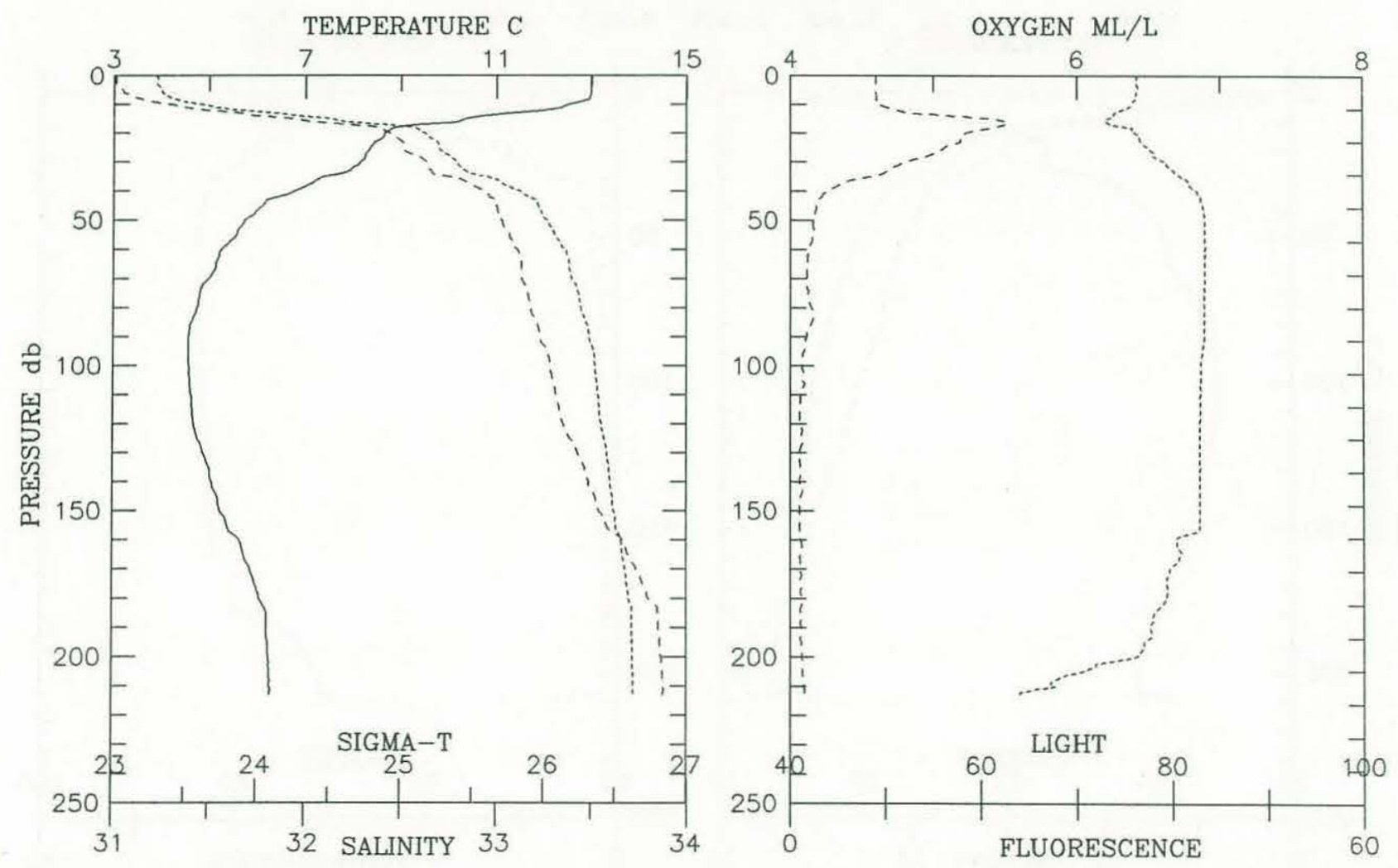
Cruise EN196 Consecutive Station \# 145 Depth m:212 Time: 1989-06-05 10:03 Position: $42^{\circ} 2.71 \mathrm{~N} 69^{\circ} 40.766^{\prime} \mathrm{W}$ Depth Deck offset: -0.85 Fish 1

PRESSURE InterP TEMP SALINITY SIGMA-T DELTA-D OXYGEN CHANNELA CHANNELB (d-bars) (deg C) $(0 / 00)$

(ml/l) (\%fluor) (\%light)

\begin{tabular}{|c|c|c|c|c|}
\hline 0.0 & $E$ & 13.484 & 30.859 & 23.093 \\
\hline 10.0 & & 12.228 & 31.188 & 23.589 \\
\hline 20.0 & & 9.198 & 32.618 & 25.223 \\
\hline 30.0 & & 6.786 & 32.935 & 25.821 \\
\hline 40.0 & & 6.191 & 33.003 & 25.951 \\
\hline 50.0 & & 5.851 & 33.032 & 26.016 \\
\hline 60.0 & & 5.275 & 33.095 & 26.134 \\
\hline 70.0 & & 5.012 & 33.132 & 26.193 \\
\hline 80.0 & & 4.819 & 33.158 & 26.235 \\
\hline 90.0 & & 4.780 & 33.167 & 26.247 \\
\hline 100.0 & & 4.585 & 33.226 & 26.315 \\
\hline 110.0 & & 4.559 & 33.260 & 26.344 \\
\hline 120.0 & & 4.623 & 33.320 & 26.385 \\
\hline 130.0 & & 4.768 & 33.392 & 26.426 \\
\hline 140.0 & & 4.928 & 33.444 & 26.450 \\
\hline 150.0 & & 5.178 & 33.525 & 26.486 \\
\hline 160.0 & & 5.523 & 33.622 & 26.522 \\
\hline 170.0 & & 5.711 & 33.678 & 26.544 \\
\hline 180.0 & & 5.953 & 33.765 & 26.583 \\
\hline 190.0 & & 6.128 & 33.822 & 26.606 \\
\hline 200.0 & & 6.168 & 33.835 & 26.611 \\
\hline 208.0 & & 6.213 & 33.856 & 26.622 \\
\hline
\end{tabular}
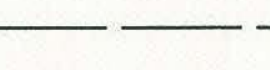

\begin{tabular}{lrrrr} 
& & & & \\
\cline { 5 - 5 } & & & & \\
0.0000 & 0.000 & 7.8 & 76.1 \\
0.0782 & 0.000 & & 9.7 & 75.5 \\
0.1026 & 0.000 & & 15.0 & 75.4 \\
0.1236 & 0.000 & 2.8 & 82.6 \\
0.1437 & 0.000 & 2.3 & 83.1 \\
0.1631 & 0.000 & 1.8 & 83.6 \\
0.1814 & 0.000 & 1.6 & 83.6 \\
0.1994 & 0.000 & 1.6 & 83.5 \\
0.2172 & 0.000 & 1.7 & 83.5 \\
0.2346 & 0.000 & 1.7 & 83.2 \\
0.2515 & 0.000 & 1.5 & 83.2 \\
0.2681 & 0.000 & 1.1 & 82.7 \\
0.2843 & 0.000 & 1.0 & 83.0 \\
0.3003 & 0.000 & 1.0 & 82.9 \\
0.3159 & 0.000 & 1.0 & 82.9 \\
0.3313 & 0.000 & 1.0 & 82.8 \\
0.3464 & 0.000 & 1.0 & 82.8 \\
0.3612 & 0.000 & 1.1 & 79.1 \\
0.3757 & 0.000 & 1.3 & 77.8 \\
0.3902 & 0.000 & 1.3 & 72.9 \\
0.4018 & 0.000 & 1.3 & 71.2 \\
& & & \\
0 & & $0 X Y G E N$ & $M L / L$
\end{tabular}
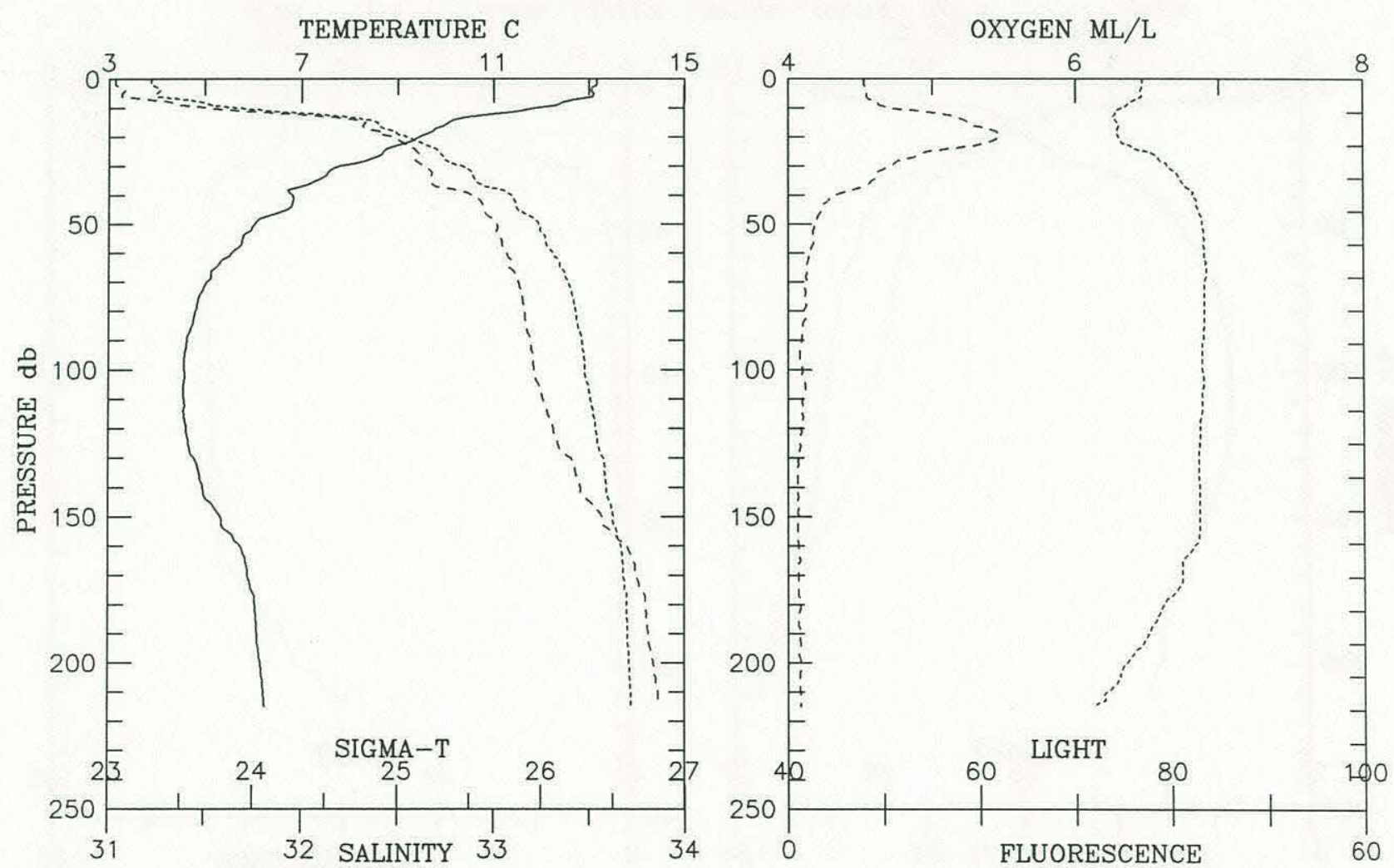


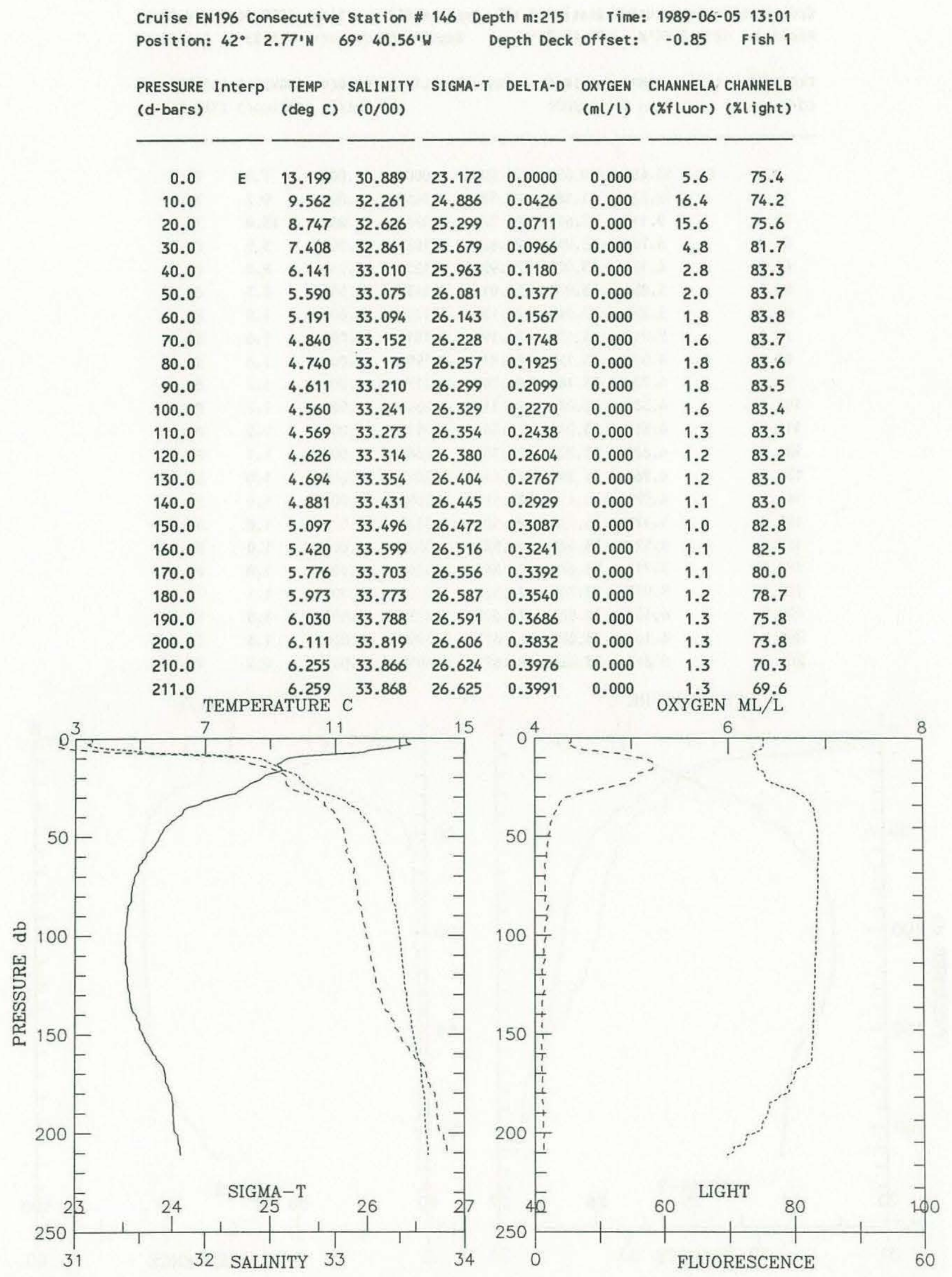




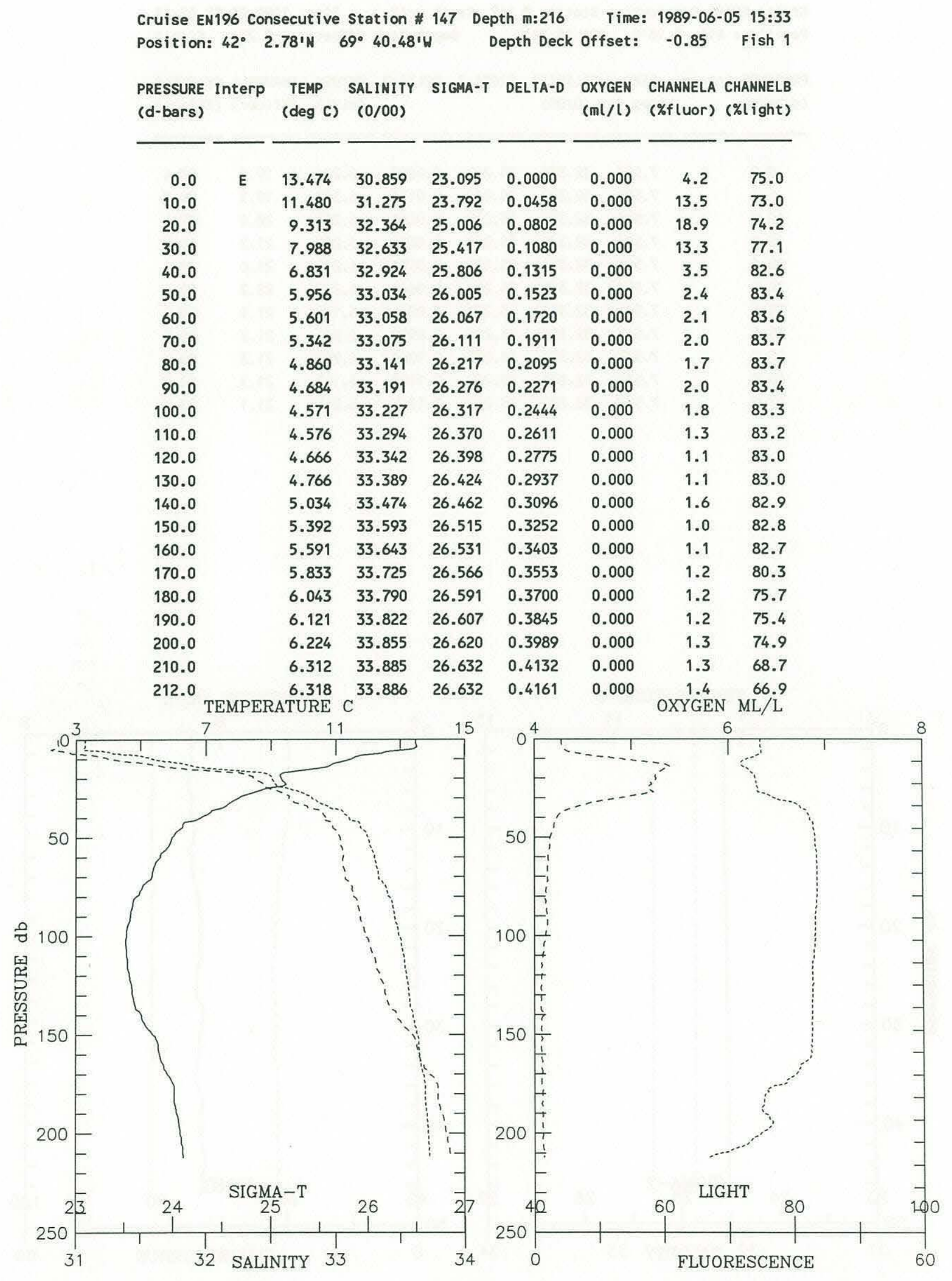




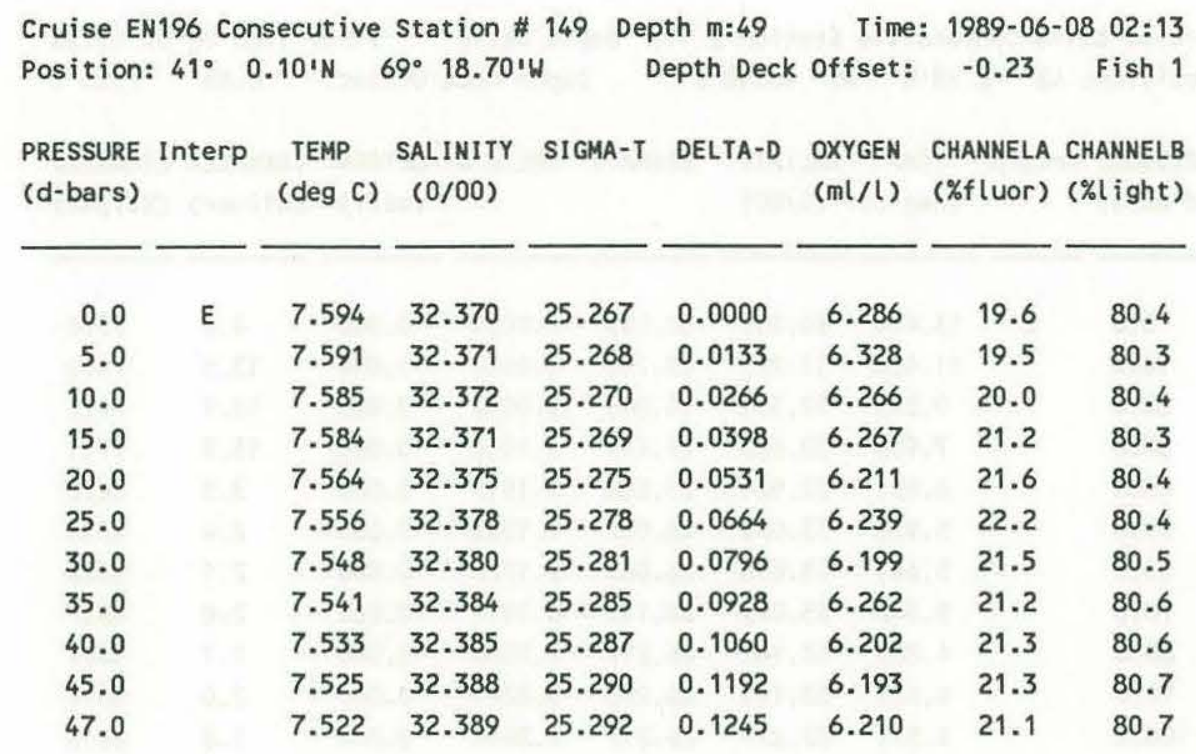
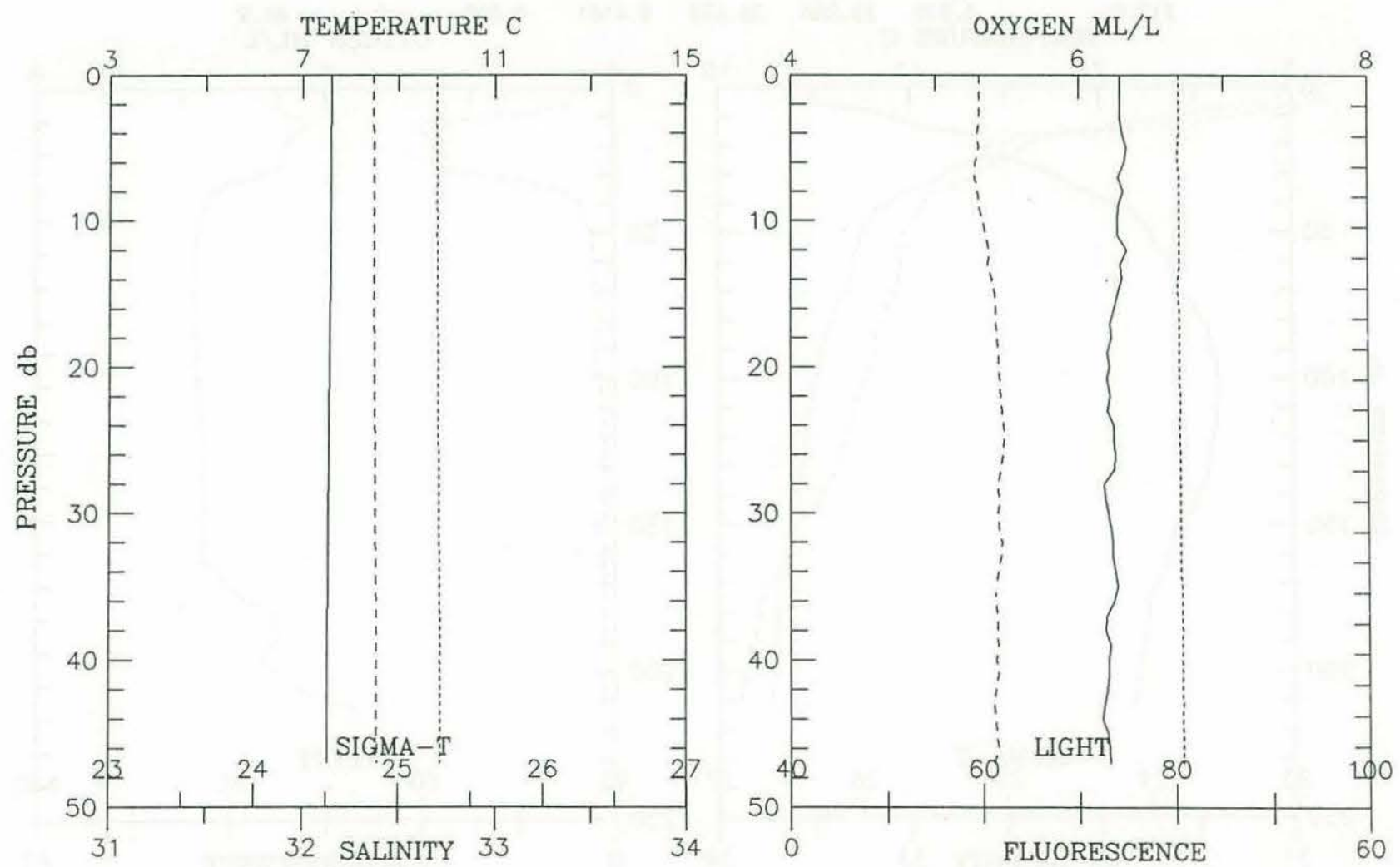


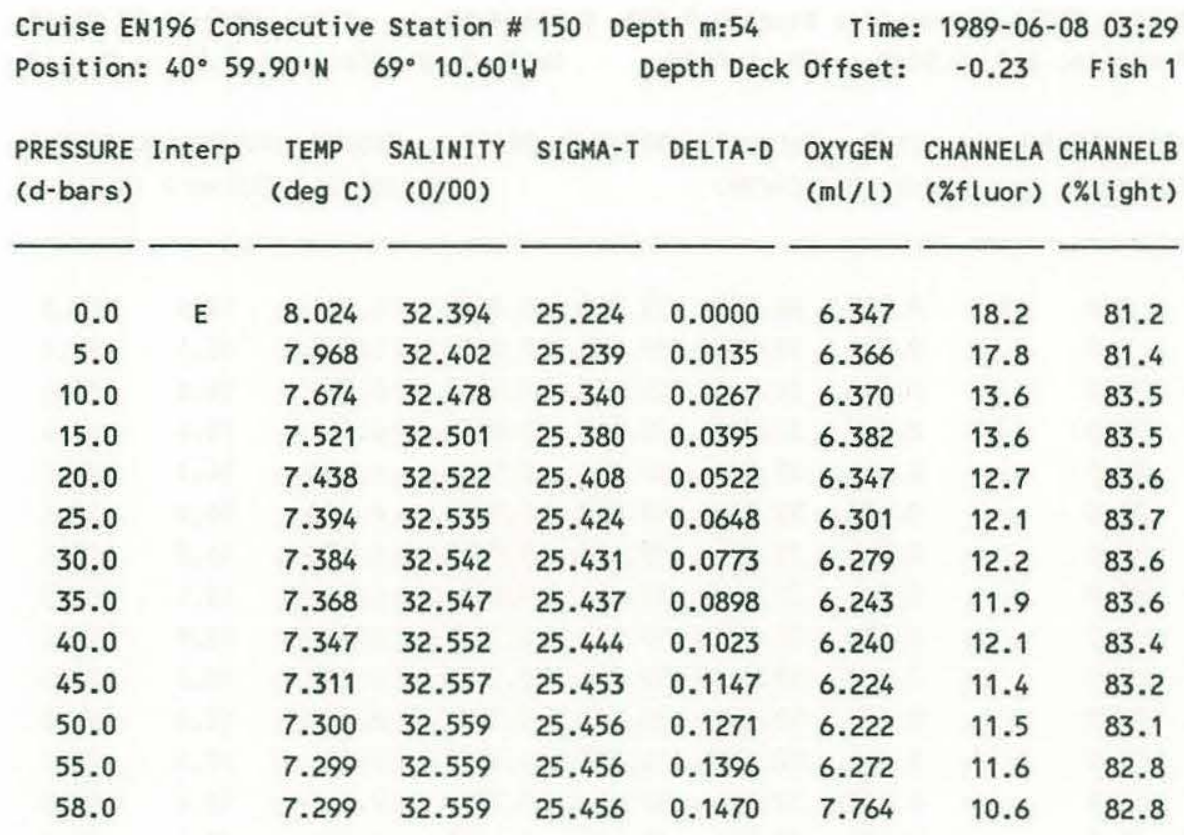
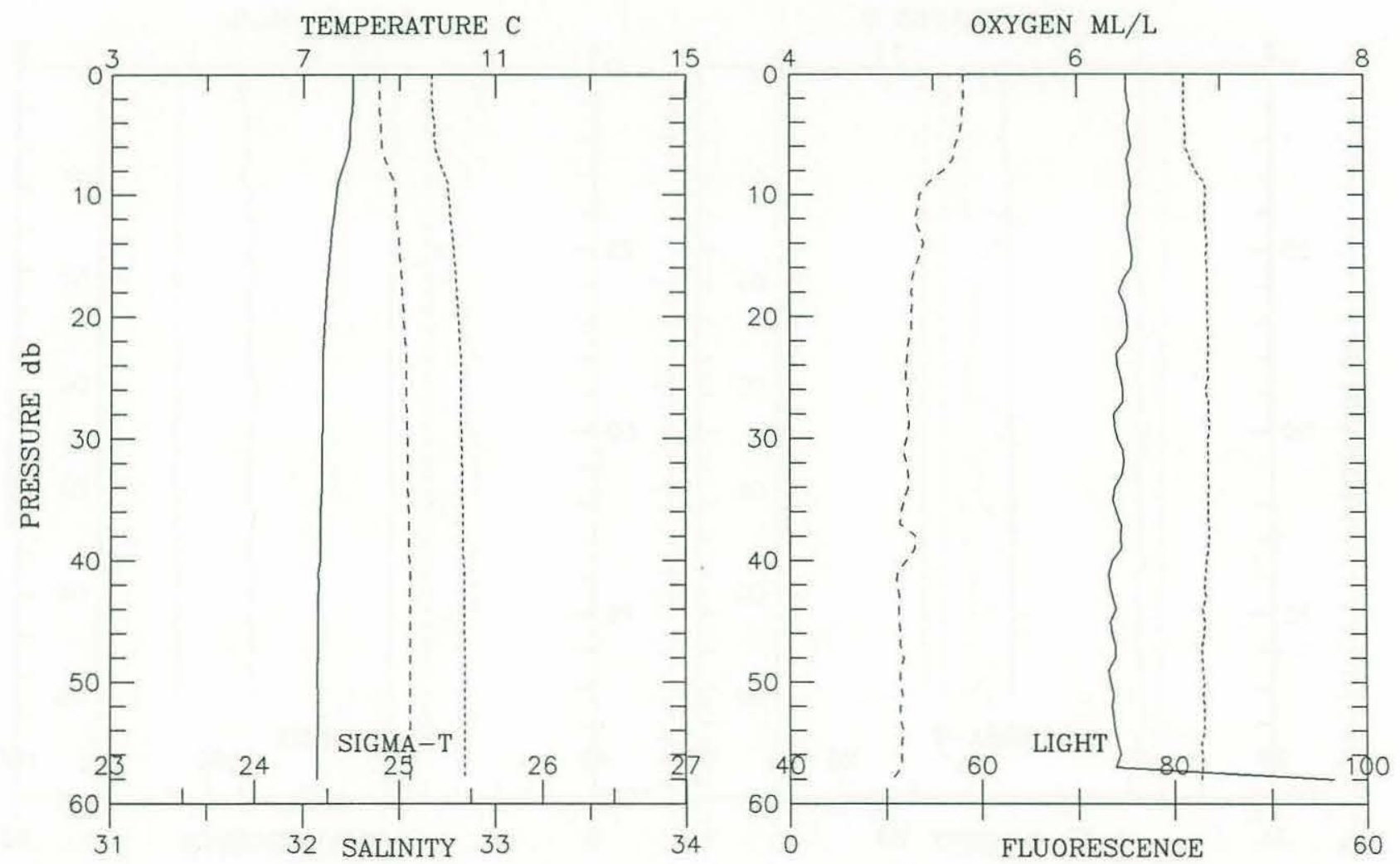
Cruise EN196 Consecutive Station \# 151 Depth m:89 Time: 1989-06-08 05:49 Position: $41^{\circ} 0.001 \mathrm{~K} 69^{\circ} 0.40^{\prime} \mathrm{W}$ Depth Deck offset: -0.23 Fish 1

\begin{tabular}{|c|c|c|c|c|c|c|c|c|}
\hline $\begin{array}{l}\text { PRESSURE } \\
\text { (d-bars) }\end{array}$ & Interp & $\begin{array}{c}\text { TEMP } \\
\text { (deg C) }\end{array}$ & $\begin{array}{l}\text { SALINITY } \\
(0 / 00)\end{array}$ & SIGMA-T & DELTA-D & $\begin{array}{l}\text { OXYGEN } \\
(\mathrm{ml} / \mathrm{l})\end{array}$ & $\begin{array}{l}\text { CHANMELA } \\
\text { ( } \% \text { fluor) }\end{array}$ & $\begin{array}{l}\text { CHANHELB } \\
\text { (\%light) }\end{array}$ \\
\hline 0.0 & $\mathbf{E}$ & 8.587 & 32.722 & 25.399 & 0.0000 & 6.452 & 12.6 & 83.3 \\
\hline 5.0 & & 8.579 & 32.722 & 25.400 & 0.0127 & 6.432 & 12.4 & 83.4 \\
\hline 10.0 & & 8.546 & 32.723 & 25.406 & 0.0253 & 6.424 & 12.8 & 83.4 \\
\hline 15.0 & & 8.479 & 32.723 & 25.416 & 0.0379 & 6.443 & 13.4 & 83.5 \\
\hline 20.0 & & 8.476 & 32.714 & 25.409 & 0.0505 & 6.422 & 14.1 & 83.5 \\
\hline 25.0 & & 8.465 & 32.722 & 25.417 & 0.0632 & 6.390 & 16.8 & 83.4 \\
\hline 30.0 & & 8.460 & 32.722 & 25.418 & 0.0758 & 6.377 & 14.8 & 83.5 \\
\hline 35.0 & & 8.479 & 32.723 & 25.416 & 0.0884 & 6.374 & 13.4 & 83.5 \\
\hline 40.0 & & 8.476 & 32.723 & 25.416 & 0.1010 & 6.379 & 12.9 & 83.4 \\
\hline 45.0 & & 8.457 & 32.722 & 25.418 & 0.1136 & 6.341 & 13.5 & 83.4 \\
\hline 50.0 & & 8.450 & 32.722 & 25.419 & 0.1262 & 6.376 & 12.8 & 83.5 \\
\hline 55.0 & & 8.441 & 32.721 & 25.420 & 0.1388 & 6.374 & 12.4 & 83.4 \\
\hline 60.0 & & 8.433 & 32.721 & 25.421 & 0.1514 & 6.369 & 18.0 & 83.3 \\
\hline 65.0 & & 8.425 & 32.720 & 25.421 & 0.1640 & 6.373 & 13.1 & 83.3 \\
\hline 70.0 & & 8.422 & 32.720 & 25.422 & 0.1766 & 6.353 & 12.4 & 83.2 \\
\hline 75.0 & & 8.422 & 32.720 & 25.422 & 0.1892 & 6.387 & 15.2 & 83.2 \\
\hline 80.0 & & 8.414 & 32.719 & 25.422 & 0.2018 & 6.360 & 13.9 & 83.3 \\
\hline 85.0 & & 8.411 & 32.719 & 25.423 & 0.2145 & 6.362 & 13.3 & 83.2 \\
\hline 86.0 & & 8.412 & 32.719 & 25.422 & 0.2170 & 6.352 & 13.0 & 83.3 \\
\hline
\end{tabular}
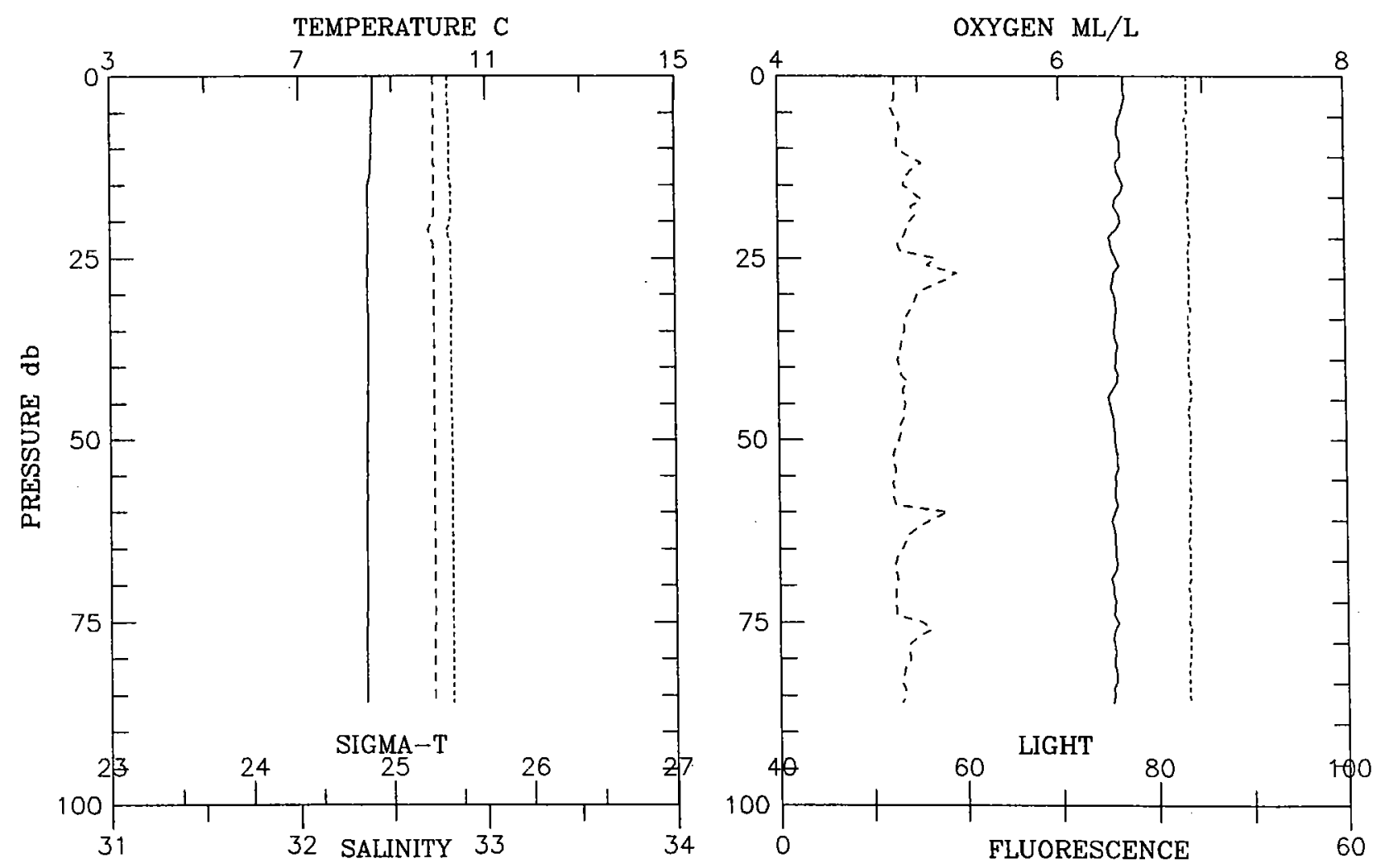


\begin{tabular}{|c|c|c|c|c|c|c|c|c|}
\hline $\begin{array}{l}\text { Cruise EN } \\
\text { Position: }\end{array}$ & $\begin{array}{l}1196 \text { Cons } \\
41^{\circ} 0\end{array}$ & .00 6 & $\begin{array}{l}\text { Station \# } \\
68^{\circ} 48.30^{\prime}\end{array}$ & $\begin{array}{r}\text { Dep } \\
\text { D }\end{array}$ & $\begin{array}{l}\text { th m:64 } \\
\text { epth Deck }\end{array}$ & $\begin{array}{l}\text { Time: } \\
\text { offset: }\end{array}$ & $\begin{array}{c}=1989-06- \\
-0.23\end{array}$ & $\begin{array}{r}08: 15 \\
\text { Fish } 1\end{array}$ \\
\hline $\begin{array}{l}\text { PRESSURE } \\
\text { (d-bars) }\end{array}$ & Interp & $\begin{array}{l}\text { TEMP } \\
\text { (deg } C)\end{array}$ & $\begin{array}{c}\text { SALINITY } \\
(0 / 00)\end{array}$ & SIGMA-T & DELTA-D & $\begin{array}{l}\text { OXYGEN } \\
(\mathrm{ml} / \mathrm{l})\end{array}$ & $\begin{array}{l}\text { CHANNELA } \\
\text { (\%fluor) }\end{array}$ & $\begin{array}{l}\text { CHANNELB } \\
\text { (\%light) }\end{array}$ \\
\hline 0.0 & $\mathbf{E}$ & 9.135 & 32.693 & 25.291 & 0.0000 & 6.542 & 18.0 & 80.7 \\
\hline 5.0 & & 9.140 & 32.692 & 25.290 & 0.0132 & 6.524 & 18.5 & 80.7 \\
\hline 10.0 & & 9.130 & 32.693 & 25.292 & 0.0264 & 6.528 & 18.4 & 80.7 \\
\hline 15.0 & & 9.102 & 32.695 & 25.298 & 0.0395 & 6.489 & 24.8 & 80.2 \\
\hline 20.0 & & 9.098 & 32.695 & 25.299 & 0.0527 & 6.524 & 20.6 & 80.3 \\
\hline 25.0 & & 9.090 & 32.691 & 25.297 & 0.0658 & 6.514 & 20.7 & 80.0 \\
\hline 30.0 & & 9.089 & 32.696 & 25.301 & 0.0790 & 6.516 & 20.0 & 80.2 \\
\hline 35.0 & & 9.086 & 32.696 & 25.301 & 0.0922 & 6.538 & 22.9 & 80.1 \\
\hline 40.0 & & 9.083 & 32.696 & 25.302 & 0.1053 & 6.516 & 21.0 & 80.0 \\
\hline 45.0 & & 9.074 & 32.697 & 25.304 & 0.1185 & 6.516 & 24.7 & 80.1 \\
\hline 50.0 & & 9.079 & 32.697 & 25.303 & 0.1316 & 6.504 & 20.2 & 79.9 \\
\hline 55.0 & & 9.080 & 32.697 & 25.303 & 0.1448 & 6.508 & 24.3 & 80.2 \\
\hline 60.0 & & 9.078 & 32.697 & 25.303 & 0.1580 & 6.538 & 22.5 & 79.7 \\
\hline 65.0 & & 9.081 & 32.696 & 25.302 & 0.1711 & 6.533 & 23.0 & 79.3 \\
\hline
\end{tabular}
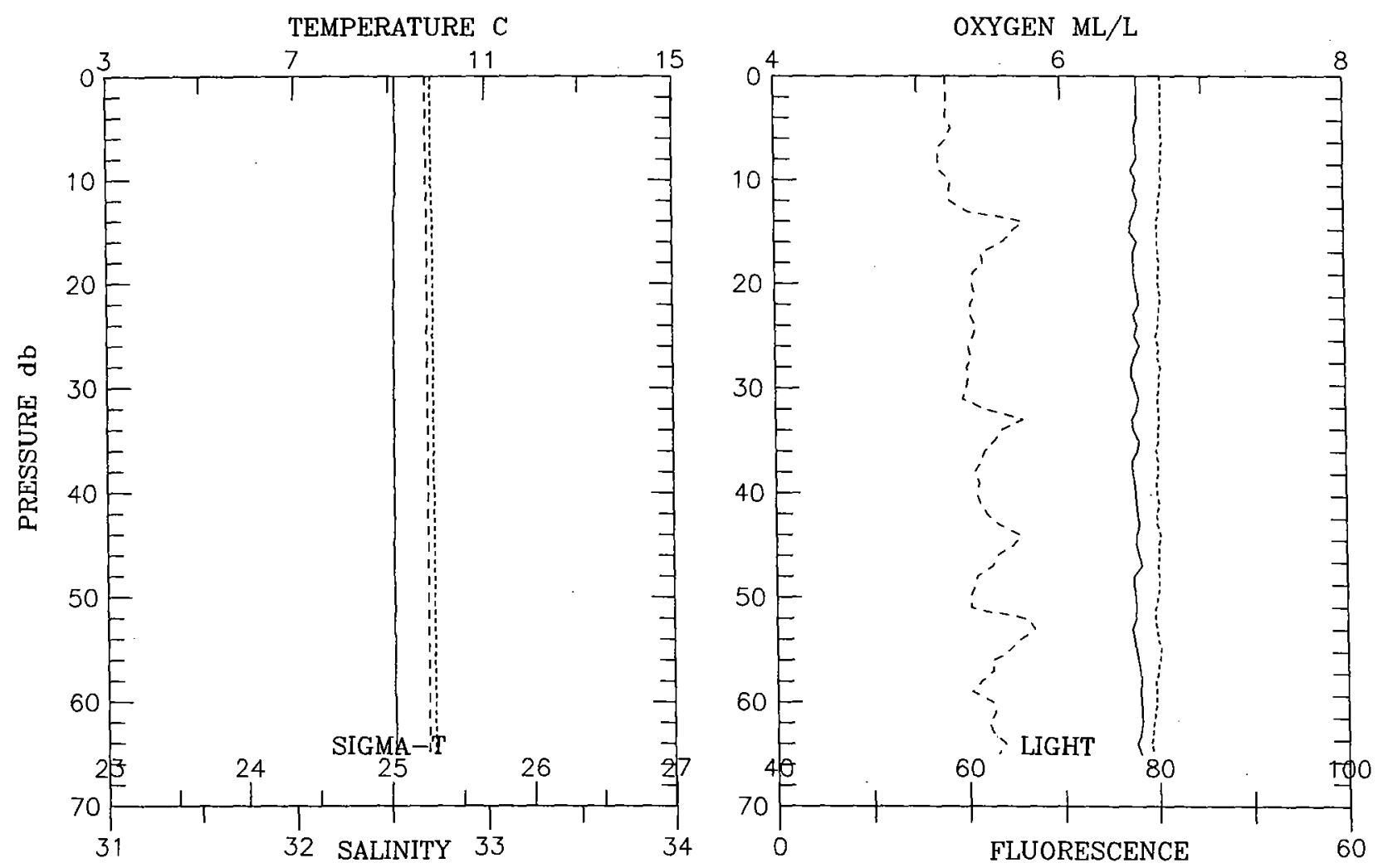


\begin{tabular}{|c|c|c|c|c|c|c|c|c|}
\hline $\begin{array}{l}\text { Cruise EN } \\
\text { Position: }\end{array}$ & $\begin{array}{l}1196 \text { Con } \\
40^{\circ} 59\end{array}$ & 90 ' 6 & $68^{\circ} 38.50^{\circ}$ & $W$ & epth Deck & offset: & -0.23 & Fish 1 \\
\hline $\begin{array}{l}\text { PRESSURE } \\
\text { (d-bars) }\end{array}$ & Interp & $\begin{array}{c}\text { TEMP } \\
(\operatorname{deg} \mathrm{C})\end{array}$ & $\begin{array}{c}\text { SALINITY } \\
(0 / 00)\end{array}$ & SIGMA-T & DELTA-D & $\begin{array}{l}\text { OXYGEN } \\
(\mathrm{ml} / \mathrm{l})\end{array}$ & $\begin{array}{l}\text { CHANNELA } \\
\text { (\%fluor) }\end{array}$ & $\begin{array}{l}\text { CHANNELB } \\
\text { (\%light) }\end{array}$ \\
\hline 0.0 & E & 9.460 & 32.646 & 25.203 & 0.0000 & 6.585 & 18.5 & 79.6 \\
\hline 5.0 & & 9.461 & 32.647 & 25.203 & 0.0136 & 6.562 & 18.4 & 79.5 \\
\hline 10.0 & & 9.450 & 32.647 & 25.205 & 0.0272 & 6.591 & 19.8 & 79.1 \\
\hline 15.0 & & 9.448 & 32.646 & 25.204 & 0.0408 & 6.590 & 19.4 & 79.2 \\
\hline 20.0 & & 9.448 & 32.647 & 25.205 & 0.0544 & 6.593 & 19.4 & 79.0 \\
\hline 25.0 & & 9.447 & 32.647 & 25.205 & 0.0680 & 6.570 & 19.5 & 78.5 \\
\hline 30.0 & & 9.450 & 32.647 & 25.205 & 0.0816 & 6.563 & 18.2 & 79.1 \\
\hline 35.0 & & 9.448 & 32.647 & 25.205 & 0.0952 & 6.572 & 19.3 & 78.3 \\
\hline 40.0 & & 9.448 & 32.647 & 25.205 & 0.1089 & 6.560 & 19.5 & 78.2 \\
\hline 45.0 & & 9.448 & 32.647 & 25.205 & 0.1225 & 6.565 & 21.4 & 78.3 \\
\hline 50.0 & & 9.447 & 32.647 & 25.205 & 0.1361 & 6.555 & 23.7 & 78.2 \\
\hline 55.0 & & 9.447 & 32.647 & 25.205 & 0.1498 & 6.555 & 23.0 & 78.2 \\
\hline 56.0 & & 9.447 & 32.647 & 25.205 & 0.1525 & 6.556 & 23.0 & 78.1 \\
\hline
\end{tabular}
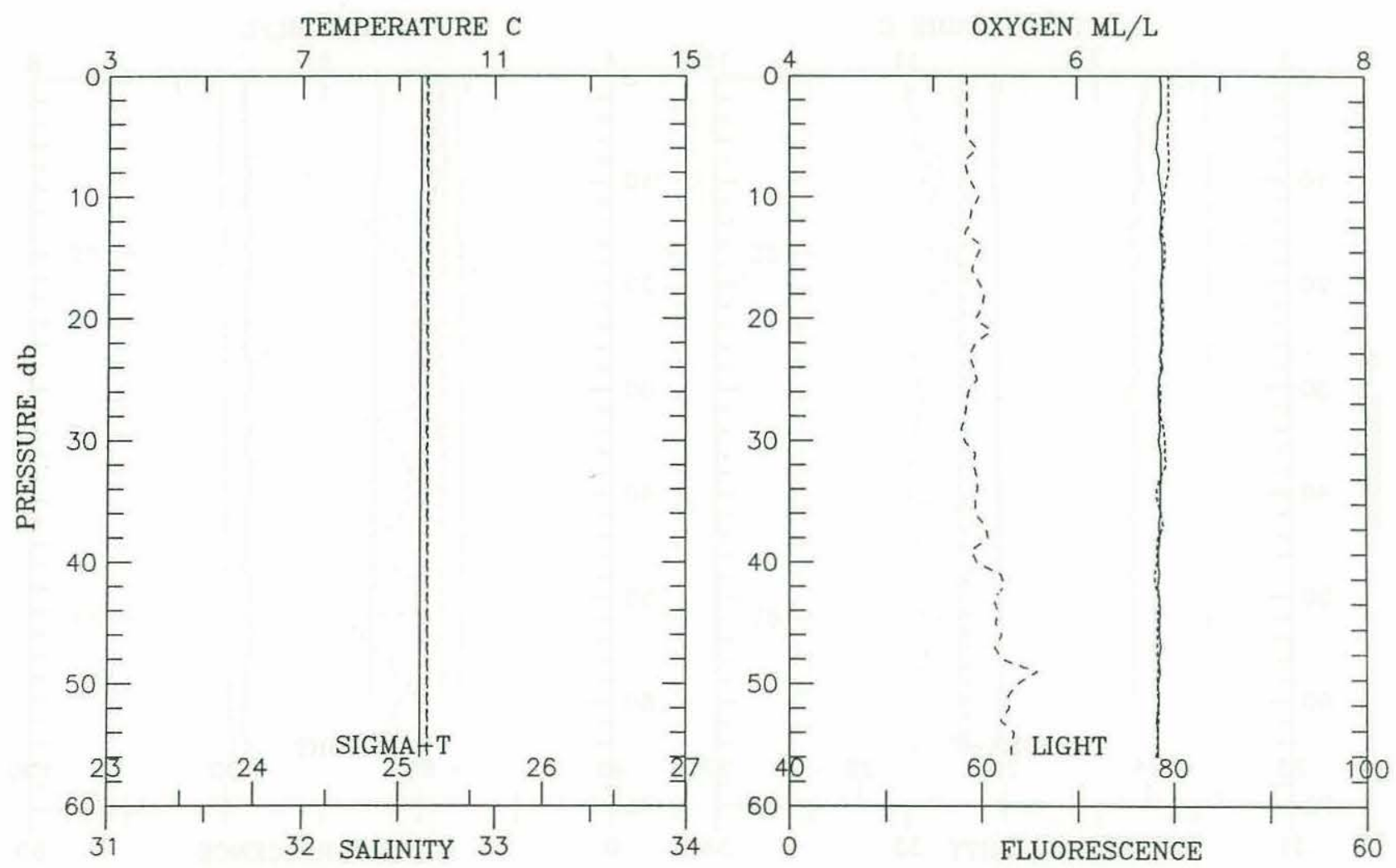


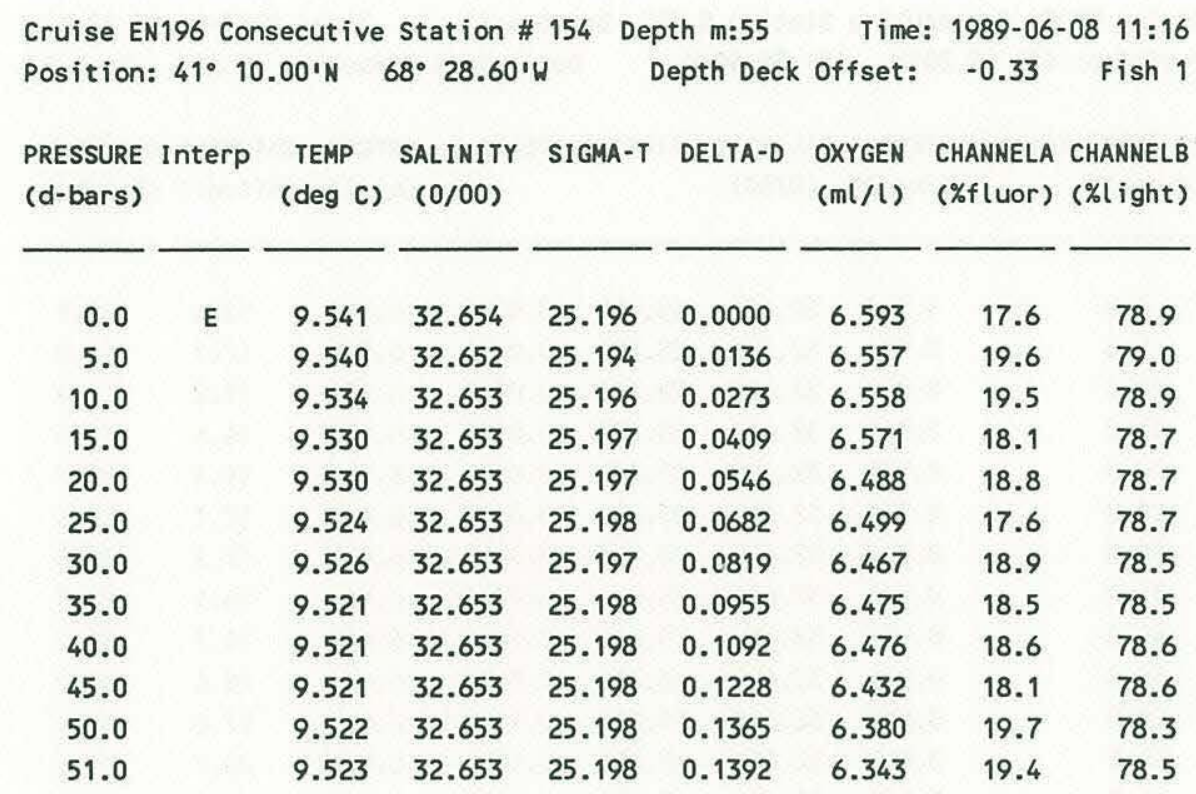
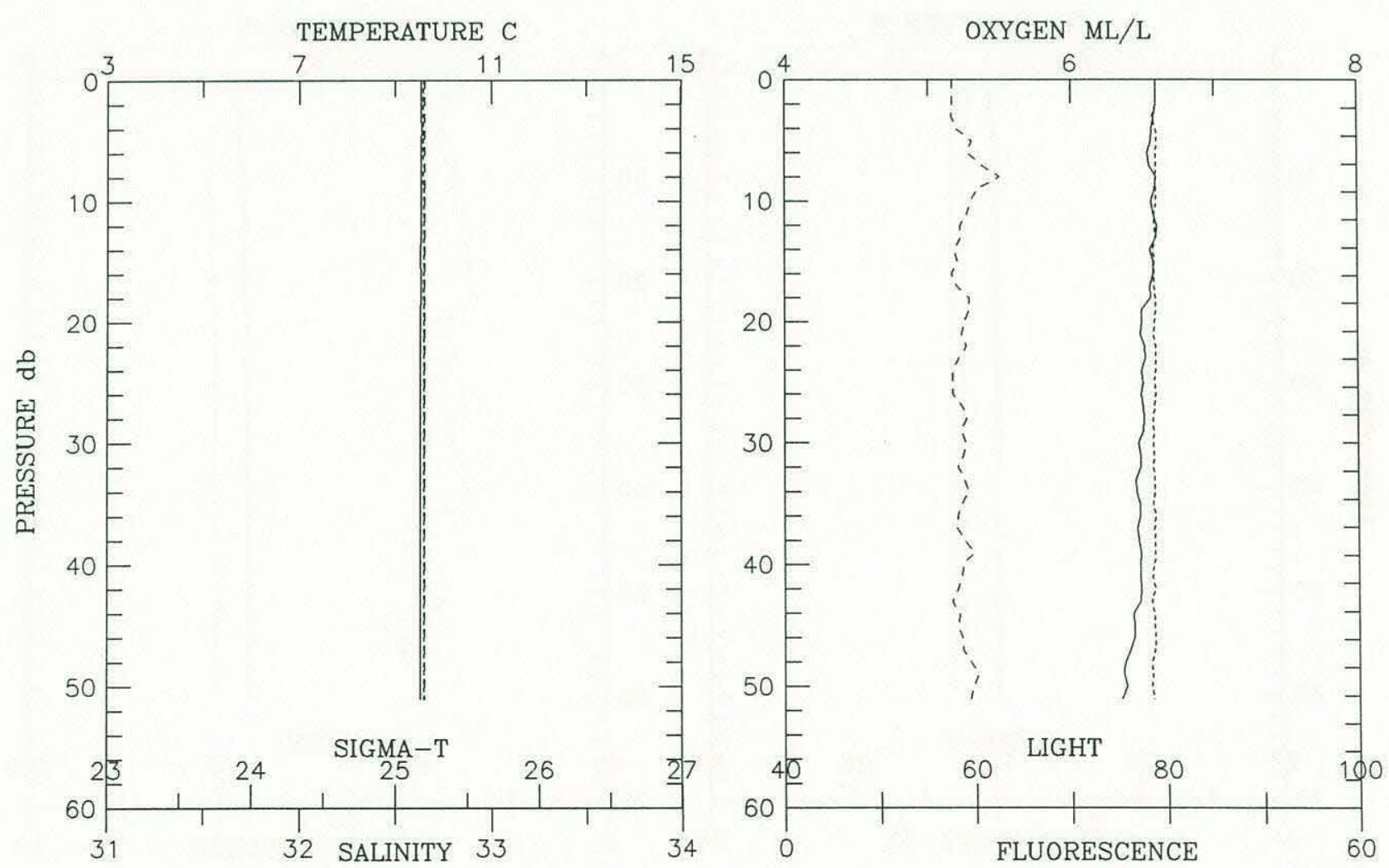


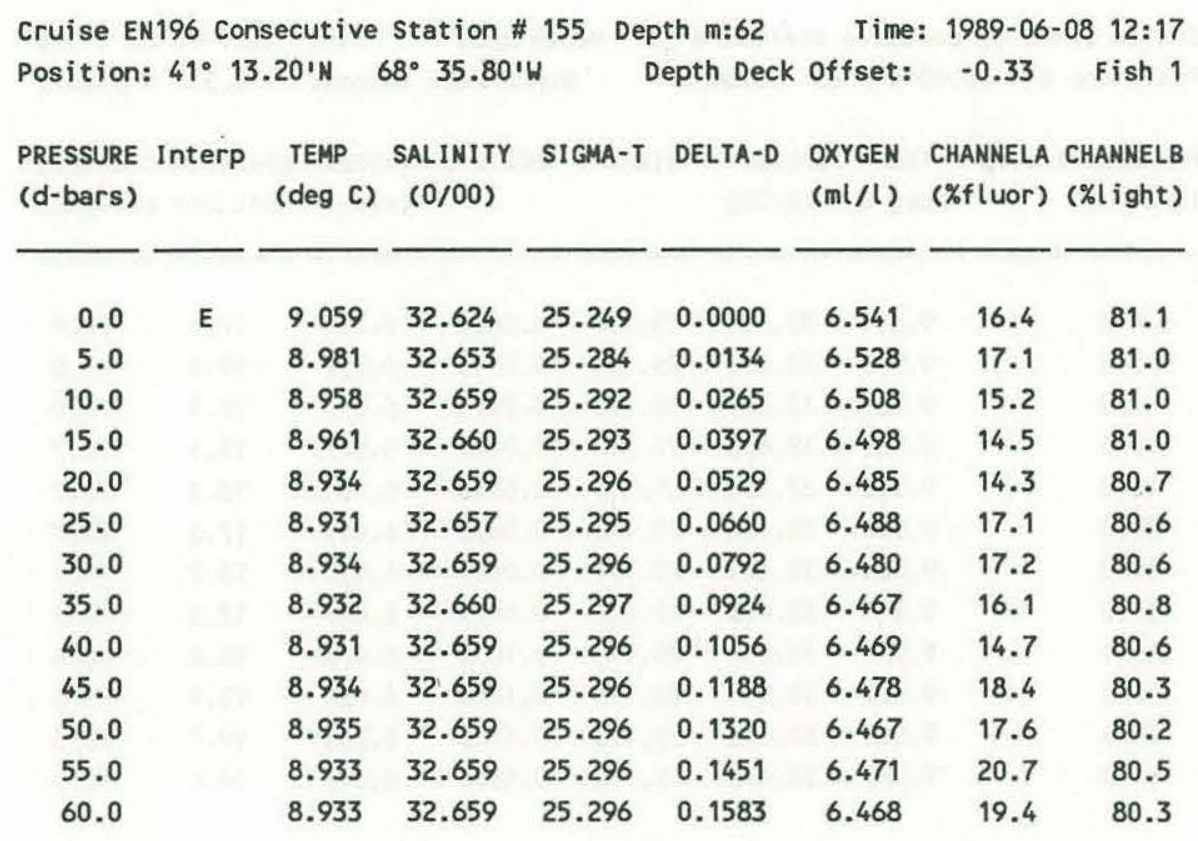
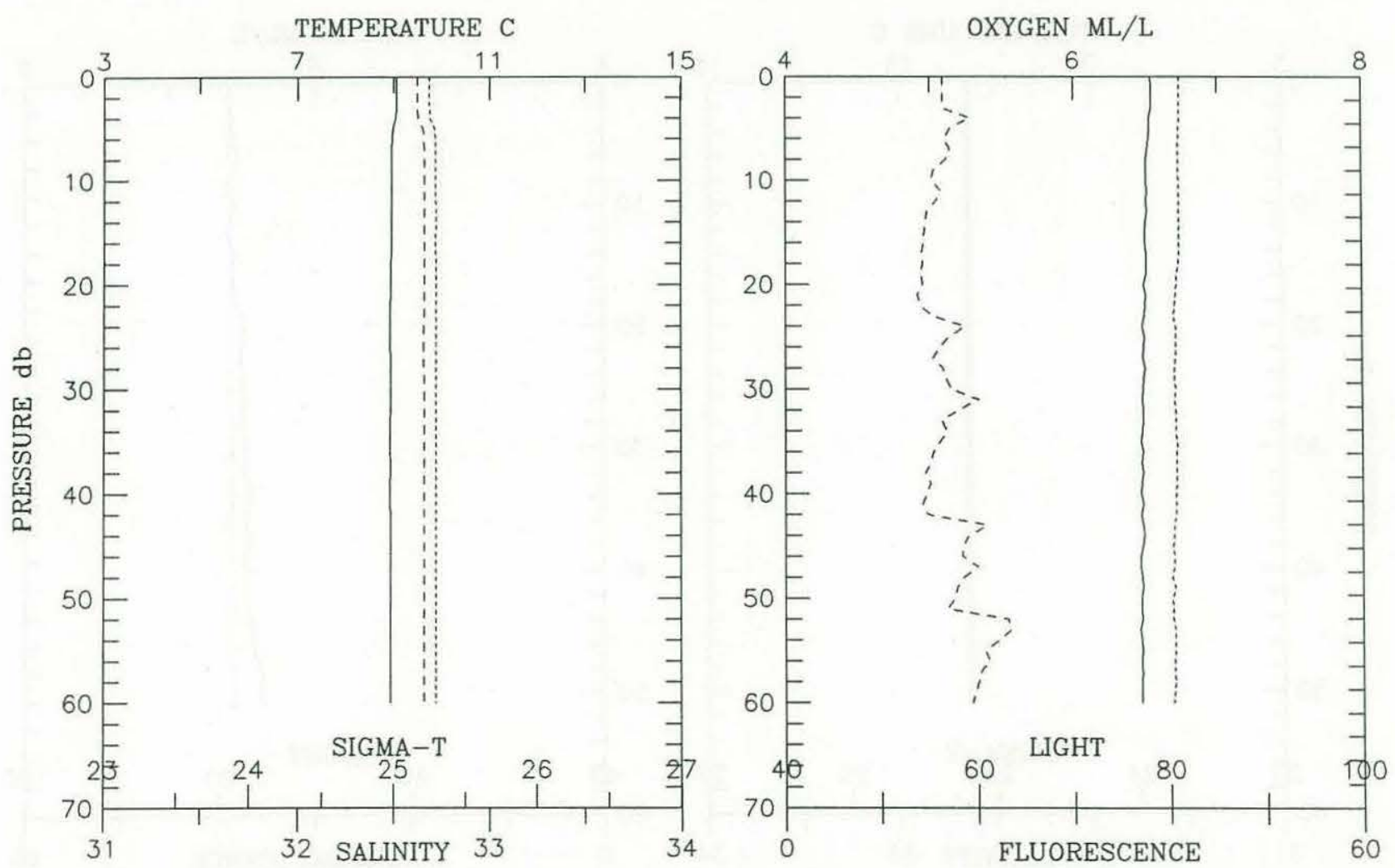
Cruise EN196 Consecutive Station \# 156 Depth m:75 Time: 1989-06-08 13:16 Position: $41^{\circ} 16.00 \mathrm{~N} 68^{\circ} 43.20^{\prime} \mathrm{W}$ Depth Deck offset: -0.15 Fish 1

\begin{tabular}{|c|c|c|c|c|c|c|c|c|}
\hline $\begin{array}{l}\text { PRESSURE } \\
\text { (d-bars) }\end{array}$ & Interp & $\begin{array}{c}\text { TEMP } \\
(\operatorname{deg} \mathrm{C})\end{array}$ & $\begin{array}{c}\text { SALINITY } \\
(0 / 00)\end{array}$ & SIGMA-T & DELTA-D & $\begin{array}{l}\text { OXYGEN } \\
(\mathrm{ml} / \mathrm{l})\end{array}$ & $\begin{array}{l}\text { CHANNELA } \\
\text { (\%fluor) }\end{array}$ & $\begin{array}{l}\text { CHANNELB } \\
\text { (\%light) }\end{array}$ \\
\hline 0.0 & E & 9.399 & 32.401 & 25.021 & 0.0000 & 6.378 & 16.8 & 80.9 \\
\hline 5.0 & & 9.278 & 32.406 & 25.044 & 0.0144 & 6.335 & 14.7 & 81.1 \\
\hline 10.0 & & 7.914 & 32.621 & 25.418 & 0.0277 & 6.583 & 12.8 & 83.4 \\
\hline 15.0 & & 7.826 & 32.641 & 25.447 & 0.0401 & 6.571 & 10.3 & 84.0 \\
\hline 20.0 & & 7.850 & 32.674 & 25.470 & 0.0525 & 6.491 & 9.6 & 84.7 \\
\hline 25.0 & & 7.756 & 32.713 & 25.513 & 0.0648 & 6.475 & 9.0 & 85.3 \\
\hline 30.0 & & 7.586 & 32.719 & 25.542 & 0.0768 & 6.433 & 9.5 & 85.5 \\
\hline 35.0 & & 7.508 & 32.721 & 25.554 & 0.0888 & 6.444 & 7.5 & 85.6 \\
\hline 40.0 & & 7.261 & 32.730 & 25.596 & 0.1006 & 6.394 & 6.8 & 85.6 \\
\hline 45.0 & & 7.039 & 32.744 & 25.637 & 0.1123 & 6.382 & 8.4 & 85.7 \\
\hline 50.0 & & 6.696 & 32.755 & 25.691 & 0.1237 & 6.351 & 5.8 & 85.0 \\
\hline 55.0 & & 6.660 & 32.757 & 25.697 & 0.1349 & 6.297 & 5.1 & 84.6 \\
\hline 60.0 & & 6.612 & 32.760 & 25.706 & 0.1462 & 6.312 & 5.2 & 84.3 \\
\hline 65.0 & & 6.594 & 32.761 & 25.709 & 0.1574 & 6.266 & 5.1 & 84.1 \\
\hline 70.0 & & 6.561 & 32.762 & 25.714 & 0.1686 & 6.267 & 5.7 & 84.0 \\
\hline 73.0 & & 6.529 & 32.765 & 25.721 & 0.1753 & 8.217 & 7.4 & 83.6 \\
\hline
\end{tabular}
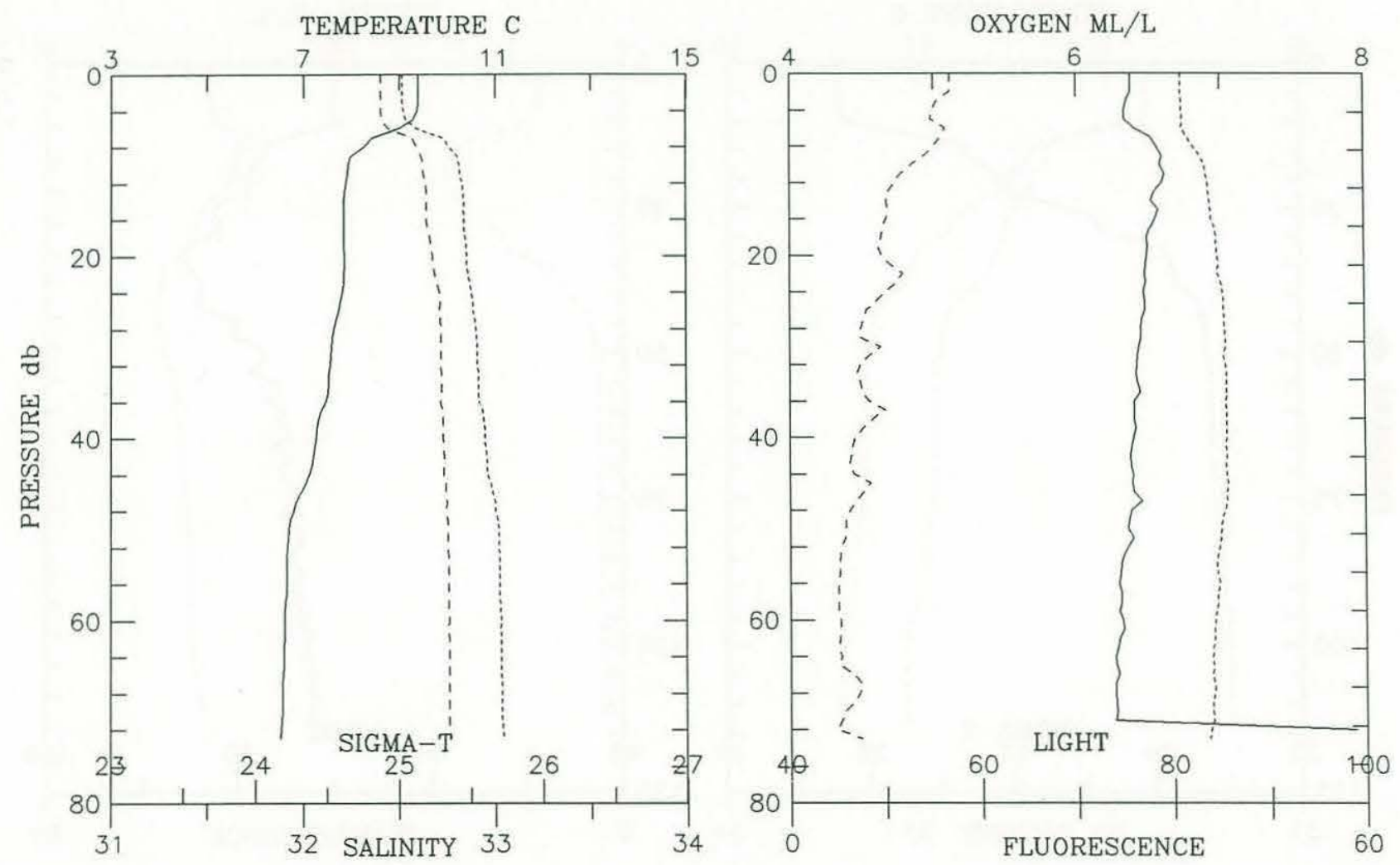


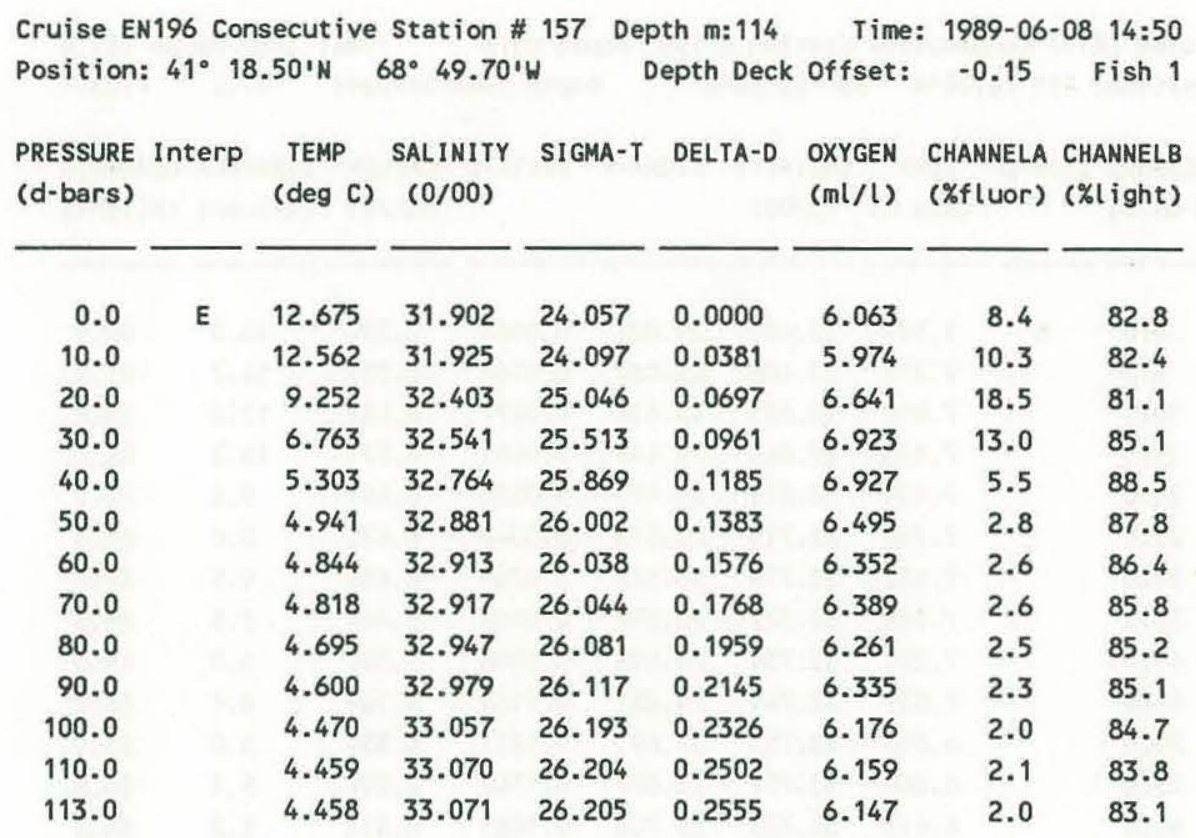
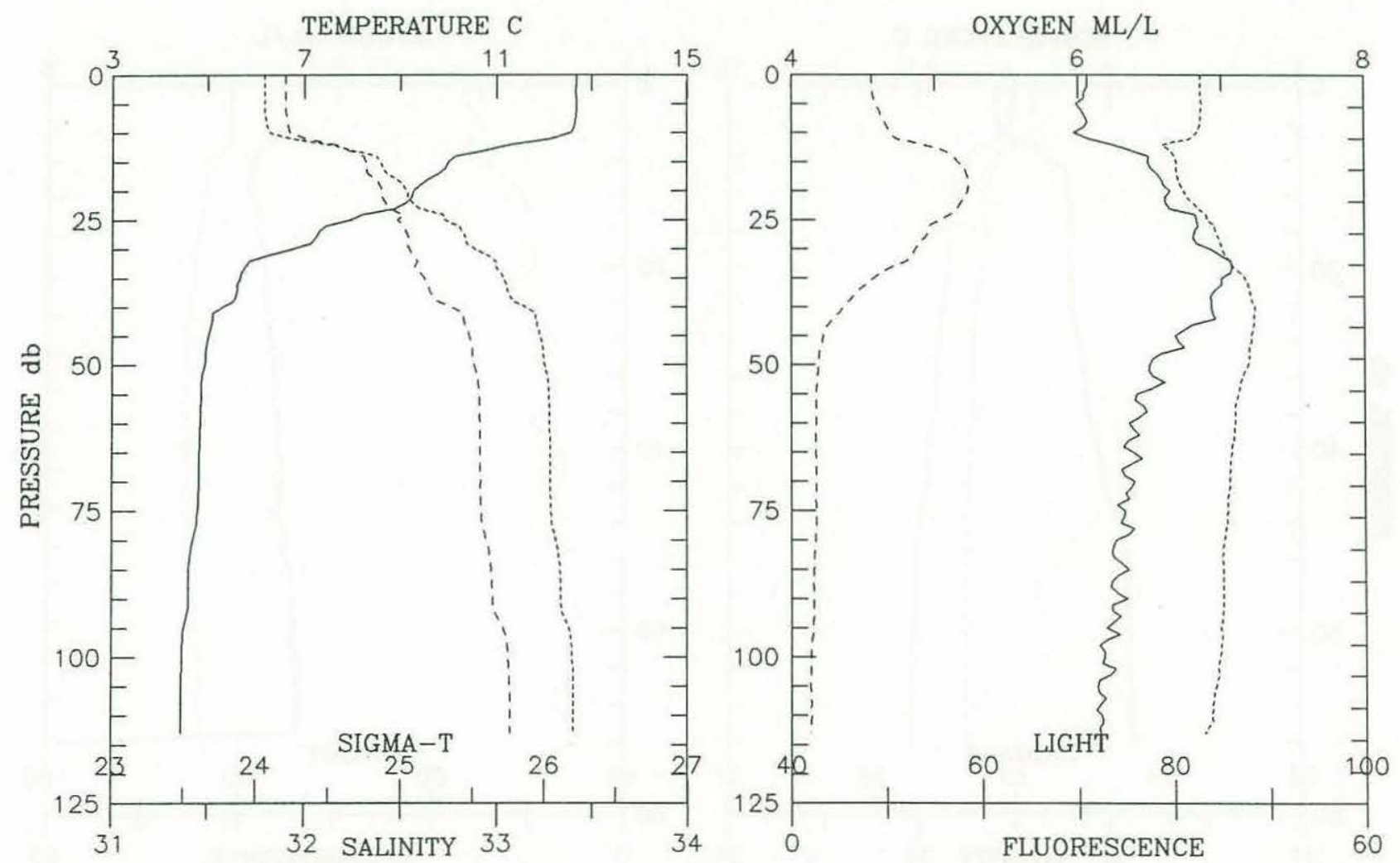
Cruise EN196 Consecutive Station \# 158 Depth m:142 Time: 1989-06-08 15:51 Position: $41^{\circ} 20.50^{\prime} \mathrm{N} 68^{\circ} 54.40^{\prime} \mathrm{W}$ Depth Deck offset: -0.15 Fish 1

\begin{tabular}{|c|c|c|c|c|c|c|c|c|}
\hline $\begin{array}{l}\text { PRESSURE } \\
\text { (d-bars) }\end{array}$ & Interp & $\begin{array}{c}\text { TEMP } \\
(\operatorname{deg} C)\end{array}$ & $\begin{array}{l}\text { SALINITY } \\
(0 / 00)\end{array}$ & SI GMA-T & DELTA-D & $\begin{array}{l}\text { OXYGEN } \\
(m l / l)\end{array}$ & $\begin{array}{l}\text { CHANNELA } \\
\text { (\%fluor) }\end{array}$ & $\begin{array}{l}\text { CHANNELB } \\
\text { (\%tight) }\end{array}$ \\
\hline 0.0 & E & 13.188 & 31.795 & 23.875 & 0.0000 & 5.964 & 7.8 & 81.4 \\
\hline 10.0 & & 13.117 & 31.806 & 23.897 & 0.0399 & 5.973 & 8.8 & 81.5 \\
\hline 20.0 & & 8.108 & 32.486 & 25.285 & 0.0739 & 7.018 & 16.0 & 82.2 \\
\hline 30.0 & & 5.462 & 32.644 & 25.756 & 0.0977 & 7.252 & 8.6 & 87.5 \\
\hline 40.0 & & 5.087 & 32.810 & 25.930 & 0.1186 & 6.928 & 3.4 & 89.6 \\
\hline 50.0 & & 4.713 & 32.897 & 26.040 & 0.1382 & 6.600 & 2.4 & 89.7 \\
\hline 60.0 & & 4.634 & 32.935 & 26.078 & 0.1572 & 6.608 & 2.0 & 89.6 \\
\hline 70.0 & & 4.460 & 33.053 & 26.190 & 0.1757 & 6.535 & 1.8 & 88.3 \\
\hline 80.0 & & 4.426 & 33.089 & 26.222 & 0.1933 & 6.316 & 1.7 & 86.1 \\
\hline 90.0 & & 4.426 & 33.090 & 26.223 & 0.2107 & 6.291 & 1.7 & 85.6 \\
\hline 100.0 & & 4.425 & 33.092 & 26.225 & 0.2282 & 6.177 & 1.8 & 85.4 \\
\hline 110.0 & & 4.423 & 33.092 & 26.225 & 0.2457 & 6.222 & 1.8 & 85.0 \\
\hline 120.0 & & 4.423 & 33.092 & 26.225 & 0.2631 & 6.195 & 1.9 & 84.9 \\
\hline 130.0 & & 4.423 & 33.092 & 26.225 & 0.2806 & 6.104 & 1.9 & 84.8 \\
\hline 137.0 & & 4.421 & 33.093 & 26.226 & 0.2929 & 6.145 & 1.9 & 84.0 \\
\hline
\end{tabular}
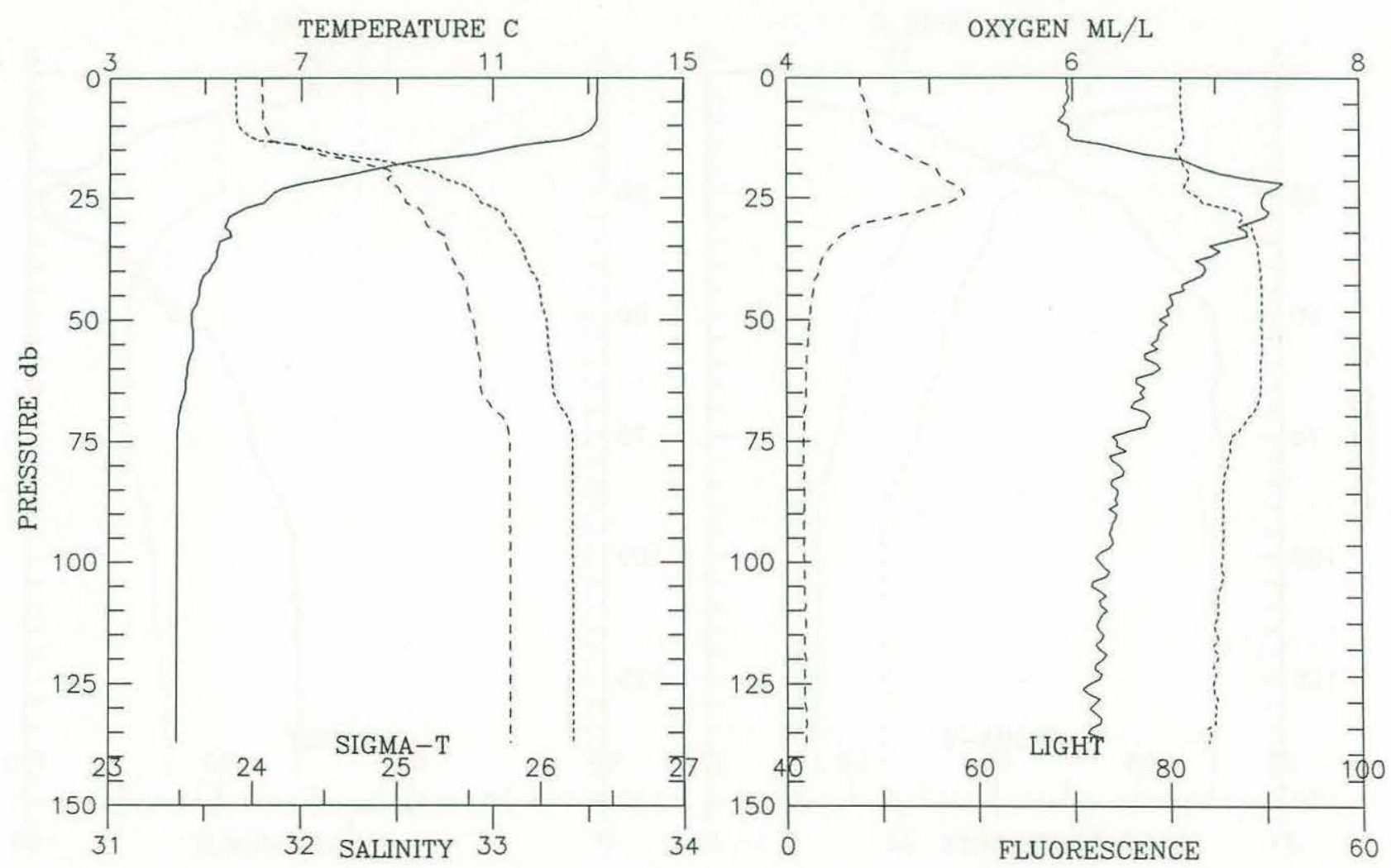


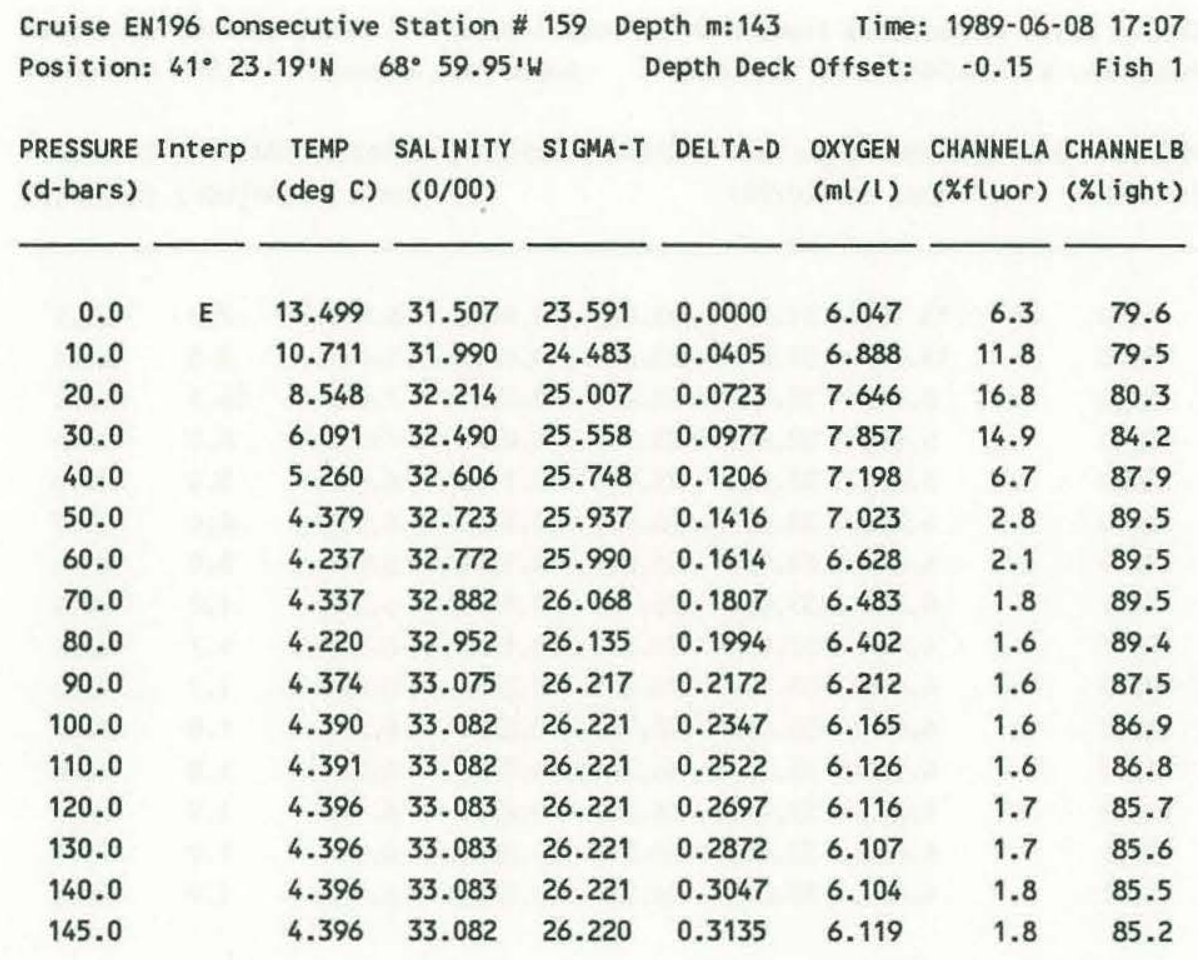
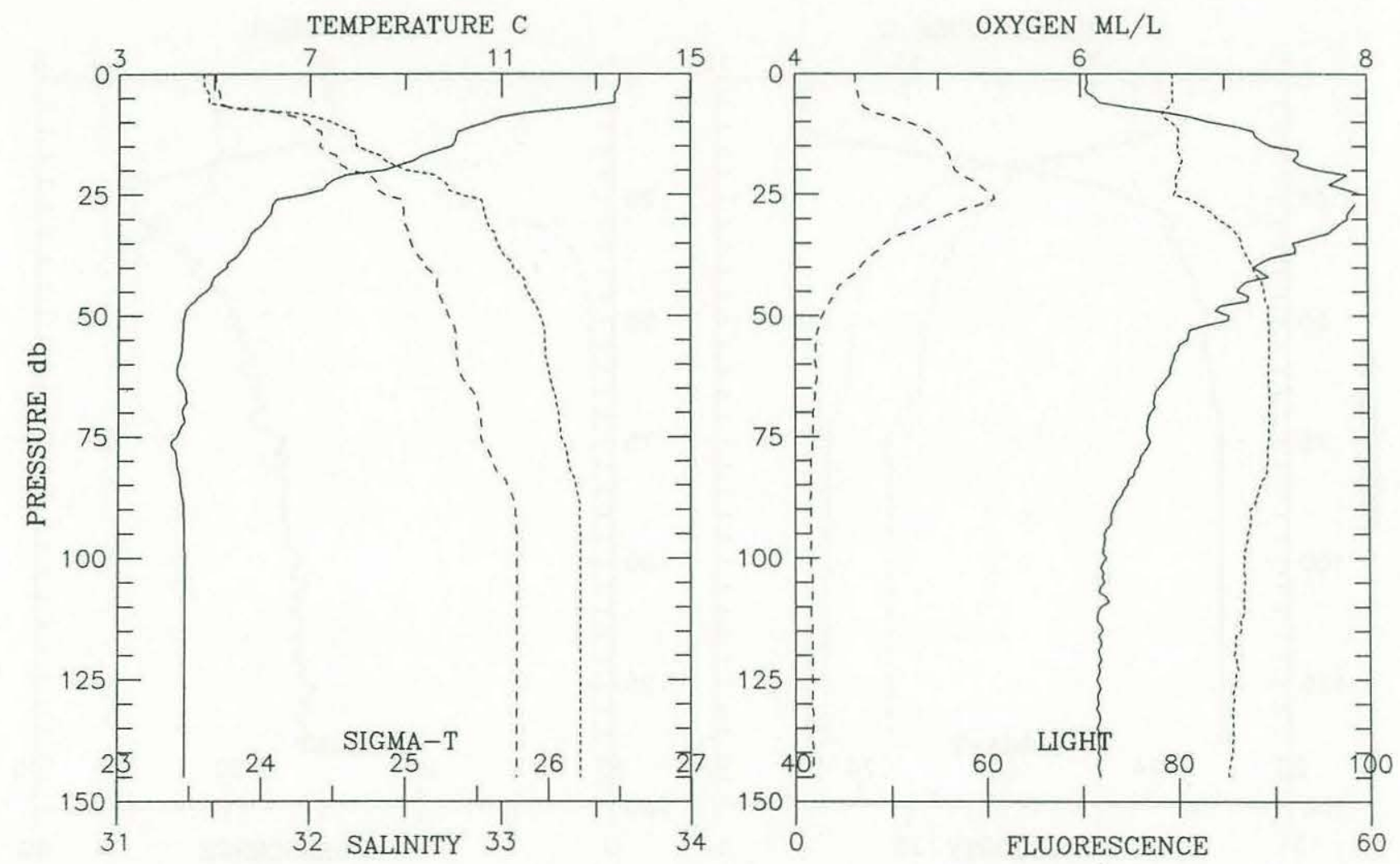
Cruise EN196 Consecutive Station \# 160 Depth m:162 Time: 1989-06-08 18:48 Position: $41^{\circ} 22.25^{\prime} \mathrm{N} 69^{\circ} 4.90^{\prime} \mathrm{W} \quad$ Depth Deck offset: -0.15 Fish 1

\begin{tabular}{|c|c|c|c|c|c|c|c|c|}
\hline $\begin{array}{l}\text { PRESSURE } \\
\text { (d-bars) }\end{array}$ & Interp & $\begin{array}{c}\text { TEMP } \\
(\operatorname{deg} C)\end{array}$ & $\begin{array}{c}\text { SALINITY } \\
(0 / 00)\end{array}$ & SIGMA-T & DELTA-D & $\begin{array}{l}\text { OXYGEN } \\
(\mathrm{ml} / \mathrm{l})\end{array}$ & $\begin{array}{l}\text { CHANNELA } \\
\text { (\%fluor) }\end{array}$ & $\begin{array}{l}\text { CHANNELB } \\
\text { (\%light) }\end{array}$ \\
\hline 0.0 & E & 13.390 & 31.586 & 23.673 & 0.0000 & 6.016 & 8.5 & 79.5 \\
\hline 10.0 & & 12.642 & 31.635 & 23.857 & 0.0416 & 6.140 & 12.5 & 78.4 \\
\hline 20.0 & & 5.765 & 32.451 & 25.567 & 0.0721 & 7.169 & 22.1 & 84.6 \\
\hline 30.0 & & 5.001 & 32.697 & 25.849 & 0.0946 & 6.795 & 5.4 & 88.8 \\
\hline 40.0 & & 4.170 & 32.771 & 25.997 & 0.1148 & 6.675 & 2.3 & 89.5 \\
\hline 50.0 & & 4.036 & 32.810 & 26.041 & 0.1342 & 6.530 & 2.1 & 89.0 \\
\hline 60.0 & & 3.791 & 32.831 & 26.082 & 0.1531 & 6.460 & 1.6 & 89.2 \\
\hline 70.0 & & 3.732 & 32.861 & 26.111 & 0.1717 & 6.483 & 1.7 & 89.0 \\
\hline 80.0 & & 4.205 & 33.014 & 26.186 & 0.1897 & 6.208 & 1.6 & 87.3 \\
\hline 90.0 & & 4.301 & 33.035 & 26.193 & 0.2075 & 6.077 & 1.6 & 85.9 \\
\hline 100.0 & & 4.305 & 33.036 & 26.193 & 0.2253 & 6.051 & 1.7 & 85.7 \\
\hline 110.0 & & 4.295 & 33.037 & 26.195 & 0.2430 & 6.074 & 2.0 & 85.6 \\
\hline 120.0 & & 4.285 & 33.040 & 26.198 & 0.2608 & 6.051 & 1.8 & 85.7 \\
\hline 130.0 & & 4.281 & 33.038 & 26.197 & 0.2785 & 6.056 & 1.8 & 85.4 \\
\hline 140.0 & & 4.273 & 33.044 & 26.203 & 0.2962 & 6.091 & 1.8 & 85.0 \\
\hline 150.0 & & 4.267 & 33.048 & 26.206 & 0.3139 & 6.082 & 2.0 & 84.7 \\
\hline 155.0 & & 4.267 & 33.049 & 26.207 & 0.3227 & 6.096 & 1.9 & 84.8 \\
\hline
\end{tabular}
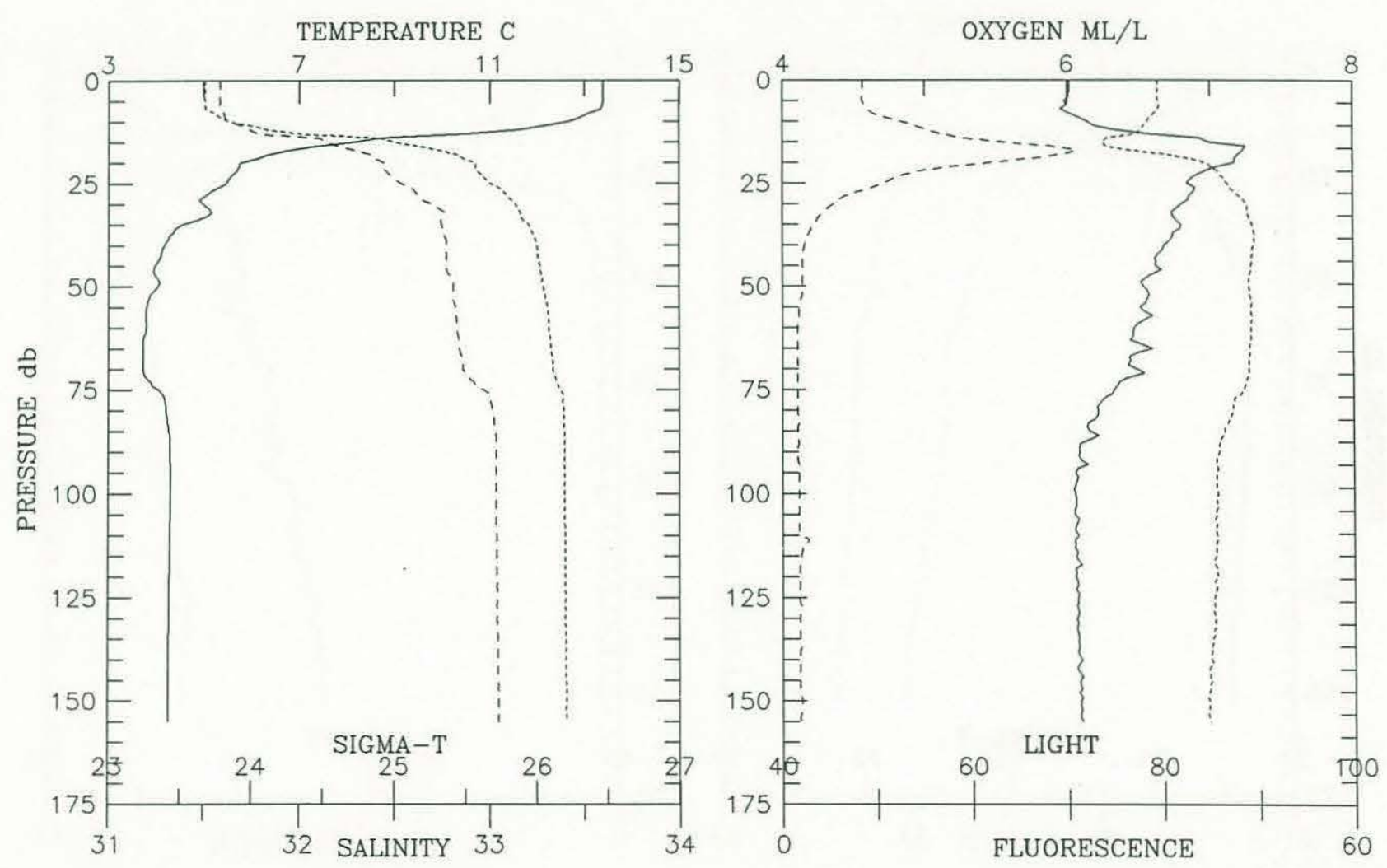


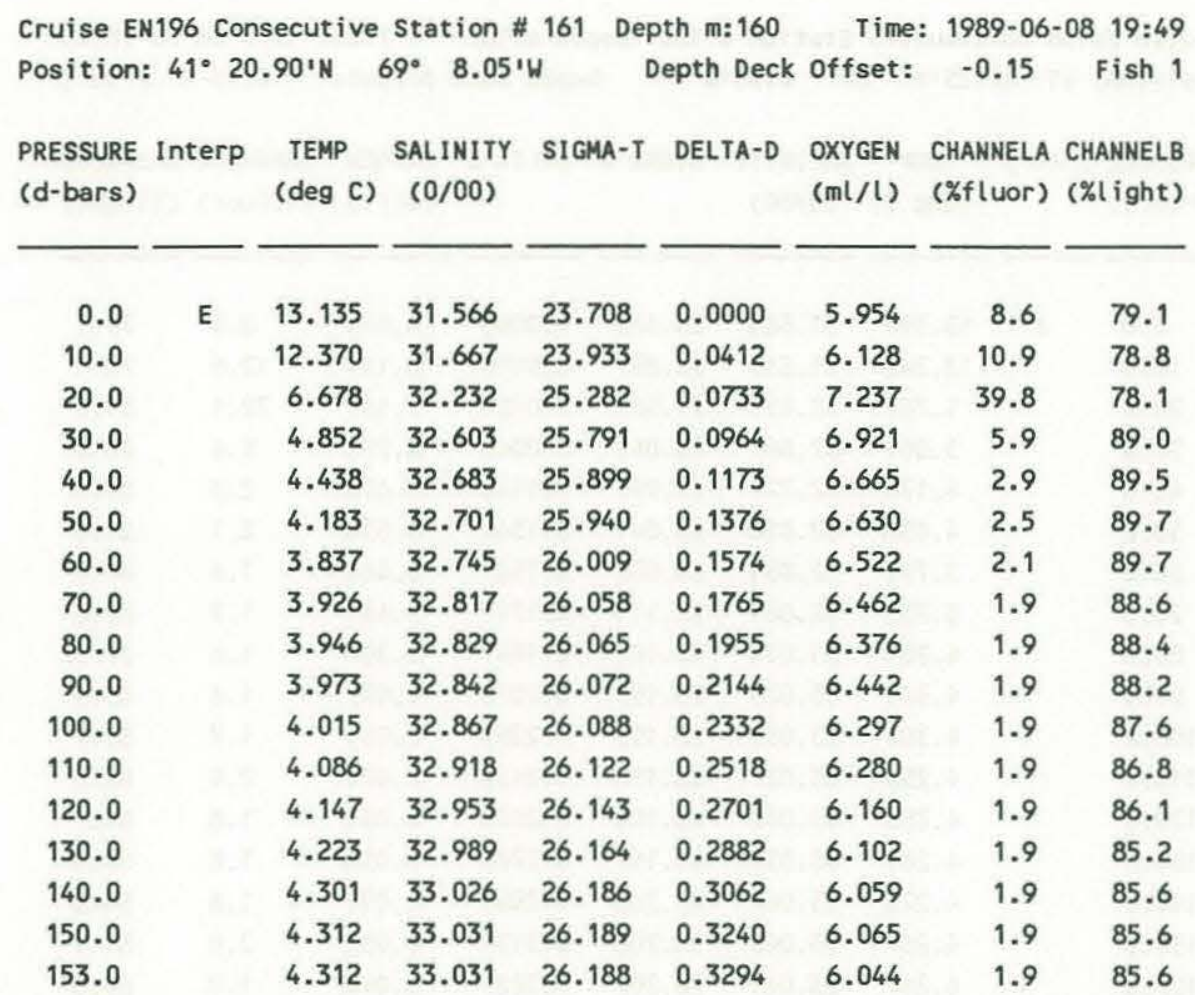
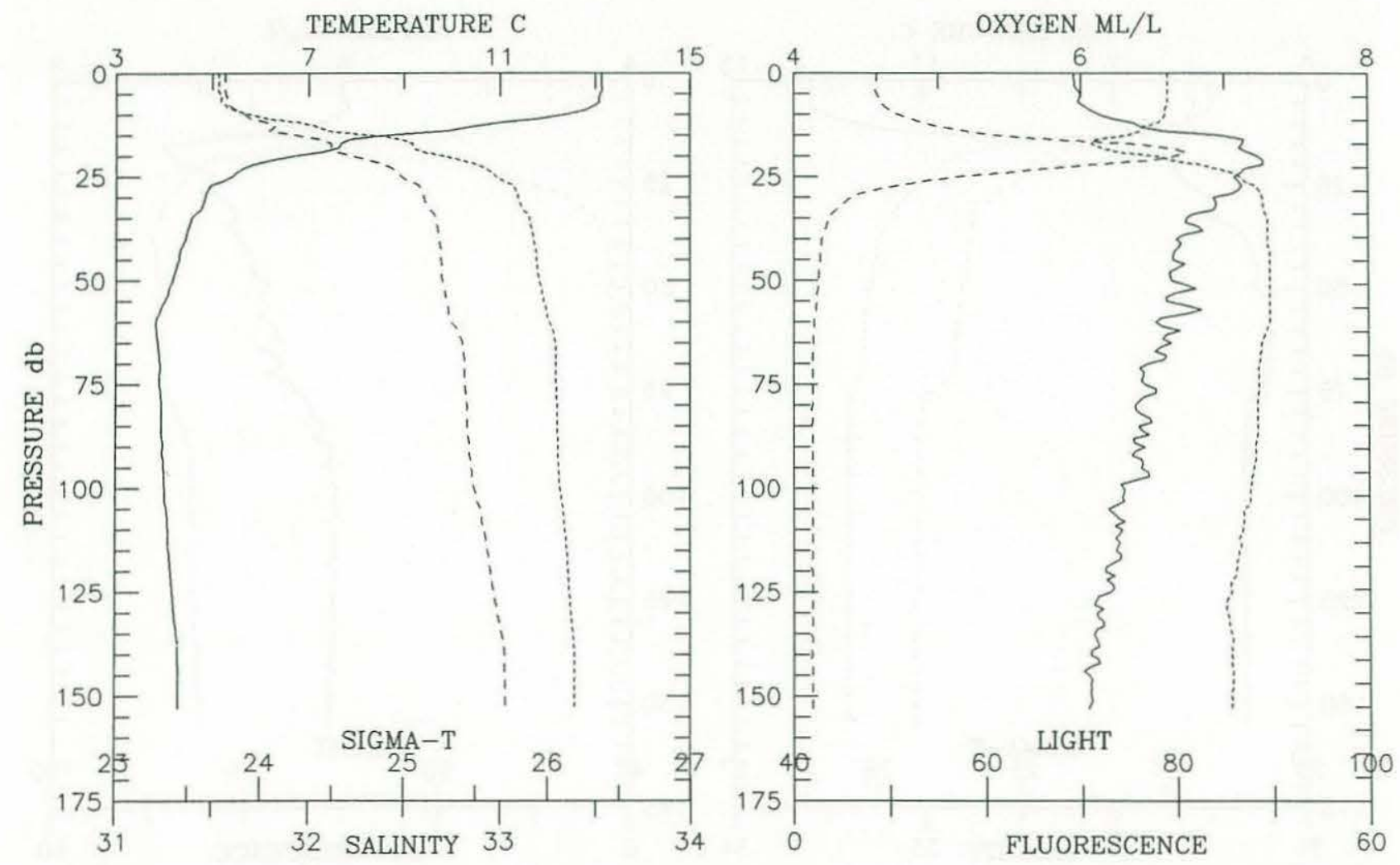
Cruise EN196 Consecutive Station \# 162 Depth m:76 Time: 1989-06-08 20:55 Position: $41^{\circ} 16.90^{\prime} \mathrm{N} 69^{\circ} 18.60^{\prime} \mathrm{W} \quad$ Depth Deck offset: -0.15 Fish 1

PRESSURE InterP TEMP SALINITY SIGMA-T DELTA-D OXYGEN CHANNELA CHANNELB (d-bars) (deg C) $(0 / 00) \quad(\mathrm{ml} / \mathrm{l})$ (\%fluor) (\%light)

\begin{tabular}{|c|c|c|c|c|c|c|c|c|}
\hline 0.0 & E & 12.843 & 31.628 & 23.813 & 0.0000 & 6.197 & 7.8 & 78.4 \\
\hline 5.0 & & 12.737 & 31.627 & 23.832 & 0.0202 & 6.070 & 9.3 & 77.9 \\
\hline 10.0 & & 12.271 & 31.675 & 23.958 & 0.0401 & 6.304 & 13.7 & 77.6 \\
\hline 15.0 & & 11.668 & 31.729 & 24.111 & 0.0593 & 6.217 & 15.6 & 77.8 \\
\hline 20.0 & & 8.435 & 32.049 & 24.894 & 0.0758 & 6.925 & 29.1 & 73.4 \\
\hline 25.0 & & 6.884 & 32.393 & 25.381 & 0.0899 & 7.049 & 34.2 & 77.8 \\
\hline 30.0 & & 5.129 & 32.624 & 25.778 & 0.1016 & 7.071 & 15.4 & 87.0 \\
\hline 35.0 & & 4.962 & 32.642 & 25.810 & 0.1124 & 6.767 & 7.3 & 87.5 \\
\hline 40.0 & & 4.459 & 32.721 & 25.927 & 0.1227 & 6.723 & 4.0 & 88.7 \\
\hline 45.0 & & 4.258 & 32.775 & 25.991 & 0.1326 & 6.667 & 2.6 & 88.1 \\
\hline 50.0 & & 4.227 & 32.792 & 26.007 & 0.1424 & 6.507 & 2.2 & 87.6 \\
\hline 55.0 & & 4.220 & 32.797 & 26.012 & 0.1521 & 6.375 & 2.2 & 87.3 \\
\hline 60.0 & & 4.214 & 32.803 & 26.017 & 0.1618 & 6.350 & 2.2 & 87.2 \\
\hline 65.0 & & 4.207 & 32.806 & 26.020 & 0.1715 & 6.371 & 2.2 & 87.1 \\
\hline 70.0 & & 4.202 & 32.810 & 26.024 & 0.1812 & 6.387 & 2.1 & 87.0 \\
\hline 75.0 & & 4.202 & 32.813 & 26.026 & 0.1908 & 6.392 & 2.2 & 86.8 \\
\hline 80.0 & & 4.200 & 32.815 & 26.028 & 0.2005 & 6.228 & 2.2 & 86.5 \\
\hline 85.0 & & 4.199 & 32.817 & 26.030 & 0.2101 & 6.243 & 2.2 & 86.5 \\
\hline 90.0 & & 4.200 & 32.818 & 26.031 & 0.2198 & 6.255 & 2.2 & 86.6 \\
\hline 95.0 & & 4.199 & 32.818 & 26.031 & 0.2294 & 6.297 & 2.2 & 86.6 \\
\hline 100.0 & & 4.198 & 32.818 & 26.031 & 0.2390 & 6.256 & 2.2 & 86.6 \\
\hline 101.0 & & 4.198 & 32.818 & 26.031 & 0.2410 & 6.302 & 2.2 & 86.5 \\
\hline
\end{tabular}
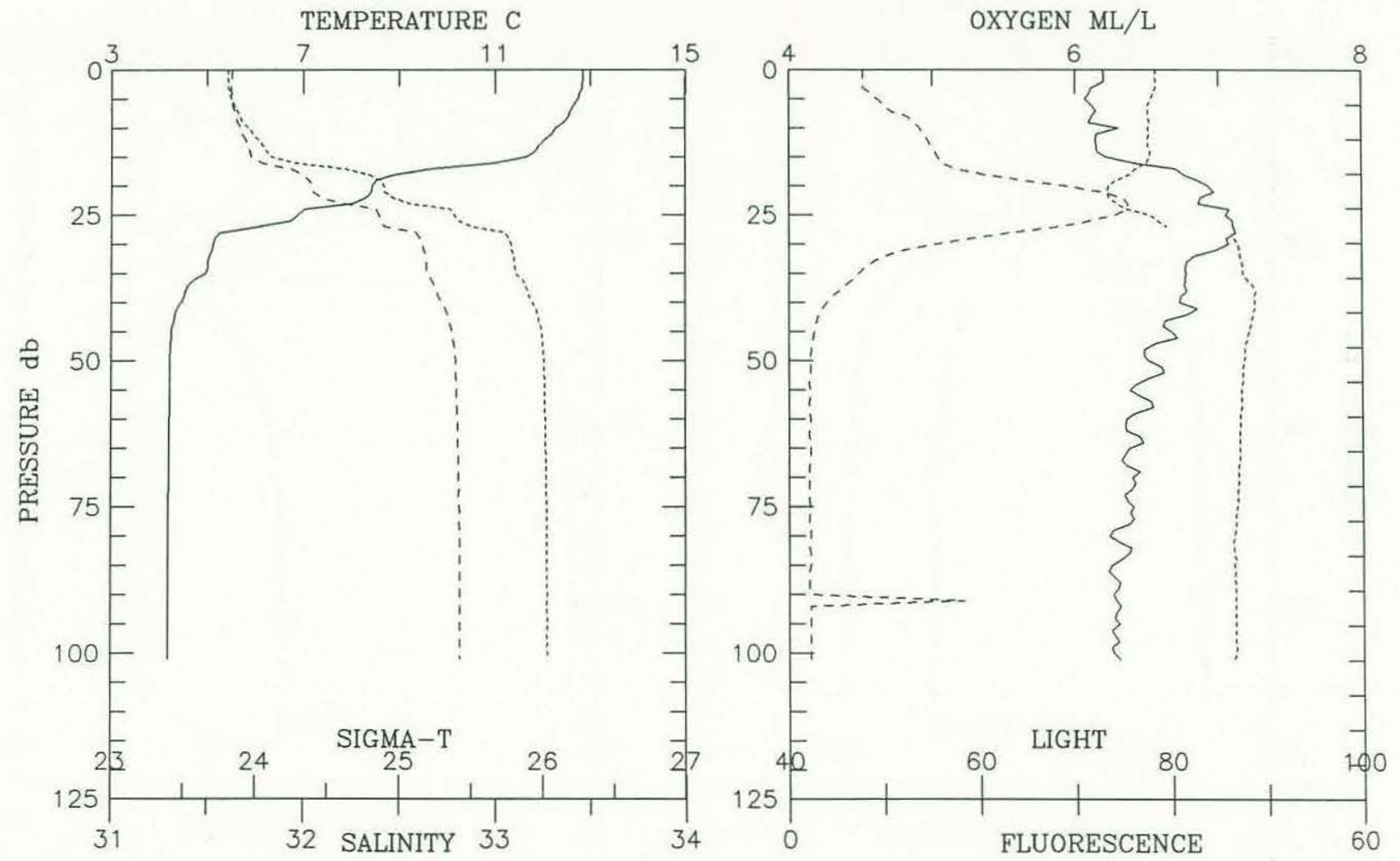
Cruise EN196 Consecutive Station \# 163 Depth m:79 Time: 1989-06-08 22:06 Position: $41^{\circ} 16.90^{\prime} \mathrm{N} 69^{\circ} 18.60^{\prime} \mathrm{W}$ Depth Deck offset: -0.15 Fish 1

\begin{tabular}{|c|c|c|c|c|c|c|c|c|}
\hline $\begin{array}{l}\text { PRESSURE } \\
\text { (d-bars) }\end{array}$ & Interp & $\begin{array}{c}\text { TEMP } \\
(\operatorname{deg} \mathrm{C})\end{array}$ & $\begin{array}{c}\text { SALINITY } \\
(0 / 00)\end{array}$ & SIGMA-T & DELTA-D & $\begin{array}{l}\text { OXYGEN } \\
(\mathrm{ml} / \mathrm{l})\end{array}$ & $\begin{array}{l}\text { CHANNELA } \\
\text { (\%fluor) }\end{array}$ & $\begin{array}{l}\text { CHANNELB } \\
\text { (\%light) }\end{array}$ \\
\hline 0.0 & E & 11.345 & 31.795 & 24.220 & 0.0000 & 6.388 & 19.0 & 75.6 \\
\hline 5.0 & & 11.199 & 31.800 & 24.250 & 0.0182 & 6.406 & 19.8 & 75.2 \\
\hline 10.0 & & 11.050 & 31.809 & 24.284 & 0.0363 & 6.426 & 20.6 & 75.1 \\
\hline 15.0 & & 10.541 & 31.860 & 24.411 & 0.0540 & 6.501 & 19.7 & 76.2 \\
\hline 20.0 & & 7.026 & 32.257 & 25.255 & 0.0697 & 7.038 & 32.6 & 72.1 \\
\hline 25.0 & & 5.672 & 32.566 & 25.670 & 0.0821 & 6.876 & 25.6 & 81.0 \\
\hline 30.0 & & 4.958 & 32.647 & 25.814 & 0.0930 & 6.586 & 8.5 & 86.6 \\
\hline 35.0 & & 4.632 & 32.719 & 25.907 & 0.1034 & 6.402 & 4.3 & 87.5 \\
\hline 40.0 & & 4.505 & 32.743 & 25.940 & 0.1135 & 6.281 & 3.1 & 87.4 \\
\hline 45.0 & & 4.465 & 32.752 & 25.951 & 0.1235 & 6.216 & 2.7 & 87.2 \\
\hline 50.0 & & 4.438 & 32.759 & 25.959 & 0.1335 & 6.187 & 2.6 & 87.3 \\
\hline 55.0 & & 4.425 & 32.762 & 25.963 & 0.1435 & 6.168 & 2.5 & 87.2 \\
\hline 60.0 & & 4.409 & 32.764 & 25.967 & 0.1534 & 6.167 & 2.5 & 87.2 \\
\hline 65.0 & & 4.402 & 32.766 & 25.969 & 0.1633 & 6.153 & 2.5 & 87.0 \\
\hline 70.0 & & 4.402 & 32.766 & 25.969 & 0.1733 & 6.135 & 2.5 & 87.0 \\
\hline 71.0 & & 4.402 & 32.766 & 25.969 & 0.1753 & 6.142 & 2.5 & 86.7 \\
\hline
\end{tabular}
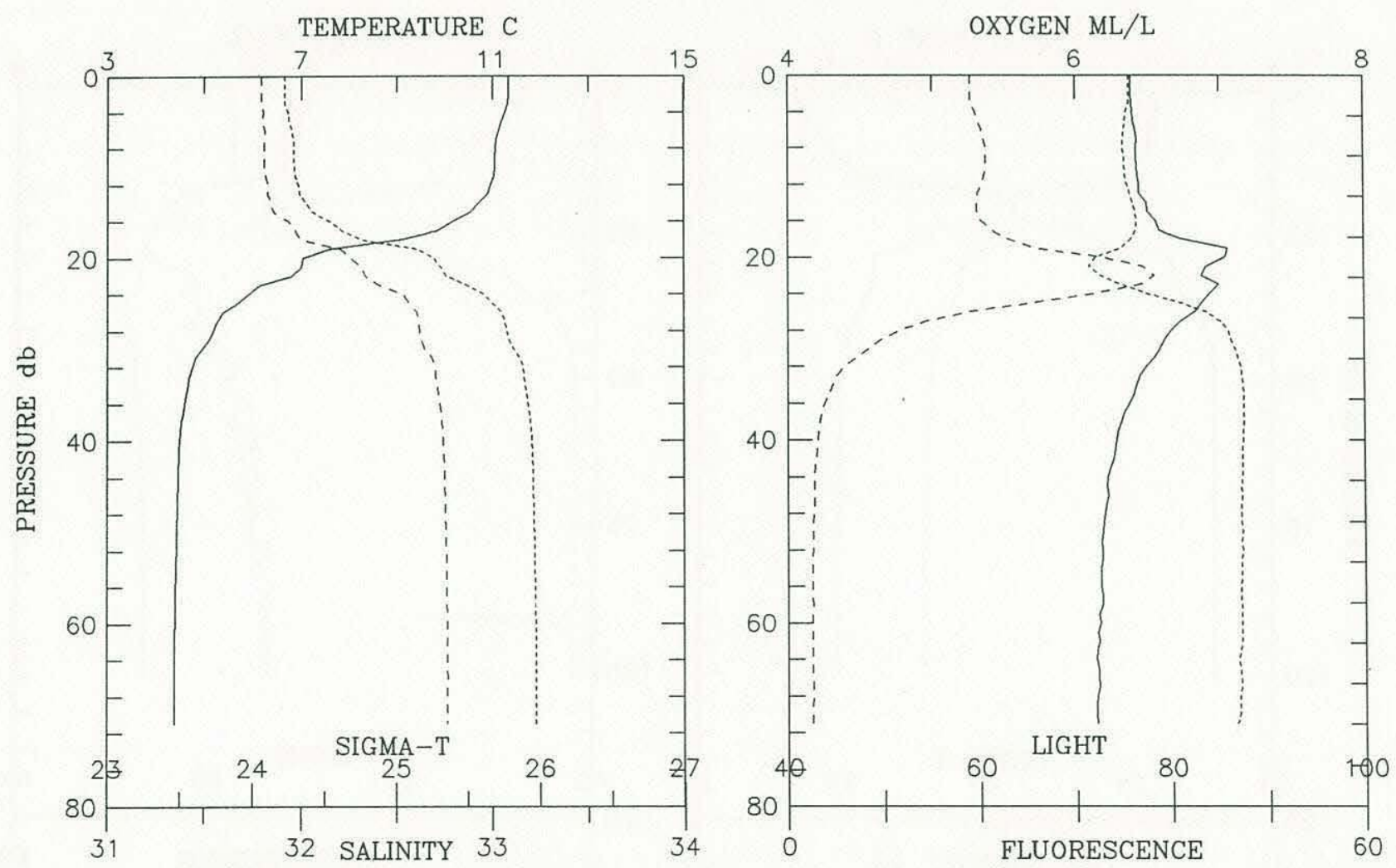


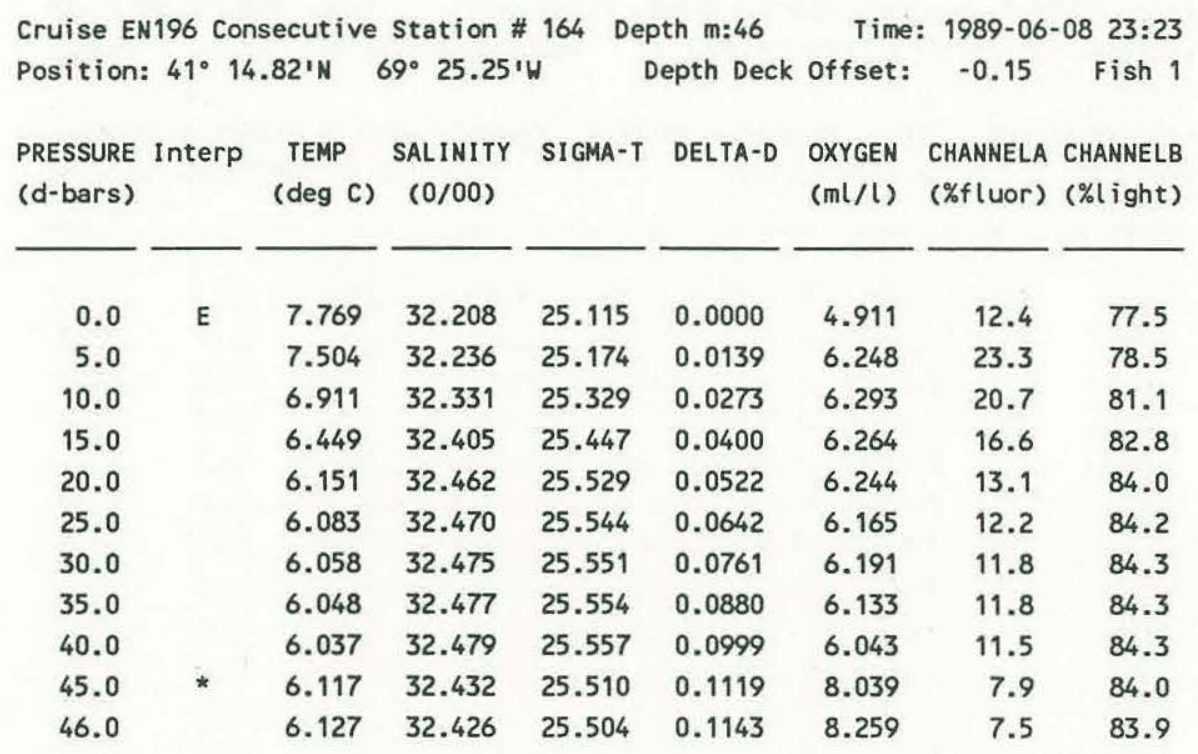
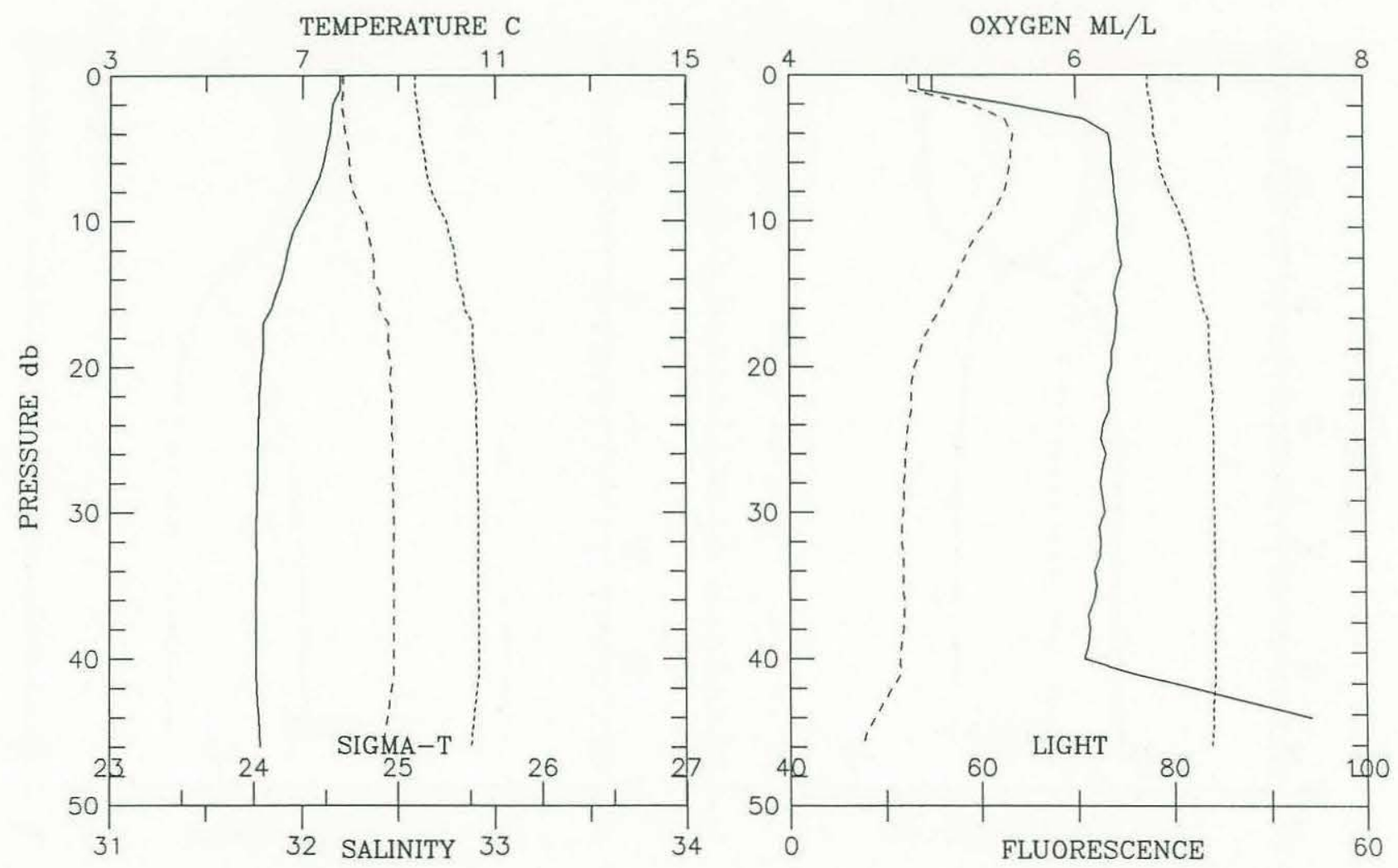
Cruise EN196 Consecutive Station \# 165 Depth m:34 Time: 1989-06-09 01:25 Position: $41^{\circ} 24.10^{\prime} \mathrm{N} 69^{\circ} 31.30^{\prime} \mathrm{W}$ Depth Deck offset: -0.15 Fish 1

\begin{tabular}{|c|c|c|c|c|c|c|c|c|}
\hline $\begin{array}{l}\text { PRESSURE } \\
\text { (d-bars) }\end{array}$ & Interp & $\begin{array}{c}\text { TEMP } \\
(\operatorname{deg} \mathrm{C})\end{array}$ & $\begin{array}{l}\text { SALINITY } \\
(0 / 00)\end{array}$ & SIGMA-T & DELTA-D & $\begin{array}{l}\text { OXYGEN } \\
(\mathrm{ml} / \mathrm{l})\end{array}$ & $\begin{array}{l}\text { CHANNELA } \\
\text { (\%fluor) }\end{array}$ & $\begin{array}{l}\text { CHANNELB } \\
\text { (\%light) }\end{array}$ \\
\hline 0.0 & E & 10.528 & 31.820 & 24.382 & 0.0000 & 6.202 & 27.6 & 73.3 \\
\hline 2.0 & E & 10.528 & 31.820 & 24.382 & 0.0070 & 6.204 & 27.6 & 73.3 \\
\hline 4.0 & & 10.585 & 31.817 & 24.370 & 0.0140 & 6.191 & 27.9 & 73.3 \\
\hline 6.0 & & 10.399 & 31.818 & 24.402 & 0.0210 & 6.253 & 27.9 & 73.4 \\
\hline 8.0 & & 9.141 & 31.905 & 24.674 & 0.0278 & 6.521 & 28.0 & 75.5 \\
\hline 10.0 & & 7.088 & 32.084 & 25.111 & 0.0337 & 6.675 & 20.8 & 82.0 \\
\hline 12.0 & & 6.649 & 32.159 & 25.228 & 0.0392 & 6.459 & 13.7 & 84.2 \\
\hline 14.0 & & 6.601 & 32.170 & 25.242 & 0.0446 & 6.299 & 11.6 & 84.7 \\
\hline 16.0 & & 6.501 & 32.197 & 25.276 & 0.0499 & 6.200 & 11.0 & 85.0 \\
\hline 18.0 & & 6.481 & 32.203 & 25.283 & 0.0552 & 6.157 & 10.6 & 85.1 \\
\hline 20.0 & & 6.470 & 32.208 & 25.289 & 0.0605 & 6.115 & 10.6 & 85.0 \\
\hline 22.0 & & 6.479 & 32.206 & 25.286 & 0.0657 & 6.098 & 10.6 & 85.1 \\
\hline 24.0 & & 6.467 & 32.210 & 25.291 & 0.0710 & 6.077 & 10.6 & 85.1 \\
\hline 26.0 & & 6.451 & 32.216 & 25.297 & 0.0763 & 6.087 & 10.4 & 85.1 \\
\hline 28.0 & & 6.398 & 32.233 & 25.318 & 0.0815 & 6.061 & 10.3 & 85.3 \\
\hline
\end{tabular}
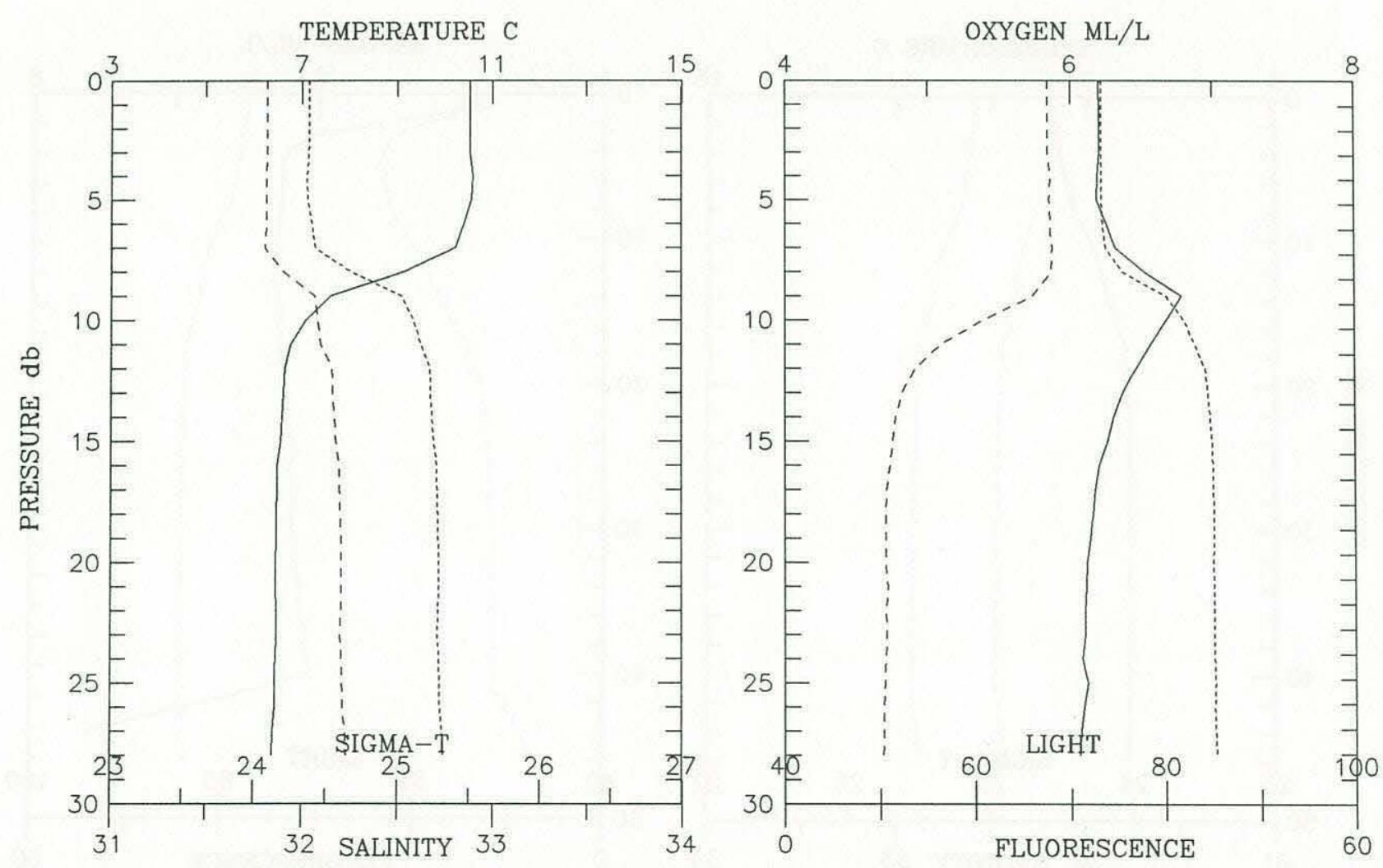


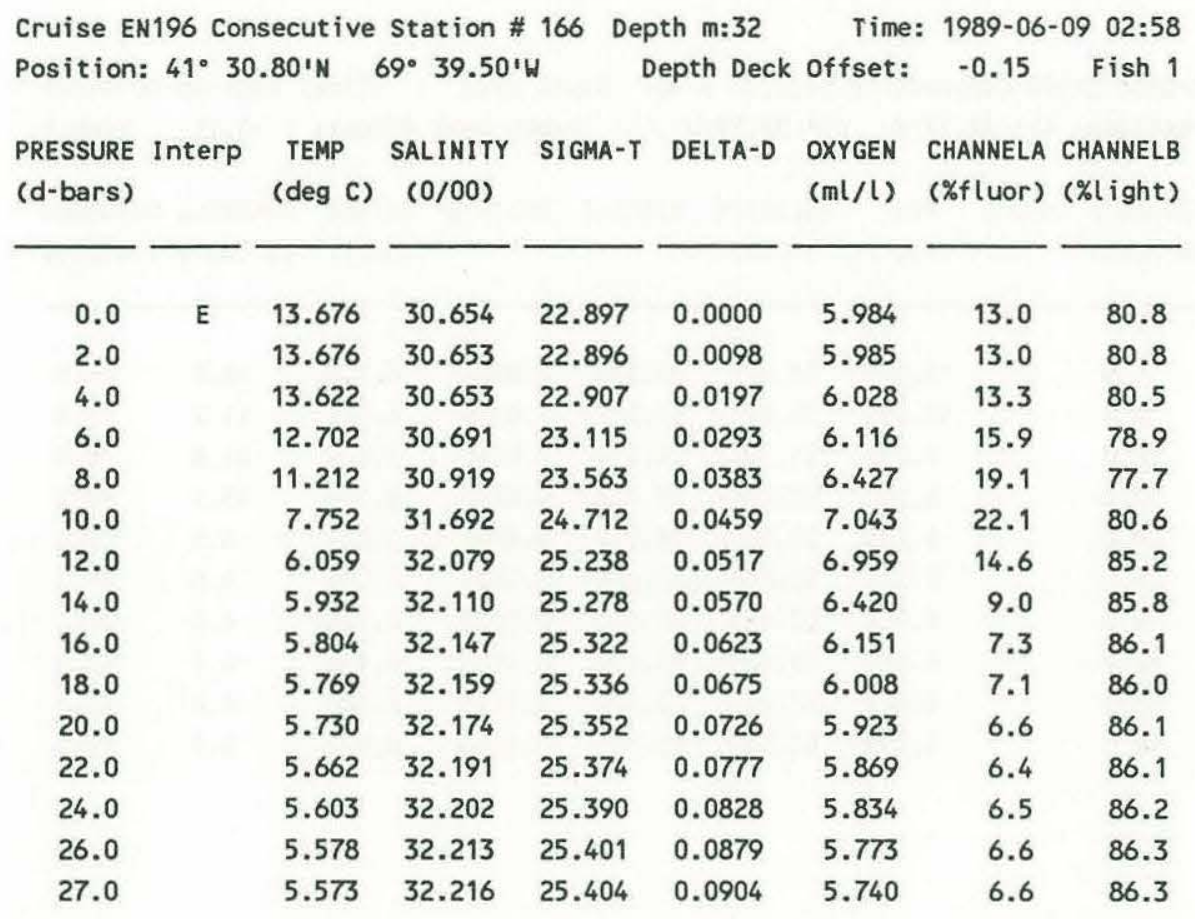
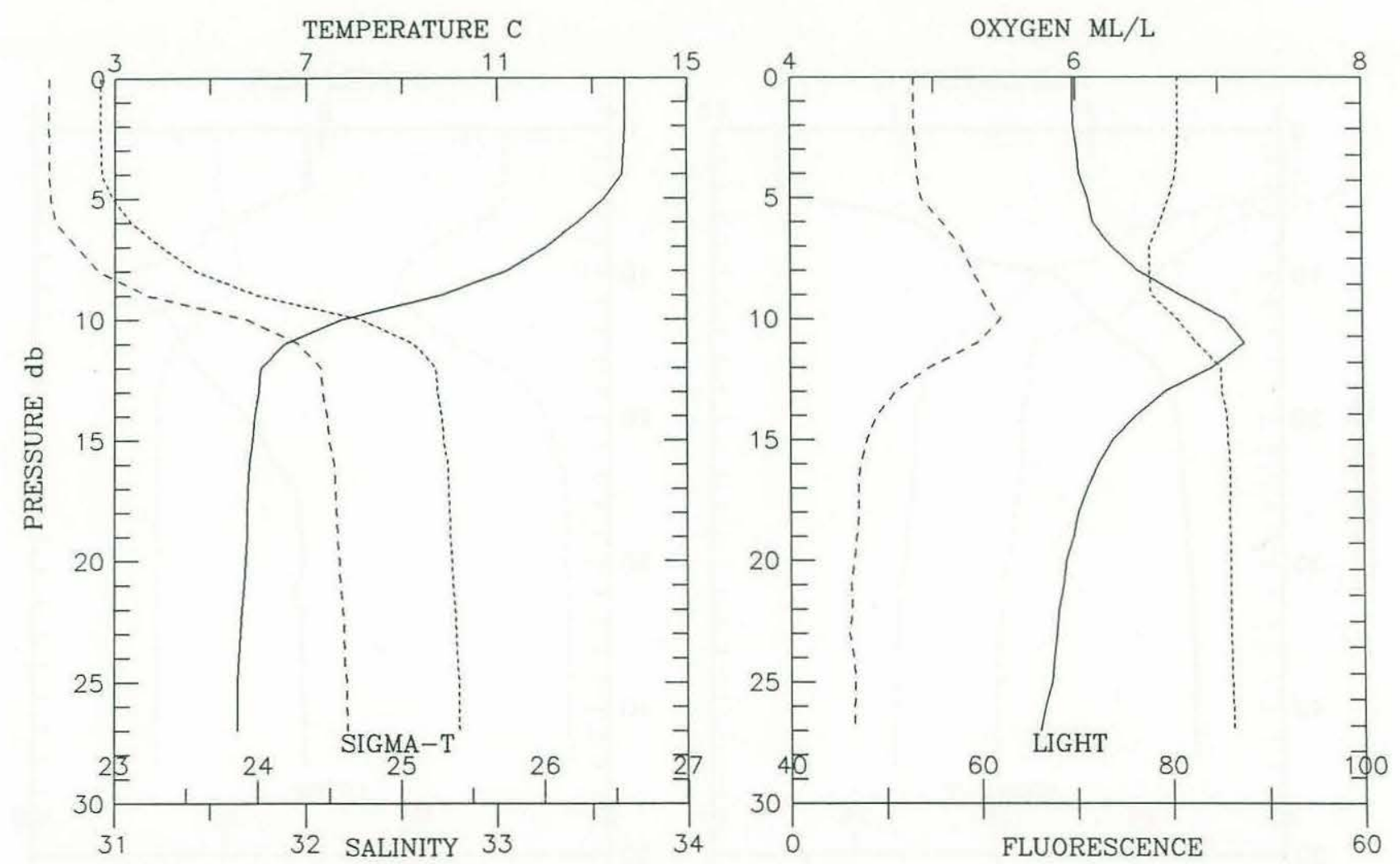


\begin{tabular}{|c|c|c|c|c|c|c|c|c|}
\hline $\begin{array}{l}\text { Cruise EN } \\
\text { Position: }\end{array}$ & $\begin{array}{l}\text { N196 Con } \\
41^{\circ} 32\end{array}$ & $\begin{array}{l}\text { secutive } \\
.17 \text { 'N }\end{array}$ & $\begin{array}{l}\text { Station \# } \\
69^{\circ} 33.59^{\prime}\end{array}$ & $w^{16 r}$ & $\begin{array}{l}\text { th } \mathrm{m}: 48 \\
\text { epth Deck }\end{array}$ & $\begin{array}{l}\text { Time: } \\
\text { offset: }\end{array}$ & $\begin{array}{c}1989-06- \\
-0.15\end{array}$ & $\begin{array}{r}-0904: 21 \\
\text { Fish } 1\end{array}$ \\
\hline $\begin{array}{l}\text { PRESSURE } \\
\text { (d-bars) }\end{array}$ & Interp & $\begin{array}{c}\text { TEMP } \\
\text { (deg C) }\end{array}$ & $\begin{array}{l}\text { SALINITY } \\
(0 / 00)\end{array}$ & SIGMA-T & DELTA-D & $\begin{array}{l}\text { OXYGEN } \\
(\mathrm{ml} / \mathrm{l})\end{array}$ & $\begin{array}{l}\text { CHANNELA } \\
\text { ( } \% \text { fluor) }\end{array}$ & $\begin{array}{l}\text { CHANNELB } \\
\text { (\%light) }\end{array}$ \\
\hline 0.0 & E & 13.608 & 30.622 & 22.885 & 0.0000 & 6.104 & 10.9 & 80.9 \\
\hline 5.0 & & 12.604 & 30.825 & 23.237 & 0.0245 & 6.337 & 11.2 & 80.8 \\
\hline 10.0 & & 7.816 & 31.707 & 24.715 & 0.0436 & 7.469 & 21.8 & 78.5 \\
\hline 15.0 & & 6.140 & 32.306 & 25.408 & 0.0582 & 6.966 & 15.5 & 85.5 \\
\hline 20.0 & & 5.204 & 32.361 & 25.561 & 0.0703 & 6.523 & 6.5 & 87.1 \\
\hline 25.0 & & 5.061 & 32.390 & 25.600 & 0.0821 & 6.167 & 4.6 & 87.1 \\
\hline 30.0 & & 4.944 & 32.440 & 25.653 & 0.0937 & 6.118 & 4.5 & 87.1 \\
\hline 35.0 & & 4.845 & 32.486 & 25.700 & 0.1049 & 6.141 & 4.1 & 87.1 \\
\hline 40.0 & & 4.827 & 32.492 & 25.706 & 0.1161 & 6.120 & 4.2 & 87.1 \\
\hline 44.0 & & 4.799 & 32.500 & 25.716 & 0.1250 & 6.093 & 3.9 & 87.2 \\
\hline
\end{tabular}
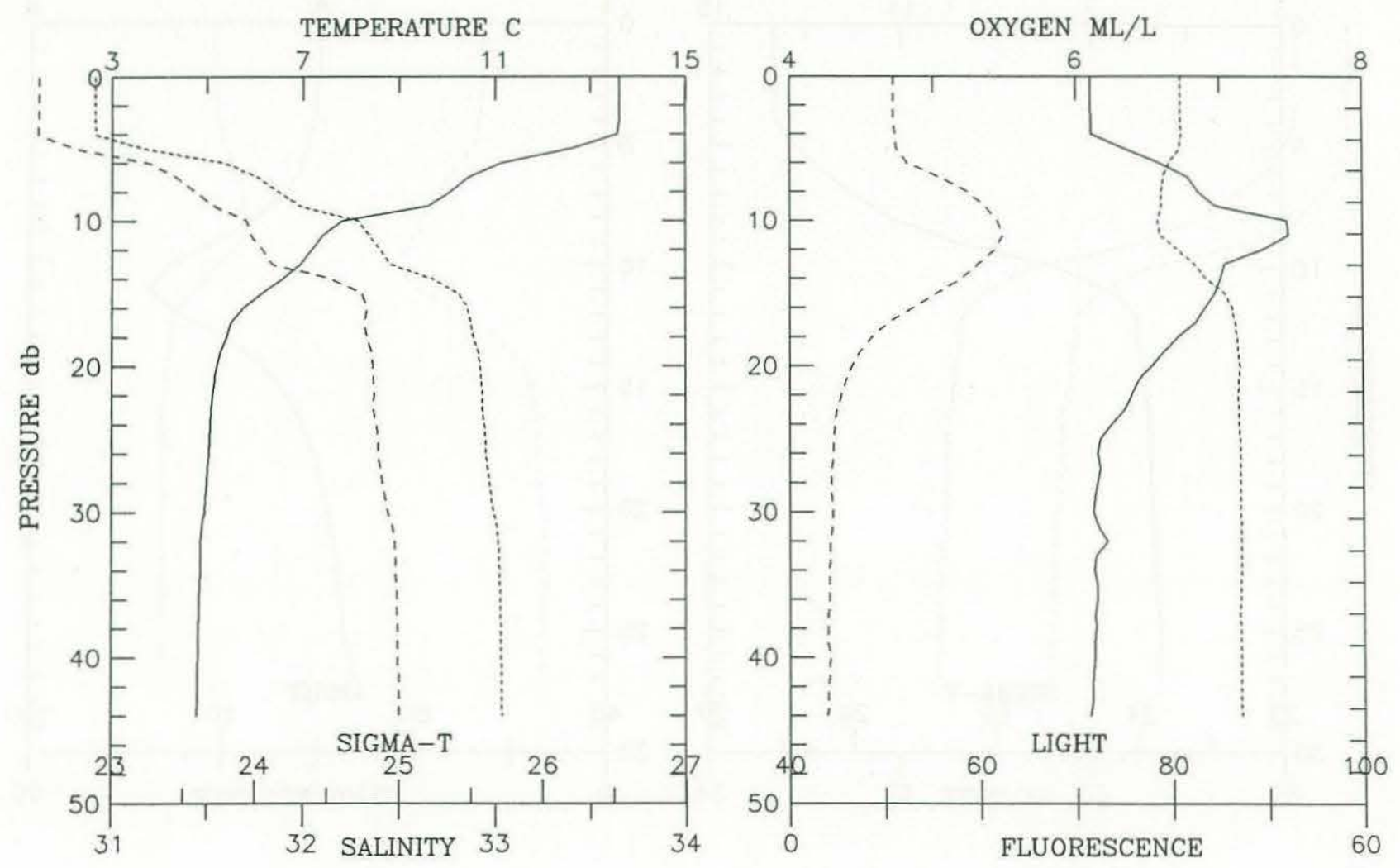


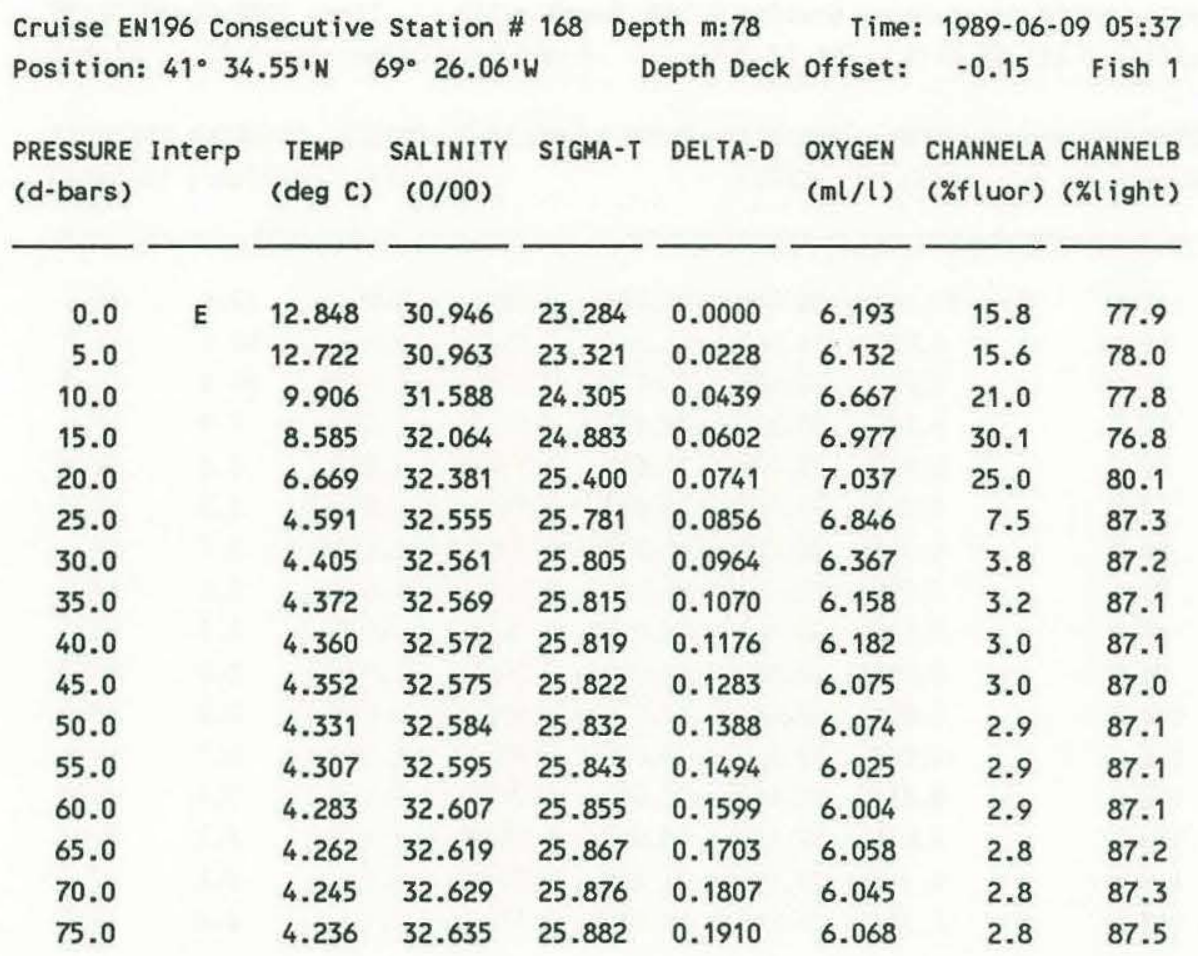
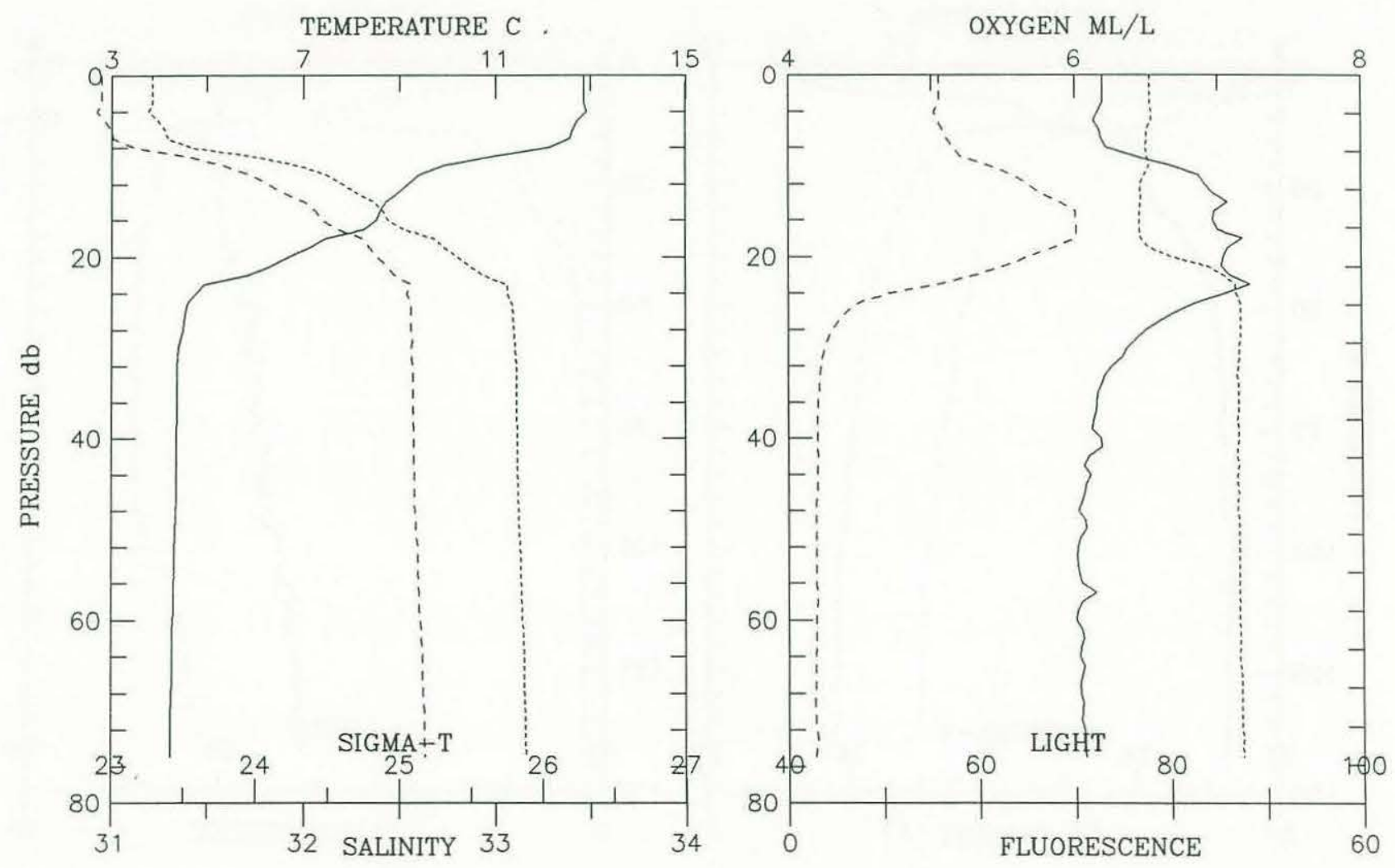


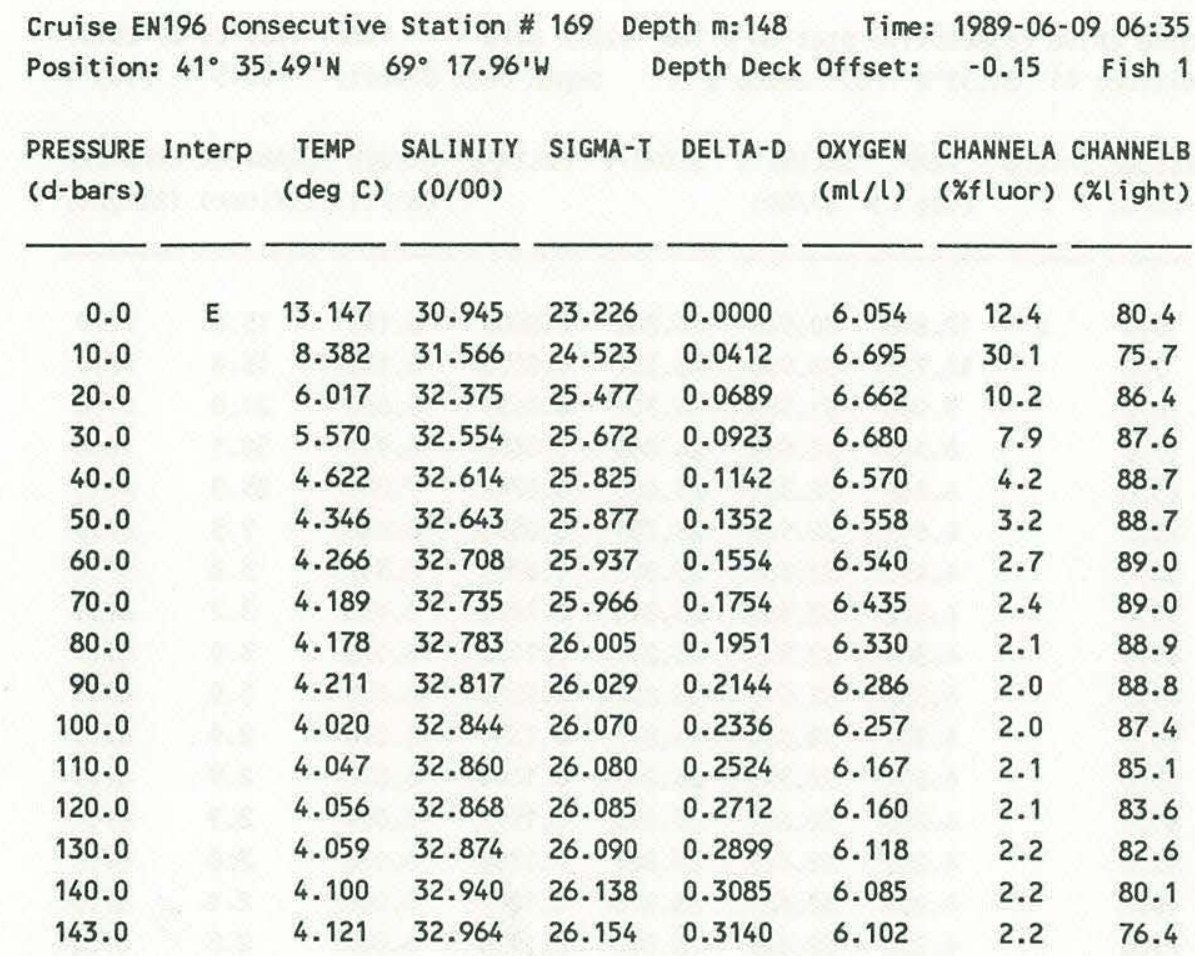
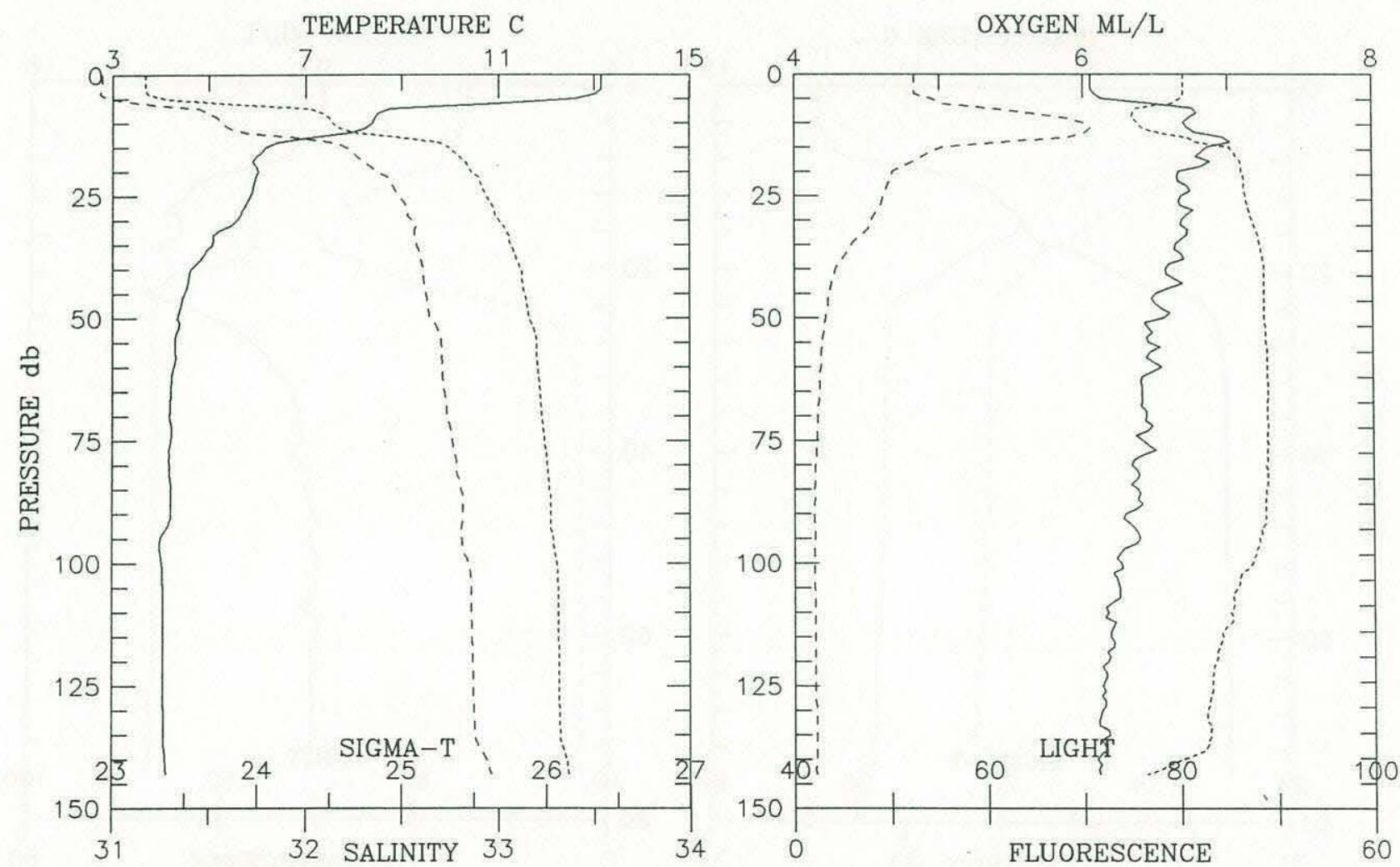


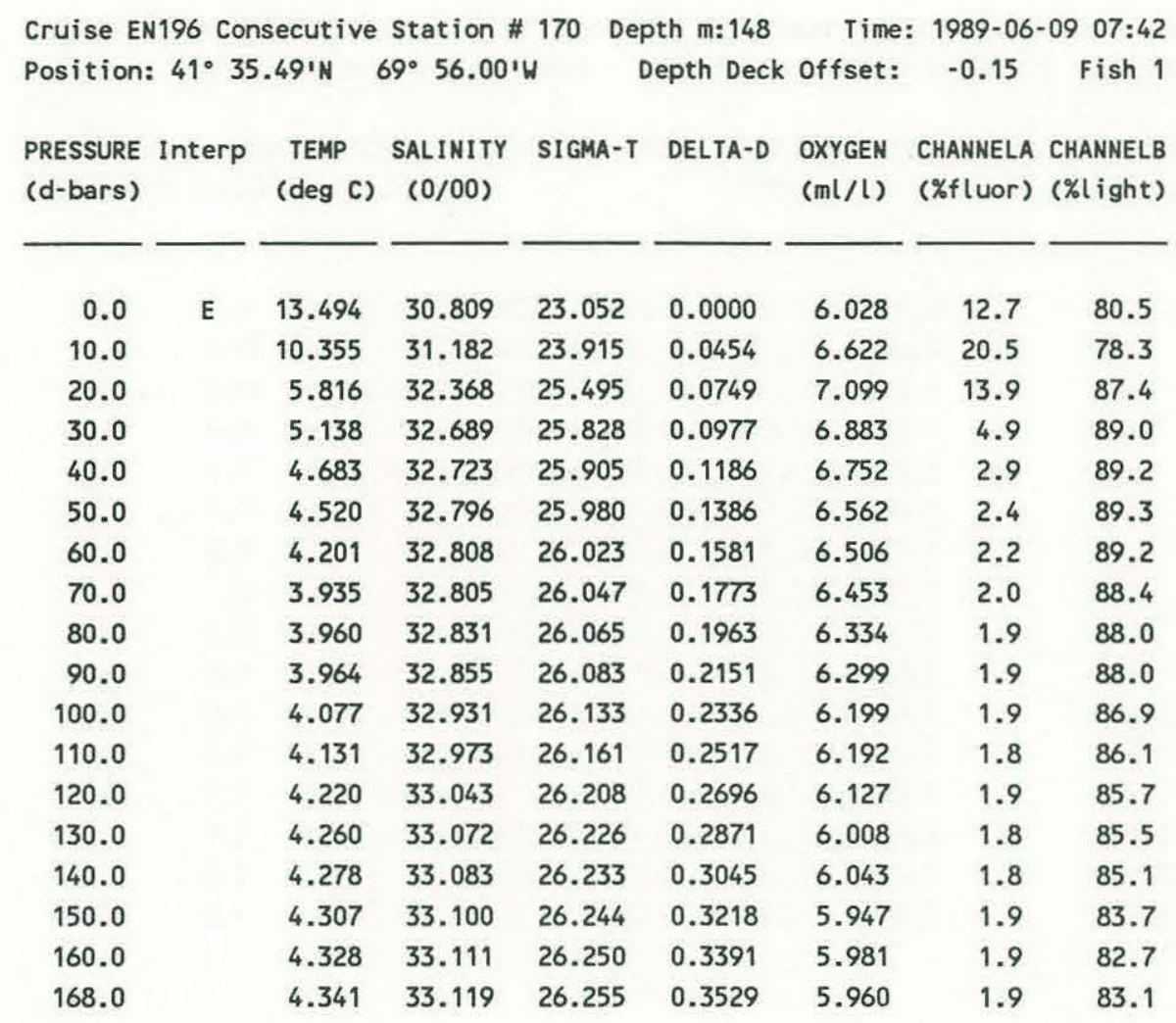
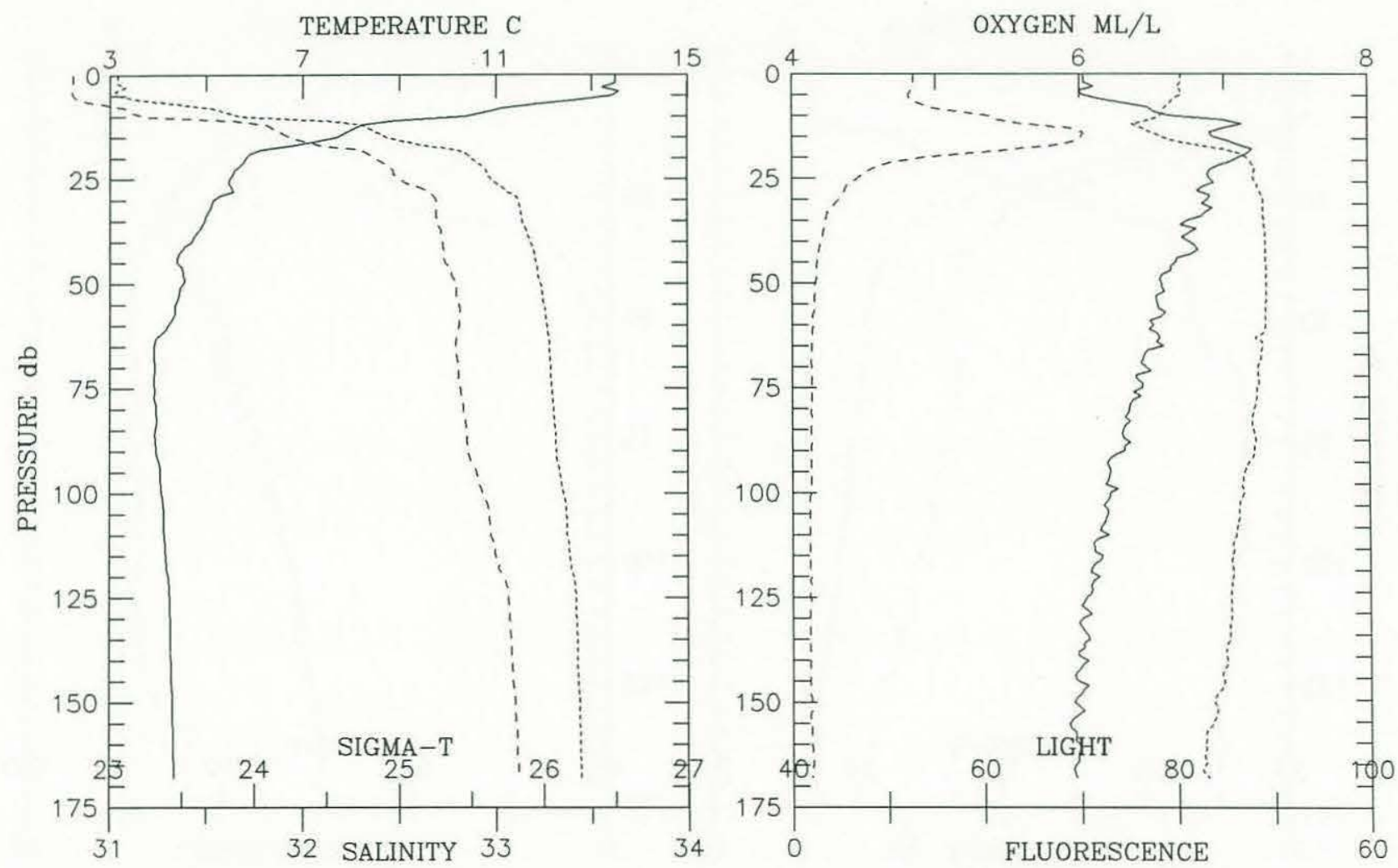


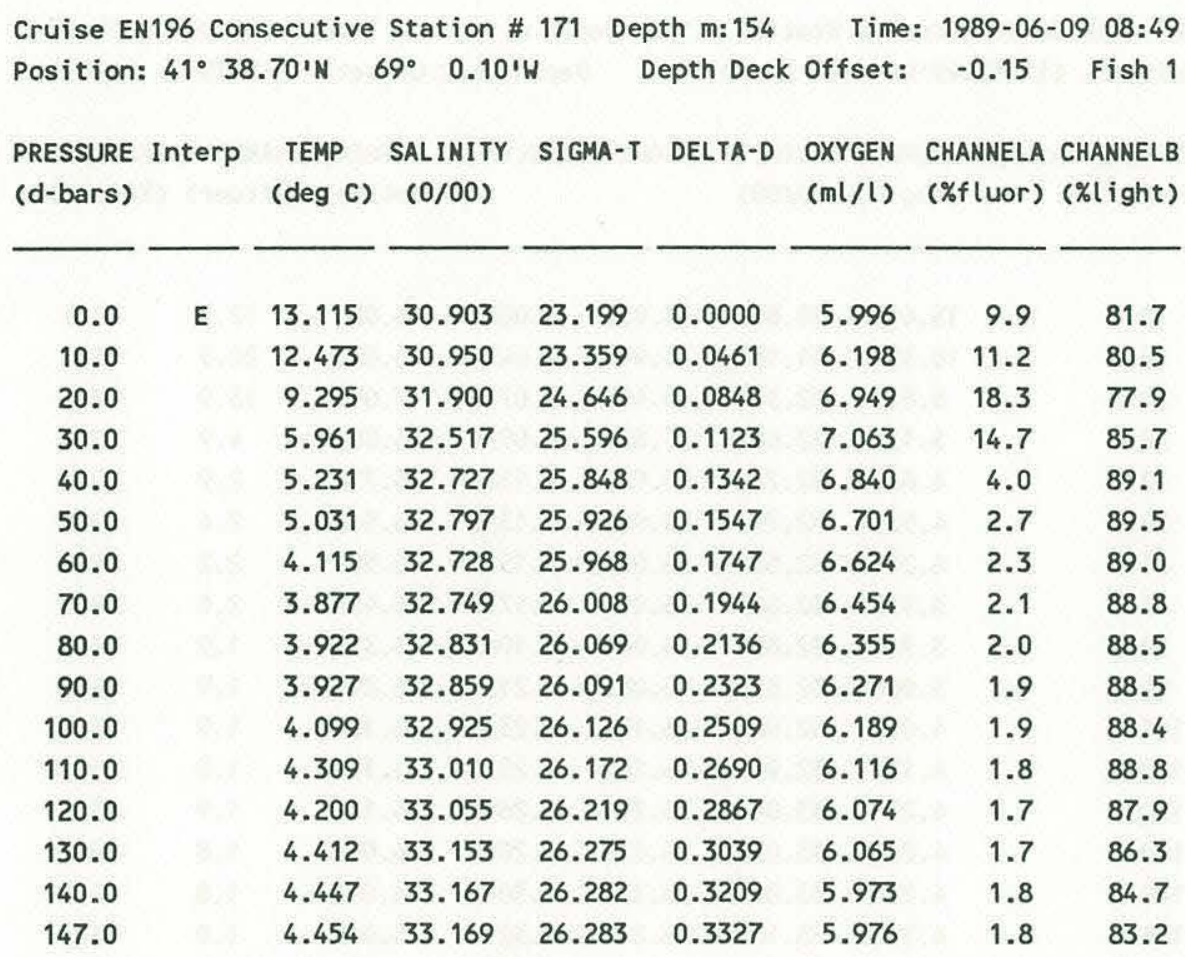
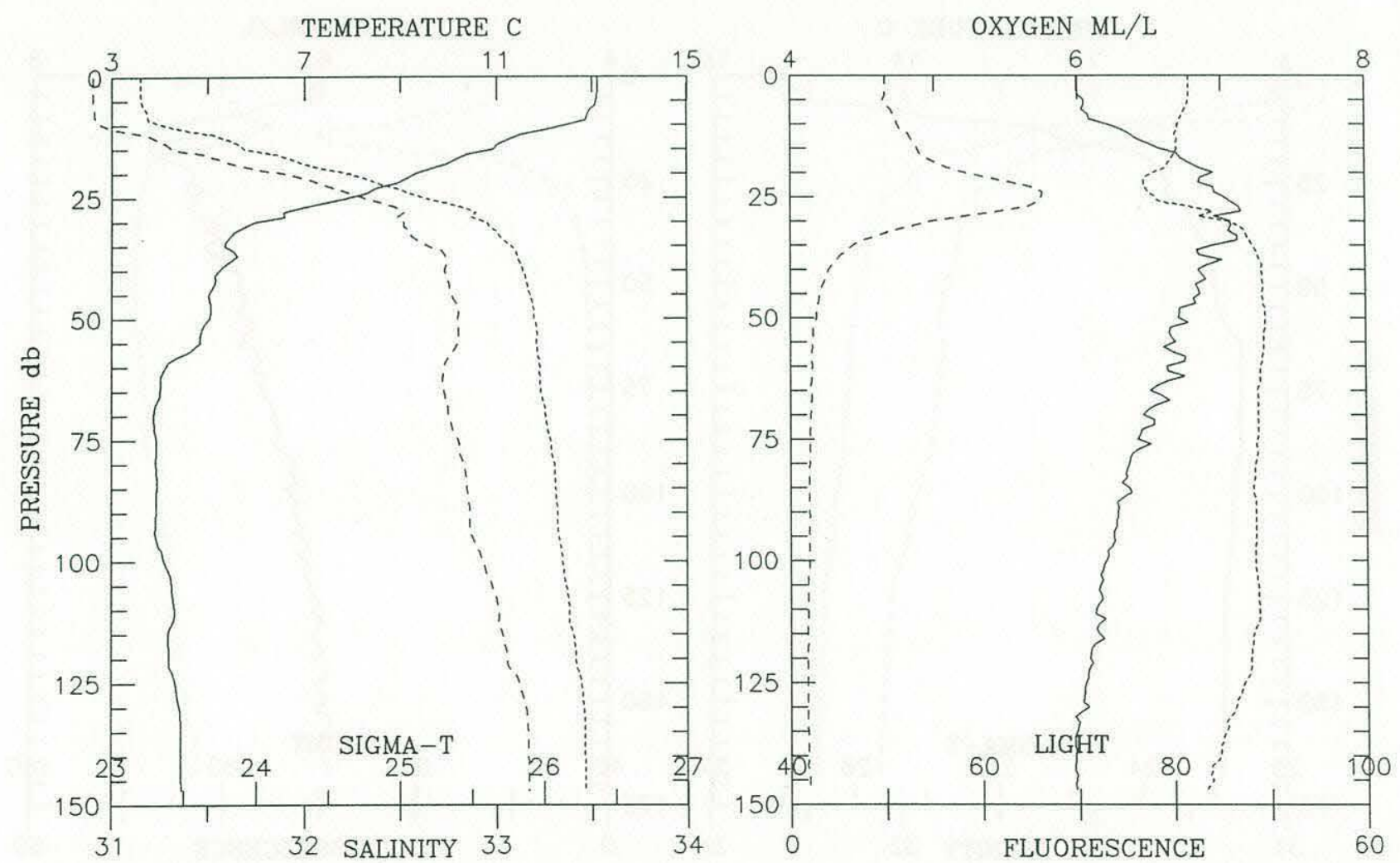


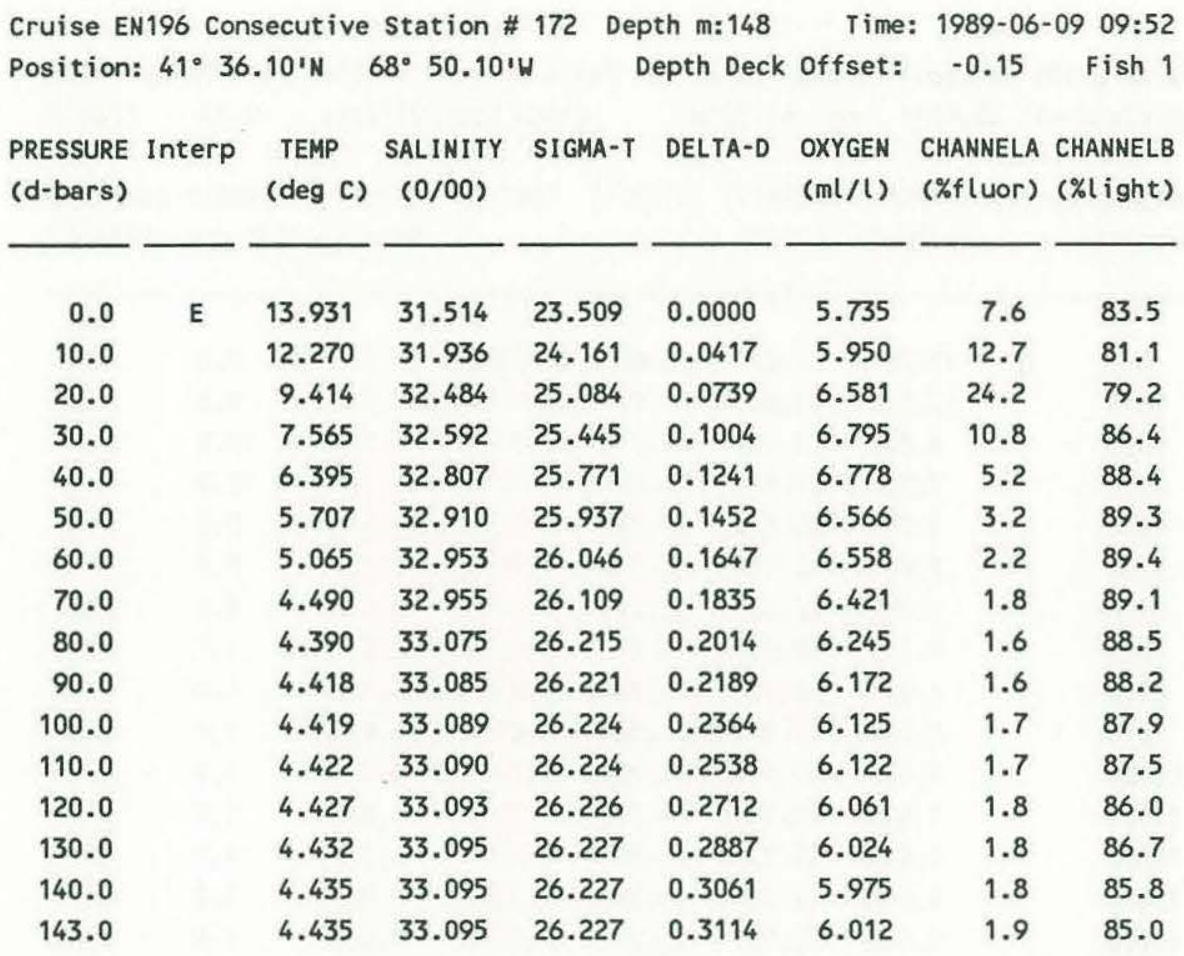
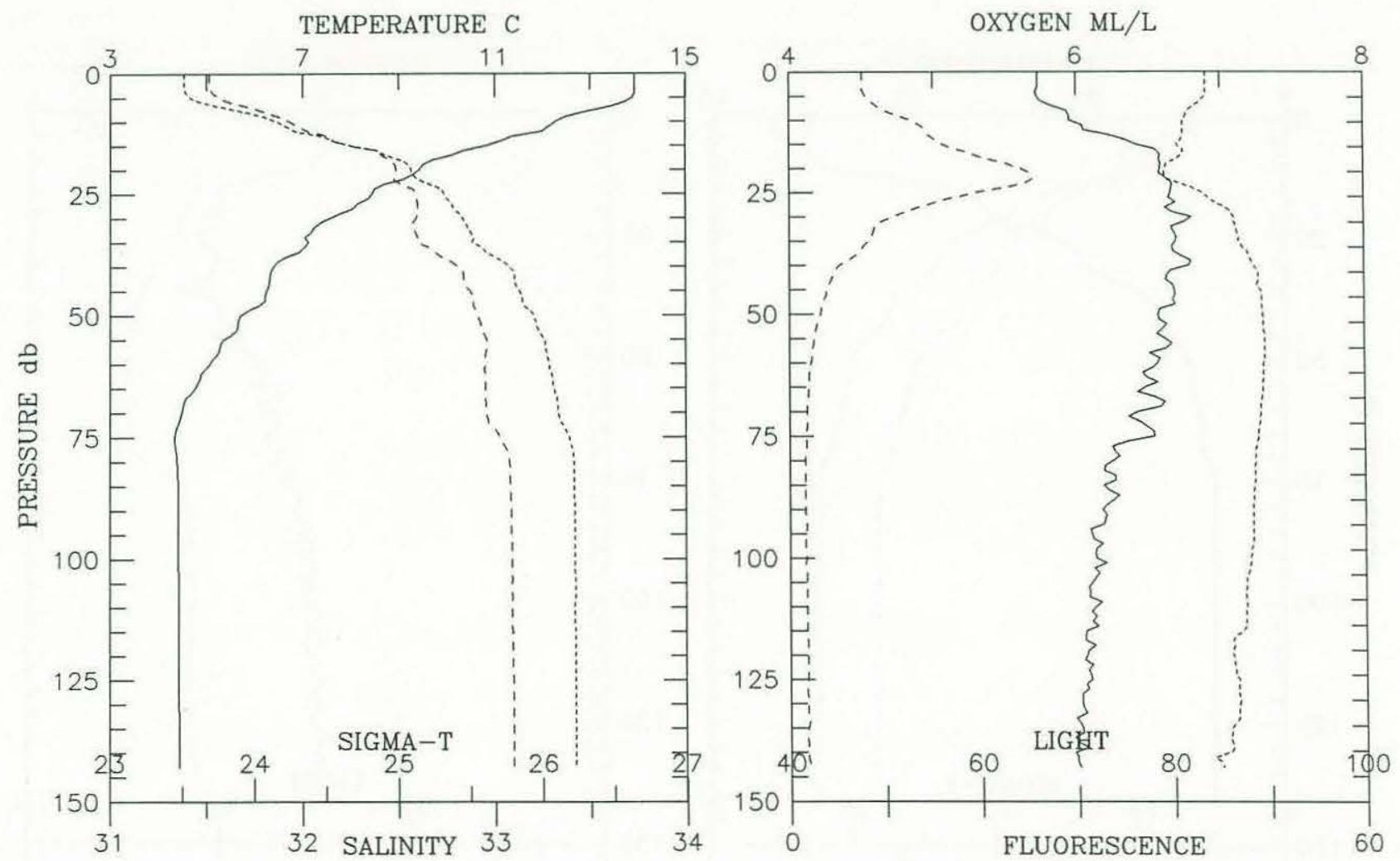


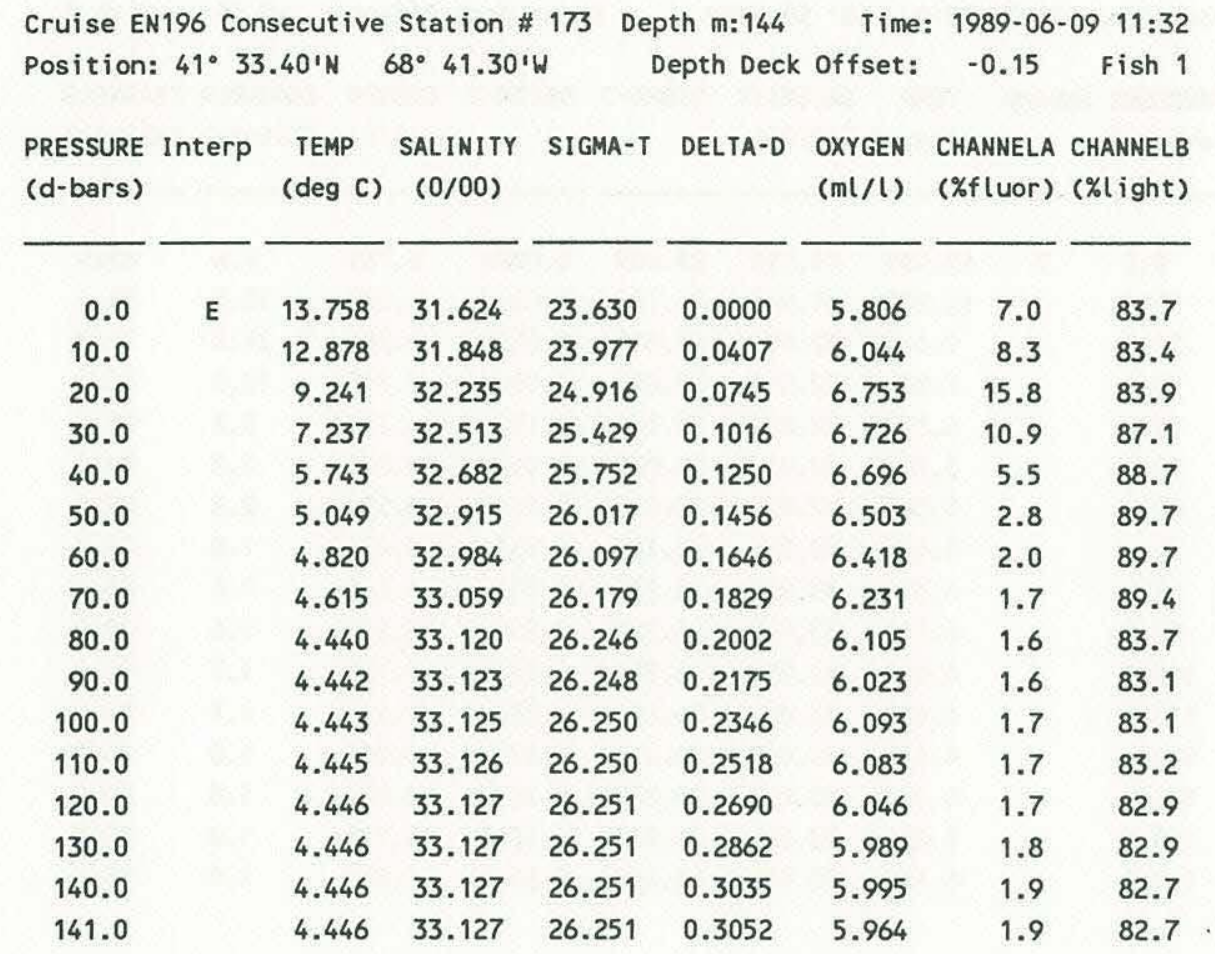
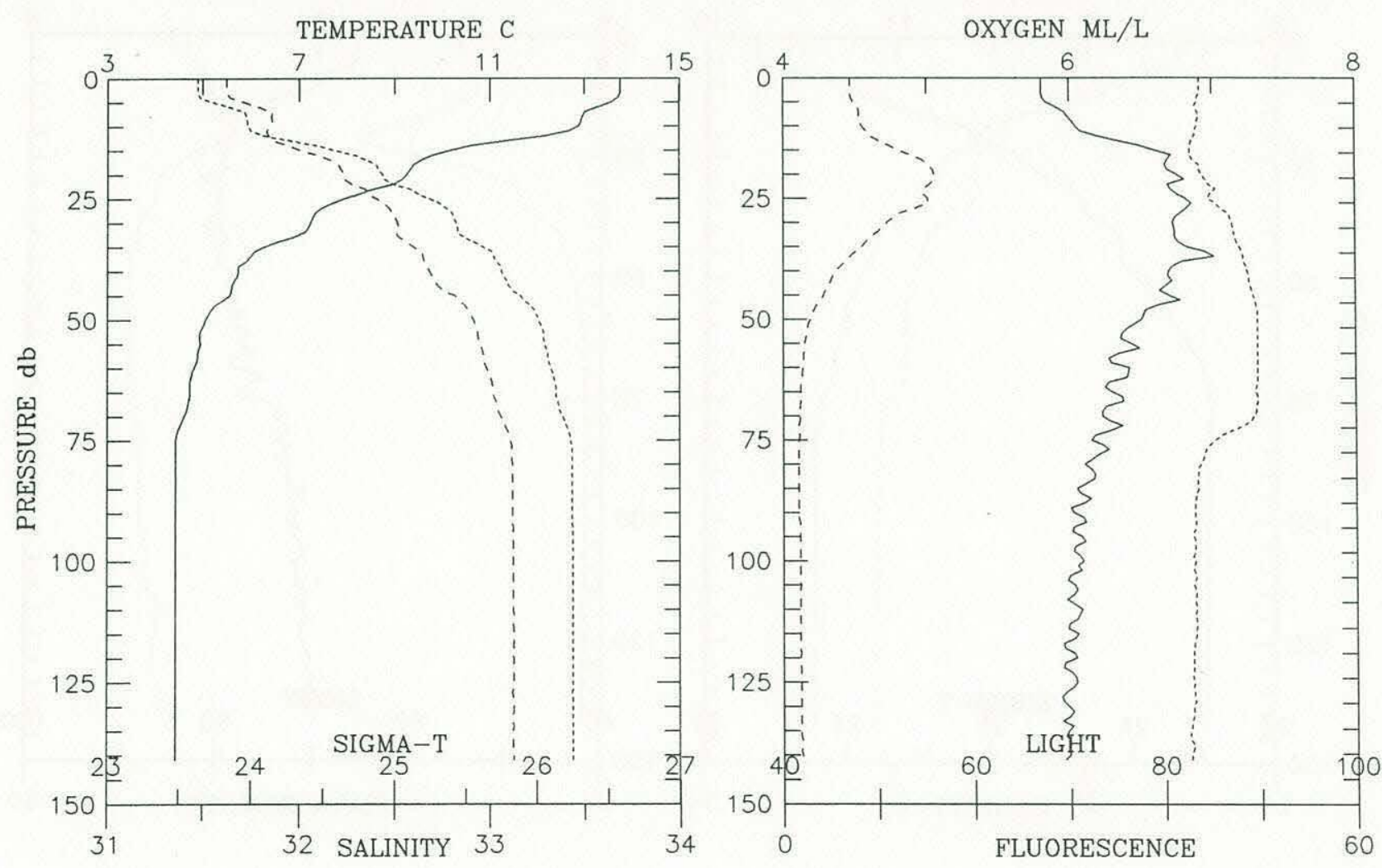


\begin{tabular}{|c|c|c|c|c|c|c|c|c|}
\hline $\begin{array}{l}\text { Cruise EN } \\
\text { Position: }\end{array}$ & $\begin{array}{l}\text { N196 Con } \\
=41^{\circ} 31\end{array}$ & $\begin{array}{l}\text { isecutive } \\
.20 \text { 'N }\end{array}$ & $\begin{array}{l}\text { Station } \\
68^{\circ} 35.20^{\circ}\end{array}$ & $H^{174}$ & $\begin{array}{l}\text { th } \mathrm{m:} 112 \\
\text { epth Deck }\end{array}$ & $\begin{array}{r}\text { Time } \\
\text { offset: }\end{array}$ & $\begin{array}{l}-0.15 \\
-1989-06\end{array}$ & 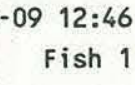 \\
\hline $\begin{array}{l}\text { PRESSURE } \\
\text { (d-bars) }\end{array}$ & Interp & $\begin{array}{c}\text { TEMP } \\
\text { (deg C) }\end{array}$ & $\begin{array}{l}\text { SALINITY } \\
(0 / 00)\end{array}$ & SIGMA-T & DELTA-D & $\begin{array}{l}\text { OXYGEN } \\
(\mathrm{ml} / \mathrm{l})\end{array}$ & $\begin{array}{l}\text { CHANNELA } \\
\text { (\%fluor) }\end{array}$ & $\begin{array}{l}\text { CHANNELB } \\
\text { (\%light) }\end{array}$ \\
\hline 0.0 & E & 13.245 & 31.763 & 23.839 & 0.0000 & 5.941 & 7.5 & 82.4 \\
\hline 10.0 & & 10.423 & 32.268 & 24.749 & 0.0383 & 6.472 & 13.5 & 81.5 \\
\hline 20.0 & & 7.393 & 32.572 & 25.454 & 0.0656 & 6.752 & 12.9 & 85.6 \\
\hline 30.0 & & 6.480 & 32.763 & 25.725 & 0.0888 & 6.727 & 6.2 & 88.3 \\
\hline 40.0 & & 4.986 & 32.891 & 26.005 & 0.1096 & 6.561 & 3.2 & 85.9 \\
\hline 50.0 & & 4.939 & 32.914 & 26.029 & 0.1289 & 6.202 & 2.7 & 84.6 \\
\hline 60.0 & & 4.915 & 32.926 & 26.041 & 0.1482 & 6.145 & 2.7 & 84.4 \\
\hline 70.0 & & 4.782 & 32.954 & 26.077 & 0.1671 & 6.066 & 2.5 & 84.6 \\
\hline 80.0 & & 4.663 & 32.987 & 26.117 & 0.1858 & 6.028 & 2.4 & 84.2 \\
\hline 90.0 & & 4.518 & 33.059 & 26.189 & 0.2040 & 6.021 & 2.1 & 82.3 \\
\hline 100.0 & & 4.495 & 33.070 & 26.200 & 0.2216 & 5.965 & 2.2 & 80.5 \\
\hline 107.0 & & 4.488 & 33.073 & 26.203 & 0.2340 & 5.975 & 2.2 & 79.6 \\
\hline
\end{tabular}
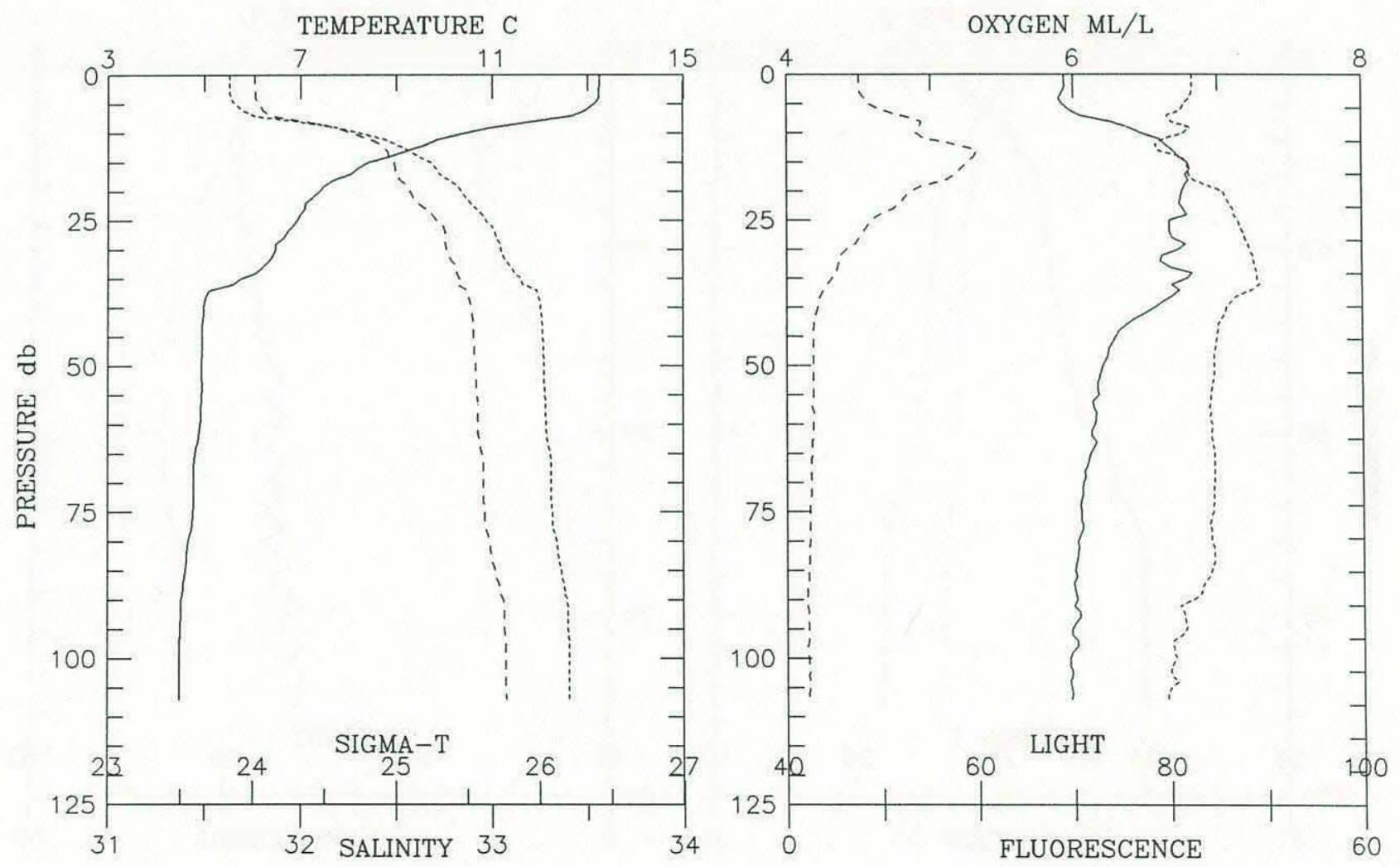


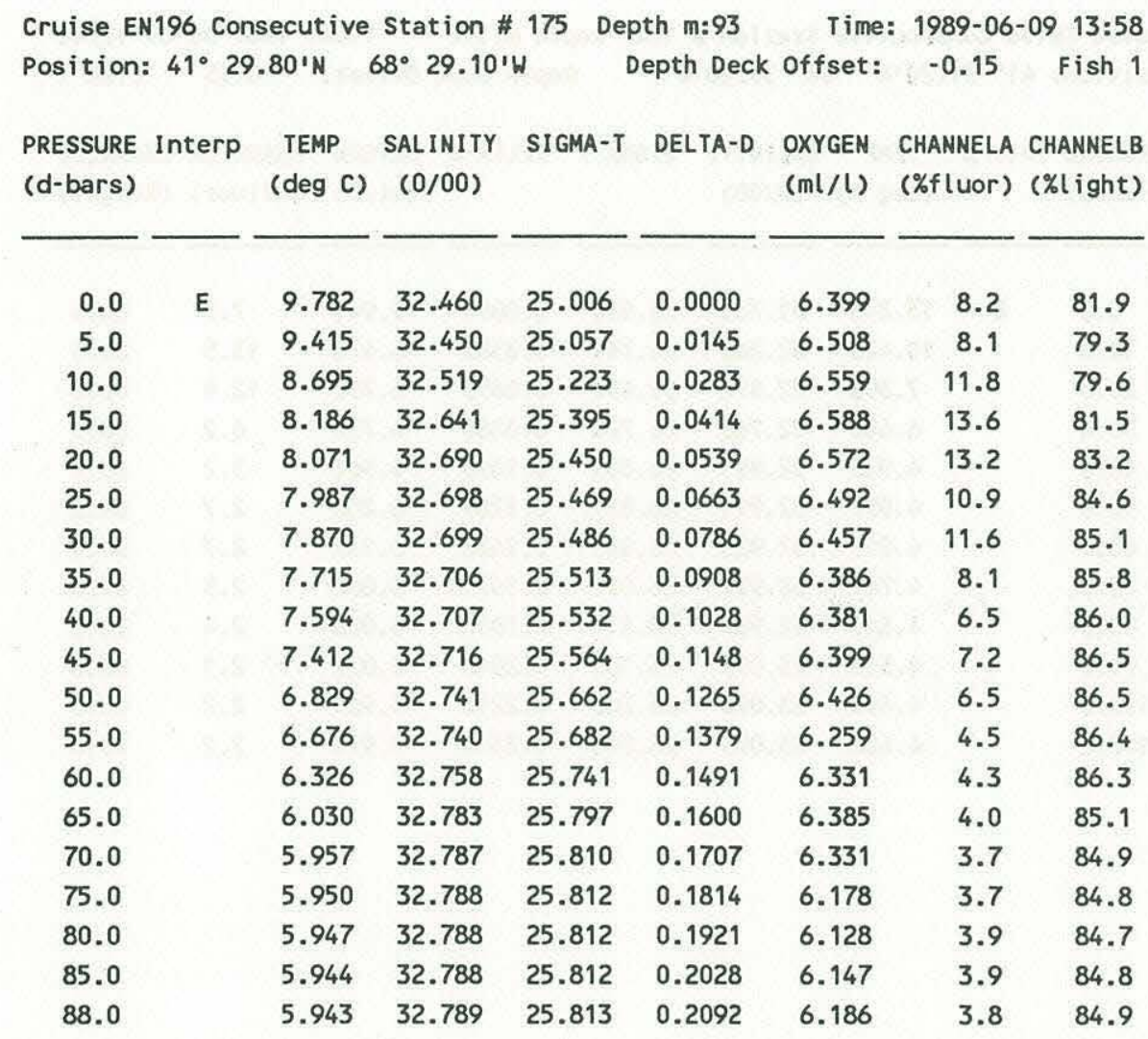
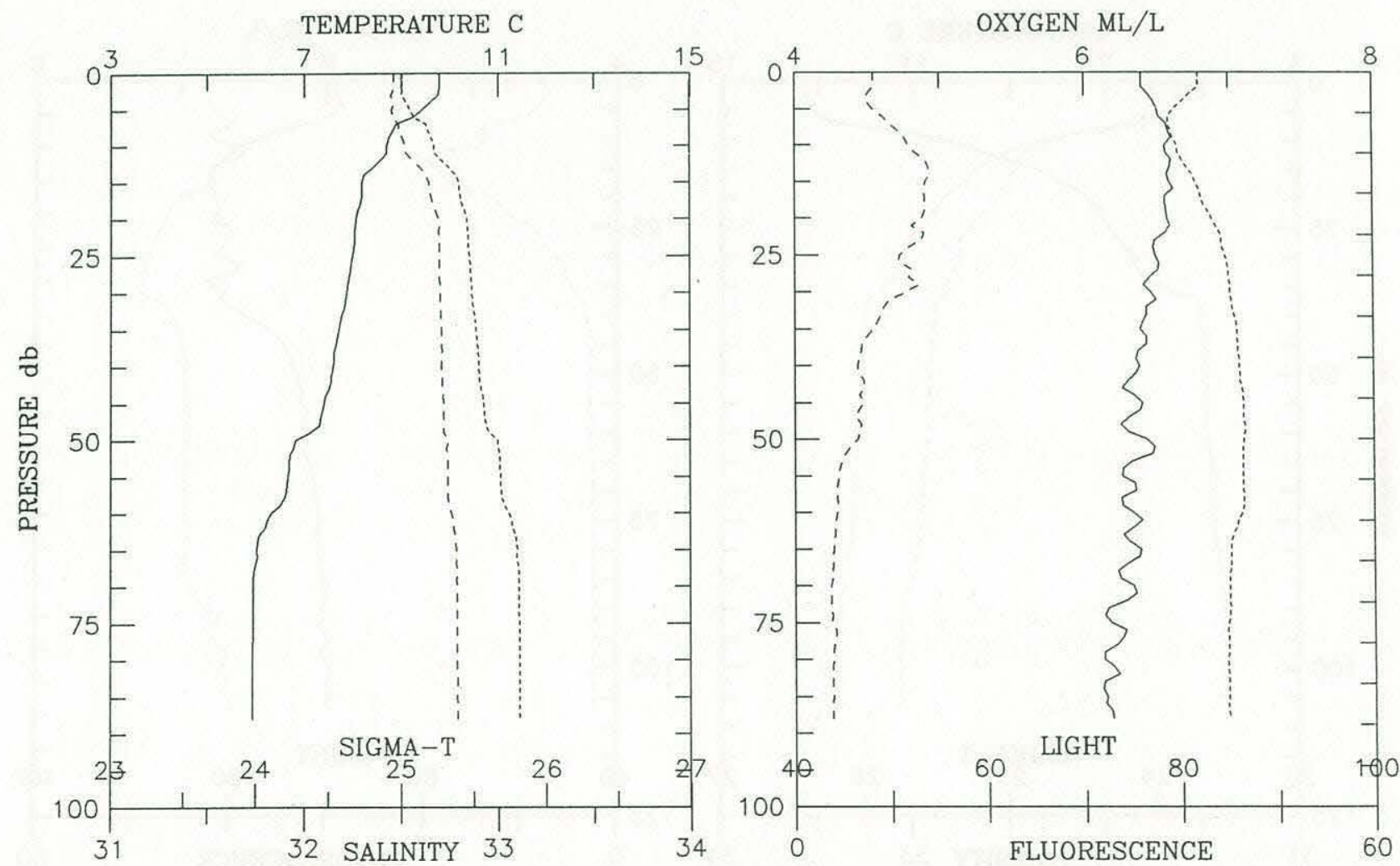
Cruise EN196 Consecutive Station \# 176 Depth m:60 Time: 1989-06-09 15:35 Position: $41^{\circ} 27.50^{\prime} \mathrm{N} 68^{\circ} 22.50^{\prime} \mathrm{W}$ Depth Deck offset: -0.15 Fish 1

\begin{tabular}{|c|c|c|c|c|c|c|c|c|}
\hline $\begin{array}{l}\text { PRESSURE } \\
\text { (d-bars) }\end{array}$ & Interp & $\begin{array}{c}\text { TEMP } \\
(\operatorname{deg} \mathrm{C})\end{array}$ & $\begin{array}{c}\text { SALINITY } \\
(0 / 00)\end{array}$ & SIGMA-T & DELTA-D & $\begin{array}{l}\text { OXYGEN } \\
(\mathrm{ml} / \mathrm{l})\end{array}$ & $\begin{array}{l}\text { CHANNELA } \\
\text { (\%fluor) }\end{array}$ & $\begin{array}{l}\text { CHANNELB } \\
\text { (\%light) }\end{array}$ \\
\hline 0.0 & E & 9.569 & 32.637 & 25.178 & 0.0000 & 6.789 & 11.1 & 79.2 \\
\hline 5.0 & & 9.552 & 32.637 & 25.181 & 0.0137 & 6.729 & 10.8 & 79.0 \\
\hline 10.0 & & 9.484 & 32.633 & 25.189 & 0.0274 & 6.820 & 14.9 & 78.9 \\
\hline 15.0 & & 9.303 & 32.634 & 25.219 & 0.0409 & 6.735 & 15.6 & 79.8 \\
\hline 20.0 & & 9.186 & 32.642 & 25.243 & 0.0543 & 6.645 & 14.7 & 80.7 \\
\hline 25.0 & & 9.139 & 32.646 & 25.254 & 0.0677 & 6.674 & 17.3 & 81.0 \\
\hline 30.0 & & 9.118 & 32.646 & 25.257 & 0.0810 & 6.642 & 17.7 & 81.1 \\
\hline 35.0 & & 8.943 & 32.654 & 25.291 & 0.0943 & 6.580 & 15.0 & 81.6 \\
\hline 40.0 & & 8.864 & 32.656 & 25.304 & 0.1075 & 6.449 & 16.9 & 81.8 \\
\hline 45.0 & & 8.725 & 32.661 & 25.329 & 0.1206 & 6.536 & 14.1 & 81.9 \\
\hline 50.0 & & 8.487 & 32.672 & 25.375 & 0.1334 & 6.532 & 11.9 & 82.4 \\
\hline 55.0 & & 8.484 & 32.672 & 25.375 & 0.1462 & 6.432 & 11.9 & 82.3 \\
\hline 57.0 & & 8.465 & 32.674 & 25.379 & 0.1513 & 6.440 & 12.0 & 82.3 \\
\hline
\end{tabular}
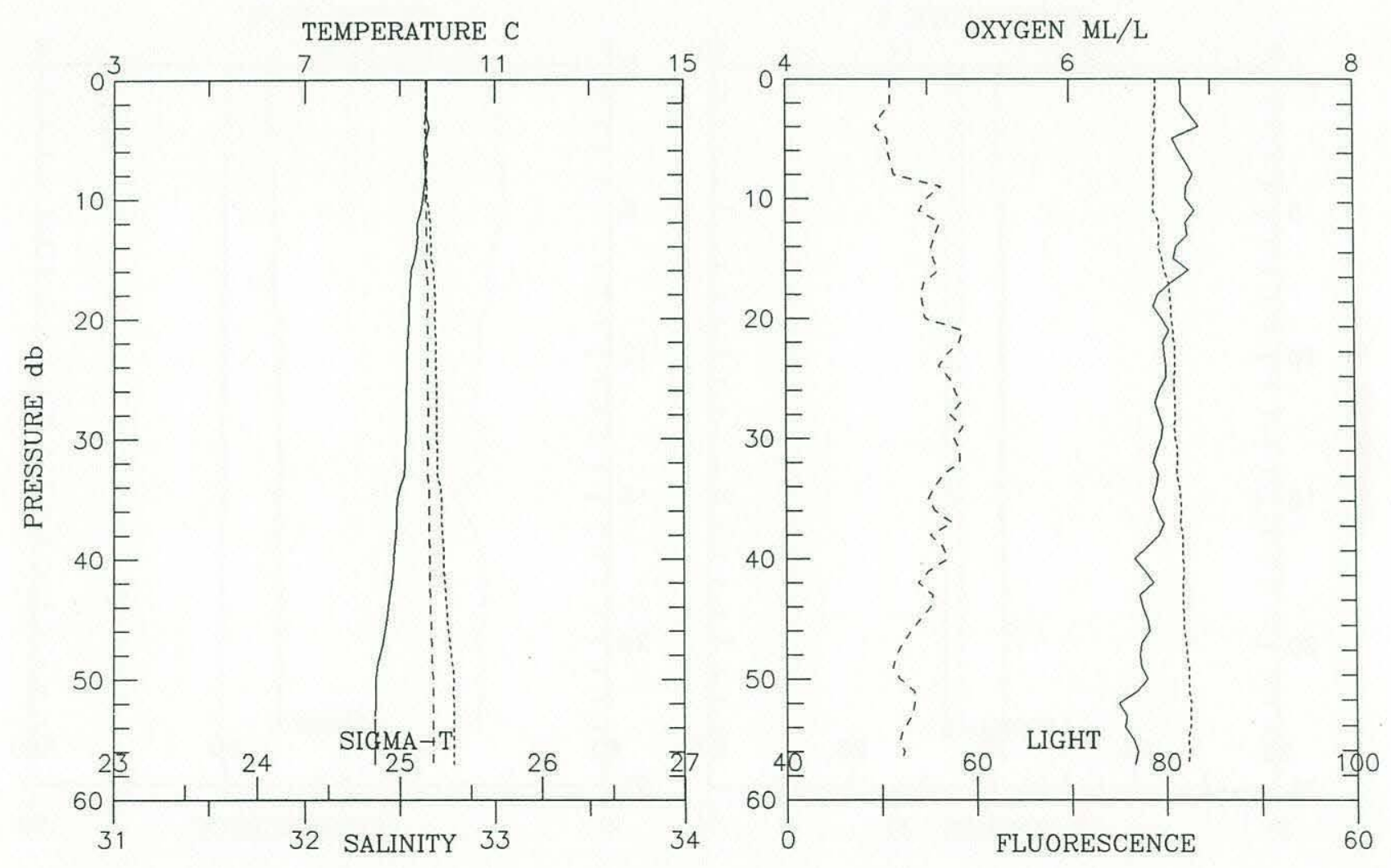


\begin{tabular}{|c|c|c|c|c|c|c|c|c|}
\hline $\begin{array}{l}\text { Cruise EN } \\
\text { Position: }\end{array}$ & $\begin{array}{l}\text { N196 Con } \\
=41^{\circ} 38\end{array}$ & & $68^{\circ} 24.10^{\prime}$ & 177 & $\begin{array}{l}\text { th } m: 25 \\
\text { epth Deck }\end{array}$ & $\begin{array}{r}\text { Time: } \\
\text { offset: }\end{array}$ & -0.15 & $\begin{array}{r}-0917: 14 \\
\text { Fish } 1\end{array}$ \\
\hline $\begin{array}{l}\text { PRESSURE } \\
\text { (d-bars) }\end{array}$ & Interp & $\begin{array}{c}\text { TEMP } \\
\text { (deg } \mathrm{C} \text { ) }\end{array}$ & $\begin{array}{l}\text { SALINITY } \\
(0 / 00)\end{array}$ & SI GMA-T & DELTA-D & $\begin{array}{l}\text { OXYGEN } \\
(\mathrm{ml} / \mathrm{l})\end{array}$ & $\begin{array}{l}\text { CHANNELA } \\
\text { (\%fluor) }\end{array}$ & $\begin{array}{l}\text { CHANNELB } \\
\text { (\%light) }\end{array}$ \\
\hline 0.0 & $E$ & 8.933 & 32.678 & 25.311 & 0.0000 & 6.391 & 11.8 & 80.3 \\
\hline 2.0 & E & 8.932 & 32.678 & 25.311 & 0.0052 & 6.392 & 11.8 & 80.3 \\
\hline 4.0 & & 8.879 & 32.673 & 25.315 & 0.0104 & 6.361 & 11.3 & 80.4 \\
\hline 6.0 & & 8.835 & 32.677 & 25.325 & 0.0156 & 6.326 & 12.0 & 80.4 \\
\hline 8.0 & & 8.814 & 32.679 & 25.330 & 0.0208 & 6.363 & 12.6 & 80.4 \\
\hline 10.0 & & 8.815 & 32.678 & 25.329 & 0.0260 & 6.307 & 13.7 & 80.4 \\
\hline 12.0 & & 8.826 & 32.678 & 25.327 & 0.0312 & 6.311 & 14.1 & 80.4 \\
\hline 14.0 & & 8.870 & 32.679 & 25.321 & 0.0364 & 6.291 & 13.0 & 80.3 \\
\hline 16.0 & & 8.805 & 32.683 & 25.334 & 0.0416 & 6.311 & 14.2 & 80.4 \\
\hline 18.0 & & 8.750 & 32.676 & 25.337 & 0.0468 & 6.309 & 12.8 & 80.4 \\
\hline 20.0 & & 8.734 & 32.674 & 25.339 & 0.0520 & 6.297 & 13.6 & 80.4 \\
\hline 22.0 & & 8.741 & 32.676 & 25.339 & 0.0572 & 6.291 & 13.1 & 80.4 \\
\hline 23.0 & & 8.735 & 32.675 & 25.339 & 0.0597 & 6.289 & 13.8 & 80.3 \\
\hline
\end{tabular}
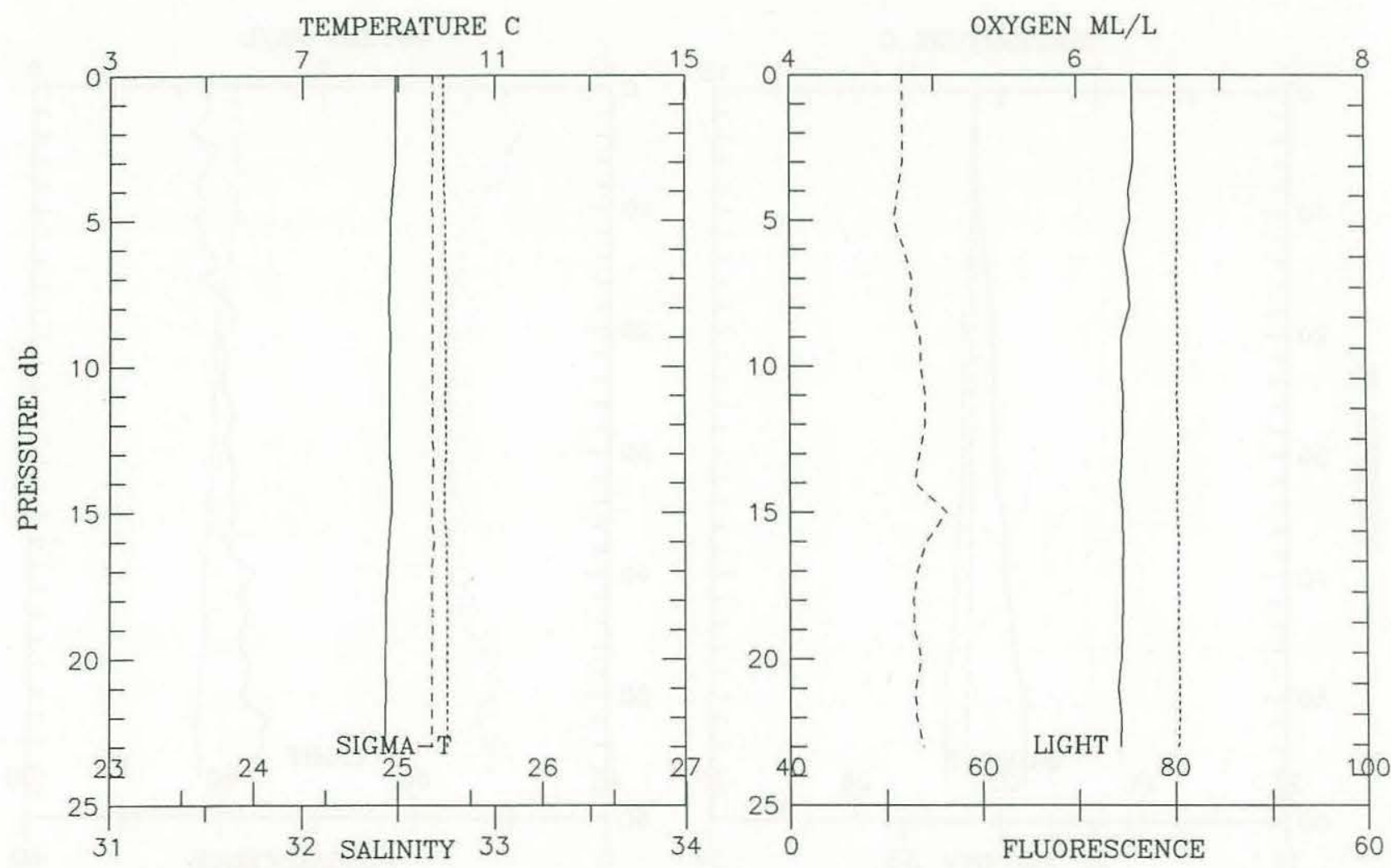


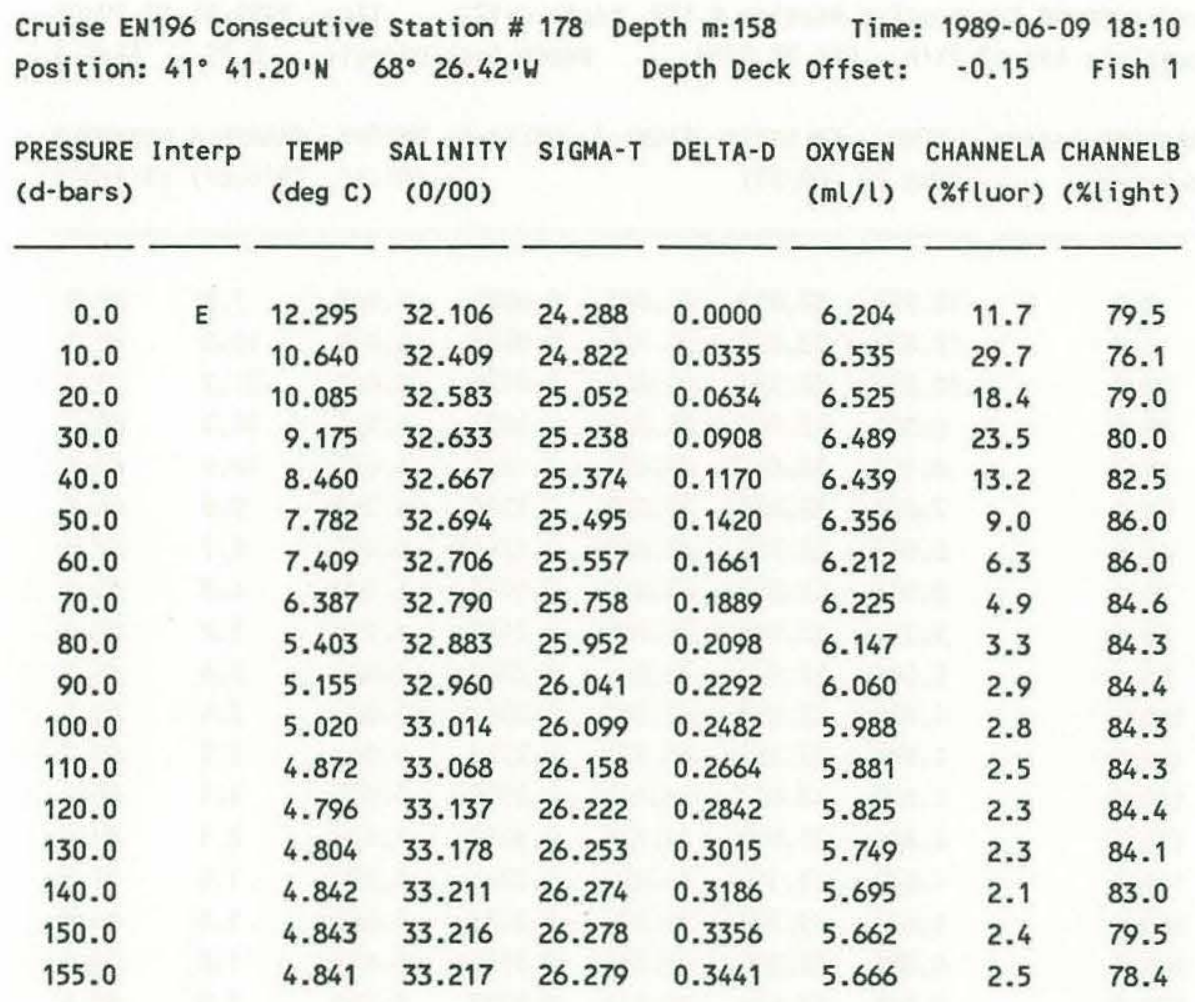
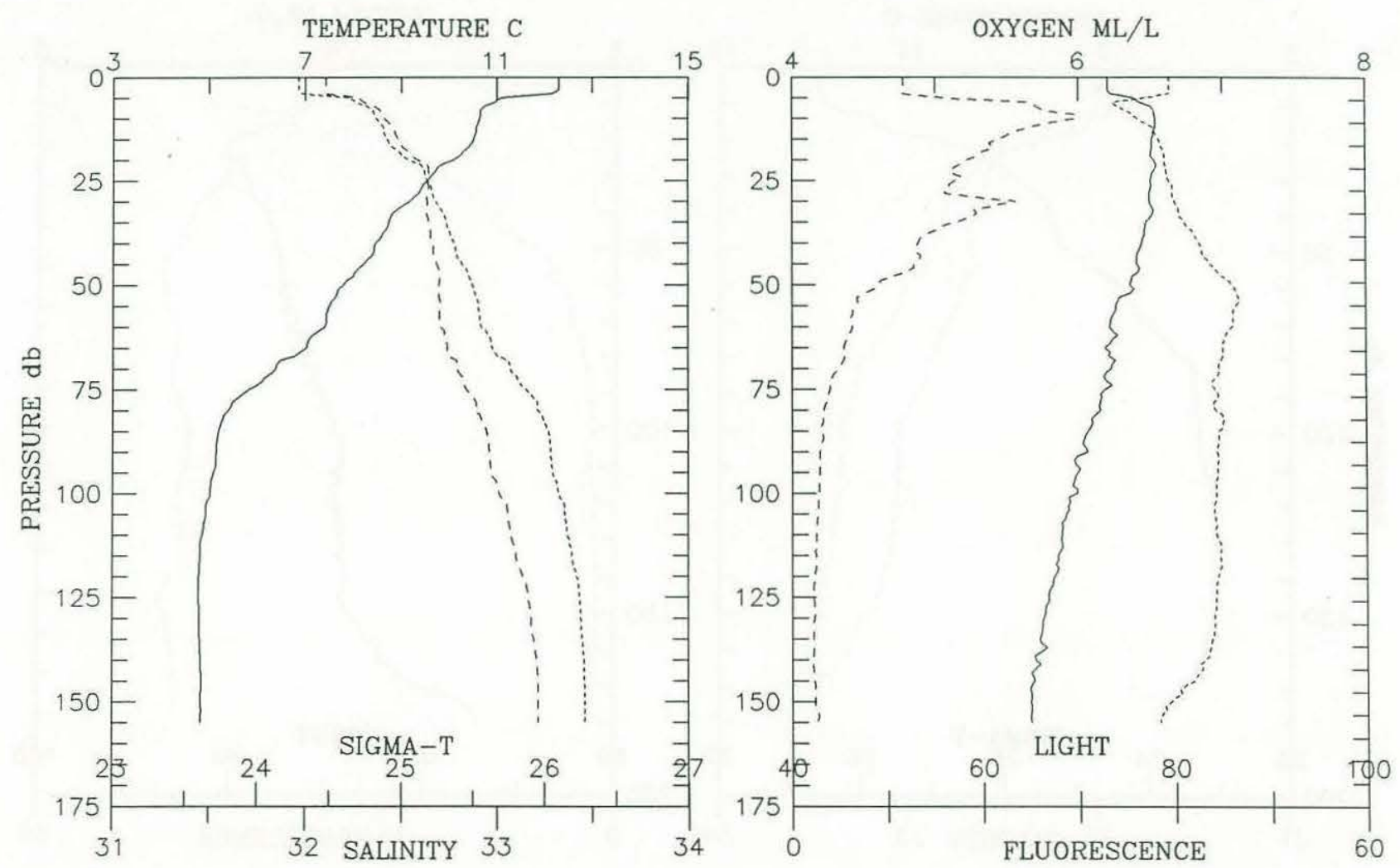


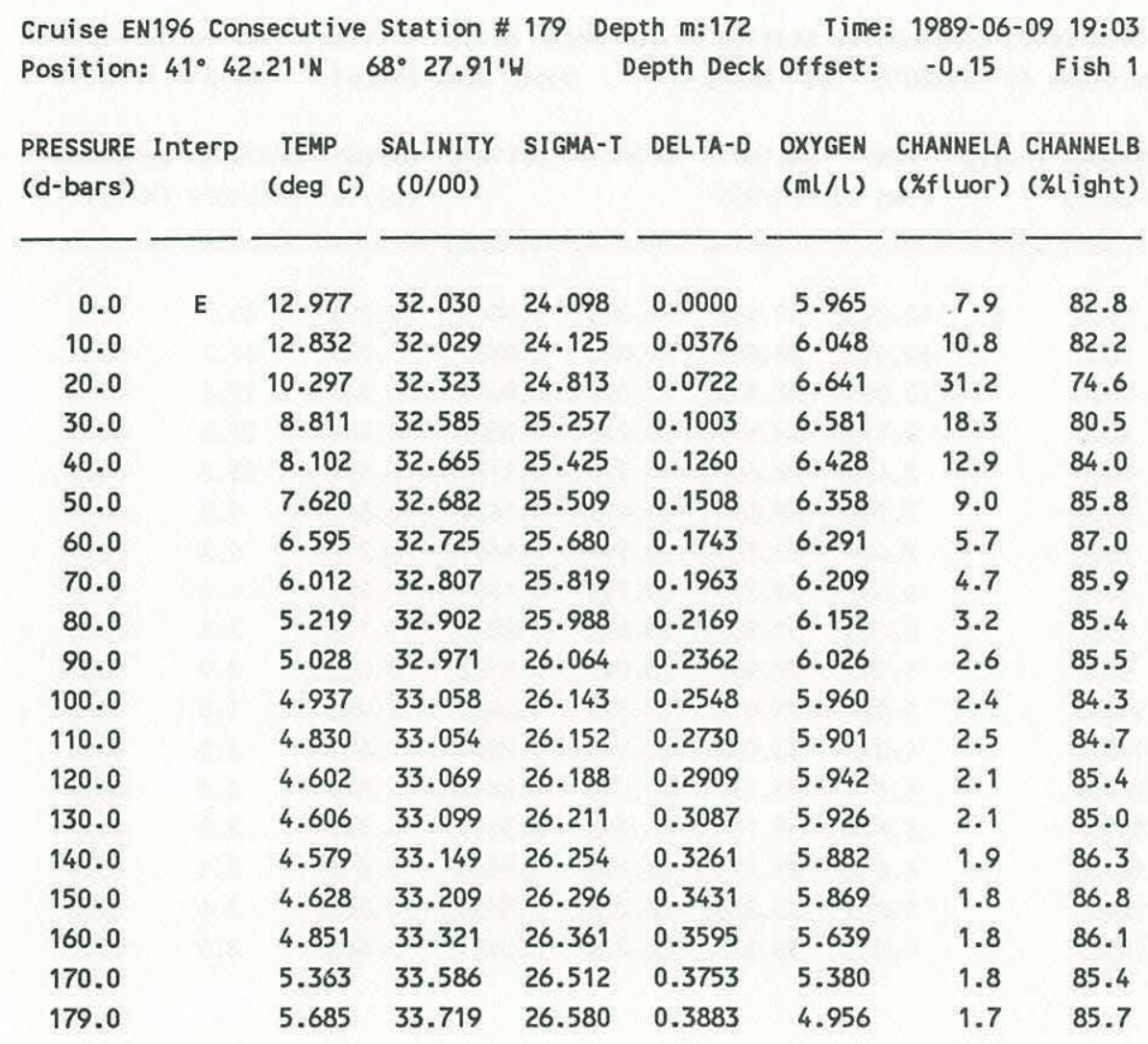
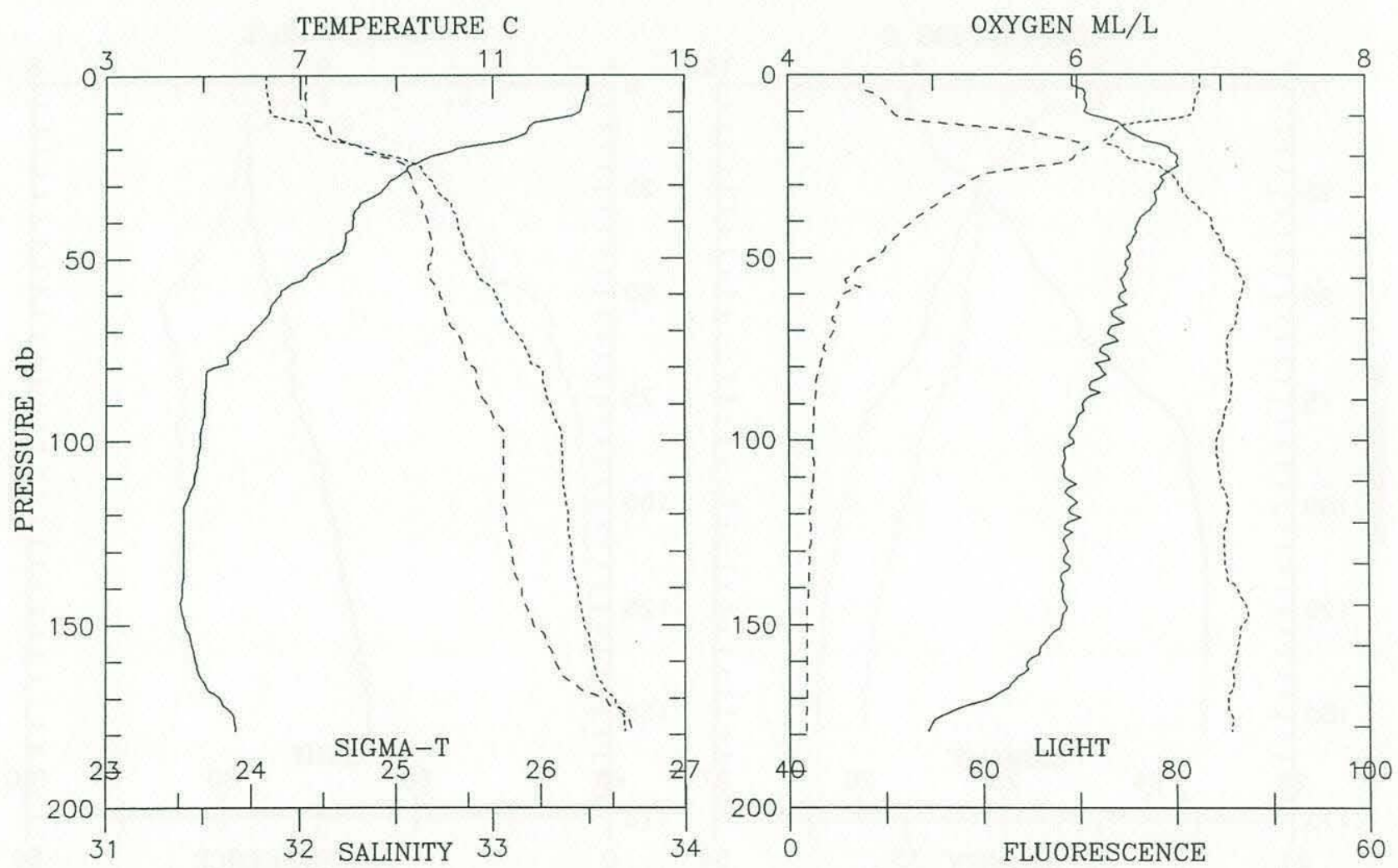
Cruise EN196 Consecutive Station \# 180 Depth m:171 Time: 1989-06-09 19:42 Position: $41^{\circ} 43.00^{\prime} \mathrm{N} \quad 68^{\circ} 29.00^{\prime} \mathrm{W} \quad$ Depth Deck offset: -0.15 Fish 1

\begin{tabular}{|c|c|c|c|c|c|c|c|c|}
\hline $\begin{array}{l}\text { PRESSURE } \\
\text { (d-bars) }\end{array}$ & Interp & $\begin{array}{c}\text { TEMP } \\
\text { (deg C) }\end{array}$ & $\begin{array}{l}\text { SALINITY } \\
(0 / 00)\end{array}$ & SIGMA-T & DELTA-D & $\begin{array}{l}\text { OXYGEN } \\
(\mathrm{ml} / \mathrm{l})\end{array}$ & $\begin{array}{l}\text { CHANNELA } \\
\text { (\%light) }\end{array}$ & $\begin{array}{l}\text { CHANNELB } \\
\text { (\%light) }\end{array}$ \\
\hline 0.0 & E & 13.233 & 31.871 & 23.925 & 0.0000 & 5.803 & 7.6 & 82.6 \\
\hline 10.0 & & 13.171 & 31.864 & 23.932 & 0.0394 & 5.926 & 8.2 & 82.4 \\
\hline 20.0 & & 11.554 & 32.150 & 24.458 & 0.0767 & 6.382 & 23.9 & 74.7 \\
\hline 30.0 & & 8.889 & 32.470 & 25.155 & 0.1071 & 6.661 & 16.2 & 82.6 \\
\hline 40.0 & & 7.413 & 32.637 & 25.501 & 0.1331 & 6.605 & 10.8 & 86.1 \\
\hline 50.0 & & 6.959 & 32.685 & 25.602 & 0.1570 & 6.329 & 6.2 & 87.4 \\
\hline 60.0 & & 6.352 & 32.716 & 25.704 & 0.1799 & 6.416 & 6.2 & 87.5 \\
\hline 70.0 & & 5.761 & 32.804 & 25.847 & 0.2013 & 6.214 & 3.8 & 86.4 \\
\hline 80.0 & & 5.444 & 32.832 & 25.906 & 0.2221 & 6.115 & 3.3 & 86.1 \\
\hline 90.0 & & 4.985 & 32.899 & 26.012 & 0.2421 & 6.078 & 2.9 & 87.4 \\
\hline 100.0 & & 4.964 & 33.020 & 26.110 & 0.2610 & 5.967 & 2.5 & 85.5 \\
\hline 110.0 & & 4.799 & 33.044 & 26.147 & 0.2793 & 5.919 & 2.4 & 85.3 \\
\hline 120.0 & & 4.610 & 33.067 & 26.186 & 0.2973 & 5.923 & 2.5 & 85.1 \\
\hline 130.0 & & 4.627 & 33.101 & 26.211 & 0.3149 & 5.869 & 2.1 & 84.0 \\
\hline 140.0 & & 4.573 & 33.125 & 26.236 & 0.3324 & 5.850 & 2.1 & 84.3 \\
\hline 150.0 & & 4.711 & 33.202 & 26.282 & 0.3496 & 5.768 & 2.0 & 85.2 \\
\hline 160.0 & & 4.761 & 33.277 & 26.336 & 0.3662 & 5.678 & 1.9 & 86.2 \\
\hline 162.0 & & 4.797 & 33.292 & 26.344 & 0.3694 & 5.660 & 1.9 & 86.1 \\
\hline
\end{tabular}
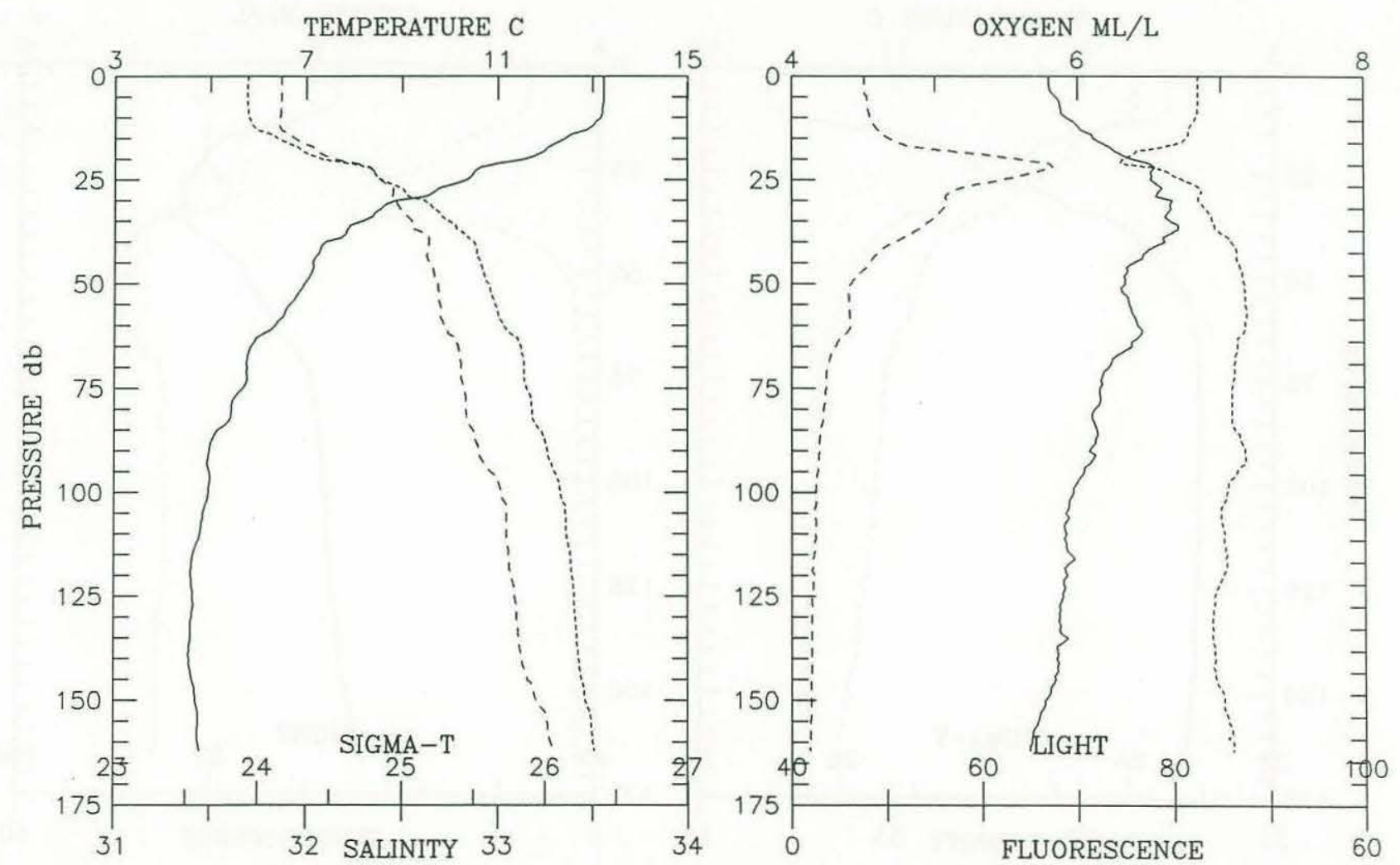
Cruise EN196 Consecutive Station \# 181 Depth m:191 Time: 1989-06-09 20:28 Position: $41^{\circ} 45.00^{\prime} \mathrm{N} 68^{\circ} 32.48^{\prime} \mathrm{W}$ Depth Deck offset: -0.15 Fish 1

\begin{tabular}{|c|c|c|c|c|c|c|c|c|}
\hline $\begin{array}{l}\text { PRESSURE } \\
\text { (d-bars) }\end{array}$ & Interp & $\begin{array}{c}\text { TEMP } \\
(\operatorname{deg} \mathrm{C})\end{array}$ & $\begin{array}{l}\text { SALINITY } \\
(0 / 00)\end{array}$ & SIGMA-T & DELTA-D & $\begin{array}{l}\text { OXYGEN } \\
(\mathrm{ml} / \mathrm{l})\end{array}$ & $\begin{array}{l}\text { CHANNELA } \\
\text { (\%fluor) }\end{array}$ & $\begin{array}{l}\text { CHANNELB } \\
\text { (\%light) }\end{array}$ \\
\hline 0.0 & $E$ & 13.644 & 31.731 & 23.735 & 0.0000 & 5.822 & 7.4 & 82.1 \\
\hline 10.0 & & 13.502 & 31.729 & 23.761 & 0.0412 & 5.865 & 7.8 & 81.8 \\
\hline 20.0 & & 9.801 & 32.056 & 24.687 & 0.0773 & 6.668 & 16.1 & 81.0 \\
\hline 30.0 & & 7.749 & 32.365 & 25.241 & 0.1071 & 6.895 & 25.6 & 78.5 \\
\hline 40.0 & & 5.888 & 32.728 & 25.772 & 0.1315 & 6.806 & 10.2 & 87.9 \\
\hline 50.0 & & 5.140 & 32.860 & 25.964 & 0.1522 & 6.457 & 3.5 & 89.5 \\
\hline 60.0 & & 4.706 & 32.915 & 26.055 & 0.1715 & 6.260 & 2.4 & 89.6 \\
\hline 70.0 & & 4.570 & 33.011 & 26.146 & 0.1899 & 6.069 & 2.0 & 87.2 \\
\hline 80.0 & & 4.541 & 33.045 & 26.176 & 0.2079 & 5.954 & 2.0 & 85.2 \\
\hline 90.0 & & 4.536 & 33.064 & 26.191 & 0.2257 & 5.919 & 2.0 & 85.2 \\
\hline 100.0 & & 4.561 & 33.080 & 26.201 & 0.2433 & 5.891 & 2.0 & 84.9 \\
\hline 110.0 & & 4.578 & 33.094 & 26.211 & 0.2610 & 5.874 & 2.0 & 84.8 \\
\hline 120.0 & & 4.589 & 33.119 & 26.229 & 0.2784 & 5.847 & 2.0 & 84.5 \\
\hline 130.0 & & 4.630 & 33.166 & 26.262 & 0.2957 & 5.805 & 2.0 & 85.3 \\
\hline 140.0 & & 4.636 & 33.177 & 26.270 & 0.3127 & 5.796 & 2.0 & 85.7 \\
\hline 150.0 & & 4.722 & 33.229 & 26.302 & 0.3296 & 5.736 & 2.0 & 85.7 \\
\hline 160.0 & & 4.778 & 33.261 & 26.322 & 0.3463 & 5.672 & 1.9 & 85.5 \\
\hline 168.0 & & 4.881 & 33.322 & 26.358 & 0.3594 & 5.586 & 2.0 & 85.6 \\
\hline
\end{tabular}
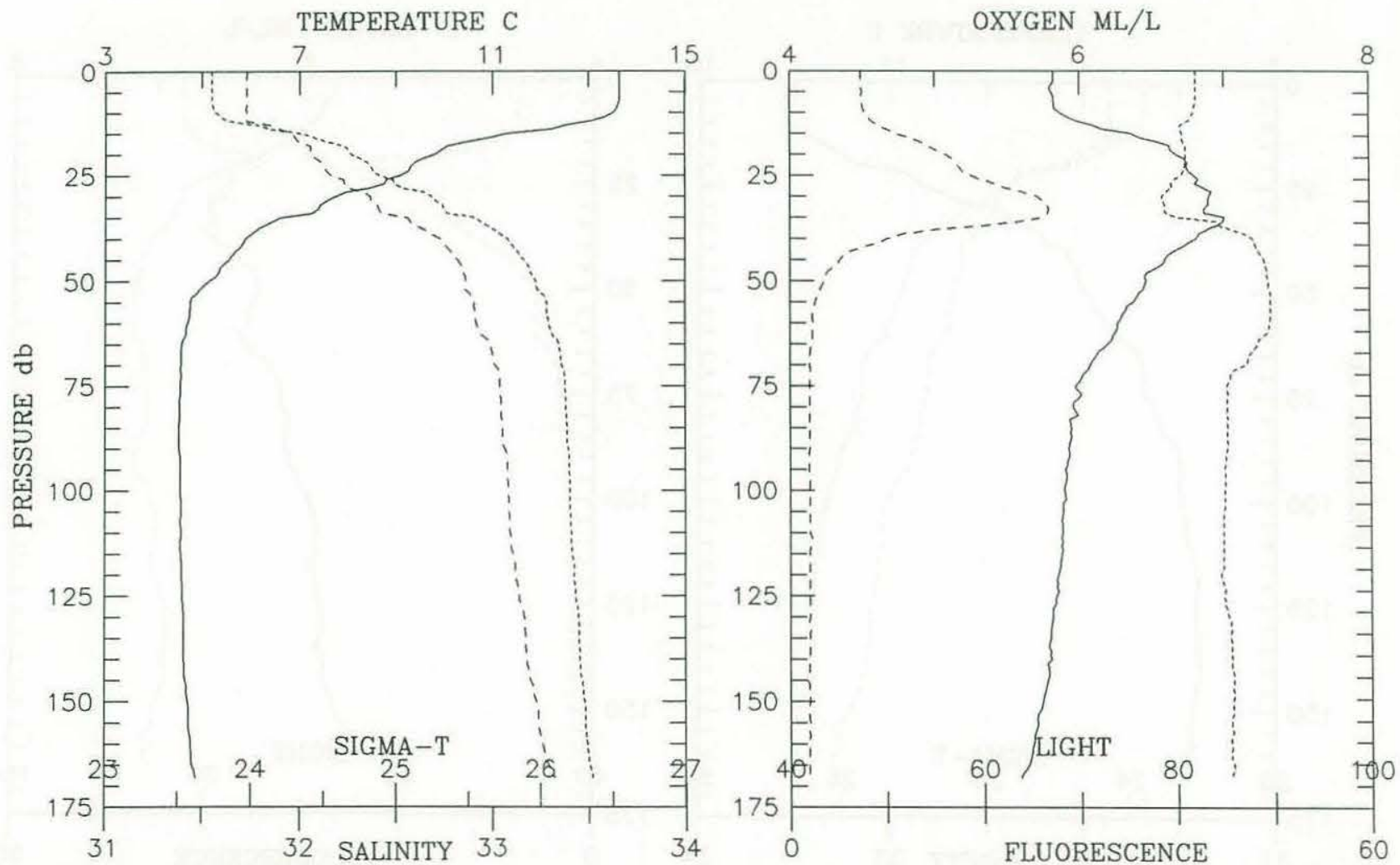


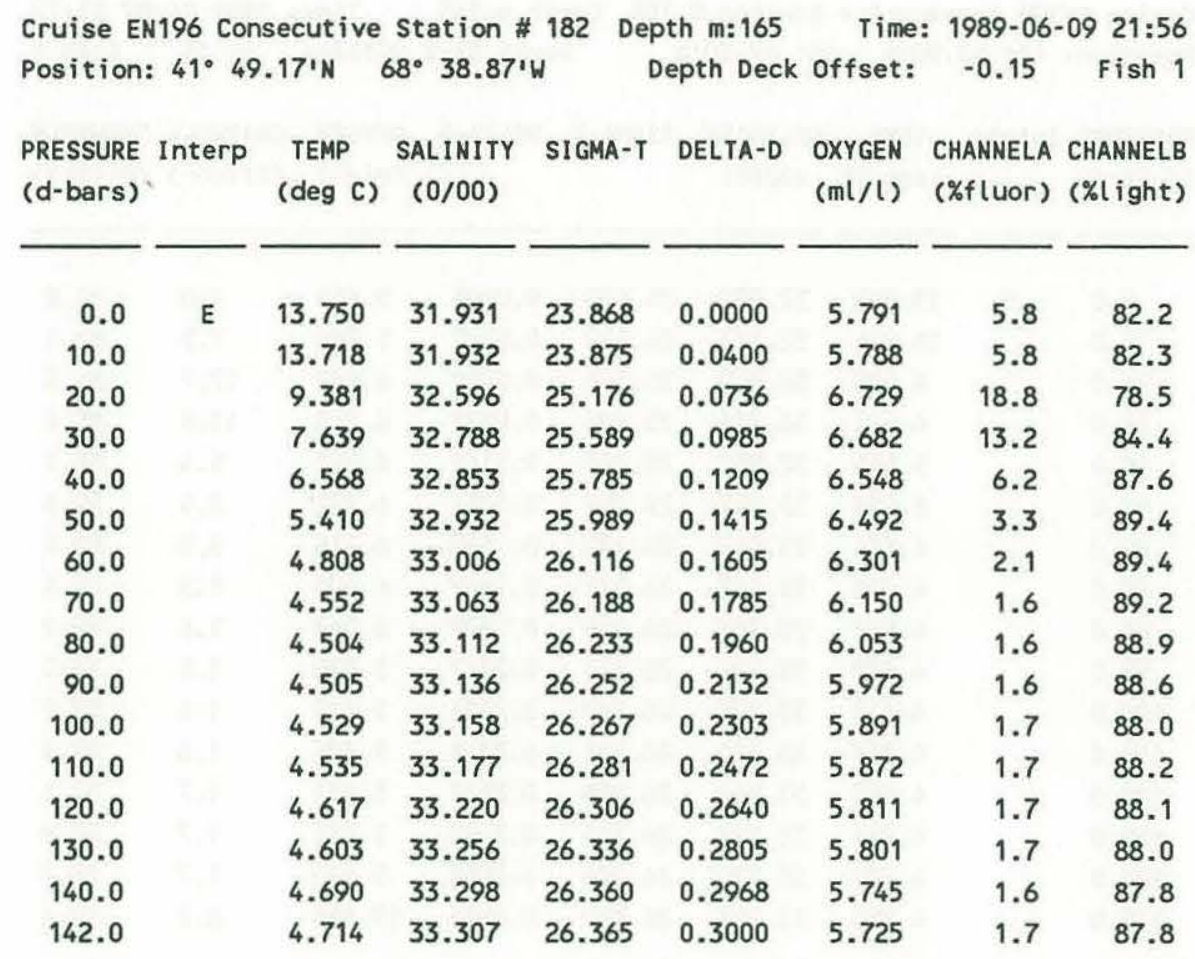
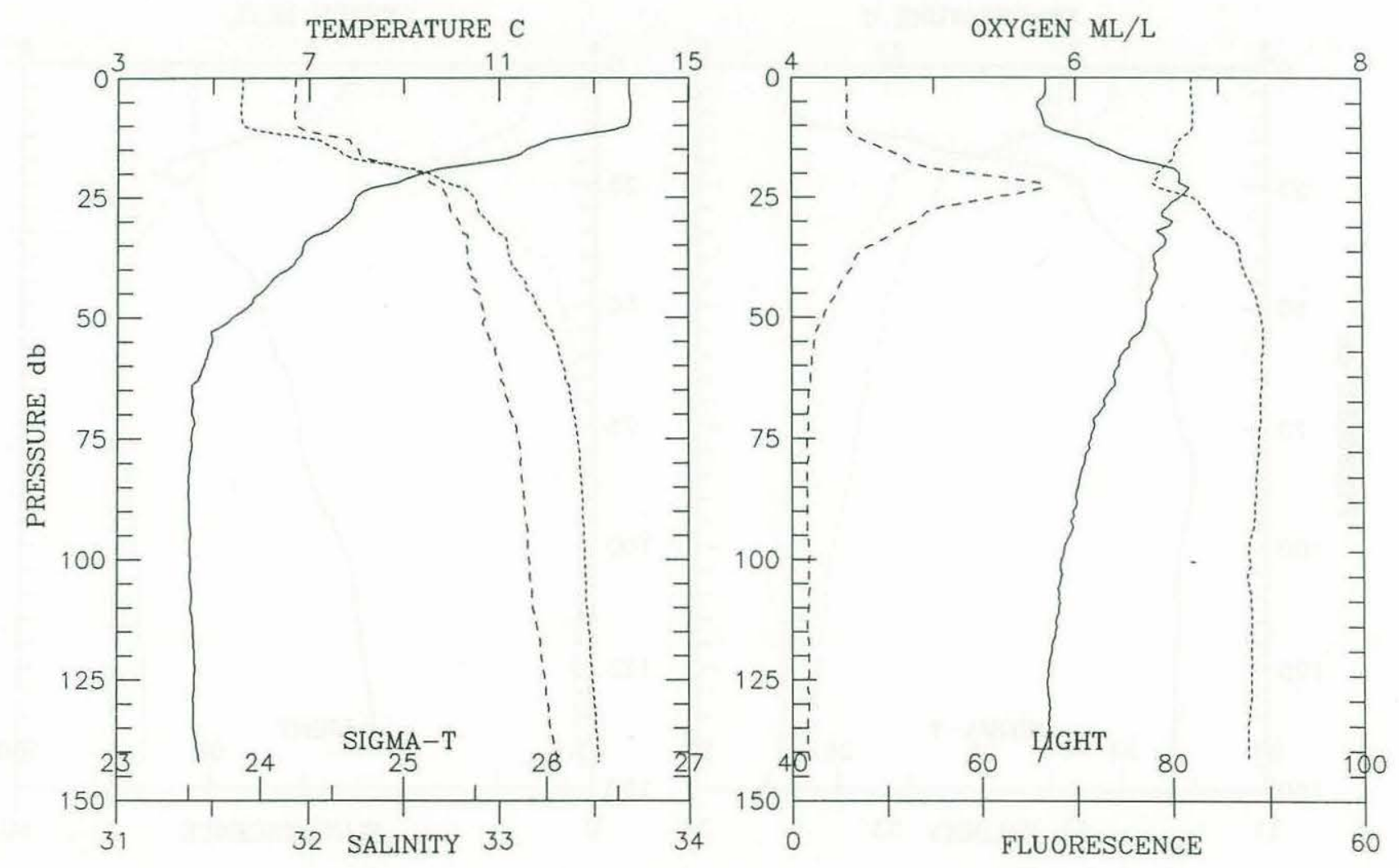


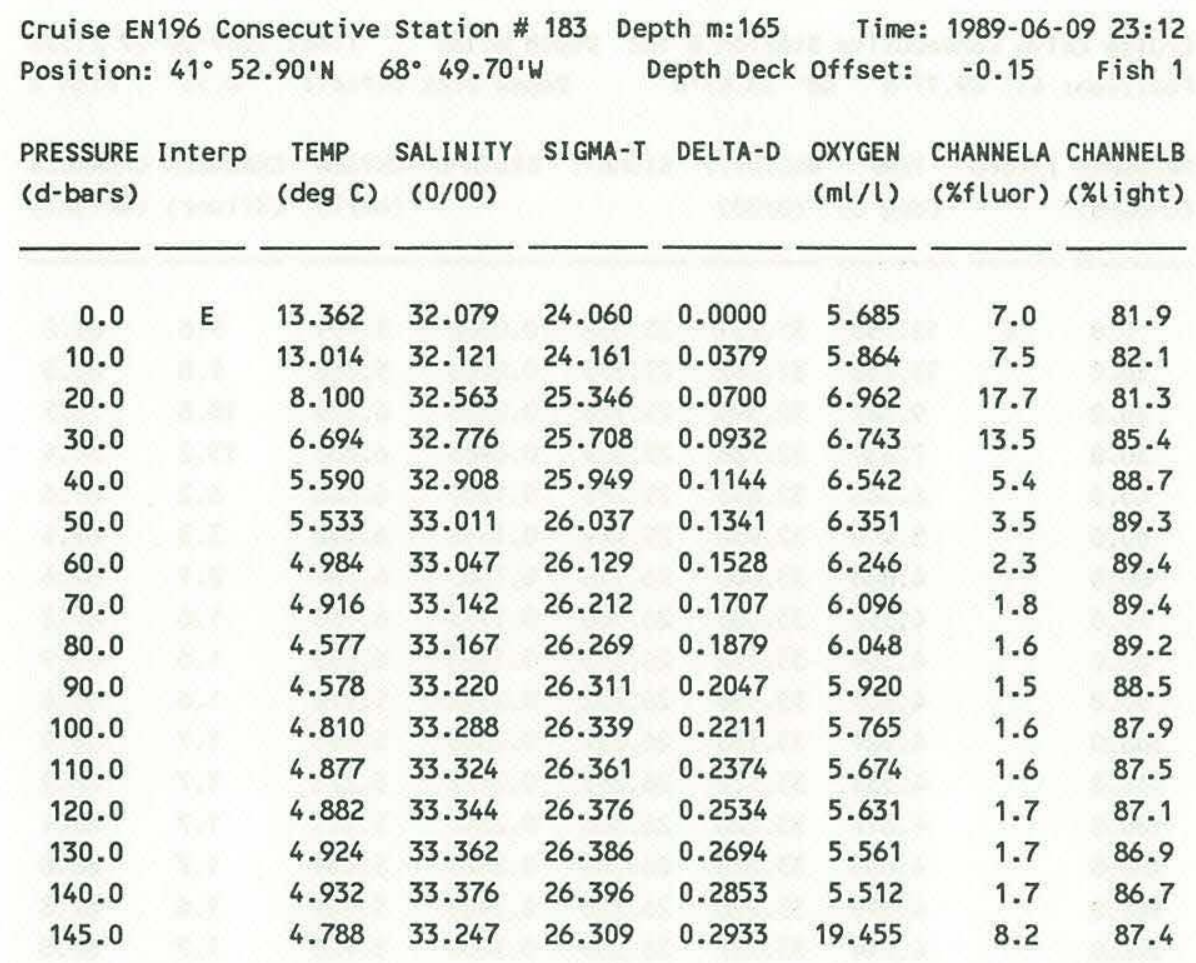
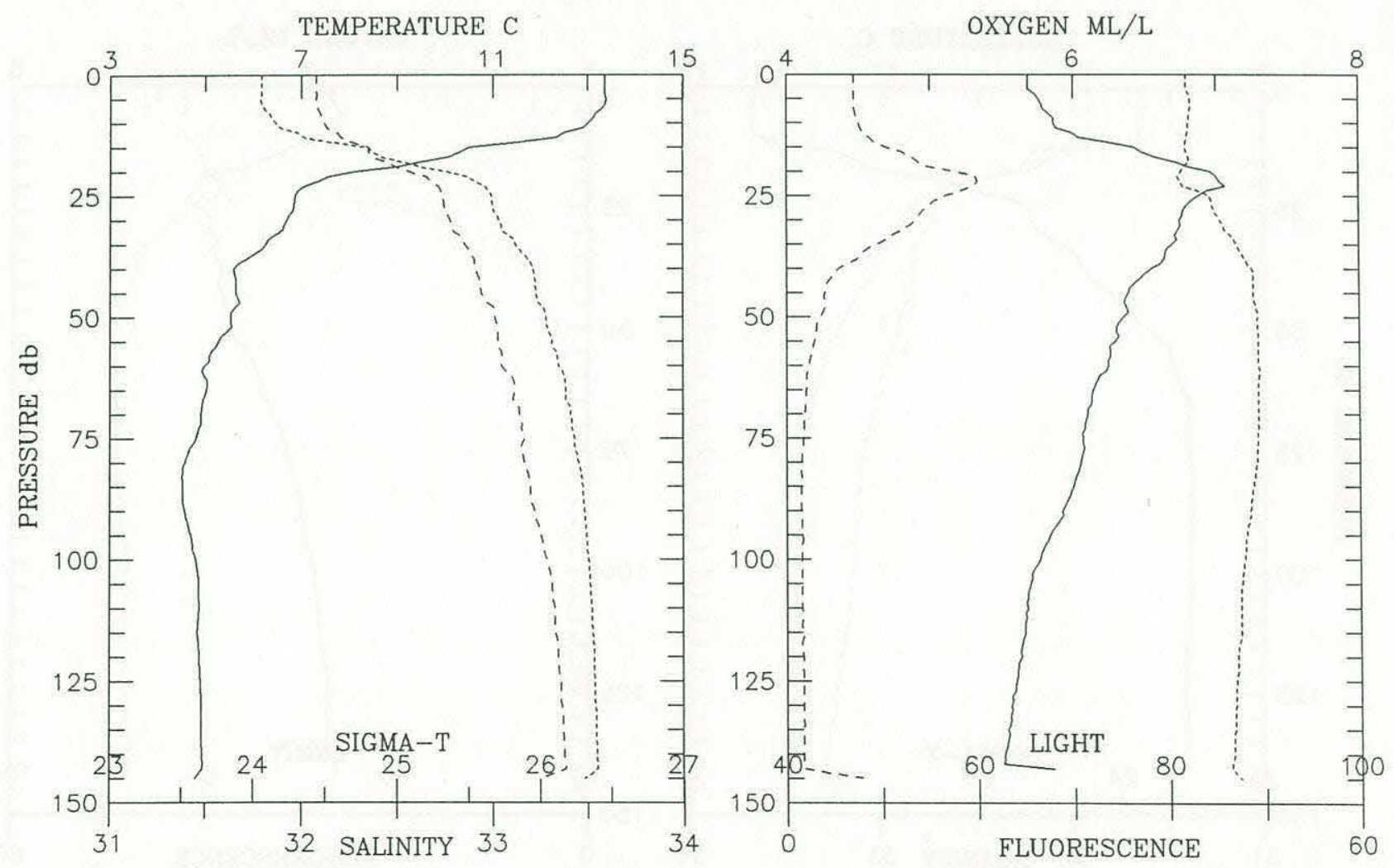
Cruise EN196 Consecutive Station \# 184 Depth m:192 Time: 1989-06-10 00:29 Position: $41^{\circ} 56.50^{\prime} \mathrm{N} 69^{\circ} 0.00^{\prime} \mathrm{W}$ Depth Deck offset: -0.15 Fish 1

\begin{tabular}{|c|c|c|c|c|c|c|c|c|}
\hline $\begin{array}{l}\text { PRESSURE } \\
\text { (d-bars) }\end{array}$ & Interp & $\begin{array}{c}\text { TEMP } \\
\text { (deg C) }\end{array}$ & $\begin{array}{l}\text { SALINITY } \\
(0 / 00)\end{array}$ & SIGMA-T & DELTA-D & $\begin{array}{l}\text { OXYGEN } \\
(\mathrm{ml} / \mathrm{l})\end{array}$ & $\begin{array}{l}\text { CHANNELA } \\
\text { ( } \% \text { fuor) }\end{array}$ & $\begin{array}{l}\text { CHANNELB } \\
\text { (\%light) }\end{array}$ \\
\hline 0.0 & E & 13.671 & 32.013 & 23.947 & 0.0000 & 5.784 & 6.3 & 82.3 \\
\hline 10.0 & & 13.629 & 32.021 & 23.962 & 0.0392 & 5.815 & 6.3 & 82.3 \\
\hline 20.0 & & 9.825 & 32.470 & 25.006 & 0.0718 & 6.688 & 20.3 & 79.1 \\
\hline 30.0 & & 7.469 & 32.753 & 25.585 & 0.0974 & 6.869 & 29.9 & 79.5 \\
\hline 40.0 & & 5.885 & 32.939 & 25.938 & 0.1192 & 6.662 & 7.3 & 88.2 \\
\hline 50.0 & & 5.207 & 33.035 & 26.094 & 0.1383 & 6.353 & 2.5 & 89.5 \\
\hline 60.0 & & 5.018 & 33.068 & 26.142 & 0.1568 & 6.223 & 2.2 & 89.5 \\
\hline 70.0 & & 4.912 & 33.156 & 26.223 & 0.1744 & 6.065 & 1.8 & 89.3 \\
\hline 80.0 & & 4.743 & 33.192 & 26.271 & 0.1916 & 5.978 & 1.7 & 89.0 \\
\hline 90.0 & & 4.705 & 33.240 & 26.313 & 0.2084 & 5.908 & 1.6 & 88.4 \\
\hline 100.0 & & 4.622 & 33.272 & 26.347 & 0.2248 & 5.848 & 1.8 & 88.4 \\
\hline 110.0 & & 4.686 & 33.327 & 26.384 & 0.2409 & 5.771 & 1.5 & 88.0 \\
\hline 120.0 & & 4.713 & 33.338 & 26.390 & 0.2568 & 5.721 & 1.6 & 87.6 \\
\hline 130.0 & & 4.725 & 33.344 & 26.393 & 0.2726 & 5.689 & 1.6 & 87.5 \\
\hline 140.0 & & 4.741 & 33.351 & 26.397 & 0.2885 & 5.669 & 1.6 & 87.3 \\
\hline 150.0 & & 4.780 & 33.370 & 26.407 & 0.3043 & 5.633 & 1.6 & 87.5 \\
\hline 160.0 & & 4.766 & 33.375 & 26.414 & 0.3200 & 5.615 & 1.7 & 87.3 \\
\hline 170.0 & & 4.759 & 33.379 & 26.417 & 0.3357 & 5.629 & 1.9 & 83.5 \\
\hline 177.0 & & 4.756 & 33.381 & 26.419 & 0.3466 & 5.650 & 1.8 & 83.0 \\
\hline
\end{tabular}
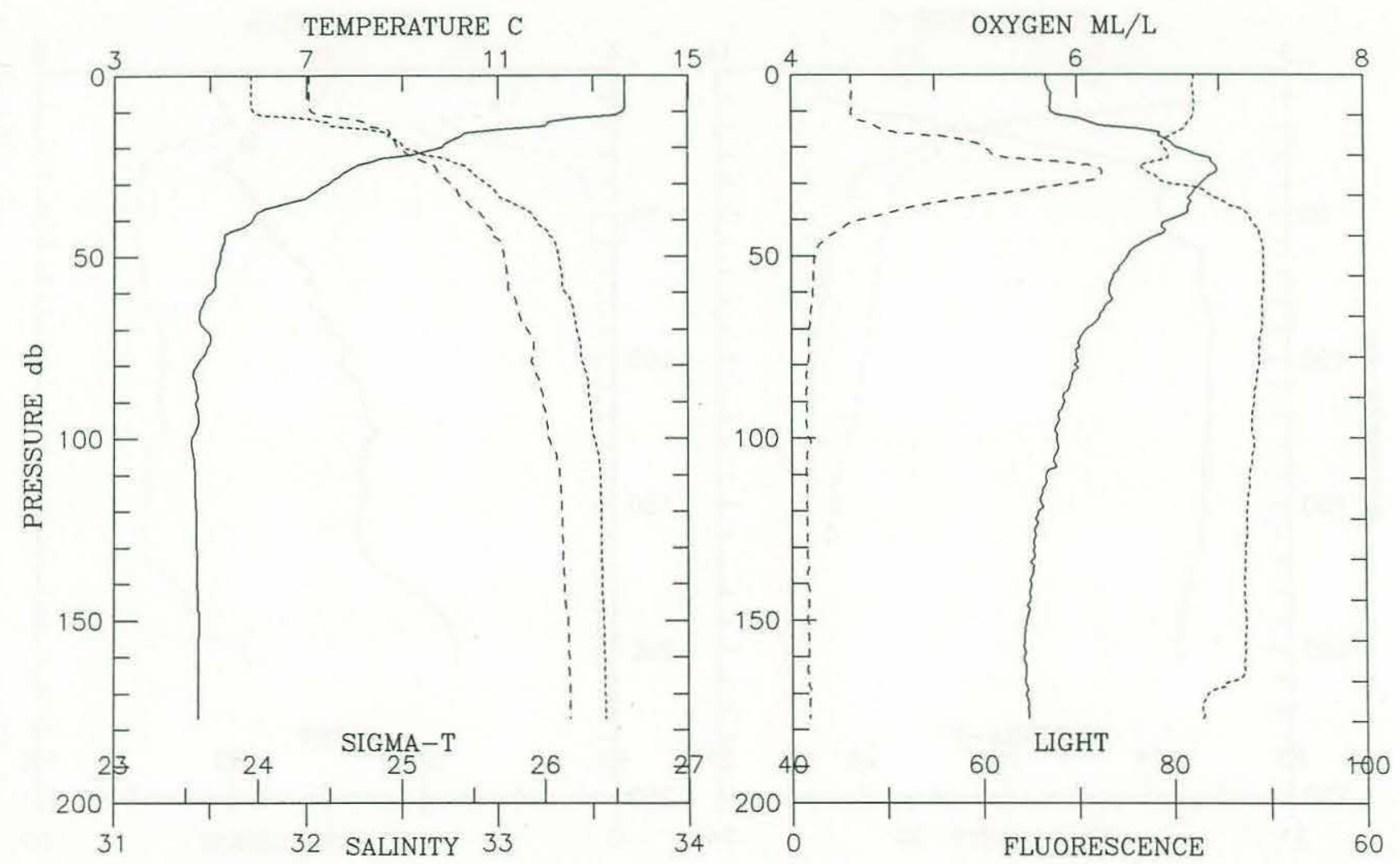
Cruise EN196 Consecutive Station \# 185 Depth m:212 Time: 1989-06-10 01:51 Position: $41^{\circ} 53.20^{\prime} \mathrm{N} 69^{\circ} 12.00^{\prime} \mathrm{W}$ Depth Deck offset: -0.15 Fish 1

\begin{tabular}{|c|c|c|c|c|c|c|c|c|}
\hline $\begin{array}{l}\text { PRESSURE } \\
\text { (d-bars) }\end{array}$ & Interp & $\begin{array}{l}\text { TEMP } \\
(\operatorname{deg} C)\end{array}$ & $\begin{array}{c}\text { SALINITY } \\
(0 / 00)\end{array}$ & SIGMA-T & DELTA-D & $\begin{array}{l}\text { OXYGEN } \\
(\mathrm{ml} / \mathrm{l})\end{array}$ & $\begin{array}{l}\text { CHANNELA } \\
\text { (\%fluor) }\end{array}$ & $\begin{array}{l}\text { CHANNELB } \\
\text { ( }(\text { i ight) }\end{array}$ \\
\hline 0.0 & E & 12.887 & 31.327 & 23.571 & 0.0000 & 5.995 & 10.2 & 81.2 \\
\hline 10.0 & & 12.796 & 31.373 & 23.625 & 0.0426 & 6.045 & 10.5 & 81.1 \\
\hline 20.0 & & 10.162 & 32.630 & 25.075 & 0.0782 & 6.497 & 21.1 & 81.4 \\
\hline 30.0 & & 7.087 & 32.848 & 25.713 & 0.1031 & 6.678 & 6.7 & 88.0 \\
\hline 40.0 & & 5.767 & 33.072 & 26.058 & 0.1232 & 6.578 & 2.9 & 89.5 \\
\hline 50.0 & & 5.646 & 33.096 & 26.091 & 0.1420 & 6.409 & 2.5 & 89.5 \\
\hline 60.0 & & 5.238 & 33.135 & 26.170 & 0.1602 & 6.268 & 2.2 & 89.6 \\
\hline 70.0 & & 4.823 & 33.161 & 26.237 & 0.1777 & 6.108 & 1.6 & 89.1 \\
\hline 80.0 & & 4.733 & 33.189 & 26.269 & 0.1948 & 6.104 & 1.6 & 88.6 \\
\hline 90.0 & & 4.578 & 33.202 & 26.296 & 0.2117 & 6.018 & 1.5 & 87.3 \\
\hline 100.0 & & 4.622 & 33.235 & 26.318 & 0.2283 & 5.874 & 1.5 & 87.1 \\
\hline 110.0 & & 4.648 & 33.249 & 26.326 & 0.2448 & 5.759 & 1.7 & 87.1 \\
\hline 120.0 & & 4.707 & 33.288 & 26.351 & 0.2611 & 5.730 & 1.7 & 86.9 \\
\hline 130.0 & & 4.725 & 33.342 & 26.391 & 0.2772 & 5.750 & 1.6 & 88.4 \\
\hline 140.0 & & 4.779 & 33.372 & 26.409 & 0.2930 & 5.775 & 1.6 & 88.5 \\
\hline 150.0 & & 4.779 & 33.393 & 26.426 & 0.3086 & 5.780 & 1.5 & 88.7 \\
\hline 160.0 & & 4.835 & 33.421 & 26.442 & 0.3241 & 5.776 & 1.4 & 88.9 \\
\hline 170.0 & & 4.956 & 33.465 & 26.463 & 0.3395 & 5.665 & 1.5 & 88.2 \\
\hline 180.0 & & 5.130 & 33.524 & 26.490 & 0.3546 & 5.374 & 1.8 & 84.3 \\
\hline 190.0 & & 5.284 & 33.571 & 26.510 & 0.3695 & 5.168 & 1.7 & 83.1 \\
\hline 200.0 & & 5.338 & 33.585 & 26.515 & 0.3843 & 5.097 & 1.8 & 79.2 \\
\hline 205.0 & & 5.345 & 33.587 & 26.515 & 0.3917 & 5.082 & 1.8 & 76.0 \\
\hline
\end{tabular}

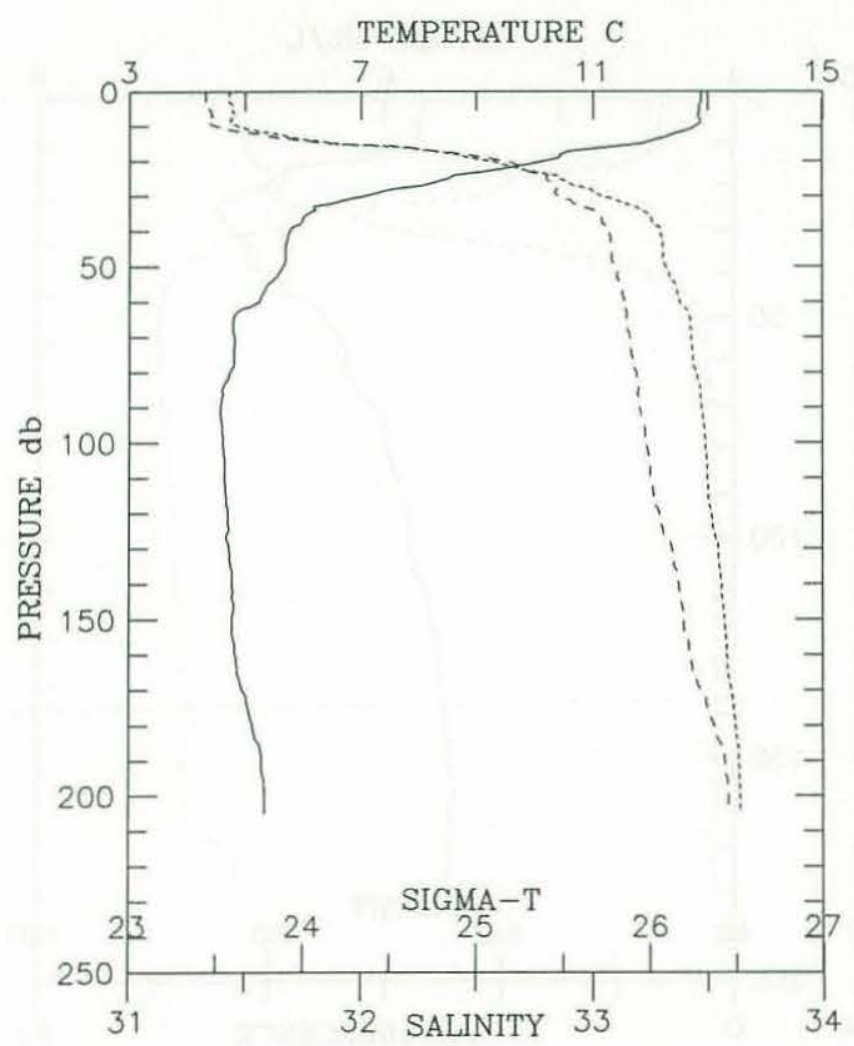
OXYGEN ML/L

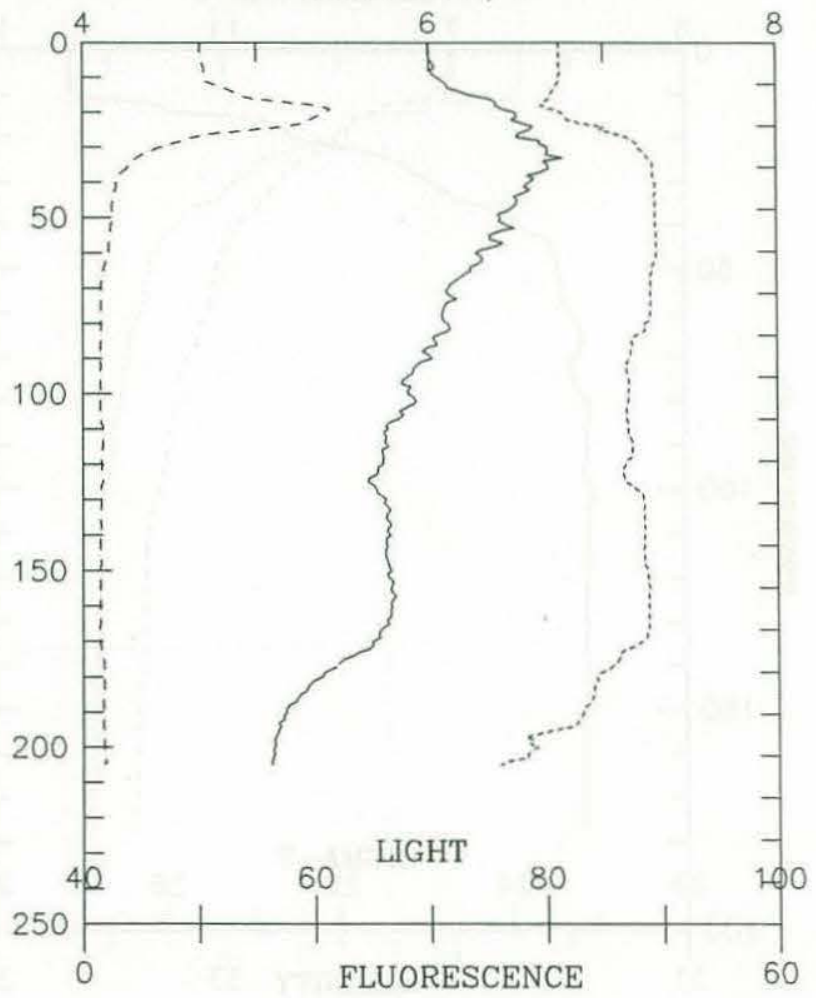




\begin{tabular}{|c|c|c|c|c|c|c|c|c|}
\hline $\begin{array}{l}\text { Cruise El } \\
\text { Position }\end{array}$ & $\begin{array}{l}\text { N196 Con } \\
=41^{\circ} 50\end{array}$ & $\begin{array}{l}\text { secutive } \\
.20 \text { 'N }\end{array}$ & $\begin{array}{l}\text { Station } \\
69^{\circ} 23.40^{\prime}\end{array}$ & & $\begin{array}{l}\text { th } \mathrm{m}: 190 \\
\text { epth Deck }\end{array}$ & $\begin{array}{r}\text { Time } \\
\text { offset: }\end{array}$ & $\begin{array}{c}=1989-06- \\
-0.15\end{array}$ & $\begin{array}{r}10 \quad 03: 21 \\
\text { Fish } 1\end{array}$ \\
\hline $\begin{array}{l}\text { PRESSURE } \\
\text { (d-bars) }\end{array}$ & Interp & $\begin{array}{c}\text { TEMP } \\
(\operatorname{deg} \mathrm{C})\end{array}$ & $\begin{array}{l}\text { SALINITY } \\
(0 / 00)\end{array}$ & SIGMA-T & DELTA-D & $\begin{array}{l}\text { OXYGEN } \\
(\mathrm{ml} / \mathrm{l})\end{array}$ & $\begin{array}{l}\text { CHANNELA } \\
\text { (\%fluor) }\end{array}$ & $\begin{array}{l}\text { CHANNELB } \\
\text { (\%light) }\end{array}$ \\
\hline 0.0 & E & 12.758 & 31.206 & 23.503 & 0.0000 & 6.047 & 12.4 & 80.1 \\
\hline 10.0 & & 12.495 & 31.280 & 23.610 & 0.0433 & 6.196 & 13.4 & 80.1 \\
\hline 20.0 & & 10.492 & 32.616 & 25.008 & 0.0790 & 6.471 & 23.7 & 80.2 \\
\hline 30.0 & & 8.263 & 32.780 & 25.492 & 0.1062 & 6.663 & 13.5 & 85.4 \\
\hline 40.0 & & 6.224 & 32.968 & 25.919 & 0.1284 & 6.662 & 5.5 & 88.6 \\
\hline 50.0 & & 5.529 & 33.066 & 26.081 & 0.1479 & 6.529 & 3.3 & 89.3 \\
\hline 60.0 & & 5.030 & 33.153 & 26.208 & 0.1660 & 6.360 & 2.4 & 89.7 \\
\hline 70.0 & & 4.704 & 33.223 & 26.299 & 0.1831 & 6.194 & 2.0 & 89.6 \\
\hline 80.0 & & 4.641 & 33.289 & 26.359 & 0.1995 & 6.059 & 1.7 & 89.5 \\
\hline 90.0 & & 4.668 & 33.332 & 26.389 & 0.2154 & 6.029 & 1.6 & 89.4 \\
\hline 100.0 & & 4.728 & 33.379 & 26.420 & 0.2311 & 5.904 & 1.5 & 89.3 \\
\hline 110.0 & & 4.876 & 33.438 & 26.451 & 0.2465 & 5.822 & 1.3 & 89.0 \\
\hline 120.0 & & 5.060 & 33.500 & 26.479 & 0.2617 & 5.684 & 1.3 & 88.8 \\
\hline 130.0 & & 5.353 & 33.588 & 26.516 & 0.2766 & 5.475 & 1.5 & 88.6 \\
\hline 140.0 & & 5.619 & 33.672 & 26.550 & 0.2912 & 5.022 & 1.4 & 87.1 \\
\hline 150.0 & & 5.964 & 33.786 & 26.598 & 0.3054 & 4.670 & 1.5 & 86.3 \\
\hline 160.0 & & 6.085 & 33.827 & 26.615 & 0.3193 & 4.443 & 1.6 & 86.1 \\
\hline 170.0 & & 6.238 & 33.881 & 26.638 & 0.3331 & 4.278 & 1.6 & 82.9 \\
\hline 179.0 & & 6.257 & 33.888 & 26.641 & 0.3454 & 4.159 & 1.7 & 80.8 \\
\hline
\end{tabular}
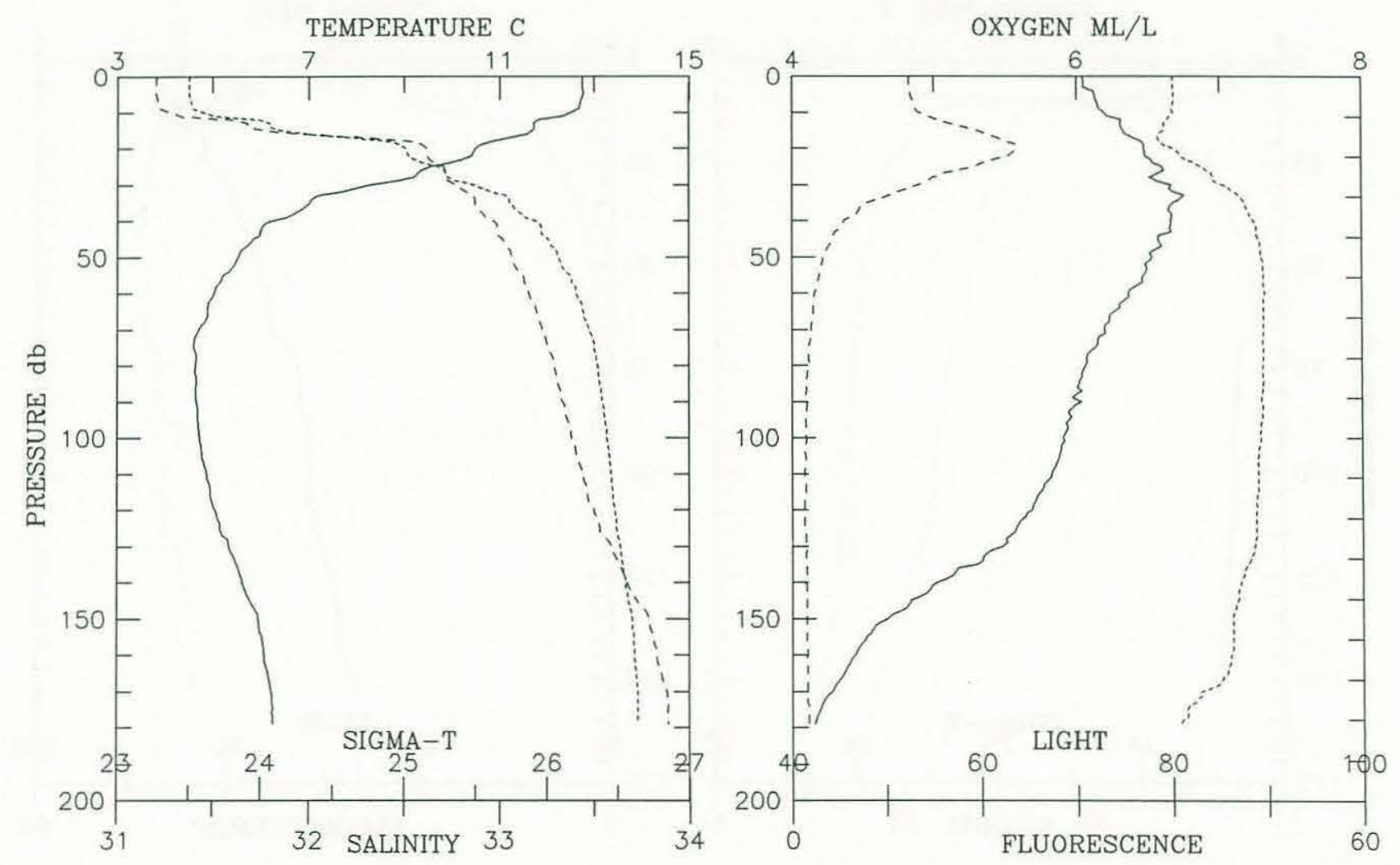
Cruise EN196 Consecutive Station \# 187 Depth m:165 Time: 1989-06-10 04:43 Position: $41^{\circ} 46.29^{\prime} \mathrm{N} 69^{\circ} 34.13^{\prime} \mathrm{W} \quad$ Depth Deck offset: -0.15 Fish 1

\begin{tabular}{|c|c|c|c|c|c|c|c|c|}
\hline $\begin{array}{l}\text { PRESSURE } \\
\text { (d-bars) }\end{array}$ & Interp & $\begin{array}{c}\text { TEMP } \\
(\operatorname{deg} C)\end{array}$ & $\begin{array}{l}\text { SALINITY } \\
(0 / 00)\end{array}$ & SIGMA-T & DELTA-D & $\begin{array}{l}\text { OXYGEN } \\
(\mathrm{ml} / \mathrm{l})\end{array}$ & $\begin{array}{l}\text { CHANNELA } \\
\text { (\%fluor) }\end{array}$ & $\begin{array}{l}\text { CHANNELB } \\
\text { (\%tight) }\end{array}$ \\
\hline 0.0 & E & 10.797 & 31.265 & 23.904 & 0.0000 & 6.443 & 19.6 & 78.7 \\
\hline 10.0 & & 7.786 & 32.030 & 24.973 & 0.0387 & 7.113 & 21.4 & 78.7 \\
\hline 20.0 & & 5.179 & 32.457 & 25.640 & 0.0629 & 6.837 & 7.4 & 87.9 \\
\hline 30.0 & & 4.336 & 32.560 & 25.812 & 0.0849 & 6.671 & 4.7 & 88.5 \\
\hline 40.0 & & 4.029 & 32.587 & 25.864 & 0.1059 & 6.559 & 2.9 & 88.9 \\
\hline 50.0 & & 3.691 & 32.655 & 25.951 & 0.1262 & 6.460 & 2.1 & 88.7 \\
\hline 60.0 & & 3.599 & 32.664 & 25.967 & 0.1460 & 6.384 & 2.0 & 88.7 \\
\hline 70.0 & & 3.962 & 32.754 & 26.004 & 0.1657 & 6.269 & 2.0 & 87.7 \\
\hline 80.0 & & 3.970 & 32.789 & 26.031 & 0.1850 & 6.171 & 2.0 & 87.6 \\
\hline 90.0 & & 4.029 & 32.820 & 26.049 & 0.2041 & 6.106 & 2.1 & 84.8 \\
\hline 100.0 & & 4.024 & 32.830 & 26.058 & 0.2231 & 6.107 & 2.0 & 86.7 \\
\hline 110.0 & & 4.057 & 32.866 & 26.083 & 0.2420 & 6.070 & 2.0 & 86.6 \\
\hline 120.0 & & 4.128 & 32.917 & 26.117 & 0.2606 & 6.002 & 2.0 & 85.2 \\
\hline 130.0 & & 4.186 & 32.973 & 26.155 & 0.2787 & 5.935 & 2.0 & 84.4 \\
\hline 140.0 & & 4.259 & 33.010 & 26.177 & 0.2967 & 5.906 & 2.0 & 85.0 \\
\hline 150.0 & & 4.433 & 33.128 & 26.253 & 0.3142 & 5.803 & 1.9 & 83.7 \\
\hline 153.0 & & 4.454 & 33.141 & 26.261 & 0.3194 & 5.784 & 1.8 & 83.7 \\
\hline
\end{tabular}
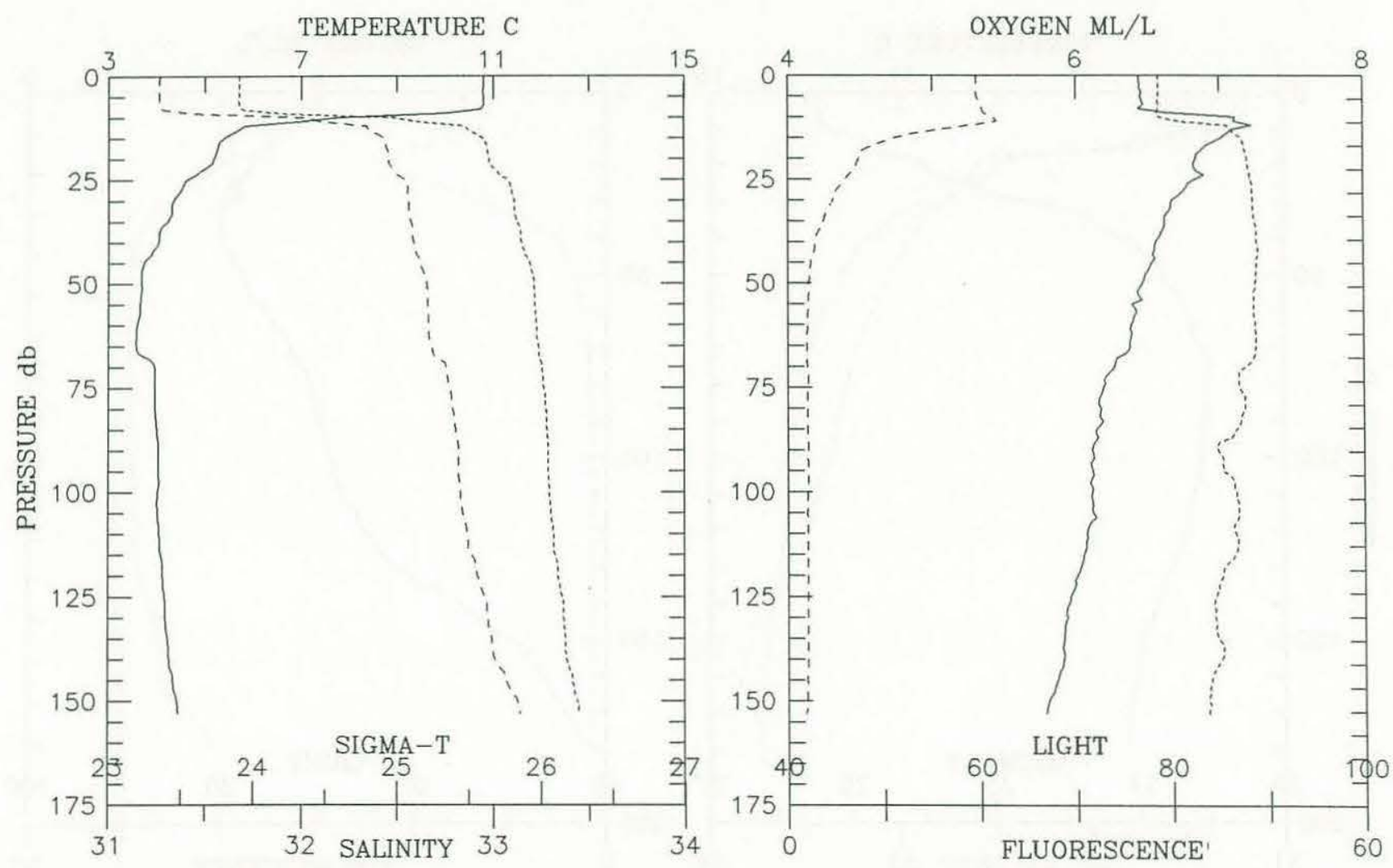
Cruise EN196 Consecutive Station \# 188 Depth m:124 Time: 1989-06-10 05:59 Position: $41^{\circ} 46.29^{\prime} \mathrm{N} 69^{\circ} 34.13^{\prime} \mathrm{W}$ Depth Deck offset: -0.15 Fish 1

\begin{tabular}{|c|c|c|c|c|c|c|c|c|}
\hline $\begin{array}{l}\text { PRESSURE } \\
\text { (d-bars) }\end{array}$ & Interp & $\begin{array}{c}\text { TEMP } \\
(\operatorname{deg} C)\end{array}$ & $\begin{array}{c}\text { SALINITY } \\
(0 / 00)\end{array}$ & SIGMA-T & DELTA-D & $\begin{array}{l}\text { OXYGEN } \\
(\mathrm{ml} / \mathrm{l})\end{array}$ & $\begin{array}{l}\text { CHANNELA } \\
\text { (\%fluor) }\end{array}$ & $\begin{array}{l}\text { CHANNELB } \\
\text { (\%light) }\end{array}$ \\
\hline 0.0 & E & 11.267 & 30.974 & 23.596 & 0.0000 & 6.190 & 14.8 & 81.0 \\
\hline 10.0 & & 11.120 & 30.999 & 23.642 & 0.0424 & 6.278 & 15.4 & 81.1 \\
\hline 20.0 & & 5.766 & 32.181 & 25.354 & 0.0765 & 6.853 & 9.4 & 86.4 \\
\hline 30.0 & & 4.564 & 32.421 & 25.678 & 0.1004 & 6.246 & 4.4 & 87.2 \\
\hline 40.0 & & 4.537 & 32.433 & 25.690 & 0.1228 & 6.018 & 3.5 & 87.3 \\
\hline 50.0 & & 4.526 & 32.449 & 25.704 & 0.1452 & 5.999 & 3.5 & 87.2 \\
\hline 60.0 & & 4.446 & 32.473 & 25.731 & 0.1674 & 5.979 & 3.5 & 86.6 \\
\hline 70.0 & & 4.443 & 32.498 & 25.751 & 0.1893 & 5.979 & 3.1 & 86.9 \\
\hline 80.0 & & 4.374 & 32.511 & 25.769 & 0.2111 & 5.956 & 3.1 & 87.0 \\
\hline 90.0 & & 4.328 & 32.543 & 25.799 & 0.2327 & 5.881 & 3.1 & 84.9 \\
\hline 100.0 & & 4.267 & 32.570 & 25.827 & 0.2540 & 5.877 & 3.0 & 84.7 \\
\hline 110.0 & & 4.182 & 32.602 & 25.861 & 0.2750 & 5.868 & 2.9 & 84.1 \\
\hline 116.0 & & 4.108 & 32.636 & 25.895 & 0.2874 & 5.904 & 2.7 & 83.4 \\
\hline
\end{tabular}
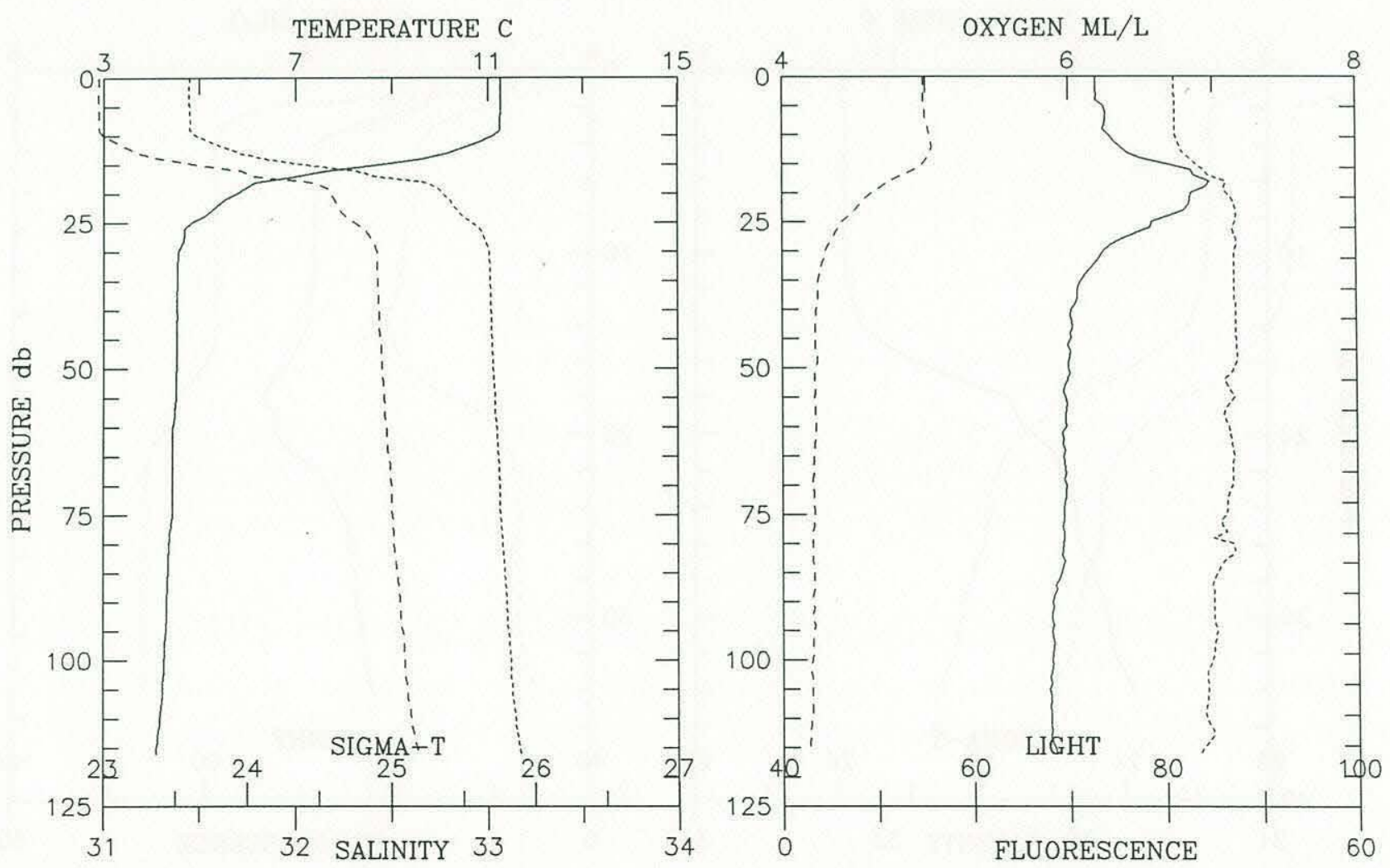


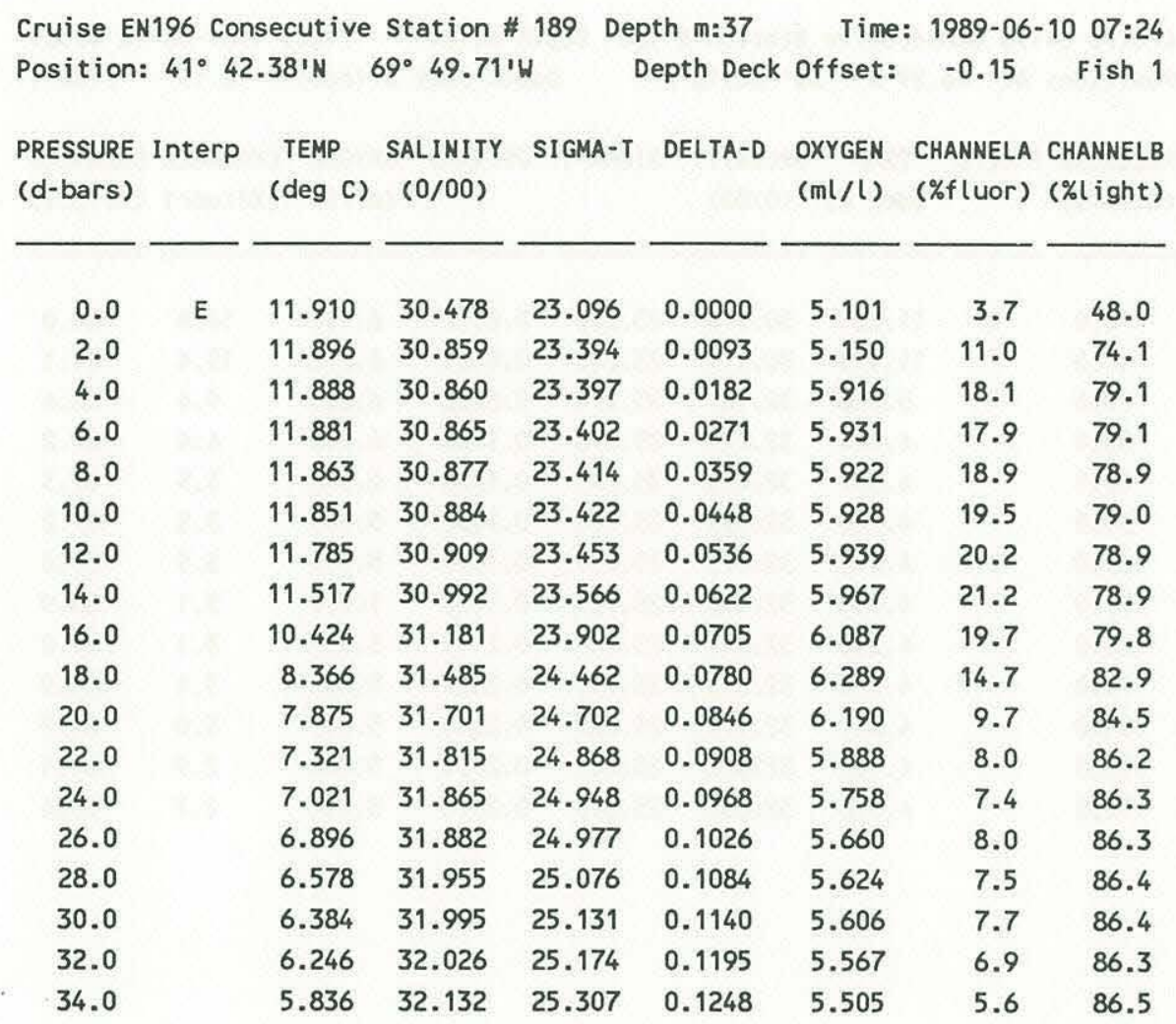
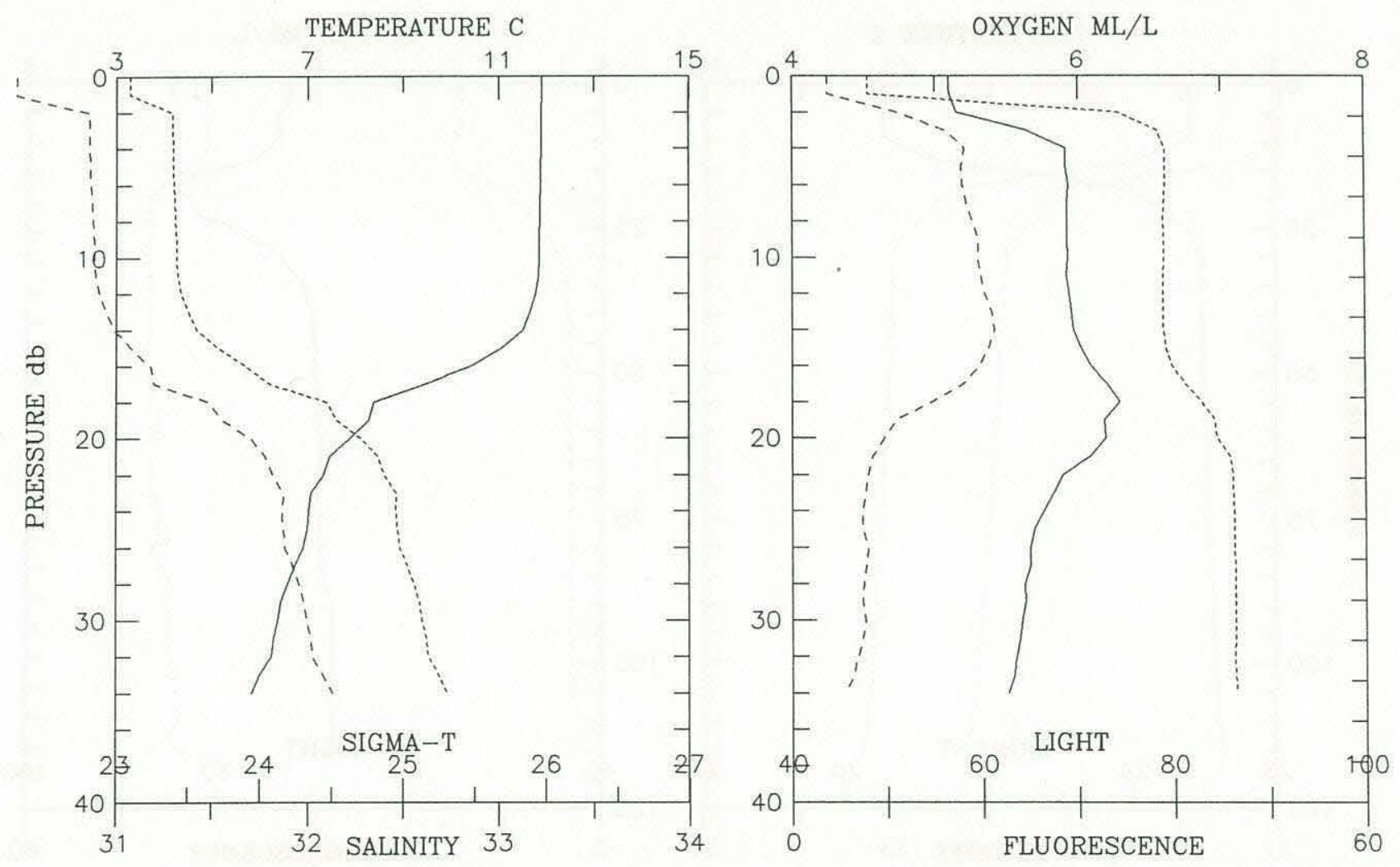
Cruise EN196 Consecutive Station \# 190 Depth m:21 Time: 1989-06-10 08:32 Position: $41^{\circ} 40.00^{\prime} \mathrm{N} 69^{\circ} 53.30^{\prime} \mathrm{H}$ Depth Deck offset: -0.15 Fish 1

\begin{tabular}{|c|c|c|c|c|c|c|c|c|}
\hline $\begin{array}{l}\text { PRESSURE } \\
\text { (d-bars) }\end{array}$ & Interp & $\begin{array}{c}\text { TEMP } \\
(\operatorname{deg} C)\end{array}$ & $\begin{array}{l}\text { SALINITY } \\
(0 / 00)\end{array}$ & SIGMA-T & DELTA-D & $\begin{array}{l}\text { OXYGEN } \\
(\mathrm{ml} / l)\end{array}$ & $\begin{array}{l}\text { CHANNELA } \\
\text { (\%fluor) }\end{array}$ & $\begin{array}{l}\text { CHANNELB } \\
(\%(\text { ight) }\end{array}$ \\
\hline 0.0 & E & 12.100 & 30.887 & 23.379 & 0.0000 & 5.597 & 12.4 & 80.9 \\
\hline 2.0 & E & 12.100 & 30.886 & 23.378 & 0.0089 & 5.598 & 12.4 & 80.9 \\
\hline 4.0 & & 12.095 & 30.886 & 23.379 & 0.0178 & 5.702 & 13.8 & 81.0 \\
\hline 6.0 & & 12.095 & 30.886 & 23.379 & 0.0267 & 5.739 & 14.8 & 80.9 \\
\hline 8.0 & & 12.088 & 30.888 & 23.382 & 0.0356 & 5.756 & 14.6 & 80.9 \\
\hline 10.0 & & 11.986 & 30.912 & 23.419 & 0.0445 & 5.756 & 15.2 & 80.6 \\
\hline 12.0 & & 11.665 & 30.976 & 23.527 & 0.0533 & 5.798 & 16.1 & 79.5 \\
\hline 14.0 & & 11.426 & 31.020 & 23.604 & 0.0618 & 5.806 & 19.1 & 78.7 \\
\hline 16.0 & & 10.774 & 31.155 & 23.822 & 0.0701 & 5.919 & 20.1 & 79.7 \\
\hline 18.0 & & 10.662 & 31.177 & 23.859 & 0.0781 & 5.830 & 20.0 & 79.8 \\
\hline 19.0 & & 10.656 & 31.179 & 23.861 & 0.0821 & 5.838 & 20.1 & 79.8 \\
\hline
\end{tabular}
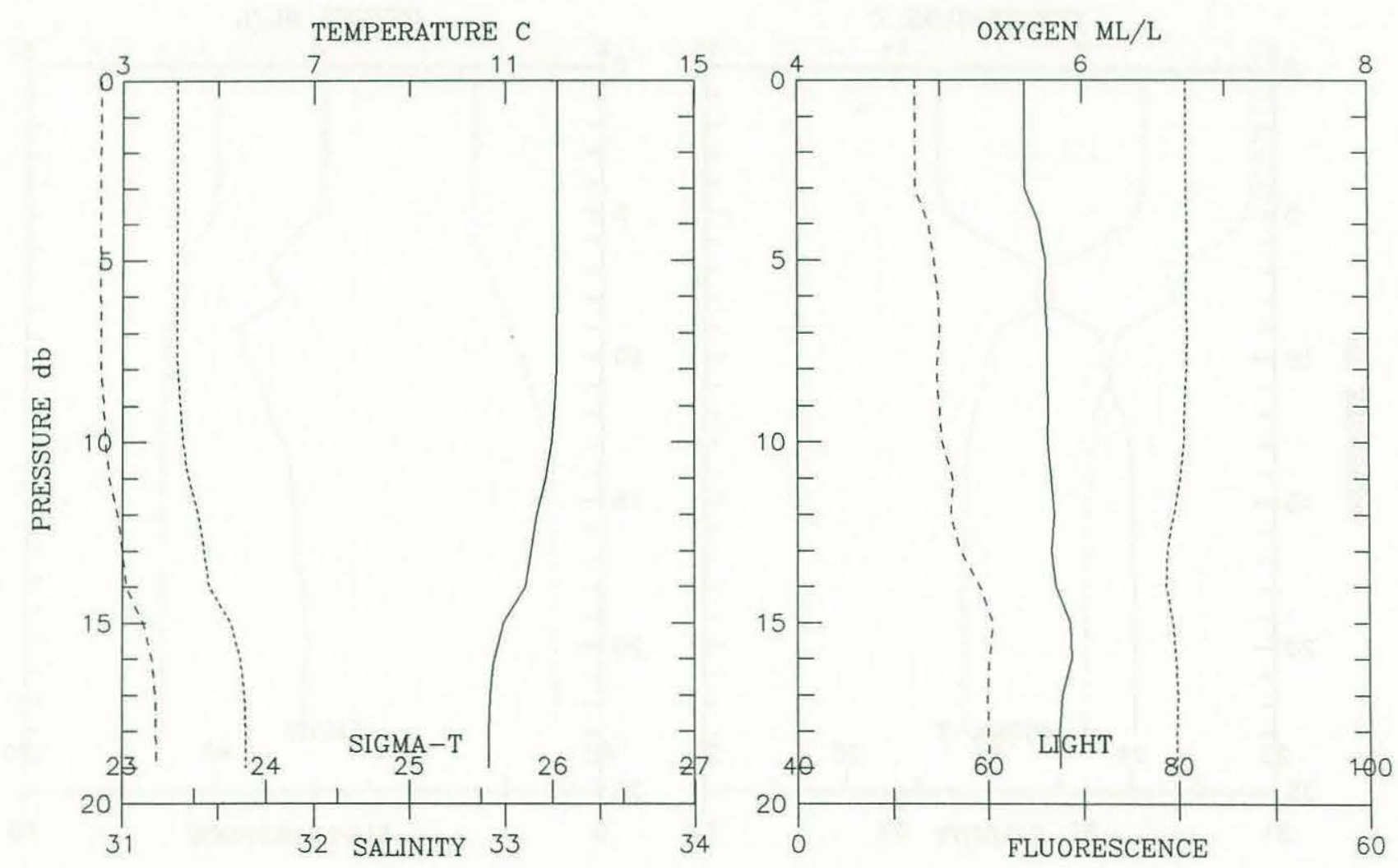


\begin{tabular}{|c|c|c|c|c|c|c|c|c|}
\hline Position: & : $41^{\circ} 58$ & $.80^{\prime} \mathrm{N}$ & $69^{\circ} 58.80^{\prime}$ & & epth Deck & Offset: & -0.15 & Fish 1 \\
\hline $\begin{array}{l}\text { PRESSURE } \\
\text { (d-bars) }\end{array}$ & Interp & $\begin{array}{c}\text { TEMP } \\
(\operatorname{deg} \mathrm{C})\end{array}$ & $\begin{array}{l}\text { SALINITY } \\
(0 / 00)\end{array}$ & SIGMA-T & DELTA-D & $\begin{array}{l}\text { OXYGEN } \\
(\mathrm{ml} / \mathrm{l})\end{array}$ & $\begin{array}{l}\text { CHANNELA } \\
\text { (\%fluor) }\end{array}$ & $\begin{array}{l}\text { CHANNELB } \\
\text { (\%light) }\end{array}$ \\
\hline 0.0 & E & 10.083 & 31.111 & 23.904 & 0.0000 & 5.943 & 13.4 & 80.2 \\
\hline 2.0 & $E$ & 10.083 & 31.110 & 23.903 & 0.0079 & 5.944 & 13.4 & 80.2 \\
\hline 4.0 & & 10.083 & 31.109 & 23.903 & 0.0158 & 5.946 & 13.4 & 80.2 \\
\hline 6.0 & & 9.014 & 31.273 & 24.199 & 0.0235 & 6.184 & 13.6 & 82.0 \\
\hline 8.0 & & 8.039 & 31.508 & 24.527 & 0.0303 & 6.220 & 11.0 & 83.5 \\
\hline 10.0 & & 6.514 & 31.849 & 25.001 & 0.0364 & 6.491 & 8.4 & 85.6 \\
\hline 12.0 & & 6.153 & 31.937 & 25.114 & 0.0421 & 6.318 & 6.7 & 85.8 \\
\hline 14.0 & & 5.964 & 31.974 & 25.167 & 0.0476 & 6.168 & 5.2 & 85.9 \\
\hline 16.0 & & 5.940 & 31.980 & 25.174 & 0.0531 & 6.095 & 4.9 & 85.9 \\
\hline 18.0 & & 5.927 & 31.985 & 25.180 & 0.0586 & 6.070 & 4.9 & 85.9 \\
\hline 20.0 & & 5.920 & 31.986 & 25.181 & 0.0640 & 6.039 & 4.9 & 85.9 \\
\hline 22.0 & & 5.912 & 31.989 & 25.185 & 0.0695 & 6.076 & 4.8 & 85.8 \\
\hline 23.0 & & 5.912 & 31.987 & 25.183 & 0.0722 & 6.038 & 4.8 & 85.5 \\
\hline
\end{tabular}
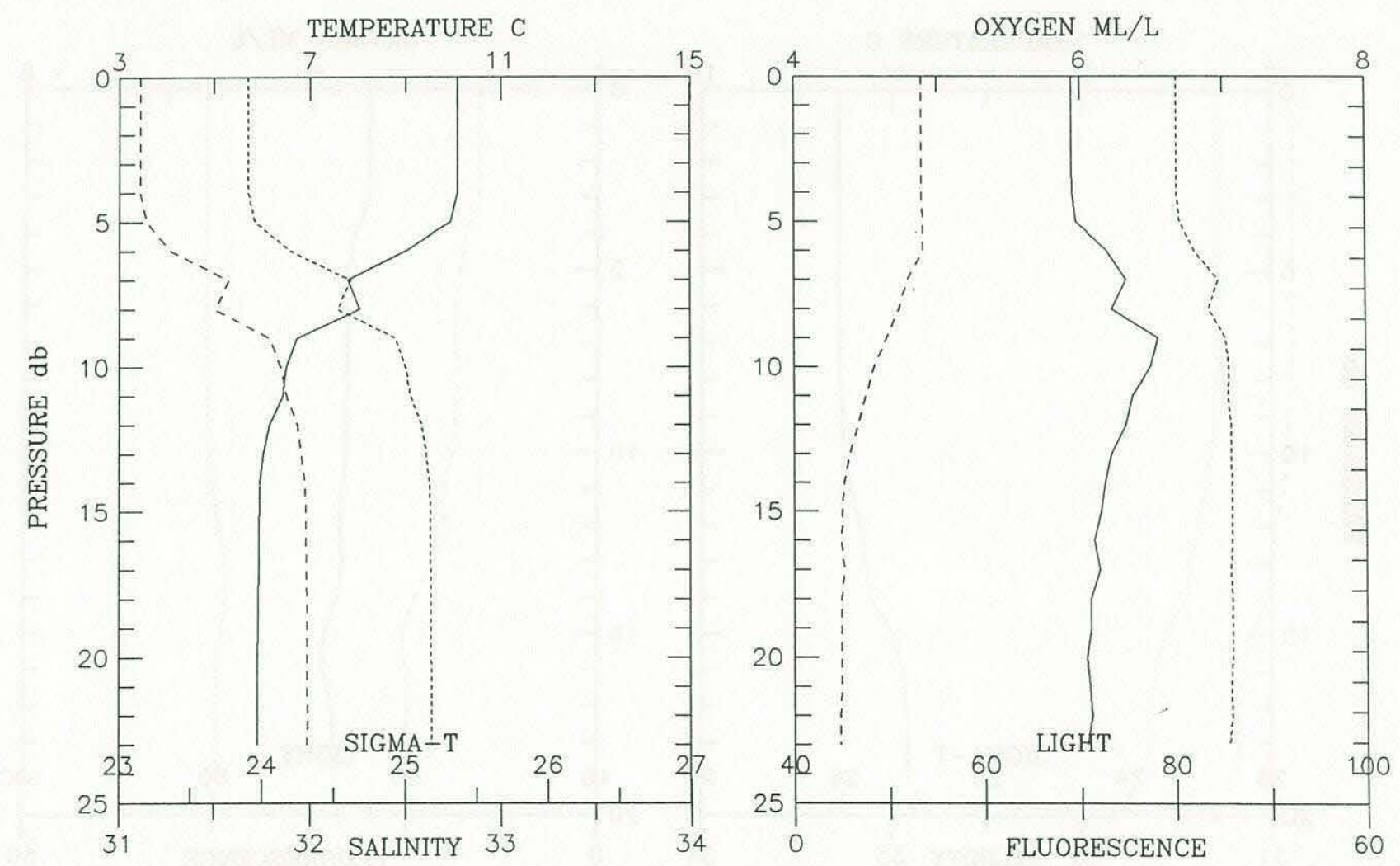
Cruise EN196 Consecutive Station \# 192 Depth m:41 Time: 1989-06-10 11:43 Position: $41^{\circ} 59.50^{\prime} \mathrm{N} 69^{\circ} 56.70^{\prime} \mathrm{W}$ Depth Deck offset: -0.15 Fish 1

\begin{tabular}{|c|c|c|c|c|c|c|c|c|}
\hline $\begin{array}{l}\text { PRESSURE } \\
\text { (d-bars) }\end{array}$ & Interp & $\begin{array}{c}\text { TEMP } \\
(\operatorname{deg} \mathrm{C})\end{array}$ & $\begin{array}{c}\text { SALINITY } \\
(0 / 00)\end{array}$ & SIGMA-T & DELTA-D & $\begin{array}{l}\text { OXYGEN } \\
(\mathrm{ml} / \mathrm{l})\end{array}$ & $\begin{array}{l}\text { CHANNELA } \\
\text { ( } \% \text { fluor) }\end{array}$ & $\begin{array}{l}\text { CHANNELB } \\
\text { (\%light) }\end{array}$ \\
\hline 0.0 & E & 11.671 & 30.759 & 23.357 & 0.0000 & 5.827 & 17.4 & 79.7 \\
\hline 2.0 & E & 11.671 & 30.759 & 23.357 & 0.0090 & 5.829 & 17.4 & 79.7 \\
\hline 4.0 & & 11.636 & 30.762 & 23.366 & 0.0179 & 5.827 & 17.3 & 79.7 \\
\hline 6.0 & & 10.720 & 30.911 & 23.641 & 0.0267 & 5.956 & 17.2 & 80.2 \\
\hline 8.0 & & 6.467 & 31.821 & 24.984 & 0.0339 & 6.861 & 15.0 & 84.2 \\
\hline 10.0 & & 5.882 & 31.962 & 25.167 & 0.0396 & 6.838 & 8.3 & 86.8 \\
\hline 12.0 & & 5.396 & 32.119 & 25.348 & 0.0448 & 6.805 & 6.0 & 87.2 \\
\hline 14.0 & & 5.159 & 32.172 & 25.417 & 0.0499 & 6.658 & 5.3 & 87.2 \\
\hline 16.0 & & 5.100 & 32.188 & 25.436 & 0.0549 & 6.363 & 5.0 & 86.8 \\
\hline 18.0 & & 5.042 & 32.197 & 25.449 & 0.0598 & 6.337 & 4.4 & 86.7 \\
\hline 20.0 & & 4.988 & 32.214 & 25.469 & 0.0647 & 6.126 & 4.2 & 86.2 \\
\hline 22.0 & & 4.940 & 32.224 & 25.481 & 0.0696 & 6.121 & 4.0 & 85.8 \\
\hline 24.0 & & 4.848 & 32.246 & 25.509 & 0.0745 & 5.935 & 4.3 & 85.6 \\
\hline 26.0 & & 4.785 & 32.262 & 25.529 & 0.0793 & 6.004 & 4.1 & 85.5 \\
\hline 28.0 & & 4.756 & 32.269 & 25.537 & 0.0841 & 5.819 & 3.8 & 85.5 \\
\hline 30.0 & & 4.713 & 32.283 & 25.553 & 0.0888 & 5.870 & 3.7 & 85.5 \\
\hline 32.0 & & 4.698 & 32.290 & 25.560 & 0.0936 & 5.843 & 3.6 & 85.3 \\
\hline 34.0 & & 4.688 & 32.293 & 25.563 & 0.0983 & 5.754 & 3.7 & 85.5 \\
\hline 36.0 & & 4.686 & 32.294 & 25.564 & 0.1030 & 5.697 & 3.7 & 85.6 \\
\hline 38.0 & & 4.687 & 32.294 & 25.564 & 0.1077 & 5.679 & 3.6 & 85.5 \\
\hline
\end{tabular}
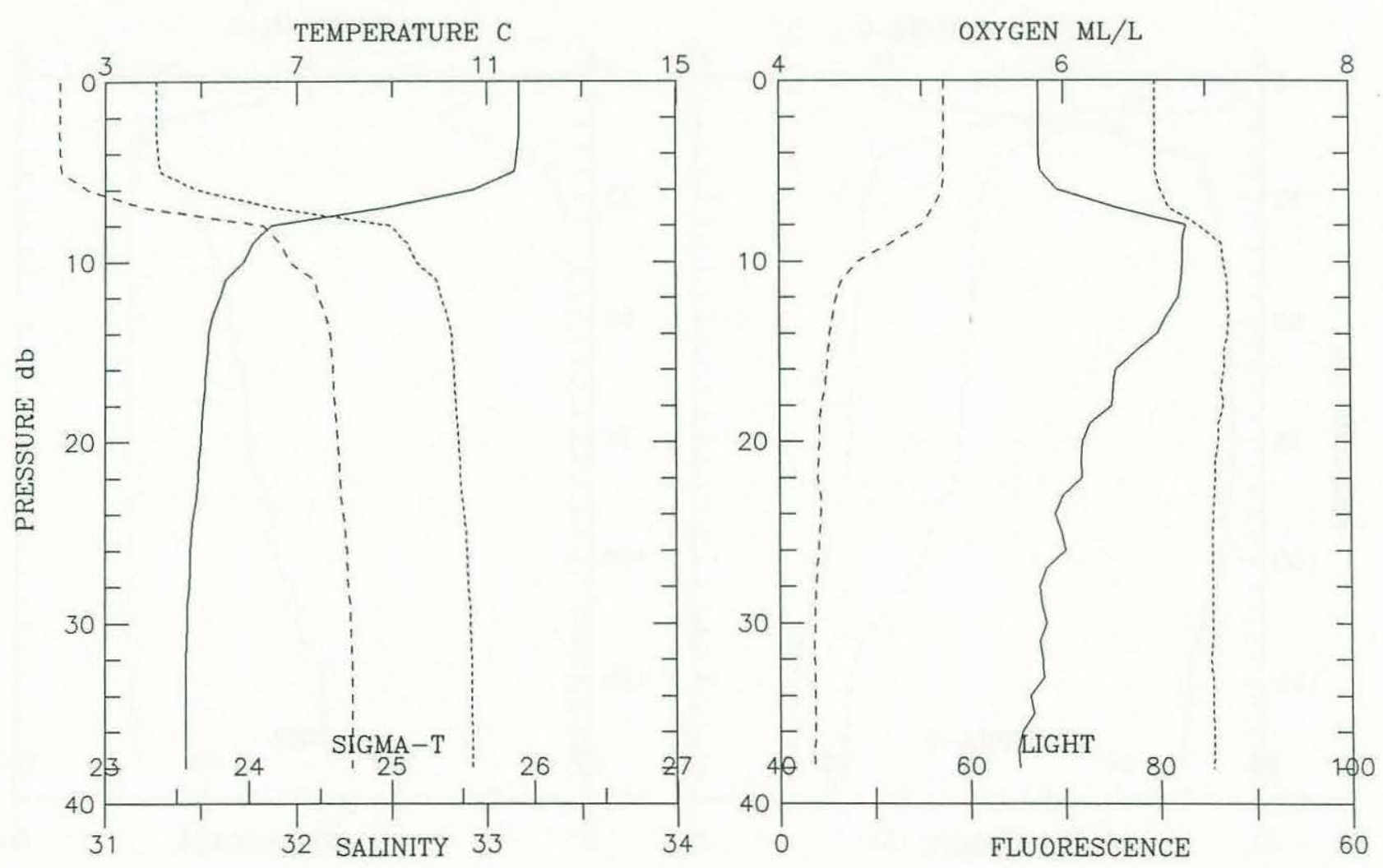
Cruise EN196 Consecutive Station \# 193 Depth m:153 Time: 1989-06-10 13:15 Position: $42^{\circ} 1.10^{\prime} \mathrm{N} 69^{\circ} 48.20^{\prime} \mathrm{W} \quad$ Depth Deck offset: -0.15 Fish 1

\begin{tabular}{|c|c|c|c|c|c|c|c|c|}
\hline $\begin{array}{l}\text { PRESSURE } \\
\text { (d-bars) }\end{array}$ & Interp & $\begin{array}{c}\text { TEMP } \\
(\operatorname{deg} \mathrm{C})\end{array}$ & $\begin{array}{l}\text { SALINITY } \\
(0 / 00)\end{array}$ & SIGMA-T & DELTA-D & $\begin{array}{l}\text { OXYGEN } \\
(\mathrm{ml} / \mathrm{l})\end{array}$ & $\begin{array}{l}\text { CHANNELA } \\
\text { (\%fluor) }\end{array}$ & $\begin{array}{l}\text { CHANNELB } \\
\text { (\%light) }\end{array}$ \\
\hline 0.0 & $E$ & 10.646 & 31.146 & 23.837 & 0.0000 & 6.272 & 12.5 & 79.0 \\
\hline 10.0 & & 6.584 & 32.019 & 25.125 & 0.0371 & 7.119 & 16.1 & 84.8 \\
\hline 20.0 & & 4.247 & 32.456 & 25.739 & 0.0611 & 6.963 & 4.5 & 88.2 \\
\hline 30.0 & & 3.958 & 32.526 & 25.823 & 0.0826 & 6.745 & 2.8 & 88.5 \\
\hline 40.0 & & 3.823 & 32.524 & 25.834 & 0.1037 & 6.639 & 2.4 & 88.3 \\
\hline 50.0 & & 3.687 & 32.547 & 25.865 & 0.1246 & 6.516 & 2.2 & 88.3 \\
\hline 60.0 & & 3.615 & 32.567 & 25.888 & 0.1452 & 6.454 & 2.0 & 88.4 \\
\hline 70.0 & & 3.627 & 32.585 & 25.901 & 0.1656 & 6.426 & 2.0 & 88.3 \\
\hline 80.0 & & 3.645 & 32.612 & 25.921 & 0.1859 & 6.365 & 1.9 & 88.4 \\
\hline 90.0 & & 3.758 & 32.735 & 26.008 & 0.2059 & 6.229 & 1.8 & 88.1 \\
\hline 100.0 & & 3.853 & 32.800 & 26.051 & 0.2251 & 6.208 & 1.8 & 88.1 \\
\hline 110.0 & & 4.210 & 32.982 & 26.160 & 0.2436 & 6.029 & 1.7 & 88.6 \\
\hline 120.0 & & 4.454 & 33.134 & 26.255 & 0.2611 & 5.930 & 1.6 & 86.8 \\
\hline 130.0 & & 4.591 & 33.181 & 26.278 & 0.2781 & 5.867 & 1.7 & 87.6 \\
\hline 140.0 & & 4.618 & 33.197 & 26.288 & 0.2950 & 5.842 & 1.7 & 87.2 \\
\hline 143.0 & & 4.636 & 33.211 & 26.297 & 0.3000 & 5.826 & 1.7 & 86.8 \\
\hline
\end{tabular}
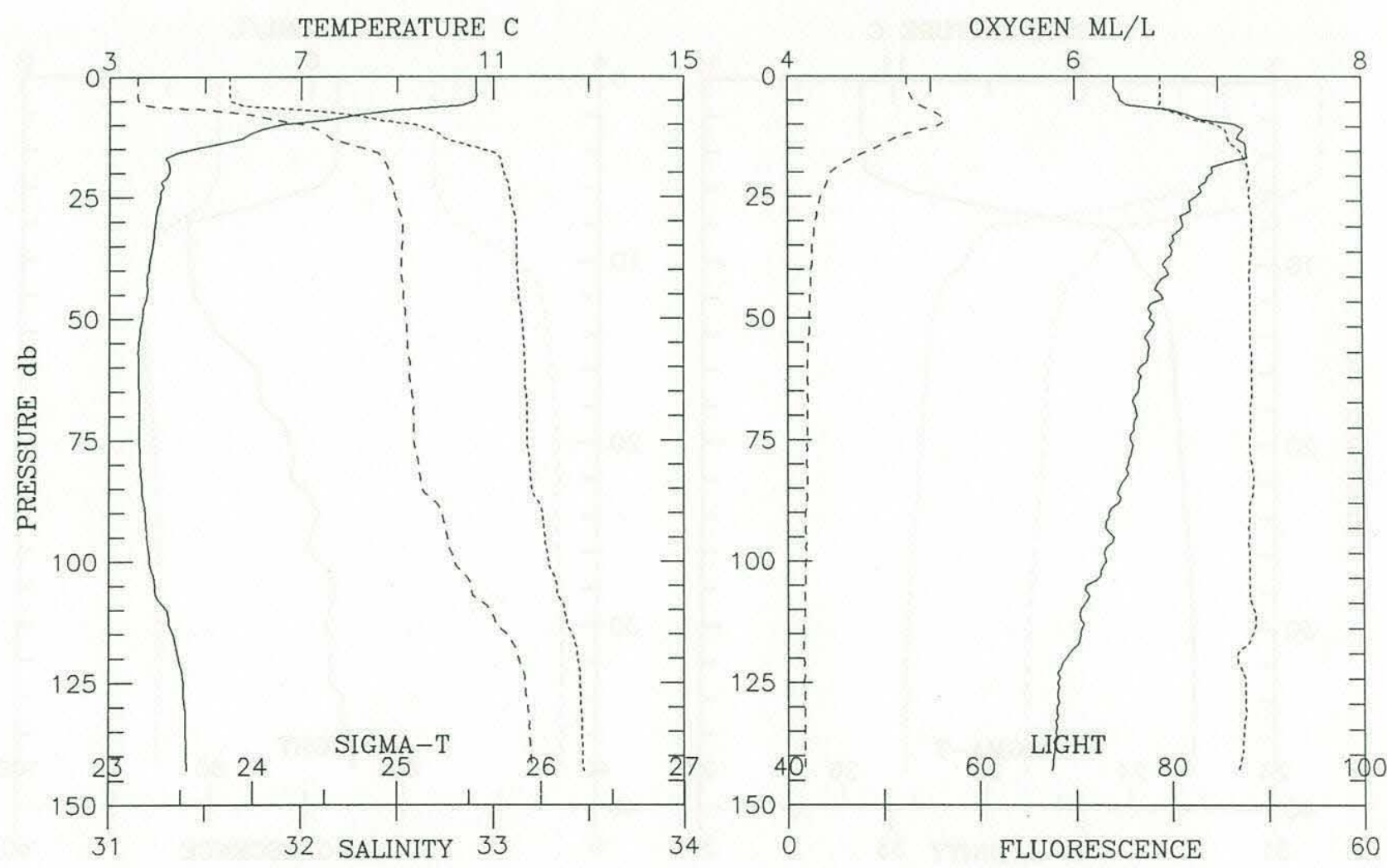


\begin{tabular}{|c|c|c|c|c|c|c|c|c|}
\hline Position: & $=42^{\circ}$ & 90 N & $69^{\circ} 41.40^{\prime}$ & & epth Deck & Offset: & -0.10 & Fish 1 \\
\hline $\begin{array}{l}\text { PRESSURE } \\
\text { (d-bars) }\end{array}$ & Interp & $\begin{array}{c}\text { TEMP } \\
(\operatorname{deg} C)\end{array}$ & $\begin{array}{l}\text { SALINITY } \\
(0 / 00)\end{array}$ & SI GMA-T & DELTA-D & $\begin{array}{l}\text { OXYGEN } \\
(\mathrm{ml} / \mathrm{l})\end{array}$ & $\begin{array}{l}\text { CHANNELA } \\
\text { ( } \% \text { fluor) }\end{array}$ & $\begin{array}{l}\text { CHANNELB } \\
\text { (\%light) }\end{array}$ \\
\hline 0.0 & E & 10.842 & 31.213 & 23.856 & 0.0000 & 6.346 & 12.4 & 78.3 \\
\hline 10.0 & & 10.723 & 31.230 & 23.889 & 0.0399 & 6.357 & 17.7 & 78.4 \\
\hline 20.0 & & 9.028 & 32.680 & 25.298 & 0.0720 & 6.770 & 18.5 & 82.2 \\
\hline 30.0 & & 6.860 & 32.916 & 25.796 & 0.0955 & 6.964 & 8.8 & 87.8 \\
\hline 40.0 & & 6.108 & 32.971 & 25.936 & 0.1163 & 6.794 & 5.8 & 88.1 \\
\hline 50.0 & & 5.378 & 33.068 & 26.101 & 0.1355 & 6.566 & 3.7 & 89.3 \\
\hline 60.0 & & 5.103 & 33.126 & 26.178 & 0.1537 & 6.424 & 2.2 & 89.5 \\
\hline 70.0 & & 5.005 & 33.152 & 26.210 & 0.1714 & 6.265 & 1.9 & 89.6 \\
\hline 80.0 & & 4.761 & 33.192 & 26.269 & 0.1885 & 6.216 & 1.8 & 89.5 \\
\hline 90.0 & & 4.608 & 33.231 & 26.316 & 0.2051 & 6.130 & 1.6 & 89.4 \\
\hline 100.0 & & 4.553 & 33.271 & 26.354 & 0.2214 & 6.198 & 1.5 & 89.2 \\
\hline 110.0 & & 4.622 & 33.327 & 26.391 & 0.2374 & 6.151 & 1.3 & 88.9 \\
\hline 120.0 & & 4.683 & 33.357 & 26.408 & 0.2532 & 6.019 & 1.3 & 89.0 \\
\hline 130.0 & & 4.731 & 33.390 & 26.429 & 0.2688 & 5.998 & 1.3 & 89.1 \\
\hline 140.0 & & 4.831 & 33.427 & 26.447 & 0.2842 & 5.959 & 1.3 & 89.1 \\
\hline 150.0 & & 5.046 & 33.491 & 26.474 & 0.2994 & 5.786 & 1.3 & 88.7 \\
\hline 160.0 & & 5.282 & 33.554 & 26.497 & 0.3144 & 5.468 & 1.5 & 87.0 \\
\hline 170.0 & & 5.543 & 33.641 & 26.535 & 0.3292 & 5.242 & 1.5 & 84.5 \\
\hline 180.0 & & 5.867 & 33.752 & 26.583 & 0.3436 & 4.723 & 1.6 & 82.3 \\
\hline 190.0 & & 6.143 & 33.848 & 26.624 & 0.3576 & 4.434 & 1.6 & 81.6 \\
\hline 200.0 & & 6.202 & 33.867 & 26.632 & 0.3713 & 4.214 & 1.7 & 79.2 \\
\hline
\end{tabular}
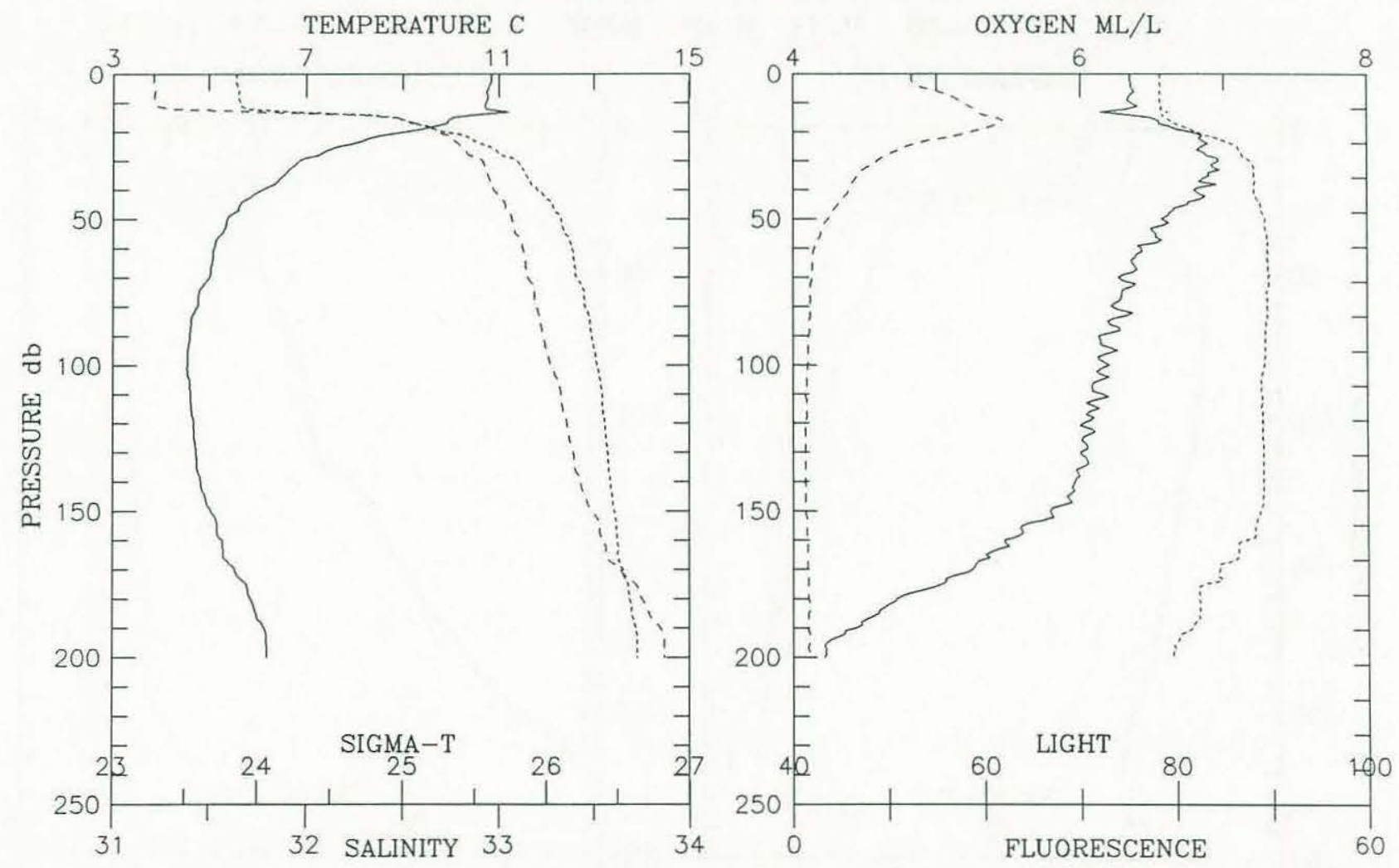
Cruise EN196 Consecutive Station \# 195 Depth m:228 Time: 1989-06-10 16:02 Position: $42^{\circ} 4.80^{\prime} \mathrm{N} 69^{\circ} 34.90^{\prime} \mathrm{W}$ Depth Deck offset: -0.10 Fish 1

PRESSURE InterP TEMP SALINITY SIGMA-T DELTA-D OXYGEN CHANNELA CHANNELB $\begin{array}{llll}\text { (d-bars) }(\operatorname{deg} C) & (0 / 00) & (\mathrm{ml} / \mathrm{l}) & (\% \text { light })(\% l i g h t)\end{array}$

\begin{tabular}{|c|c|c|c|c|c|c|c|c|}
\hline 0.0 & E & 13.506 & 32.044 & 24.004 & 0.0000 & 5.948 & 5.8 & 76.7 \\
\hline 10.0 & & 13.380 & 32.054 & 24.037 & 0.0385 & 5.910 & 8.1 & 75.8 \\
\hline 20.0 & & 11.837 & 32.316 & 24.536 & 0.0756 & 6.241 & 17.2 & 77.0 \\
\hline 30.0 & & 6.258 & 32.950 & 25.901 & 0.1008 & 6.878 & 10.5 & 87.8 \\
\hline 40.0 & & 5.356 & 33.068 & 26.103 & 0.1202 & 6.574 & 3.1 & 89.3 \\
\hline 50.0 & & 4.919 & 33.146 & 26.214 & 0.1382 & 6.374 & 1.8 & 89.6 \\
\hline 60.0 & & 4.625 & 33.202 & 26.291 & 0.1553 & 6.278 & 1.6 & 89.2 \\
\hline 70.0 & & 4.574 & 33.238 & 26.325 & 0.1719 & 6.164 & 1.6 & 89.1 \\
\hline 80.0 & & 4.654 & 33.330 & 26.390 & 0.1880 & 6.086 & 1.3 & 89.0 \\
\hline 90.0 & & 4.689 & 33.356 & 26.406 & 0.2038 & 6.081 & 1.3 & 89.0 \\
\hline 100.0 & & 4.732 & 33.380 & 26.421 & 0.2194 & 6.015 & 1.2 & 89.0 \\
\hline 110.0 & & 4.794 & 33.410 & 26.438 & 0.2348 & 5.981 & 1.2 & 89.2 \\
\hline 120.0 & & 5.011 & 33.476 & 26.466 & 0.2501 & 5.852 & 1.3 & 88.5 \\
\hline 130.0 & & 5.128 & 33.512 & 26.481 & 0.2652 & 5.631 & 1.3 & 88.1 \\
\hline 140.0 & & 5.197 & 33.531 & 26.489 & 0.2801 & 5.534 & 1.4 & 87.9 \\
\hline 150.0 & & 5.316 & 33.571 & 26.506 & 0.2950 & 5.398 & 1.4 & 87.4 \\
\hline 160.0 & & 5.405 & 33.596 & 26.515 & 0.3098 & 5.255 & 1.4 & 87.5 \\
\hline 170.0 & & 5.522 & 33.634 & 26.532 & 0.3245 & 5.103 & 1.5 & 86.8 \\
\hline 180.0 & & 5.640 & 33.672 & 26.548 & 0.3390 & 4.967 & 1.6 & 86.9 \\
\hline 190.0 & & 5.832 & 33.727 & 26.568 & 0.3534 & 4.782 & 1.5 & 86.9 \\
\hline 200.0 & & 6.048 & 33.801 & 26.600 & 0.3676 & 4.593 & 1.6 & 85.7 \\
\hline 210.0 & & 6.214 & 33.861 & 26.625 & 0.3815 & 4.296 & 1.6 & 84.2 \\
\hline 220.0 & & 6.253 & 33.874 & 26.631 & 0.3953 & 4.172 & 1.7 & 82.1 \\
\hline 221.0 & & 6.255 & 33.874 & 26.631 & 0.3967 & 4.157 & 1.7 & 81.8 \\
\hline
\end{tabular}
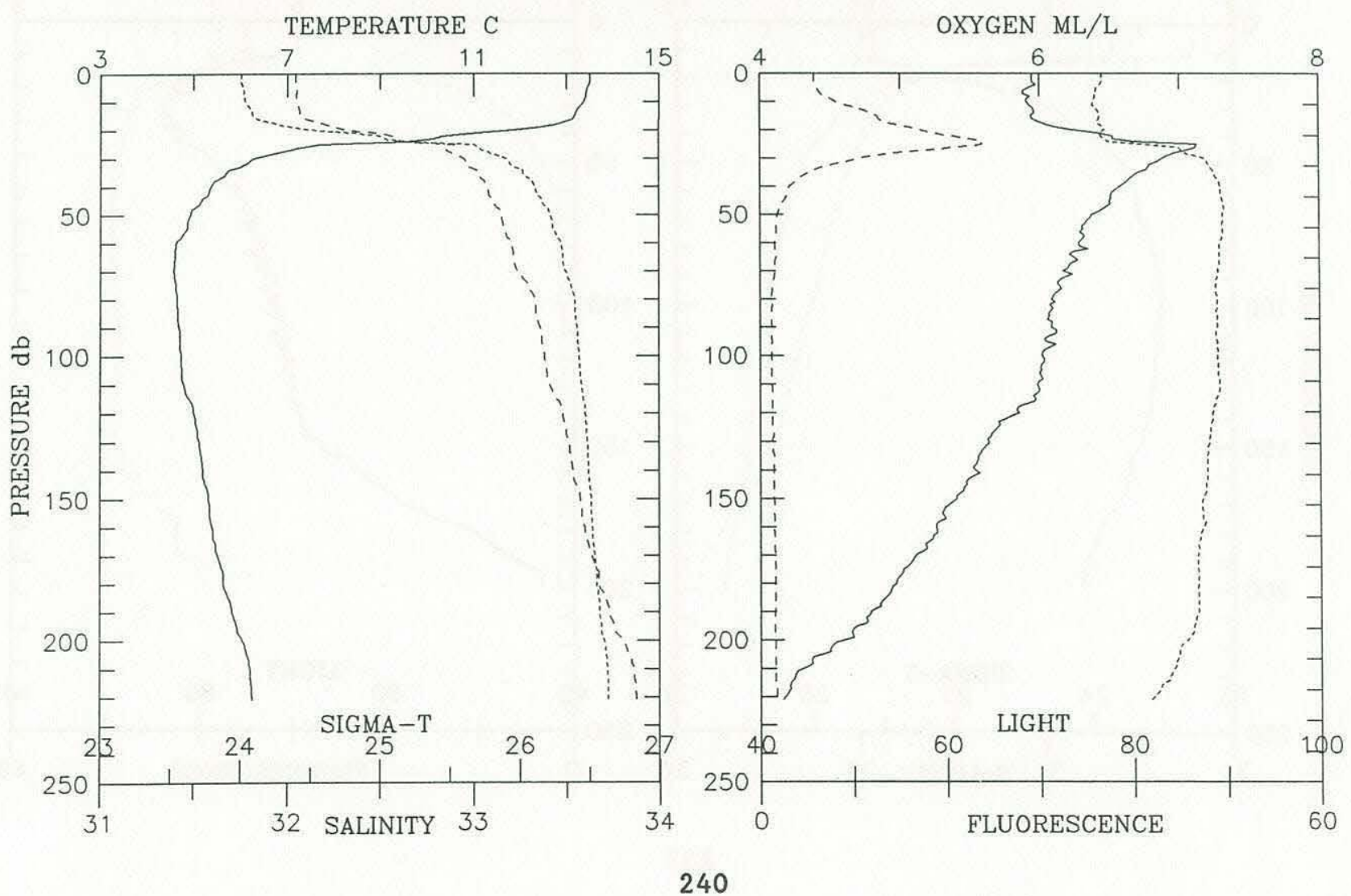


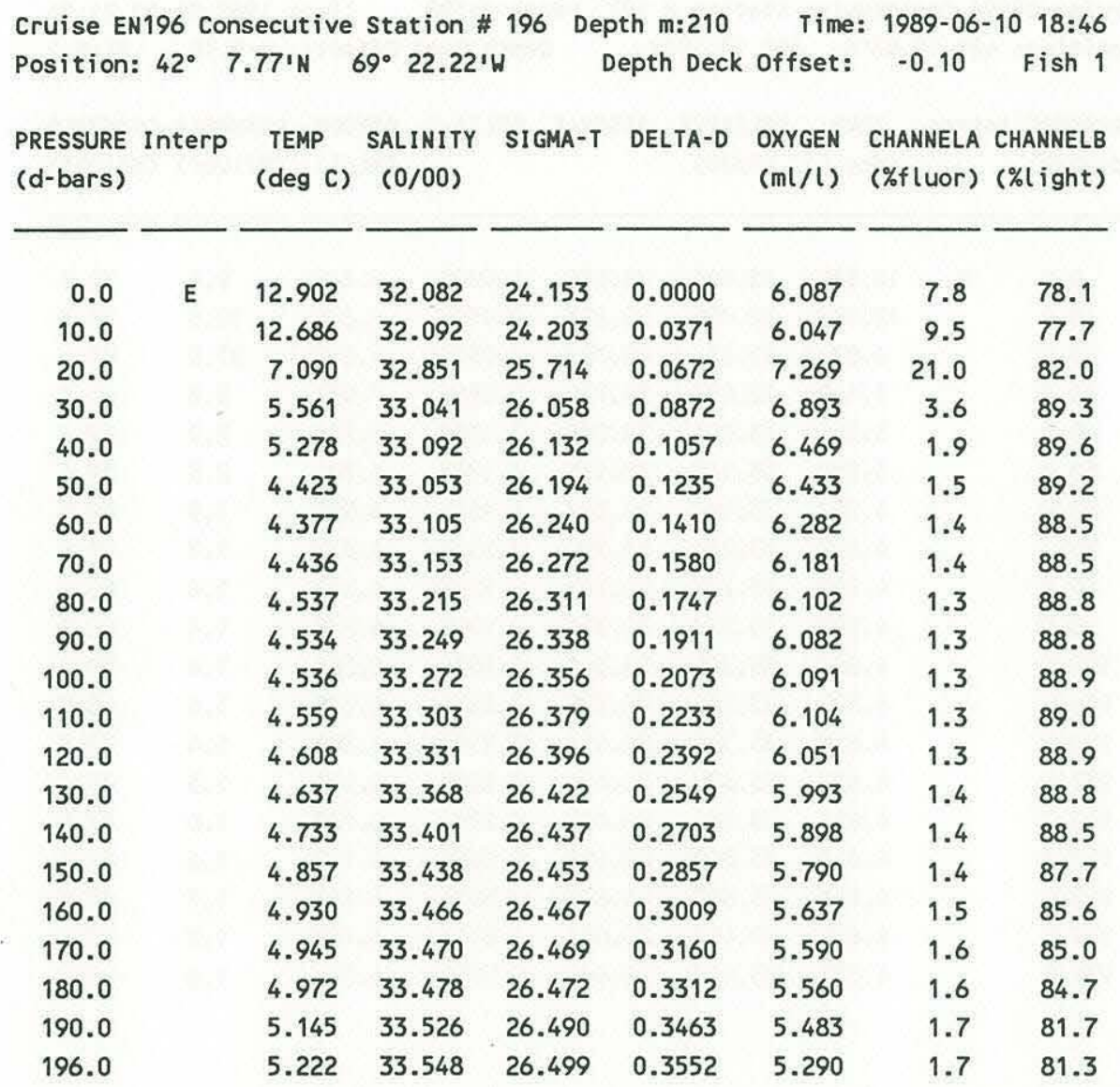
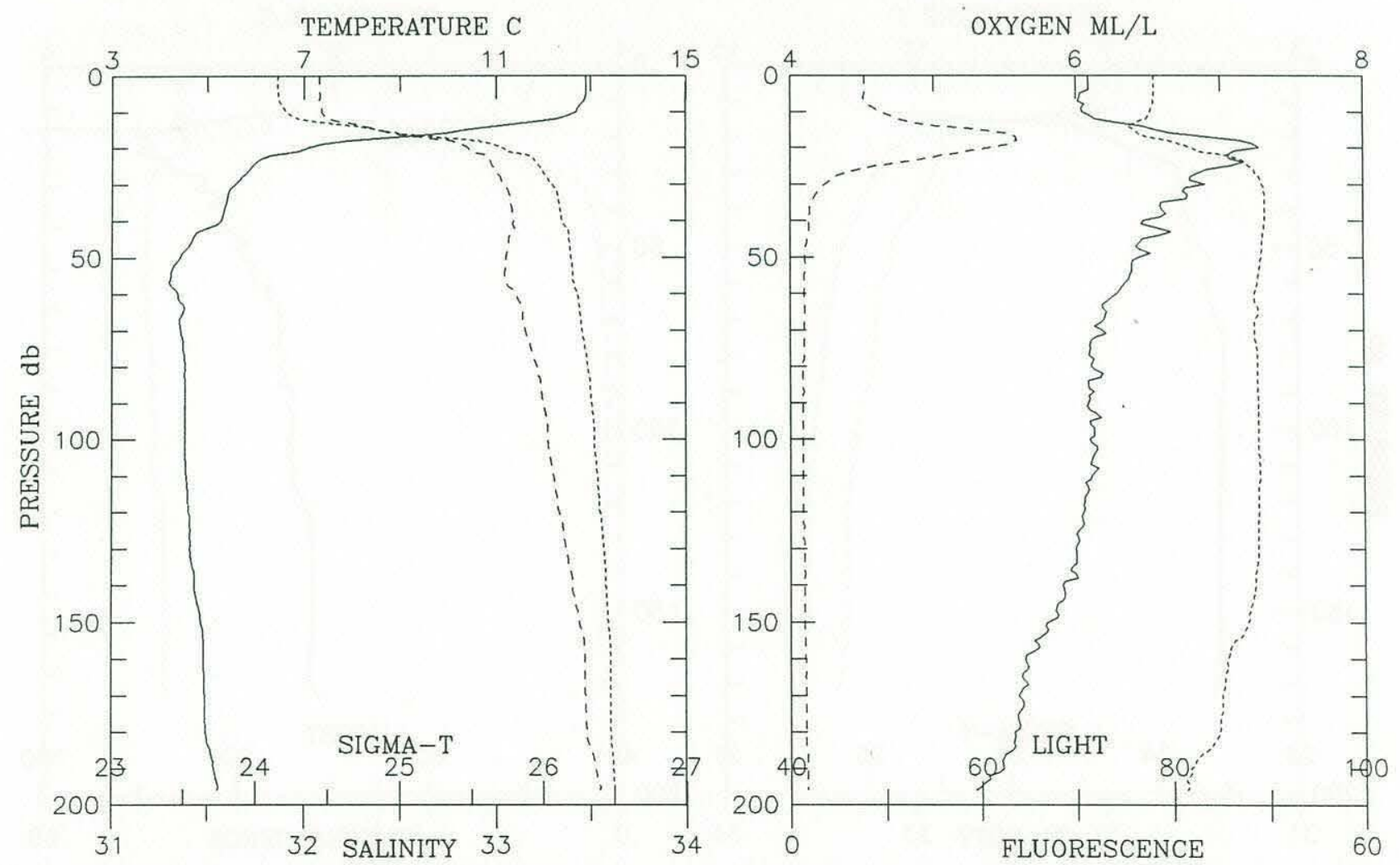


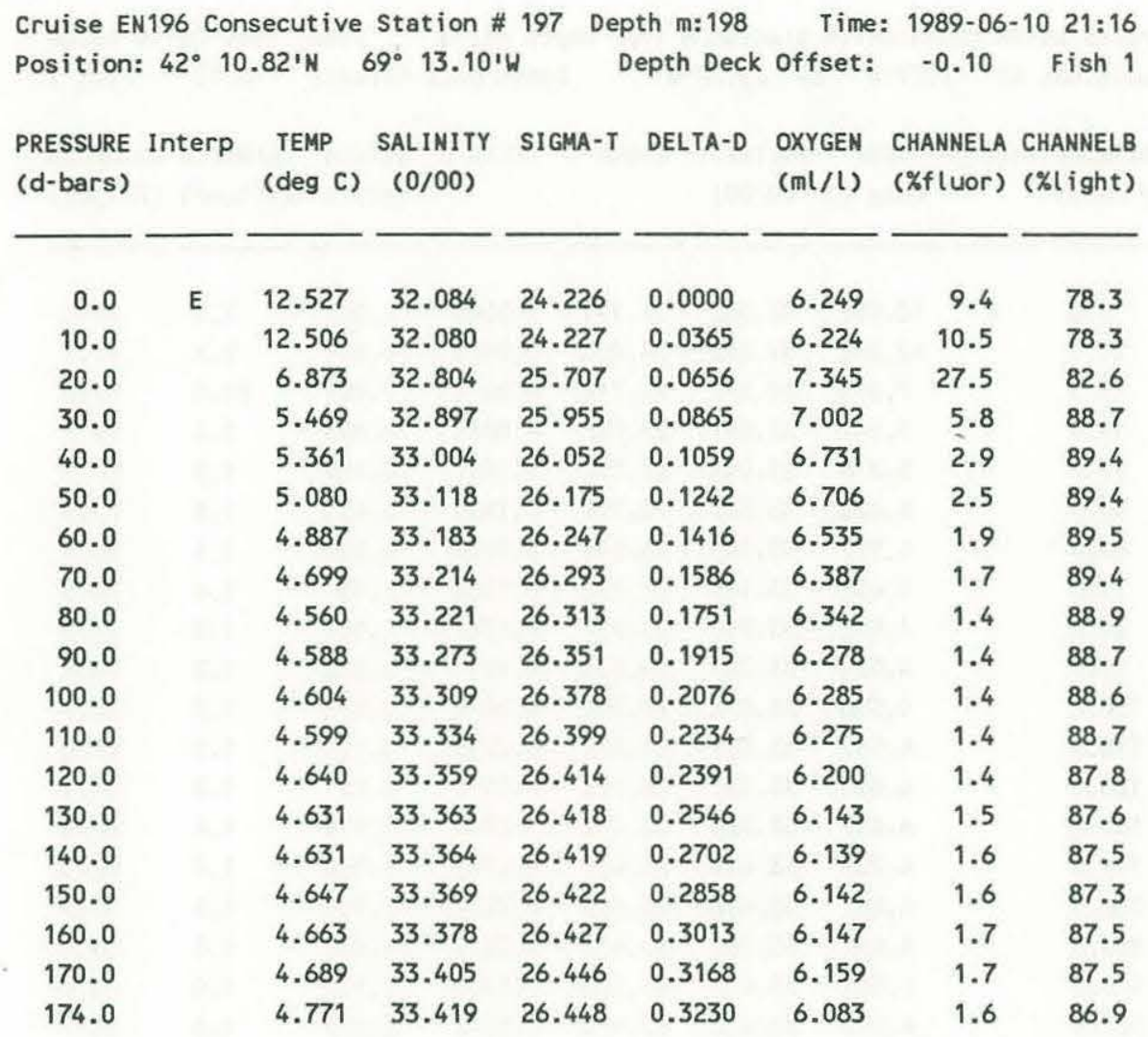
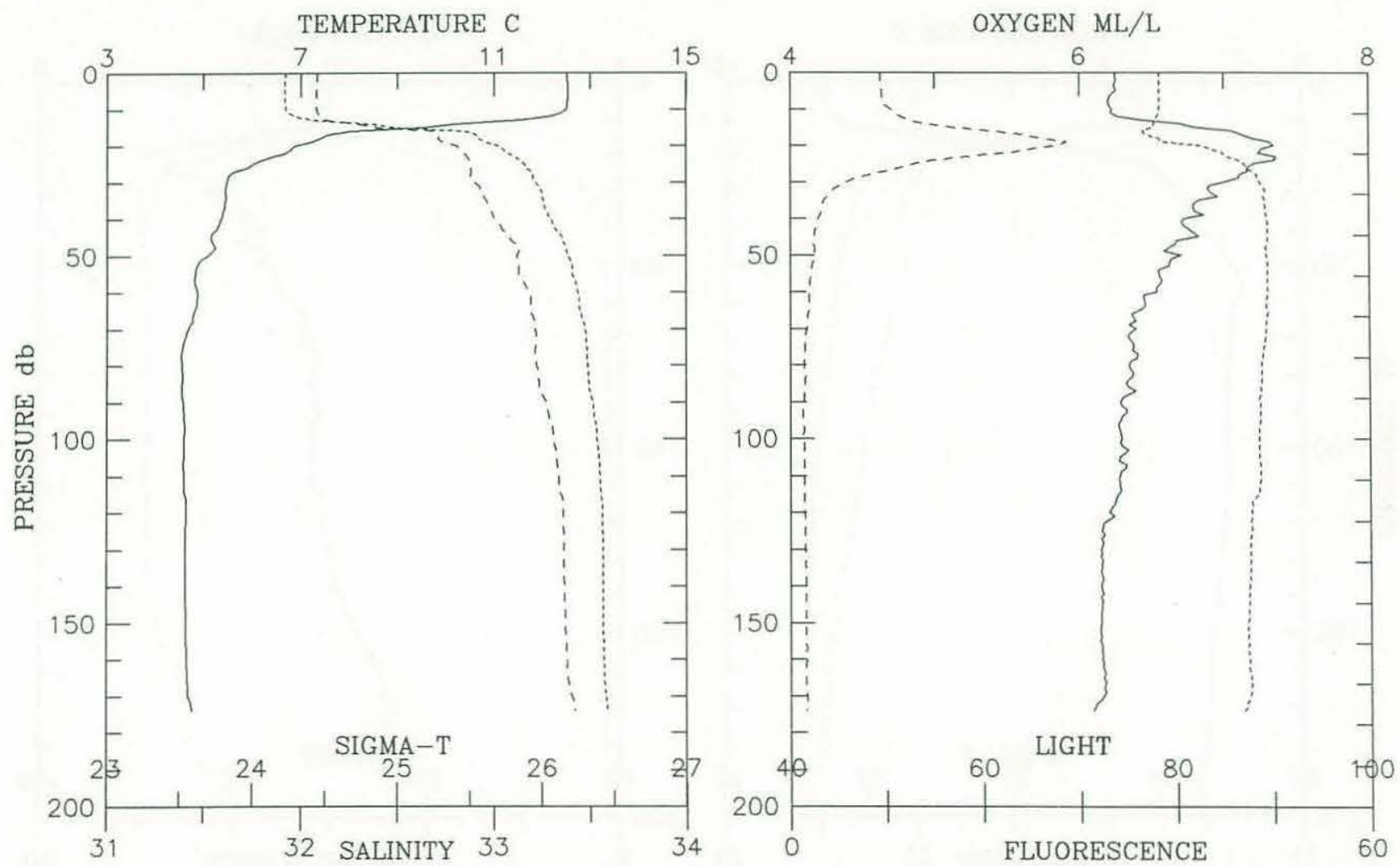


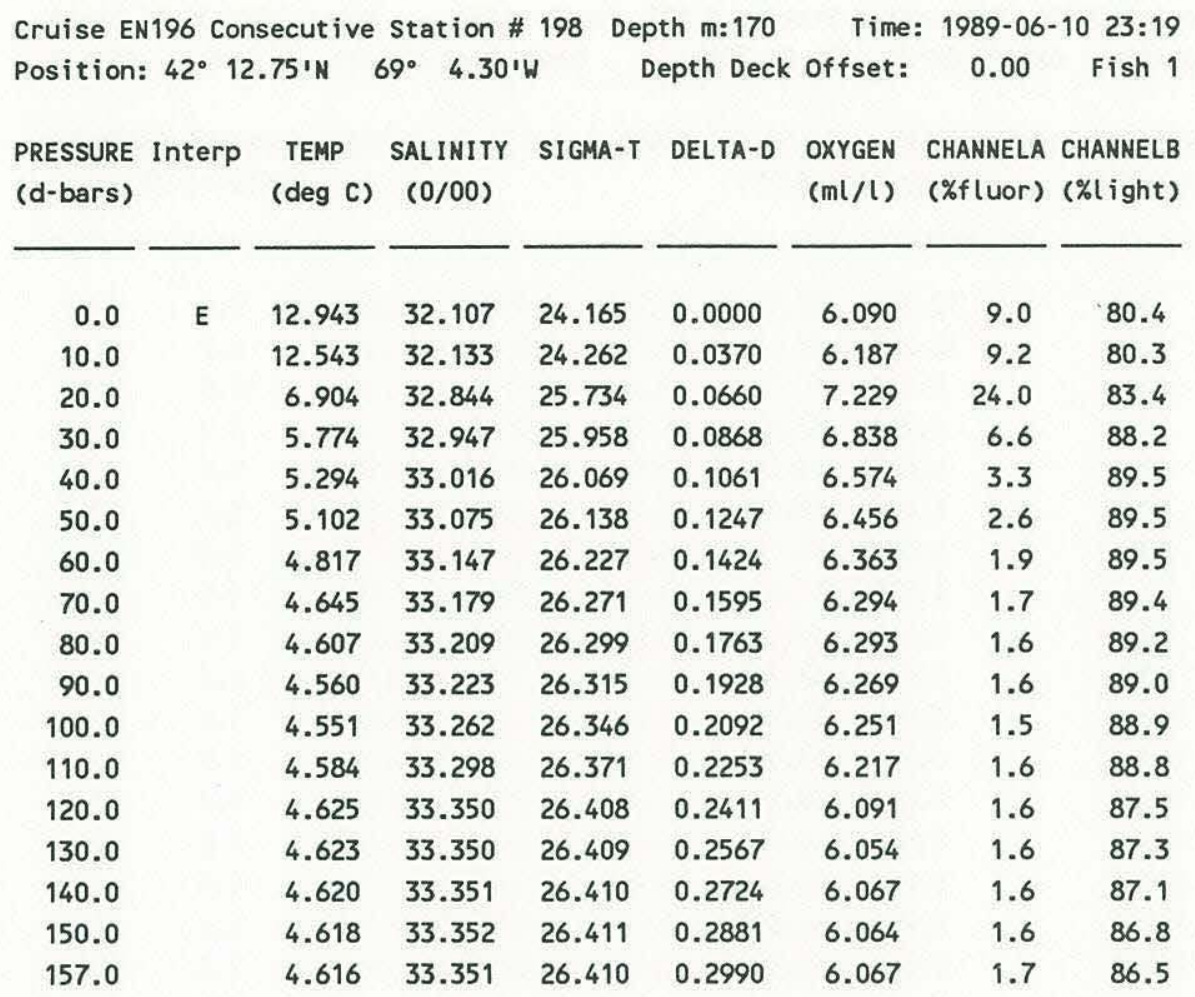
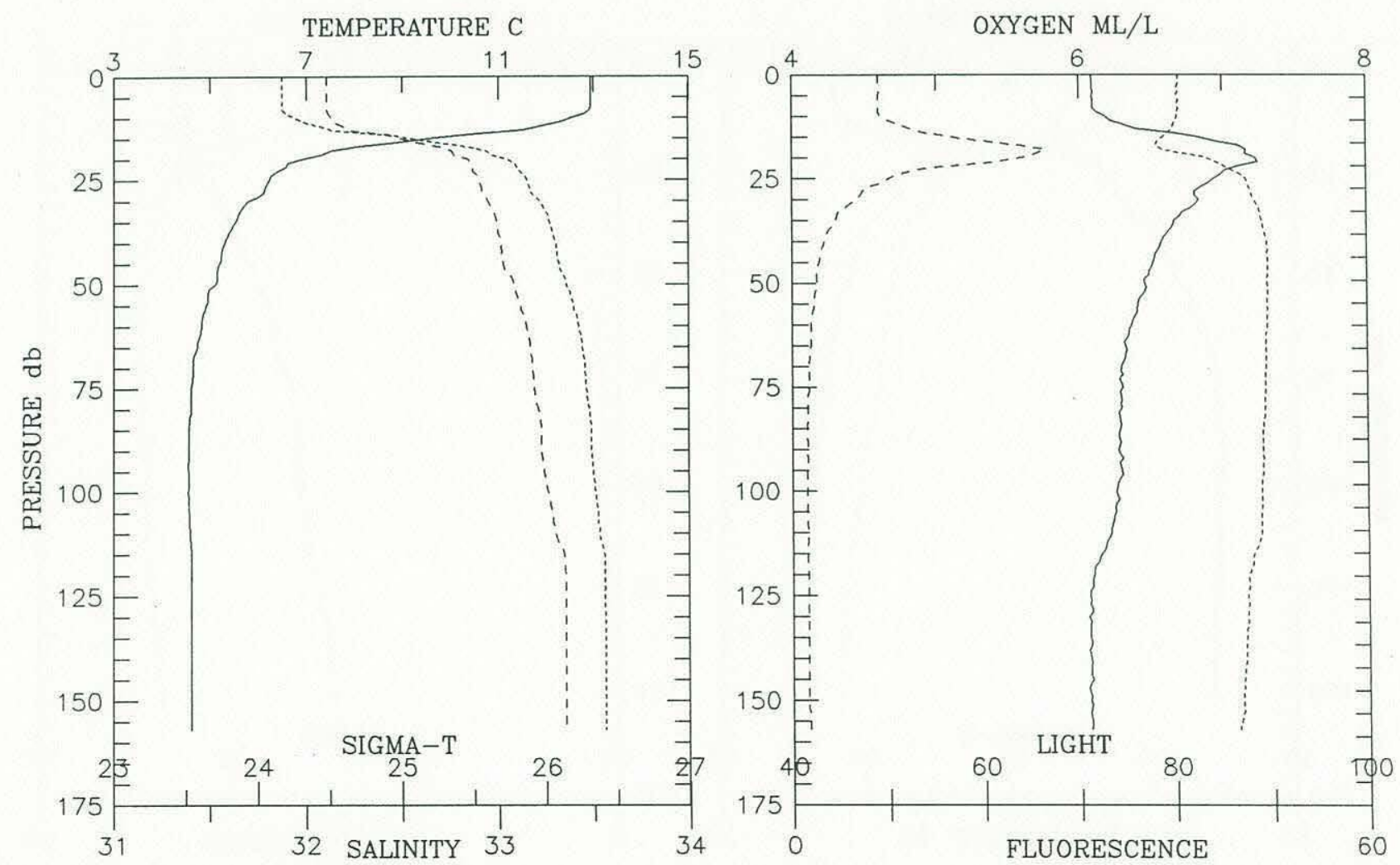


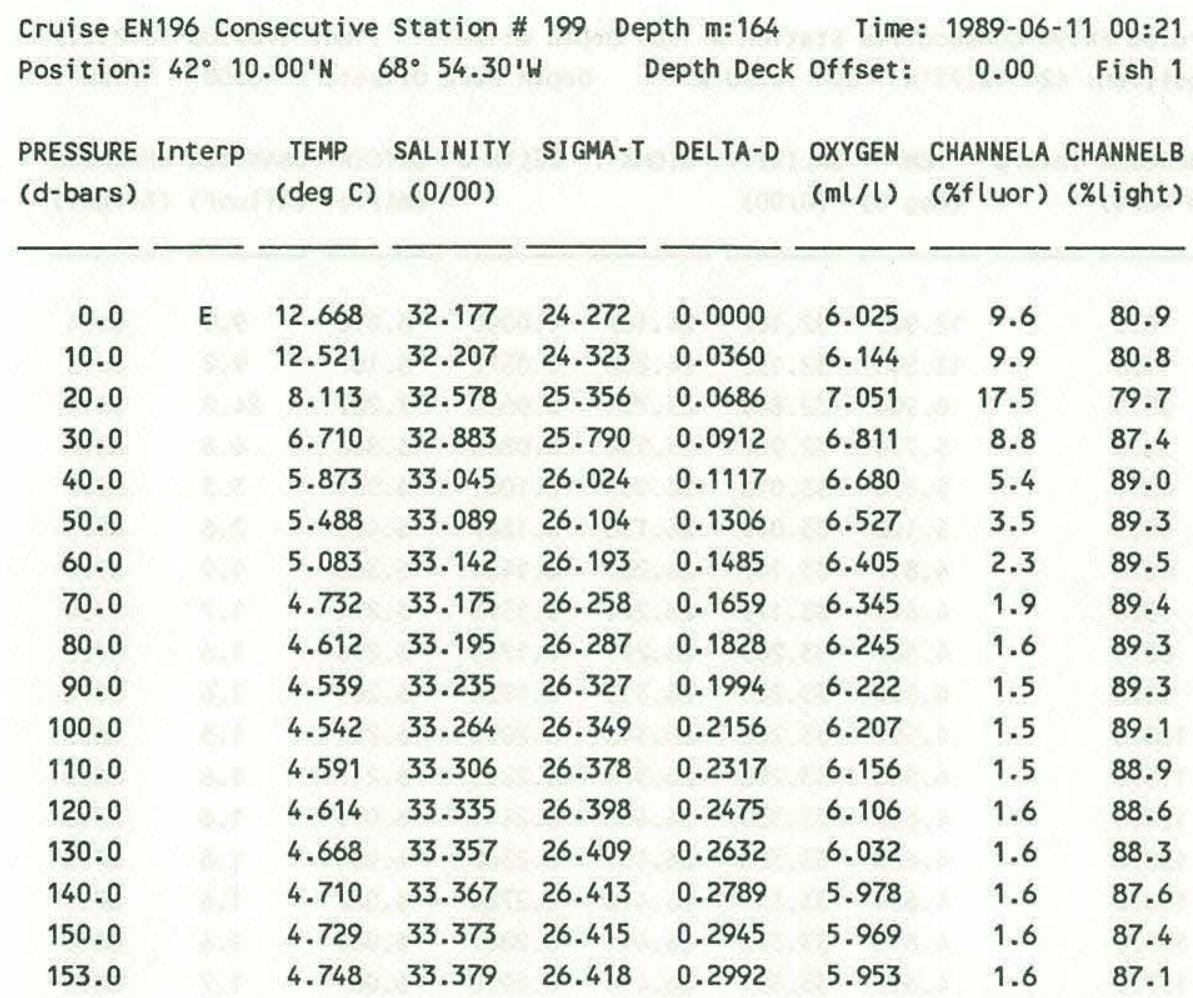
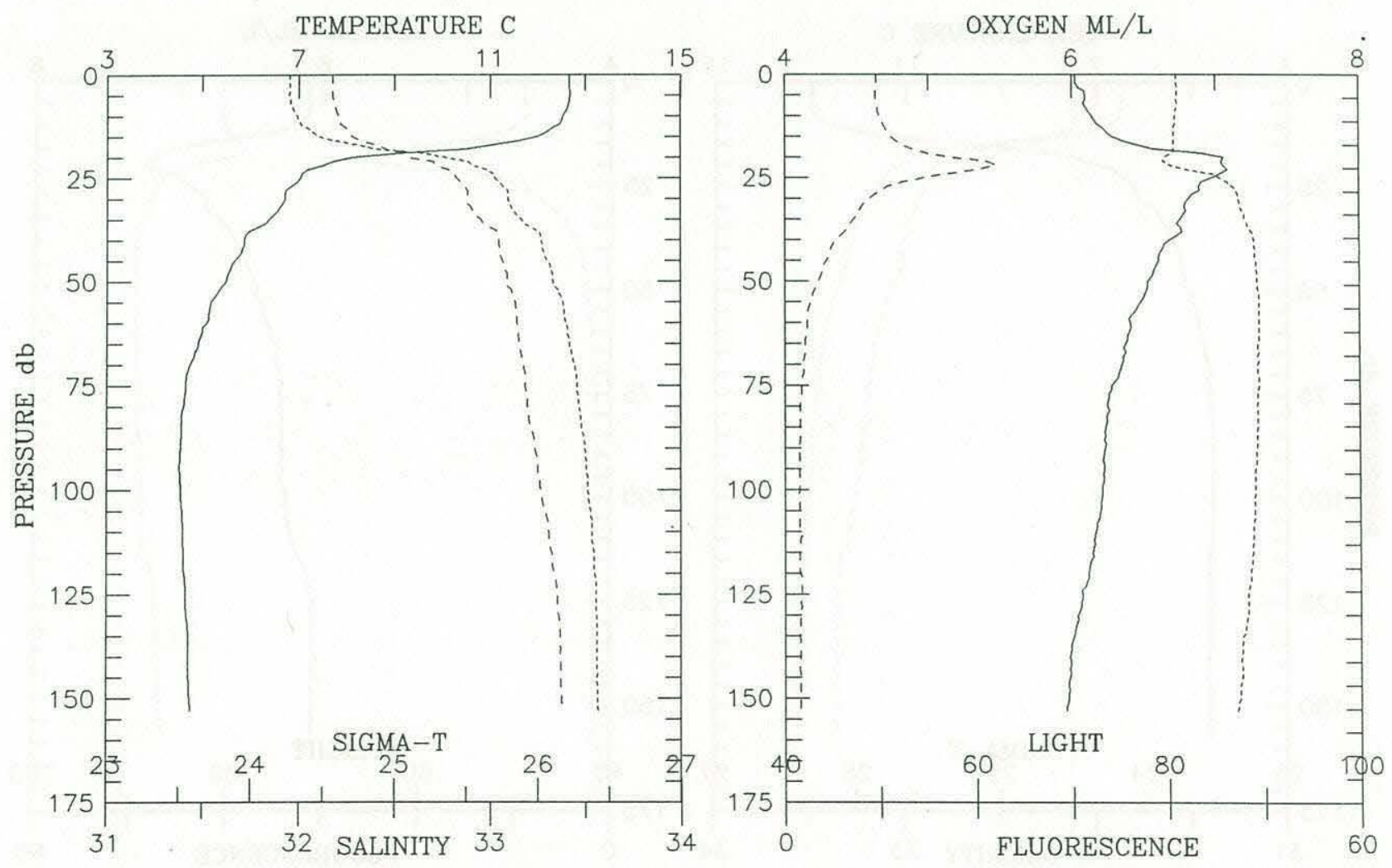
Cruise EN196 Consecutive Station \# 200 Depth m:183 Time: 1989-06-11 01:28 Position: $42^{\circ} 7.90^{\prime} \mathrm{N} 68^{\circ} 44.00^{\prime} \mathrm{W} \quad$ Depth Deck offset: $-0.10 \quad$ Fish 1

\begin{tabular}{|c|c|c|c|c|c|c|c|c|}
\hline $\begin{array}{l}\text { PRESSURE } \\
\text { (d-bars) }\end{array}$ & Interp & $\begin{array}{c}\text { TEMP } \\
(\operatorname{deg} \mathrm{C})\end{array}$ & $\begin{array}{l}\text { SALINITY } \\
(0 / 00)\end{array}$ & SI GMA-T & DELTA-D & $\begin{array}{l}\text { OXYGEN } \\
(\mathrm{ml} / \mathrm{l})\end{array}$ & $\begin{array}{l}\text { CHANNELA } \\
\text { (\%fluor) }\end{array}$ & $\begin{array}{l}\text { CHANNELB } \\
\text { (\%light) }\end{array}$ \\
\hline 0.0 & $E$ & 11.824 & 32.474 & 24.661 & 0.0000 & 6.124 & 9.5 & 82.4 \\
\hline 10.0 & & 11.810 & 32.472 & 24.662 & 0.0323 & 6.146 & 9.9 & 82.5 \\
\hline 20.0 & & 9.669 & 32.609 & 25.140 & 0.0639 & 6.701 & 13.3 & 81.6 \\
\hline 30.0 & & 6.903 & 32.927 & 25.800 & 0.0866 & 6.762 & 11.7 & 86.6 \\
\hline 40.0 & & 5.490 & 32.974 & 26.013 & 0.1075 & 6.959 & 7.8 & 88.7 \\
\hline 50.0 & & 5.070 & 33.041 & 26.115 & 0.1266 & 6.538 & 2.7 & 89.6 \\
\hline 60.0 & & 4.841 & 33.116 & 26.199 & 0.1445 & 6.360 & 2.0 & 89.5 \\
\hline 70.0 & & 4.965 & 33.185 & 26.241 & 0.1619 & 6.210 & 1.8 & 89.1 \\
\hline 80.0 & & 4.830 & 33.200 & 26.267 & 0.1790 & 6.159 & 1.7 & 89.1 \\
\hline 90.0 & & 4.627 & 33.252 & 26.331 & 0.1956 & 6.141 & 1.6 & 89.0 \\
\hline 100.0 & & 4.652 & 33.314 & 26.377 & 0.2117 & 6.094 & 1.6 & 88.9 \\
\hline 110.0 & & 4.731 & 33.368 & 26.412 & 0.2275 & 6.032 & 1.4 & 88.7 \\
\hline 120.0 & & 4.771 & 33.390 & 26.424 & 0.2430 & 5.960 & 1.4 & 88.5 \\
\hline 130.0 & & 4.807 & 33.404 & 26.431 & 0.2585 & 5.893 & 1.5 & 87.8 \\
\hline 140.0 & & 4.819 & 33.410 & 26.435 & 0.2740 & 5.873 & 1.5 & 87.9 \\
\hline 150.0 & & 4.826 & 33.415 & 26.438 & 0.2894 & 5.851 & 1.5 & 87.9 \\
\hline 160.0 & & 4.906 & 33.441 & 26.450 & 0.3048 & 5.800 & 1.5 & 87.3 \\
\hline 168.0 & & 4.954 & 33.456 & 26.457 & 0.3170 & 5.715 & 1.5 & 87.2 \\
\hline
\end{tabular}
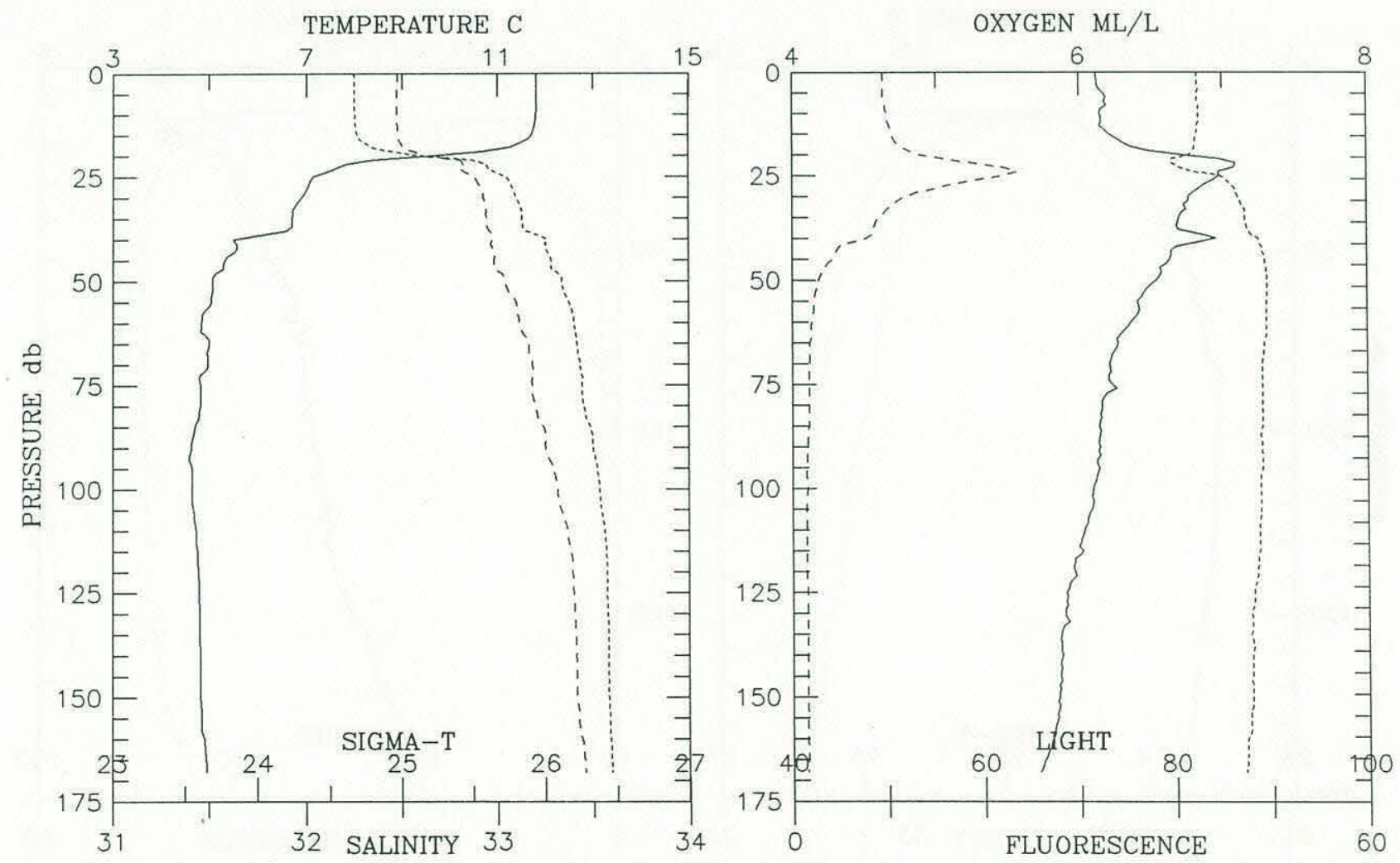


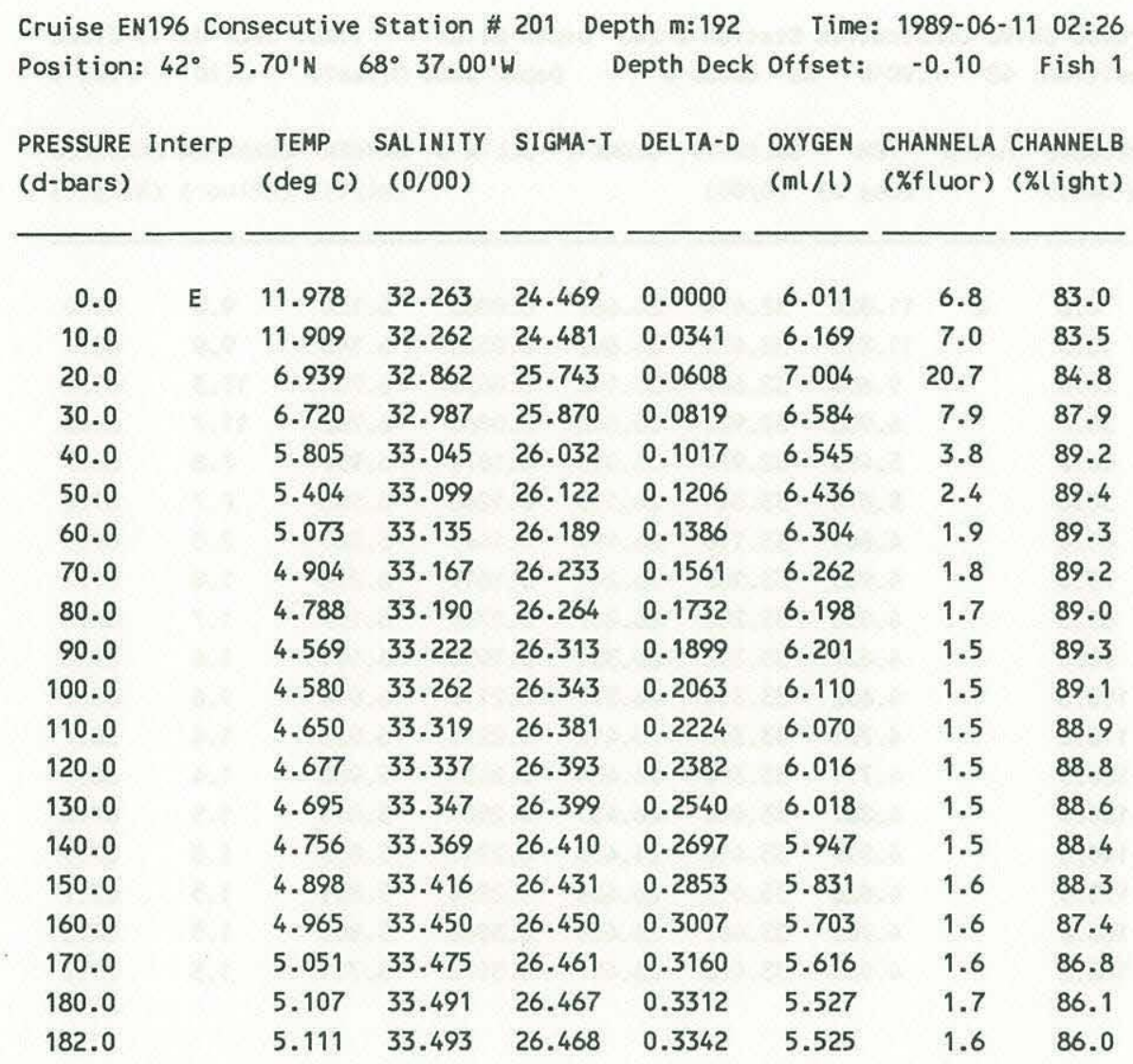
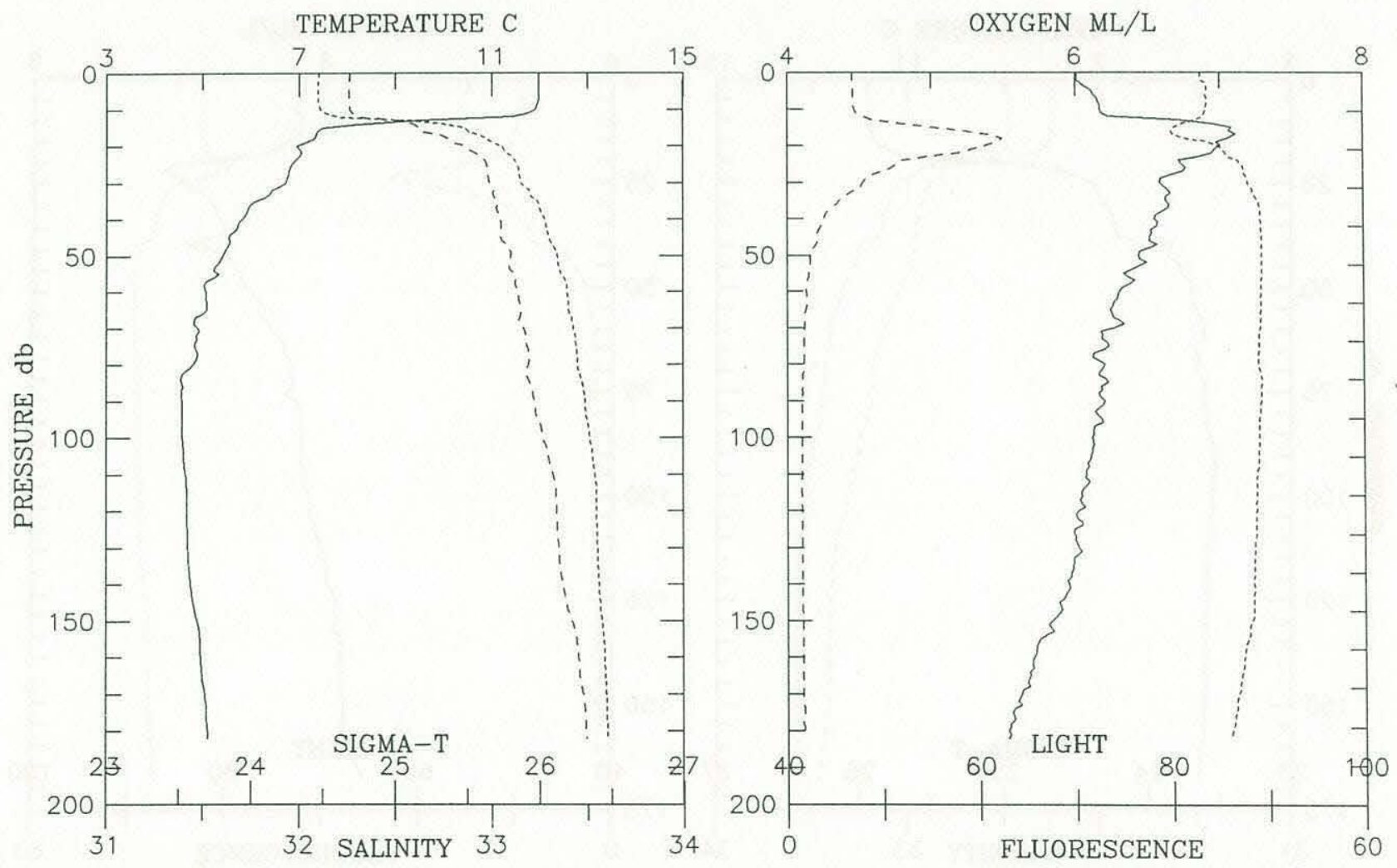


\begin{tabular}{|c|c|c|c|c|c|c|c|c|}
\hline Position: & $42^{\circ}$ & $.60^{\prime} \mathrm{N}$ & $68^{\circ} 28.20^{\prime}$ & & epth Deck & Offset: & -0.10 & Fish 1 \\
\hline $\begin{array}{l}\text { PRESSURE } \\
\text { (d-bars) }\end{array}$ & Interp & $\begin{array}{c}\text { TEMP } \\
\text { (deg C) }\end{array}$ & $\begin{array}{l}\text { SALINITY } \\
(0 / 00)\end{array}$ & SIGMA-T & DELTA-D & $\begin{array}{l}\text { OXYGEN } \\
(\mathrm{ml} / \mathrm{l})\end{array}$ & $\begin{array}{l}\text { CHANNELA } \\
\text { (\%fluor) }\end{array}$ & $\begin{array}{l}\text { CHANNELB } \\
\text { (\%light) }\end{array}$ \\
\hline 0.0 & $E$ & 11.745 & 32.171 & 24.440 & 0.0000 & 6.139 & 12.9 & 81.0 \\
\hline 10.0 & & 11.725 & 32.173 & 24.445 & 0.0344 & 6.189 & 13.6 & 81.0 \\
\hline 20.0 & & 10.955 & 32.242 & 24.637 & 0.0682 & 6.322 & 16.8 & 81.0 \\
\hline 30.0 & & 8.518 & 32.373 & 25.135 & 0.0992 & 6.765 & 20.7 & 81.9 \\
\hline 40.0 & & 7.065 & 32.606 & 25.525 & 0.1246 & 6.829 & 19.1 & 85.3 \\
\hline 50.0 & & 6.137 & 32.882 & 25.862 & 0.1470 & 6.654 & 7.0 & 88.5 \\
\hline 60.0 & & 5.749 & 32.996 & 26.000 & 0.1669 & 6.492 & 3.7 & 89.4 \\
\hline 70.0 & & 5.354 & 33.052 & 26.091 & 0.1858 & 6.374 & 2.7 & 89.6 \\
\hline 80.0 & & 4.976 & 33.110 & 26.180 & 0.2040 & 6.267 & 2.0 & 89.6 \\
\hline 90.0 & & 4.653 & 33.136 & 26.235 & 0.2215 & 6.226 & 1.6 & 89.6 \\
\hline 100.0 & & 4.540 & 33.167 & 26.273 . & 0.2386 & 6.160 & 1.6 & 89.2 \\
\hline 110.0 & & 4.543 & 33.207 & 26.304 & 0.2553 & 6.086 & 1.7 & 88.7 \\
\hline 120.0 & & 4.708 & 33.279 & 26.343 & 0.2718 & 5.946 & 1.6 & 88.3 \\
\hline 130.0 & & 5.008 & 33.428 & 26.428 & 0.2877 & 5.629 & 1.7 & 87.2 \\
\hline 140.0 & & 5.100 & 33.468 & 26.449 & 0.3031 & 5.437 & 1.7 & 86.2 \\
\hline 150.0 & & 5.171 & 33.497 & 26.464 & 0.3183 & 5.366 & 1.7 & 85.9 \\
\hline 160.0 & & 5.362 & 33.582 & 26.509 & 0.3333 & 5.219 & 1.8 & 84.4 \\
\hline
\end{tabular}
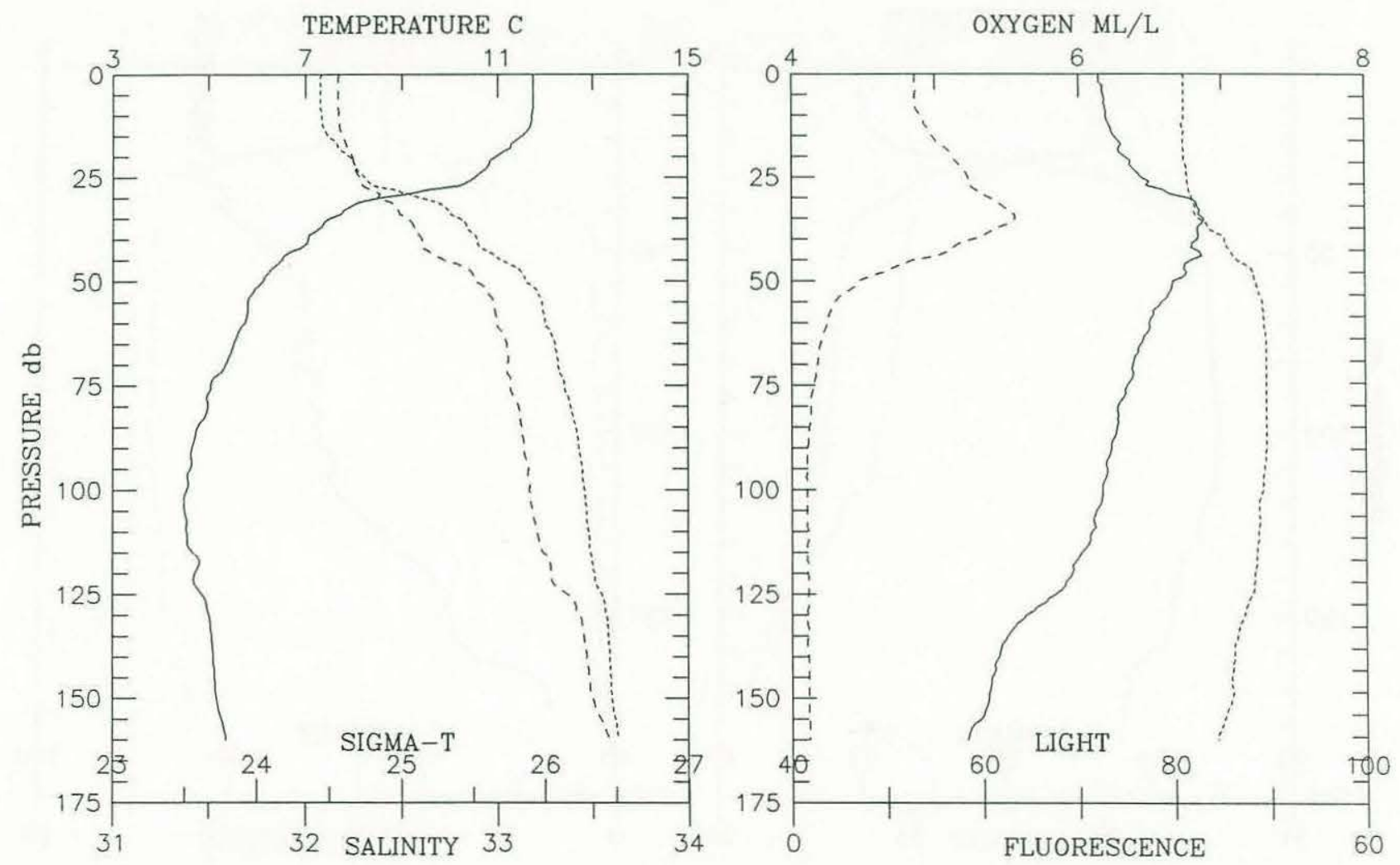


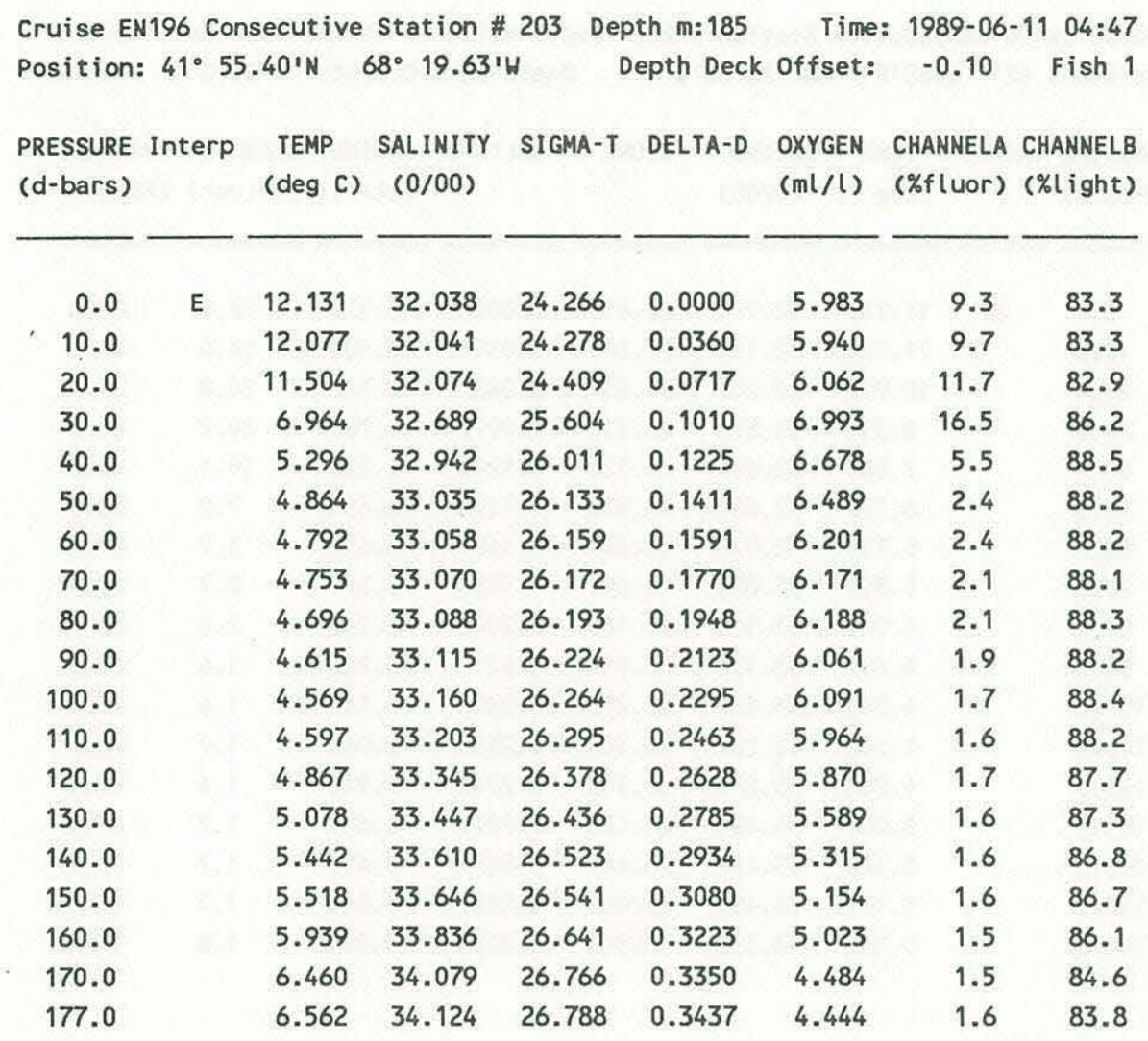
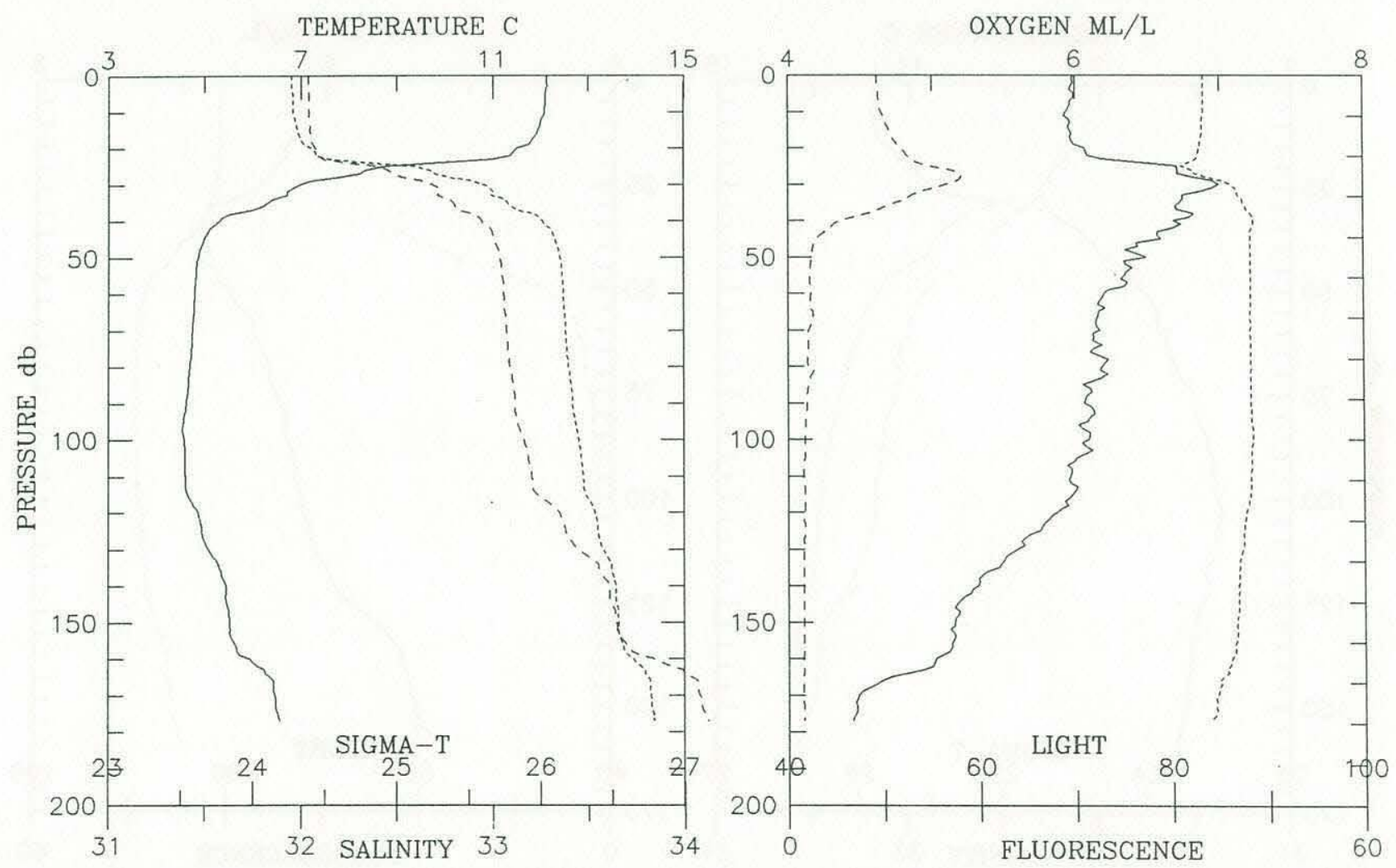


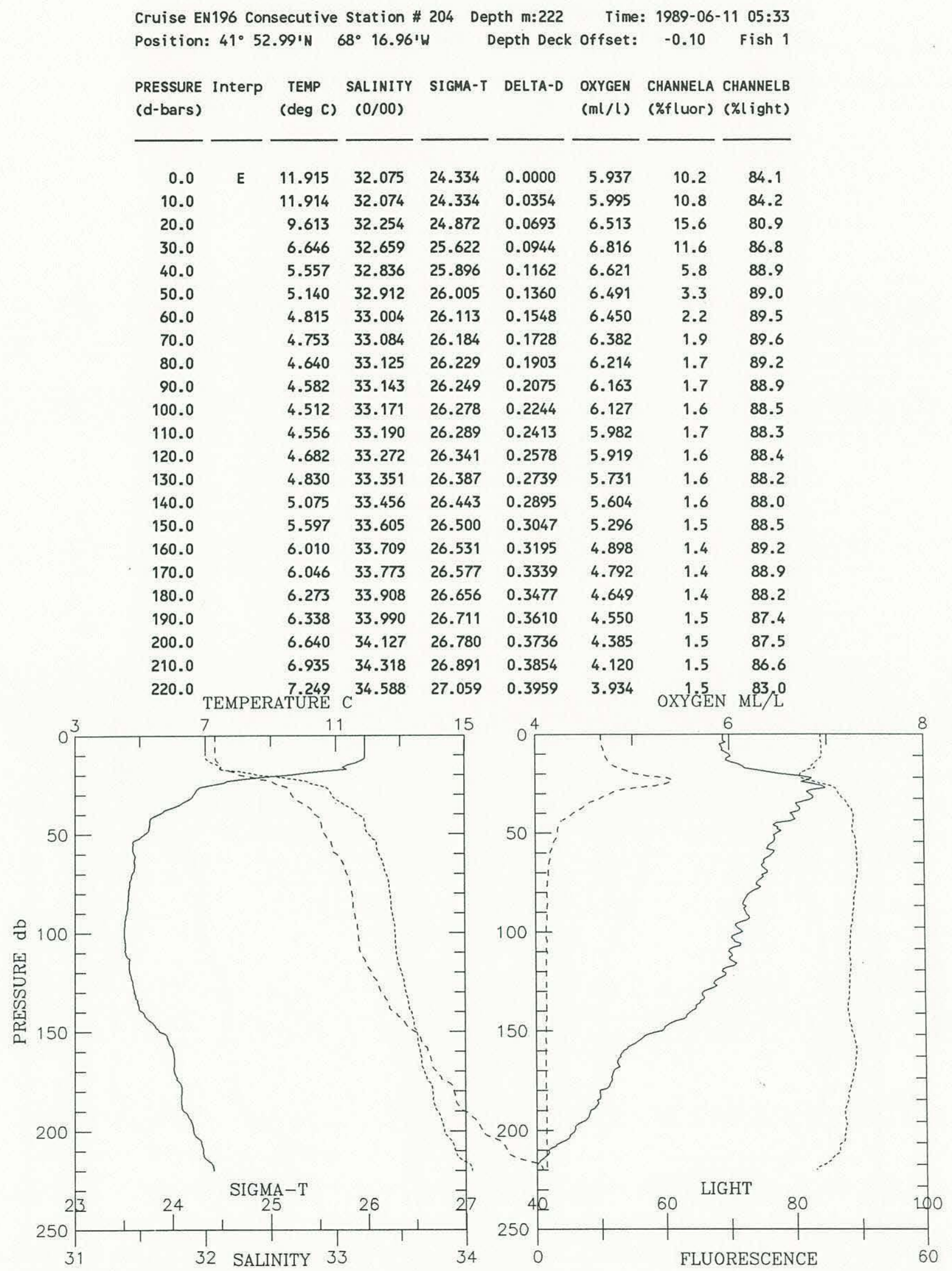




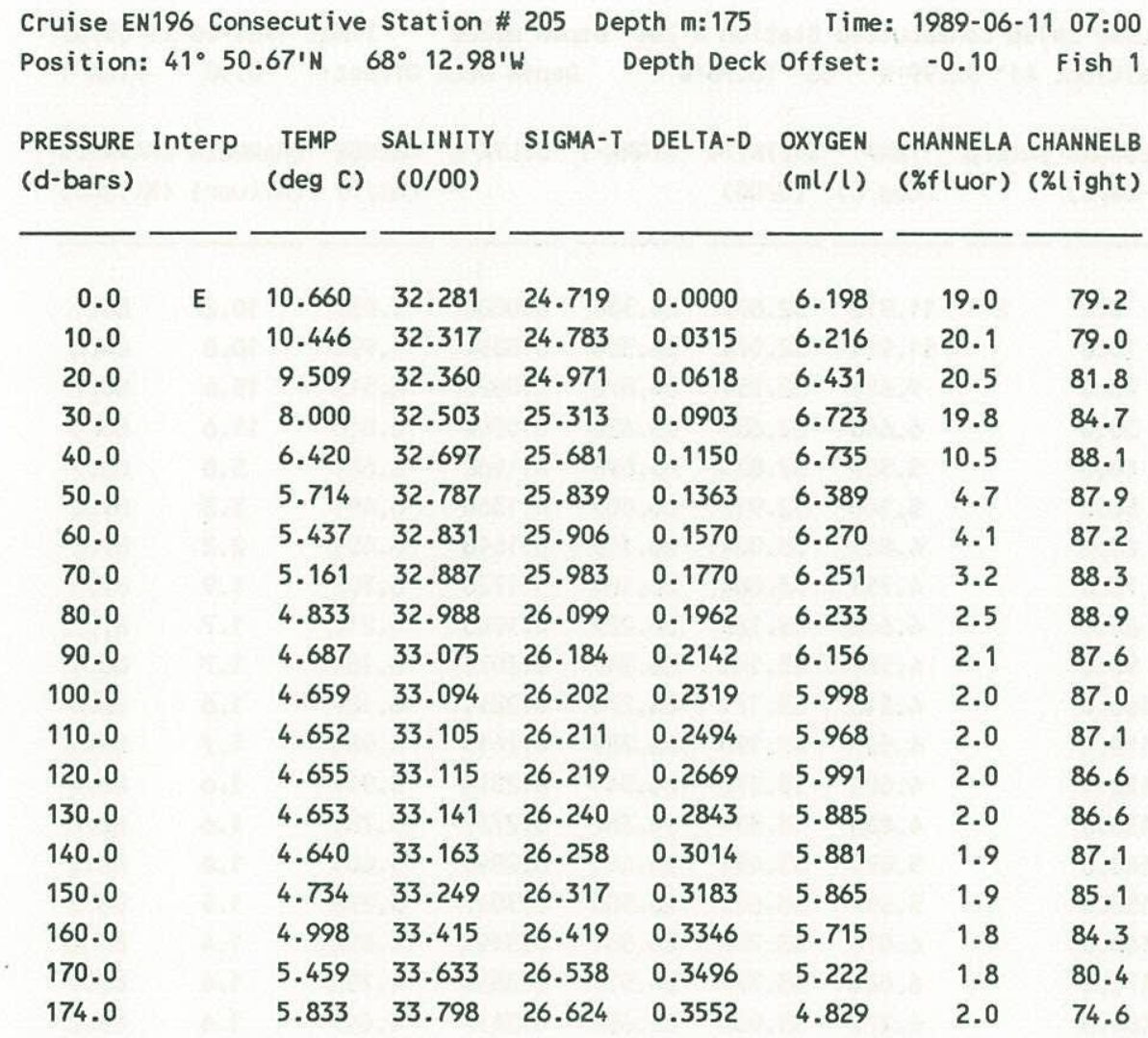
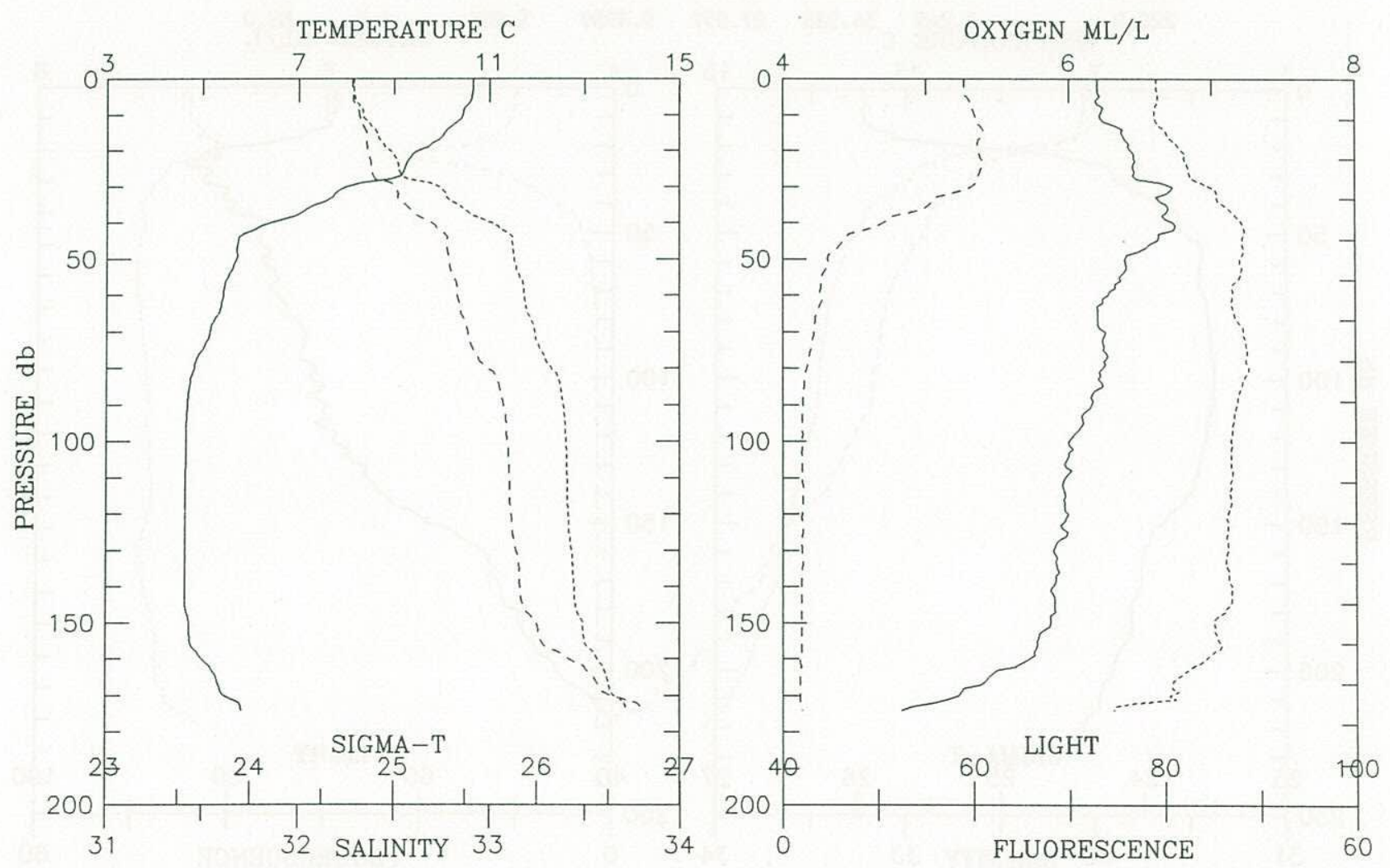


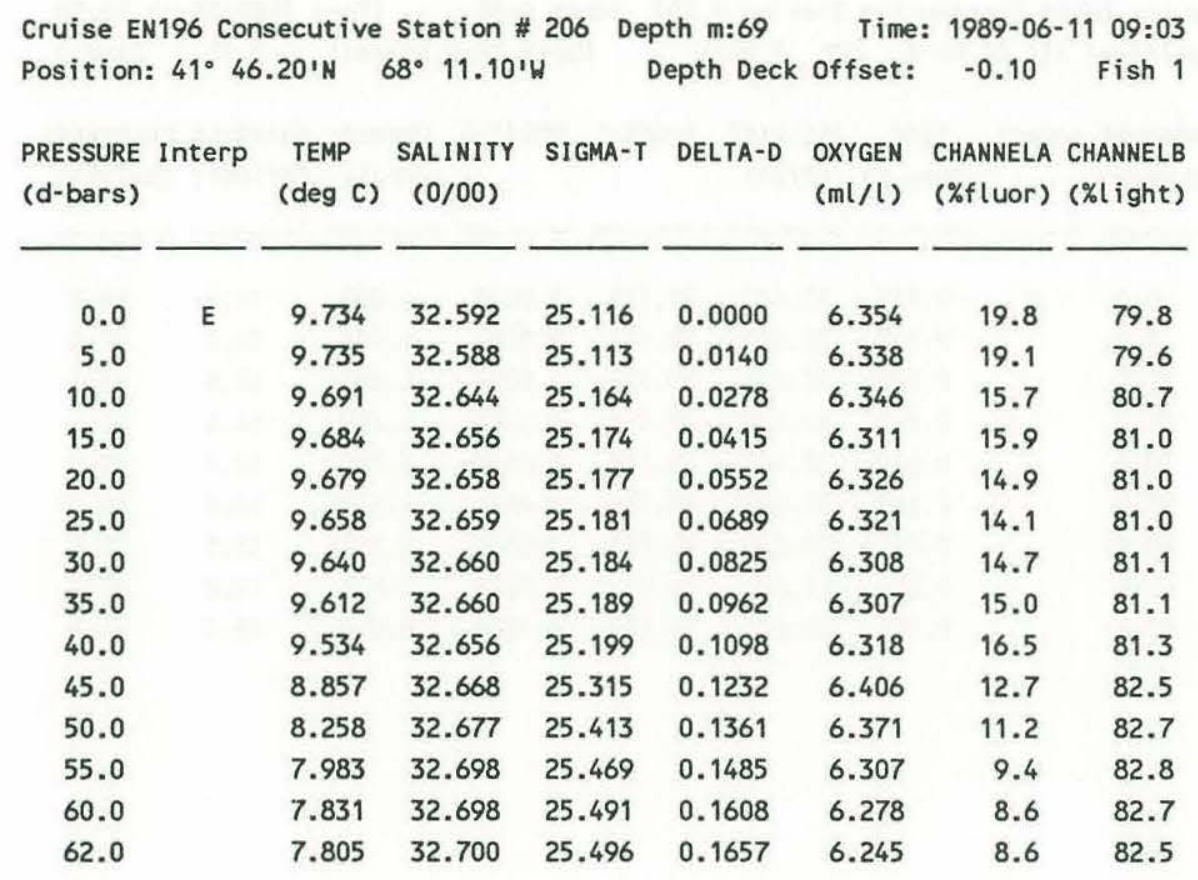
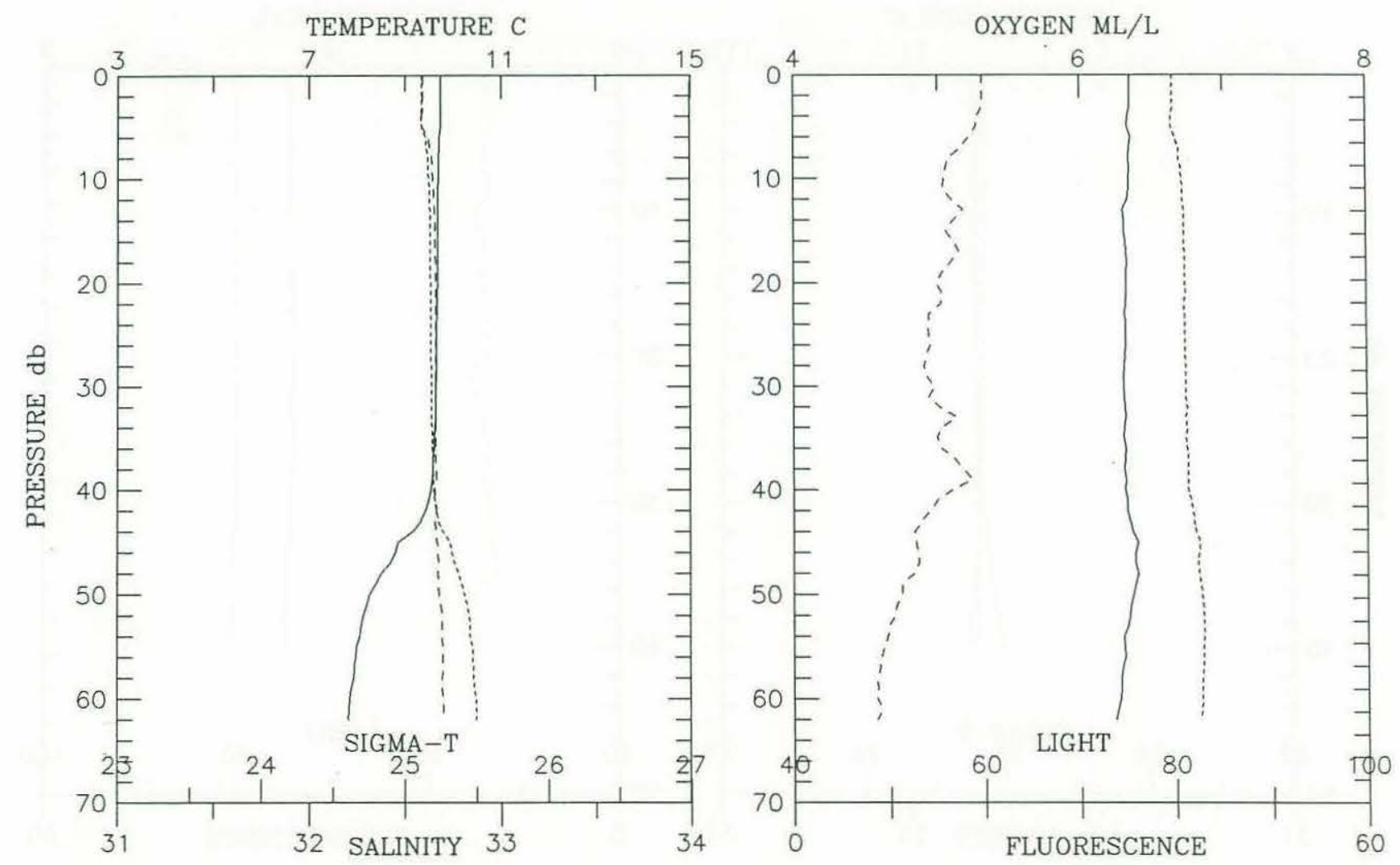


\begin{tabular}{|c|c|c|c|c|c|c|c|c|}
\hline \multicolumn{5}{|c|}{ Cruise EN196 Consecutive Station \# 207} & epth $m: 50$ & Time: & \multicolumn{2}{|c|}{ : $1989-06-11 \quad 09: 58$} \\
\hline Position: & $: 41^{\circ} 44$ & .00 'N 6 & $68^{\circ} 8.20^{\prime}$ & & epth Deck & offset: & -0.10 & Fish 1 \\
\hline $\begin{array}{l}\text { PRESSURE } \\
\text { (d-bars) }\end{array}$ & Interp & $\begin{array}{l}\text { TEMP } \\
(\operatorname{deg} \mathrm{C})\end{array}$ & $\begin{array}{l}\text { SALINITY } \\
(0 / 00)\end{array}$ & SIGMA-T & DELTA-D & $\begin{array}{l}\text { OXYGEN } \\
(\mathrm{mL} / \mathrm{l})\end{array}$ & $\begin{array}{l}\text { CHANNELA } \\
\text { (\%fluor) }\end{array}$ & $\begin{array}{l}\text { CHANNELB } \\
\text { (\%tight) }\end{array}$ \\
\hline 0.0 & E & 9.732 & 32.667 & 25.175 & 0.0000 & 6.308 & 14.0 & 80.3 \\
\hline 5.0 & & 9.733 & 32.665 & 25.173 & 0.0137 & 6.300 & 14.3 & 80.2 \\
\hline 10.0 & & 9.730 & 32.666 & 25.174 & 0.0274 & 6.295 & 15.3 & 80.4 \\
\hline 15.0 & & 9.715 & 32.667 & 25.178 & 0.0411 & 6.289 & 14.4 & 80.1 \\
\hline 20.0 & & 9.668 & 32.665 & 25.184 & 0.0547 & 6.285 & 13.7 & 80.2 \\
\hline 25.0 & & 9.620 & 32.661 & 25.189 & 0.0684 & 6.269 & 14.0 & 80.1 \\
\hline 30.0 & & 9.524 & 32.661 & 25.204 & 0.0820 & 6.287 & 13.5 & 80.0 \\
\hline 35.0 & & 9.333 & 32.658 & 25.232 & 0.0955 & 6.307 & 12.9 & 80.3 \\
\hline 40.0 & & 9.105 & 32.661 & 25.271 & 0.1088 & 6.319 & 15.4 & 80.4 \\
\hline
\end{tabular}
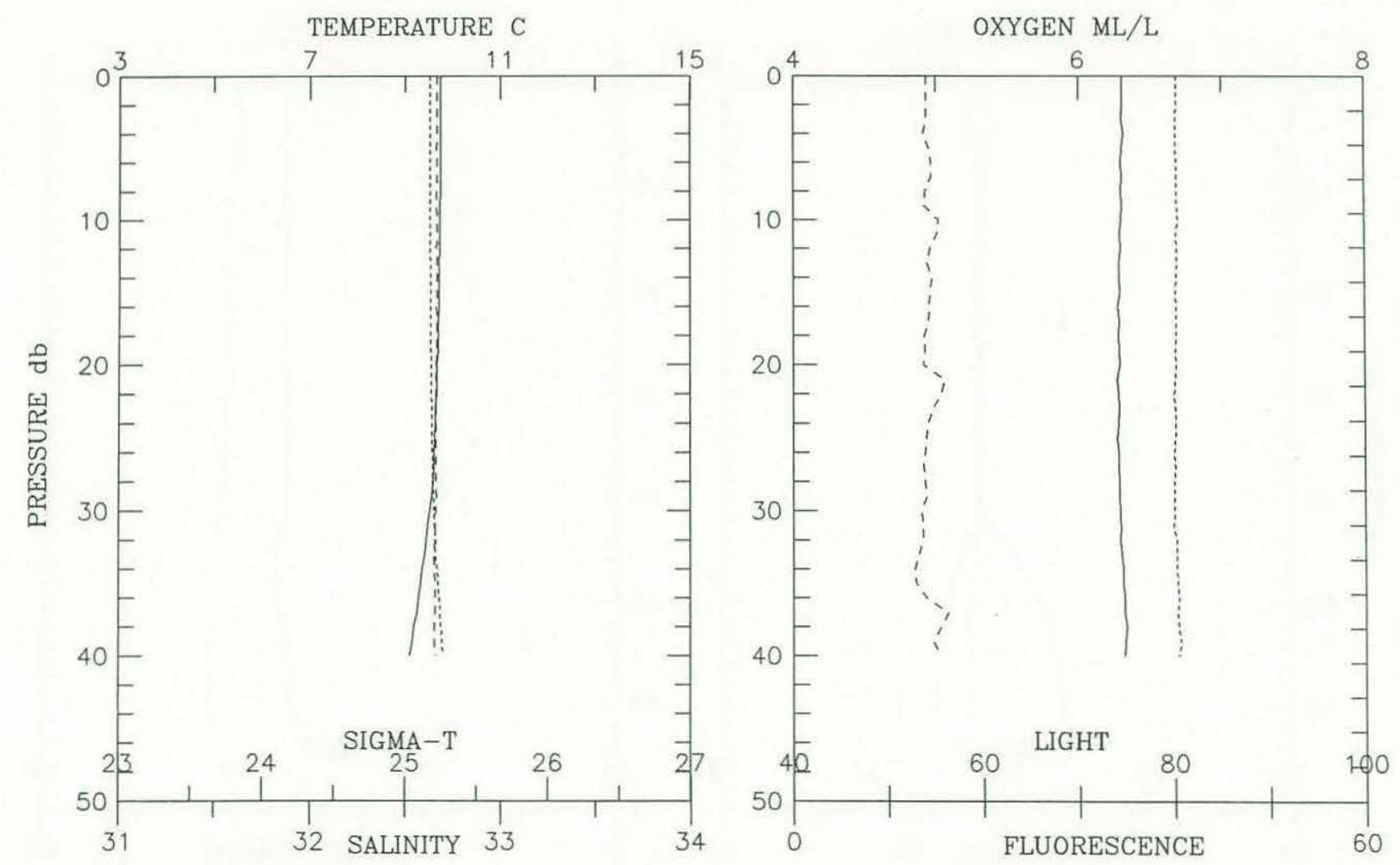
DOCUMENT LIBRARY

July 5, 1989

Distribution List for Technical Report Exchange

Attn: Stella Sanchez-Wade

Documents Section

Scripps Institution of Oceanography

Library, Mail Code C-075C

La Jolla, CA 92093

Hancock Library of Biology \& Oceanography

Alan Hancock Laboratory

University of Southern California

University Park

Los Angeles, CA 90089-0371

Gifts \& Exchanges

Library

Bedford Institute of Oceanography

P.O. Box 1006

Dartmouth, NS, B2Y 4A2, CANADA

Office of the International

Ice Patrol

c/o Coast Guard R \& D Center

Avery Point

Groton, CT 06340

\section{Library}

Physical Oceanographic Laboratory

Nova University

8000 N. Ocean Drive

Dania, FL 33304

NOAA/EDIS Miami Library Center 4301 Rickenbacker Causeway

Miami, FL 33149

\section{Library}

Skidaway Institute of Oceanography

P.O. Box 13687

Savannah, GA 31416

Institute of Geophysics

University of Hawaii

Library Room 252

2525 Correa Road

Honolulu, HI 96822

Library

Chesapeake Bay Institute

4800 Atwell Road

Shady Side, MD 20876

MIT Libraries

Serial Journal Room 14E-210

Cambridge, MA 02139
Director, Ralph M. Parsons Laboratory

Room 48-311

MIT

Cambridge, MA 02139

Marine Resources Information Center

Building E38-320

MIT

Cambridge, MA 02139

Library

Lamont-Doherty Geological

Observatory

Colombia University

Palisades, NY 10964

Library

Serials Department

Oregon State University

Corvallis, OR 97331

Pell Marine Science Library

University of Rhode Island

Narragansett Bay Campus

Narragansett, RI 02882

Working Collection

Texas A\&M University

Dept. of Oceanography

College Station, TX 77843

Library

Virginia Institute of Marine Science

Gloucester Point, VA 23062

Fisheries-Oceanography Library

151 Oceanography Teaching Bldg.

University of Washington

Seattle, WA 98195

Library

R.S.M.A.S.

University of Miami

4600 Rickenbacker Causeway

Miami, FL 33149

Maury Oceanographic Library

Naval Oceanographic Office

Bay St. Louis

NSTL, MS 39522-5001

Marine Sciences Collection

Mayaguez Campus Library

University of Puerto Rico

Mayagues, Puerto Rico 00708 


\begin{tabular}{|c|c|c|c|}
\hline $\begin{array}{l}\text { REPORT DOCUMENTATION } \\
\text { PAGE }\end{array}$ & $\begin{array}{l}\text { 1. REPORT NO. } \\
\text { WHOI-89-51 }\end{array}$ & 2. & 3. Recipient's Accession No. \\
\hline \multirow{2}{*}{\multicolumn{3}{|c|}{$\begin{array}{l}\text { 4. Title and Subtitle } \\
\text { CTD Observations in the Great South Channel During the South Channel Ocean Productivity } \\
\text { EXperiment, SCOPEX, May - June } 1989\end{array}$}} & $\begin{array}{l}\text { 5. Report Date } \\
\text { November, } 1989\end{array}$ \\
\hline & & & 6. \\
\hline \multicolumn{3}{|c|}{$\begin{array}{l}\text { 7. Author(s) } \\
\text { Richard Limeburner and Robert C. Beardsley }\end{array}$} & $\begin{array}{l}\text { 8. Performing Organization Rept. No. } \\
\text { WHOI-89-51 }\end{array}$ \\
\hline \multirow{2}{*}{\multicolumn{3}{|c|}{$\begin{array}{l}\text { 9. Performing Organization Name and Address } \\
\text { The Woods Hole Oceanographic Institution } \\
\text { Woods Hole, Massachusetts } 02543\end{array}$}} & 10. Project/Task/Work Unit No. \\
\hline & & & $\begin{array}{l}\text { 11. Contract(C) or Grant(G) No. } \\
\text { (C) } \\
\text { (G) OCE } 87-13988\end{array}$ \\
\hline \multirow{2}{*}{\multicolumn{3}{|c|}{$\begin{array}{l}\text { 12. Sponsoring Organization Name and Address } \\
\text { Funding was provided by the National Science Foundation }\end{array}$}} & $\begin{array}{l}\text { 13. Type of Report \& Period Covered } \\
\text { Technical Report }\end{array}$ \\
\hline & & & 14. \\
\hline
\end{tabular}

15. Supplementary Notes

This report should be cited as: Woods Hole Oceanog. Inst. Tech. Rept., WHOI-89-51.

16. Abstract (Limit: 200 words)

CTD and acoustic Doppler current profiler (ADCP) observations were made in the Great South Channel (GSC) off the New England coast during R/V Endeavor cruise EN196 May 18 to June 12, 1989 as part of the South Channel Ocean Productivity EXperiment (SCOPEX). These observations were obtained using several sampling plans -a series of small-scale surveys in support of biological sampling and a large-scale survey of five cross-channel transects extending from Nantucket Shoals and the coast of Cape Cod to Georges Bank. The maximum sampling depth at each station was within a few meters of the bottom.

The primary objectives of the hydrographic measurement program were to (a) observe and characterize the temperature, salinity, density, oxygen, fluorescence and light transmission fields and their spatial variability in the Great South Channel off the New England coast, (b) resolve the low salinity surface plume-like structure usually observed east of Cape Cod in late spring, (c) define the front or boundary between the vertically well-mixed water over Nantucket Shoals, the GSC, Georges Bank, and the stratified water in the deeper southwestern Gulf of Maine, and (d) characterize water properties in regions of enhanced biological productivity. This report presents a summary in graphic and tabular form of the hydrographic observations made during cruise EN196 on the R/V Endeavor.

\section{Document Analysis a. Descriptors}

1. hydrography/CTD

2. Great South Channel, Gulf of Maine, Nantucket Shoals, Georges Bank

3. SCOPEX (South Channel Ocean Productivity EXperiment)

b. Identifiers/Open-Ended Terms

\section{c. COSATI Field/Group}

18. Availability Statement

Approved for publication; distribution unlimited.

\begin{tabular}{|l|l|}
\hline $\begin{array}{c}\text { 19. Security Class (This Report) } \\
\text { UNCLASSIFIED }\end{array}$ & $\begin{array}{c}\text { 21. No. of Pages } \\
252\end{array}$ \\
\hline 20. Security Class (This Page) & 22. Price \\
\hline
\end{tabular}

